


\title{
Evaluation of the NASA \\ Langley Research Center \\ Mode-Stirred Chamber Facility
}

\author{
John Ladbury \\ Galen Koepke \\ Dennis Camell
}

Radio-Frequency Technology Division

Electronics and Electrical Engineering Laboratory

National Institute of Standards and Technology

325 Broadway

Boulder, Colorado 80303-3328

January 1999

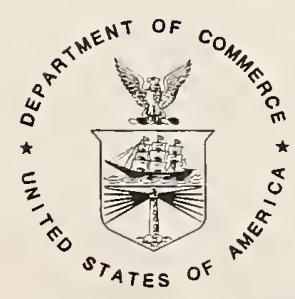

U.S. DEPARTMENT OF COMMERCE, William M. Daley, Secretary 
Nationa! Institute of Standards and Technology Technical Note Natl. Inst. Stand. Technol., Tech. Note 1508, 288 pages (January 1999) CODEN:NTNOEF

\section{U.S. GOVERNMENT PRINTING OFFICE \\ WASHINGTON: 1999}

For sale by the Superintendent of Documents, U.S. Government Printing Office, Washington, DC 20402-9325 


\section{CONTENTS}

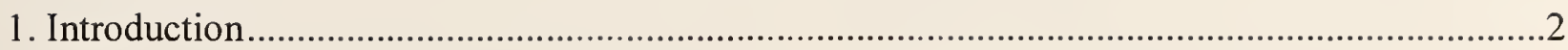

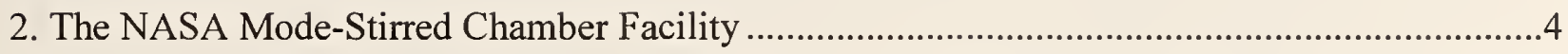

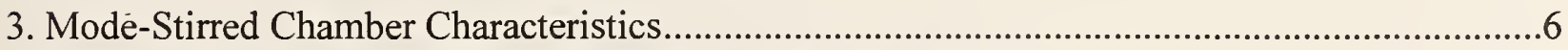

3.1 Electromagnetic Fields in a Mode-Stirred Chamber....................................................10

3.1.1 Mechanisms of Power Loss in a Mode-Stirred Chamber …….....................................14

3.2 Statistical Description of Mode-Stirred Chambers ....................................................18

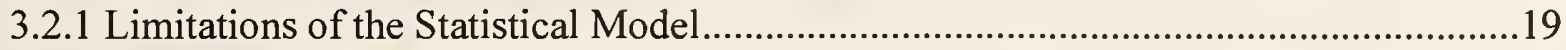

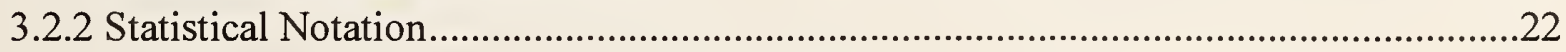

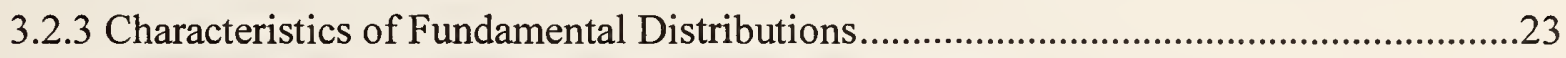

3.2.4 Characteristics of Distributions Expressed in Decibels ....................................................29

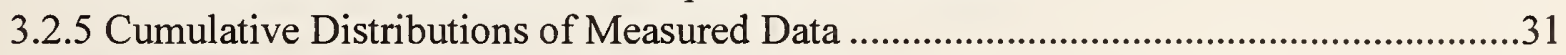

3.2.6 Extreme Values of Fundamental Distributions ..............................................................33

3.2.7 Ideal Models Applied to Mode-Stirred Chambers ..........................................................52

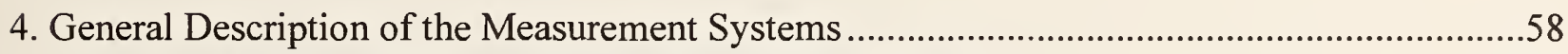

4.1 Power Meters and Isotropic Probes (The Probe System) ....................................................59

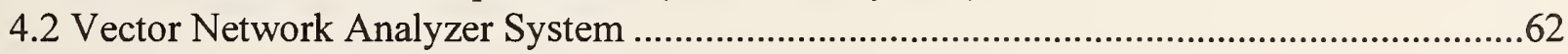

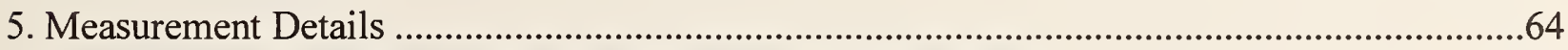

5.1 Selection of Paddle Positions and Number of Paddle Steps ..................................................68

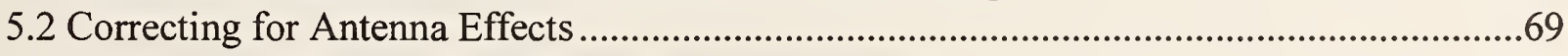

5.2.1 Antenna Effects in Free-Space Measurements ..........................................................69

5.2.2 Antenna Effects in Mode-Stirred Chambers .................................................................71

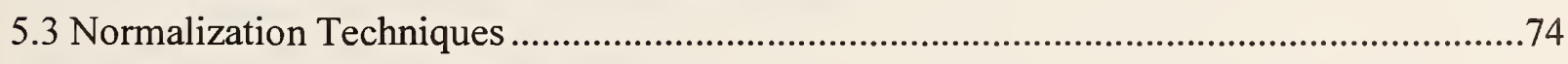

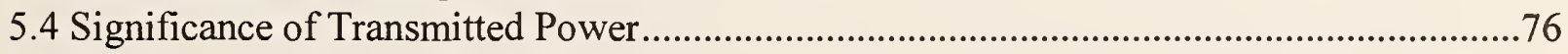

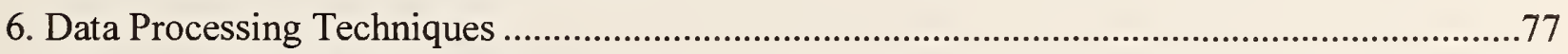

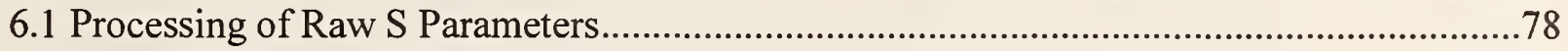

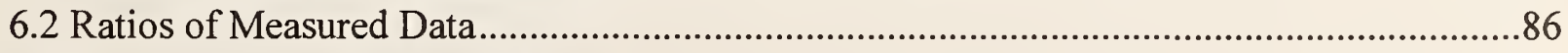

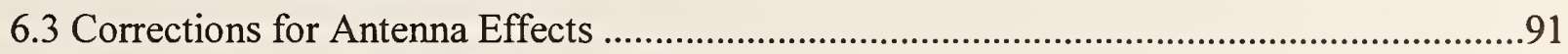

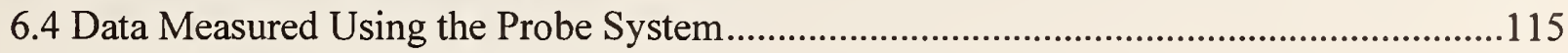

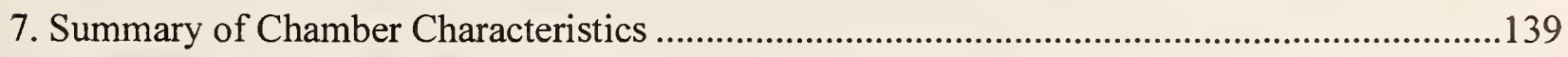

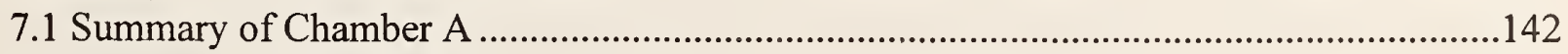

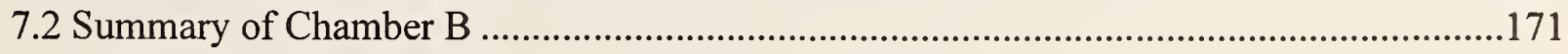

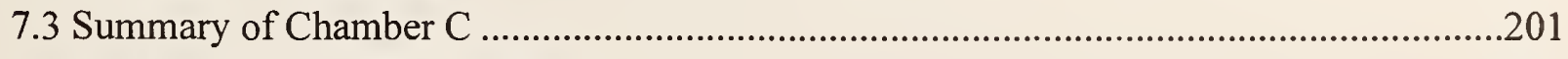

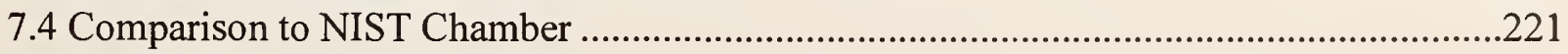

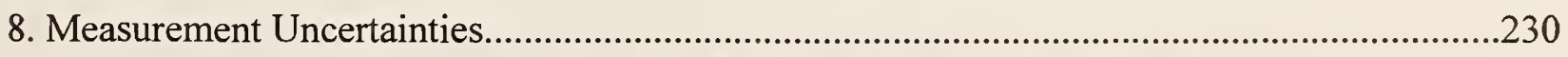

8.1 Presentation of Uncertainties and Uncertainties about Uncertainties ...............................231

8.2 Uncertainty due to Random Nature of Mode-Stirred Chamber Measurements..................234 


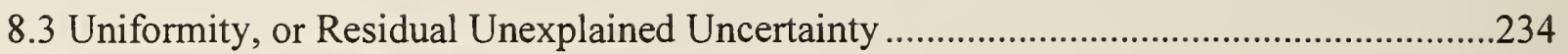

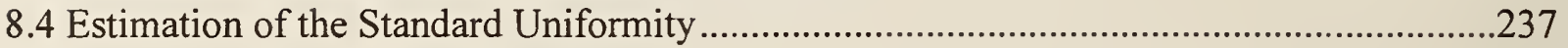

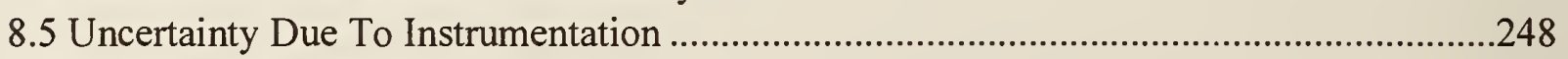

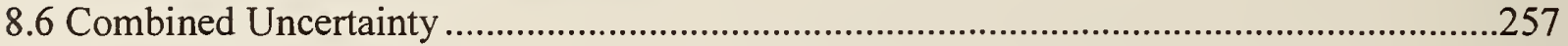

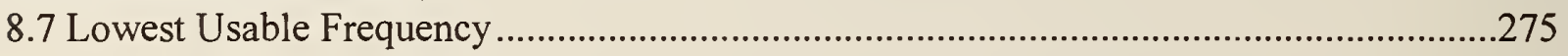

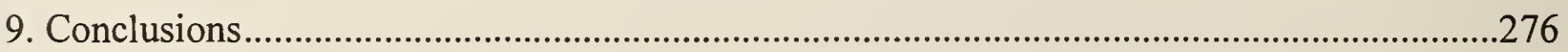

9.1 Electromagnetic Theory .................................................................................................276

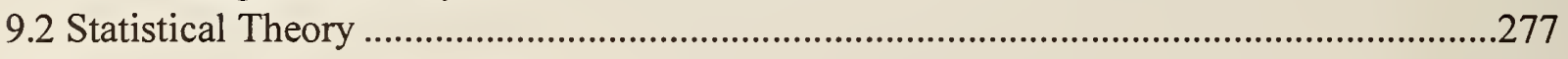

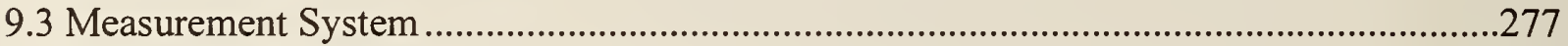

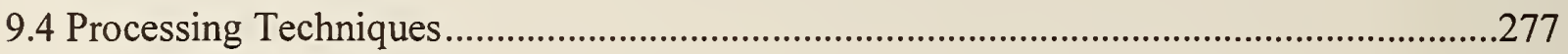

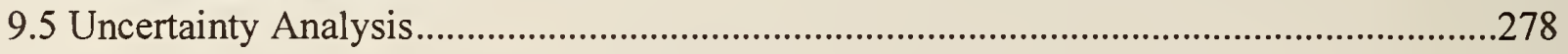

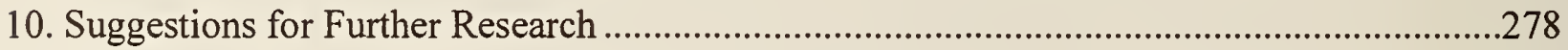

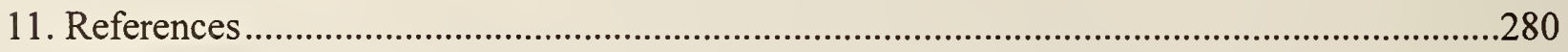




\title{
Evaluation of the NASA Langley Research Center Mode-Stirred Chamber Facility
}

\author{
J. Ladbury, G. Koepke, D. Camell \\ Radio-Frequency Technology Division \\ Electronics and Electrical Engineering Laboratory \\ National Institute of Standards and Technology \\ 325 Broadway \\ Boulder, Colorado 80303-3328
}

We performed extensive tests on three mode-stirred (reverberation) chambers operated by the High Intensity Radiation Laboratory located at the National Aeronautics and Space Administration Langley Research Center (NASA/LaRC) in Hampton, Virginia. The goal of these tests was to determine the performance characteristics of the chambers; specifically, we were interested in evaluating the characteristics of the electromagnetic environment inside the chambers when the chambers were excited by a continuous wave signal between $80 \mathrm{MHz}$ and $18 \mathrm{GHz}$. The combination of well designed facilities and the new measurement procedures developed for this project have resulted in data which show unprecedented reproducibility (as good as $\pm 0.8 \mathrm{~dB}$ from $200 \mathrm{MHz}$ to $17 \mathrm{GHz}$ ) for some measurements, and excellent agreement with theory. As a result of the unexpectedly good chamber performance, we were forced to improve our data processing, and the results are a simple two-parameter model of mode-stirred chamber characteristics which predicts the behavior of the fundamental chamber parameters between $300 \mathrm{MHz}$ and $18 \mathrm{GHz}$ within $\pm 1 \mathrm{~dB}$ for the average and $\pm 2 \mathrm{~dB}$ for the maximum.

Keywords: chamber evaluation; chamber models; electric field; mechanical stirring; modestirred chamber; order statistics; Q; quality factor; reverberation chamber; statistical analysis; statistical electromagnetics; uncertainty analysis 


\section{Introduction}

The National Aeronautics and Space Administration Langley Research Center's (NASA/LaRC) High Intensity Radiation Laboratory in Hampton, Virginia serves the Federal Aviation Administration (FAA) and the aircraft and aerospace industry with research and development of metrology techniques for certification to HIRF (high intensity radiated electromagnetic fields) standards. In support of this mission, the High Intensity Radiation Laboratory at NASA/LaRC has constructed a new mode-stirred chamber facility. The decision to invest in this facility is supported by evidence which indicates that aircraft cavities behave as electromagnetic mode-stirred chambers [1]. This characteristic, along with other desirable features of mode-stirred chambers, such as the ability to generate high fields for modest input power, makes this technology one of the most practical and cost-effective methods for testing airborne systems for HIRF immunity. The new mode-stirred chamber facility at NASA/LaRC, consisting of three separate chambers and a centrally located control room, will allow simultaneous testing of three independent systems. Alternatively, by connecting the systems together but leaving them in three separate chambers, NASA/LaRC has the unprecedented ability to test a single system, consisting of up to three subsystems, with each subsystem exposed to different field conditions. This could be useful for simulating the interior of an aircraft, which is assumed to have subsections (cockpit, body, tail section), each with different electromagnetic characteristics.

The Fields and Interference Metrology Group of the National Institute of Standards and Technology (NIST) has performed extensive measurements in the NASA/LaRC chambers to establish the operational parameters and characteristics of these chambers. This characterization was necessary to determine values for such parameters as unloaded chamber power loss, chamber quality factor, field strength for a given input power, effectiveness of the mechanical stirrers, and lowest recommended operating frequency. These measurements can then be used to establish confidence in future tests performed in the chambers and as baseline data to measure the effects on this environment due to the placement of the equipment under test (EUT) in the chamber.

The NASA/LaRC facility was designed to support modern aircraft designers and manufacturers. These designers and manufacturers increasingly rely on sophisticated electronic systems to provide critical flight control and necessary safety features for the next generation aircraft and for upgrades to existing aircraft. These systems must meet stringent safety requirements for operability and performance under adverse conditions, including exposure to high intensity radiated electromagnetic fields which may exist around airports and within flight paths. Measurement standards such as RTCA/DO-160D, Section 20 [2] require interference and immunity testing of airborne equipment to electric fields of up to several thousand volts per meter.

A detailed description of the NASA facilities is given in Section 2. Here we give dimensions and general layout of the facilities, as well as descriptions of some of the safety features and capabilities of the facilities.

In Section 3, we describe the theoretical framework behind all of the measurements. Originally, we had planned to process the data based on the theory presented in NBS Technical Note 1092 [3], along with some improvements [4] which have been made since reference [3] was 
published. Preliminary analysis of the data measured in the NASA chambers, however, showed that the new data were significantly "better behaved" (the random component of the uncertainty was significantly smaller) than any data we had taken before. Small errors, which were undetectable in data that had been taken previously, were now apparent. As a result, we were forced to reformulate much of the theory that had been used to describe the operation of modestirred chambers. The theoretical description on which we based our analysis is divided into two sections: the general electromagnetic theory is presented in Section 3.1, and the general statistical theory is presented in Section 3.2. Much of the information presented here is a summary of results that have been published elsewhere for different applications. We provide this information so that those aspects that are directly applicable to mode-stirred chamber measurements can be easily referenced.

In addition to material that has been previously published, we also include information that, to our knowledge, is unique and presented here for the first time. These can be divided into two general groups, which coincide with information in Sections 3.1 and 3.2: electromagnetic theory and statistical theory applied to mode-stirred chamber applications. In Section 3.1, we present a simple two-parameter model of the characteristics of a mode-stirred chamber. The twoparameter model allows a closed-form expression to be used to predict chamber behavior. This is more practical and more accurate than looking up previously measured results in a table. In Section 3.2, we present the statistical characteristics of the extreme values of the electric field and the power received by a reference antenna inside a mode-stirred chamber. Combining these statistical characteristics with the two-parameter model of the chamber, we are able to predict the characteristics of the maximum and minimum electric field and the maximum and minimum power received from a reference antenna.

Two test methods were employed for the evaluation of the chambers. These methods are described in Section 4. The first method, described in Section 4.1, estimated field characteristics using an array of 10 calibrated isotropic field sensors (each consisting of 3 orthogonal dipoles) placed in several locations in the test volume of the chamber, a transmitting antenna, and a receiving antenna. This measurement setup has been used in the past for the evaluation of other mode-stirred chamber facilities. The second method, described in Section 4.2, estimated the same parameters using a vector network analyzer and the same transmitting and receiving antennas used in the first method. The network analyzer method is not able to measure the electric field at multiple locations simultaneously, but it is extremely fast and accurate. This allowed measurements at a greater number of frequencies than was possible with the probe system, and also allowed repeated measurements with the antennas placed at different locations in the chamber. Also, the use of a vector network analyzer allowed us to measure complex phasor data, which gave substantially more information than was available using scalar techniques.

Information on details specific to the measurements performed in the NASA chambers are given in Section 5. We discuss the number and distribution of the paddle steps in each chamber, as well as our reasons for selecting the values used in the evaluations of the chambers in Section 5.1. One contribution which we think is a significant improvement over other modestirred chamber evaluations comes from corrections for the effects of imperfect antennas. These are presented in Section 5.2. In Sections 5.3 and 5.4, we discuss the two primary methods of power normalization used in mode-stirred chamber measurements, normalization to a constant incident power and to a constant net input power, and the effects this normalization can have on the estimation of the transmitted power. 
The techniques we used to process the measured data are given in Section 6. Using data we collected in a chamber as an example, we step through each procedure and stage of analysis. The data from other chambers were analyzed similarly, and a summary of the characteristics of the NASA/LaRC mode-stirred chambers is presented in Section 7. The majority of the information we present on each chamber is contained in a large number of figures for each parameter in each chamber. We also include equations that can be used to estimate the descriptive parameters of each empty chamber as a function of input power.

An estimate of the uncertainties associated with our results is presented in Section 8. Here we show that the component of uncertainty due to random fluctuations in the data is only slightly greater than what is predicted by the statistical model. This indicates that the NASA chambers, when used in conjunction with precise instrumentation, will give measurement results that are nearly optimal.

\section{The NASA Mode-Stirred Chamber Facility}

The NASA facility is shown in Figure 1. This facility consists of one large shielded enclosure which has been divided into five subenclosures: Chamber A, Chamber B, Chamber C, the amplifier room, and the control room. This setup allows simultaneous testing of three systems, each subjected to a different electromagnetic environment. Alternatively, if a single system is made up of components that can operate in separate locations, those components can be placed in separate chambers. Any cabling required for communication between the components can be connected by bulkhead feedthroughs, and the separate components can be tested in three different electromagnetic environments simultaneously. This allows testing of complex systems in a setting that is more realistic than subjecting all components to the same environment, or subjecting only one component in the system to a harsh environment while the remainder of the system is not tested at all.

The dimensions of each chamber are shown in Table 1, along with the respective surface areas and volumes. For reference, the dimensions of the NIST chamber are also included in this table.

Table 1. Dimensions of the NASA chambers and the NIST chamber.

\begin{tabular}{|c|c|c|c|c|c|}
\hline & Height (m) & Width (m) & Depth (m) & Surface Area $\left(\mathrm{m}^{2}\right)$ & Volume $\left(\mathrm{m}^{3}\right)$ \\
\hline Chamber A & 2.90 & 7.01 & 14.33 & 324.42 & 290.80 \\
\hline Chamber B & 2.90 & 3.96 & 7.01 & 119.10 & 80.43 \\
\hline Chamber C & 2.90 & 2.13 & 2.72 & 39.70 & 16.80 \\
\hline$\overline{\text { NIST }} \overline{\mathrm{Ch}} \overline{\mathrm{Cmb}} \overline{\mathrm{r}}$ & $\overline{2.74}$ & $3 . \overline{05}$ & $4 . \overline{57}$ & $6 \overline{9.63}$ & $\overline{38.1} \overline{9}$ \\
\hline
\end{tabular}




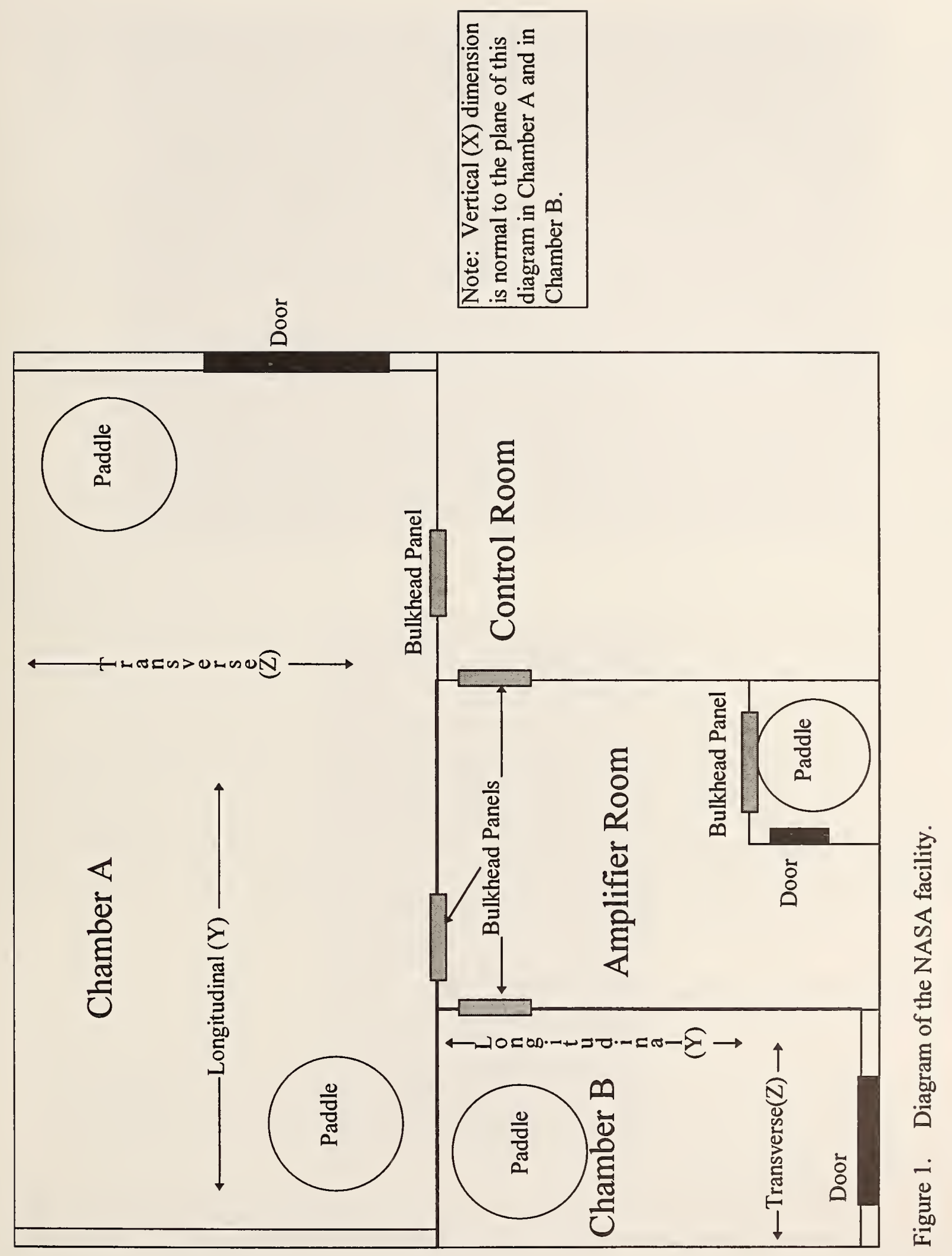


The size of the paddle(s) used in a chamber is generally a compromise between the desire for the largest possible test volume and the largest possible paddle. A large paddle is desirable because large paddles appear to result in better chamber performance than small paddles (this will be demonstrated in Section 7.4). The paddles used in Chambers A and B are identical, but two independent paddles are used in Chamber A, whereas a single paddle is used in Chamber B. The paddle used in Chamber B is shown in Figure 2a. The paddle used in Chamber $C$ is significantly smaller than those used in the other chambers. The paddle used in Chamber $\mathrm{C}$ is shown in Figure 2b. The location of each paddle is indicated in Figure 1, but the unique design of the paddles and drive system allow them to be placed anywhere in the chamber. The size and placement of the paddles in the NASA chambers appears to be a good compromise between paddle size and size of the test volume.

The doors are opened and closed using an automatic air piston. The doors are operated from inside the chamber using a pressure relief button, ensuring that personnel will be able to exit a chamber at will. From the outside, however, the doors are operated by card locks. This helps prevent unauthorized access to a chamber during a measurement and also allows a monitoring computer to identify any person accessing the chamber from the outside, as well as the time at which the chamber access occurred. The door is sealed by inflating an air bladder between two metal plates. As the bladder inflates, the plates are pressed against two opposing metal surfaces in the walls, resulting in a high pressure seal. This seal ensures good electrical contact between the door and the walls and also keeps the door from being opened inadvertently. In the unlikely event that the doors are opened during a measurement or if air pressure drops in the door bladder, sensors detect this event and automatically shut down all rf amplifiers. This significantly decreases the possibility of personnel being exposed to high levels of rf radiation. As an additional safety precaution, $r$ sensors are placed outside each chamber. These sensors will sound an alarm and shut down the amplifiers if rf levels exceed safety thresholds.

The chambers are outfitted with shielded incandescent lights. Filtered ac power is also available, so typical electronic systems can be tested in any of the chambers. Small shielded cameras can be placed in the chambers, if necessary, so visual indicators on instruments inside a chamber can be observed during measurements. Other special features can be added to the chambers if necessary. The combination of features listed above increases the capabilities and flexibility of this facility beyond those found in less sophisticated facilities.

\section{Mode-Stirred Chamber Characteristics}

A mode-stirred chamber is an electrically large, highly conductive enclosed cavity or chamber used to measure electromagnetic compatibility (both emissions and immunity) of electronic devices. Any facility that fits this description can be considered a mode-stirred or reverberation chamber. Other conditions, however, may be required before such a facility can be used with acceptable uncertainty, as discussed later in this report.

A typical measurement setup is shown in Figure 3. In this figure, the term sensor is used to indicate that any device that is capable of measuring the characteristics of an rf signal can be used. Typically, either power meters or spectrum analyzers are used, but receivers, rf voltmeters, or oscilloscopes could also be used. 


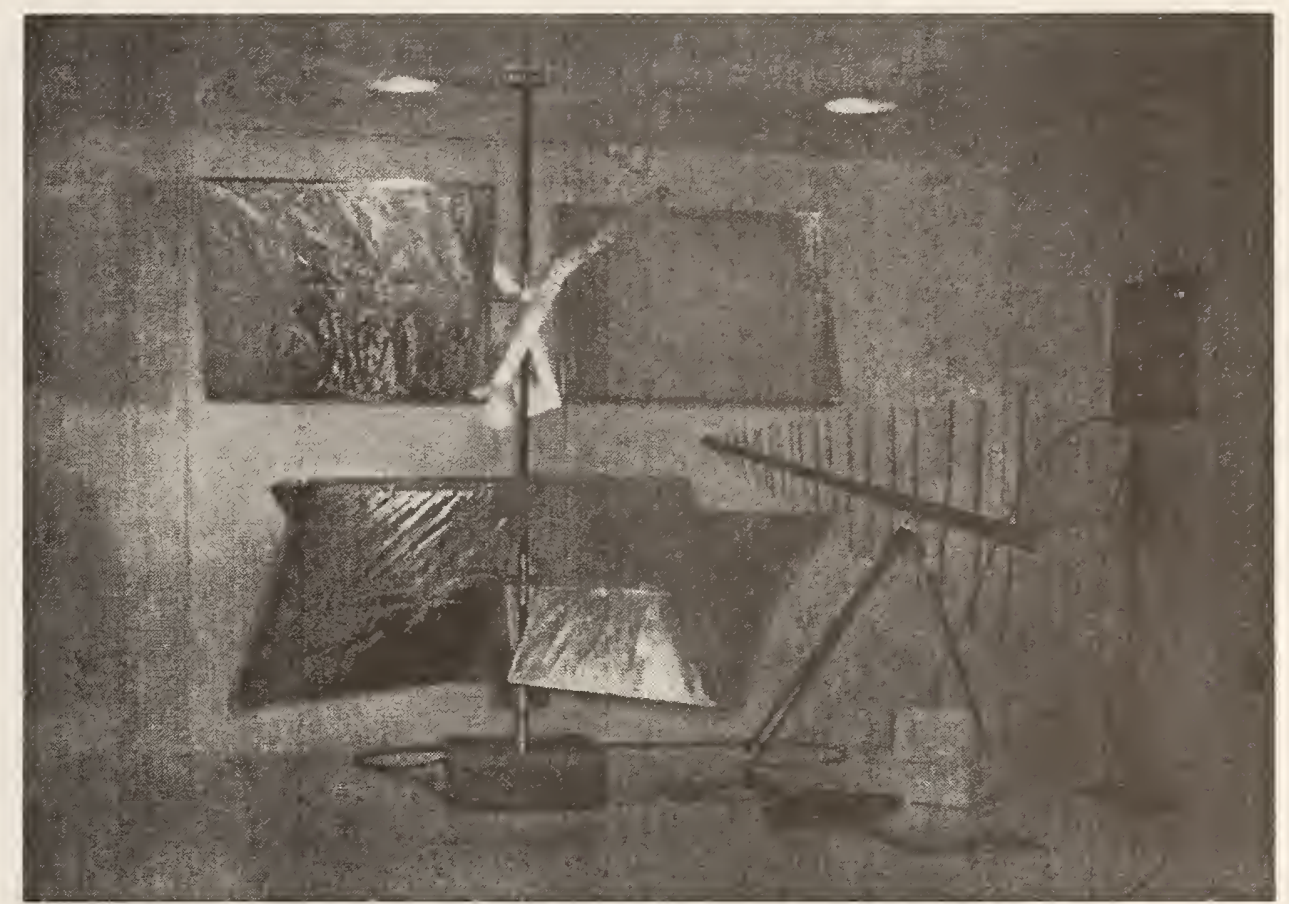

(a)

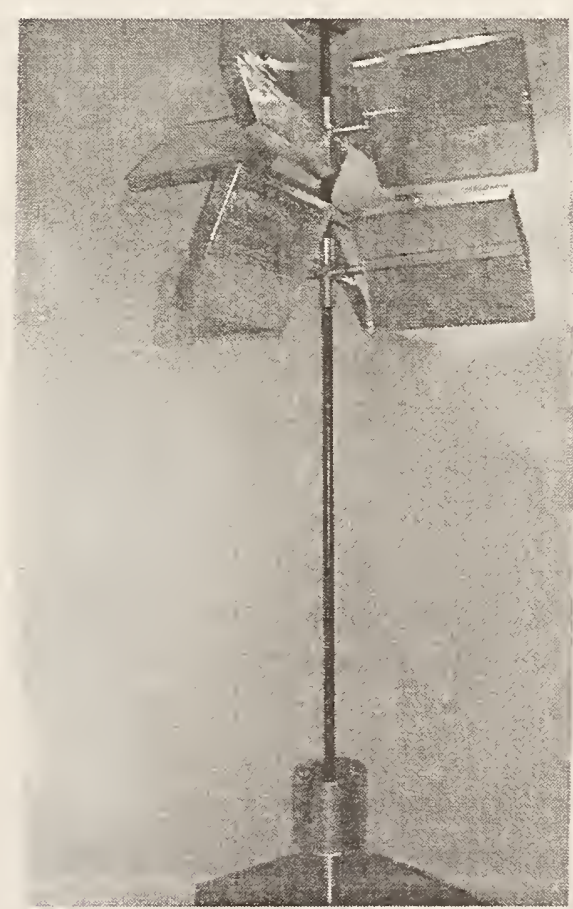

(b)

Figure 2. Pictures of stirrer in (a) chamber B. (b) chamber C. 
Electromagnetic energy is introduced into the chamber by a transmitting antenna. This energy is reflected off the walls and eventually reaches steady-state. The electromagnetic fields inside the chamber satisfy boundary conditions on the conducting surfaces and may be described as the summation of cavity modes which exist in the cavity. The modes that can exist in the cavity depend on the geometry of the cavity, placement of the antennas, and placement of any other objects inside the cavity. The electrically large nature of the cavity means that it will support a large number of possible modes, each of which will contribute to the resultant fields at any given location depending on how effectively that mode is excited by the source. Calculations of the electromagnetic fields for any realistic test setup in such a complex environment are impractical, if not impossible. This complexity, while it may stymie any direct calculation of the steady-state electromagnetic fields, can be used to our advantage. For a complex cavity, any change in the boundary conditions or frequency will produce different, almost random field structures in the chamber. If the fields in the cavity can indeed be treated as if they are random, the field parameters associated with the highly complex field structures in the chamber can now be analyzed statistically. The objective in characterizing a mode-stirred chamber is no longer a matter of calculating deterministic field equations or measuring a single steady-state field quantity, but rather determining the underlying probability distributions and statistical descriptions. Traditionally, the fields have been randomized (perturbed) by changing the electrical boundary conditions. This has been done using a rotating metal paddle or stirrer [3] (hence the popular term for a reverberation chamber is the "mode-stirred" chamber). Other techniques which have been proposed and used include changing the drive frequency some small amount (frequency stirring) [5], and transmitting a time variant (noisy) signal of some fixed bandwidth and center frequency (noise stirring) [6]. For this report, we will be concerned with paddle stirring only.

Theory suggests [7] and measurements confirm [8] that the randomized fields inside the chamber can be approximated by a simple statistical model, as will be discussed in Section 3.2. As long as certain operating conditions (which will be discussed later) are met, the statistical properties appear to be independent of location and orientation over most of the test volume inside the chamber. For an ideal chamber, the magnitude of the electric field will be independent of location inside the chamber, and there should be no dependence on orientation of a probe or test device (that is, there should be no preferred polarization to the electric field). Thus, one method of evaluating a chamber is by measuring the magnitude of the electric field at a number of different points and orientations inside the chamber, and determining whether or not the measurements are consistent with the hypothesis that the observed values are independent. A chamber is considered acceptable if any observed dependence is less than some limit.

Given these requirements, the operating conditions can be determined in terms of these requirements. For example, the characteristics of the electric field are generally closer to ideal at high frequencies than at low frequencies. The lowest usable frequency of a chamber is the frequency at which the dependence of the electric field on location and orientation is within the specified limit, but below which the dependence is unacceptable.

The concept of spatial independence is fundamental in the analysis of mode-stirred chambers. If the measurements are independent of location, we can use a reference antenna or probe to sample the fields at one location in the chamber and be assured that the fields at any other location, including locations surrounding any equipment under test, will be similar. 


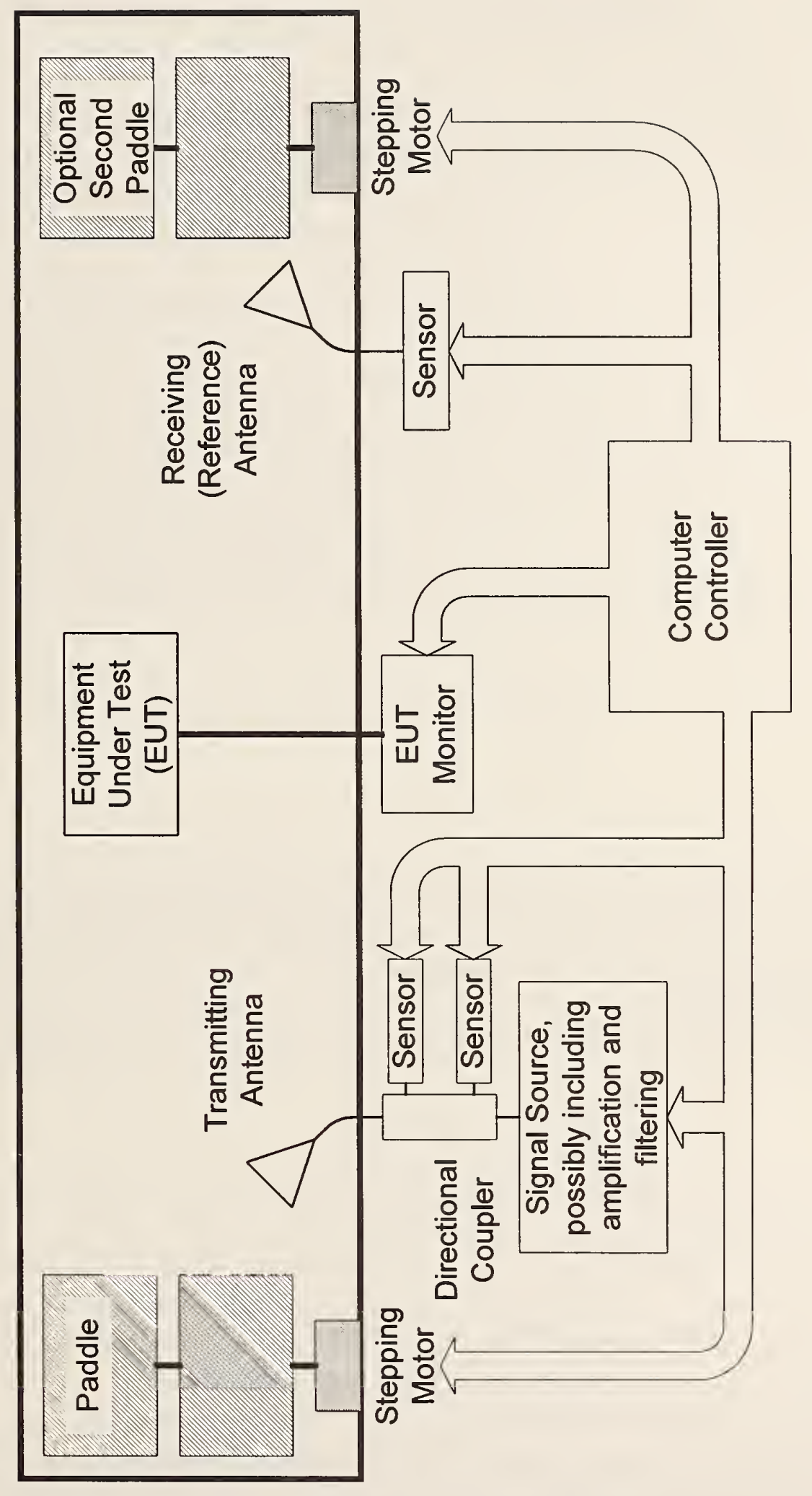

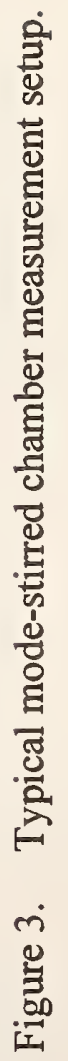




\subsection{Electromagnetic Fields in a Mode-Stirred Chamber}

Several related electromagnetic parameters are derived from measurements in a modestirred chamber. We are initially interested in the energy density $W$ and electric field $E$ inside the chamber, and the quality factor $Q$ of the chamber. In general, all parameters associated with mode-stirred chamber measurements will be functions of frequency, paddle position, and location in the chamber. If the input signal varies with time, then the parameters will also be a function of time. As a result, we cannot simply refer to the electric field, for example, inside the chamber without specifying all variables of which electric field is a function. To avoid this complication we will refer to the average or expected value of the parameter. In this paper, we are interested primarily in the average over all paddle positions and will refer to this as the ensemble average. We will use the symbols $<>$ to indicate ensemble average in the following equations, with $\langle W>$ indicating the value of $W$ averaged over all paddle positions. It is also possible to perform spatial averaging [9], frequency averaging [5], and time averaging [6], and these have all been addressed in the cited references. Ideally, all of these methods will result in the same estimate for the parameter of interest. In reality, however, some differences have been observed, and these will require further investigation.

If we assume that $\langle W\rangle$ is uniform throughout the cavity volume, we can express $\langle W\rangle$ as the average steady state energy $\left\langle U_{S}>\right.$ in the cavity divided by the chamber volume $V$ :

$$
<W>=\frac{<U_{S}>}{V} .
$$

This may appear to be a substantial assumption, but experiments demonstrate the uniformity of the average energy density [3]. Ideally, the energy stored in the cavity is constant and ensemble averaging is unnecessary, but we will continue to use the ensemble averaging notation to allow for the possibility of fluctuations.

The quality factor is defined as $2 \pi$ times the ratio of the energy stored to the energy dissipated per cycle, or

$$
Q=\frac{\omega<U_{S}>}{\left\langle P_{d}\right\rangle}=\frac{\left.\omega<U_{S}\right\rangle}{<P_{T}>},
$$

where $\omega$ is the excitation (radian) frequency, $\left\langle P_{d}\right\rangle$ is the dissipated power and is equal to the average transmitted power $\left\langle P_{T}\right\rangle$ in steady state. Here, we have intentionally left off the symbols for ensemble averaging (we used $Q$ instead of $\langle Q\rangle$ ) to indicate that $Q$ is an intrinsic property of the chamber we are measuring, and should be constant.

Ideally, $P_{T}$ will be constant for all paddle positions, in which case ensemble averaging is unnecessary. It is not always possible to maintain a constant transmitted power, however, if the antennas used in the measurement are imperfect, as discussed in Section 5. Therefore, we will continue to use the ensemble average of the transmitted power in all equations. 
An average scalar power density $<S_{C}>$ can be defined as

$$
\left\langle S_{C}>=c<W>=\frac{\lambda \omega}{2 \pi}<W>,\right.
$$

where $\lambda$ is the free-space wavelength, $c=\lambda f=\lambda \omega / 2 \pi$ is the speed of light in free space, and $f$ is the frequency in hertz. We use the term scalar power density to differentiate it from the standard Poynting vector $(E \times H)$ definition of power density. Although the units are correct $\left(\mathrm{W} / \mathrm{m}^{2}\right)$, these parameters have very different interpretations. Ideally, some term other than power density should be used to avoid confusion, but, unfortunately, the scalar power density as defined here is commonly used in the mode-stirred chamber literature [3]. In fact, the scalar modifier is generally ignored in historical discussions of mode-stirred chambers. Because of this, we will acknowledge the use of this term, as well as the fact that it can be useful in the derivation of some of the fundamental equations that describe the electromagnetic theory of mode-stirred chambers [10], but we will avoid using it in our derivations, choosing instead to base our equations on energy density $W$.

By combining eqs (1) through (3), we can calculate the average energy density in terms of the transmitted power:

$$
<W>=\frac{\lambda Q<P_{T}>}{c 2 \pi V} .
$$

We define the average received power $\left\langle P_{R}\right\rangle$ as the power that would be received by an impedance-matched lossless antenna at an arbitrary location and orientation inside the cavity. If the realized effective area of the receiving antenna is independent of the energy density, we can apply the plane-wave spectrum model of the fields inside a mode-stirred chamber [10] to give a simple expression for $\left\langle P_{R}\right\rangle$. The received power is the product of the speed of light in free space, the average energy density inside the cavity, and the average effective area $\left\langle A_{e}>\right.$ of the receiving antenna

$$
<P_{R}>=c<W><A_{e}>\text {. }
$$

Hill et al. [10] predict that the effective area of the antenna averaged over all paddle positions is equal to the effective area of the same antenna located in free space and averaged over all incidence angles and polarizations. The averaged effective area is

$$
<A_{e}>=\frac{\lambda^{2}}{8 \pi} .
$$

$<A_{e}>$ is one half of the effective area of an isotropic antenna, due to a polarization mismatch factor of one half [10]. 
Equation (6) does not depend on antenna pattern. This somewhat surprising result comes from averaging over all incidence angles and polarizations. This independence of pattern has been verified experimentally [3].

Combining eqs (5) and (6), we can write the received power as

$$
<P_{R}>=c<W>\frac{\lambda^{2}}{8 \pi}=<S_{C}>\frac{\lambda^{2}}{8 \pi} .
$$

We can rearrange this equation to give a measure of the average scalar power density as a function of received power:

$$
<S_{C}>=\frac{8 \pi<P_{R}>}{\lambda^{2}}
$$

This quantity is occasionally specified in $\mathrm{mW} / \mathrm{cm}^{2}$. If power is measured in watts and wavelength is measured meters, the conversions are straightforward. As a shortcut, $\left\langle S_{C}\right\rangle$ can be computed in $\mathrm{W} / \mathrm{m}^{2}$, and the result can be divided by 10 to give $\left\langle S_{C}\right\rangle$ in $\mathrm{mW} / \mathrm{cm}^{2}$. Although the derivation is trivial, it is easy to forget that any conversion is necessary when using eq (8) (we speak from experience).

Combining eqs (4) and (7), we can express $Q$ as

$$
Q=\frac{16 \pi^{2} V}{\lambda^{3}} \frac{<P_{R}>}{<P_{T}>}
$$

Equation (9) requires us to calculate the ensemble average of the received and transmitted powers before the ratio is computed. Alternatively, if it is possible to measure the transmitted power at each paddle position, we could calculate the ratio first, and then compute the ensemble average of these ratios. This approach is intuitively appealing because it is equivalent to forcing the transmitted power to be constant over all paddle positions. In most real cases, however, we do not know the transmitted power at each paddle step, and we are forced to use eq (9) and either calculate or estimate the average transmitted power. Additional research needs to be performed to assess which method yields the best results and what the resultant effects on the final uncertainty estimates will be. For now, the difference needs to be addressed experimentally.

It helpful to write the energy density as

$$
W=\frac{1}{2}\left(\varepsilon_{0}\left|E_{T}\right|^{2}+\mu_{0}\left|H_{T}\right|^{2}\right)
$$

where $\left|E_{T}\right|^{2}$ is the squared magnitude of the total electric field, $\left|H_{T}\right|^{2}$ is the squared magnitude of the total magnetic field, $\varepsilon_{0}$ is the permittivity of free space, $\mu_{0}$ is the permeability of free space, $\varepsilon_{0}\left|E_{T}\right|^{2}$ is the electric energy density, and $\mu_{0}\left|H_{T}\right|^{2}$ is the magnetic energy density. If we 
assume that the ensemble average of the electric and magnetic energy densities are equal, we can write the ensemble average of the energy density as

$$
<W>=\frac{1}{2}\left(\varepsilon _ { 0 } \left\langle\left|E_{T}\right|^{2}>+\mu_{0}\left\langle\left|H_{T}\right|^{2}>\right)=\varepsilon_{0}\left\langle\left|E_{T}\right|^{2}>=\mu_{0}<\left|H_{T}\right|^{2}>\right.\right.\right.
$$

We can rewrite eq (11) as

$$
<W>=\frac{\left\langle\left. E_{T}\right|^{2}>\right.}{c \eta_{0}}=\frac{\eta_{0}\left\langle\left|H_{T}\right|^{2}>\right.}{c}
$$

where $\eta_{0}=1 / c \varepsilon_{0}=c \mu_{0} \approx 120 \pi$ is the intrinsic wave impedance of free space. (It may be better to use eq (11) instead of eq (12), thus avoiding some of the confusion associated with the use of the free-space wave impedance. Either way, the results are the same.) Combining eqs (7) and (12) yields an expression for the squared magnitude of the total electric field in terms of the received power:

$$
<\left|E_{T}\right|^{2}>=\frac{8 \pi \eta_{0}<P_{R}>}{\lambda^{2}}=\frac{960 \pi^{2}<P_{R}>}{\lambda^{2}} .
$$

Similarly, we could write an expression for the average squared magnitude of the magnetic field, but in this paper we will restrict our attention to the electric field.

The magnitude of the total electric field can be measured using a set of three electrically short orthogonal dipoles which have been calibrated under plane-wave conditions. Ideally, the centers of these three dipoles should be located at a single point in space, although differences will be negligible if they are separated by an electrically short distance. Each dipole measures a single rectangular component of the total electric field, which we will refer to as $E_{R 1}, E_{R 2}$, and $E_{R 3}$. An arbitrary rectangular component of the electric field will be called simply $E_{R}$. The squared magnitude of the total electric field is

$$
\left|E_{T}\right|^{2}=\left|E_{R 1}\right|^{2}+\left|E_{R 2}\right|^{2}+\left|E_{R 3}\right|^{2}
$$

and the magnitude of the total electric field is

$$
\left|E_{T}\right|=\sqrt{\left|E_{R 1}\right|^{2}+\left|E_{R 2}\right|^{2}+\left|E_{R 3}\right|^{2}} \text {. }
$$


In a mode-stirred chamber, the average electric field is assumed to be independent of orientation. This implies that

$$
<\left|E_{R 1}\right|>=\left\langle\left|E_{R 2}\right|>=\left\langle\left|E_{R 3}\right|>\right.\right.
$$

and

$$
\left\langle\left|E_{R 1}\right|^{2}>=\left\langle\left|E_{R 2}\right|^{2}>=\left\langle\left|E_{R 3}\right|^{2}>\right.\right.\right.
$$

Therefore, we can write

$$
<\left|E_{T}\right|^{2}>=3<\left|E_{R}\right|^{2}>
$$

Combining eq (13) and eq (18), we can solve for $\left\langle\left|E_{R}\right|^{2}>\right.$ in terms of the received power:

$$
\left\langle\left. E_{R}\right|^{2}>=\frac{320 \pi^{2}<P_{R}>}{\lambda^{2}}\right.
$$

The equations given above are valid only for the average of the squared magnitude of the electric field. In general, substituting the maximum or minimum received power for the average received power is not valid. Also, it is not valid to simply take the square root of eqs (18) or (19) and generate equations for the average magnitude of the electric field instead of the average squared magnitude. These approximations have been used in the past [3] and have resulted in erroneous data and poor agreement with theory.

\subsubsection{Mechanisms of Power Loss in a Mode-Stirred Chamber}

Once power has entered a chamber, it must either escape through an aperture (leakage) or be absorbed and converted to heat (absorption). A good mode-stirred chamber should have negligible leakage, so this term will not be considered further in this paper. Three separate identifiable absorption methods deserve special mention: absorption by a lossy object paced in the chamber, absorption by chamber walls, and absorption by receiving antennas (actually, this is absorption by loads connected to the antennas, but we will call these losses antenna losses). The chambers we evaluated were empty except for the paddles and the transmitting and receiving antennas. Thus we will ignore the contribution caused by lossy objects and concentrate on wall losses and antenna losses.

Dunn shows [11] that the average power absorbed by a highly conductive wall per unit area is

$$
<P_{\text {wall }}>\text { (per unit area) } \approx \frac{2 \omega \delta(\omega) \mu_{r}(\omega)<W>}{3},
$$


where $\delta(\omega)$ is the skin depth of the fields in the metal walls, and $\mu_{r}(\omega)$ is the relative permeability of the wall material. We write $\delta(\omega)$ and $\mu_{r}(\omega)$ as explicit reminders that the skin depth and permeability are, in general, functions of frequency. Equation (20) is a first order approximation for the power absorbed by the walls, but the higher order terms contribute very little to the total for typical operating frequencies and will therefore be ignored. The values of $\delta(\omega)$ and $\mu_{r}(\omega)$ are given by

$$
\delta(\omega)=\sqrt{\frac{2}{\omega \mu_{W} \sigma_{W}}}
$$

and

$$
\mu_{r}(\omega)=\frac{\mu_{W}(\omega)}{\mu_{0}}
$$

where $\mu_{W}(\omega)$ is the wall permeability, $\mu_{0}$ is the permeability of free space, and $\sigma_{W}$ is the wall conductivity (generally assumed to be constant with frequency). For walls made of non-magnetic materials, the wall permeability will be equal to the permeability of free space $\left(\mu_{W}=\mu_{0}\right.$ and $\mu_{r}=1$ ) for all frequencies. For walls made of ferromagnetic materials, the permeability exhibits a strong dependence on frequency. This dependence is well documented at low frequencies (below a few megahertz) [12], but information on the properties of ferromagnetic materials at microwave frequencies is very difficult to find [13]. Thus, until more accurate data are available on the microwave properties of ferromagnetic materials, we will assume that $\mu_{r}$ is independent of frequency. We will continue to include it in our equations as a reminder that this factor may prove to be important in the future.

If we multiply eq (20) by the chamber surface area $S$ to get the average total power dissipated by the walls, we can write the average total power $\left\langle P_{\text {wall }}\right\rangle$ dissipated by the walls as

$$
<P_{\text {wall }}>\approx \frac{2 \omega \delta(\omega) \mu_{r}(\omega) S<W>}{3}
$$

into eq (23)

$$
<P_{\text {wall }}>\approx \frac{2 \mu_{r} S<W>}{3} \sqrt{\frac{2 \omega}{\mu_{W} \sigma_{W}}} .
$$

The average power absorbed by the walls is proportional to the square root of frequency. Thus, the walls will absorb only low power at low frequencies and much higher power at high 
frequencies. Equation (24) is more cumbersome to use than eq (23) and will therefore only be used when it is necessary to demonstrate the frequency dependence of the power absorbed by the walls.

The average power absorbed by an impedance-matched lossless receiving antenna is given above in eq (7) as

$$
<P_{R}>=\frac{c<W>\lambda^{2}}{8 \pi}=\frac{c^{3} \pi<W>}{2 \omega^{2}} .
$$

Comparing eqs (24) and (25), we see that most of the power will be dissipated by the antennas at low frequencies, whereas the walls will be the dominant loss mechanism at high frequencies.

If more than one receiving antenna is present, the average total power absorbed by all of the antennas is simply the sum of powers absorbed by the individual antennas. By superposition, the transmitting antenna will also act as a receiving antenna unless an external control system is used to maintain a constant net power into the chamber.

The average total power dissipated inside the chamber is given by

$$
\begin{aligned}
<P_{T}>=<P_{d}> & =\frac{2 \omega \delta(\omega) \mu_{r} S<W>}{3}+N \frac{c^{3} \pi<W>}{2 \omega^{2}} \\
& =\frac{2 \omega \delta(\omega) \mu_{r} S<W>}{3}+N<P_{R}>,
\end{aligned}
$$

where $N$ is the number of receiving antennas in the chamber, and we again make use of the fact that, for steady state conditions, the transmitted power must be equal to the dissipated power.

We define the ratio of the power received by an ideal antenna to the transmitted power at any given paddle position as the chamber gain $G_{C}$ :

$$
G_{C}=\frac{P_{R}}{P_{T}} .
$$

The average chamber gain is given by

$$
\begin{aligned}
\left.<G_{C}\right\rangle & =\frac{\left\langle P_{R}\right\rangle}{\left\langle P_{T}\right\rangle}=\frac{\left\langle P_{R}\right\rangle}{\left.N<P_{R}\right\rangle+\frac{2 \omega \delta(\omega) \mu_{r} S<W>}{3}} \\
& =\frac{1}{N+\frac{2 \omega \delta(\omega) \mu_{r} S<W>}{3<P_{R}>}} \\
& =\frac{1}{N+\frac{4 \omega^{3} \delta(\omega) \mu_{r} S}{3 c^{3} \pi}} .
\end{aligned}
$$


If we assume that the relative permeability of the wall material is approximately constant with frequency, then $\left\langle G_{C}\right\rangle$ will have some simple and well defined characteristics. A plot of the chamber gain should be approximately constant at low frequencies and, since $\omega$ is proportional to $f$, should decrease as $f^{-5 / 2}$ at high frequencies. Thus we can write a general equation for $\left\langle G_{C}\right\rangle$ with one unknown:

$$
<G_{C}>=\frac{1}{N+b f^{5 / 2}}
$$

where $b$ depends primarily on the characteristics of the walls. If we know the characteristics of the antennas and the wall materials, $\left\langle G_{C}\right\rangle$ should be predictable. Alternatively, it should be possible to compute these characteristics based on measurements performed in the chamber.

One of the most common test setups inside a mode-stirred chamber involves one well matched transmitting antenna and one well matched receiving antenna. In this case, the chamber gain reduces to

$$
\begin{aligned}
<G_{C}> & =\frac{1}{2+\frac{4 \omega^{3} \delta(\omega) \mu_{R} S}{3 c^{3} \pi}} \\
& =\frac{1}{2+b f^{5 / 2}} .
\end{aligned}
$$

For low frequencies, on average, a receiving antenna will receive half of the incident power radiated by the transmitting antenna, and the other half will be received by the transmitting antenna. Alternatively, if we define the net input power $P_{N E T}$ as the difference between the incident and reflected powers at the transmitting test port, the received power should be equal to the net input power at low frequencies where antenna losses are the dominant loss mechanism. variable $a$ :

Equation (29) can be made more flexible by replacing the constant $N$ by an unknown

$$
<G_{C}>=\frac{1}{a+b f^{5 / 2}}
$$

where $a$ is approximately equal to the number of receiving antennas in the chamber, but is no longer restricted to this value. We use $a$ instead of $N$ to allow for nonideal antennas and for losses in the chamber due to mechanisms other than the antennas.

Given the definition for chamber gain from eq (27), we can define other quantities in terms of $\left\langle G_{C}\right\rangle$. For example, the quality factor is

$$
Q=\frac{16 \pi^{2} V}{\lambda^{3}}<G_{C}>
$$


and loss $L$ is

$$
L=\frac{1}{\left\langle G_{C}\right\rangle} .
$$

Also, it is often convenient to normalize the data for a constant transmitted power. If the transmitted power is normalized to a constant $1 \mathrm{~W}$, then we can write the scalar power density, the mean-squared total electric field, and the mean-squared rectangular component of the electric field as functions of the normalized average chamber gain $\left\langle\widetilde{G}_{C}\right\rangle$,

$$
\begin{gathered}
<S_{C}>=\frac{8 \pi}{\lambda^{2}}<\widetilde{G}_{C}>, \\
<E_{T}^{2}>=\frac{960 \pi^{2}}{\lambda^{2}}<\widetilde{G}_{C}>,
\end{gathered}
$$

and

$$
<E_{R}^{2}>=\frac{320 \pi^{2}}{\lambda^{2}}<\widetilde{G}_{C}>.
$$

Unfortunately, we cannot write the average electric field directly as a function of chamber gain, since chamber gain is related to the average value of the squared electric field (total or rectangular component), not the average value of the electric field. This problem will be dealt with later in Section 3.2.3.

Equations (32) through (36) show that all of the basic parameters associated with modestirred chamber evaluations, with the exception of electric field, can be calculated from any of the others. Thus, we can analyze the statistical properties of any one of these parameters (repeatability, uncertainty), and the other parameters will have the same properties.

\subsection{Statistical Description of Mode-Stirred Chambers}

Due to the almost random nature of the fields inside a mode-stirred chamber, a solid understanding of basic statistics is essential. For a review of basic statistics, refer to Papoulis [14]. In this section, we will assume that you are familiar with the concept of a random variable, standard statistical terms such as mean, standard deviation, and both Gaussian (normal) and nonGaussian distributions. Also, a solid understanding of network analysis and scattering or $\mathrm{S}$ parameters is helpful. For a review of these topics, refer to reference [15].

Several references $[7,8]$ have shown that the total electric field at a point inside a modestirred chamber can be characterized by six parameters: the in-phase and quadrature components 
in each of three orthogonal directions. For a chamber operating at a sufficiently high frequency (to be determined later), the chamber will behave as a complex cavity, and each component can be approximated as an independent and identically distributed (iid) random variable. By the central limit theorem, the distribution which best describes each component is the normal or Gaussian distribution [7]. A short dipole will interact with only two of the six components: the in-phase and quadrature components which match the polarization of the dipole. By using a vector network analyzer, we can measure each of these two components separately. The in-phase component is the real part of $S_{21}$, and the quadrature component is the imaginary part of $S_{21}$. Our measurements indicate that an extended linear antenna (log-periodic, horn) behaves much like a short dipole as long as the power received by the antenna does not significantly decrease the quality factor of the chamber (this situation will be discussed in detail in Section 6). Therefore, measurements of $S_{21}$ using an extended antenna will have similar statistical characteristics to measurements of $S_{21}$ made using a short dipole, which in turn has the same statistical characteristics as measurements of the electric field inside the chamber. Based on these approximations, we can construct a very simple model of the fields inside a mode-stirred chamber and also the signals received by an antenna. Before proceeding with the analysis based on these assumptions, we note that these are idealized assumptions. There are some conditions where these assumptions are not and, in fact, cannot be valid.

\subsubsection{Limitations of the Statistical Model}

The assumption of randomness is not completely justified. Consider a chamber which is stirred by a single paddle. For a given paddle position, the system is not random; it is possible, although extremely difficult, to completely characterize the fields at all points within the chamber. Once the paddle has made one complete revolution, the same field distribution will be present. This implies that the fields inside the chamber are not random at all, but are in fact deterministic and calculable at each paddle position.

Another related item of concern is the assumption of statistical independence. For small changes in paddle position, we expect only small changes in measured values. This effect will be most pronounced at low frequencies, where a small change in the paddle position results in a small change in the position of the ends of the paddle, relative to the wavelength of the excitation frequency. At high frequencies, where wavelengths are short, even small changes in paddle position result in electrically large changes in boundary conditions, so this problem will be significant only at low frequencies or for very small paddle steps. Figure 4 shows four traces of measured values of $S_{21}$ as a function of paddle position. These measurements were performed at 225 equally spaced ( $1.6^{\circ}$ per step) paddle positions. The horizontal axis is the real part of $S_{21}$, and the vertical axis is the imaginary part of $S_{21}$. This type of plot will be called a scatter plot. Figure $4 \mathrm{a}$ shows a scatter plot for an idealized, completely independent case generated with a random number generator. Figures $4 \mathrm{~b}$ through $\mathrm{d}$ show measurements in Chamber $\mathrm{B}$ at $90 \mathrm{MHz}$, $200 \mathrm{MHz}$, and $1 \mathrm{GHz}$. We used data from Chamber B because that chamber had only one paddle. The results using two paddles in Chamber A are presented in Section 5.1. The data measured at low frequencies $(90 \mathrm{MHz})$ are smoother than data measured at high frequencies $(1 \mathrm{GHz})$, 


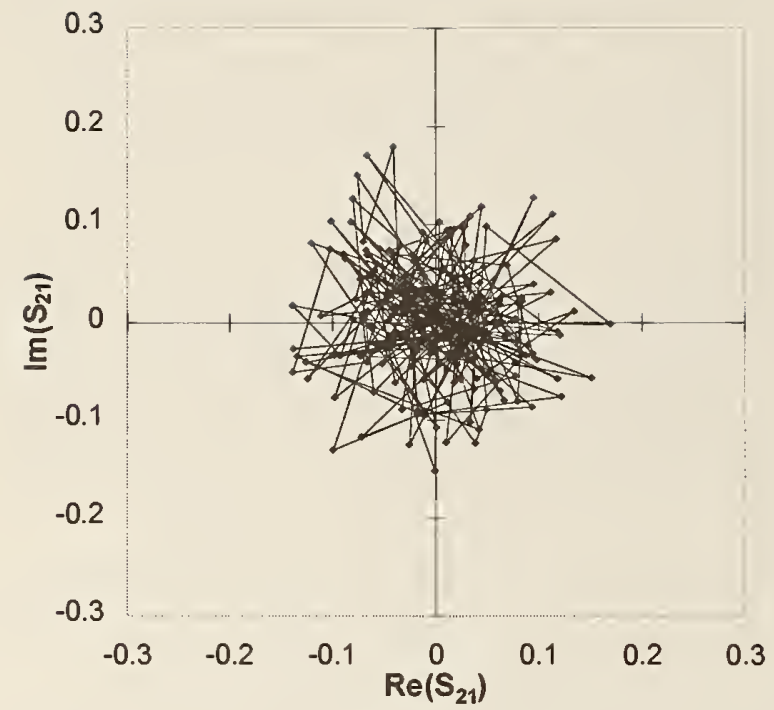

(a)

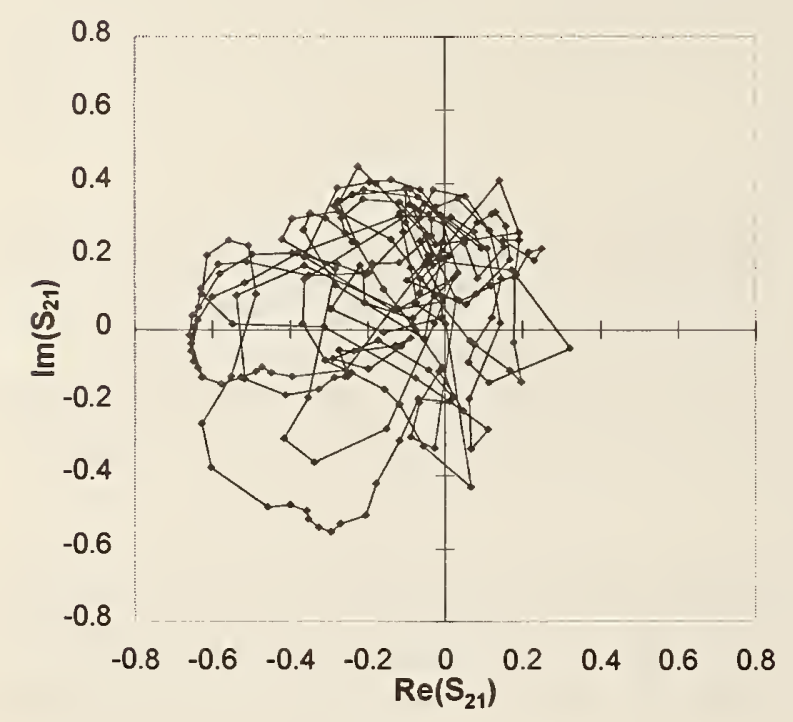

(c)

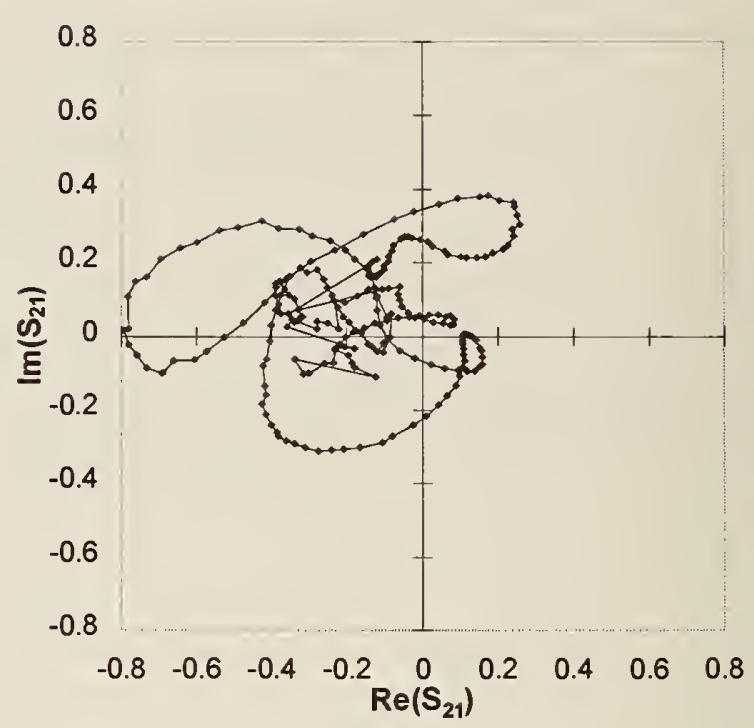

(b)

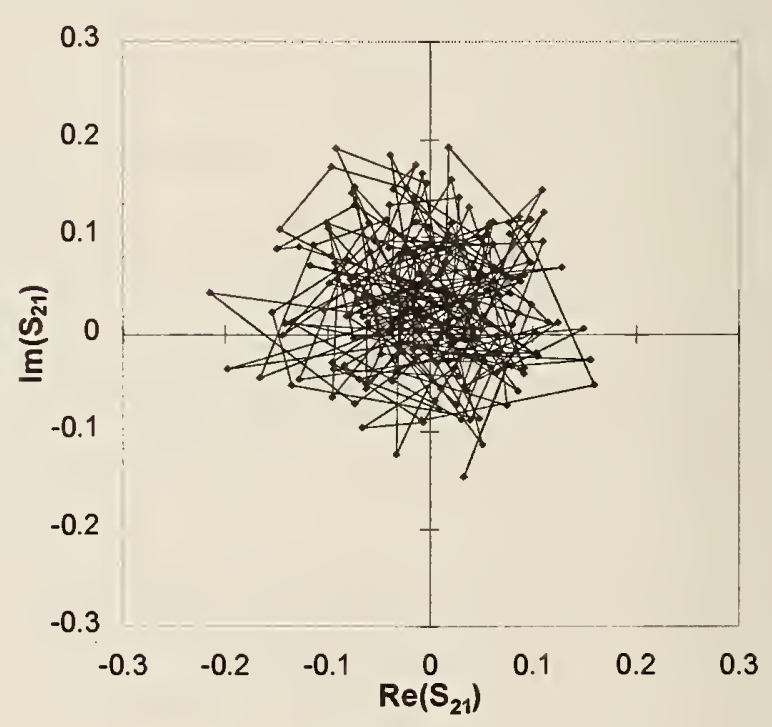

(d)

Figure 4. Scatter plots of $S_{21}$ using one paddle. (a) Simulated data. (b) $90 \mathrm{MHz}$ in chamber B. (c) $200 \mathrm{MHz}$ in chamber B. (d) $1 \mathrm{GHz}$ in chamber $\mathrm{B}$. 


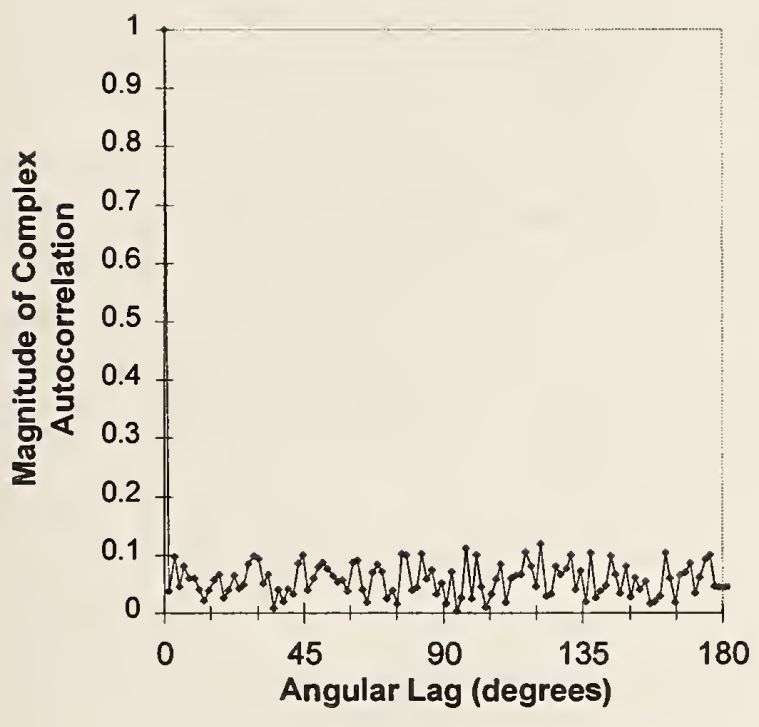

(a)

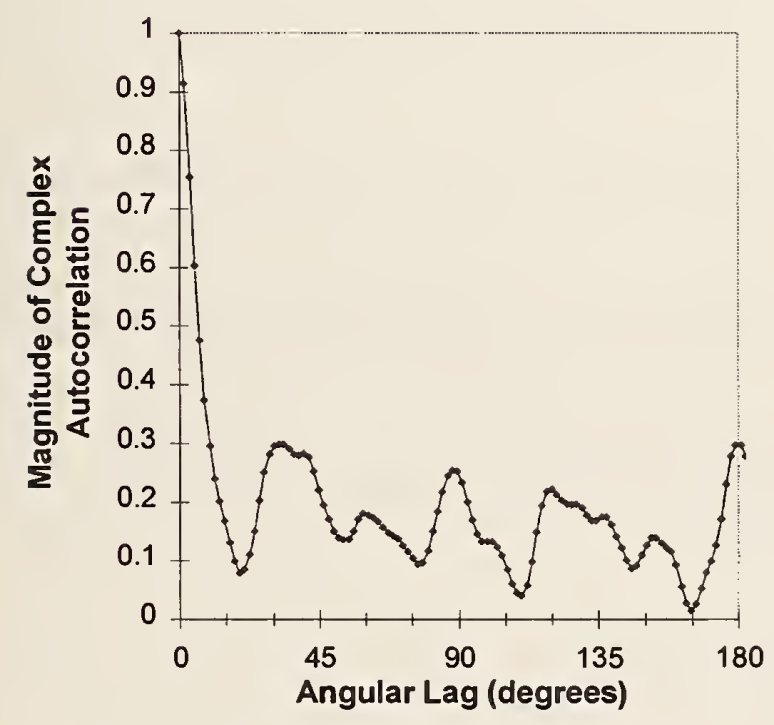

(c)

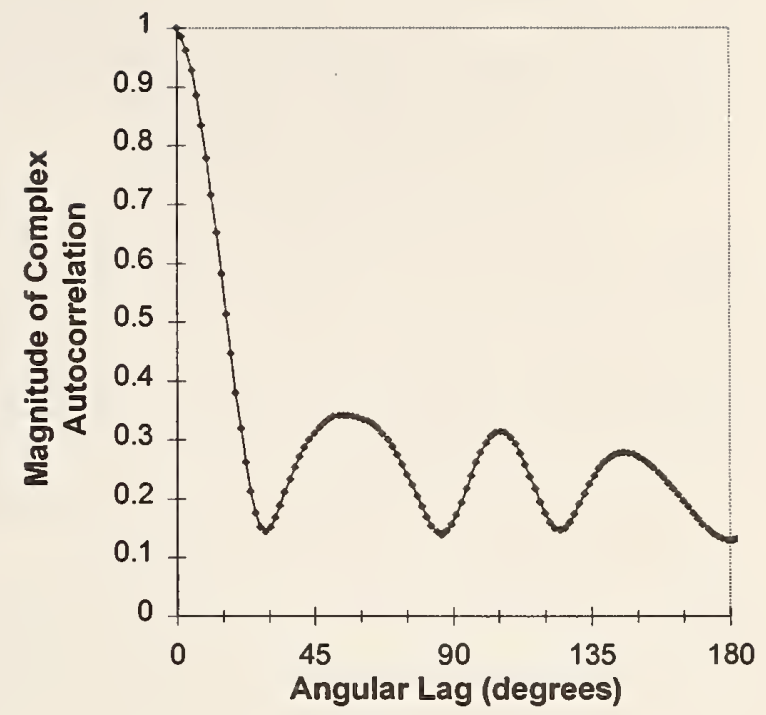

(b)

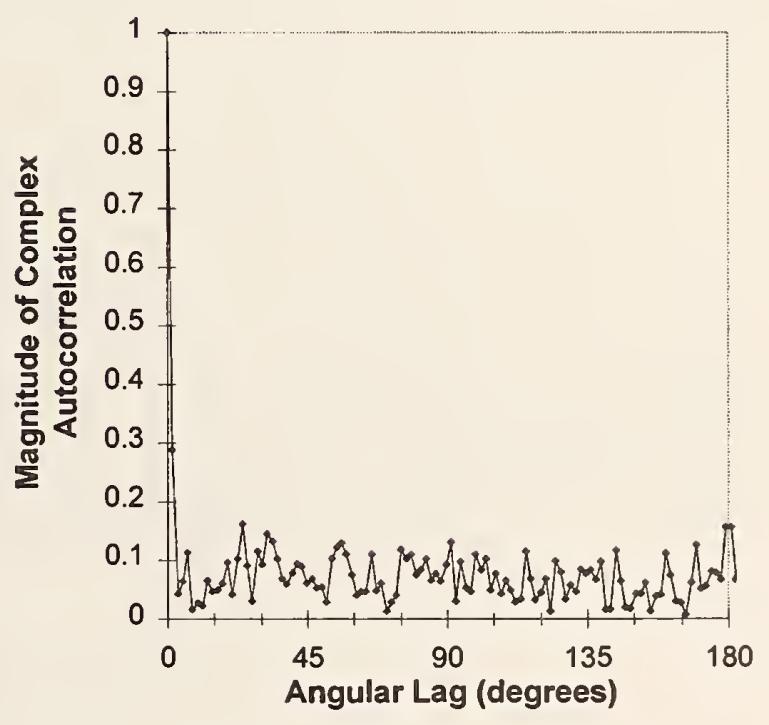

(d)

Figure 5. Autocorrelation of $S_{21}$ using one paddle. (a) Simulated data. (b) $90 \mathrm{MHz}$ in chamber B. (c) $200 \mathrm{MHz}$ in chamber B. (d) $1 \mathrm{GHz}$ in chamber B. 
indicating that successive data points are less correlated as frequency increases, as expected. One tool for measuring the independence of the data is the autocorrelation function [14]. Given a sequence of $\mathrm{N}$ samples, the autocorrelation function measures the relative correlation between the sequence and a shifted version of the same sequence. It is assumed that the mean is removed before the autocorrelation is calculated.

For no shift, the correlation will be perfect, and the autocorrelation function will have a value of 1 . For all other shift values, the autocorrelation function will have a magnitude between 0 and 1 , with values near 1 indicating strong correlation (poor independence) and values near 0 indicating weak correlation (good independence). Figure 5 shows the autocorrelation of the data shown in Figure 4. As expected, there is weak correlation for small changes in paddle position at high frequencies, but there is strong correlation at low frequencies. In this case, the assumption of independence is violated, and our model may behave poorly.

Given these potential problems with the idealized statistical model, it is still beneficial to analyze the model in great detail. This gives a baseline for comparison and allows us to evaluate when a statistical analysis is appropriate. To this end, we will now evaluate the characteristics of the idealized model, based on the assumption that the in-phase and quadrature components of the signal picked up by the receiving antenna are indeed independent, random and normally distributed. We will show in Section 6.3 that the statistical models do indeed accurately predict the behavior of the signals in a mode-stirred chamber.

\subsubsection{Statistical Notation}

Notation for the probability density function (pdf) and cumulative distribution function (cdf) for a given distribution is notoriously cumbersome. In this paper, a pdf will be written with a lower case $f$ followed by a subscripted letter or group of letters indicating the distribution. For example, $f_{N}(x)$ will represent the probability density function for the normal distribution. The variable $(x)$ is simply a place holder and chosen arbitrarily. Similarly, we will write the cdf for a specific distribution, defined as the integral of the pdf evaluated from $-\infty$ to $x$ (again, the choice of $x$ is arbitrary), as an uppercase $F$ followed by the same subscripted letter. For example, the cdf for a normal distribution is defined as

$$
F_{N}(x)=\int_{-\infty}^{x} f_{N}(y) d y .
$$

If we wish to denote some property of an arbitrary distribution, we will denote the pdf as $f_{A}(x)$ and the cdf as $F_{A}(x)$, where the subscript $A$ means arbitrary. The mean of distribution $A$ will be indicated as $\langle A\rangle$, and the variance will be indicated as $\operatorname{var}(A)$.

Occasionally we will state that a random variable has a specific distribution. For example, we may say that the random variable $\mathbf{y}$ has a normal distribution or is distributed normally. In this case, the fact that $\mathbf{y}$ is a random variable will be indicated by making the variable bold. For any random variable $\mathbf{y}$, the expected value of $\mathbf{y}$ will be indicated as $E(\mathbf{y})$, and this will be assumed to be the average of an infinite number of samples, each with the same distribution. 
Another useful notation is related to the concept of quantiles [16]. For any continuous distribution with cdf $F_{A}(x)$, there will be some value of $x$, call it $\xi_{q}$, such that $F_{A}\left(\xi_{q}\right)$ is equal to some desired value $q$. For example, if $q=0.025$, then, after a large number of measurements have been made, approximately $2.5 \%$ of the data will be less than $\xi_{0.025}$. We are particularly interested in the values of $\xi_{0.025}$ and $\xi_{0.975}$ because we expect $95 \%$ of our data to lie between these values. We will call the interval $\left(\xi_{0.025}, \xi_{0.975}\right)$ the $95 \%$ tolerance interval for the distribution we are interested in. In general, the values of $\xi_{0.025}$ and $\xi_{0.975}$ can be found for a particular value of $q$ by solving the equation $F_{A}\left(\xi_{q}\right)=q$ for $\xi_{q}$. This can be done in closed form in most cases, or the equation can be solved numerically. In general, the distribution for which we are calculating the quantiles should be obvious. If there is some ambiguity, we will attempt to specify the particular distribution. For example, $\xi_{0.025}(A)$ is the value such that $F_{A}\left(\xi_{0.025}(A)\right)=0.025$. Alternatively, if we specify a random variable $\mathbf{x}$ instead of a specific distribution, we will use the notation $\xi_{0.025}^{\mathbf{x}}$, with a superscript to indicate the particular random variable for which the quantile was calculated. This notation is cumbersome, however, so we will use it only when necessary.

Quantiles have one feature that is of great practical importance to us. Given any random variable $\mathbf{x}$ and any monotonic function $g$, we can construct a new random variable $\mathbf{y}=g(\mathbf{x})$. The quantiles of the new random variable $\mathbf{y}$ are simply given by $g$ applied to the previous quantiles calculated for the random variable $\mathbf{x}$, that is, $\xi_{0.025}^{\mathbf{y}}=g\left(\xi_{0.025}^{\mathbf{x}}\right)$. This is not necessarily true for the mean or variance of $\mathbf{y}, \mathrm{E}(\mathbf{y}) \neq g(\mathrm{E}(\mathbf{x}), \operatorname{var}(\mathbf{y}) \neq g(\operatorname{var}(\mathbf{x})$.

\subsubsection{Characteristics of Fundamental Distributions}

Given this notation, we can now examine some of the characteristics of the distributions that describe the operation of a mode-stirred chamber. The probability density function for a general normal random variable is given as the familiar bell curve equation,

$$
f_{N}(x)=\frac{1}{\sigma \sqrt{2 \pi}} e^{-(x-\mu)^{2} / 2 \sigma^{2}}
$$

where $\mu$ is the mean or expected value, $\sigma$ is the standard deviation, and $\sigma^{2}$ is the variance. For the normal distribution, $\xi_{0.025} \approx \mu-1.96 \sigma$ and $\xi_{0.975} \approx \mu+1.96 \sigma$.

The general $\chi^{2}$ distribution, or chi square distribution, is derived from the normal distribution as follows. Given $N$ random variables, $\mathbf{x}_{1}, \mathbf{x}_{2}, \ldots, \mathbf{x}_{N}$, each distributed normally with a mean of 0 and a variance of $\sigma^{2}$, another random variable $\mathbf{y}=\sum_{i=1}^{N}\left(\mathbf{x}_{i}\right)^{2}$ will have a $\chi^{2}$ distribution with $N$ degrees of freedom (dof). We will use the shorthand notation $\chi_{N}^{2}$ for this 
distribution. The normal distribution from which the random variable $\mathbf{x}_{i}$ are drawn will be called the parent normal distribution. If we define another random variable $\mathbf{z}=\sqrt{\mathbf{y}}=\sqrt{\sum_{i=1}^{N}\left(\mathbf{x}_{i}\right)^{2}}, \mathbf{z}$ will have a $\chi_{N}$ distribution, or a chi distribution with $N$ dof.

When we refer to a $\chi$ or $\chi^{2}$ distribution, we are actually referring to a family of distributions. That is, we specify the shape of the distribution but not the mean or scale factor of the distribution. Any of the $\chi$ or $\chi^{2}$ can be characterized by a single parameter, and throughout the majority of the report we will assume the these distributions are parametrized by the standard deviation $\sigma$ of the parent normal distribution. Thus, all statistical characteristics such as mean, variance, and quantiles will be written in terms of $\sigma$. More formally, we should write $\chi(\sigma)$ and $\chi^{2}(\sigma)$ to indicate that these distributions are parametrized by $\sigma$, but we will generally use the shorter notation. Statistical texts [17] often assume that $\sigma=1$, which removes the ambiguity but is not as general, so we will not follow this convention.

To complicate matters, other parameterizations are also useful. Specifically, although the $\chi$ or distributions can be specified by $\sigma$, it is sometimes more helpful to specify them by some characteristic of the distributions themselves, such as the mean. For example, it is sometimes better to say the $\chi_{2}^{2}$ distribution that has a mean of 1 than to say the $\chi_{2}^{2}$ distribution that is derived from a parent normal with $\sigma=1 / \sqrt{2}$, resulting in a mean of 1 . To differentiate these cases, we will use the following notation. When we need to specify a value for the standard deviation of the parent normal, we will use $\sigma_{p}=\alpha$, where $\alpha$ is an arbitrary constant. When we need to specify a value for the mean some distribution, we will use $\mu_{d}=\beta$, where $\beta$ is also an arbitrary constant. We will show that a distribution is parameterized by $\sigma_{p}$ using the notation $\left.\chi^{2}\right|_{\sigma_{p}=\alpha}$, and we will show that a distribution is parameterized by $\mu_{d}$ using the notation $\left.\chi^{2}\right|_{\mu_{d}=\beta}$. This notation is cumbersome, so we will use it only when necessary.

Both the in-phase and quadrature components of $S_{21}$ are assumed to be described by the normal distribution. We also assume that both the magnitude and phase of the electric field are independent, which implies that the in-phase and quadrature components of $S_{21}$ will be independent and identically distributed (iid). Since the magnitude is independent of the phase, the mean of the in-phase and quadrature components of $S_{21}$ will be 0 (the bivariate distribution is circularly symmetric). Thus, for statistically ideal data, a scatter plot of the measured values of $S_{21}$ will form a roughly circular pattern with a center (measured as $\left|\left\langle S_{21}\right\rangle\right|$ ) located at the origin (refer to Figure 4a). This does not imply that the measured mean will always be 0 , however. Page [18] asserts that a significant deviation from the origin can be indicative of poor stirring. Specifically, a deviation from the origin suggests that there is some component of $S_{21}$ that is not randomized. A simple example is a case of direct coupling between the transmitting and receiving antennas. This will result in a deterministic component which is superimposed on the random data, and the result will be a shift in the center of the scatter plot. Even with good stirring it is unlikely that the measured or sample mean will be exactly 0 . Consequently, we need a 
method for evaluating whether the offset is significant. This will be discussed in detail in Section 6.

For our analysis here, we will assume that all measurements are performed using a vector network analyzer (VNA), because this technique gives more information about the measurement process than scalar techniques. If only scalar data are available, as is the case when signals are measured using a power meter or spectrum analyzer, some of this analysis will not be possible, and, as a result, the estimates of the chamber parameters based on these measurements will have greater uncertainties associated with them. These considerations will be examined in Section 8 .

The power received by a network analyzer is equal to

$$
P_{\text {rec }}=P_{\text {inc }}\left|S_{21}\right|^{2}=P_{i n c}\left[\left(\operatorname{Re}\left(S_{21}\right)\right)^{2}+\left(\operatorname{Im}\left(S_{21}\right)\right)^{2}\right],
$$

where $P_{i n c}$ is the incident power available from the transmitting test port of the network analyzer, and $\operatorname{Re}\left(S_{21}\right)$ and $\operatorname{Im}\left(S_{21}\right)$ are the real and imaginary parts of $S_{21}$. Similarly, the reflected power is

$$
P_{\text {refl }}=P_{\text {inc }}\left|S_{11}\right|^{2}=P_{\text {inc }}\left[\left(\operatorname{Re}\left(S_{11}\right)\right)^{2}+\left(\operatorname{Im}\left(S_{11}\right)\right)^{2}\right]
$$

If the roles of the transmitting and receiving antennas are reversed, then these definitions will be $P_{\text {rec }}=P_{\text {inc }}\left|S_{12}\right|^{2}$ and $P_{\text {refl }}=P_{\text {inc }}\left|S_{22}\right|^{2}$. For now, we will restrict ourselves to the single-direction case, but the results are applicable regardless of which antenna is the transmitter and which is the receiver.

If the real and imaginary components of $S_{21}$ are independent and normally distributed, each with a mean of 0 and a variance of $\sigma^{2}$, the received power can be described by the $\chi_{2}^{2}$ distribution:

$$
f_{\chi_{2}^{2}}(x)=\frac{1}{2 \sigma^{2}} e^{-x / 2 \sigma^{2}} U(x),
$$

where $U(x)$ is the unit step function given by

$$
U(x)= \begin{cases}1 & x \geq 0 \\ 0 & x<0\end{cases}
$$

The $\chi_{2}^{2}$ distribution is also known as the exponential distribution. The $\chi_{2}^{2}$ distribution given in eq (41) has a mean of $2 \sigma^{2}$, a standard deviation of $2 \sigma^{2}$, and a variance of $4 \sigma^{4}$ [14]. For this distribution, $\xi_{0.025} \approx 0.051 \sigma^{2}$ and $\xi_{0.975} \approx 7.38 \sigma^{2}$. 
Since the mean and standard deviation of a $\chi_{2}^{2}$ random variable are both equal to $2 \sigma^{2}$, we can use a quick test to verify that measured data are consistent with the assumption of a $\chi_{2}^{2}$ distribution. The ratio of the sample standard deviation to the sample mean, which we will refer to as the normalized standard deviation should be approximately equal to 1 . For now this is simply a qualitative test. A more rigorous analysis must be performed if we wish to be more specific than "approximately equal to 1." A plot of the normalized standard deviation of data measured in Chamber A is shown in Figure 6. Figure 6 shows that the measured data are consistent with the assumption of a $\chi_{2}^{2}$ distribution for frequencies greater than $1 \mathrm{GHz}$, but there may be problems at lower frequencies.

The magnitude of a rectangular component of the electric field inside the chamber, defined as the square root of the sum of the squares of the in-phase and quadrature components, is proportional to the square root of the received power (this is true for a short dipole and assumed true for an extended antenna). We will call such an electric field a "single axis" electric field or a rectangular component of the electric field. The distribution of the magnitude of a rectangular component of the electric field is described by the $\chi_{2}$ distribution, also known as the Rayleigh distribution:

$$
f_{\chi_{2}}(x)=\frac{x}{\sigma^{2}} e^{-x^{2} / 2 \sigma^{2}} U(x)
$$

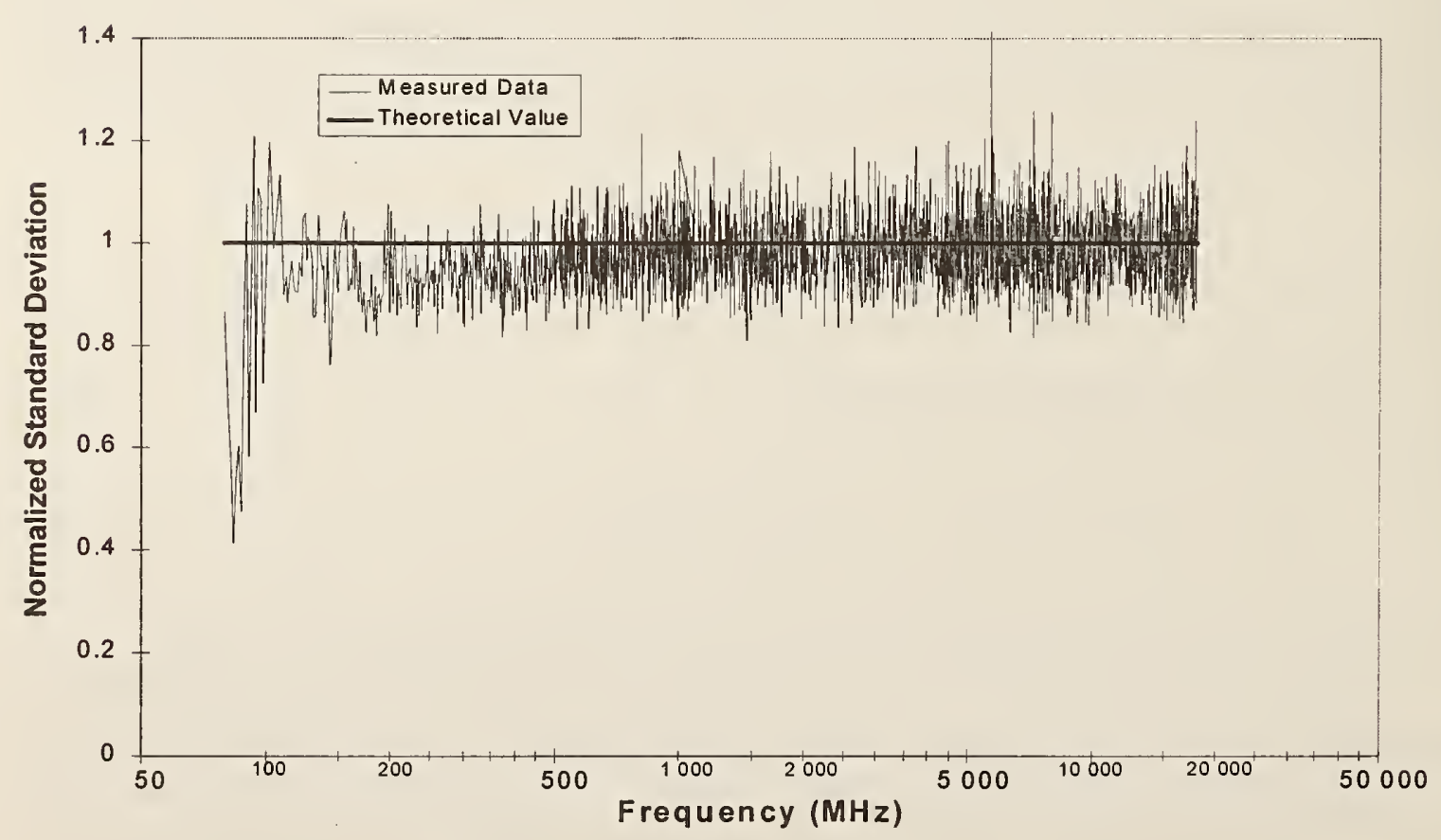

Figure 6. Normalized standard deviation of received power as measured in NASA chamber A. 
The mean of this distribution is $\sigma \sqrt{\pi / 2}$, the standard deviation is $\sigma \sqrt{2-\pi / 2}$, and the variance is $\sigma^{2}(2-\pi / 2)$ [14]. For this distribution, $\xi_{0.025} \approx 0.225 \sigma$ and $\xi_{0.975} \approx 2.72 \sigma$. These quantiles are simply the square root of the quantiles of the $\chi_{2}^{2}$ distribution. This is not true of the means, however. In fact, if $\mathbf{z}$ is a $\chi_{2}$ random variable and $\mathbf{y}$ is a $\chi_{2}^{2}$ random variable,

$$
E(z)=\frac{\sqrt{\pi}}{2} \sqrt{E(y)}
$$

We mentioned in Section 3.1 that the average squared rectangular component of the electric field can be calculated directly from the normalized chamber gain, but the average magnitude of a rectangular component of the electric field is somewhat more difficult. Equation (44) now gives us a method of calculating this quantity, assuming that the assumed distributions are valid:

$$
<\left|E_{R}\right|>=\frac{4 \pi}{\lambda} \sqrt{5 \pi<P_{R}>}
$$

Equation (44) shows that simply assuming that the average magnitude of the electric field is equal to the square root of the average squared magnitude of the electric field would result in an error of a factor of $\sqrt{\pi} / 2$. On a decibel scale, this is equivalent to an error of $20 \cdot \log _{10}(\sqrt{\pi} / 2)$ or approximately $1 \mathrm{~dB}$. Had the square root been calculated before the average, that is $\left\langle\sqrt{P_{R}}>\right.$ instead of $\sqrt{\left\langle P_{R}\right\rangle}$, the equation would be different. We would then have

$$
<\left|E_{R}\right|>=\frac{8 \pi}{\lambda}<\sqrt{5 P_{R}}>\text {. }
$$

Occasionally it is helpful to estimate the maximum or minimum magnitude of a rectangular component of the electric field as a function of the maximum or minimum received power. Ideally, we would like to base this estimate on either eq (45) or eq (46), but which is the better choice? We base our answer on the knowledge that the power received by an antenna at any given paddle position is approximately proportional to the square of the magnitude of a rectangular component of the electric field. From this, we can deduce that the magnitude of a rectangular component of the electric field has approximately the same distribution as the square root of the received power. As a result, we can generalize eq (46) to

$$
\left|E_{R}\right| \approx \frac{8 \pi}{\lambda} \sqrt{5 P_{R}}
$$


Since eq (47) is valid at each paddle position, then we can write the maximum and minimum of the magnitude of a rectangular component of the electric field as

$$
\operatorname{MAX}\left(\left|E_{R}\right|\right) \approx \frac{8 \pi}{\lambda} \sqrt{5 M A X\left(P_{R}\right)}
$$

and

$$
\operatorname{MIN}\left(\left|E_{R}\right|\right) \approx \frac{8 \pi}{\lambda} \sqrt{5 M I N\left(P_{R}\right)}
$$

The squared magnitude of the total electric field $\left|E_{T}\right|^{2}$, as defined in eq (14) is the sum of the squared magnitudes of the three orthogonal rectangular components of the electric field $\left|E_{R 1}\right|^{2}+\left|E_{R 2}\right|^{2}+\left|E_{R 3}\right|^{2}$. Since the magnitude of each rectangular component is assumed to be iid, and each rectangular component is assumed to be the sum of the squares of two normal iid random variable, $\left|E_{T}\right|^{2}$ is the sum of the squares of six normal iid random variables, and can be described by the $\chi_{6}^{2}$ distribution. Similarly, $\left|E_{T}\right|$ can be described by the $\chi_{6}$ distribution.

The pdf of the $\chi_{6}^{2}$ is

$$
f_{\chi_{6}^{2}}(x)=\frac{x^{2}}{16 \sigma^{6}} e^{-x / 2 \sigma^{2}} U(x)
$$

The $\chi_{6}^{2}$ distribution given in eq (50) has a mean of $6 \sigma^{2}$, a standard deviation of $\sigma^{2} \sqrt{12}$, and a variance of $12 \sigma^{4}$ [14]. For this distribution, $\xi_{0.025} \approx 1.237 \sigma^{2}$ and $\xi_{0.975} \approx 14.449 \sigma^{2}$.

The pdf of the $\chi_{6}$ is

$$
f_{\chi_{6}}(x)=\frac{x^{5}}{8 \sigma^{6}} e^{-x^{2} / 2 \sigma^{2}} U(x)
$$

The $\chi_{6}$ distribution given in eq (51) has a mean of $15 \sigma \sqrt{2 \pi} / 16$, a standard deviation of $\sigma \sqrt{6-(225 \pi / 128)}$, and a variance of $6 \sigma^{2}-\left(225 \pi \sigma^{2} / 128\right)$ [14]. For this distribution, $\xi_{0.025} \approx 1.112 \sigma$ and $\xi_{0.975} \approx 3.801 \sigma$.

Since the average squared magnitude of the total electric field is 3 times the average squared magnitude of a rectangular component of the electric field, as in eq (18), it has been assumed that the average magnitude of the total electric field is simply $\sqrt{3}$ times the average magnitude of a rectangular component of the electric field. We can now see that this is close, but not exactly correct. Specifically, if we compare the mean of the $\chi_{6}$ distribution given in eq (51) 
with the mean of the $\chi_{2}$ distribution given in eq (43), we see that the average magnitude of the total electric field is

$$
\left\langle\left|E_{T}\right|>=\left\langle\left|E_{R}\right|>\frac{15 \sigma \sqrt{2 \pi} / 16}{\sigma \sqrt{\pi / 2}}=\left\langle\left|E_{R}\right|>\frac{15}{8} .\right.\right.\right.
$$

Thus, by combining eqs (45) and (52), the average magnitude of the total electric field can be written as

$$
<\left|E_{T}\right|>=\frac{15}{8} \frac{4 \pi}{\lambda} \sqrt{5 \pi<P_{R}>}=\frac{15 \pi}{2 \lambda} \sqrt{5 \pi<P_{R}>} .
$$

\subsubsection{Characteristics of Distributions Expressed in Decibels}

Quantities associated with electromagnetic measurements are often expressed on a logarithmic (decibel) scale. This can affect the apparent underlying statistical properties of those quantities, so we need to examine this conversion in more detail. The general problem is, given some random variable $\mathbf{x}$ with a pdf $f_{A}(x)$, what is the pdf of another random variable $\mathbf{y}=k \log _{10}(\mathbf{x})$, where $k$ is 10 if $\mathbf{x}$ is a power quantity (received power, scalar power density), and $k$ is 20 if $\mathbf{x}$ is a voltage or current quantity (voltage, electric field, magnetic field). We will write the pdf of $\mathbf{y}$ as $f_{\mathrm{dB} A}(x)$, where the variable $y$ is arbitrary. We can calculate the pdf of $f_{\mathrm{dB} A}(x)$ using standard transformation techniques [14]. In general, given any pdf $f_{A}(x)$ that is known to be 0 for $x<0$, we can write $f_{\mathrm{dB} A}(x)$ as

$$
f_{\mathrm{dB} A}(y)=\frac{10^{y / k} \ln (10) f_{A}\left(10^{y / k}\right)}{k}
$$

Specifically, since $f_{\chi_{2}^{2}}$ describes the exponentially distributed squared magnitude of a rectangular component of the electric field and $f_{\chi_{2}}$ describes the Rayleigh distributed magnitude of a rectangular component of the electric field inside the chamber, we can write $f_{\mathrm{dB}_{2}^{2}}$ (which we will call the pdf of the $\mathrm{dB} \chi_{2}^{2}$ distribution) and $f_{\mathrm{dB} \chi_{2}}$ (which we will call the pdf of the $\mathrm{dB} \chi_{2}$ distribution) as 


$$
f_{\mathrm{dB} \chi_{2}^{2}}(y)=\frac{10^{y / 10} \ln (10) f_{\chi_{2}^{2}}\left(10^{y / 10}\right)}{10}
$$

and

$$
f_{\mathrm{dB} \chi_{2}}(y)=\frac{10^{y / 20} \ln (10) f_{\chi_{2}}\left(10^{y / 20}\right)}{20} .
$$

Substituting eq (41) into eq (55) and eq (43) into eq (56), we see that both of these distributions are identical:

$$
f_{\mathrm{dB} \chi_{2}^{2}}(y)=f_{\mathrm{dB} \chi_{2}}(y)=\frac{10^{y / 10} \ln (10) \exp \left(-10^{y / 10} / 2 \sigma^{2}\right)}{20 \sigma^{2}} .
$$

Similarly, by applying eq (54) to eqs (50) and (51), we can show that

$$
f_{\mathrm{dB} \chi_{6}^{2}}(y)=f_{\mathrm{dB} \chi_{6}}(y)=\frac{10^{3 y / 10} \ln (10) \exp \left(-10^{y / 10} / 2 \sigma^{2}\right)}{160 \sigma^{6}} .
$$

This means that the statistical characteristics of both the measured squared magnitude of the electric field (either total or rectangular component) and the measured electric field, when expressed in decibels, will be based on the same distribution. This is intuitively satisfying since conversion of a measured magnitude of the electric field to decibels requires us to square the measured value before the logarithm is computed, effectively converting the field measurement into a power measurement.

A more subtle interpretation of these results is that, if all measurements are made in terms of decibels (decibels with respect to $1 \mathrm{~V} / \mathrm{m}$, decibels with respect to $1 \mathrm{~W}$, etc.), then a statistical evaluation of the measured values will give identical results, except for a possible offset. Thus, the standard deviation, variance, and all central moments will be identical. If the measurements are made using linear units (volts per meter, watts, etc.) this may not be the case. As a result, it is important to verify that any piece of automated test equipment averages linear values and not logarithmic values. One example where this may be a problem is a spectrum analyzer where the "video average" of the measured data is displayed on a logarithmic scale. If measurements are made while the paddle is stationary, the problem should be negligible, but if measurements are made while the paddle is moving, this could result in a biased estimate of the average received power.

We computed some of the properties of the $\mathrm{dB} \chi_{2}^{2}$ distribution using numerical techniques. The mean of this distribution is approximately $10 \cdot \log _{10}\left(2 \sigma^{2}\right)-2.507$, the standard deviation is independent of $\sigma$ and has a value of approximately 5.57, and the variance is 
approximately 31.02 . Thus, $\sigma$ influences the value of the mean, but not the standard deviation or the variance. For this distribution, $\xi_{0.025} \approx 10 \cdot \log _{10}\left(0.051 \sigma^{2}\right)$ and $\xi_{0.975} \approx 10 \cdot \log _{10}\left(7.38 \sigma^{2}\right)$. The $\mathrm{dB} \chi_{2}^{2}$ distribution is a good example of a distribution with properties that are somewhat different from what would be expected based on the linear version of the same distribution. The mean of the $\chi_{2}^{2}$ distribution given in eq (41) is $2 \sigma^{2}$, so it might appear reasonable to assume that the mean of the $\mathrm{dB} \chi_{2}^{2}$ distribution would be $10 \cdot \log _{10}\left(2 \sigma^{2}\right)$, but this estimate is off by slightly more than $2.5 \mathrm{~dB}$.

The mean of the $\mathrm{dB} \chi_{6}^{2}$ distribution is approximately $10 \cdot \log _{10}\left(6 \sigma^{2}\right)-0.764$, the standard deviation is independent of $\sigma$ and has a value of approximately 2.729 , and the variance is approximately 7.449. Thus again, $\sigma$ influences the value of the mean, but not the standard deviation or the variance. For this distribution, $\xi_{0.025} \approx 10 \cdot \log _{10}\left(1.237 \sigma^{2}\right)$ and $\xi_{0.975} \approx 10 \cdot \log _{10}\left(14.449 \sigma^{2}\right)$.

We can apply eq (54) to any distribution that is known to be nonnegative, that is, any distribution for which the probability of getting a value of less than 0 is 0 . Technically, eq (54) cannot be applied to the normal distribution because there is always a nonzero probability that a measurement will result in a data point with a value that is less than or equal to 0 , and the logarithm of this nonpositive number is undefined. If the standard deviation is small relative to the mean $(\sigma / \mu<0.3)$, then the probability of measuring a negative value is very small ( < $0.043 \%$ ), and we can make a good approximation. Assuming $\mathbf{x}$ is normally distributed with $\sigma / \mu$ $<0.3$, and using standard uncertainty analysis techniques [19], $k \log _{10}(\mathbf{x})$ will also be approximately normal, with mean $k \log _{10}(\mu)$ and standard deviation $k \sigma /(\mu \ln (10))$.

\subsubsection{Cumulative Distributions of Measured Data}

The cumulative distribution of measured received power is occasionally compared to the theoretical cumulative distribution as a method of verifying the statistical model [20]. To simplify the comparison, the measured data are normalized to a mean of one by dividing each measured value by the sample mean. These normalized data are then compared to a normalized version of the $\chi_{2}^{2}$ distribution (denoted $\left.\chi^{2}\right|_{\mu_{d}=1}=\hat{\chi}_{2}^{2}$ ), which is simply the general $\chi_{2}^{2}$ distribution given in eq (41) with mean $2 \sigma^{2}=1$ :

$$
f_{\hat{\chi}_{2}^{2}}(x)=e^{-x} U(x)
$$

The cumulative distribution is

$$
F_{\hat{\chi}_{2}^{2}}(x)=\left(1-e^{-x}\right) U(x) .
$$




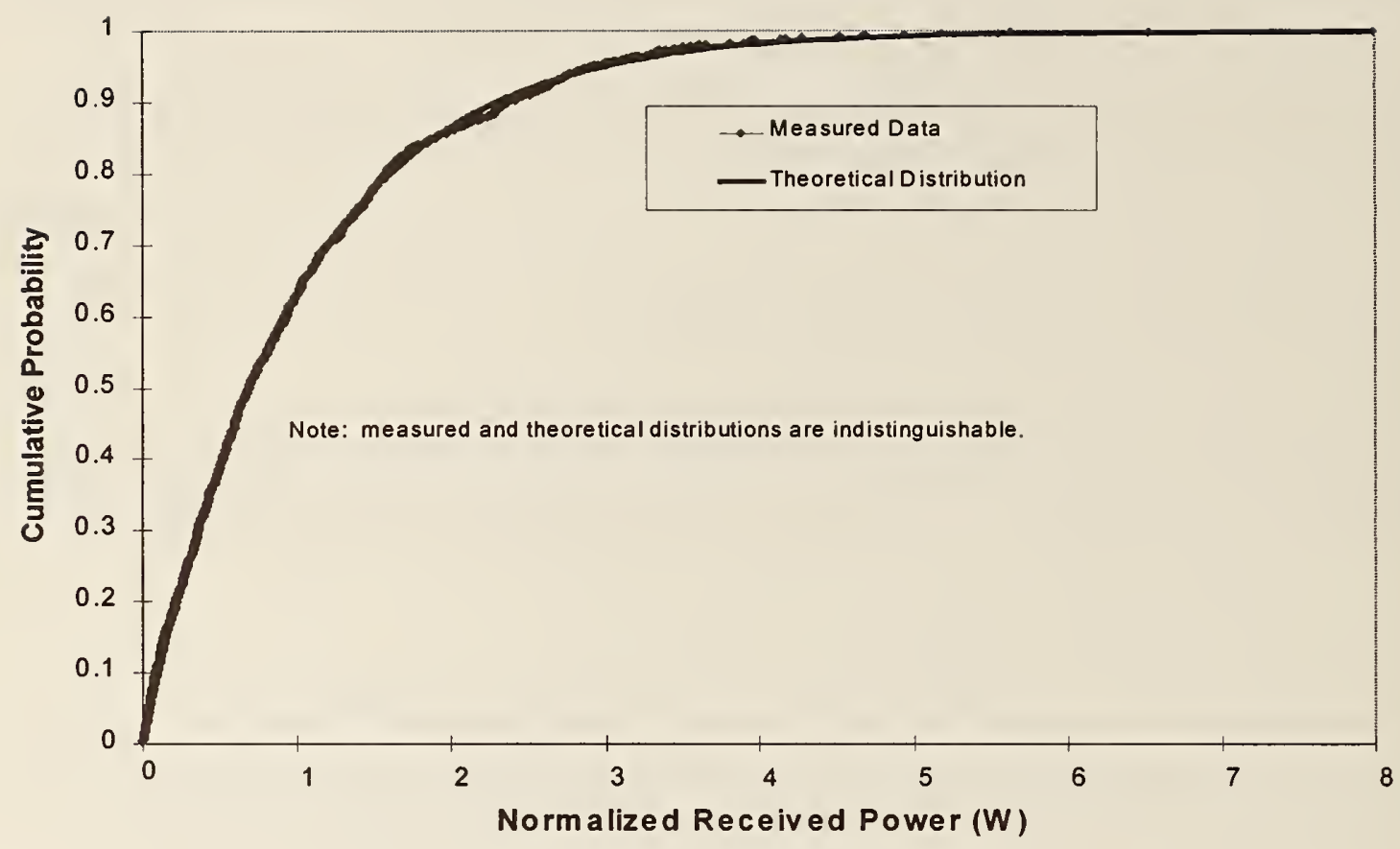

(a)

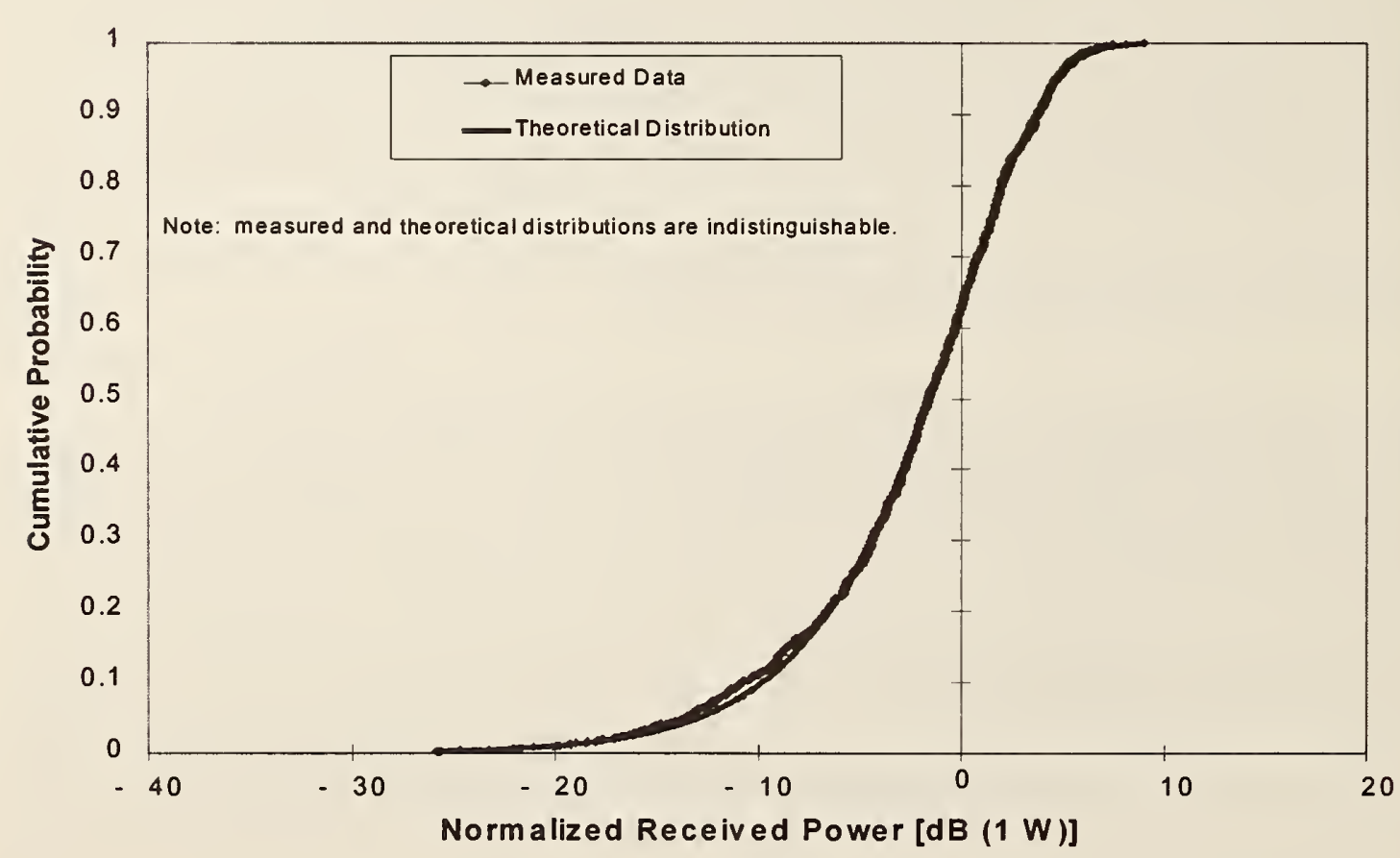

(b)

Figure 7. Comparison of theoretical cumulative distribution with measured cumulative distribution. (a) Comparison with the standard $\chi_{2}^{2}$ distribution. (b) Comparison with the standard $\mathrm{dB} \chi_{2}^{2}$ distribution. 
Figure 7a shows a comparison between the theoretical and measured cumulative distribution of data measured in Chamber A.

We can also compare the cumulative distribution of the normalized data expressed in decibels to the theoretical $\mathrm{dB} \hat{\chi}_{2}^{2}$ distribution. The standard $\mathrm{dB} \hat{\chi}_{2}^{2}$ distribution is the general $\mathrm{dB} \chi_{2}^{2}$ distribution given in eq (57) with $2 \sigma^{2}=1$ :

$$
f_{\mathrm{dB} \hat{\chi}_{2}^{2}}(y)=\frac{10^{y / 10} \ln (10) \exp \left(-10^{y / 10}\right)}{10} .
$$

The cumulative $\mathrm{dB} \hat{\chi}_{2}^{2}$ distribution is

$$
F_{\mathrm{dB} \hat{\chi}_{2}^{2}}(x)=\int_{-\infty}^{x} f_{\mathrm{dB} \hat{\chi}_{2}^{2}}(y) d y=1-\exp \left(-10^{x / 10}\right)=1-\exp \left(-\exp \left(\frac{x \ln 10}{10}\right)\right) .
$$

Figure $7 \mathrm{~b}$ shows a comparison between the theoretical and measured cumulative distribution of the same data shown in Figure 7a, but expressed on a logarithmic scale. We will plot all subsequent comparisons of the cumulative distribution on a logarithmic scale in an effort to be consistent with Freyer et al. [20]. 


\subsubsection{Extreme Values of Fundamental Distributions}

In addition to the basic distributions, it is helpful to examine the properties of the extremes of these distributions. If $N$ independent samples are taken from some known arbitrary distribution with probability density function $f_{A}(x)$ and cumulative distribution $F_{A}(x)$, it is possible to write the probability density function of the minimum and maximum of those samples, based on the theory of order statistics [14]. We will use the notation $\lfloor A\rfloor_{N}$ to indicate the minimum of $N$ independent samples, each taken from distribution $A$. Similarly, we will use the notation $\lceil A\rceil_{N}$ to indicate the maximum of $N$ independent samples, each taken from distribution $A$. This notation is used to specify distributions, not numbers. That is, $\lfloor A\rfloor_{N}$ and $\lceil A\rceil_{N}$ are distributions themselves, derived from the distribution $A$. The distribution of the minimum of $N$ samples is

$$
f_{\lfloor A\rfloor_{N}}(x)=N\left[1-F_{A}(x)\right]^{N-1} f_{A}(x)
$$

and the distribution of the maximum of $N$ samples is

$$
f_{\lceil A\rceil_{N}}(x)=N\left[F_{A}(x)\right]^{N-1} f_{A}(x) .
$$

Also, for any given distribution, averaging $N$ values together will not alter the expected value, but the standard deviation will be reduced by a factor of $\sqrt{N}$. If $N$ is large, the distribution of the average of $N$ samples will be approximately normal [14].

We will now examine the extreme values of the $\chi$ and $\chi^{2}$ distributions, expressed in both linear units and in decibels, in greater detail. Fortunately, all of these linear distributions have been analyzed extensively [17], and the decibel distributions are easy to evaluate numerically. For brevity, we will not explicitly write out the pdf for any of the logarithmic density functions except for those that are easy to compute and write, since they are bulky and can be evaluated numerically using only the linear forms and eq (54). Also, to simplify matters, the characteristics of the $\mathrm{dB} \chi$ distributions will not be specifically addressed here, since these distributions are identical to the $\mathrm{dB} \chi^{2}$ distributions. Finally, we will not spend much time discussing the quantiles of the logarithmic distributions, as these values can be easily obtained from the linear quantiles as discussed above. 
The probability density functions for the minimum and the maximum of $N \chi_{2}^{2}$ samples are

$$
f_{\left\lfloor\chi_{2}^{2}\right\rfloor_{N}}(x)=\frac{N}{2 \sigma^{2}} e^{-x N / 2 \sigma^{2}} U(x)
$$

and

$$
f_{\left\lceil\chi_{2}^{2}\right\rceil_{N}}(x)=\frac{N}{2 \sigma^{2}}\left[1-e^{-x / 2 \sigma^{2}}\right]^{N-1} e^{-x / 2 \sigma^{2}} U(x)
$$

The distribution for $f\left\lfloor\chi_{2}^{2}\right\rfloor_{N}(x)$ is a scaled $\chi_{2}^{2}$ distribution. Thus the mean of $\left\lfloor\chi_{2}^{2}\right\rfloor_{N}$ is $2 \sigma^{2} / N$, the standard deviation is $2 \sigma^{2} / N$, and the variance is $4 \sigma^{4} / N^{2}$. For this distribution, $\xi_{0.025} \approx 0.051 \sigma^{2} / N$ and $\xi_{0.975} \approx 7.38 \sigma^{2} / N$.

Since $\left\lfloor\chi_{2}^{2}\right\rfloor_{N}$ is exponentially distributed, the properties of $\left[\mathrm{dB}_{2}^{2}\right\rfloor_{N}$ are easy to compute. The mean is approximately $10 \cdot \log \left(2 \sigma^{2} / N\right)-2.507$, the standard deviation is approximately 5.57 , and the variance is approximately 31.02 . The quantiles can be calculated by calculating 10 times the common log of the quantiles of $\left\lfloor\chi_{2}^{2}\right\rfloor_{N}$.

The properties of $\left\lceil\chi_{2}^{2}\right\rceil_{N}$ are more difficult to derive. The mean and variance are given by Johnson [17], and the quantiles are exact. The results are presented below:

$$
\begin{gathered}
\left\langle\left\lceil\chi_{2}^{2}\right\rceil_{N}\right\rangle=2 \sigma^{2} \sum_{i=1}^{N} \frac{1}{i} \approx 2 \sigma^{2}\left(0.577+\ln (N)+\frac{1}{2 N}\right), \quad N>1, \\
\operatorname{var}\left(\left[\chi_{2}^{2}\right\rceil_{N}\right)=4 \sigma^{4} \sum_{i=1}^{N} \frac{1}{i^{2}} \approx 4 \sigma^{4}\left(\frac{\pi^{2}}{6}-\frac{1}{N}\right), \quad N>5, \\
\xi_{0.025}\left(\left[\chi_{2}^{2}\right\rceil_{N}\right)=-2 \sigma^{2} \ln \left(1-0.025^{1 / N}\right) \\
\xi_{0.975}\left(\left[\chi_{2}^{2}\right\rceil_{N}\right)=-2 \sigma^{2} \ln \left(1-0.975^{1 / N}\right) .
\end{gathered}
$$

The approximations in eqs (67) and (68) are from Gradshteyn [21]. 
Table 2. Properties of the $\left\lceil\chi_{2}^{2}\right\rceil_{N}$ distribution for $\sigma=1$.

\begin{tabular}{rrrrrr}
\hline$N$ & Mean & $\begin{array}{r}\text { Standard } \\
\text { deviation }\end{array}$ & Variance & $\xi_{0.025}$ & $\xi_{0.975}$ \\
\hline 1 & 2.000 & 2.000 & 4.000 & 0.051 & 7.378 \\
2 & 3.000 & 2.236 & 5.000 & 0.344 & 8.752 \\
5 & 4.566 & 2.420 & 5.856 & 1.301 & 10.576 \\
10 & 5.858 & 2.490 & 6.200 & 2.352 & 11.960 \\
20 & 7.196 & 2.526 & 6.381 & 3.562 & 13.346 \\
50 & 8.998 & 2.550 & 6.503 & 5.286 & 15.178 \\
100 & 10.374 & 2.558 & 6.543 & 6.636 & 16.564 \\
200 & 11.756 & 2.562 & 6.564 & 8.004 & 17.950 \\
225 & 11.992 & 2.562 & 6.564 & 8.238 & 18.184 \\
400 & 13.140 & 2.564 & 6.574 & 9.382 & 19.336 \\
500 & 13.586 & 2.564 & 6.574 & 9.826 & 19.782 \\
800 & 14.524 & 2.564 & 6.574 & 10.764 & 20.720 \\
1000 & 14.970 & 2.564 & 6.574 & 11.208 & 21.160 \\
1024 & 15.018 & 2.564 & 6.574 & 11.256 & 21.220 \\
1600 & 15.910 & 2.564 & 6.574 & 12.148 & 22.100 \\
2000 & 16.356 & 2.564 & 6.574 & 12.594 & 22.560 \\
3200 & 17.296 & 2.564 & 6.574 & 13.532 & 23.500 \\
5000 & 18.190 & 2.564 & 6.574 & 14.424 & 24.380 \\
10000 & 19.576 & 2.566 & 6.584 & 15.810 & 25.780 \\
\hline
\end{tabular}

We cannot generate plots of parameters for all values of $\sigma$, so it is helpful to assume a specific value for the standard deviation of the original normal from which all of the distributions are generated. For all plots and tables we will assume that $\sigma=1$, except where noted. The normalized mean and $95 \%$ tolerance interval of the $\left\lceil\chi_{2}^{2}\right\rceil_{N}$ distribution are plotted as a function of $N$ in Figure 8a, and the standard deviation is plotted in Figure 8b. Values for $N<50$ were generated by numerical integration. The values of the normalized mean, variance, and the $2.5 \%$ and $97.5 \%$ quantiles are given in Table 2 . To use Table 2 for arbitrary values of $\sigma$, multiply the mean, standard deviation, and quantile terms by $\sigma^{2}$, and multiply the variance term by $\sigma^{4}$.

Equations (67) and (68) show that the mean of $\left\lceil\chi_{2}^{2}\right\rceil_{N}$ diverges as $N$ increases, and that the variance of $\left[\chi_{2}^{2}\right]_{N}$ approaches a nonzero constant as $N$ increases. Thus, taking more data will increase the variance or standard deviation. 


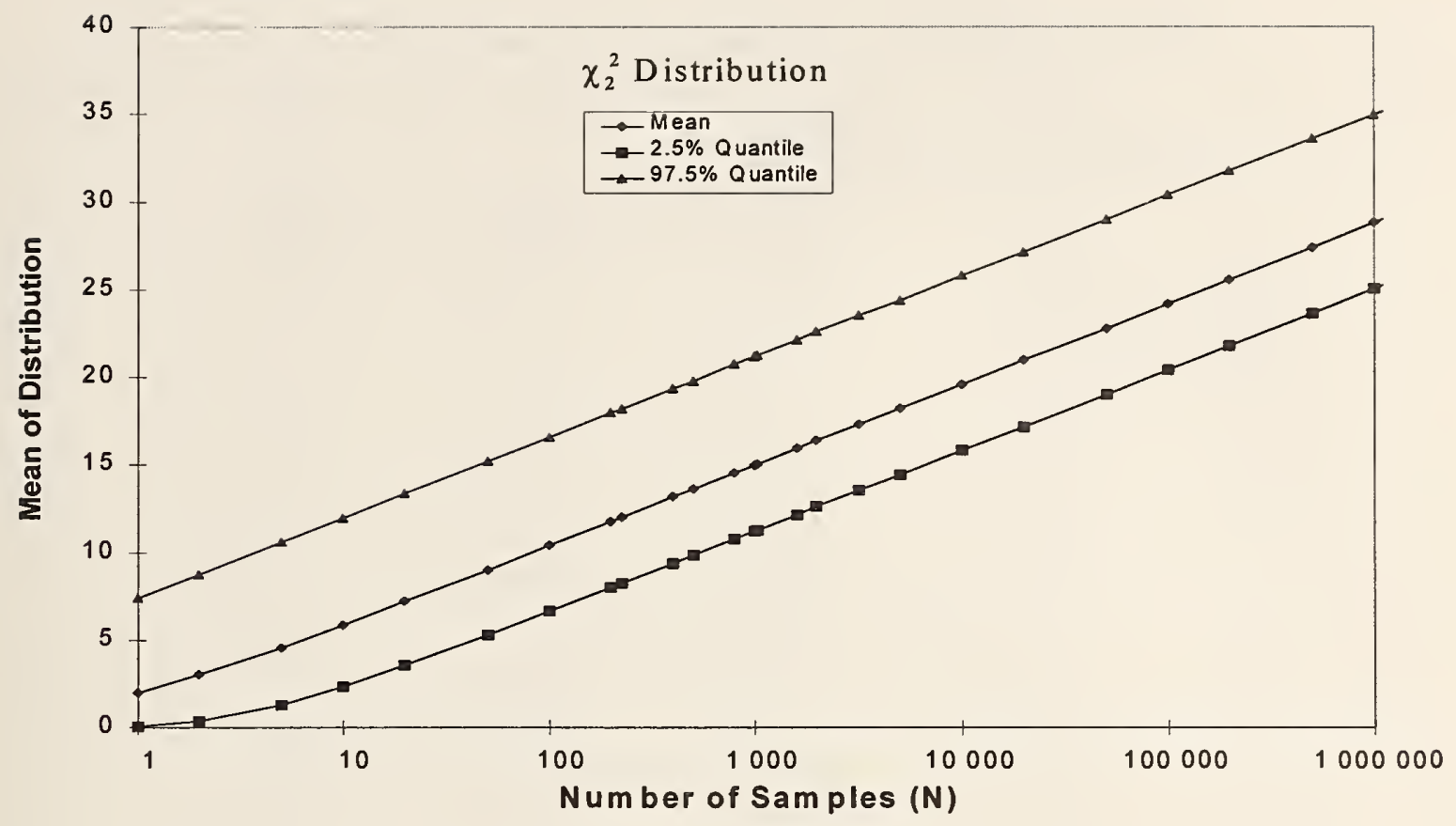

(a)

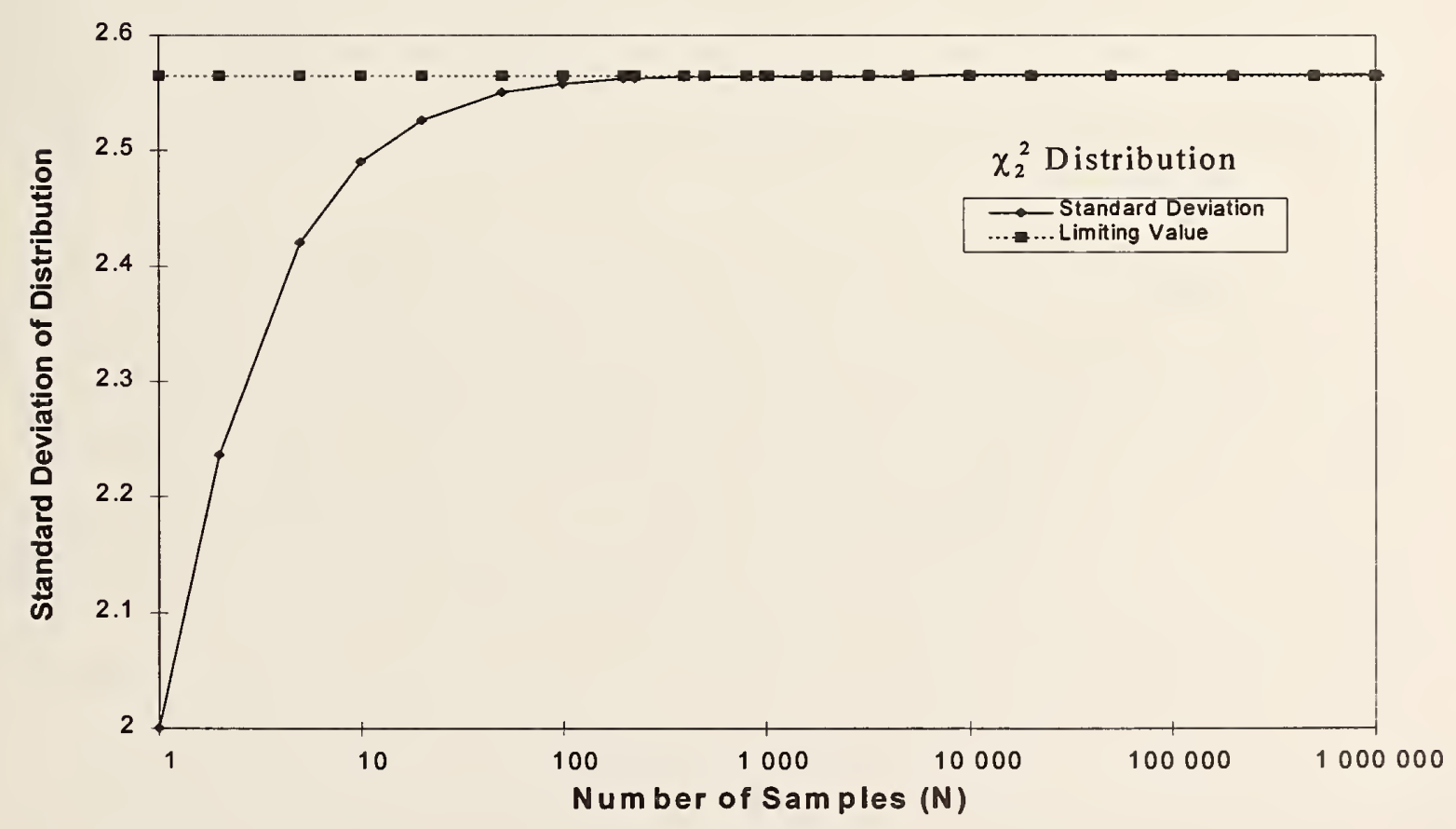

(b)

Figure 8. Statistical properties of the $\mathrm{N}^{\text {th }}$ order statistic (maximum) of a standard $\chi_{2}^{2}$ distribution, assuming $\mathrm{N}$ samples. (a) Mean, $2.5 \%$ quantile, and $97.5 \%$ quantile. (b) Standard deviation. 
Table 3. Properties of the $\left\lceil\mathrm{dB} \chi_{2}^{2}\right\rceil_{N}$ distribution for $\sigma=1$.

\begin{tabular}{rrrrrr}
\hline$N$ & Mean & $\begin{array}{r}\text { Standard } \\
\text { deviation }\end{array}$ & Variance & $\xi_{0.025}$ & $\xi_{0.975}$ \\
\hline 1 & 0.503 & 5.569 & 31.020 & -12.960 & 8.679 \\
2 & 3.514 & 3.592 & 12.900 & -4.632 & 9.420 \\
5 & 6.007 & 2.318 & 5.373 & 1.142 & 10.243 \\
10 & 7.309 & 1.798 & 3.233 & 3.714 & 10.777 \\
20 & 8.322 & 1.461 & 2.134 & 5.517 & 11.253 \\
50 & 9.381 & 1.167 & 1.363 & 7.232 & 11.812 \\
100 & 10.038 & 1.013 & 1.026 & 8.219 & 12.191 \\
200 & 10.607 & 0.895 & 0.801 & 9.033 & 12.540 \\
225 & 10.697 & 0.878 & 0.770 & 9.158 & 12.597 \\
400 & 11.109 & 0.802 & 0.643 & 9.722 & 12.863 \\
500 & 11.259 & 0.776 & 0.602 & 9.923 & 12.962 \\
800 & 11.558 & 0.727 & 0.528 & 10.319 & 13.160 \\
1000 & 11.693 & 0.706 & 0.498 & 10.495 & 13.260 \\
1024 & 11.707 & 0.703 & 0.495 & 10.514 & 13.270 \\
1600 & 11.964 & 0.665 & 0.442 & 10.844 & 13.450 \\
2000 & 12.087 & 0.647 & 0.419 & 11.001 & 13.530 \\
3200 & 12.335 & 0.613 & 0.375 & 11.313 & 13.710 \\
5000 & 12.557 & 0.583 & 0.340 & 11.591 & 13.870 \\
10000 & 12.882 & 0.543 & 0.295 & 11.989 & 14.110 \\
\hline
\end{tabular}

We will not attempt to derive closed-form expressions for the properties of $\left\lceil\mathrm{dB} \chi_{2}^{2}\right\rceil_{N}$. Instead, we calculated the properties at specific values of $N$ using numerical methods. The normalized mean and $95 \%$ tolerance interval as a function of $N$ are presented in Figure $9 \mathrm{a}$, and the standard deviation of $\left\lceil\mathrm{dB} \chi_{2}^{2}\right\rceil_{N}$ is presented in Figure $9 \mathrm{~b}$. The mean, standard deviation, variance, and $95 \%$ tolerance interval are listed in Table 3 . To use Table 3 for arbitrary values of $\sigma$, the standard deviation and variance terms are unchanged, and $10 \cdot \log _{10}\left(\sigma^{2}\right)$ should be added to the mean and quantile terms.

Several interesting results come from Table 2 and Table 3. First, the standard deviation of $\left\lceil\mathrm{dB} \chi_{2}^{2}\right\rceil_{N}$ decreases as $N$ increases, whereas the standard deviation of $\left\lceil\chi_{2}^{2}\right\rceil_{N}$ approaches a constant. Thus, while there is no apparent reason to use large values of $N$ for data plotted on a linear scale, there is an improvement if the data are plotted on a logarithmic scale. This unusual result comes from the fact that, for $\left\lceil\chi_{2}^{2}\right\rceil_{N}$, the mean continues to increase even though the standard deviation approaches a constant, and this results in a relative standard deviation (standard deviation divided by the mean) that decreases as $N$ increases. Second, if we were to convert the mean of $\left\lceil\chi_{2}^{2}\right\rceil_{N}$ to decibels by taking the common logarithm and multiplying by 10 , 


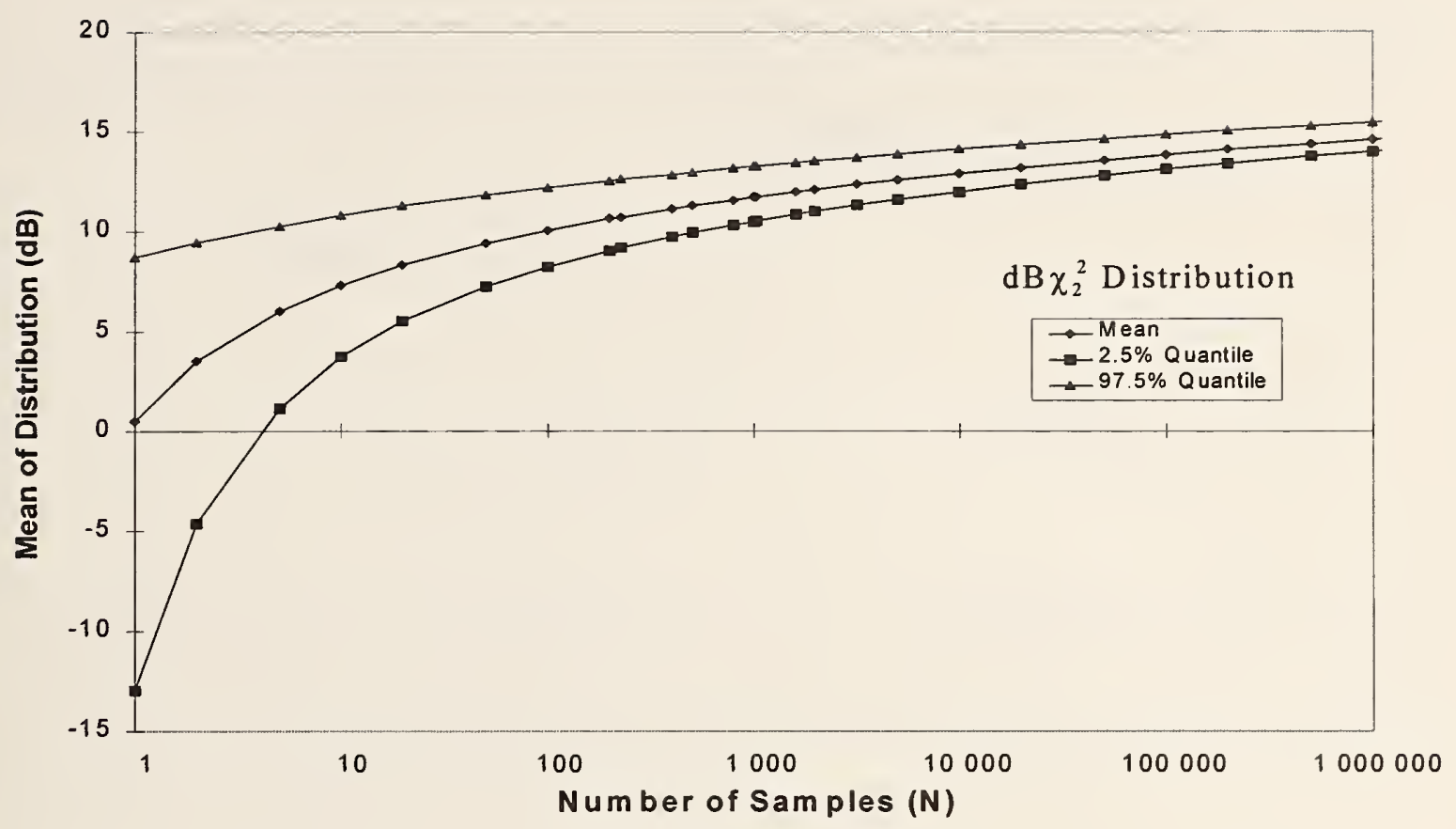

(a)

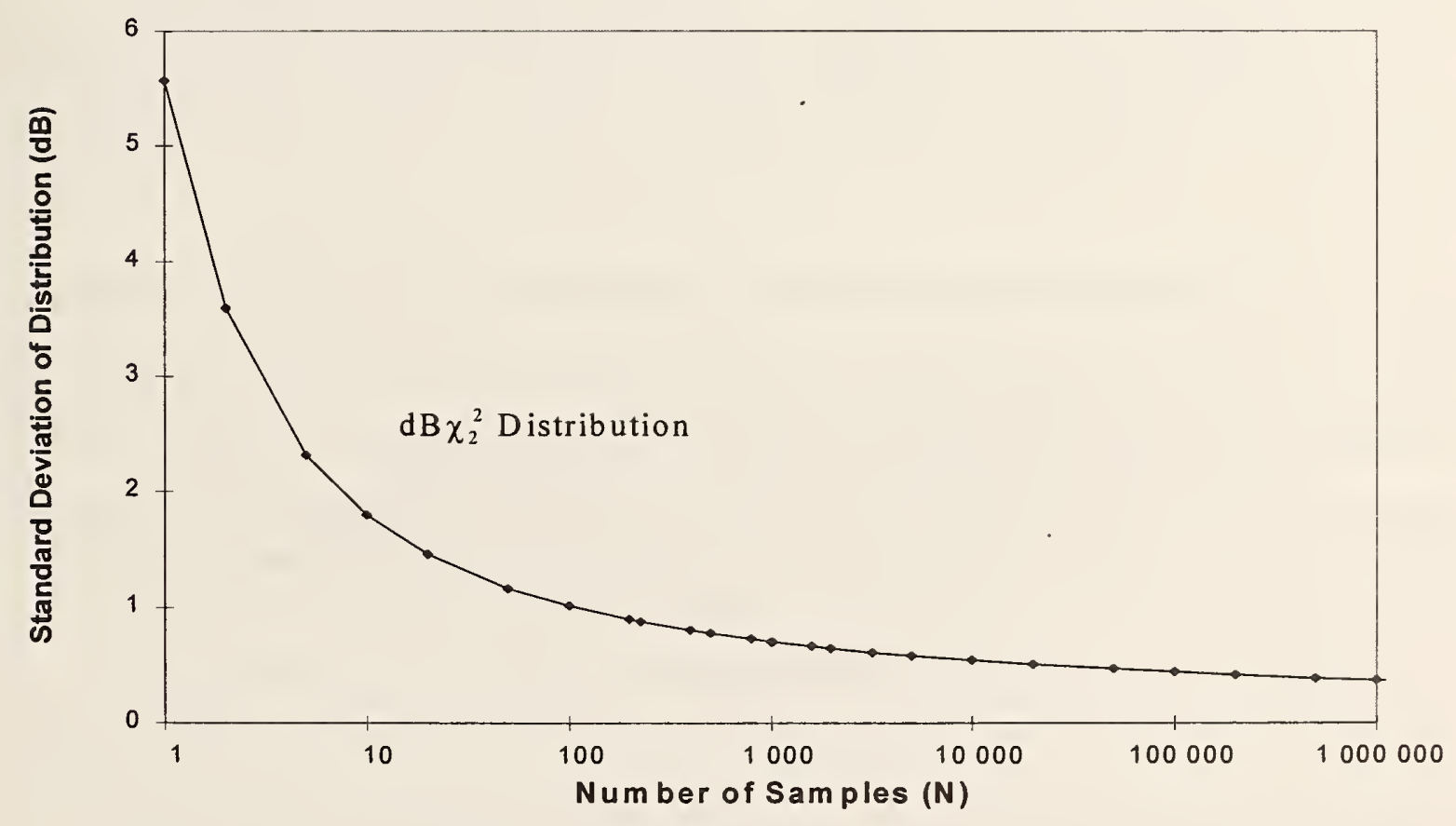

(b)

Figure 9. Statistical properties of the $\mathrm{N}^{\text {th }}$ order statistic (maximum) of a standard $\mathrm{dB} \chi_{2}^{2}$ distribution, assuming $\mathrm{N}$ samples. (a) Mean, $2.5 \%$ quantile, and $97.5 \%$ quantile. (b) Standard deviation. 


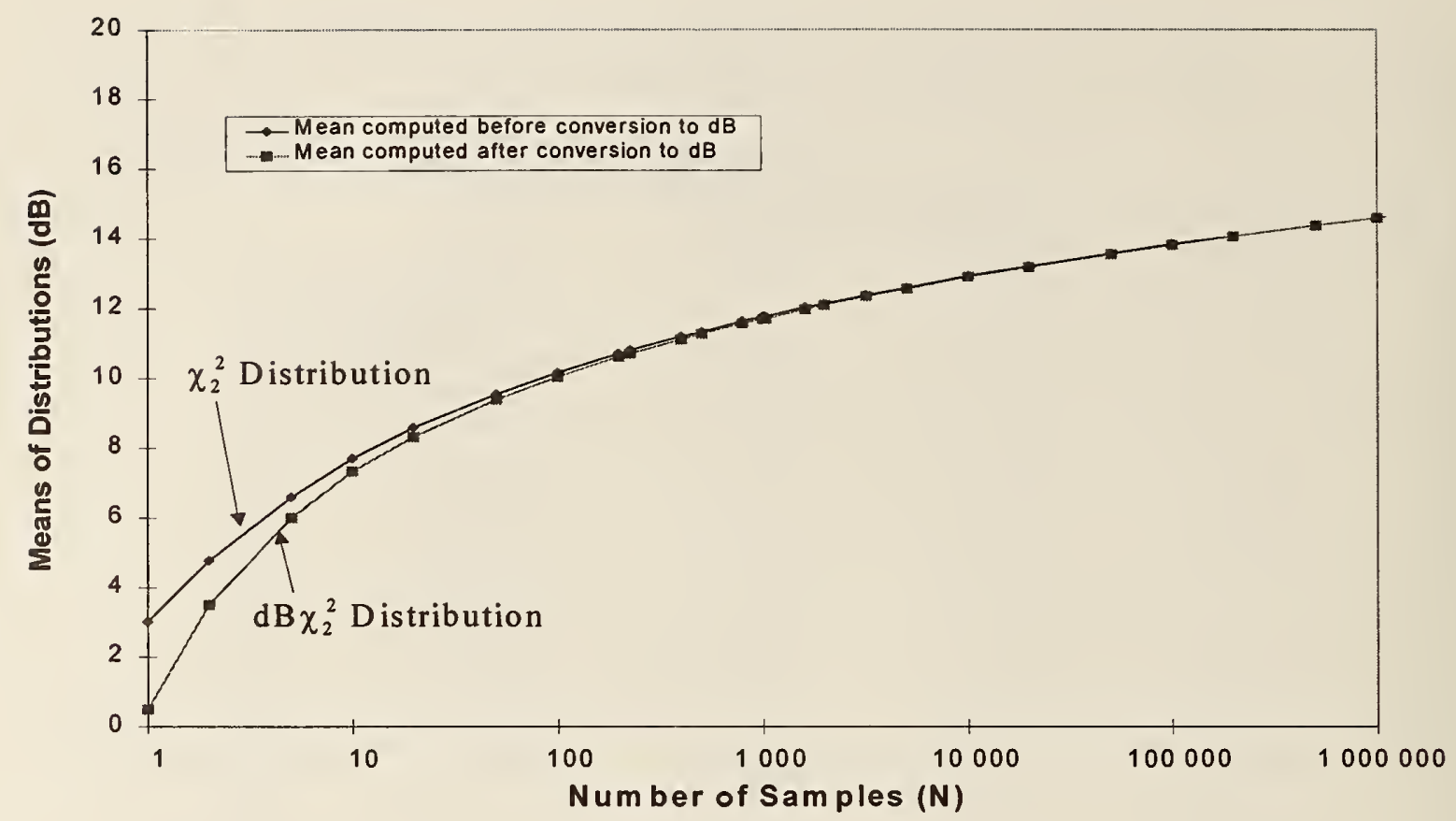

Figure 10. Comparison of means of the $\mathrm{N}^{\text {th }}$ order statistics (maximums) of a standard $\chi_{2}^{2}$ distribution and a standard $\mathrm{dB} \chi_{2}^{2}$ distribution, assuming $\mathrm{N}$ samples. Both means are plotted on a decibel scale for comparison.

the result is similar (the difference is less than $0.1 \mathrm{~dB}$ ) to the mean of $\left\lceil\mathrm{dB} \chi_{2}^{2}\right\rceil_{N}$ for $N \geq 200$. Both of these means are shown in Figure 10. Finally, if we examine the quantiles of $\left\lceil\chi_{2}^{2}\right\rceil_{N}$, we see that they do not appear to be symmetric about the mean. The quantiles of $\left\lceil\mathrm{dB} \chi_{2}^{2}\right\rceil_{N}$ on the other hand are more symmetric. This implies that the logarithmic distributions may be more similar to a normal distribution than the linear distributions, and the simple assumptions of general uncertainty analysis [19] (the data are normally distributed with a small standard deviation) may be more applicable to $\left[\mathrm{dB} \chi_{2}^{2}\right\rceil_{N}$ than to $\left\lceil\chi_{2}^{2}\right\rceil_{N}$. 
We now turn our attention to the $\chi_{2}$ distribution. The probability density functions for the minimum and the maximum of $N \chi_{2}$ samples are

$$
f_{\left\lfloor\chi_{2}\right\rfloor_{N}}(x)=\frac{N x}{\sigma^{2}} e^{-x^{2} N / 2 \sigma^{2}} U(x)
$$

and

$$
f_{\left\lceil\chi_{2}\right\rceil_{N}}(x)=\frac{N x}{\sigma^{2}}\left[1-e^{-x^{2} / 2 \sigma^{2}}\right]^{N-1} e^{-x^{2} / 2 \sigma^{2}} U(x) .
$$

The distribution for $\left\lfloor\chi_{2}\right\rfloor_{N}$ is a scaled $\chi_{2}$ distribution. Thus the mean of $\left\lfloor\chi_{2}\right\rfloor_{N}$ is $\sigma \sqrt{\pi / 2 N}$, the standard deviation is $(\sigma \sqrt{2-\pi / 2}) / \sqrt{N}$, and the variance is $\sigma^{2}(2-\pi / 2) / N$. For $\left\lfloor\chi_{2}\right\rfloor_{N}$, $\xi_{0.025} \approx 0.225 \sigma / \sqrt{N}$ and $\xi_{0.975} \approx 2.72 \sigma / \sqrt{N}$. For details on the properties of $\left\lfloor\mathrm{dB} \chi_{2}\right\rfloor_{N}$, refer to the properties of $\left\lfloor\mathrm{dB} \chi_{2}^{2}\right\rfloor_{N}$.

Unfortunately, the statistics for $\left[\chi_{2}\right]_{N}$ are more difficult to come by. To research this distribution, it helps to know that the Rayleigh distribution is a special case of the Weibull distribution, which is well documented by Johnson [17] (there is a typographical error in the equations of interest, eqs (14) and (15) on pages 254 and 255 of this reference. The equation should have $+j$ instead of $-j$ ). The results for both the mean and variance are cumbersome and difficult to compute. For now, we recommend calculating the moments of $f_{\left\lceil_{\chi 2}\right\rceil_{N}}$ by numerically evaluating the moment integrals of eq (72). Figure 11a shows a plot of the mean and the $95 \%$ tolerance interval of $\left\lceil\chi_{2}\right\rceil_{N}$ versus $N$, and the standard deviation of $\left\lceil\chi_{2}\right\rceil_{N}$ versus $N$ is given in Figure $11 \mathrm{~b}$. The values plotted in these figures were generated numerically assuming that $\sigma=1$. The mean, standard deviation, variance, and the $2.5 \%$ quantile and the $97.5 \%$ quantile values are presented in Table 4 for various values of $N$. We hope to present a simple equation for the computation of these quantities, or at least a good approximation, in a future report.

A comparison of the mean of $\left\lceil\chi_{2}\right\rceil_{N}$ converted to decibels and the mean of $\left\lceil\mathrm{dB} \chi_{2}\right\rceil_{N}$ is shown in Figure 12. The difference between the two means is small (less than $0.1 \mathrm{~dB}$ ) for $N \geq 50$.

To use Table 4 with an arbitrary value of $\sigma$, multiply the mean, standard deviation, and quantile terms by $\sigma$, and multiply the variance term by $\sigma^{2}$. 
Table 4. Properties of the $\left\lceil\chi_{2}\right\rceil_{N}$ distribution for $\sigma=1$.

\begin{tabular}{rrrrrr}
\hline$N$ & Mean & $\begin{array}{r}\text { Standard } \\
\text { deviation }\end{array}$ & Variance & $\xi_{0.025}$ & $\xi_{0.975}$ \\
\hline 1 & 1.253 & 0.655 & 0.429 & 0.225 & 2.717 \\
2 & 1.621 & 0.612 & 0.374 & 0.587 & 2.959 \\
5 & 2.068 & 0.540 & 0.292 & 1.141 & 3.253 \\
10 & 2.370 & 0.492 & 0.242 & 1.533 & 3.458 \\
20 & 2.645 & 0.451 & 0.203 & 1.888 & 3.653 \\
50 & 2.971 & 0.408 & 0.166 & 2.300 & 3.896 \\
100 & 3.199 & 0.382 & 0.146 & 2.577 & 4.070 \\
200 & 3.410 & 0.360 & 0.130 & 2.830 & 4.237 \\
225 & 3.445 & 0.357 & 0.127 & 2.871 & 4.264 \\
400 & 3.609 & 0.342 & 0.117 & 3.063 & 4.397 \\
500 & 3.670 & 0.336 & 0.113 & 3.135 & 4.448 \\
800 & 3.797 & 0.326 & 0.106 & 3.281 & 4.552 \\
1000 & 3.857 & 0.321 & 0.103 & 3.347 & 4.600 \\
1024 & 3.862 & 0.320 & 0.103 & 3.355 & 4.606 \\
1600 & 3.977 & 0.312 & 0.097 & 3.485 & 4.702 \\
2000 & 4.033 & 0.308 & 0.095 & 3.548 & 4.749 \\
3200 & 4.148 & 0.299 & 0.090 & 3.678 & 4.847 \\
5000 & 4.255 & 0.292 & 0.085 & 3.799 & 4.938 \\
10000 & 4.415 & 0.282 & 0.080 & 3.977 & 5.077 \\
\hline
\end{tabular}




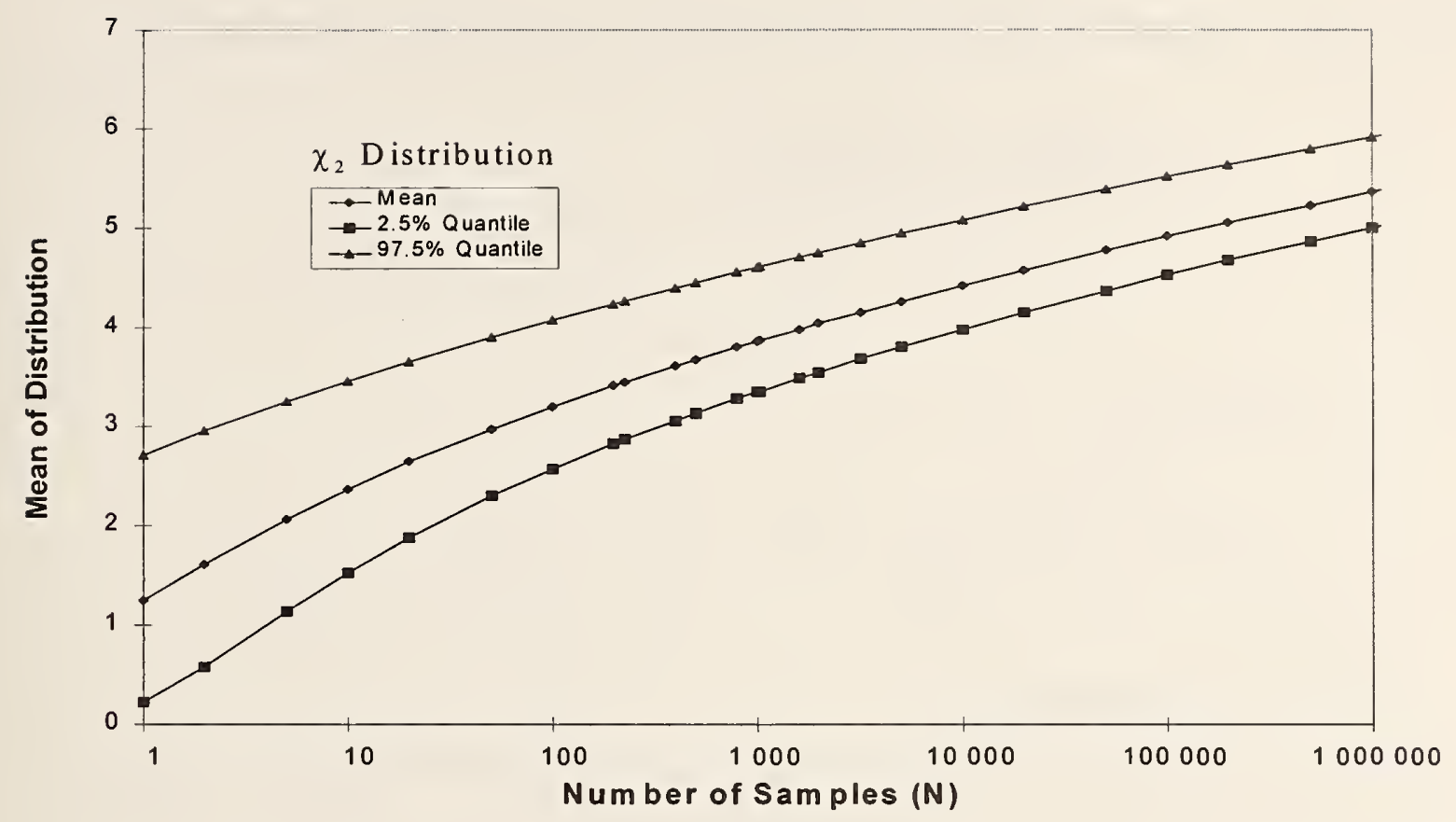

(a)

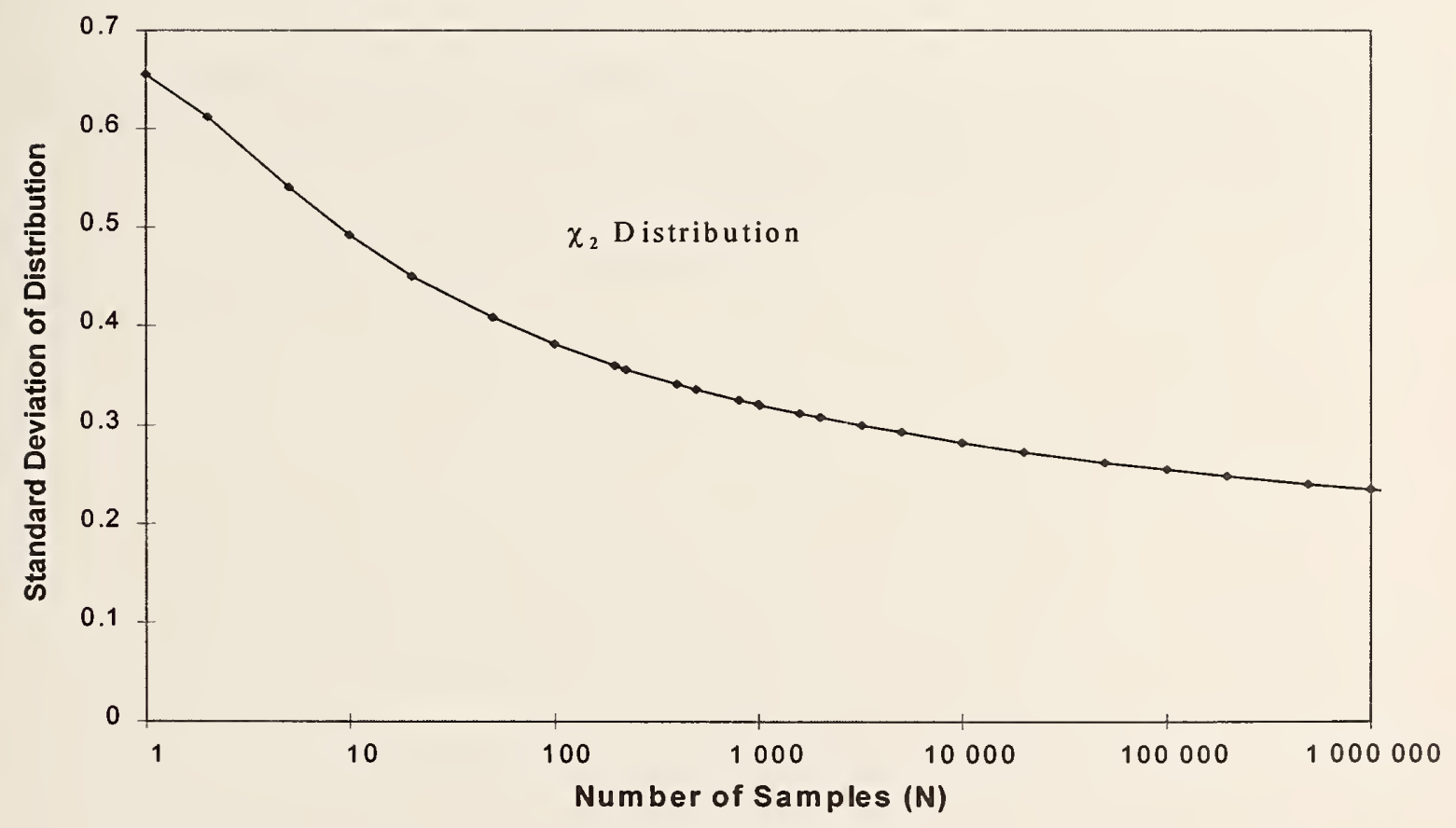

(b)

Figure 11. Statistical properties of the $\mathrm{N}^{\text {th }}$ order statistic (maximum) of a standard $\chi_{2}$ distribution, assuming $\mathrm{N}$ samples. (a) Mean, $2.5 \%$ quantile, and $97.5 \%$ quantile. (b) Standard deviation. 


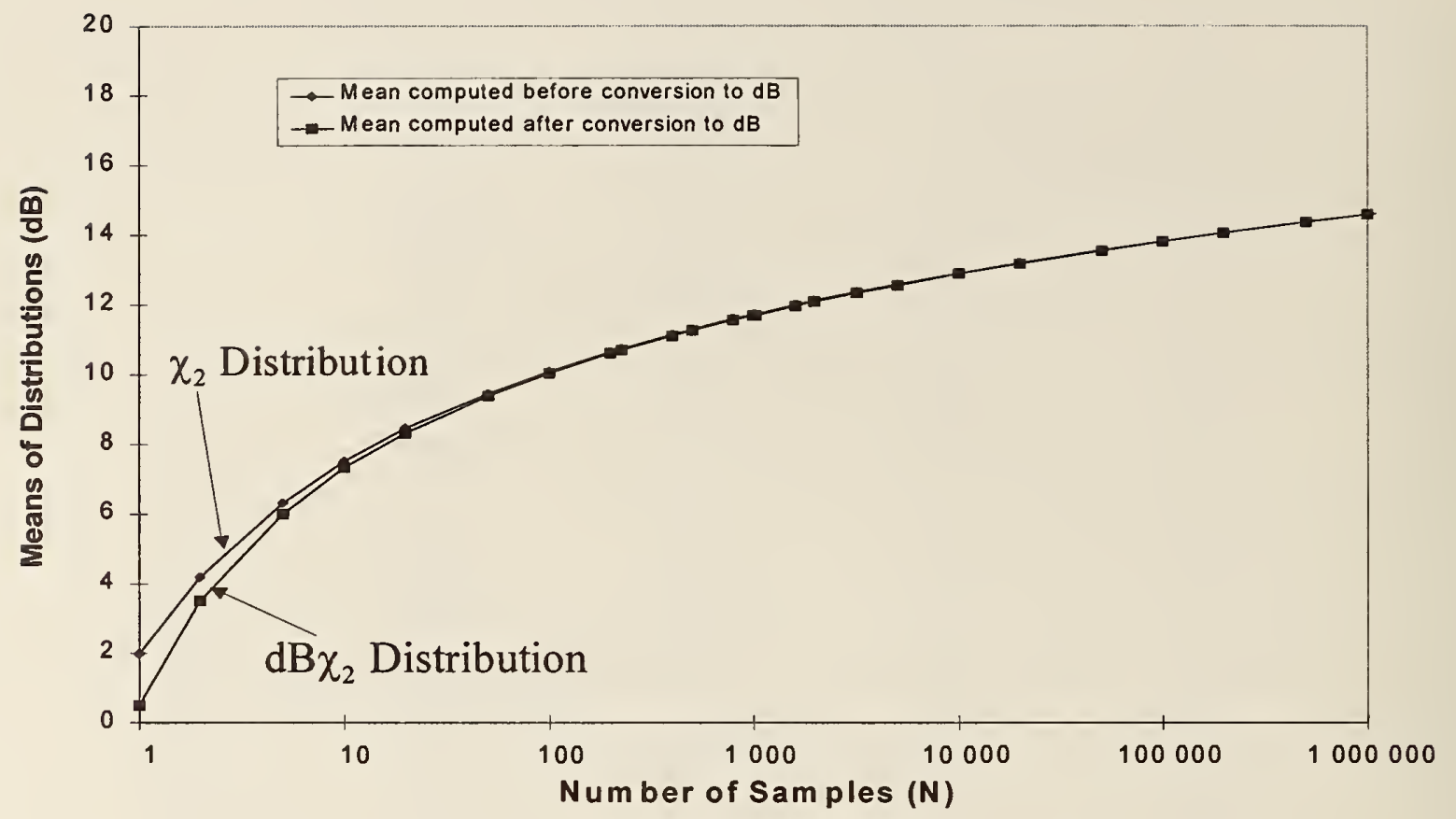

Figure 12. Comparison of means of the $\mathrm{N}^{\text {th }}$ order statistics (maximums) of a standard $\chi_{2}$ distribution and a standard $\mathrm{dB} \chi_{2}$ distribution, assuming $\mathrm{N}$ samples. Both means are plotted on a decibel scale for comparison. 
The probability density functions for the minimum and the maximum of $N \chi_{6}^{2}$ samples $[17]$ are

$$
f_{\left\lfloor\chi_{6}^{2}\right\rfloor_{N}}(x)=\frac{N x^{2}}{16 \sigma^{6}} e^{-N x / 2 \sigma^{2}}\left[\sum_{k=0}^{2} \frac{\left(x / 2 \sigma^{2}\right)^{k}}{k !}\right]^{N-1} U(x)
$$

and

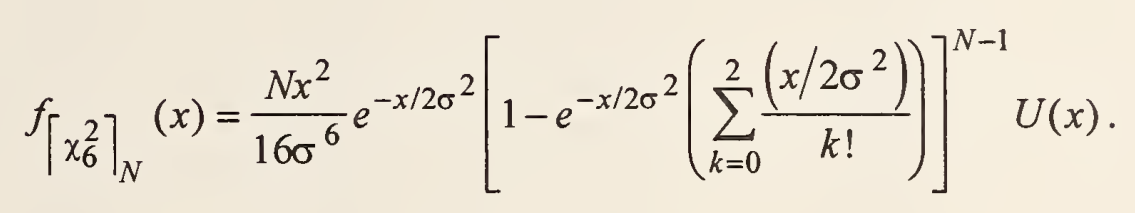

We could think of no practical use for estimates of the minimum of $N \chi_{6}^{2}$ samples, so we will not examine the properties of the $\left\lfloor\chi_{6}^{2}\right\rfloor_{N}$ distribution. We included eq (73) for completeness, only.

We calculated the mean, standard deviation, variance, and the $2.5 \%$ quantile and the $97.5 \%$ quantile of the $\left\lceil\chi_{6}^{2}\right\rceil_{N}$ distribution for $\sigma=1$ as a function of $N$ using numerical methods, and the results are shown in Figures 13a and b, and listed in Table 5. To use Table 5 for arbitrary values of $\sigma$, multiply the mean, standard deviation, and quantile terms by $\sigma^{2}$, and multiply the variance term by $\sigma^{4}$. 
Table 5. Properties of the $\left\lceil\chi_{6}^{2}\right\rceil_{N}$ distribution for $\sigma=1$.

\begin{tabular}{rrrrrr}
\hline$N$ & Mean & $\begin{array}{r}\text { Standard } \\
\text { deviation }\end{array}$ & Variance & $\xi_{0.025}$ & $\xi_{0.975}$ \\
\hline 1 & 6.000 & 3.464 & 12.000 & 1.237 & 14.450 \\
2 & 7.876 & 3.498 & 12.236 & 2.730 & 16.228 \\
5 & 10.394 & 3.436 & 11.806 & 5.174 & 18.522 \\
10 & 12.274 & 3.362 & 11.303 & 7.136 & 20.220 \\
20 & 14.110 & 3.288 & 10.811 & 9.092 & 21.900 \\
50 & 16.474 & 3.198 & 10.227 & 11.616 & 24.080 \\
100 & 18.218 & 3.142 & 9.872 & 13.466 & 25.700 \\
200 & 19.928 & 3.094 & 9.573 & 15.268 & 27.320 \\
225 & 20.220 & 3.086 & 9.523 & 15.570 & 27.580 \\
400 & 21.620 & 3.052 & 9.315 & 17.028 & 28.900 \\
500 & 22.140 & 3.042 & 9.254 & 17.588 & 29.420 \\
800 & 23.280 & 3.018 & 9.108 & 18.754 & 30.500 \\
1000 & 23.800 & 3.008 & 9.048 & 19.302 & 31.000 \\
1024 & 23.860 & 3.006 & 9.036 & 19.360 & 31.060 \\
1600 & 24.900 & 2.988 & 8.928 & 20.440 & 32.060 \\
2000 & 25.440 & 2.978 & 8.868 & 20.980 & 32.580 \\
3200 & 26.540 & 2.960 & 8.762 & 22.120 & 33.640 \\
5000 & 27.560 & 2.946 & 8.679 & 23.180 & 34.640 \\
10000 & 29.160 & 2.924 & 8.550 & 24.820 & 36.200 \\
\hline
\end{tabular}




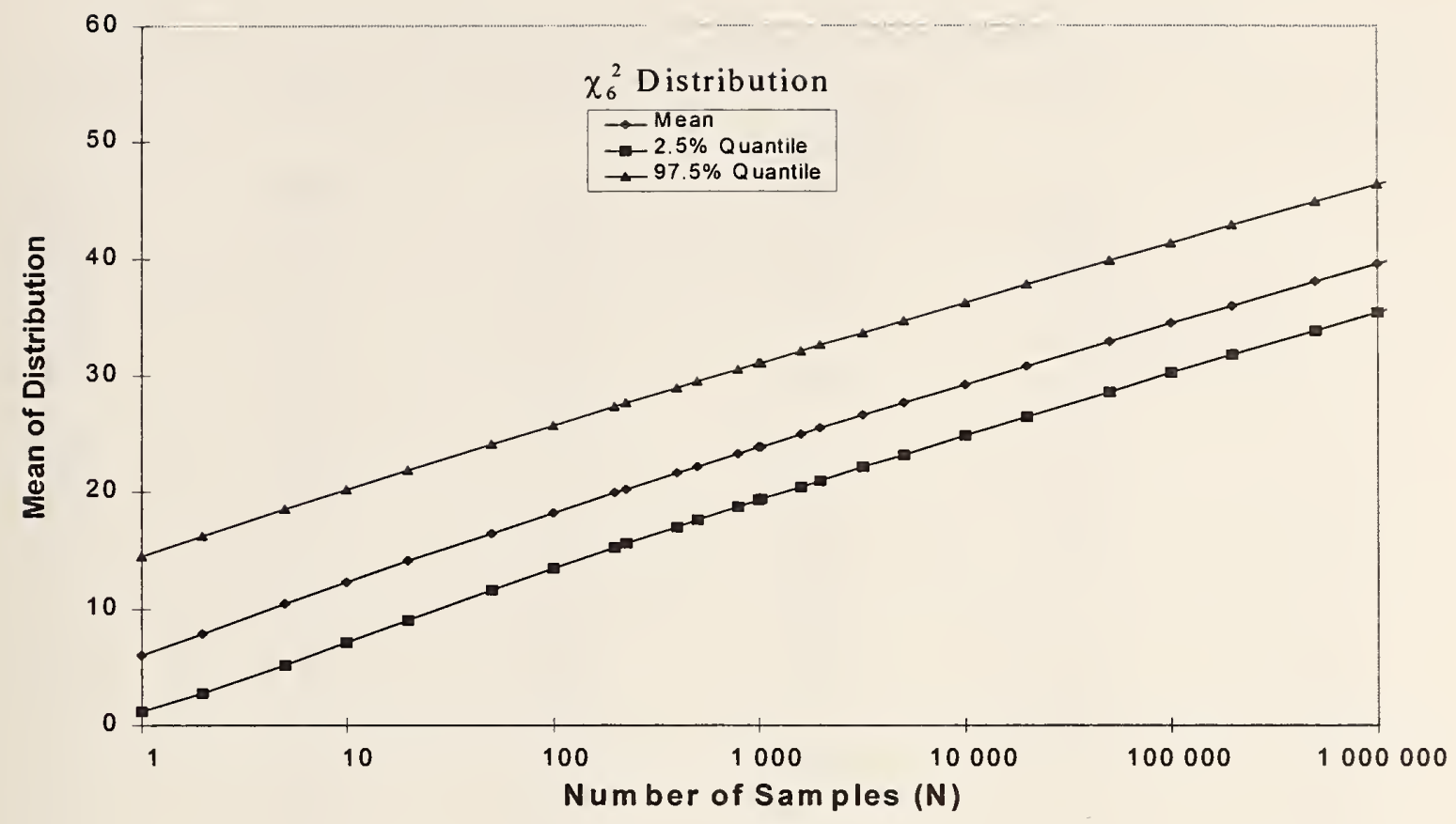

(a)

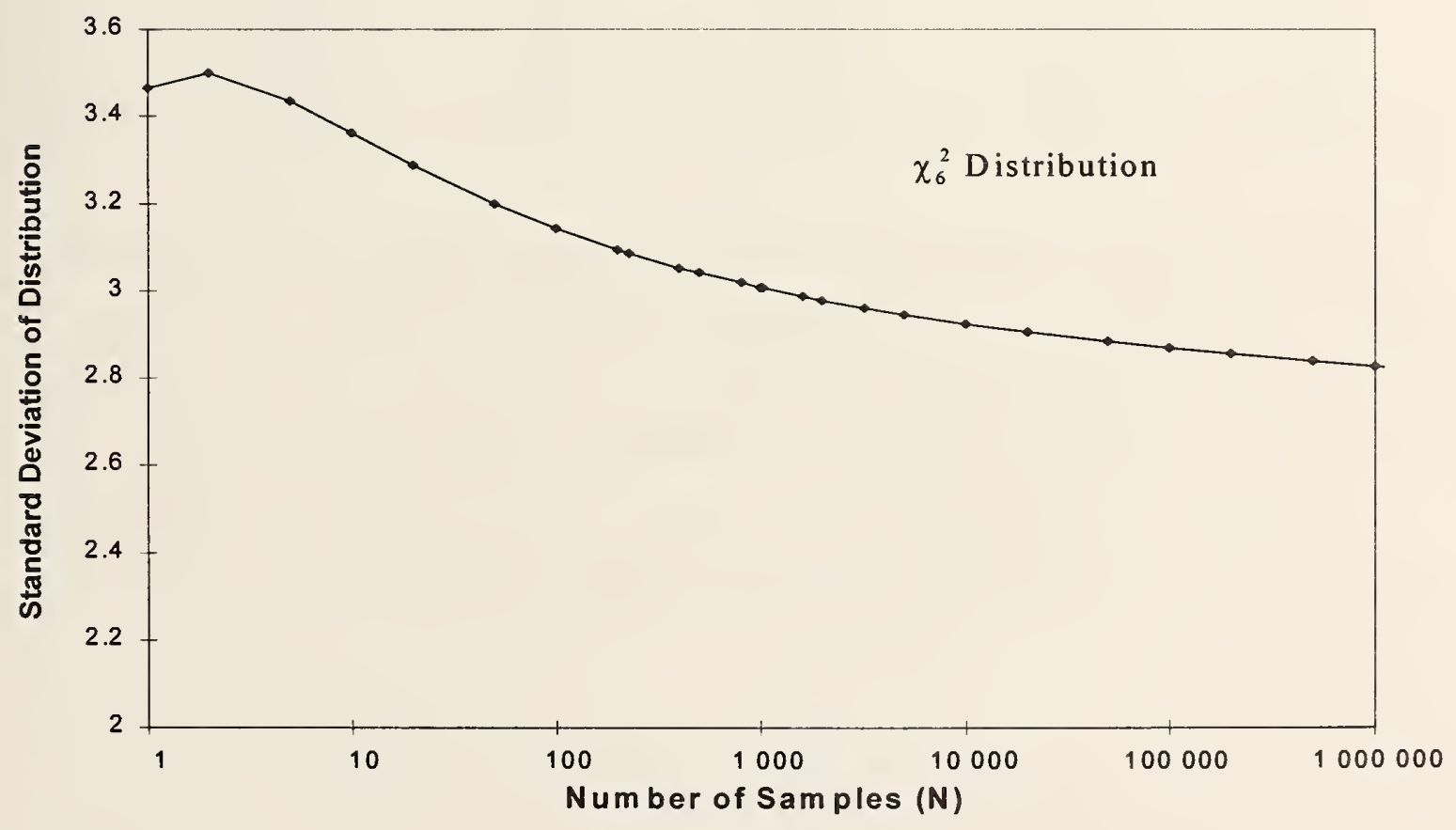

(b)

Figure 13. Statistical properties of the $\mathrm{N}^{\text {th }}$ order statistic (maximum) of a standard $\chi_{6}^{2}$ distribution, assuming $\mathrm{N}$ samples. (a) Mean, $2.5 \%$ quantile, and $97.5 \%$ quantile. (b) Standard deviation. 
Table 6. Properties of the $\left\lceil\mathrm{dB} \chi_{6}^{2}\right\rceil_{N}$ distribution for $\sigma=1$.

\begin{tabular}{rrrrrr}
\hline$N$ & Mean & $\begin{array}{r}\text { Standard } \\
\text { deviation }\end{array}$ & Variance & $\xi_{0.025}$ & $\xi_{0.975}$ \\
\hline 1 & 7.018 & 2.729 & 7.449 & 0.925 & 11.598 \\
2 & 8.535 & 1.973 & 3.894 & 4.361 & 12.102 \\
5 & 9.940 & 1.410 & 1.989 & 7.137 & 12.677 \\
10 & 10.735 & 1.152 & 1.327 & 8.534 & 13.060 \\
20 & 11.384 & 0.972 & 0.945 & 9.587 & 13.400 \\
50 & 12.091 & 0.807 & 0.651 & 10.650 & 13.820 \\
100 & 12.544 & 0.716 & 0.513 & 11.292 & 14.100 \\
200 & 12.945 & 0.645 & 0.416 & 11.837 & 14.360 \\
225 & 13.009 & 0.634 & 0.402 & 11.922 & 14.410 \\
400 & 13.310 & 0.587 & 0.345 & 12.311 & 14.610 \\
500 & 13.410 & 0.571 & 0.326 & 12.452 & 14.690 \\
800 & 13.630 & 0.540 & 0.291 & 12.731 & 14.840 \\
1000 & 13.730 & 0.526 & 0.277 & 12.856 & 14.910 \\
1024 & 13.740 & 0.525 & 0.275 & 12.869 & 14.920 \\
1600 & 13.930 & 0.500 & 0.250 & 13.110 & 15.060 \\
2000 & 14.030 & 0.488 & 0.238 & 13.220 & 15.130 \\
3200 & 14.210 & 0.466 & 0.217 & 13.450 & 15.270 \\
5000 & 14.380 & 0.446 & 0.199 & 13.650 & 15.400 \\
10000 & 14.630 & 0.419 & 0.176 & 13.950 & 15.590 \\
\hline
\end{tabular}

Similarly, the mean, standard deviation, variance, $2.5 \%$ quantile, and $97.5 \%$ quantile of the $\left\lceil\mathrm{dB} \chi_{6}^{2}\right\rceil_{N}$ distribution for $\sigma=1$ as a function of $N$ are shown in Figures $14 \mathrm{a}$ and $\mathrm{b}$, and listed in Table 6 . To use Table 6 for arbitrary values of $\sigma$, the standard deviation and variance terms are unchanged, and $10 \cdot \log _{10}\left(\sigma^{2}\right)$ should be added to the mean and quantile terms.

A comparison of the mean of $\left\lceil\chi_{6}^{2}\right\rceil_{N}$ converted to decibels and the mean of $\left\lceil\mathrm{dB} \chi_{6}^{2}\right\rceil_{N}$ is shown in Figure 15. The difference between the two means is less than $0.1 \mathrm{~dB}$ for $N \geq 50$. 


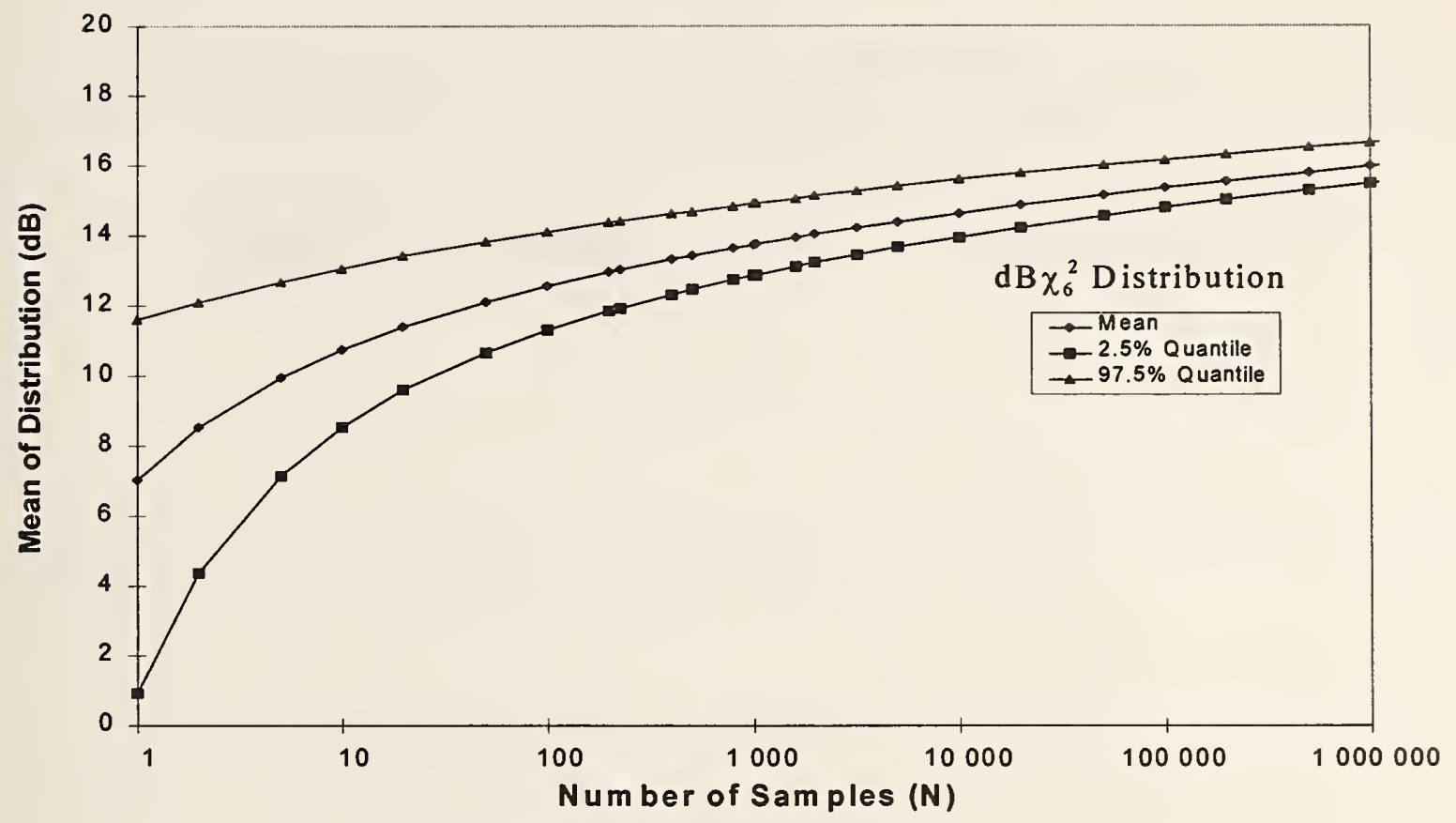

(a)

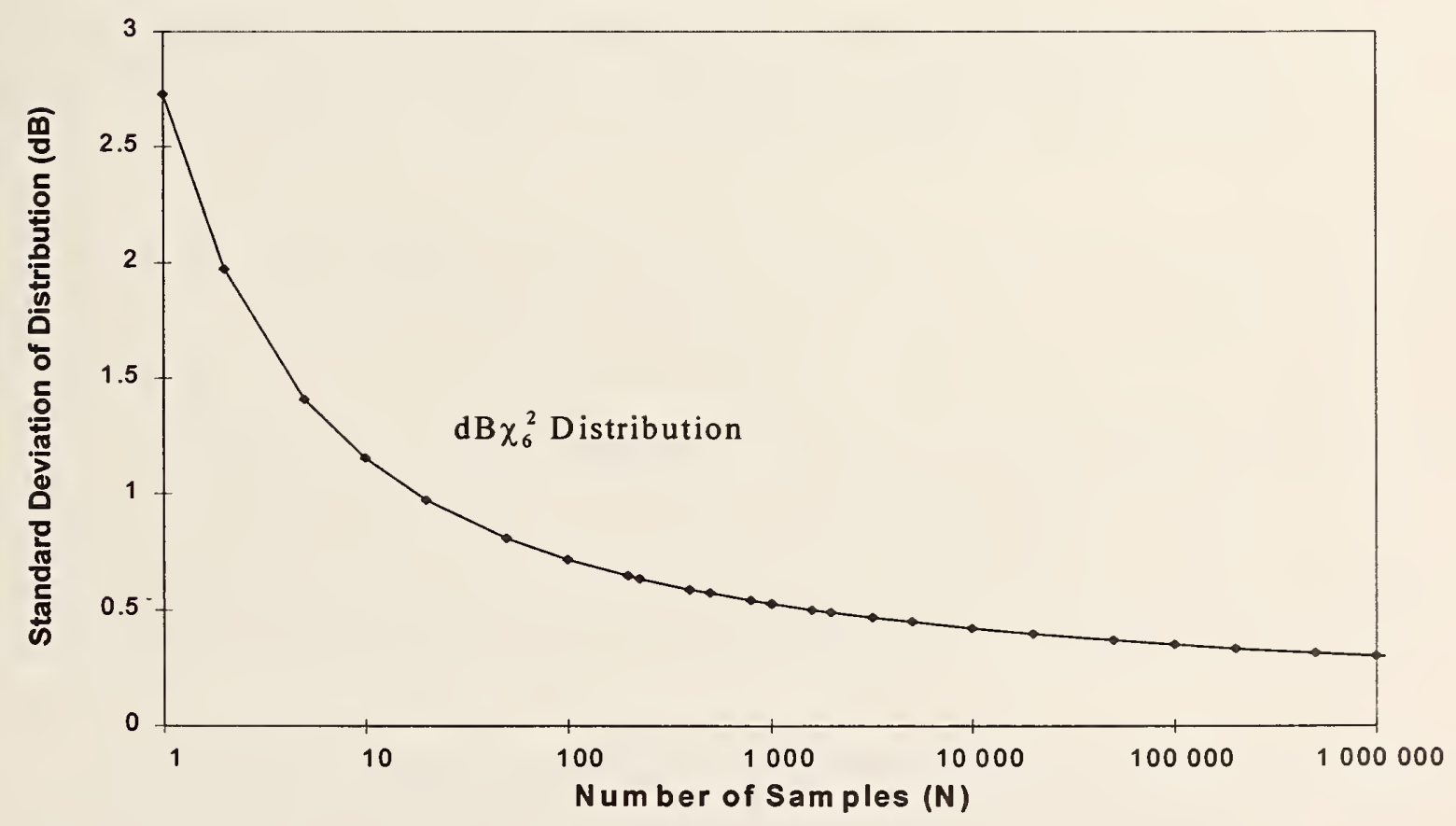

(b)

Figure 14. Statistical properties of the $\mathrm{N}^{\text {th }}$ order statistic (maximum) of a standard $\mathrm{dB} \chi_{6}^{2}$ distribution, assuming $\mathrm{N}$ samples. (a) Mean, $2.5 \%$ quantile, and $97.5 \%$ quantile. (b) Standard deviation. 


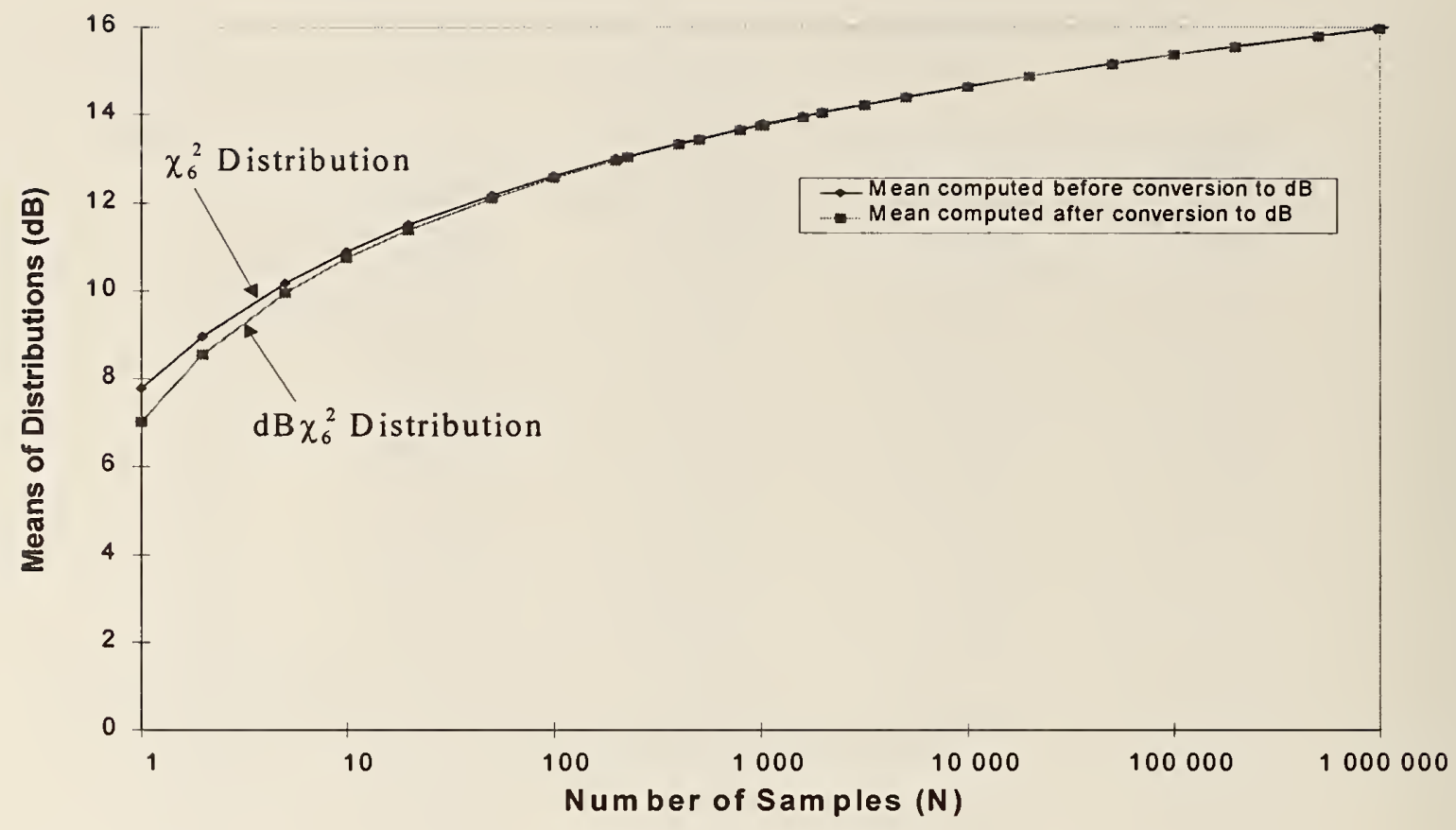

Figure 15. Comparison of means of the $\mathrm{N}^{\text {th }}$ order statistics (maximums) of a standard $\chi_{6}^{2}$ distribution and a standard $\mathrm{dB} \chi_{6}^{2}$ distribution, assuming $\mathrm{N}$ samples. Both means are plotted on a decibel scale for comparison. 
The probability density functions for the minimum and the maximum of $N \chi_{6}$ samples are difficult to find, but they can be derived from the corresponding probability density functions for a $\chi_{6}^{2}$ random variable (eqs (73) and (74)). Since a $\chi_{v}$ random variable is simply the square root of a $\chi_{v}^{2}$ random variable, we can use simple transformation techniques [14] to show that the pdf of a $\chi_{v}$ random variable is given by

$$
f_{\chi_{v}}(x)=2 x f_{\chi_{v}^{2}}\left(x^{2}\right)
$$

and the same relationship holds for the extreme values of a $\chi_{v}$ random variable. Applying the relationship in eq (75) to eqs (73) and (74), we obtain

$$
f_{\left\lfloor x_{6}\right\rfloor_{N}}(x)=\frac{N x^{5}}{8 \sigma^{6}} e^{-N x^{2} / 2 \sigma^{2}}\left[\sum_{k=0}^{2} \frac{\left(x^{2} / 2 \sigma^{2}\right)^{k}}{k !}\right]^{N-1} U(x)
$$

and

$$
f_{\left\lceil\chi \chi_{6}\right\rceil_{N}}(x)=\frac{N x^{5}}{8 \sigma^{6}} e^{-x^{2} / 2 \sigma^{2}}\left[1-e^{-x^{2} / 2 \sigma^{2}}\left(\sum_{k=0}^{2} \frac{\left(x^{2} / 2 \sigma^{2}\right)}{k !}\right)\right]^{N-1} U(x) .
$$

Once again, we could think of no practical use for estimates of the minimum of $N \chi_{6}$ samples, so we will not examine the properties of the $\left\lfloor\chi_{6}\right\rfloor_{N}$ distribution. We included eq (76) for completeness, only.

We calculated the mean, standard deviation, variance, $2.5 \%$ quantile, and $97.5 \%$ quantile of the $\left\lceil_{\chi_{6}}\right\rceil_{N}$ distribution for $\sigma=1$ as a function of $N$ using numerical methods, and the results are shown in Figures 16a and b, and listed in Table 7. To use Table 7 for arbitrary values of $\sigma$, multiply the mean, standard deviation, and quantile terms by $\sigma$, and multiply the variance term by $\sigma^{2}$.

A comparison of the mean of $\left\lceil\chi_{6}\right\rceil_{N}$ converted to decibels and the mean of $\left\lceil\mathrm{dB} \chi_{6}\right\rceil_{N}$ is shown in Figure 17. The difference between the two means is less than $0.1 \mathrm{~dB}$ for $N \geq 10$. 
Table 7. Properties of the $\left\lceil\chi_{6}\right\rceil_{N}$ distribution for $\sigma=1$.

\begin{tabular}{rrrrrr}
\hline$N$ & Mean & $\begin{array}{r}\text { Standard } \\
\text { deviation }\end{array}$ & Variance & $\xi_{0.025}$ & $\xi_{0.975}$ \\
\hline 1 & 2.350 & 0.691 & 0.478 & 1.112 & 3.801 \\
2 & 2.739 & 0.608 & 0.369 & 1.652 & 4.029 \\
5 & 3.182 & 0.518 & 0.268 & 2.274 & 4.303 \\
10 & 3.472 & 0.466 & 0.217 & 2.671 & 4.497 \\
20 & 3.732 & 0.425 & 0.180 & 3.015 & 4.680 \\
50 & 4.040 & 0.383 & 0.147 & 3.408 & 4.906 \\
100 & 4.253 & 0.358 & 0.128 & 3.670 & 5.070 \\
200 & 4.452 & 0.337 & 0.114 & 3.907 & 5.226 \\
225 & 4.484 & 0.334 & 0.112 & 3.946 & 5.252 \\
400 & 4.637 & 0.320 & 0.102 & 4.127 & 5.377 \\
500 & 4.695 & 0.315 & 0.099 & 4.193 & 5.424 \\
800 & 4.814 & 0.305 & 0.093 & 4.330 & 5.523 \\
1000 & 4.869 & 0.301 & 0.090 & 4.394 & 5.568 \\
1024 & 4.875 & 0.300 & 0.090 & 4.400 & 5.573 \\
1600 & 4.982 & 0.292 & 0.085 & 4.523 & 5.663 \\
2000 & 5.035 & 0.288 & 0.083 & 4.582 & 5.708 \\
3200 & 5.143 & 0.281 & 0.079 & 4.704 & 5.800 \\
5000 & 5.244 & 0.274 & 0.075 & 4.815 & 5.886 \\
10000 & 5.394 & 0.265 & 0.070 & 4.982 & 6.016 \\
\hline
\end{tabular}




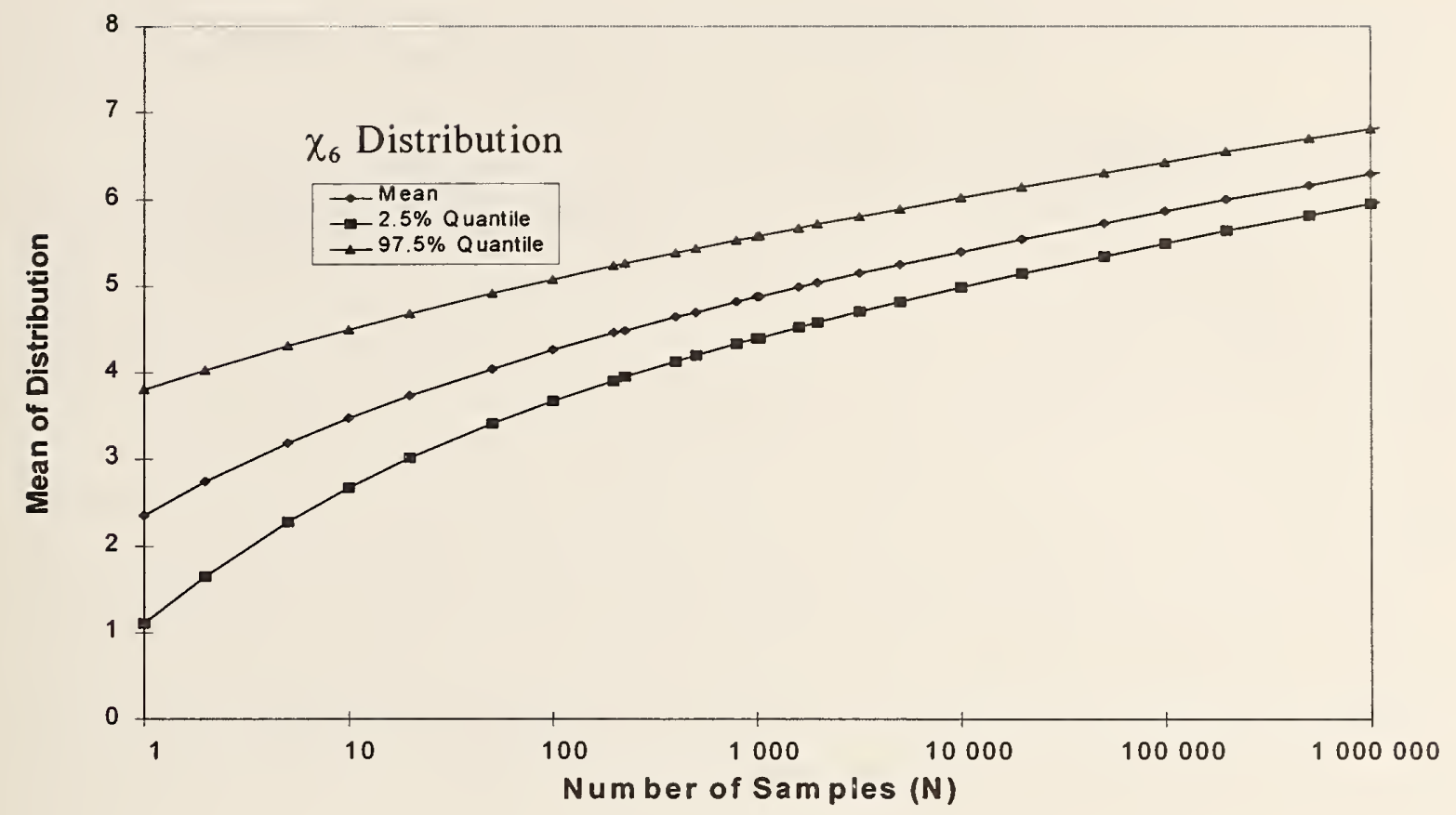

(a)

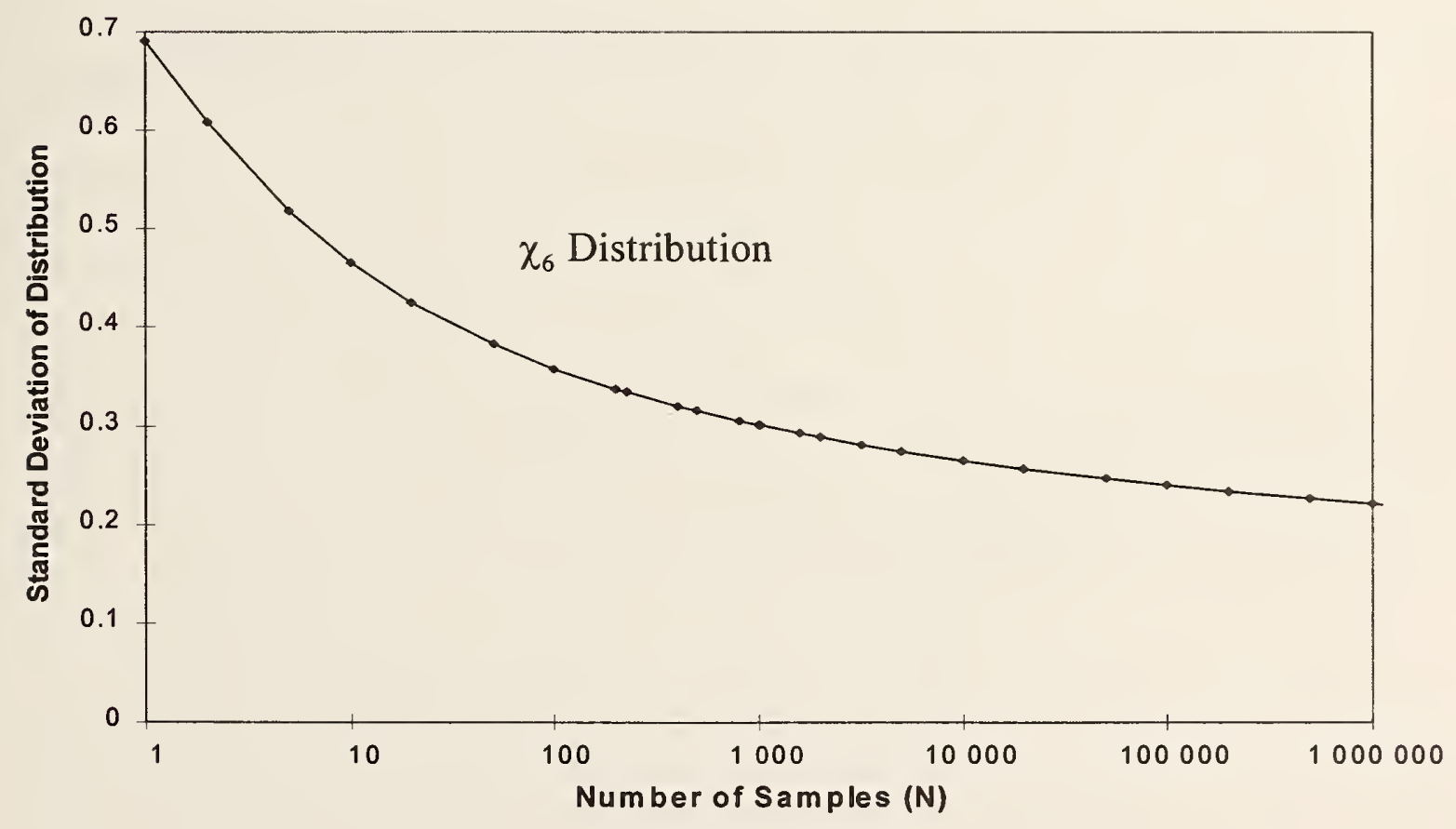

(b)

Figure 16. Statistical properties of the $\mathrm{N}^{\text {th }}$ order statistic (maximum) of a standard $\chi_{6}$ distribution, assuming $\mathrm{N}$ samples. (a) Mean, $2.5 \%$ quantile, and $97.5 \%$ quantile.

(b) Standard deviation. 


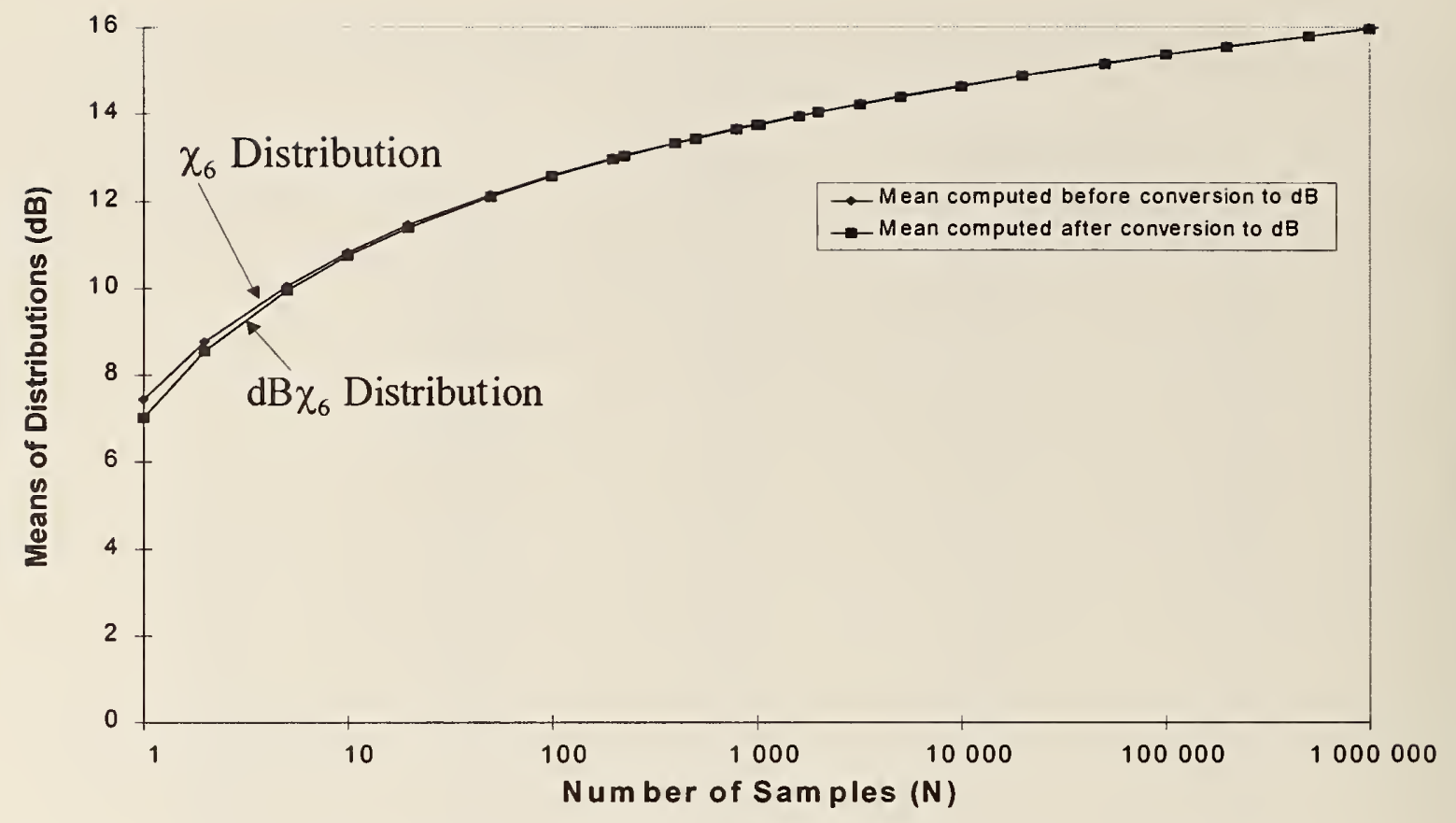

Figure 17. Comparison of means of the $\mathrm{N}^{\text {th }}$ order statistics (maximums) of a standard $\chi_{6}$ distribution and a standard $\mathrm{dB} \chi_{6}$ distribution, assuming $\mathrm{N}$ samples. Both means are plotted on a decibel scale for comparison. 


\subsubsection{Ideal Models Applied to Mode-Stirred Chambers}

Now that we have a description of the properties of these distributions, we will now explore the implications for a typical mode-stirred chamber measurement. In NBS Tech. Note 1092 [3], Crawford noticed that the ratio of the maximum measured received power to the average measured received power (referred to as the peak-to-average ratio or the maximum-toaverage ratio) was typically between 7 and $9 \mathrm{~dB}$. Actually, this is just $a$ maximum-to-average ratio. Any distribution will have a maximum-to-average ratio, and the maximum-to-average ratio of the magnitude of a rectangular component of the electric field will be different from the maximum-to-average ratio of the magnitude of the total electric field, which will be different from the maximum-to-average ratio of the received power. This said, the ratio of the maximum received power to the average received power, measured in linear units (not decibels), has become known as the maximum-to-average ratio, and we will continue with this convention. Any other reference to any other maximum-to-average ratio will explicitly refer to the parameter on which the ratio was computed. Similarly, any other ratios (maximum-to-minimum, averageto-minimum) will also be based on received power as measured in linear units, unless otherwise noted.

Lehman [4] observed that, if the received power can be described by the $\chi_{2}^{2}$ distribution, the ratio of the mean of $\left\lceil\chi_{2}^{2}\right\rceil_{N}$ to the mean of $\chi_{2}^{2}$ is a good estimate of the maximum-toaverage ratio (note that the maximum and average of $N$ samples from any distribution are not independent, so the ratio of the sample maximum to the sample average will be slightly different than the ratio of the expected value of the maximum to the expected value of the average). If we take the mean values of $\left\lceil\chi_{2}^{2}\right\rceil_{N}$ presented in Table 2 and divide by 2 (the average of a $\chi_{2}^{2}$ random variable for $\sigma=1$ ), we see that the maximum-to-average ratio can range from approximately 1 to $10(0$ to $10 \mathrm{~dB})$ for values of $N$ between 1 and 10000 . For values of $N$ which would typically be used in a mode-stirred chamber measurement (between 100 and 2000), the peak to average ratio should be between 5 and $8(7 \mathrm{~dB}$ and $9 \mathrm{~dB})$. Thus the relationship between the maximum received power and the average received power observed by Crawford agrees with the statistical model.

NBS Tech. Note 1092 assumed that there was a "true" peak signal, and that any measured peak value below this true peak was an error. This assumption is reasonable at low frequencies, where fluctuations in the received signal are small for small changes in paddle position. At high frequencies, however, it is extremely difficult to locate the true maximum because the received signal can fluctuate wildly for a small change in paddle position. Even at low frequencies, the received signal had to be sampled at a large number of paddle positions to ensure that the measured peak was close to the true peak. For these reasons, even if a true peak exists, it cannot practically be measured. Crawford [3] recommended that a minimum of 200 paddle steps should be used below $1 \mathrm{GHz}$, a minimum of 400 paddle steps should be used between $1 \mathrm{GHz}$ and $2 \mathrm{GHz}$, and more than 5000 paddle steps should be used above $2 \mathrm{GHz}$. For this reason, the modestirred technique, which samples the fields while the paddle continuously turns at a fixed rate of 
rotation, was considered to be the most accurate measurement method for frequencies greater than $2 \mathrm{GHz}$.

From a statistical point of view, there is no true peak value. Instead, there is only the largest measured value. The statistical theory of mode-stirred chambers gives us a tool for estimating the largest measured value without needing to know the true peak value. In fact, we can predict the peak based on any number of measurements. This means that we can use a fixed number of measurements at all frequencies without loss of accuracy. As we will show in Section 8 , it is possible to obtain acceptable uncertainty with less than 200 paddle positions, even at (actually, especially at) frequencies greater than $2 \mathrm{GHz}$.

Given this background, we will give a few examples of what can be expected of measurements in an ideal chamber with ideal equipment. For these examples, we will assume that the measurements were made with 225 paddle positions, and that the measurements were repeated a large number of times (say 1000 or more). We will not assume a specific frequency or a specific input or received power. Instead we will investigate the behavior of an arbitrary ideal signal. The question we will attempt to answer is, for a specific parameter, what is the expected maximum-to-average ratio, and what range of values do I expect to see over a large number of measurements. We will restrict our attention to the power received by a reference antenna, which is assumed to have a $\chi_{2}^{2}$ distribution. For this analysis we will use Table 2. Other characteristics, such as average or maximum electric field (rectangular component or total) can also be easily derived using the appropriate table and the procedures outlined here.

We expect a maximum-to-average ratio of the received power to be approximately $11.992 / 2=5.996$, or $10 \cdot \log _{10}(5.996)=7.78 \mathrm{~dB}$. Of course, every measurement will not give us a maximum-to-average ratio of exactly $7.78 \mathrm{~dB}$, but this should be the average over a large number of measurements. The observant reader may notice that the value of $7.78 \mathrm{~dB}$ does not agree with the value predicted in Table 3 of $10.697-0.503=10.194 \mathrm{~dB}$. This difference is due to the fact that the maximum and average received power were computed in linear units and then converted to decibels, whereas the data in Table 3 assumes that the maximum and average were computed in decibels. Although a difference of $2.4 \mathrm{~dB}$ may be surprising, it is real.

Next we examine the properties of the average received power. Although we are interested in the average received power, we are also interested in "how close" we can expect individual measurements to be to the average. This concept of how close an individual measurement will be to the average is best described statistically. Typically, standard deviations and tolerance intervals, as discussed in Section 3.2.2, are used to characterize the amount of variation that can be expected in a measurement. Specifically, if we assume that the results of a large number of measurements are normally distributed, then we know that approximately $68 \%$ of the results will be within one standard deviation of the mean, and approximately $95 \%$ of the results will be within two standard deviations of the mean. Thus, for normally distributed data, the $68 \%$ tolerance interval is the range of values that are less than one standard deviation away from the mean, and the $95 \%$ tolerance interval is the range of values that are less than two standard deviation away from the mean. (Technically, any range that can be expected to contain $95 \%$ of the data can be considered a $95 \%$ tolerance interval, and therefore there are an infinite number of possible $95 \%$ tolerance intervals. For our purposes here, we will consider the $95 \%$ tolerance interval to be the symmetric tolerance interval such that the probability that a measured 
value is greater than the upper limit of the interval is equal to the probability the a measured value is less than the lower limit of the interval.) If the measured data are not normally distributed, the correspondence between standard deviations and tolerance intervals is no longer exact, but the approximation is still good for most distributions discussed in this report.

After a large number of averages (paddle positions), the average received power will be approximately normally distributed. For a single paddle position, the mean and standard deviation are equal (refer to Section 3.2.3). After $N$ paddle positions, the mean is unchanged but the standard deviation is reduced by a factor of $\sqrt{N}$. For 225 paddle positions, the standard deviation is reduced by a factor of 15 . Thus, assuming an average received power of $P \mathrm{~W}$ (a power $P$, measured in watts), we expect to see most (approximately 68\%, assuming a normal distribution) of our measured values to fall within the range of $\left(P \pm \frac{P}{15}\right) \mathrm{W}=P \cdot\left(1 \pm \frac{1}{15}\right) \mathrm{W}$. This can also be written as $P \mathrm{~W} \pm 6.7 \%$. Notice that this range is independent of the mean. Since both the mean and the standard deviation are proportional to the same constant, it is sometimes useful to express this range in decibels: $10 \cdot \log _{10}(P)+10 \cdot \log _{10}\left(1 \pm \frac{1}{15}\right)$ dB relative to $1 \mathrm{~W}$. This form is similar to writing standard uncertainties in the form $\alpha \pm \beta$, except that the second term is not symmetric about the mean, that is $10 \cdot \log _{10}\left(1+\frac{1}{15}\right) \approx 0.28 \mathrm{~dB}$, whereas $10 \cdot \log _{10}\left(1-\frac{1}{15}\right) \approx-0.30 \mathrm{~dB}$. This minor problem can be handled in a number of ways. Two of the more common ways are to either choose symmetric bounds and accept a larger estimated standard deviation $\left(10 \cdot \log _{10}(P) \pm 0.30 \mathrm{~dB}\right)$, or to use an awkward notation and keep track of the separate converted standard deviations $(10 \log (P)+0.28$. 0.30 . As long as the standard deviations are small, it makes little difference which method is used. We will generally use the latter format wherever possible as an explicit reminder that the bounds are not symmetric when expressed in decibels. In figures, however, whenever we compare a measured standard deviation with an ideal theoretical standard deviation, we will use a third, less common approach: we will compute the average of the absolute value of the two bounds. For the situation presented here, we would assume a standard deviation of $0.29 \mathrm{~dB}$. Although this is somewhat unconventional, it should be roughly equivalent to the value that would be observed if the various measurements of average received power (or maximum received power, or electric field, etc.) were converted to decibels, and then the average and standard deviation of these data were computed.

For a $95 \%$ tolerance interval, the procedure is similar. Assuming an average received power of $P \mathrm{~W}$, we expect to see $95 \%$ of our measured values to fall within the range of (assuming 2 standard deviations and normally distributed data) $\left(P \pm 2 \cdot \frac{P}{15}\right) \mathrm{W}$ $=P \cdot\left(1 \pm 2 \cdot \frac{1}{15}\right) \mathrm{W}$, or $P \mathrm{~W} \pm 13.3 \%$. When expressed in decibels, we have $10 \cdot \log _{10}(P)_{-0.62}^{+0.54} \mathrm{~dB}$ relative to $1 \mathrm{~W}$. 
We now analyze the characteristics of the maximum received power, also based on information from Table 2. Assuming that the expected maximum received power is $\alpha \mathrm{W}$ and $N=225$, we can calculate similar characteristics. We expect approximately $68 \%$ of the measurements of the maximum received power to fall with one standard deviation of the mean. (Note: since the distribution of the maximum received power is not normal; all bounds presented here are only approximations.) Thus we expect to see $68 \%$ of our measured values of the maximum received power to fall within a range of $\alpha \pm \frac{2.256 \alpha}{11.992} \mathrm{~W}=\alpha \pm 0.188 \alpha \mathrm{W}$ $=\alpha \cdot(1 \pm 0.188) \mathrm{W}$, or $\alpha \mathrm{W} \pm 18.8 \%$. When dealing with the maximum measured value, we are not averaging, so we cannot reduce the standard deviation by the $\sqrt{N}$. When expressed in decibels, we have $10 \log (\alpha)+0.75$ dB relative to $1 \mathrm{~W}$. For the $95 \%$ tolerance interval, we expect $95 \%$ of the measured data to fall within $\pm 37.6 \%$ of the average, or $95 \%$ of the data should be less than $1.39 \mathrm{~dB}$ above the average and less than $2.05 \mathrm{~dB}$ below the average $(+1.39 /-2.05 \mathrm{~dB})$.

Now that we have these estimates of the $95 \%$ tolerance interval of the maximum received power assuming normally distributed data, it is interesting to compare the bounds with those computed specifically for this distribution and listed in the last two columns of Table 2 . The actual upper bound is $\frac{18.184}{11.992}=1.52$ or $52 \%$ greater than the average, and the actual lower bound is $\frac{8.238}{11.992}=0.69$ or $31 \%$ less than the average. When these bounds are expressed in decibels, we have $+1.81 /-1.63 \mathrm{~dB}$. Notice that these true bounds are not symmetric about the mean when expressed in either linear or logarithmic terms, and that the estimated bounds are significantly different from those derived assuming normally distributed data. The width of the tolerance interval when expressed in decibels, however, is the same: $(1.39+2.05=1.81+1.63=3.44 \mathrm{~dB})$.

Similar properties could easily be derived for values of $N$ other than 225 using the procedures outlined above and values from the appropriate table. For values of $N$ that are not included in the tables, interpolation should provide results with adequate accuracy.

\section{General Description of the Measurement Systems}

Several methods are available for evaluating a mode-stirred chamber. A system must have some means of introducing an electromagnetic signal into the chamber and must have some means of measuring the electromagnetic parameters of interest. The goal of a chamber evaluation is to measure the parameters of interest under as many different conditions as possible, given the constraints on time available to do the measurement. For a CW evaluation, the different test conditions include different frequencies, different paddle positions, and different spatial coordinates inside the chamber. The electromagnetic parameters that can be easily measured are the characteristics of the transmitted signal (incident power, incident voltage (magnitude and phase), reflected power, reflected voltage (magnitude and phase), received power from an antenna, received voltage from an antenna (magnitude and phase), and electric field. The electric 
field can be measured using a calibrated short dipole that either converts the electric field to an if signal or a rectified DC signal. Rectified DC probes are typically easier to manufacture and use, so only these types of probes will be discussed further.

The ideal instrumentation for this measurement would simultaneously measure the received voltage or power from a large number of sensors (antennas or probes), would not require external amplification (changing amplifiers is time consuming and limits the ability to automate the measurement), and could rapidly evaluate the measured data at a large number of frequencies. The instrumentation should also be accurate, and should not perturb the testing environment

The instrumentation available for performing the measurements all have associated strengths and weaknesses. The NIST probe system $[3,22,23]$ can simultaneously measure the rectangular component of the electric field at 30 different location, or can measure the three orthogonal rectangular components of the electric field at 10 different locations. This makes it ideal for sampling the fields inside a mode-stirred chamber. Unfortunately, the probe system also has several disadvantages. It is slow, perturbs the test environment because the high resistance lines connected to the probes increase the losses in the chamber, and is somewhat insensitive, meaning that large amounts of power must be transmitted into the chamber before a detectable signal is produced. As a result, vector network analyzers, which typically generate only a few milliwatts of power, cannot easily be used to generate a signal that can be accurately detected with the probes. Instead, separate power sensors and instrumentation must be used to generate and monitor the transmitted and received signals, resulting in increased uncertainty and measurement time. The combined probe system, signal source, and sensors are referred to collectively as "the probe system."

A vector network analyzer, on the other hand, is fast and accurate, does not significantly perturb the test environment (except at low frequencies where the power removed from the chamber by the antennas is significant), and does not require external amplifiers. The biggest disadvantage of the vector network analyzer is that it can generally monitor only one sensor at a time. Consequently, the transmitting and receiving antennas must be moved to a number of different locations to give the same information on the spatial fluctuations of measured parameters that can be obtained with a single set of measurements using multiple sensors.

Given the advantages and disadvantages of both systems, we decided to take advantage of the benefits of each system. The network analyzer system was used to evaluate the performance of the chambers at a large number of frequencies and paddle positions, whereas the probe system was used to evaluate the spatial variations at a smaller number of frequencies. The combination of systems gave us more and better information than could be obtained by either system individually. These systems are described in more detail below. 


\subsection{Power Meters and Isotropic Probes (The Probe System)}

The probe system consists of several discrete instruments, each connected to a computer which is programmed to control and read each instrument in sequence. The block diagram in Figure 18 details the instruments and the interconnections. There is little change in this system compared to that described in NBS Tech Note 1092 [3] other than an updated computer, newer instruments, and more complicated software. All the basic functions and processes are unchanged.

The signal generator produces a single frequency continuous wave (cw) signal which is amplified by the power amplifier and fed into the transmitting antenna in the chamber. The incident (input to the transmitting antenna) and reflected (from the transmitting antenna) components of this signal are measured by the directional coupler and power meters. The readings were corrected for all losses in the signal path up to the terminals of the transmitting antenna. The power delivered to the antenna is set at a level which provides stable readings on the power meters and also sufficient electric fields in the chamber for the isotropic probes. The typical values for incident power were between 1 and $10 \mathrm{~W}$. All measured chamber parameters were then mathematically scaled (normalized) to a net input power (the difference between the incident and reflected powers) of $1 \mathrm{~W}$ based on the actual measured net input power at every measurement point. There was no attempt to level the net power delivered to the chamber. Although it is possible to normalize to a constant incident power instead of a constant net power, we do not present any data processed in this way. We hope to present results comparing the two normalization methods in a future report.

The receiving part of the system consists of an antenna (similar to the transmitting antenna), cables, attenuators, and a spectrum analyzer. The spectrum analyzer is calibrated to a reference power meter at all the settings (resolution bandwidth, reference level, video bandwidth, etc.) and frequencies used during the measurements. (Note: failure to do this calibration can result in substantially greater uncertainty than can be achieved when the calibration is performed.) As was done on the transmitting side, all readings were corrected for cable and attenuator losses up to the terminals of the receiving antenna.

We can determine many of the chamber parameters from the incident and reflected power at the terminals of the transmitting antenna and the received power at the terminals of the receiving antenna. If we interchange the roles of the transmitting and receiving antennas by swapping test ports (and their associated equipment), we would have a complete two-port scalar network analyzer measurement. There is little doubt that an automatic scalar network analyzer would be much more efficient and accurate than this system of power meters, a spectrum analyzer, and individually calibrated components. This is not the main strength of this system, however. The real power of this system comes from the ability to sample the electric field using an array of 10 isotropic probes using the NIST probe system. 


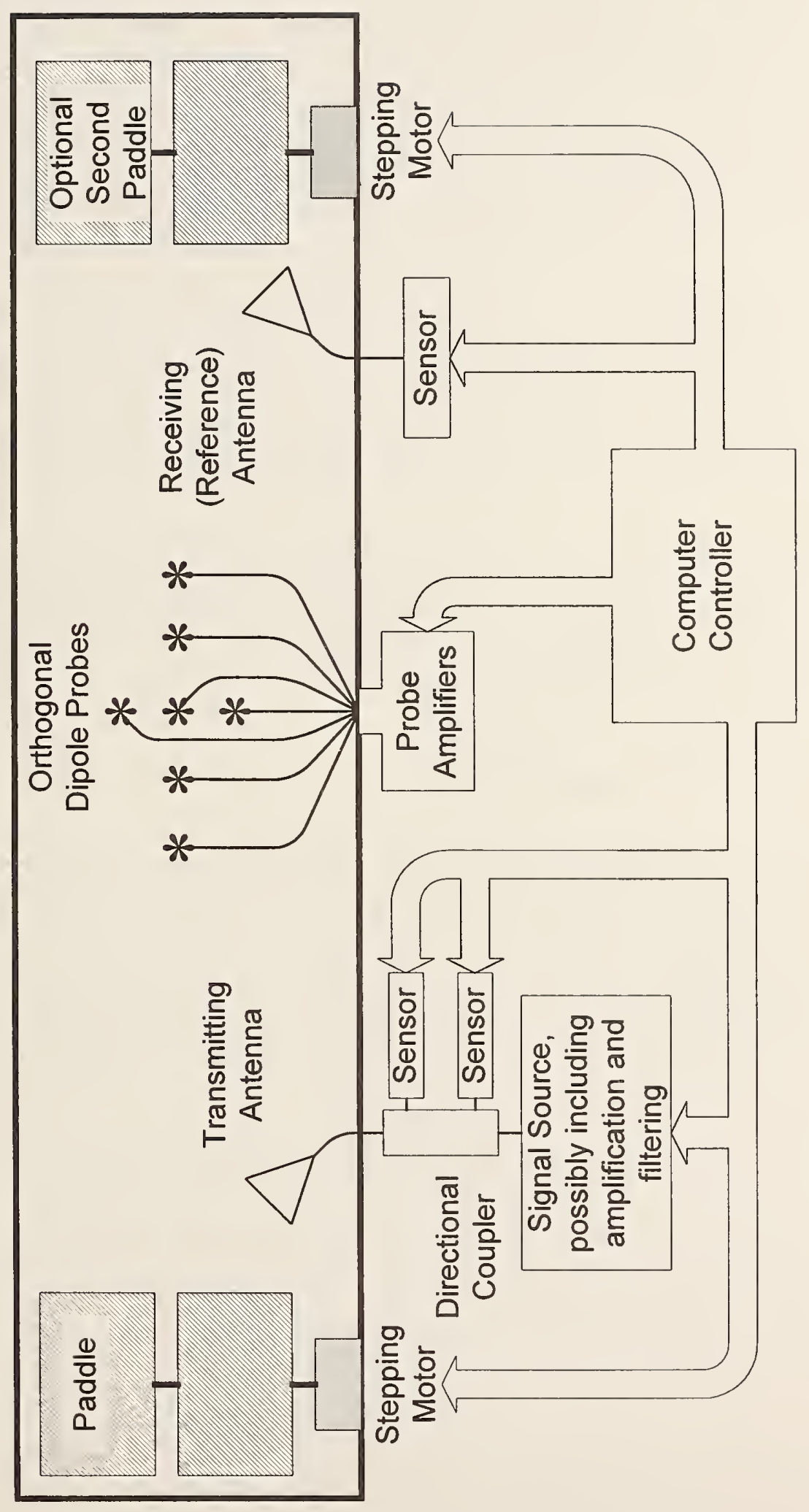

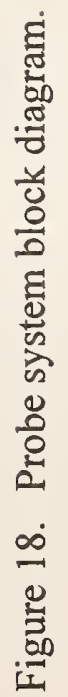


The NIST probe system is a set of 30 direct current (dc) voltmeters, each measuring the rectified dc voltage induced by the electric field on a single very short dipole antenna. The radio frequency (rf) voltage generated by an incident electric field is rectified by a diode across the dipole center gap and filtered by a simple RC filter. A feedline made of a carbon impregnated plastic (tetrafluoroethylene) filament carries the open-circuit voltage to the high-impedance input of the voltmeters. Each isotropic probe is composed of three orthogonal (imagine three adjacent edges of a cube) dipoles very closely spaced and arranged with the feedlines (and the handle) routed along the diagonal of this imaginary cube. This arrangement measures the three orthogonal components of the electric field at a given point in space using three independent antennas bundled into one probe. The 30 voltmeters are then used to measure the voltage from the 10 isotropic probes, each with three outputs corresponding to $E_{\mathrm{x}}, E_{\mathrm{y}}$, and $E_{\mathrm{z}}$. For the measurements described in this report, the $\mathrm{X}, \mathrm{Y}$, and $\mathrm{Z}$ axes of the probes were aligned with the vertical, longitudinal, and transverse axes (respectively) of the rectangular chambers, as defined in Figure 1. For more information on the placement of the probes in each chamber, refer to Section 6.4. A more detailed analysis of the probe construction and frequency characteristics is available in the literature [22] and a description of the electronic design of the voltmeters is given in [23].

The dc voltage generated by an element of a probe antenna and filtered by the diode detector/filter assembly is a nonlinear function of the electric field incident on the dipole. These nonlinearities are compensated for by calibrating the probes. The probe calibrations were performed in a TEM (transverse electromagnetic) cell for frequencies below $300 \mathrm{MHz}$, and in the NIST anechoic chamber using the standard fields system for higher frequencies.

\subsection{Vector Network Analyzer System}

The block diagram of the vector network analyzer measurement is shown in Figure 19. A network analyzer is an instrument designed to automatically sample and record the magnitude and phase of the incident, reflected, and transmitted signals from two test ports. The measurements are made at a number of discrete frequencies selected by the operator. The instrument will drive a signal into one test port and measure the incident and reflected signals at that port and the signal received by the other port. The process is repeated by driving the opposite port and recording a similar set of data. These measurements are usually used to determine the four network S-parameters associated with a two-port network.

The test ports for the network analysis measurements are the cable ends which connect to the antennas inside the chamber. The system is calibrated before each measurement by connecting the calibration standards to the test ports and performing a system calibration as described in the manuals for the network analyzer. This technique allows us to remove the effects of losses and mismatches in the cables, connectors, adapters, and bulkhead feedthroughs. The errors introduced by losses and mismatches will not be completely removed, but the effects will be greatly diminished. 


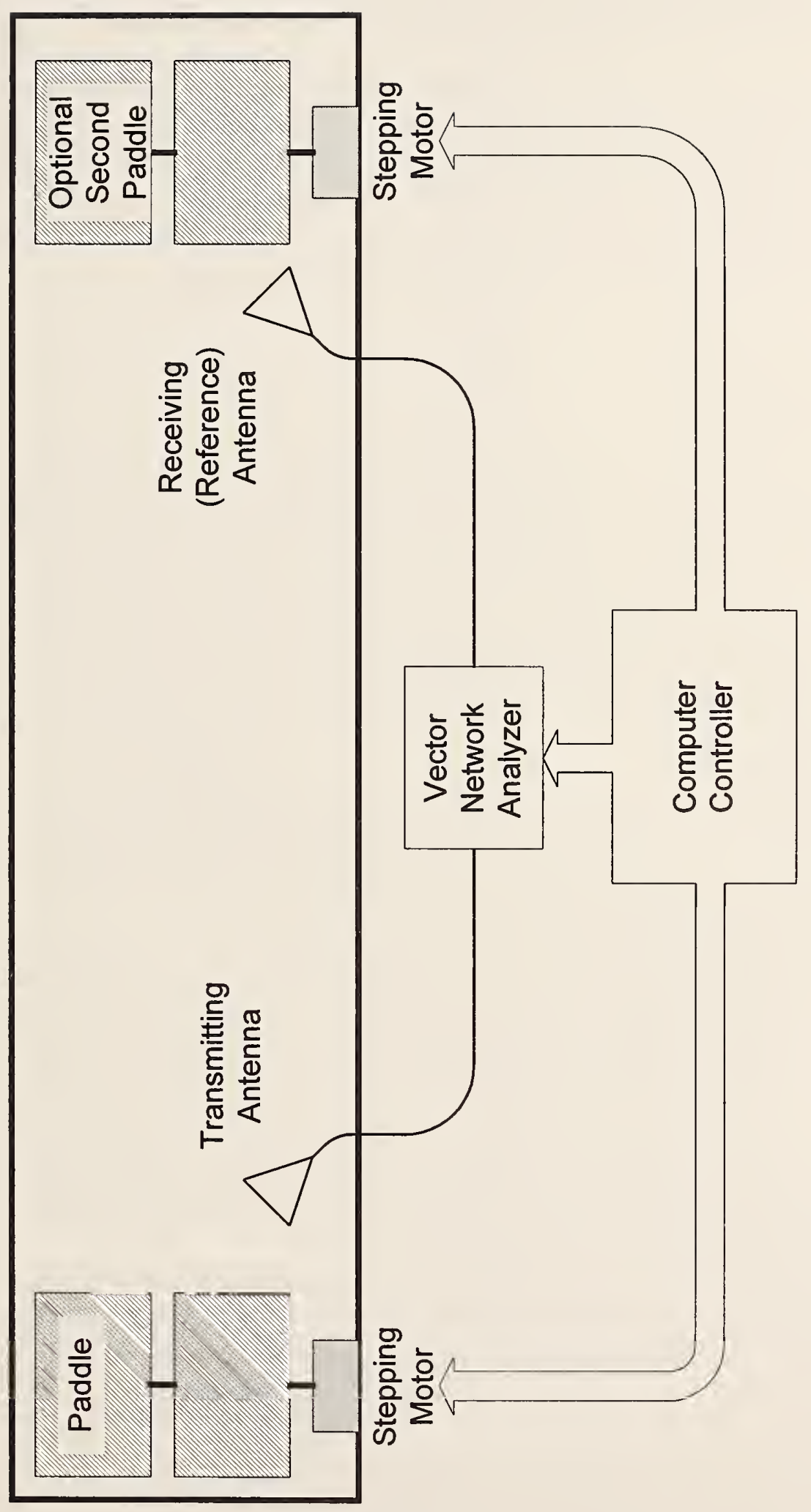

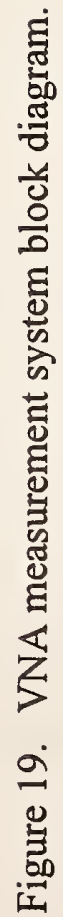


Ideally, we would like to measure only the characteristics of the chamber. At this point, however, there is no way to calibrate out the effects of the antennas. As a result, we are forced to measure the antennas and the chamber as a system and then attempt to remove the antenna effects from the measured data (described in Section 6), resulting in an estimate of the chamber parameters alone.

The network analyzer system operates at relatively low power (less than $100 \mathrm{~mW}$ ) and is not easily configured to generate the field levels required by the NIST isotropic probes. However, the information obtained with the network analyzer is ideal for determining most of the fundamental chamber parameters discussed in Section 3.1. With additional hardware, it may be possible to use the network analyzer with an amplifier, in which case all the measurements described in this report could be performed by a single measurement system.

\section{Measurement Details}

All measurements described in this report were performed using mode-tuned techniques. This means that all measurements were made under static conditions. Any time the paddle was moved, the measurement was paused to allow any mechanical oscillations to die out. We chose the mode-tuned approach rather than the mode-stirred techniques, where the paddle moved continuously, because some of the equipment used in the measurements require steady-state conditions. The mode-tuned approach also allows us to ignore the interplay between a constantly varying signal and the narrow bandwidth of our instrumentation. These issues need to be investigated in greater detail to thoroughly evaluate the advantages and disadvantages of each approach.

Although two different systems were used to characterize the NASA chambers, the general measurement procedure was identical for both systems. A flow diagram of the measurement procedure is shown in Figure 20. First the instrumentation and equipment (cables, couplers, attenuators, etc.) are calibrated.

For the probe system, this calibration is performed beforehand at NIST. The power meters and spectrum analyzer are calibrated using a standard power meter, and only differences between power measured by the standard power meter and the test instrument are recorded. No attempt is made to correct for the mismatch characteristics of the instrumentation, although a test is performed to verify that reflections from the instrumentation are small. The cables, attenuators, and other passive devices are calibrated using a vector network analyzer. Only attenuation values are recorded and used in subsequent measurements. No attempt is made to correct for the phase or mismatch characteristics of the passive devices, but these effects are generally small and have little impact on the total uncertainty of the measurement.

For the VNA measurement system, the calibration is performed immediately before the measurement, and all calibration information (magnitude and phase of both the transmission characteristics and the reflection characteristics) is used to correct for imperfections in the instrumentation and equipment. 


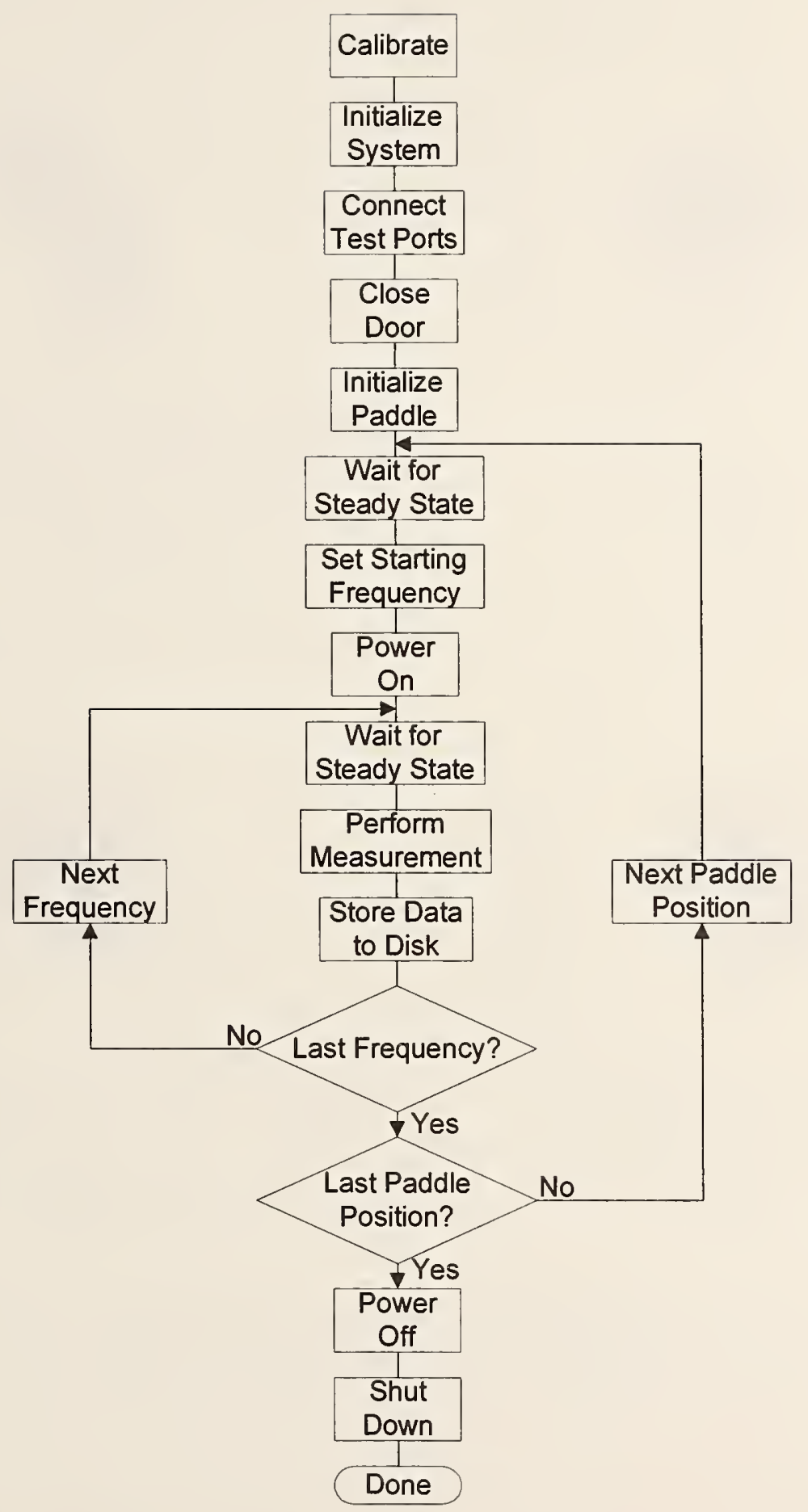

Figure 20. Measurement flow diagram. 


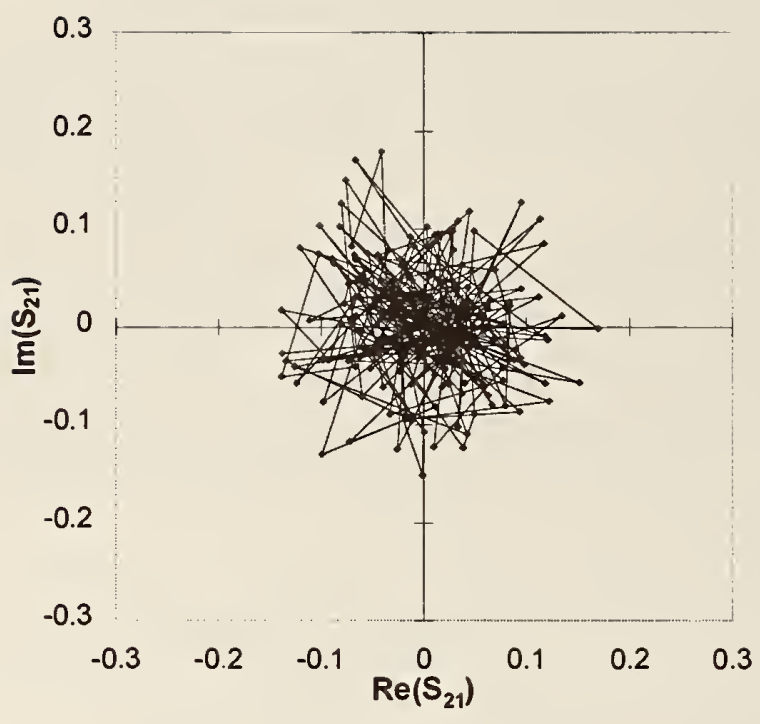

(a)

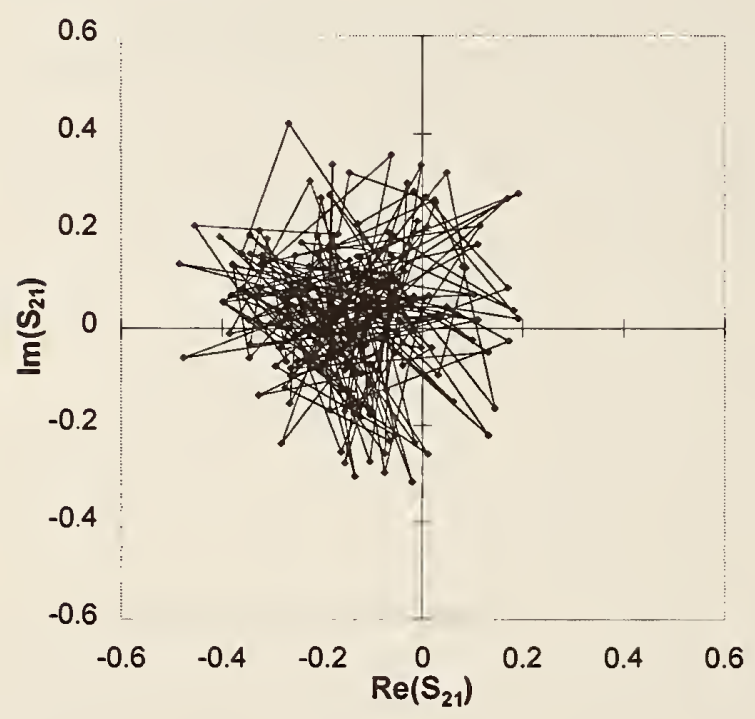

(c)

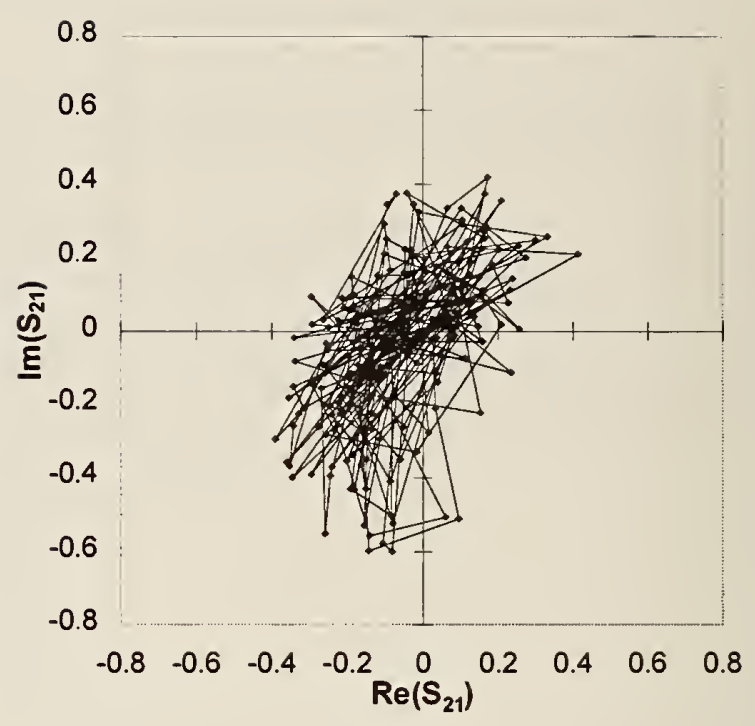

(b)

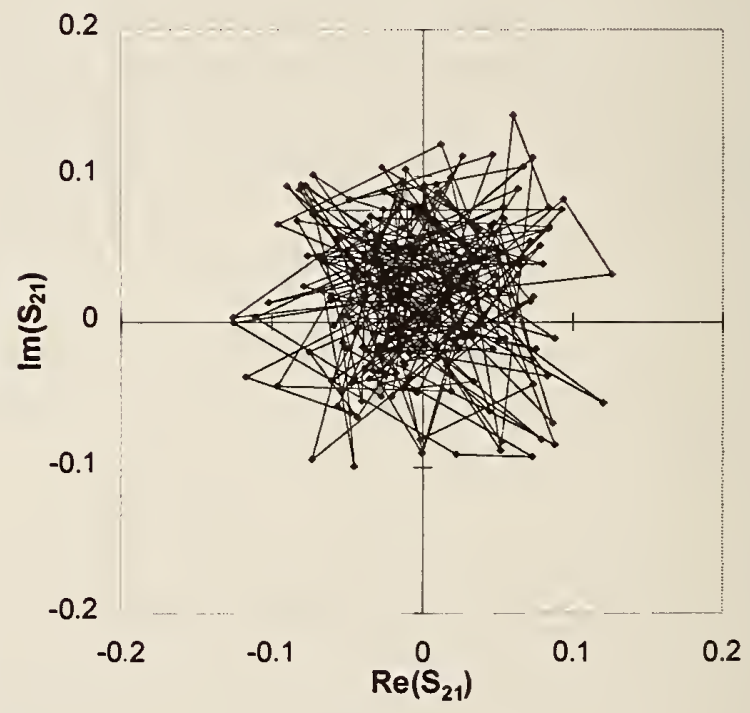

(d)

Figure 21. Scatter plots of $S_{21}$ using two paddles. (a) Simulated data. (b) $90 \mathrm{MHz}$ in chamber A. (c) $200 \mathrm{MHz}$ in chamber $\mathrm{A}$. (d) $1 \mathrm{GHz}$ in chamber $\mathrm{A}$. 


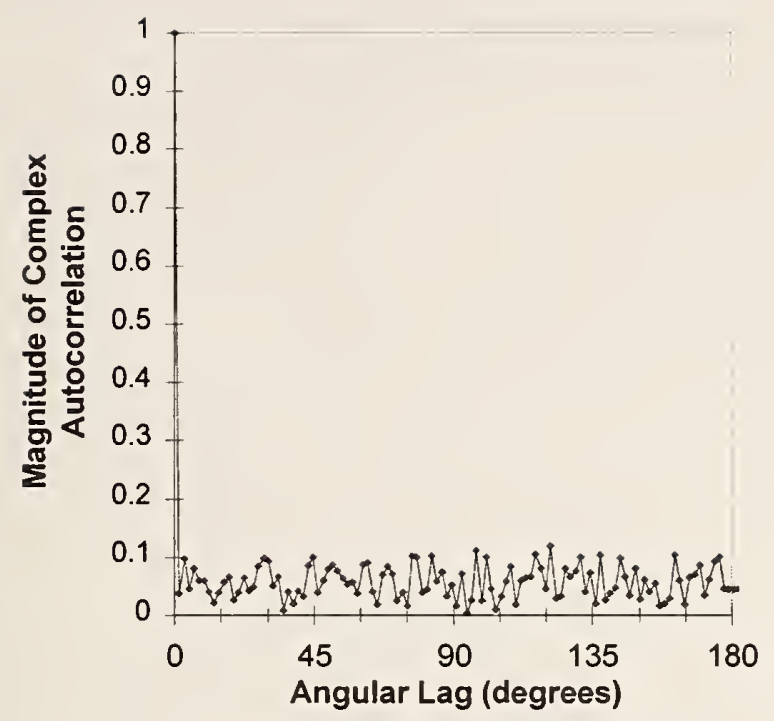

(a)

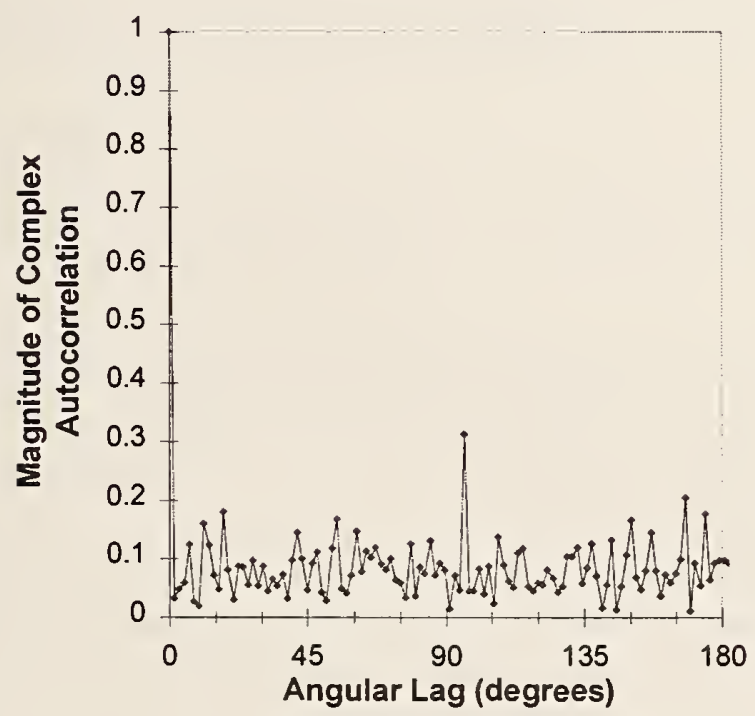

(c)

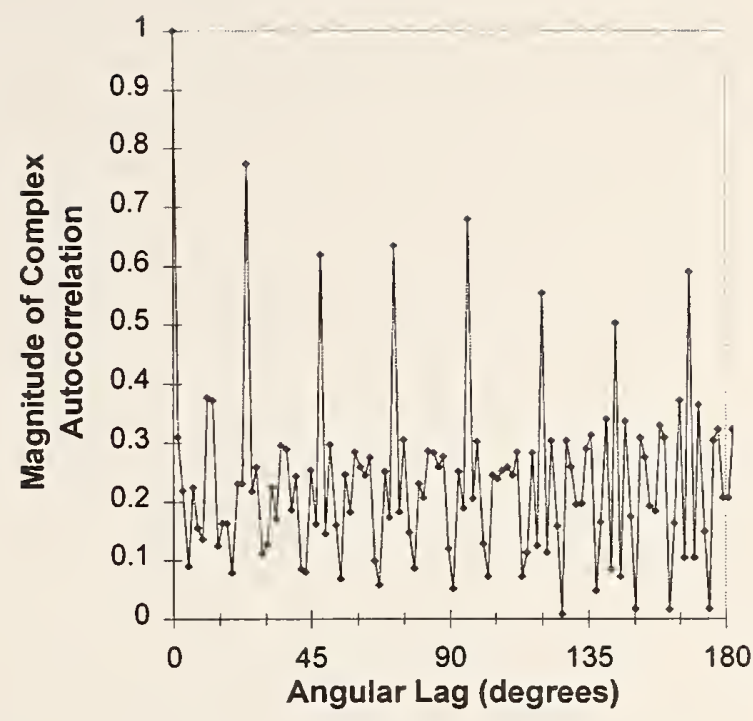

(b)

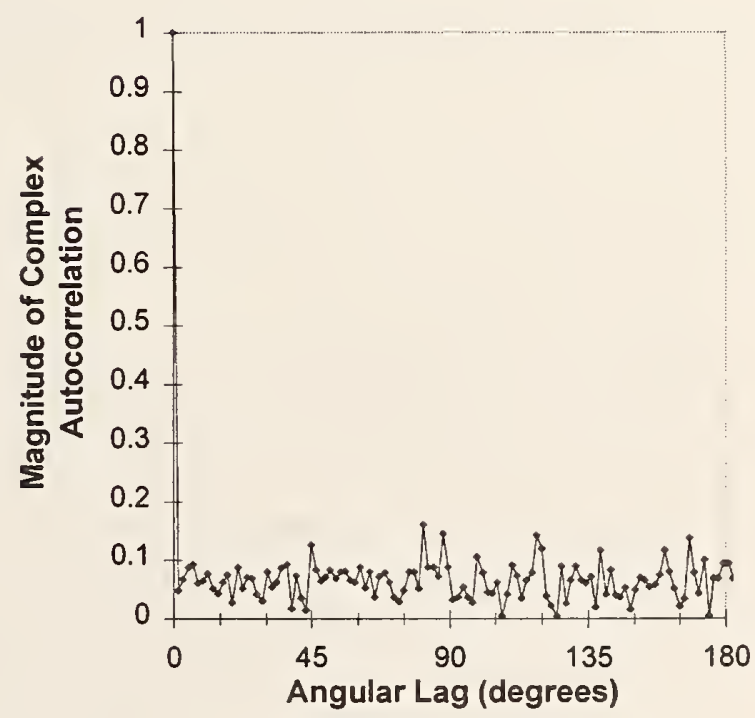

(d)

Figure 22. Autocorrelation of $S_{21}$ using two paddles. (a) Simulated data. (b) $90 \mathrm{MHz}$ in chamber A. (c) $200 \mathrm{MHz}$ in chamber A. (d) $1 \mathrm{GHz}$ in chamber A. 
After the measurement system is calibrated, it is initialized, the test ports are connected to the antennas, the chamber door is closed, and the paddle is set to an initial location. Once the chamber has reached steady-state conditions (the door is closed, the paddle position is stationary), the systems measure the parameters of interest at each requested frequency and store the data. These measurements are being made in a large, high-quality-factor environment; any transient (change in amplitude, change in frequency) may not die out rapidly. For this reason, a delay should be programmed into the system so that any transients will die out before a measurement is made. Failure to do this may result in significant errors [24].

Once the measured data are recorded at each frequency of interest, the paddle is moved and the measurement is suspended until the mechanical oscillations of the paddle die out. This sequence is repeated until measurements have been made at all of the desired paddle positions. After all the data have been collected, the rf power is turned off, and the measurement is complete. The final result is a computer file that contains the data, measured at a large number of frequencies and paddle positions.

\subsection{Selection of Paddle Positions and Number of Paddle Steps}

Before a measurement is started, the operator must select the number of unique paddle locations and how those locations will be distributed. For the case of a single paddle, the distribution of paddle locations is reasonably simple: choose $N$ paddle positions, and divide a single revolution into steps of $360^{\circ} / \mathrm{N}$. Other, more complicated distributions could be used, but the expected gains were outweighed by the increased complexity of other methods. For more than one paddle, the choice of paddle locations is more difficult. Possible examples include leaving one paddle stationary while stepping the other as described above, or stepping both paddle with equal steps simultaneously. Based on the autocorrelation plots given in Figure 5, we expect that a large change in the position of any individual paddle will result in low sequential correlation. The challenge is choosing an algorithm for moving two or more paddles that takes advantage of this effect. Obviously, moving only one paddle will not be much of an improvement, and moving two paddles simultaneously by the same increment will probably not be much better. One possibility would be to move one paddle in large increments and the other paddle in small increments. Eventually, the paddle which is stepped in large increments will have rotated through one full revolution. When this occurs, the paddle which is stepped in small increments should have moved far enough that the overall correlation is small. One possible implementation of this scheme and the implementation we used is to move one paddle in steps of $360^{\circ} / N$ and the other in steps of $360^{\circ} / \sqrt{N}$. As for the choice of $N$, we wanted a value close to 200 in order to be compatible with NBS Tech. Note 1092 [3], and also a perfect square to make the paddle step size easy to calculate. The best candidates were $N=14^{2}=196$ and $N=15^{2}=225$, and we chose a value for $N$ of 225 for all of our measurements, including those made in chambers with only a single paddle. The choice of $N$ and of the step sizes listed above are arbitrary, and other choices may result in data that is in some way "better" than what we measured. Figure 21 shows some scatter plots of measured data. Figure 21a shows a scatter plot for an idealized, completely independent case generated with a random number generator. 
Figures $21 \mathrm{~b}$ through d show measurements in Chamber A at $90 \mathrm{MHz}, 200 \mathrm{MHz}$, and $1 \mathrm{GHz}$. Figure 22 shows the autocorrelation plots of the data presented in Figure 21. We saw an apparent improvement in the autocorrelation of the data in the chamber with two paddles relative to the chamber with one paddle (Figure 5), but we saw some other interesting results as well. Based on the number of paddle steps and the step sizes described above, one of the paddles will make a complete revolution every 15 steps. If the correlation is large even for a movement of $1 / 15$ revolution or $24^{\circ}$, then the autocorrelation will have spikes every 15 steps. We think this is the cause of the strange behavior shown in Figure 22b. Based on this plot, some procedure of moving the paddles which does not require one of the paddles to go to the same location repeatedly will probably give better results.

\subsection{Correcting for Antenna Effects}

The primary difficulties in evaluating the measured data come from estimating the effects the transmitting and receiving antennas can have on the overall measurement, and from estimating the value of the power transmitted into the chamber. These problems occur in freespace measurements as well as mode-stirred measurements, so we first examine the characteristics of antennas used in free-space measurements. Many of the techniques used in free-space measurements can be applied to mode-stirred chamber measurements as well, and this will be discussed in Section 5.2.2.

\subsubsection{Antenna Effects in Free-Space Measurements}

To better understand how the antennas used in mode-stirred applications behave, it is helpful to examine typical free-space measurements, based on IEEE standard definitions and terms for antennas [25]. An idealized free-space transmission measurement is shown in Figure 23 . We can accurately measure the power available to the transmitting antenna and the power reflected by the antenna, referenced to the input port of the antenna. Assuming the antenna is located in free space, these reflections are only due to mismatch effects inside the antenna (scattering effects will be negligible). The difference between the power available to the antenna $P_{i n c}$ (the incident power) and the power reflected by the antenna $P_{\text {reff }}$ is equal to the net input power $P_{N E T}$. $P_{N E T}$ is also equal to the power accepted by the antenna. Some of this power will be dissipated by resistive losses in the antenna, and the remainder will be radiated (transmitted). The ratio of the total power radiated by an antenna to the net power accepted by the antenna is defined as the radiation efficiency $\eta$ of the antenna. For an antenna with arbitrary efficiency, the transmitted power is equal to the net input power times the antenna efficiency. If the free-space reflection coefficient of the transmitting antenna is $S_{11}^{0}, P_{\text {refl }}$ is simply the product of $P_{i n c}$ and $S_{11}^{0}$, and the transmitted power $P_{T}$ is given as

$$
P_{T}=\eta P_{N E T}=\eta P_{i n c}\left(1-\left|S_{11}^{0}\right|^{2}\right)
$$



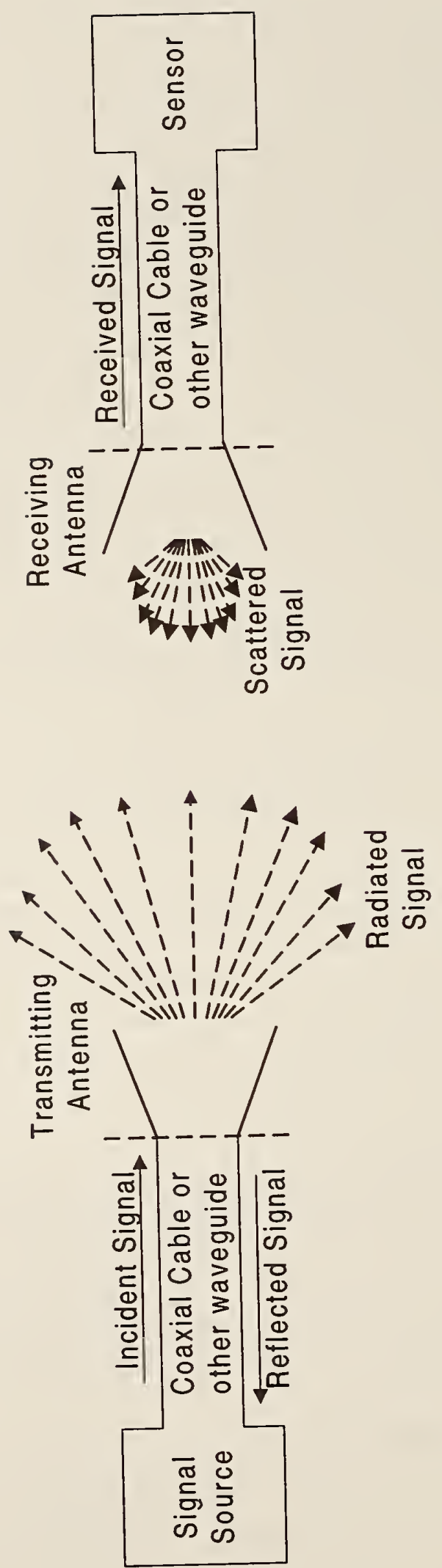

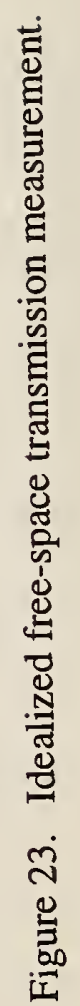


In general, $P_{i n c}, P_{N E T}$, and $S_{11}^{0}$ can be measured accurately and easily. The antenna efficiency $\eta$ is extremely difficult to measure, and has traditionally been assumed to be nearly 1 for all antennas. Because of the assumed value for $\eta$, the transmitted power cannot be known exactly, but can be estimated fairly well. Once the transmitted power has been estimated, other terms related to the pattern of the antenna are then applied to give a measure of the field generated at any location relative to the location of the transmitting antenna. Thus, if we know $P_{N E T}, \eta$, and the antenna pattern, we can calculate the electric field generated by the transmitting antenna at any point in space.

The receiving antenna can be analyzed similarly. If a receiving antenna is lossless and perfectly matched, then the power measured $P_{\text {meas }}$ by a matched sensor attached to the antenna will be equal to the power received $P_{R}$ or accepted by the antenna from the environment.

If the receiving antenna is perfectly matched but not lossless (not perfectly efficient), the power measured by the sensor will be reduced by the efficiency of the antenna: $P_{\text {meas }}=\eta P_{R}$.

On the other hand, if the receiving antenna is perfectly efficient but not perfectly matched, then the power measured by the sensor will again be decreased. Technically, the receiving antenna accepts less power from the environment because it is not perfectly matched. Reciprocity tells us that the measured power should be given by $P_{\text {meas }}=P_{R}\left(1-\left|S_{22}^{0}\right|^{2}\right)$ where $S_{22}^{0}$ is the free-space reflection coefficient of the antenna measured "looking into" the antenna terminal.

Finally, if the receiving antenna is neither perfectly matched nor perfectly efficient, the situation is somewhat more difficult to evaluate. The most reasonable approach is to assume that the measured power is given by

$$
P_{\text {meas }}=\eta P_{R}\left(1-\left|S_{22}^{0}\right|^{2}\right) \text {. }
$$

Using eq (79), we can estimate the power that would have been received by a perfect antenna as

$$
P_{R}=\frac{P_{\text {meas }}}{\eta\left(1-\left|S_{22}^{0}\right|^{2}\right)}
$$

Unfortunately, the losses in the antenna could affect measurements of $S_{22}^{0}$, and estimates of $P_{R}$ based on measurements of $P_{\text {meas }}$ could be either higher or lower than the power that would actually be accepted by a perfect antenna. To minimize this effect, a receiving antenna that is as efficient as possible should be selected. Once a receiving antenna has been selected, eq (80) should be used to estimate the power that would have been accepted by a perfect antenna. 


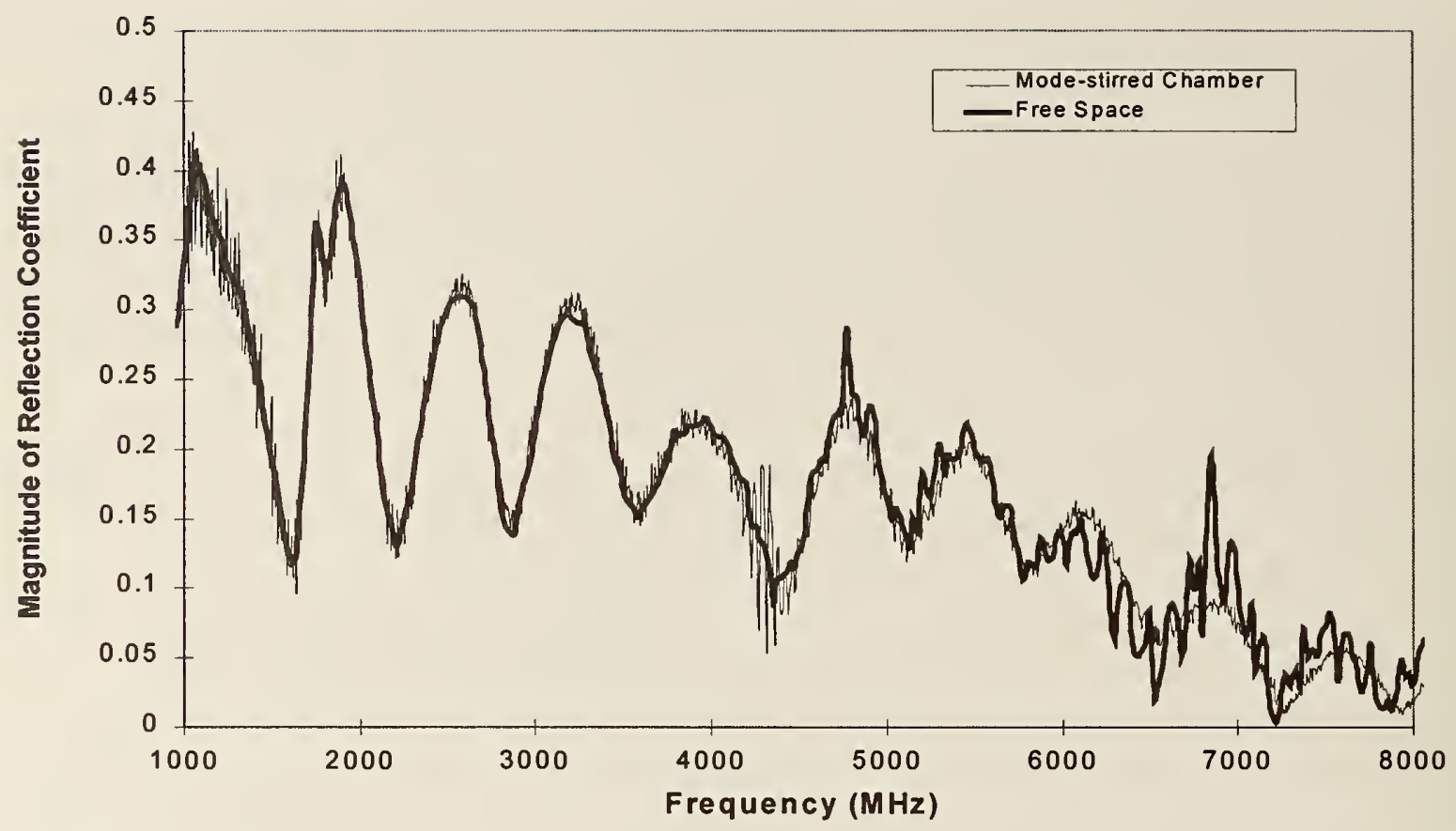

Figure 24. Comparison of $S_{22}$ measured in free space and in a mode-stirred chamber.

\subsubsection{Antenna Effects in Mode-Stirred Chambers}

Once an antenna is placed inside a mode-stirred chamber, things become more complicated. The reflections measured at the input of the transmitting antenna will consist of two components: the intrinsic reflection caused by the antenna, and the reflections caused by the transmitted signal interacting with the chamber and returning to the antenna. Now the definition of transmitted power is somewhat more complex. Measurements have shown that the average electromagnetic environment inside a mode-stirred chamber behaves much like free space [3], with the average wave impedance inside the chamber equal to that of free space. Unfortunately, this does not tell us what the average reflection coefficient of an antenna might be if it is placed inside a mode-stirred chamber, but a good guess might be that, on average, the antenna will behave as if it were in free space. Thus, the magnitude of the average reflection coefficient $\left(\left|<S_{11}\right\rangle \mid\right.$ or $\left.\left|<S_{22}>\right|\right)$ measured in a mode-stirred chamber and averaged over a large number of paddle positions should be similar to the magnitude of the reflection coefficient of the antenna in free space $\left(\left|S_{11}^{0}\right|\right.$ or $\left|S_{22}^{0}\right|$, where the superscript 0 will be used to indicate the value measured in free space). Figure 24 shows measured values of $\left|\left\langle S_{22}\right\rangle\right|$ measured in NASA Chamber A and $\left|S_{22}^{0}\right|$ for a dual-ridged horn between the frequencies of $1 \mathrm{GHz}$ and $8 \mathrm{GHz}$. Given the dramatic difference between the two measurement methods, the agreement is remarkable. 
Thus, if we are interested in maintaining a constant net input power, we can write the transmitted power as

$$
P_{T}=\eta P_{N E T}=\eta P_{\text {inc }}\left(1-\left|S_{11}\right|^{2}\right),
$$

where $S_{11}$ is measured at each paddle position, and adjust $P_{i n c}$ such that $P_{N E T}$ stays constant. Alternatively, if we are interested in maintaining a constant incident power, we can write the transmitted power as

$$
P_{T}=\eta P_{\text {inc }}\left(1-\left|<S_{11}>\right|^{2}\right),
$$

which does not require any change to $P_{i n c}$.

The agreement between $\left|<S_{22}>\right|$ and $\left|S_{22}^{0}\right|$ is important for two reasons. First, if an antenna has been calibrated in free space (or an anechoic chamber), it may not be necessary to measure reflections during the measurement. Alternatively, measurement data inside the chamber can be compared with the reference data during the measurement to verify performance during the test. Second, if the antennas have not been calibrated, it may be possible to calibrate them inside a mode-stirred chamber facility, or at least roughly determine their characteristics.

Figure 24 shows that the magnitude of the average reflection coefficient inside a modestirred chamber is a good approximation of the magnitude of the free-space reflection coefficient, and the average wave impedance inside a mode-stirred chamber has been previously shown to be approximately equal to the free-space wave impedance [3]. Based on these two observations, we guess that the average transmitted power is the power which would be transmitted if the antenna were placed in free space, as given in eq (78). If this guess is valid (and it has not yet been verified in detail), we are faced with an obvious problem. Equation (78) gives two ways of calculating the power transmitted by an antenna in free space: one based on the net input power $P_{N E T}$, and the other based on the incident power. In free space, these equations give the same result, but in a mode-stirred chamber the differences can be substantial. The net input power $P_{N E T}$ will fluctuate as the paddle rotates, whereas $P_{i n c}$ will remain approximately constant. The measurement systems used to evaluate the NASA chambers were not equipped to hold any of these measures of power constant. Instead, they measured the actual incident and reflected powers and using this information, they normalized the received signals mathematically as if either the net input power or the incident power were held constant. We are now faced with the problem of determining if either of the two options for normalizing the received signals is correct, or deciding on another option. We will not attempt to answer this question here. Instead, we will present some of the advantages and disadvantages associated with each option, and present data processed using both techniques. In the discussion below, we will address the different types of mathematical normalization techniques we have available to us. It is important to remember, however, that a test performed in a mode-stirred chamber cannot rely on mathematical normalization, but must actually maintain a constant transmitted power. This can result in a significant increase in the time required for measurement.

The receiving antenna can also be treated in a similar manner. We will apply the same corrections that we would apply in a free-space measurement. Assuming that the magnitude of 
the free-space reflection coefficient of the receiving antenna can be determined in a mode-stirred chamber as the as the magnitude of the average measured reflection coefficient, we can rewrite eq $(80)$ as

$$
P_{R} \approx \frac{P_{\text {meas }}}{\eta\left(1-\left|<S_{22}>\right|^{2}\right)} \text {. }
$$

Poor efficiency may cause the estimate of $P_{R}$ to be different from the power that would have been received by an ideal antenna, but, for now, this appears to be the best approximation available to us.

\subsection{Normalization Techniques}

The net input power has traditionally been used as the more accurate estimate of transmitted power and has been used in all previous work done at NIST [3]. The basic argument justifying the use of $P_{N E T}$ is that the only power available for interacting with a device inside the chamber is equal to the net power entering the chamber, and any reflected power is a measure of the "amount" of the transmitted signal that never actually entered the chamber. Calculating the transmitted power based on $P_{N E T}$ has several advantages. First, there is a historical precedent, and much data have been measured based on a constant value for $P_{N E T}$. This should not keep us from examining alternative methods, but can be a compelling argument for maintaining the status quo. Second, the net input power is easy to calculate and is well defined at each paddle position. If we assume that the transmitting antenna is perfectly efficient, the net power coupled into the antenna will be equal to the net power coupled into the chamber. If we were to hold $P_{N E T}$ constant over all paddle positions (which requires us to measure $P_{\text {refl }}$ at every paddle position and compensate for it by changing $P_{i n c}$ ), we would have a system that was equivalent to placing an ideal power source into the chamber. This ideal source can transmit a constant power into the chamber, but will not remove any power from the chamber. In this case, the transmitting antenna will not act like a receiving antenna. Also, the experiment should be better controlled by holding $P_{N E T}$ constant, which could result in lower uncertainties.

The primary disadvantages of this technique are complexity and the potential for extremely high power requirements. During a typical measurement, the reflected power can vary in a range between 0 and values near the incident power. For each new paddle position or frequency, the reflected power must be measured and compensated for. If the reflected power is only slightly less than the incident power, it may be necessary to transmit large amounts of power just to maintain a relatively small net input power. Typically, only a fixed power is available, and this fixed power may not be sufficient to hold $P_{N E T}$ constant. In this case, the operator must decide if the test is still valid even though a constant transmitted power could not be maintained for the entire test. Finally, if the magnitude of the reflected power is approximately equal to the magnitude of the incident power, the difference between $P_{i n c}$ and 
$P_{\text {refl }}$ will be very sensitive to noise and calibration errors, and this may translate into large measurement errors.

Finally, if the efficiency of the transmitting antenna is not perfect, there will be a difference between the net power coupled into the antenna and the net power transmitted into the chamber. In fact, the power transmitted into the chamber can fluctuate even if the net power coupled into the antenna is held constant. As an extreme example, consider a situation in which there is no net power coupled into the chamber (all power transmitted by the antenna is reflected back into it). For a lossless antenna, this would mean that no net power is coupled into the antenna. For a real antenna, some of the power coupled into the antenna will be converted to heat by the resistive losses of the antenna, resulting in a nonzero value for the net power coupled into the antenna, even though the net power coupled into the chamber is 0 . Thus, even holding the net input power constant does not result in a well defined value for the instantaneous transmitted power (at a specific paddle position).

The incident power has been suggested as an alternative to the net input power as a measure of the transmitted power. The incident power has generally been used at high frequencies where the fluctuations in reflected power are small relative to the value of the incident power. This technique for estimating the transmitted power is used because it is less time consuming and easier to implement than the technique which estimates the transmitted power based on the net input power. These advantages are also present at low frequencies, and are therefore worth considering. First, however, we will take a closer look at the assumption that the only power available to interact with a device inside the chamber is the net input power. If the net power into the chamber is 0 , no power can be received by another antenna inside a chamber. This condition is reasonable and consistent. This situation can be viewed differently, however. The transmitting antenna can be viewed using superposition as both a transmitter and a receiver. From this perspective, a net input power of 0 is caused by the transmitting antenna receiving all of the power which has been transmitted into the chamber. Once again, this interpretation is reasonable and consistent. Thus, either of these views can be justified.

Note, however, that when we calculate the transmitted power based on the incident power we ignore the power received by the transmitting antenna. By superposition, we expect the transmitting antenna to receive approximately the same power as the receiving antenna. Thus, maintaining a constant incident power results in two antennas removing power from the chamber, whereas maintaining a constant net input power results in only one antenna removing power. Consequently, the two methods of normalization will result in different estimates of the fundamental properties of the chamber, with higher estimates of $Q$, electric field, scalar power density, and chamber gain when $P_{N E T}$ is held constant than when $P_{i n c}$ is held constant.

The primary advantages of using $P_{i n c}$ to estimate the transmitted power are mentioned above. This technique is simple to implement and does not require that the reflected power be monitored. Also, since the incident power does not fluctuate with paddle position (assuming a matched source), the power requirements for a given measurement can be known ahead of time. This allows an accurate estimation of the power densities or electric fields that can be produced given a specific set of amplifiers.

The disadvantages of calculating the transmitted power based on $P_{i n c}$ are a lack of historical precedent, loss of knowledge about the reflected power, and possible increased 
uncertainty as a result of ignoring fluctuations in the reflected power. As mentioned before, the lack of a historical precedent is not a strong argument. The other two, however, could be important. It seems reasonable that as much information should be used as possible, and neglect of any component will give a less reliable result. This of course assumes that the component is used correctly in the evaluation of the final result.

Another problem occurs if the transmitting antenna is not perfect, especially if it is not perfectly impedance matched. For any given incident power into the transmitting antenna, it is possible for the transmitting antenna to receive a signal that has a magnitude and phase such that the reflection caused by the intrinsic mismatch of the antennas is exactly canceled, resulting in complete transmission of the incident signal. Similarly, a portion of the received signal could be reflected (actually scattered) by the transmitting antenna in such a way that the entire amount of incident power is canceled, resulting in no transmitted power. Once again, this definition may not result in a well defined value for the instantaneous value of the transmitted power (at each individual paddle position) but the result may be adequate on average.

\subsection{Significance of Transmitted Power}

Given the uncertainty as to the best way to estimate the transmitted power discussed in the previous section, we now examine the question of why we are even interested in the transmitted power. The primary electromagnetic parameters, such as electric field, scalar power density, and power received by an antenna, can be measured independently and do not require knowledge of the transmitted power. All that is required is that some measure of the transmitted power is held constant so that the test can be repeated if necessary. The quality factor and chamber gain, which are defined in terms of both the transmitted and received power, are useful simply because they give us a mechanism for estimating the power received by an antenna or the field measured by a probe as a function of transmitted power. If a reference antenna or probe is used to establish and measure a particular electromagnetic parameter, then the definition of transmitted power is unimportant.

In general, however, the transmitted power is used to establish known operating conditions inside the chamber. Once the operating conditions are known, the only requirement for establishing the same operating conditions (assuming the chamber is unchanged) is that the transmitted power, however it was previously measured, be set to the same value, and that the same transmitting antenna is used in establishing and reestablishing the operating conditions. For most tests inside a mode-stirred chamber, the actual method of estimating the transmitted power is unimportant, as long as the same method is used consistently. The only reason to choose one over the other is if the uncertainties are substantially smaller or one method is substantially less cumbersome to implement.

Occasionally we are interested in the chamber parameters themselves. For example, we may be interested in evaluating the conductivity of the chamber walls. In this case, we must be more concerned with the exact definition of all of the components involved in calculating the final quantities. In this case, the definition of transmitted power may be significant in the final evaluation. 
Alternatively, if we are interested in which definitions of transmitted power gives the best results, we could construct a chamber with known properties, and then see which technique gives us results that are similar to the expected results. The problems of determining the optimal processing techniques are generally theoretical in nature, and can be ignored for the majority of measurements that can be made using a mode-stirred chamber. For this reason, we will not attempt to resolve this issue here. Instead, we will present data processed using different techniques and leave the final choice to the reader.

\section{Data Processing Techniques}

We developed several methods of processing the measured data in an effort to use the measured data as efficiently as possible. These methods include techniques for verifying the validity of the statistical models, predicting and correcting for the effects of the antennas used in the measurements, and comparisons of the probe system and the measurement system. All of the processing techniques will be described in this section. For examples, we will use data taken in NASA Chamber A with 225 paddle steps. These figures are representative of all measurements we made, including additional measurements in Chamber $\mathrm{A}$ as well as in the other two chambers, and the final results of the analysis of the other chambers will be presented in detail in Section 7.

All measurements performed using a vector network analyzer returned measurements of system S parameters. Typically, mode-stirred chamber results have been presented in terms of measured power. To simplify the discussion, we will assume an incident power of $1 \mathrm{~W}$. Assuming the system is linear (which appears to be well justified), the received power at any given paddle position is given as $\left|S_{21}\right|^{2}$, the reflected power is $\left|S_{11}\right|^{2}$, and the net input power is $1-\left|S_{11}\right|^{2}$. If we want to normalize the received power to a constant incident power (which we are already assuming), no correction is necessary. If we want to normalize to a constant net input power, then the received power at each paddle position would be $\left|S_{21}\right|^{2} /\left(1-\left|S_{11}\right|^{2}\right)$. From this point on, we will discuss the measurements in terms of measured power instead of measured $S$ parameters, except as noted.

Initially, we will present data that depend on measured values and do not require normalization or correction. These include raw measurements of $S$ parameters and simple functions of those $S$ parameters. The measured reflection parameters will be used to give measurements of the minimum, average, and maximum reflected power, estimates of the freespace reflection coefficient, the minimum, average, and maximum VSWR of the antennas and an estimate of the free-space VSWR of the antennas. The transmission coefficients will be used to measure the minimum, average, and maximum power received by an antenna in the chamber, and also to evaluate the quality of the mechanical stirrer.

We will assume that any correction factors we apply to the data will be applied at each paddle position. This is equivalent to assuming that the efficiency and mismatch characteristics of the antennas are independent of paddle position. Therefore, these corrections will affect the minimum, average, and maximum measured values equally, and a ratio of any of these measured values will also be independent of the correction factor. These measurements include the 
maximum-to-minimum ratio, maximum-to-average ratio, as well as the average-to-minimum ratio which we introduce here.

The average received power is then processed to correct for the estimated characteristics of the antennas used in the measurements. Here we present a simple two-parameter model of the characteristics of the chamber and several methods for evaluating those two parameters. These parameters are then used to evaluate our ability to correct for antenna characteristics. We correct for the mismatch effects of both the transmitting and receiving antenna, as well as the efficiencies of the antennas. To do this, we present a new approach for estimating the relative efficiencies of antennas. The final corrected values of received power are then used as an estimate of the average chamber gain. The average chamber gain is then used to estimate the average chamber quality factor, as well as the average magnitude of the electric field (both rectangular component and total) and the scalar power density for an input power of $1 \mathrm{~W}$. We then use the statistical model to predict the maximum chamber gain and discuss the difficulties associated with estimating the maximum of other parameters.

The antenna characteristics and the correction factors we derive from them are approximately representative of the characteristics and correction factors that would be expected for any set of typical antennas, but these results should not be used for processing or evaluating any data that were not obtained using the exact same antennas that were used for the evaluation. Even antennas that are the same make and model as those used in the evaluation can have different characteristics, as can be seen in the differences between the forward and reverse measurements presented below. These antennas had sequential serial numbers but still had different characteristics.

Finally, we use the probe system to evaluate the characteristics of the electric field (both rectangular component and total) inside the chamber, and show that the power received from a reference antenna can be used to predict both the average and maximum magnitudes of both measures of the electric field.

\subsection{Processing of Raw S Parameters}

To begin with, we will present the unprocessed data we have available to us for evaluating the chamber. Figure 25 shows a plot of the minimum, average, and maximum measured value for the reflected power. Figure $25 \mathrm{a}$ is the reflected power when port 1 is transmitting (the forward direction), and Figure 25b is the reflected power when port 2 is transmitting (the reverse direction). For these figures, the reflection coefficient was measured at each paddle position, the squared magnitude was computed, and the minimum, average, and maximum values were stored.

The plots of reflected power are included to give an indication of the power that can be expected on the reflected sidearm of a directional coupler or the power a source amplifier may be required to dissipate at some point during a measurement. If, for example, a maximum reflected power of $0.8 \mathrm{~W}$ was measured for an input power of $1 \mathrm{~W}$, then a $100 \mathrm{~W}$ amplifier may be forced to dissipate up to $80 \mathrm{~W}$ of reflected power, and a power sensor connected to the reflected sidearm of a $20 \mathrm{~dB}$ directional coupler will require a rating of greater than $0.8 \mathrm{~W}$. 


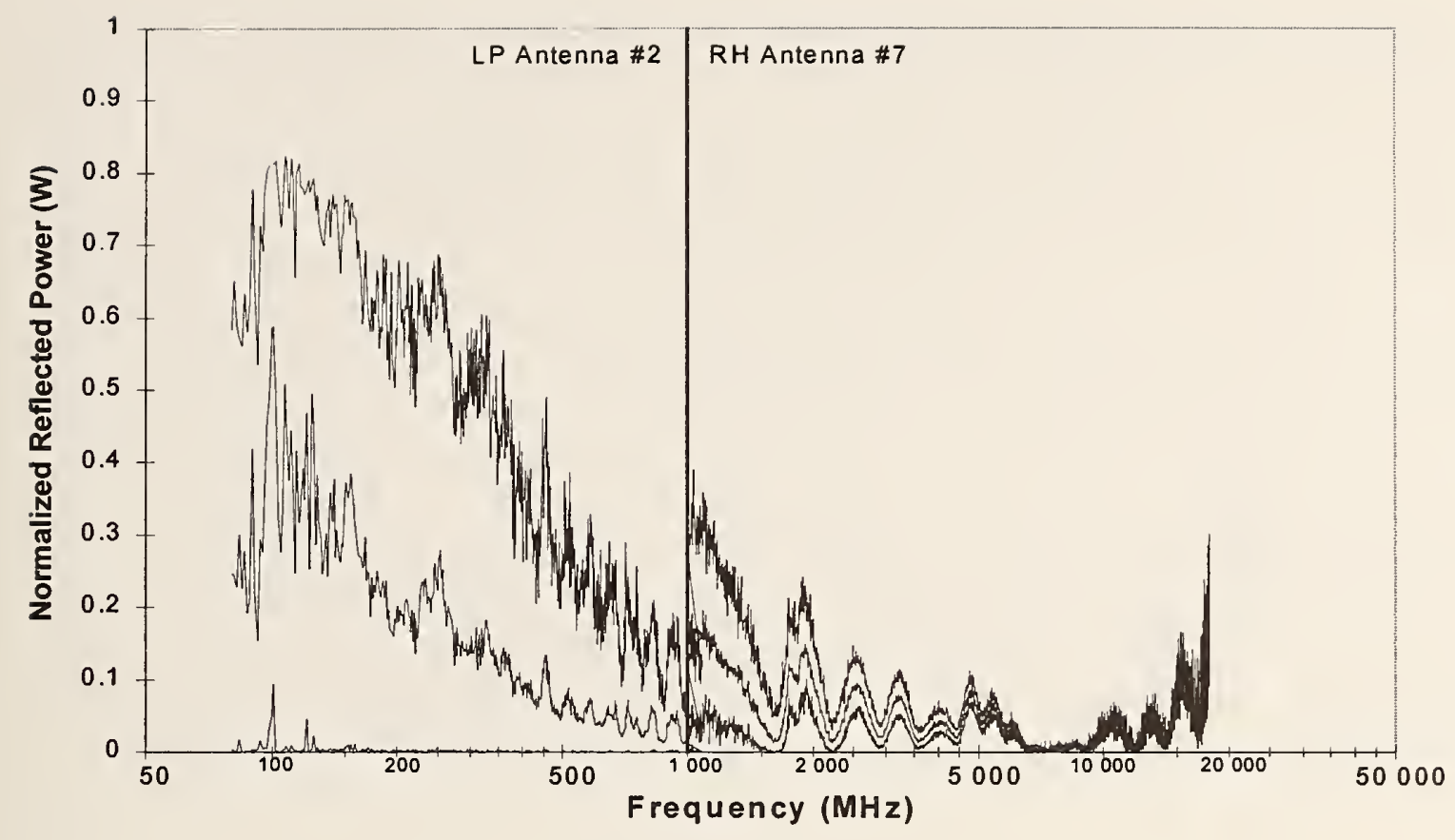

(a)

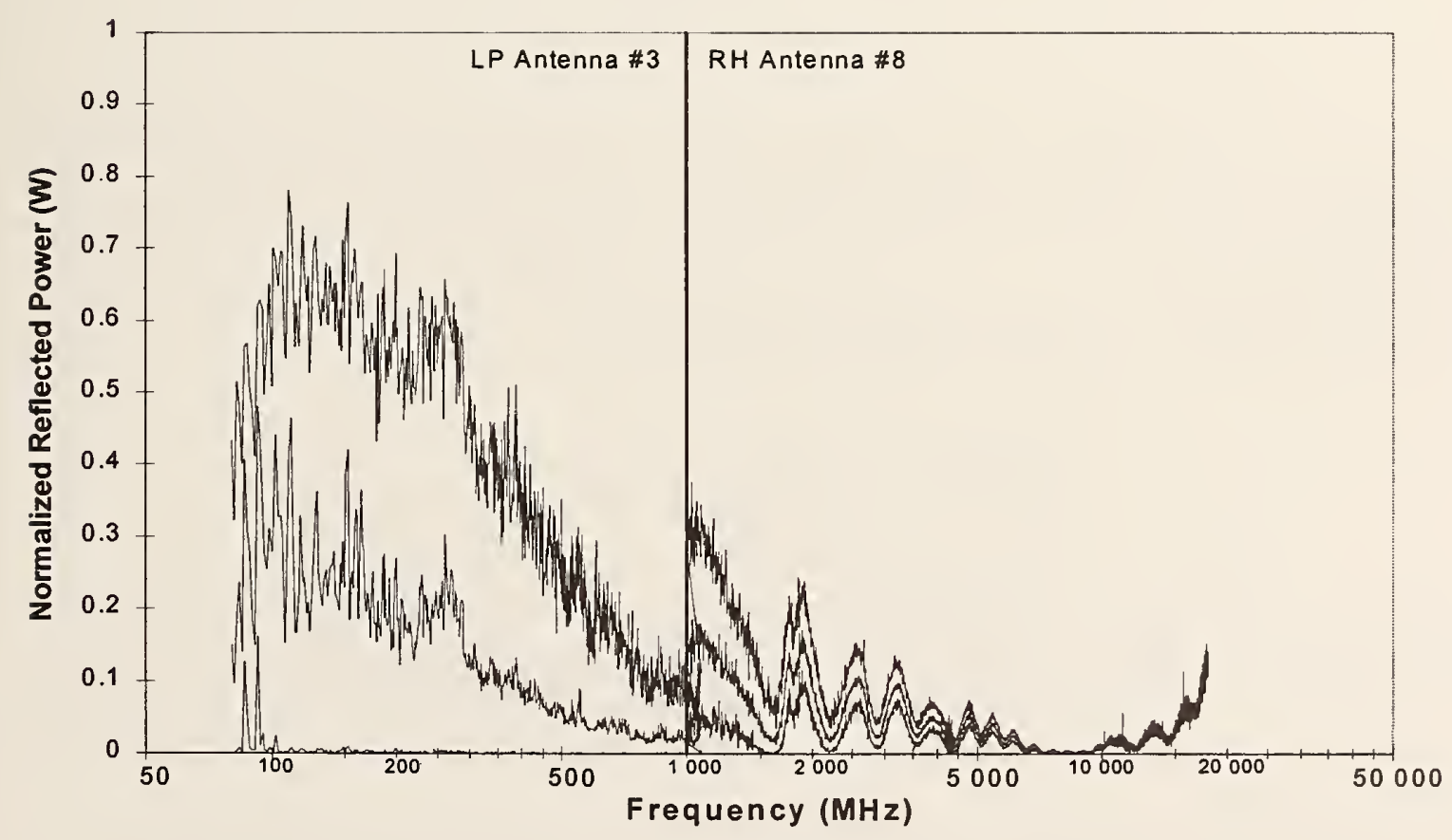

(b)

Figure 25. Minimum, average, and maximum reflected power for an incident power of $1 \mathrm{~W}$ in chamber A. (a) Port 1 transmitting (forward). Log periodic antenna 2, $80 \mathrm{MHz}$ to $1.08 \mathrm{GHz}$. Ridged horn antenna 7, $1 \mathrm{GHz}$ to $18 \mathrm{GHz}$. (b) Port 2 transmitting (reverse). Log periodic antenna 3, $80 \mathrm{MHz}$ to $1.08 \mathrm{GHz}$. Ridged horn antenna 8, $1 \mathrm{GHz}$ to $18 \mathrm{GHz}$. 
Another interesting parameter which can be generated based on the reflection coefficient measured at each paddle position is the magnitude of the average reflection coefficient $\left|\left\langle S_{i i}\right\rangle\right|$, where $i$ can be 1 (forward reflection coefficient) or 2 (reverse reflection coefficient). We will use the notation $S_{i i}$ to indicate an arbitrary reflection coefficient whenever signal direction is unimportant. As discussed before, this is a complex quantity, the magnitude of which is very similar to the free-space reflection coefficient of the antenna. The magnitude of the average reflection coefficient is given in Figure 26a for the forward direction and Figure 26b for the reverse direction. The magnitude of the average reflection coefficient is used to estimate corrections for the transmitted and received power. Although it may be possible to generate the same corrections based on measurements of the free-space reflection coefficient of the antennas, we recommend against this procedure because it will not account for any deterministic components of the reflections caused by placing the antennas in an imperfect chamber.

Given measurements of the magnitude of the average reflection coefficients of the antennas, we can calculate the voltage standing wave ratio (VSWR) of the antennas in two ways. The first method calculates the VSWR at each paddle position. For an incident power equal to 1 W and a reflected power equal to $P_{\text {refl }}$, the VSWR at each paddle position is calculated as

$$
\text { VSWR }=\frac{1+\sqrt{P_{\text {refl }}}}{1-\sqrt{P_{\text {refl }}}} .
$$

Since this quantity is calculated at each paddle position, we can compute the minimum, average, and maximum VSWR over all paddle positions, and these values are shown in Figures 27a (forward) and $b$ (reverse).

In addition to the point by point calculation of the VSWR, the free-space value VSWR ${ }^{0}$ can be estimated from the average reflection coefficient as

$$
\operatorname{VSWR}^{0}=\frac{1+\left|S_{i i}^{0}\right|}{1-\left|S_{i i}^{0}\right|} \approx \frac{1+\left|<S_{i i}>\right|}{1-\left|<S_{i i}>\right|},
$$

where the superscript 0 indicates the value that would have been measured in free space. The estimated free-space VSWR is given in Figures 28a (forward, $i=1$ ) and b (reverse, $i=2$ ).

Plots of the VSWR of the antennas are included simply to indicate the characteristics of the antennas when placed inside a mode-stirred chamber. The actual values of the VSWR are not used in any calculation.

Once again, we stress that all measurements related to reflected power depend on the specific antennas used in the measurements and will be different if other antennas are used. The plots presented here are typical of what can be expected for general antennas, and should be used for reference purposes only. The actual characteristics of the antennas used in any measurement should be evaluated, and these characteristics should be used for any subsequent corrections which depend on the reflection coefficient of the antennas. 


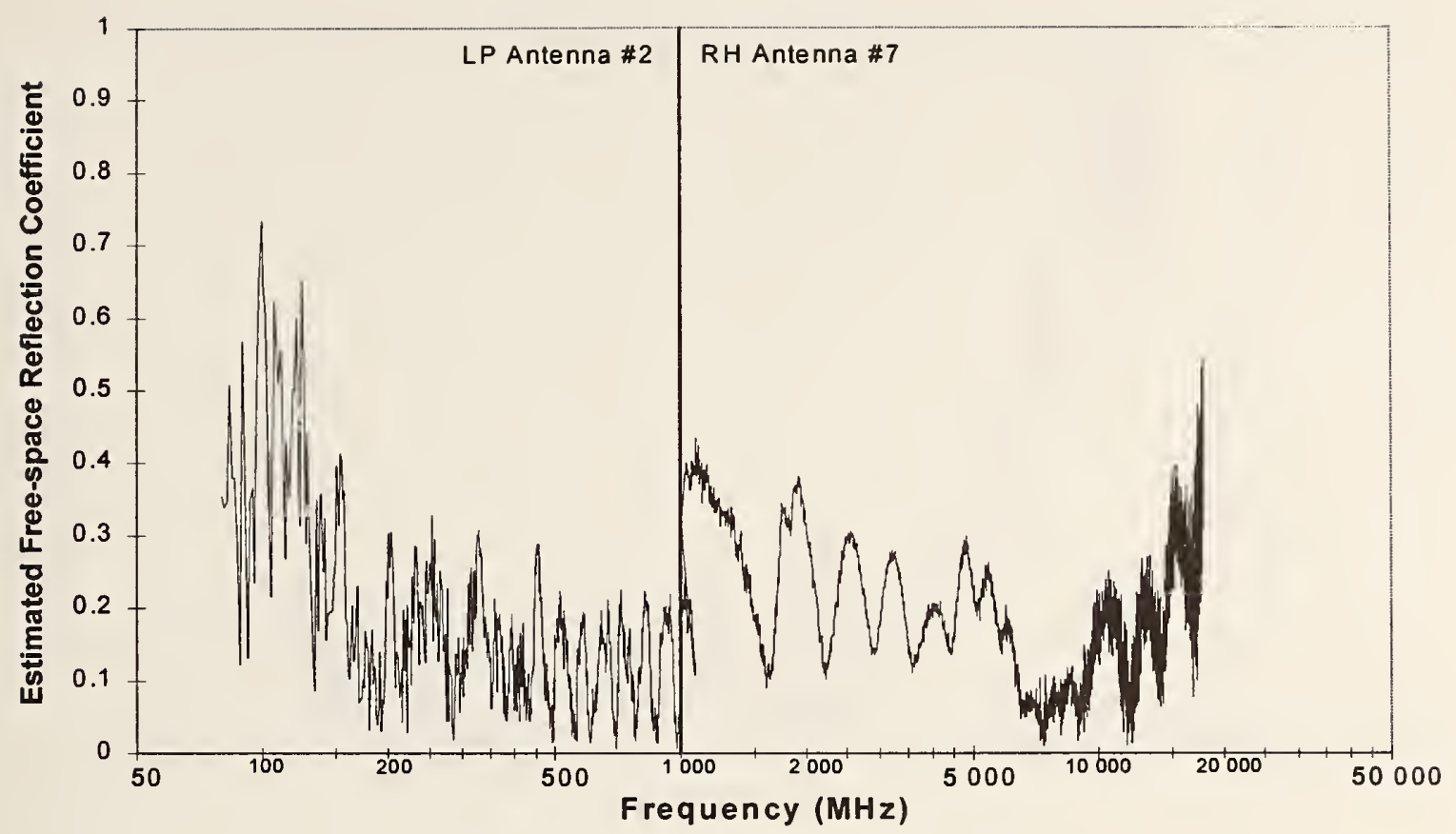

(a)

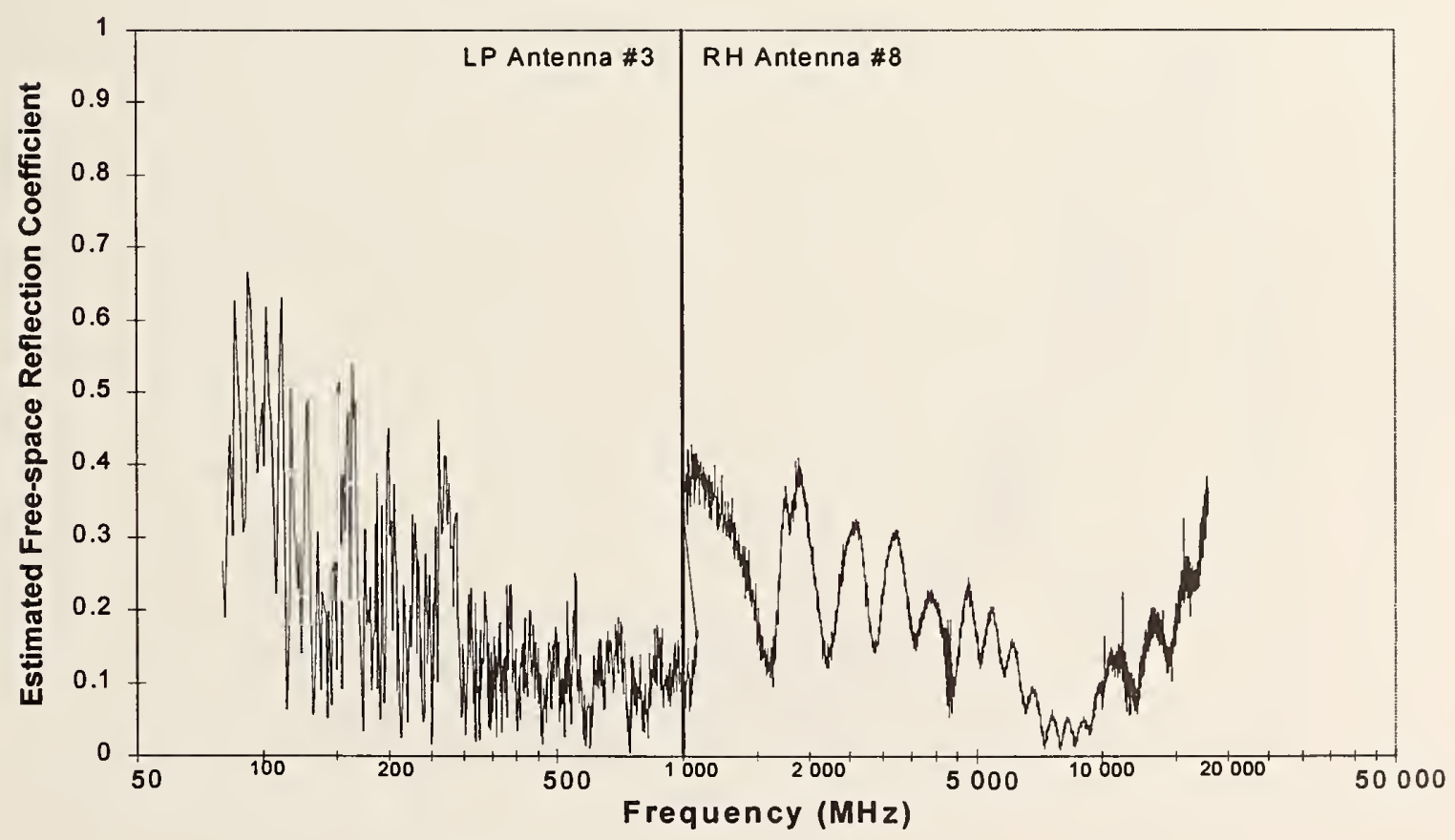

(b)

Figure 26. Estimated free-space reflection coefficient. (a) Port 1 transmitting (forward). Log periodic antenna 2, $80 \mathrm{MHz}$ to $1.08 \mathrm{GHz}$. Ridged horn antenna $7,1 \mathrm{GHz}$ to $18 \mathrm{GHz}$. (b) Port 2 transmitting (reverse). Log periodic antenna 3, $80 \mathrm{MHz}$ to $1.08 \mathrm{GHz}$. Ridged horn antenna $8,1 \mathrm{GHz}$ to $18 \mathrm{GHz}$. 


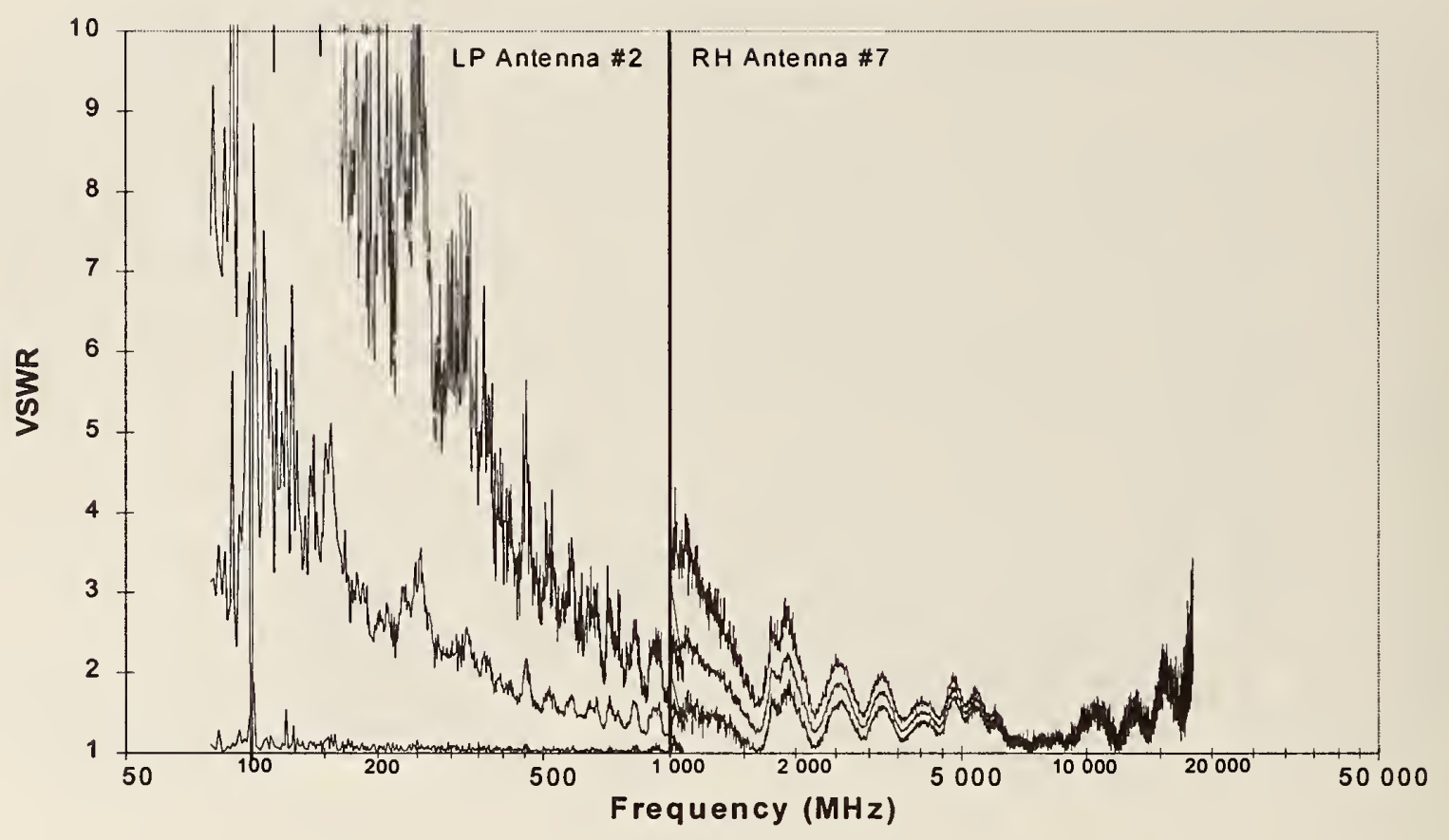

(a)

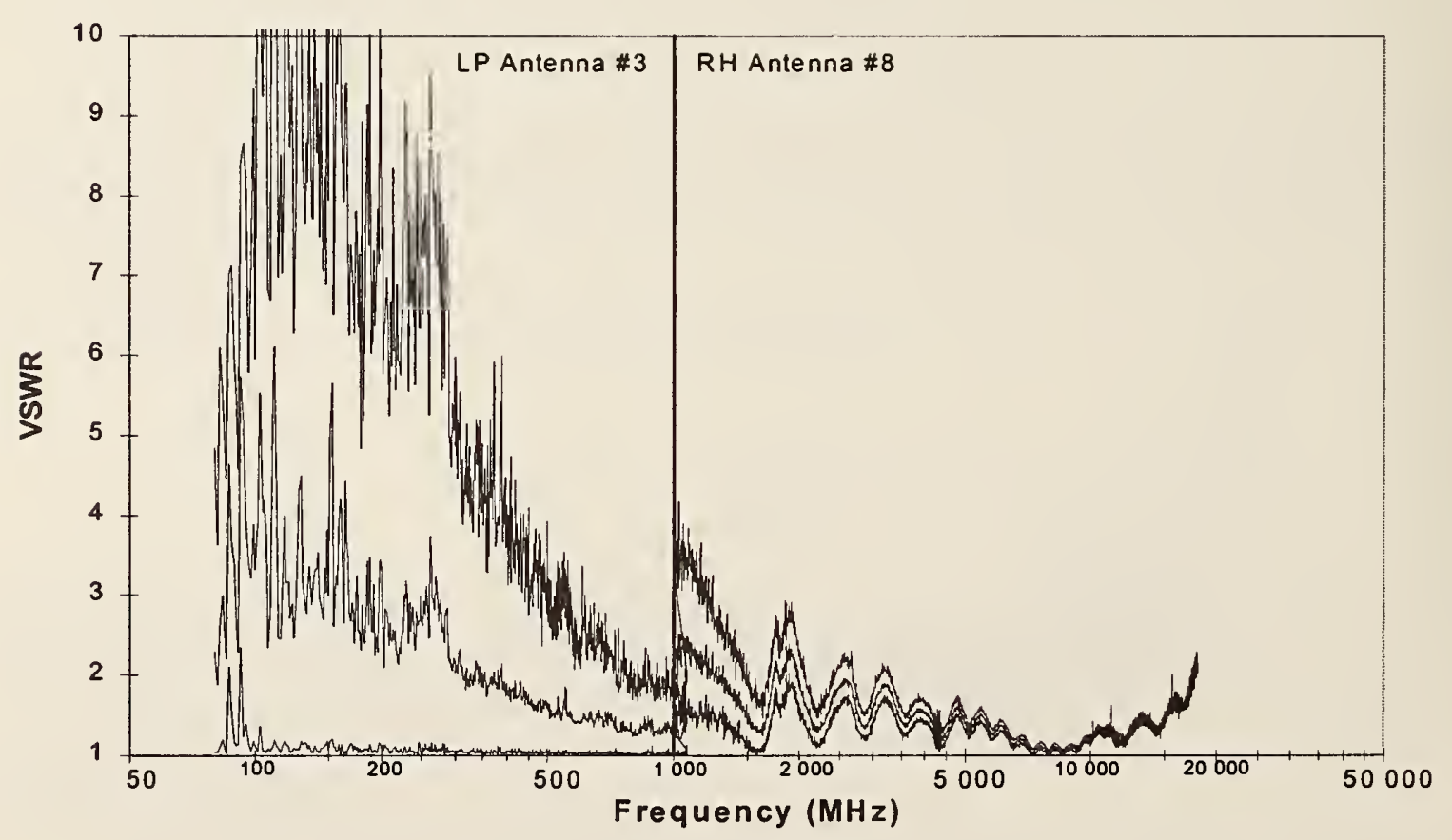

(b)

Figure 27. Minimum, average, and maximum VSWR. (a) Port 1 transmitting (forward). Log periodic antenna $2,80 \mathrm{MHz}$ to $1.08 \mathrm{GHz}$. Ridged horn antenna 7,1 GHz to $18 \mathrm{GHz}$. (b) Port 2 transmitting (reverse). Log periodic antenna 3, $80 \mathrm{MHz}$ to $1.08 \mathrm{GHz}$. Ridged horn antenna $8,1 \mathrm{GHz}$ to $18 \mathrm{GHz}$. 


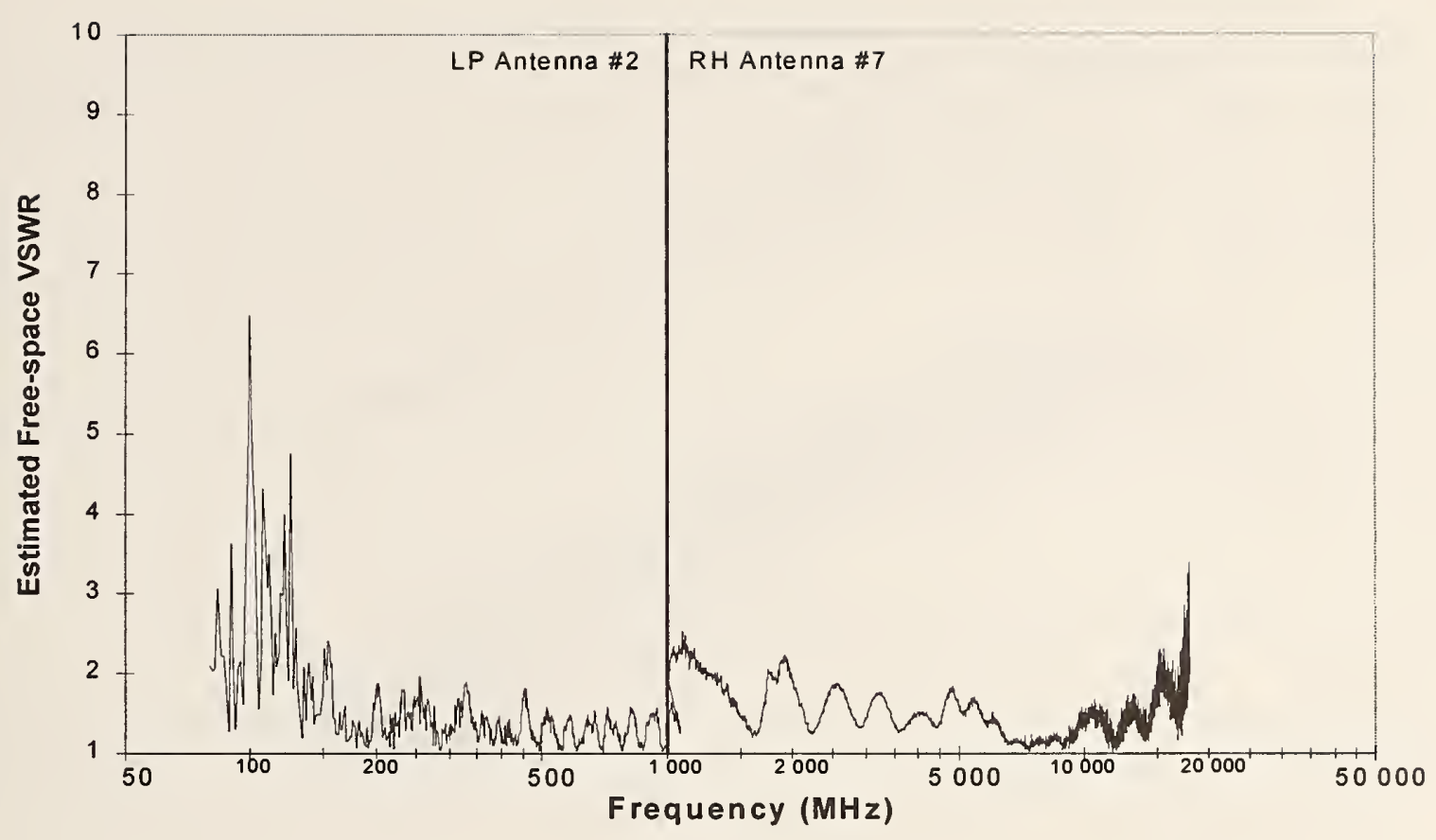

(a)

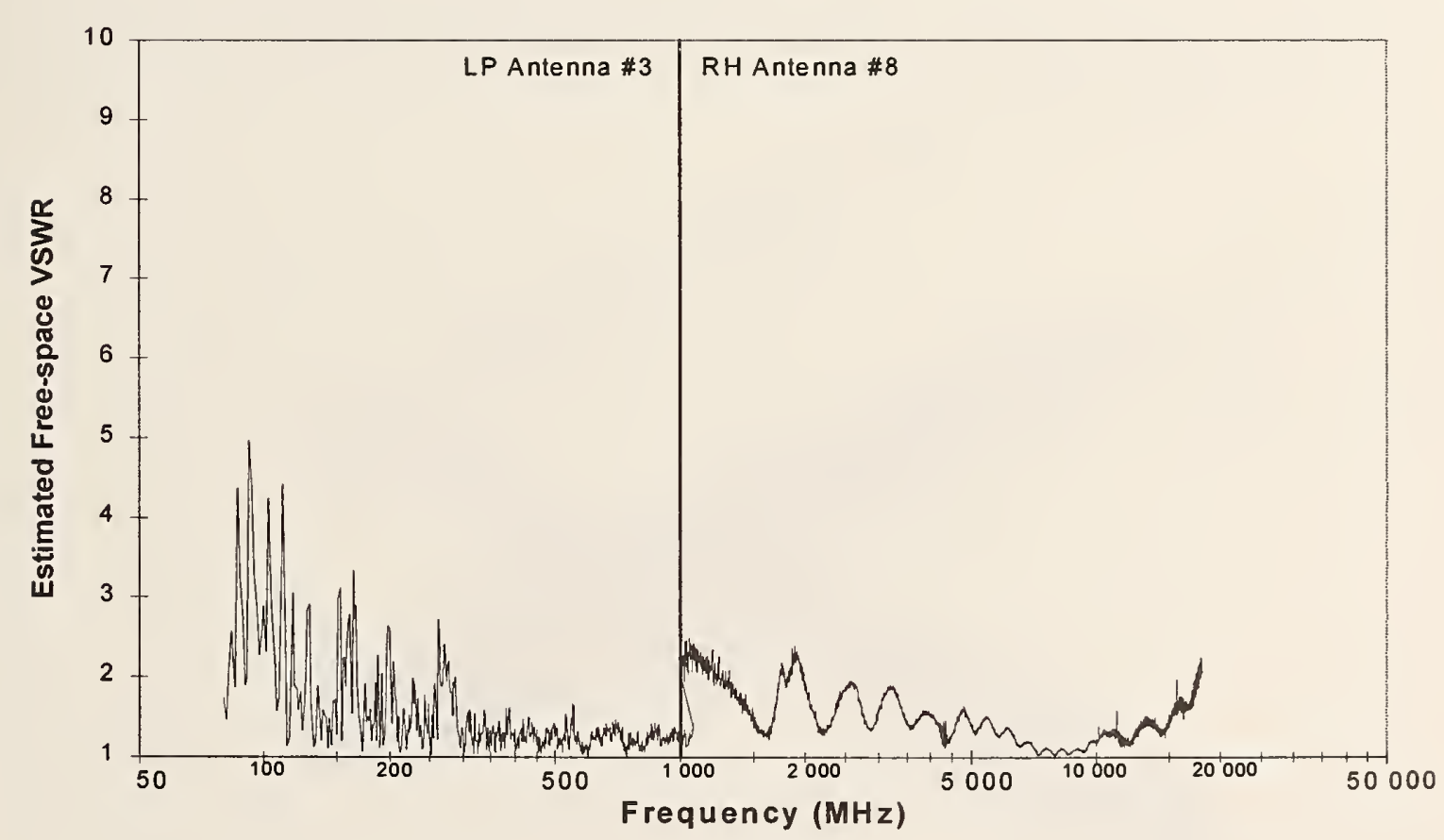

(b)

Figure 28. Estimated free-space VSWR. (a) Port 1 transmitting (forward). Log periodic antenna 2, $80 \mathrm{MHz}$ to $1.08 \mathrm{GHz}$. Ridged horn antenna 7,1 GHz to $18 \mathrm{GHz}$. (b) Port 2 transmitting (reverse). Log periodic antenna $3,80 \mathrm{MHz}$ to $1.08 \mathrm{GHz}$. Ridged horn antenna $8,1 \mathrm{GHz}$ to $18 \mathrm{GHz}$. 


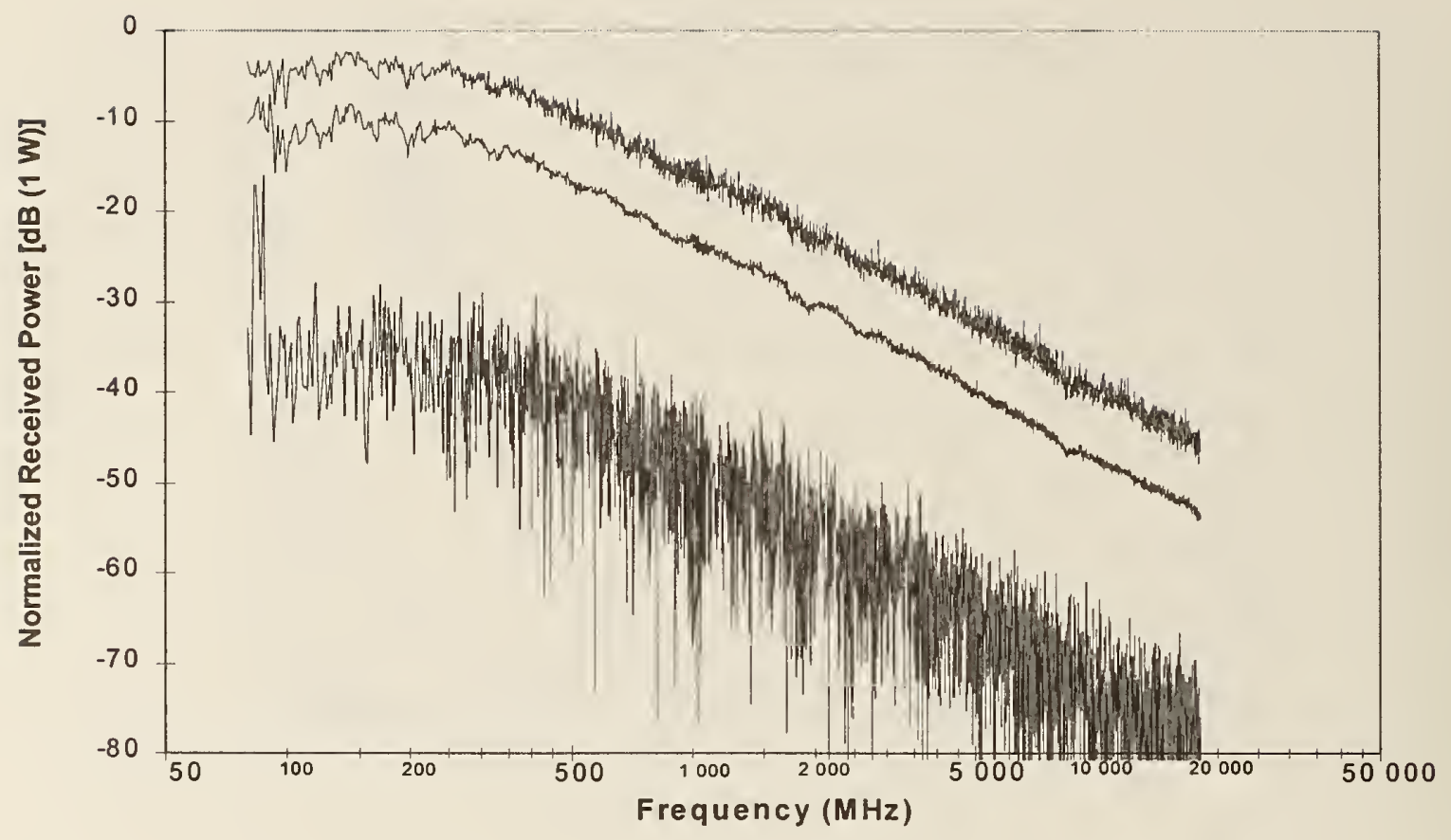

(a)

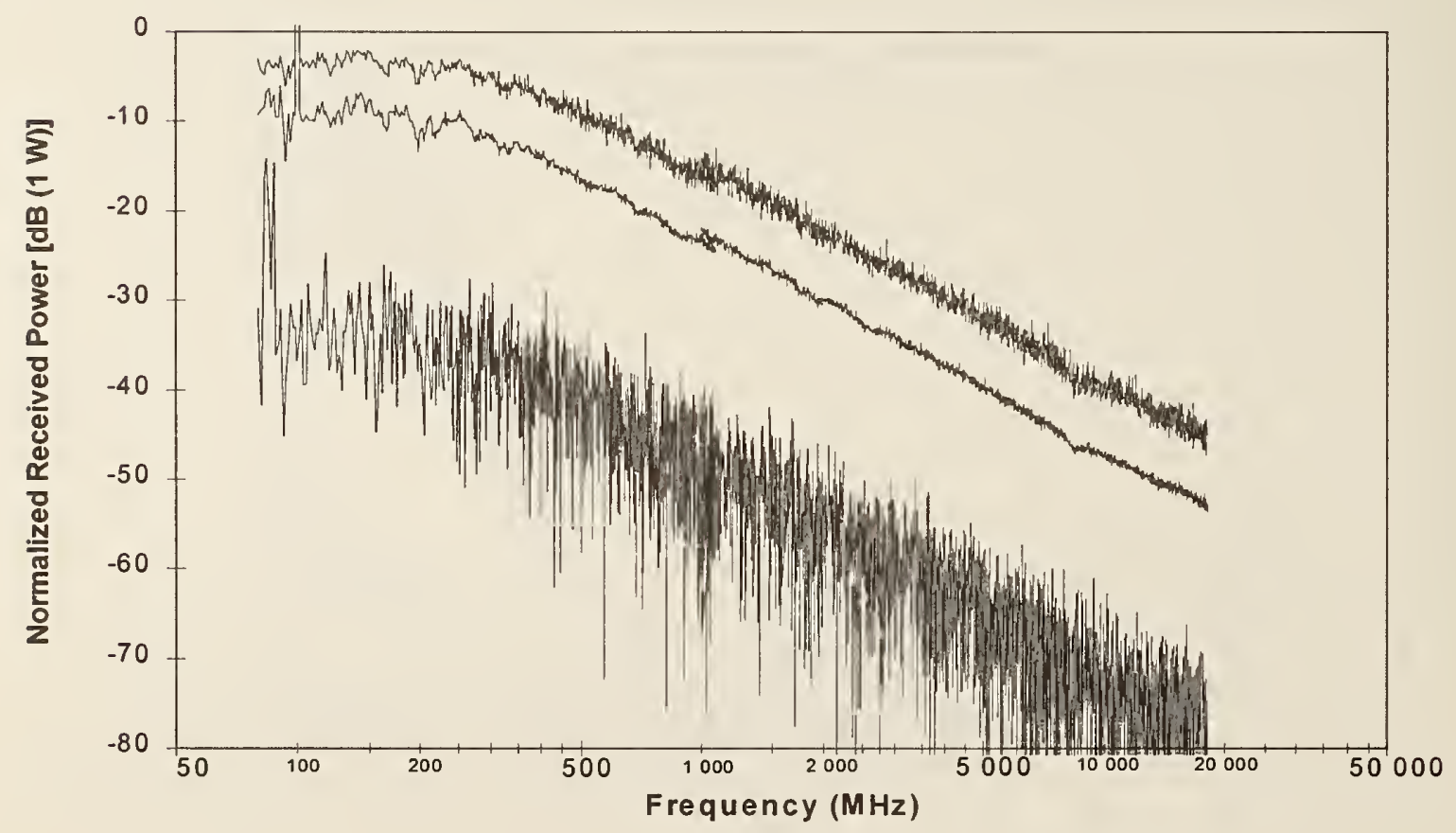

(b)

Figure 29. Minimum, average, and maximum received power for a constant input power of $1 \mathrm{~W}$. (a) Constant incident power of $1 \mathrm{~W}$. (b) Constant net input power of $1 \mathrm{~W}$. 
The transmission coefficients $\left(S_{21}\right.$ and $\left.S_{12}\right)$ are presented next. The two measured transmission coefficients are essentially indistinguishable from each other (as expected for a reciprocal linear system) and we will therefore only present the forward transmission coefficient $\left(S_{21}\right)$. Unfortunately, since the value of the received power normalized to a constant net input power depends on the reflection coefficient of the antennas, this quantity is not independent of the signal direction. The differences are small, however, and we therefore present results for the forward measurements only, except as noted.

Figure 29a shows the minimum, average, and maximum received power measured in the chamber, normalized based on a constant incident power. The same parameter based on a constant net input power is given in Figure 29b. These plots have the same general shape that we expect for chamber gain (approximately constant at low frequencies, decreasing linearly (on a logarithmic plot) at high frequencies).

We can also compute the magnitude of the average transmission coefficient $\left|<S_{21}\right\rangle \mid$. As mentioned before, Page [18] refers to this quantity as a measure of the "unstirred energy" in the cavity. We modify and, we hope, improve on this interpretation somewhat. First, we suggest a different name. This quantity does appear to be indicative of the quality of the stirrer (paddle), but the reference to energy is difficult to justify given the units of the measured quantities. Instead, we suggest a name based on one possible interpretation of a scatter plot of $S_{21}$ (as given in Figure 4). A scatter plot consists of two components: the unstirred (or possibly deterministic or systematic) component given by the complex mean of the measured data, and the stirred (or random) component, given by the measured data less the mean. Given this interpretation, we

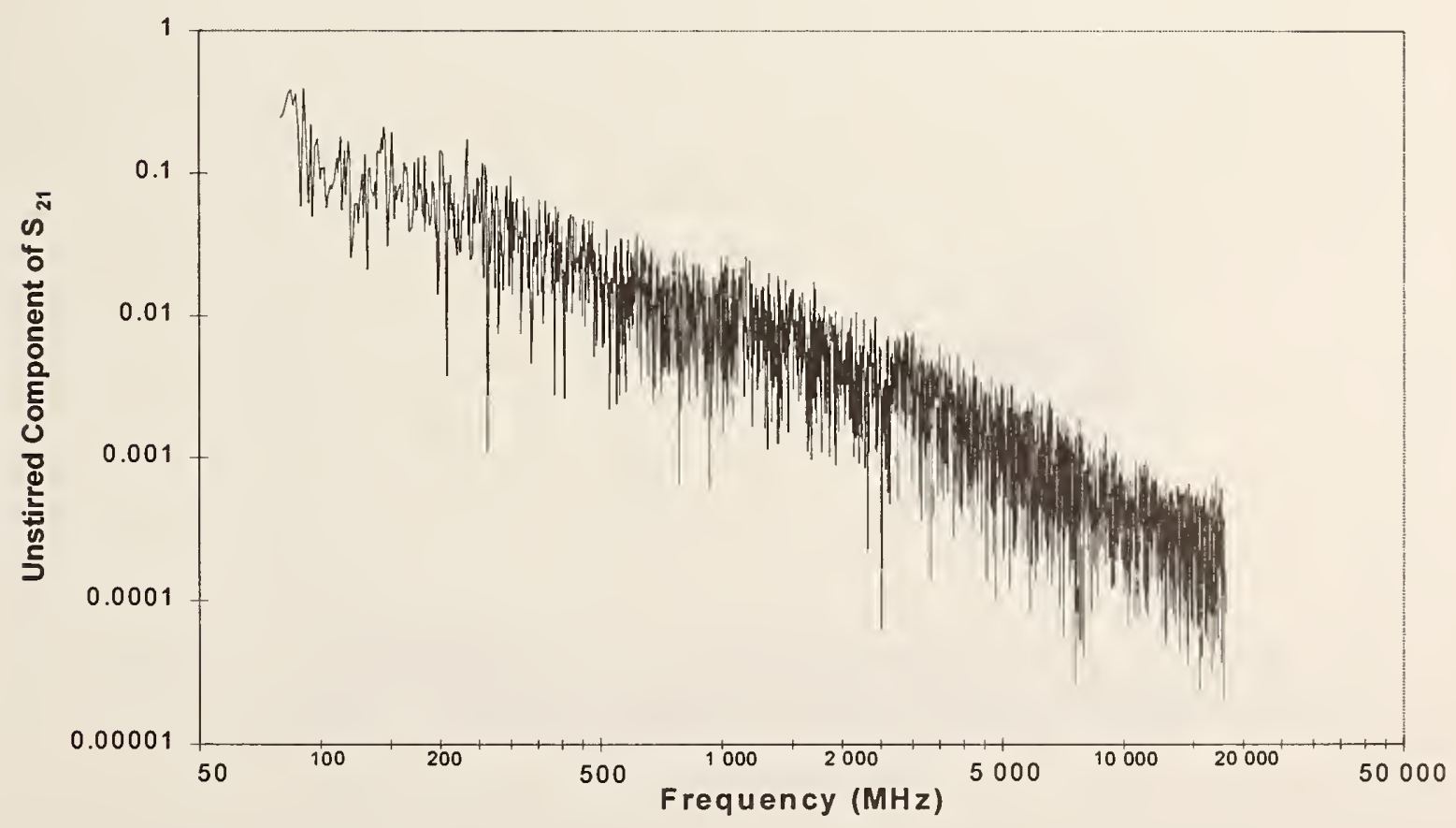

Figure 30. Unstirred component of $S_{21}$. 
suggest the name "unstirred component of $S_{21}$ " instead of "unstirred energy." Since $S_{21}$ is assumed to be approximately proportional to the electric field in the vicinity of the receiving antenna, another reasonable name would be "the unstirred component of the electric field," but to maintain consistent units, we will use "unstirred component of $S_{21}$." The unstirred component of $S_{21}$ is shown in Figure 30.

One problem with using $\left|<S_{21}>\right|$ as a measure of the unstirred component of $S_{21}$ is demonstrated in Figures 31a and b. For both plots, $\left|<S_{21}\right\rangle \mid=0.1$; that is, both plots have the same value for the unstirred component of $S_{21}$. The offset appears to be much less significant, however, in Figure 31a, where there is a large spread to the data, than in Figure 31b, where there is a small spread to the data. This suggests that we should normalize $\left|\left\langle S_{21}\right\rangle\right|$ based on some measure of the spread of the data. A reasonable normalization factor is the average of the standard deviation of the real and imaginary parts of $S_{21}$. The standard deviation of the real part of $S_{21}$ is nearly identical to the standard deviation of the imaginary part, and in the plot of the standard deviation given in Figure 32, they are indistinguishable. The normalized unstirred component of $S_{21}$ is given in Figure 33. For now, this value is used for qualitative purposes only. Large values are indicative of insufficient stirring, whereas small values correspond to a well randomized field. A quantitative interpretation should be possible, but a full analysis has not yet been completed.

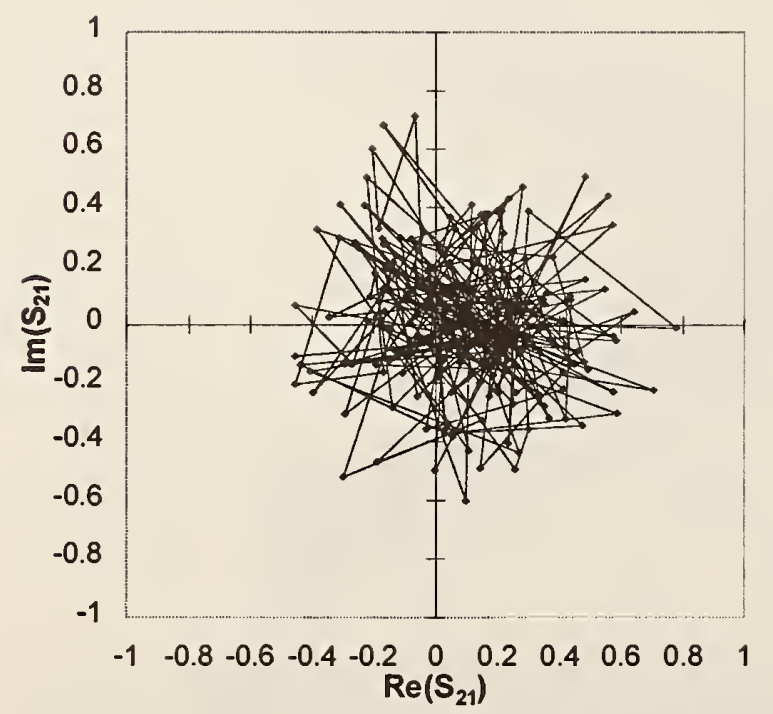

(a)

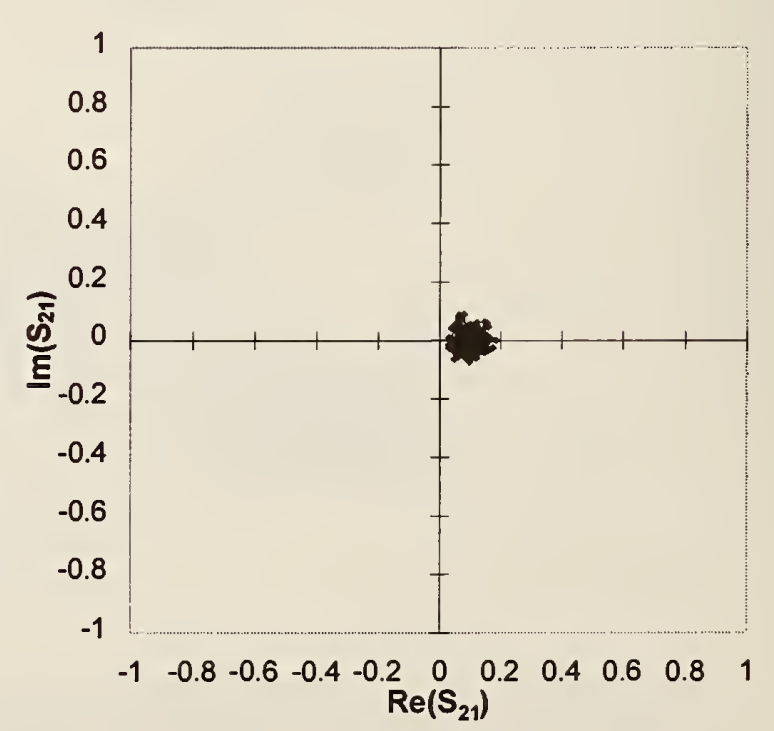

(b)

Figure 31. Simulated scatter plots with mean of $0.1+j 0$. (a) large spread in data. (b) small spread in data. 


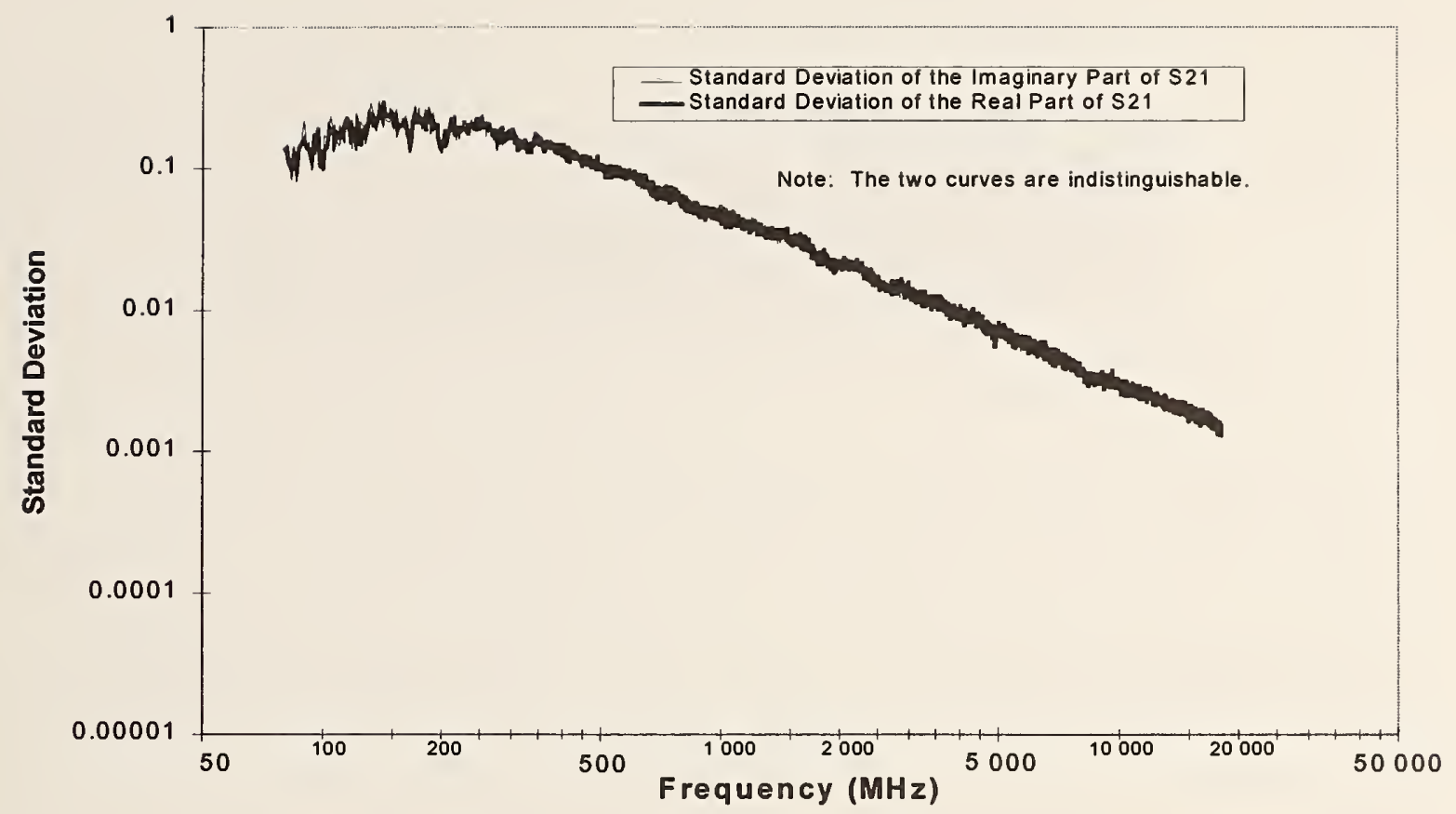

Figure 32. Sample standard deviation of the real and imaginary parts of $S_{21}$.

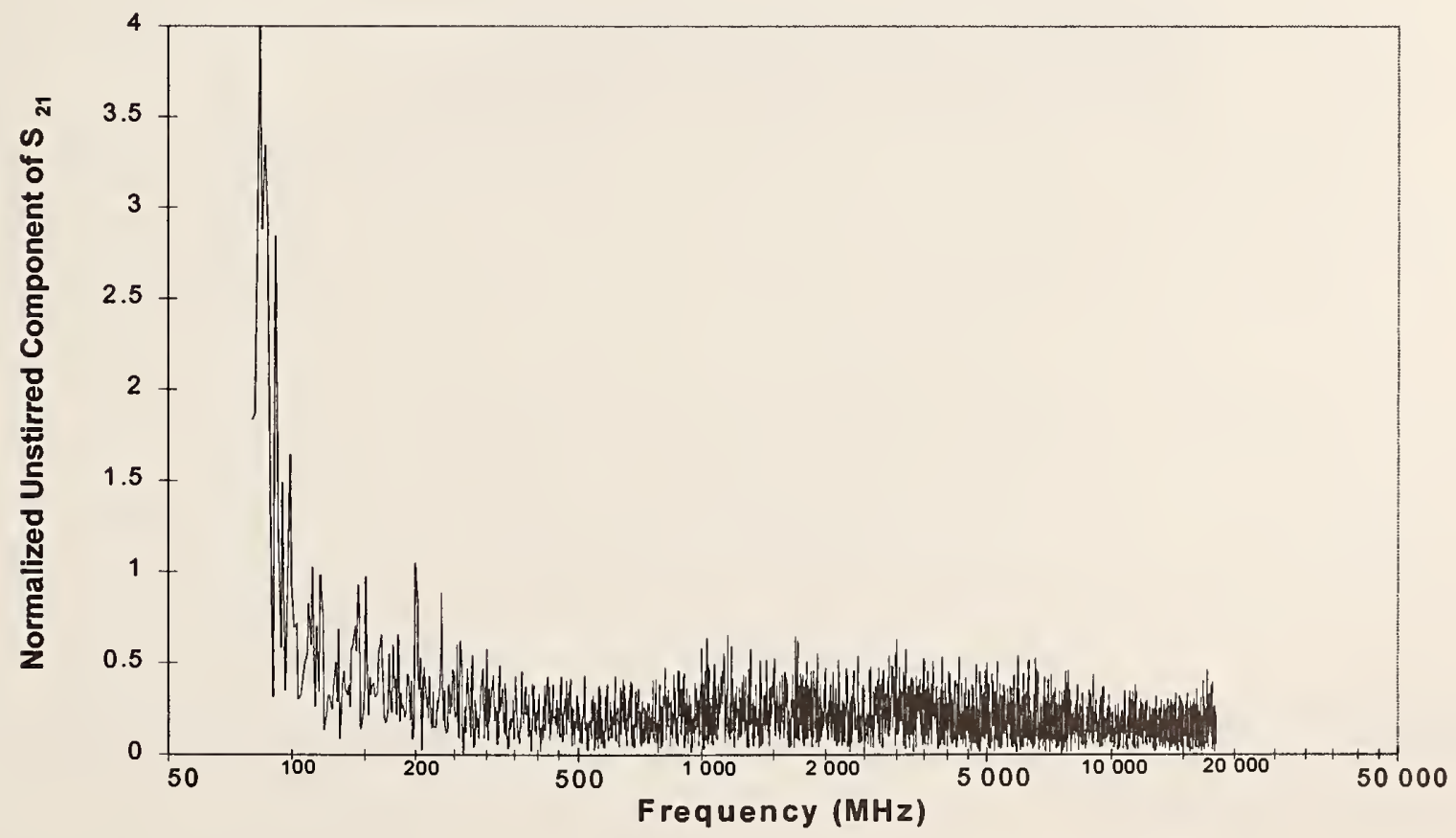

Figure 33. Normalized unstirred component of $S_{21}$. 


\subsection{Ratios of Measured Data}

We now examine the data presented in Figure 29 in more detail. Crawford observed [3] that the maximum received power was approximately 7 to $9 \mathrm{~dB}$ (a multiplicative factor between 5 and 8 ) greater than the average received power, regardless of chamber type or location. This difference has become known as the peak-to-average ratio or the maximum-to-average ratio. (We will refer to this as the maximum-to-average ratio.) Based on the statistical analysis presented in Section 3.2, we know that 225 paddle positions should result in a maximum-to-average ratio of approximately $7.8 \mathrm{~dB}$, independent of frequency. The maximum-to-average ratio of the measured received power for a constant incident power is presented in Figure 34a, and the same parameter evaluated assuming a constant net input power is presented in Figure $34 \mathrm{~b}$. The agreement between the measured value and the expected value indicates that the statistical model appears to be justified for frequencies greater than $500 \mathrm{MHz}$. Below $500 \mathrm{MHz}$, the power received by the receiving antenna approaches the maximum theoretical limit (all of the transmitted power is received), and this physical limit is influencing the results. This deviation is more apparent in Figure 34b than in Figure 34a because maintaining a constant net input power does not allow the transmitting antenna to remove power from the chamber, resulting in a greater value for the average received power, but the maximum received power is unchanged because of the physical limits. Thus, the measured data appear to deviate more from the ideal statistical model when a constant net input power is maintained than when a constant incident power is maintained, but the data in both cases still deviate from the ideal model. As a result, the theoretical model does not apply exactly to the signal received from a large obtrusive antenna in Chamber A for frequencies below $500 \mathrm{MHz}$, although it may still apply to unobtrusive antennas, and the electric field in general. The model may still be useful for predicting the signals received from an obtrusive antenna, but approximations and modifications must be made. One such modification is described below.

Crawford also observed [3] that the maximum received power was almost always at least $20 \mathrm{~dB}$ (a multiplicative factor of at least 100) greater than the minimum received power. This difference (known as the maximum-to-minimum ratio, paddle effectiveness, tuner effectiveness, or tuning ratio) indicates that changes in the paddle position resulted in significant changes in the electromagnetic environment in the vicinity of the receiving antenna, and therefore indicated that the paddle was operating. A minimum difference of $20 \mathrm{~dB}$ was recommended as a guideline for the proper operation of the paddle, although no theoretical justification was given. For 225 paddle positions, the maximum-to-minimum ratio should be approximately $33.8 \mathrm{~dB}$ for all frequencies. The maximum-to-minimum ratio for the measured received power is given in Figure $35 \mathrm{a}$ for data measured with a constant incident power, and in Figure 35b for data measured with a constant net input power. Once again, the agreement between the expected and observed values is excellent. The difference between data measured assuming a constant incident power and a constant incident power below $500 \mathrm{MHz}$ is once again apparent for the same reasons mentioned above, but the magnitude of the difference is less important because it is small relative to the overall spread in the data 


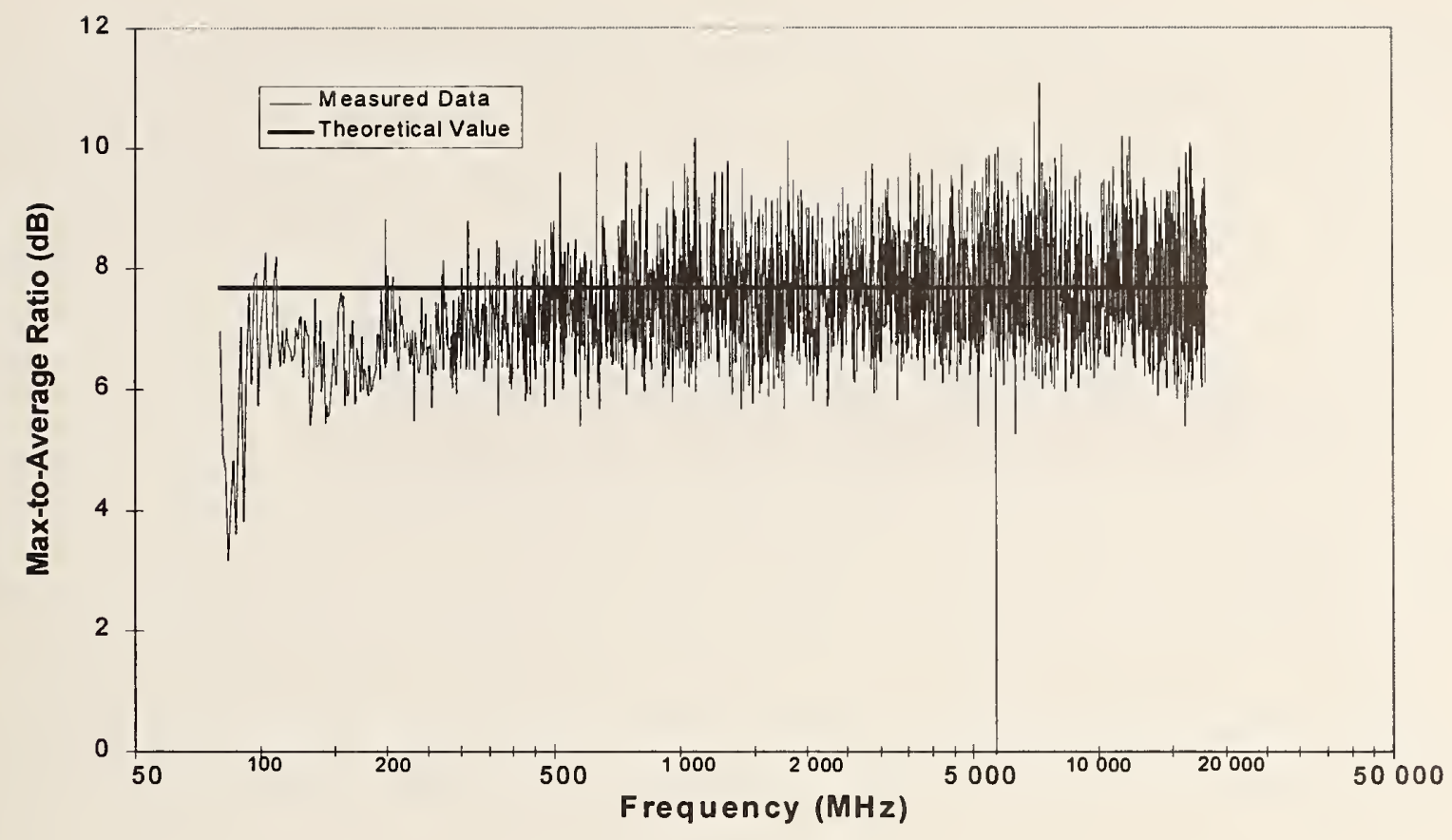

(a)

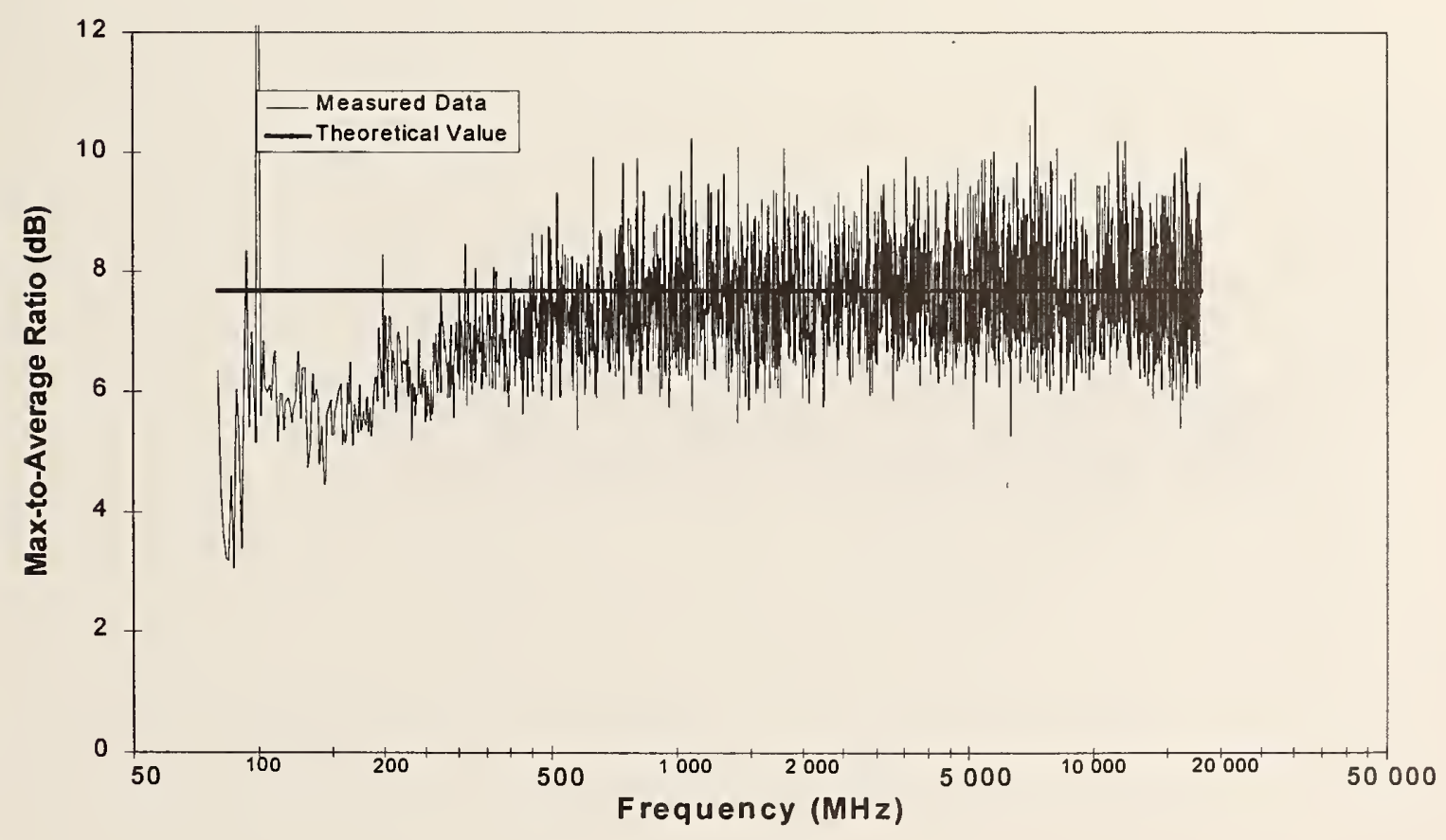

(b)

Figure 34. Max-to-average ratio. (a) Constant incident power. (b) Constant net input power. 


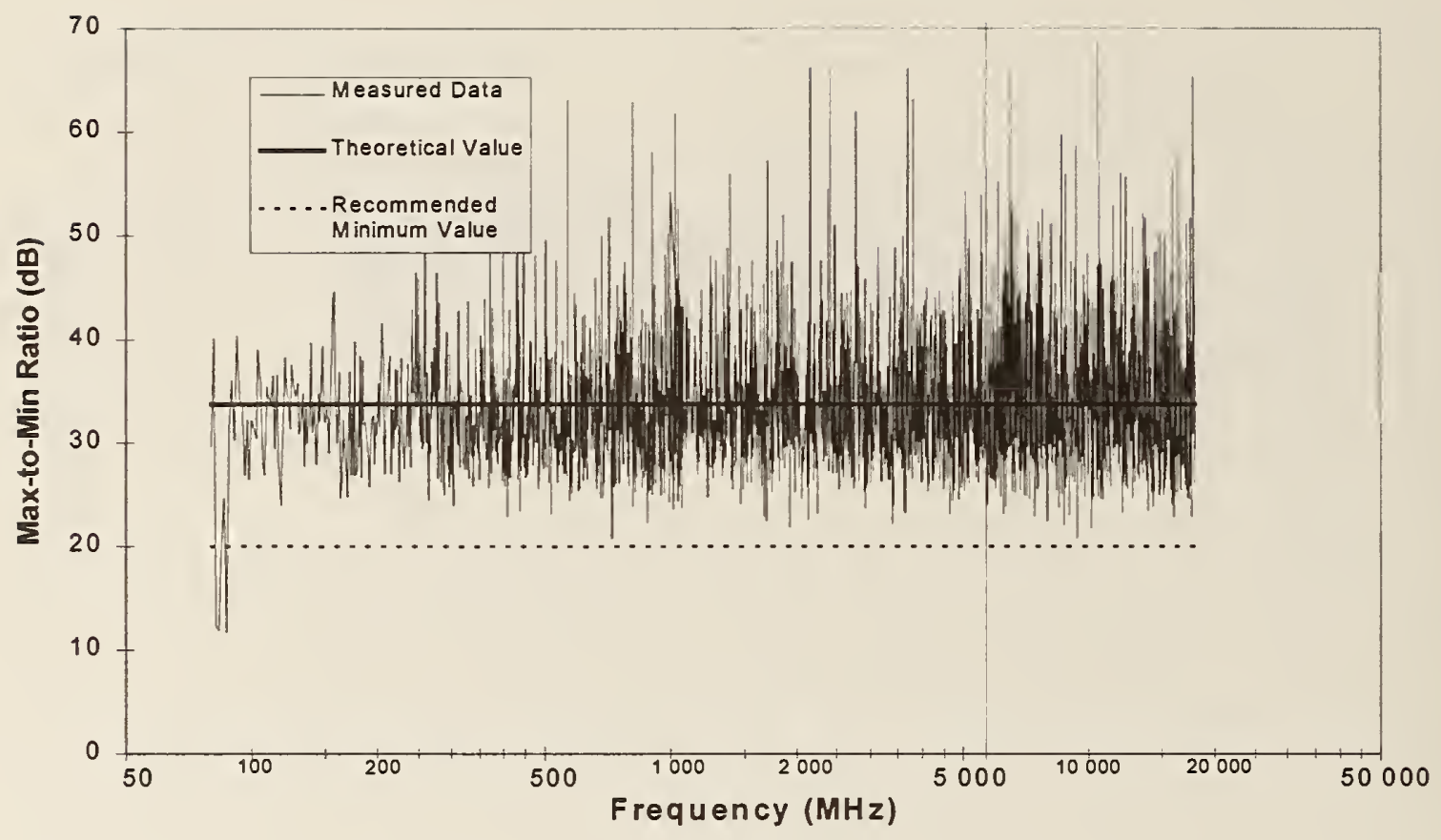

(a)

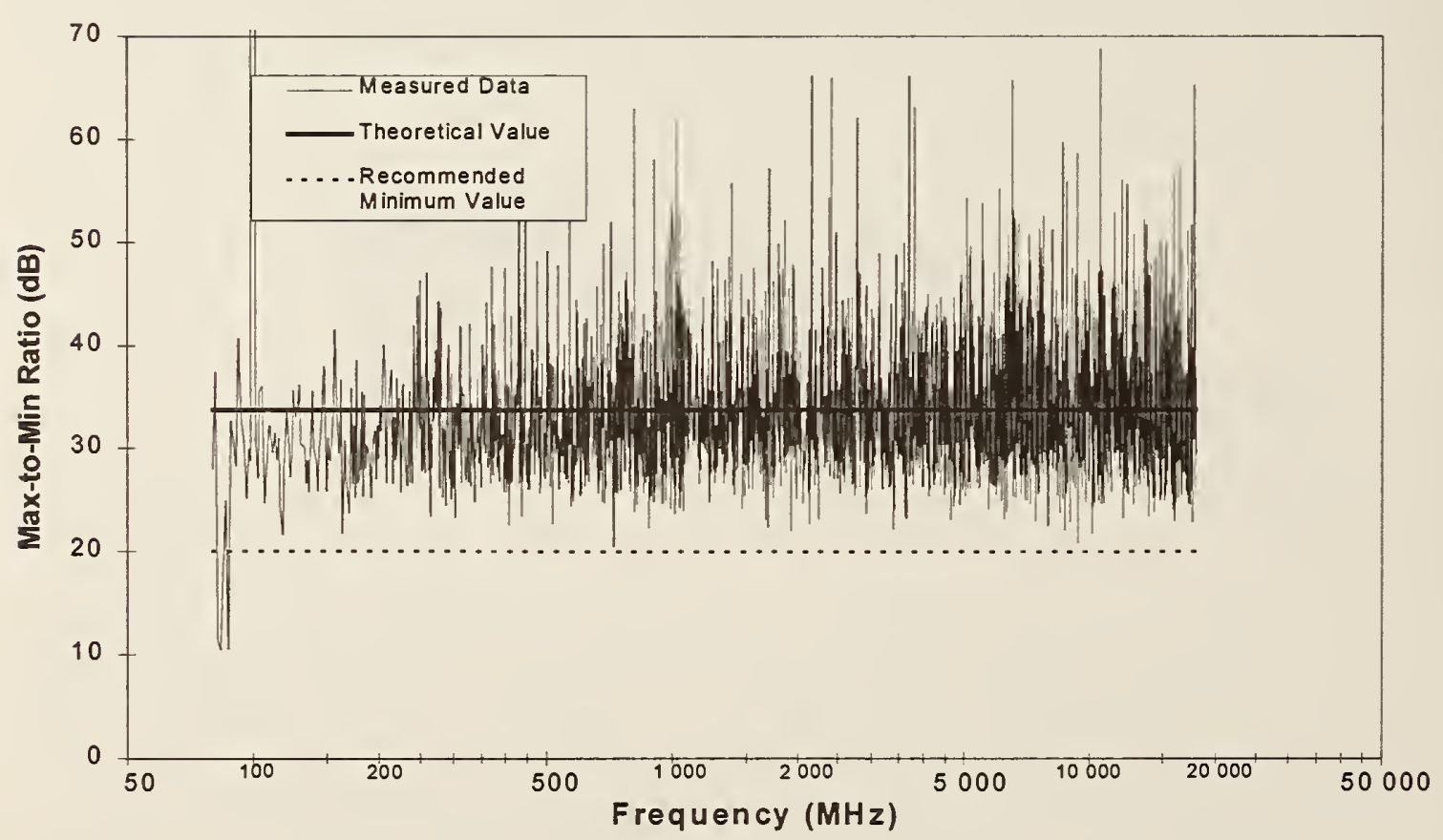

(b)

Figure 35. Max-to-min ratio. (a) Constant incident power. (b) Constant net input power. 
Finally, we present one additional ratio, which we call the average-to-minimum ratio. This ratio should also be independent of frequency. Although this ratio appears to have little practical significance due to the wide fluctuations in the minimum measured power, at least three pieces of information provided by this ratio have a valuable interpretation. First, since the minimum measured power will be sensitive to the noise floor of the measurement system, any reduction in this ratio indicates that the noise floor may be influencing the results. Second, the ratio in linear units should be approximately equal to the number of paddle steps $N$. When the data are converted to decibels, the difference between the average and the minimum will be $10 \cdot \log _{10}(N)+2.5 \mathrm{~dB}$, where the additional $2.5 \mathrm{~dB}$ comes from the conversion of the noisy minimum from linear units to logarithmic units, as discussed in Section 3.2. Last, since the average received power is typically less noisy than the maximum received power, the average-tominimum ratio should also be (somewhat) less noisy than the maximum-to-average ratio. If the maximum-to-minimum ratio is indeed a good indicator of the quality of the paddle, then the average-to-minimum ratio may be a better indicator. The average-to-minimum ratio for a constant incident power is presented in Figure 36a, and the average-to-minimum ratio for a constant net input power is presented in Figure 36b. The difference between these curves is small, even below $500 \mathrm{MHz}$. because neither the average nor the minimum received power is approaching a physical limitation of the system.

\subsection{Corrections for Antenna Effects}

We now turn our attention to the properties of a mode-stirred chamber that depend on the characteristics of the antennas and therefore may need correction. To simplify the analysis, we will concentrate on a single parameter. Any of the typical mode-stirred chamber parameters (quality factor, scalar power density, electric field, chamber gain) could be analyzed, and the results will generally be applicable to the other parameters. We analyzed chamber gain $G_{C}$ since it has a simple, well-defined theoretical description. We will restrict our attention to the average value of $G_{C}$, which should be better behaved (have a smaller uncertainty) than either the maximum or minimum values of $G_{C}$. Due to the wide range of values (a difference of approximately $50 \mathrm{~dB}$ between the data measured at $200 \mathrm{MHz}$ and $18 \mathrm{GHz}$ ), it is difficult to evaluate the fine details. To rectify this problem, we first assume that the characteristics of received power are similar to those of the ideal theoretical chamber gain. If this is the case, then the received power can be described by eq (31), for some value of $a$ and $b$. Using nonlinear leastsquares curve-fitting and iterative optimization, we estimated the values of $a$ and $b$. We examined three methods for estimating the curve parameters $a$ and $b$, and will describe these here. 


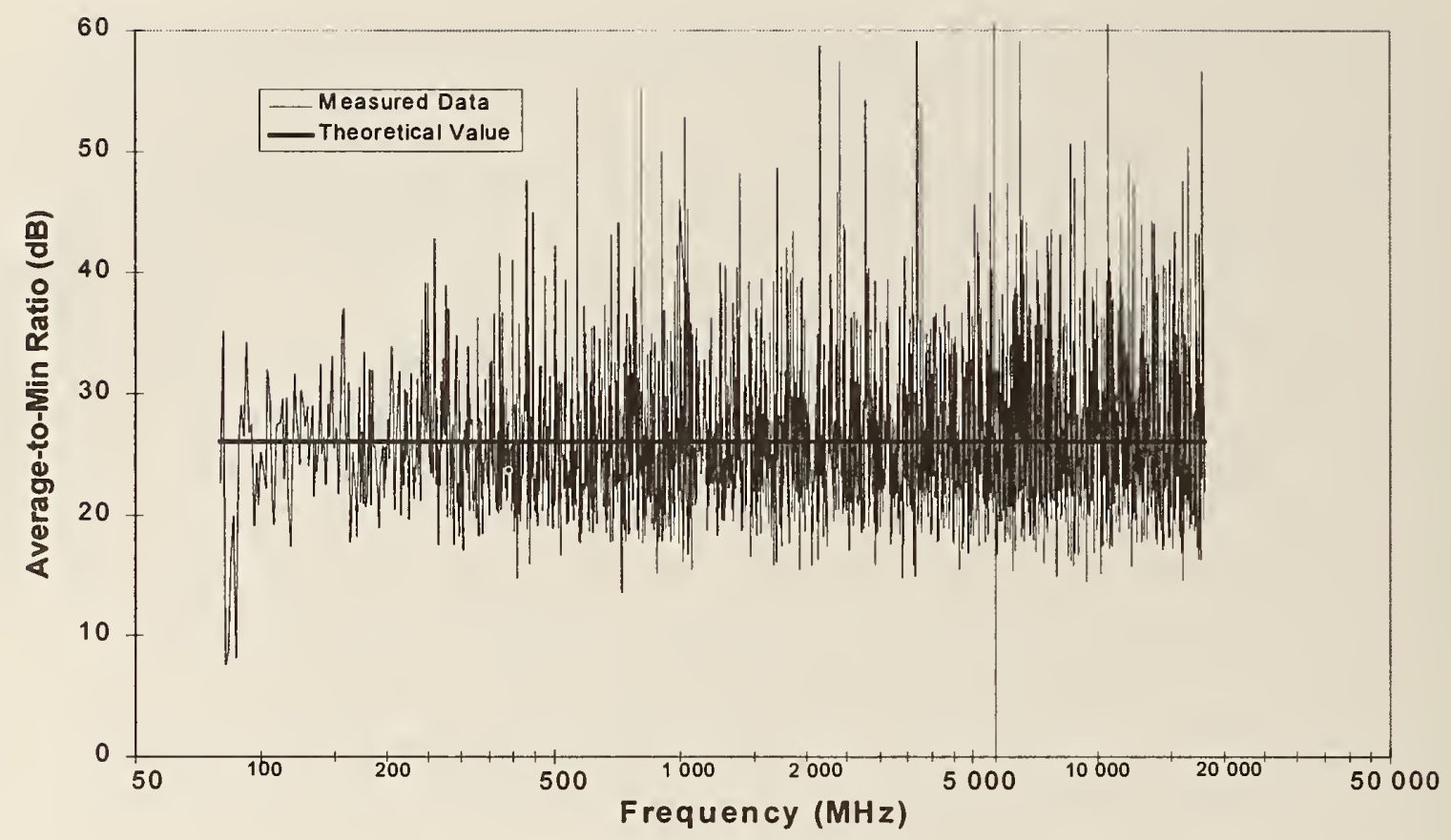

(a)

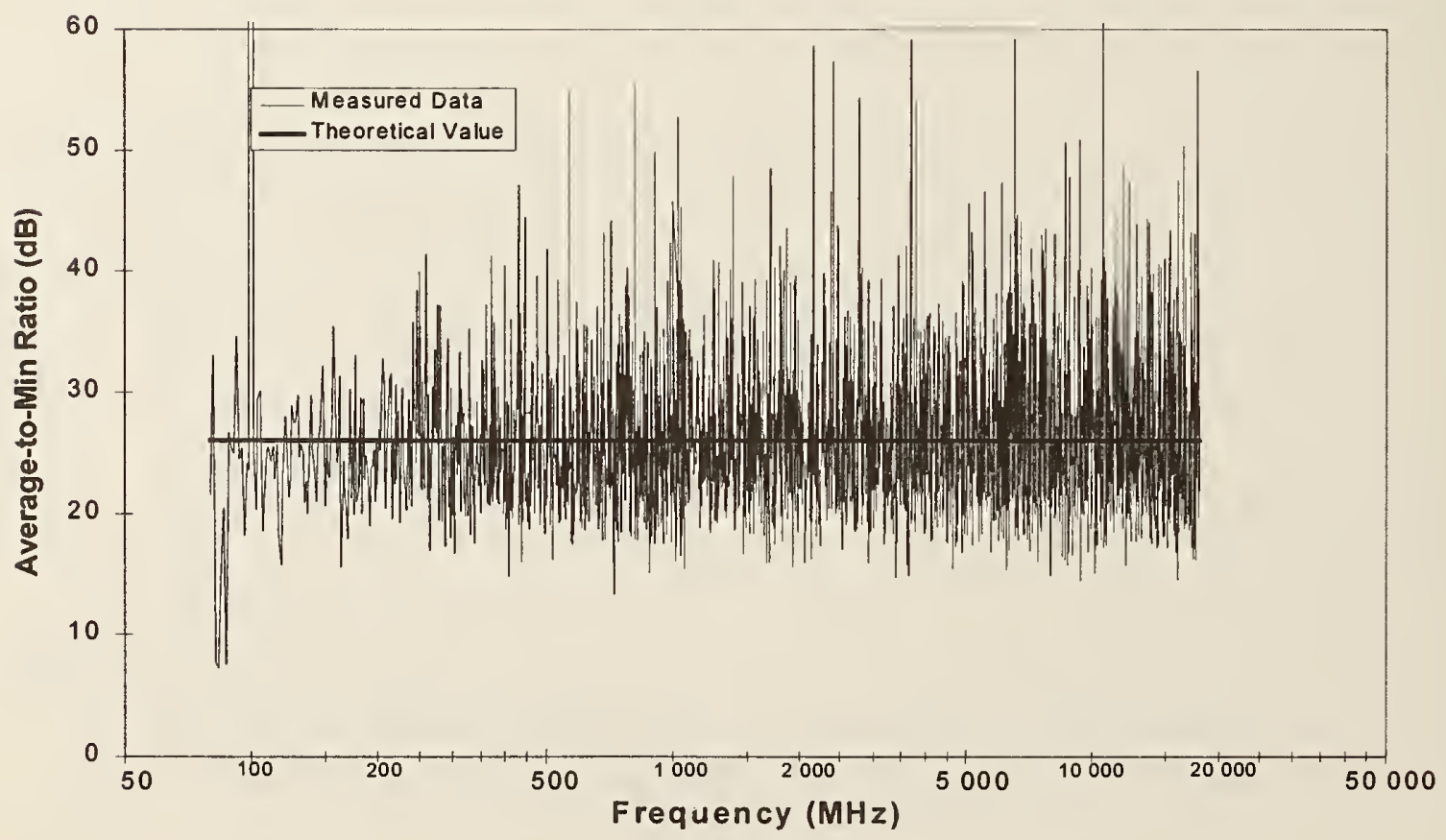

(b)

Figure 36. Average-to-min ratio. (a) Constant incident power. (b) Constant net input power. 
We have shown that the received power at any given paddle position can be described by the $\chi_{2}^{2}$ distribution, and as a result the mean is approximately equal to the standard deviation. The received power is averaged over $N$ paddle positions, so the standard deviation of the average is a factor of $\sqrt{N}$ less than the standard deviation of the individual components, but is still proportional to the mean. Since the mean decreases with frequency according to eq (31), the standard deviation will also decrease with frequency. Standard least-squares fitting requires that the standard deviations be approximately equal at each point, so we must use some modified technique. The first technique we tried was based on the observation that the data, when plotted on a decibel scale, appeared to have a constant standard deviation. This comes from the fact that we can write the mean plus or minus the standard deviation, using linear units, as $\mu \pm c \mu=\mu(1 \pm c)$, where $\mu$ is the mean, and $c$ is some constant. Taking the natural logarithm, we get $\ln (\mu)+\ln (1 \pm c)$. For small values of $c$ (which we should have after a large number of averages), $\ln (1 \pm c) \approx \pm c$, resulting in a standard deviation that is approximately constant. Since the natural logarithm of a number is proportional to the common logarithm of that number, the linear values expressed as decibels will also have a standard deviation that is approximately constant.

Now we can use traditional least-squares techniques to fit $-10 \cdot \log _{10}\left(a+b f^{2.5}\right)$ to the data expressed in decibels. For the average received power, this results in estimates of $a=10.3$ and $b=5.7 \cdot 10^{-21}$ (the values of $b$ presented are based on frequency measured in hertz). Although the values of $b$ given here seem to be too small to be significant, the combined value $b f^{2.5}$ is significant and even dominant at typical operating frequencies.

Another way of dealing with nonconstant standard deviations is to fit the data directly to eq (31) using the method of weighted least squares [19]. This technique requires an estimate of the standard deviation associated with the measurements at each frequency, or at least an estimate that is proportional to the standard deviation. Two options are available. After a measurement has been completed, we should have a good estimate of the standard deviation at each frequency. Alternatively, since we know that the standard deviation is approximately equal to the mean, we could use the measured mean as the estimate for the standard deviation. Either one should result in a similar value, and indeed, using the calculated standard deviation results in estimates of $a=12.0$ and $b=5.8 \cdot 10^{-21}$, and using the standard deviation based on the mean results in estimates of $a=12.8$ and $b=5.8 \cdot 10^{-21}$.

Finally, we could fit the reciprocal of the data to $a+b f^{2.5}$. This may seem like an unusual suggestion, but it allows us to calculate the parameters as a simple function of the data instead of using iterative optimization routines. Also, it greatly simplifies the uncertainty analysis associated with the estimation of the parameters $a$ and $b$. In this situation we must estimate the standard deviation of the data, and once again we have two options for this estimate: the measured standard deviation, or the average, which is assumed to be proportional to the standard deviation. We need to make one correction, however, if we want to use the measured standard deviation, because we did not measure the standard deviation of the reciprocal of the data. Fortunately, we can approximate it as the reciprocal of the standard deviation. Once again, the two methods should give similar results. Using the mean for the estimate of the standard 
deviation, we get estimates of $a=8.94$ and $b=5.6 \cdot 10^{-21}$. The reciprocal of the standard deviation gives results of $a=9.39$ and $b=5.6 \cdot 10^{-21}$.

The data and the five fitted curves are presented in Figure 37a, and an expanded version of the same data is given in Figure 37b. Comparing the results from the five methods we see that the estimate of the value of $b$ is reasonably insensitive to the processing method, but the value of $a$ is very sensitive. Also, we see that fitting to the log of the data results in estimates for $a$ and $b$ that fall between the estimates using the other techniques, and these estimates are the most intuitively satisfying. Fitting to the actual measured data results in estimates of the parameters that are higher than estimates obtained by fitting the parameters based on the logarithm of the data, and fitting to the reciprocal of the data results in estimates that are lower. All the results are similar enough, however, that any advantage we could gain by fitting the parameters to either the data or the logarithm of the data was not worth the increased complexity and computation time, so we chose to fit to the reciprocal of the data. We estimated the standard deviation as the reciprocal of the measured standard deviation since this gave the result that was closer to the result based on the logarithm of the data. For now, this choice should not have a major impact on the processing of the data since we need only a rough approximation of the data. The final values will be important, however, if we attempt to perform any quantitative analysis based on these values.

We present the average received power for a constant incident power in Figure 38a, along with the estimated curve fit. If we compute the difference (in decibels) between the measured data and the fitted estimate, the result should have a range of values significantly smaller than the original curve, and we will be able to observe the relatively small fluctuations in the data. The difference between the data and an estimate of the data is referred to as the residuals. The residuals of the received power are presented in Figure 38b. A similar pair of graphs based on a constant net input power is given in Figures $39 \mathrm{a}$ and $\mathrm{b}$. Based on the graphs in Figure $38 \mathrm{~b}$ and Figure 39b, we can make several observations. The most surprising result is that, regardless of normalization technique, we can estimate the power received by an antenna, based on the input power and rough estimates of the parameters in eq (31), and that estimate will be off by less than $2 \mathrm{~dB}$ for frequencies between $250 \mathrm{MHz}$ and $18 \mathrm{GHz}$. Also, the notch at $8.5 \mathrm{GHz}$ caused us a number of problems, and the causes of this notch forced us to carefully examine our model. We will discuss this notch in more detail below. Another interesting result is the apparent $1 \mathrm{~dB}$ step at $1 \mathrm{GHz}$ in Figures 39a and $\mathrm{b}$, where a constant net input power was used. This offset was not present, or at least was much smaller, when a constant incident power was maintained, as in Figures $38 \mathrm{a}$ and $\mathrm{b}$. As mentioned before, log-periodic antennas were used as transducers for one set of measurements between $80 \mathrm{MHz}$ and $1.08 \mathrm{GHz}$, and dual-ridged horns were used for measurements between $1 \mathrm{GHz}$ and $18 \mathrm{GHz}$. The overlap between $1 \mathrm{GHz}$ and $1.08 \mathrm{GHz}$ allows us to compare the measurements made with the different antennas. Assuming that there is a difference in the measured received power depending on which antenna was used, we must develop some explanation as to what is causing the difference, and also attempt to explain the difference in this effect caused by the different methods of normalization. We have shown in Section 5.2.2 that the amount of power transmitted and received by an antenna is a function of the internal reflections of the antenna. Figure 25 and Figure 26 show that, in the vicinity of 1 $\mathrm{GHz}$, the reflected power is lower for the log-periodic than for the dual-ridged horn. Thus we 


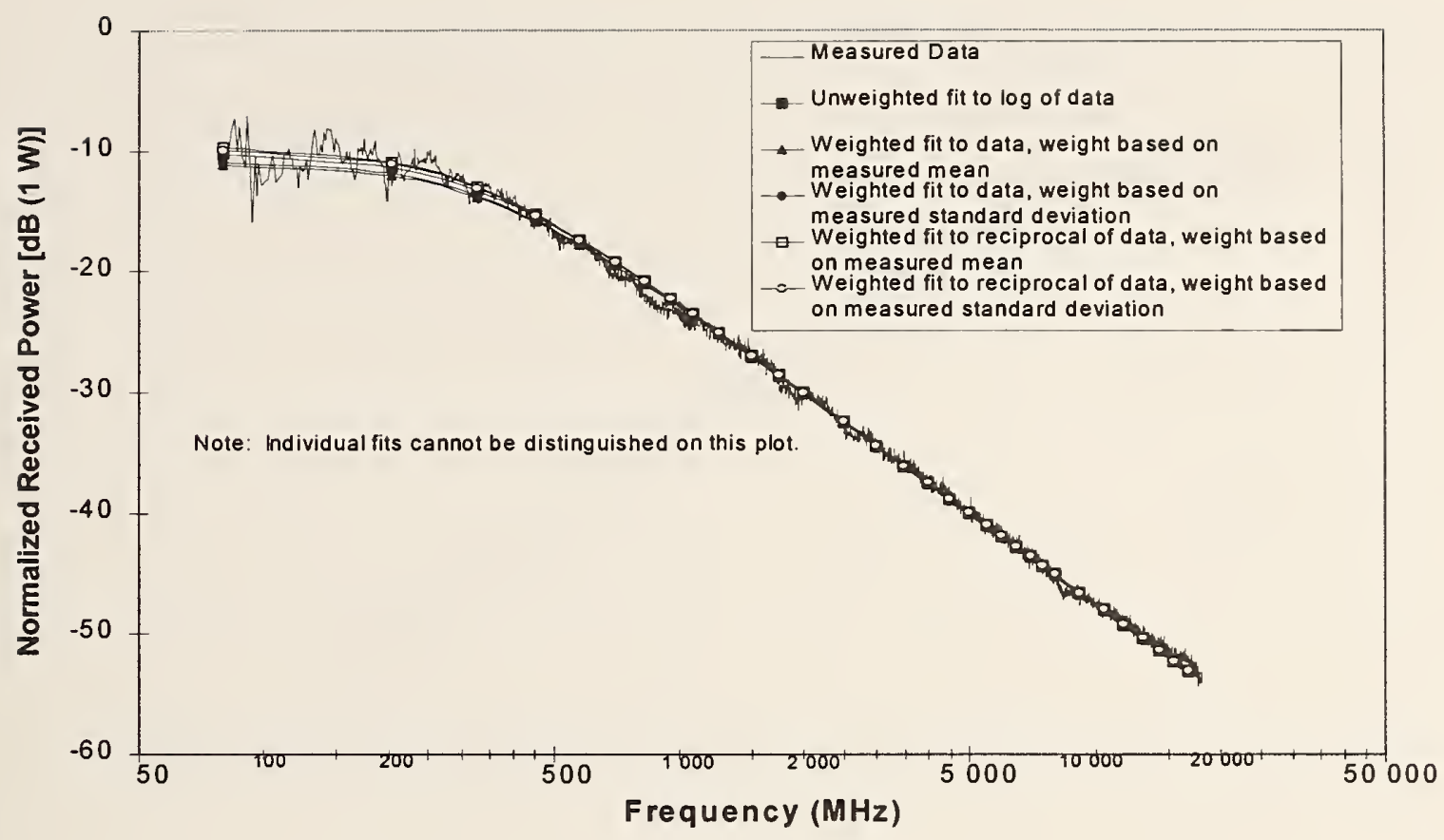

(a)

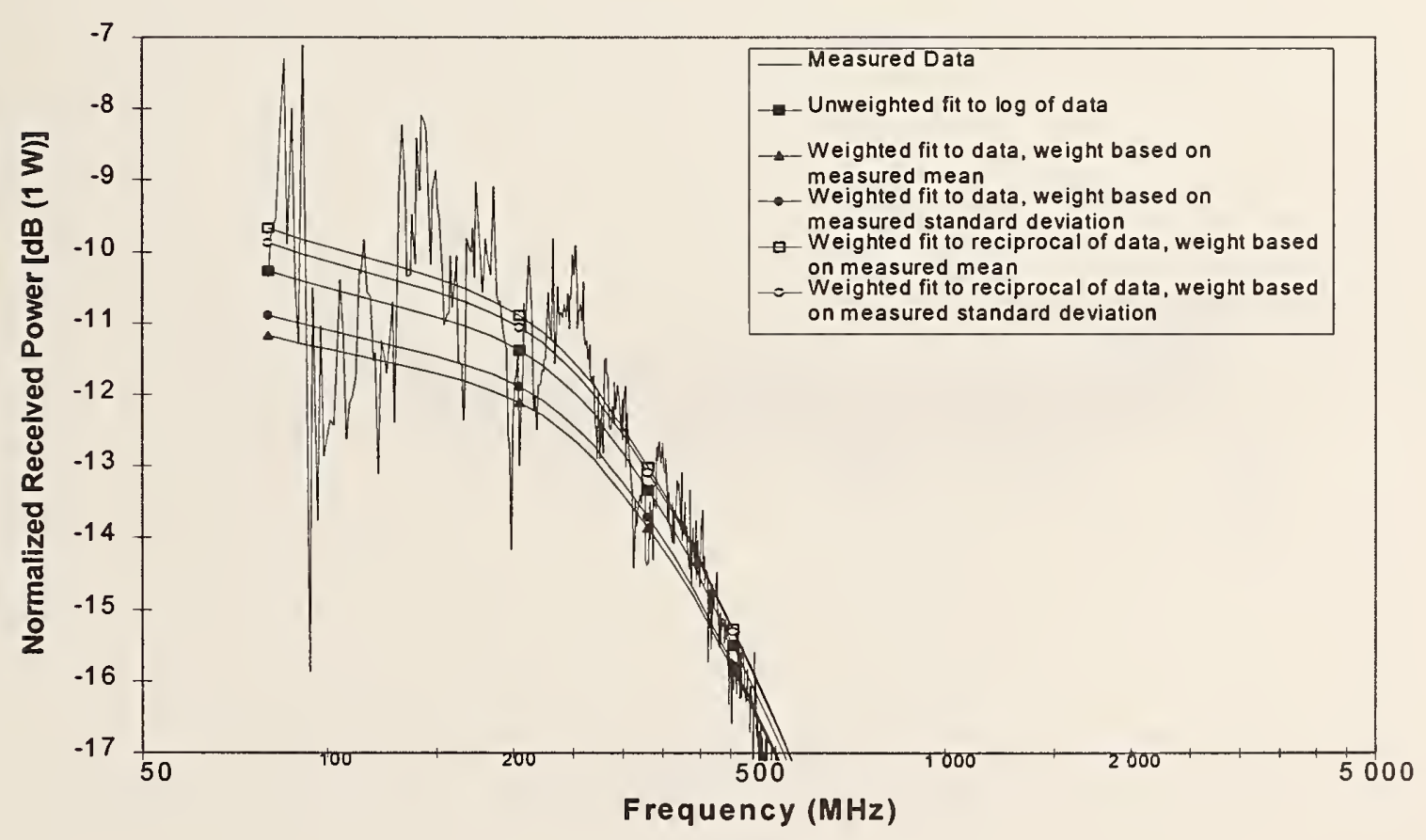

(b)

Figure 37. Average received power for a constant incident power of $1 \mathrm{~W}$ and five possible curve fits. (a) Standard scale. (b) Expanded scale to show detail. 


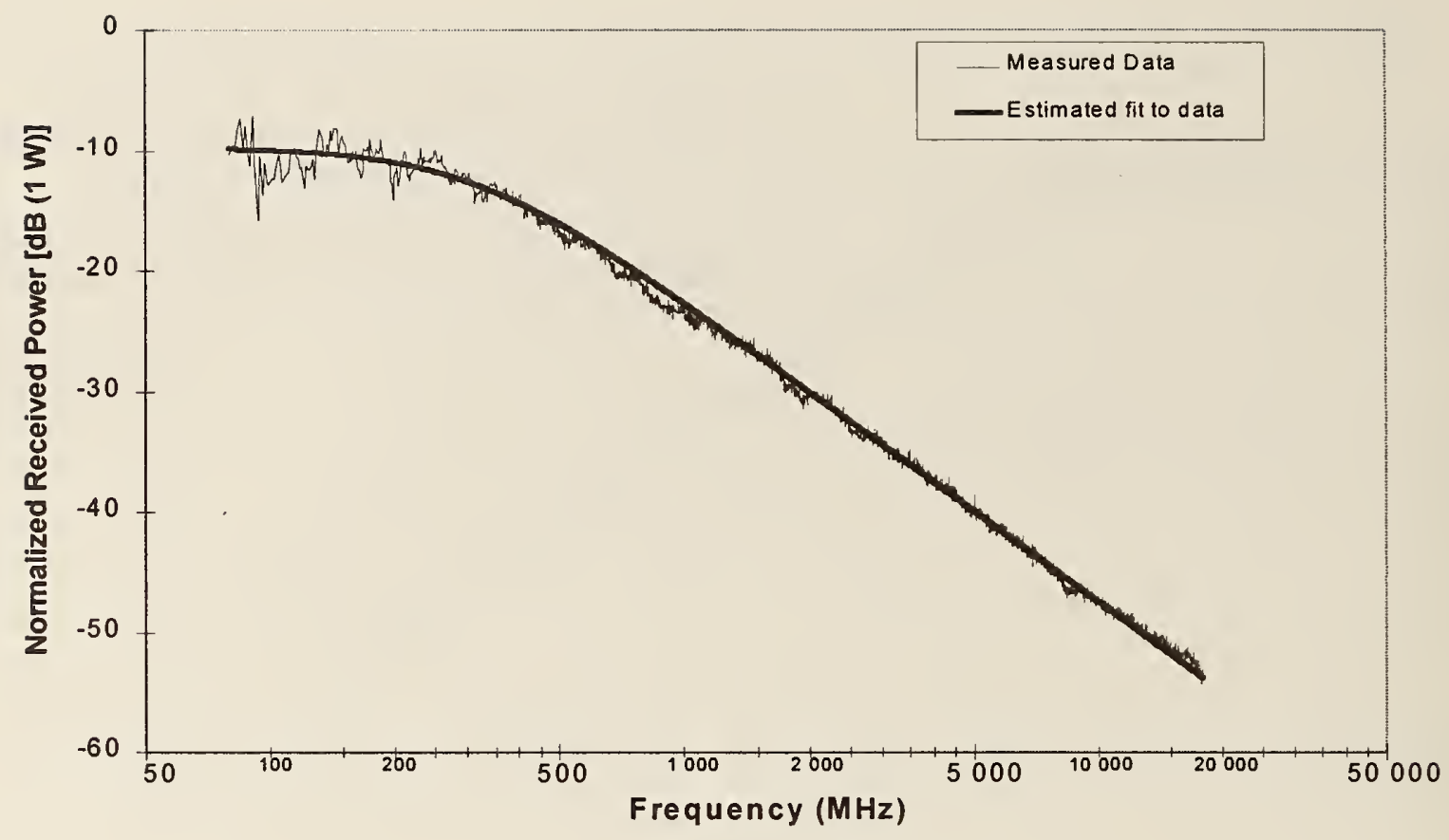

(a)

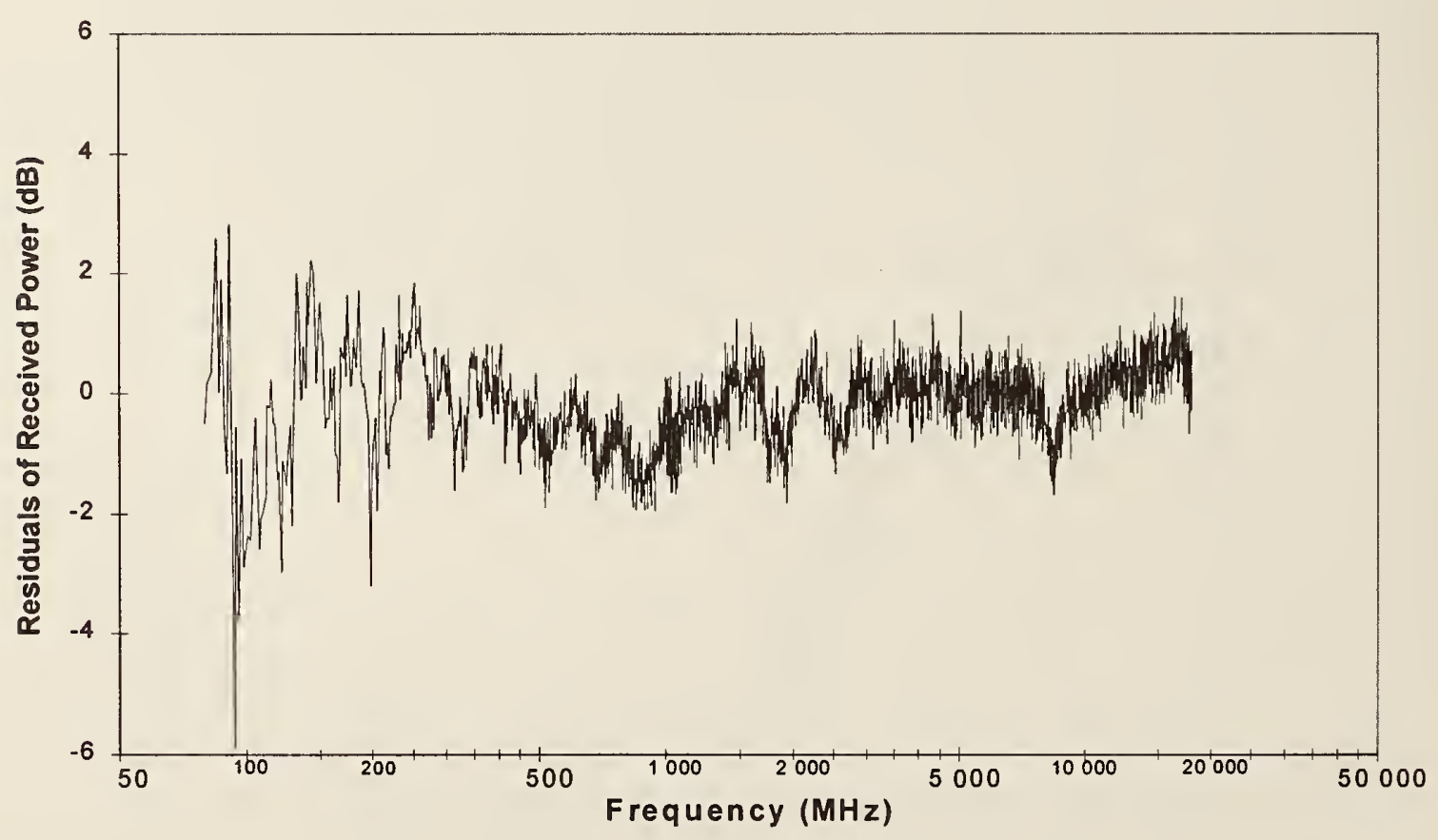

(b)

Figure 38. Average received power for a constant incident power of $1 \mathrm{~W}$ and approximate curve fit. (a) Received power. (b) Residuals of received power. 


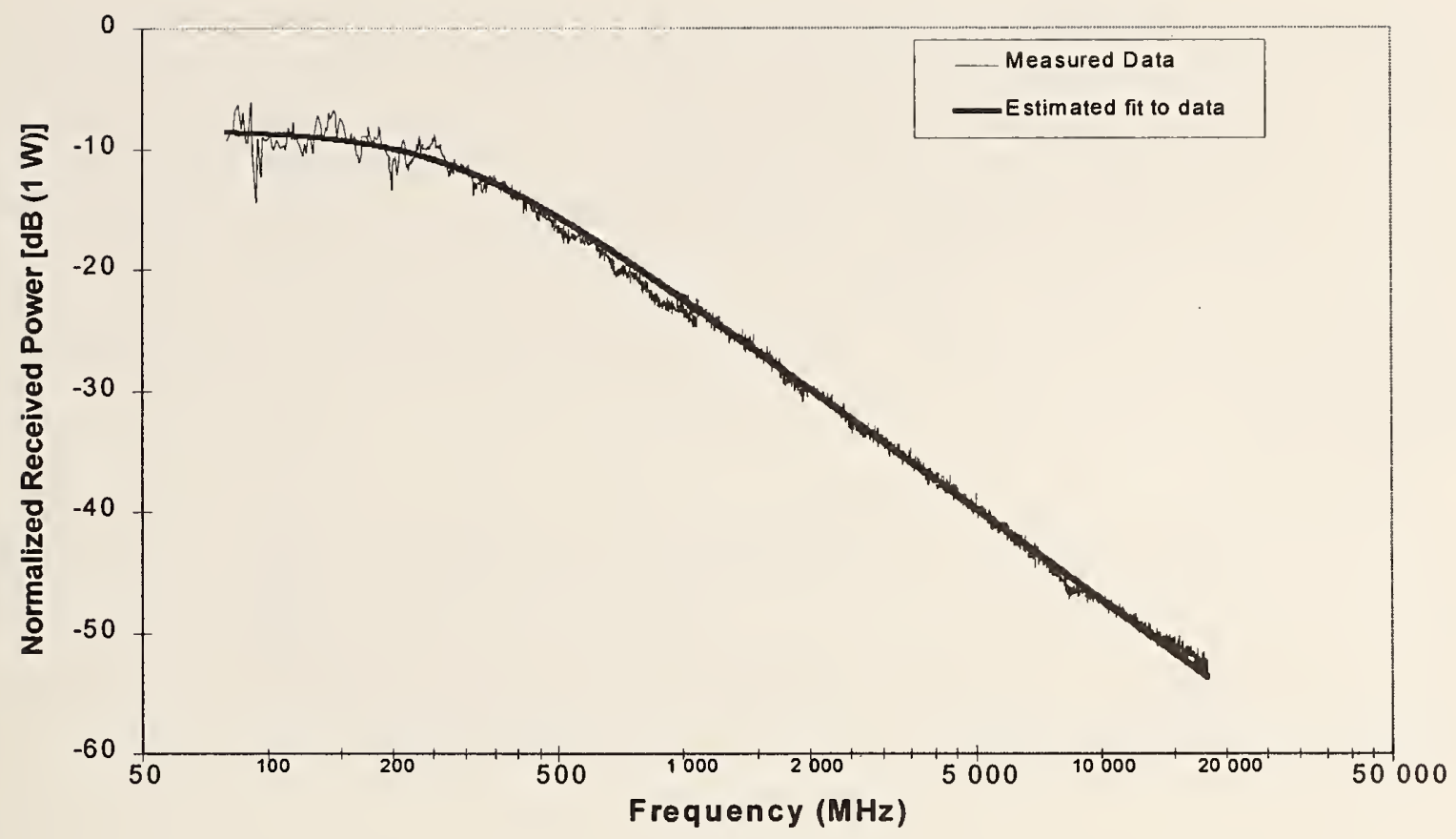

(a)

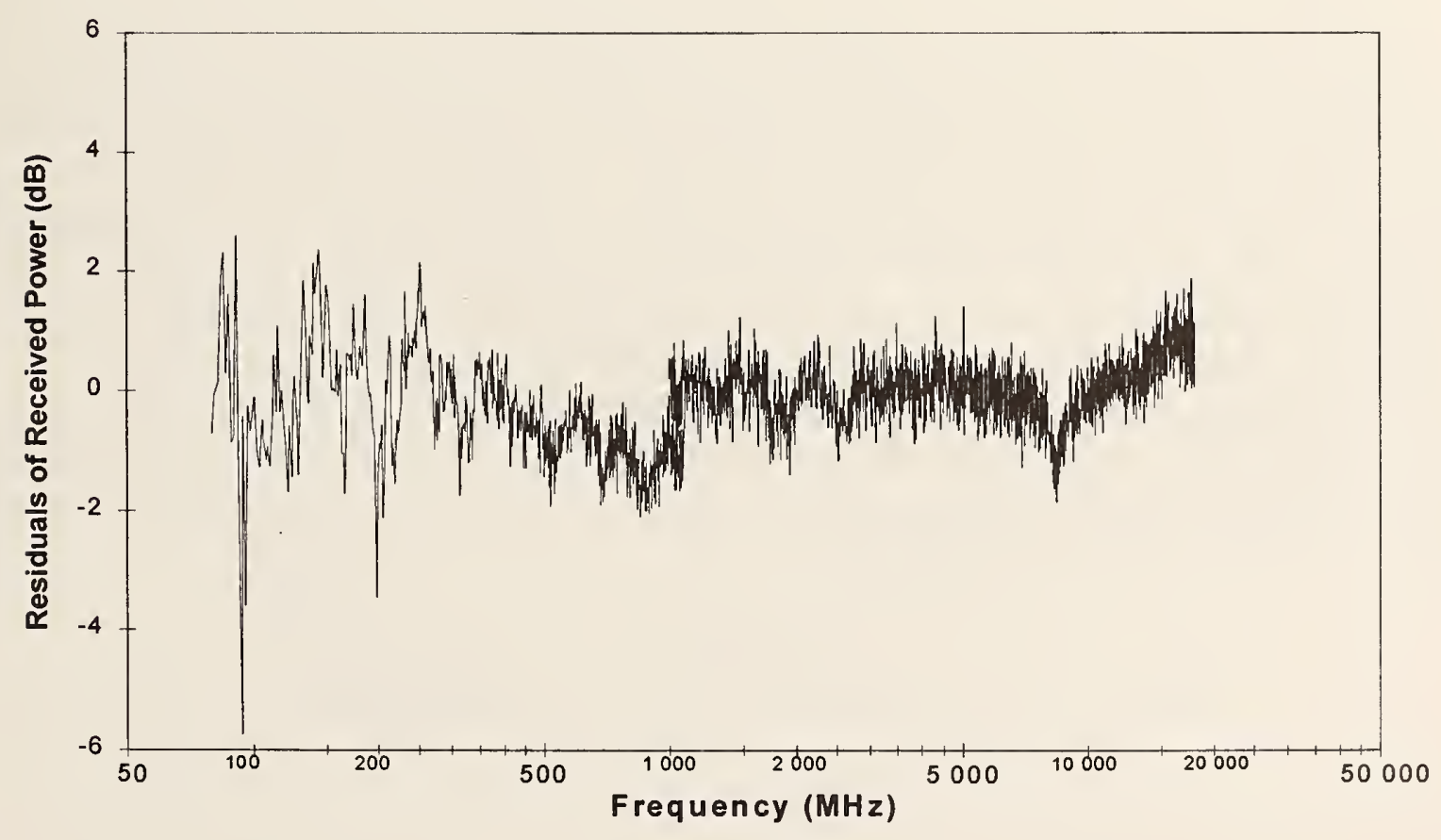

(b)

Figure 39. Average received power for a constant net input power of $1 \mathrm{~W}$ and approximate curve fit. (a) Received power. (b) Residuals of received power. 
would expect the power received by the dual-ridged horns to be less than that received by the log-periodic antennas, not greater. This implies that the differences in received power must have some other explanation. These differences are not a function of instrumentation or the chamber, since the same chamber and equipment (other than antennas) were used in both cases. This leaves two main possibilities: absorptive loading of the chamber by the antennas, or antenna efficiency. Absorptive loading implies that components in the antenna are lossy enough to actually lower the quality factor of the chamber. Although this is a possibility, we consider it unlikely because the same effect has been observed in other chambers that have significant differences in their quality factors. For these reasons, we feel that the most likely culprit is antenna efficiency.

To verify this conclusion, we attempted to correct for all other antenna effects, to the extent possible. We first corrected for the mismatch of the transmitting antenna. Since this is important only if we are attempting to maintain a constant incident power (maintaining a constant net input power already compensates for mismatches in the transmitting antenna), we multiplied the average received power, normalized to a constant incident power, by a mismatch correction factor of $1 /\left(1-\left|<S_{11}>\right|^{2}\right)$ :

$$
P_{R} \approx \frac{P_{\text {meas }}}{1-\left|<S_{11}>\right|^{2}}
$$

The forward mismatch correction factor is shown in Figure 40a, and the reverse mismatch correction factor (based on $S_{22}$ ) is shown in Figure 40b. In general, the correction factor is less than $1 \mathrm{~dB}$ for most frequencies, with larger values below $150 \mathrm{MHz}$ and above $15 \mathrm{GHz}$. This parameter can be approximated based on measurements of the free-space reflection coefficient of the antenna, as Figure 24 shows that the magnitude of the reflection coefficient measured in a mode-stirred chamber is similar to the free-space reflection coefficient. Unfortunately, any deterministic offset caused by placing the antenna in a chamber that is not sufficiently randomized can only be detected by measurement of the reflection coefficient inside the chamber. Also, these measurements can only be made using a vector network analyzer. It may be possible to estimate the mismatch correction factor based on scalar measurements, but we do not attempt to calculate such an estimate here. 


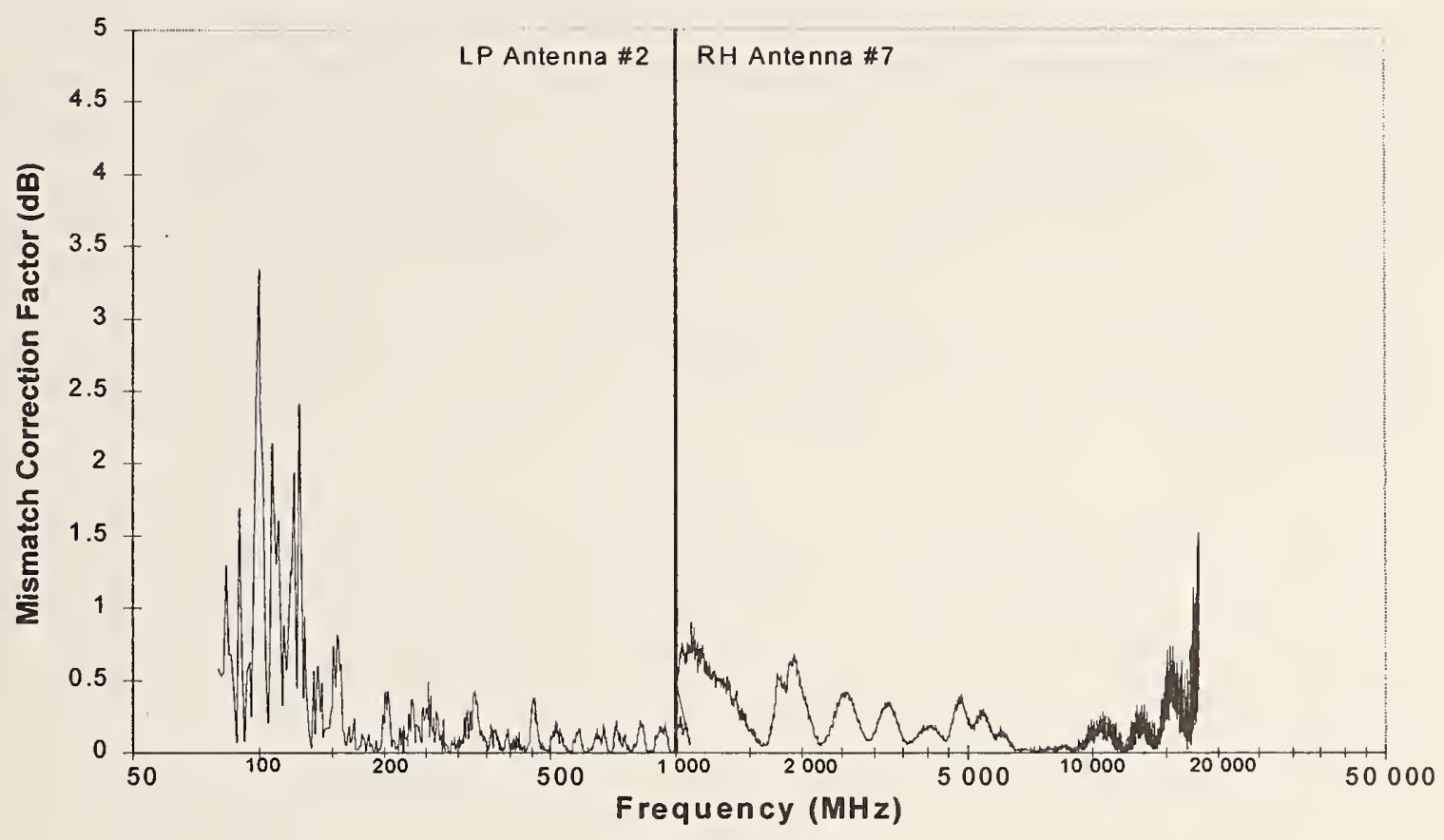

(a)

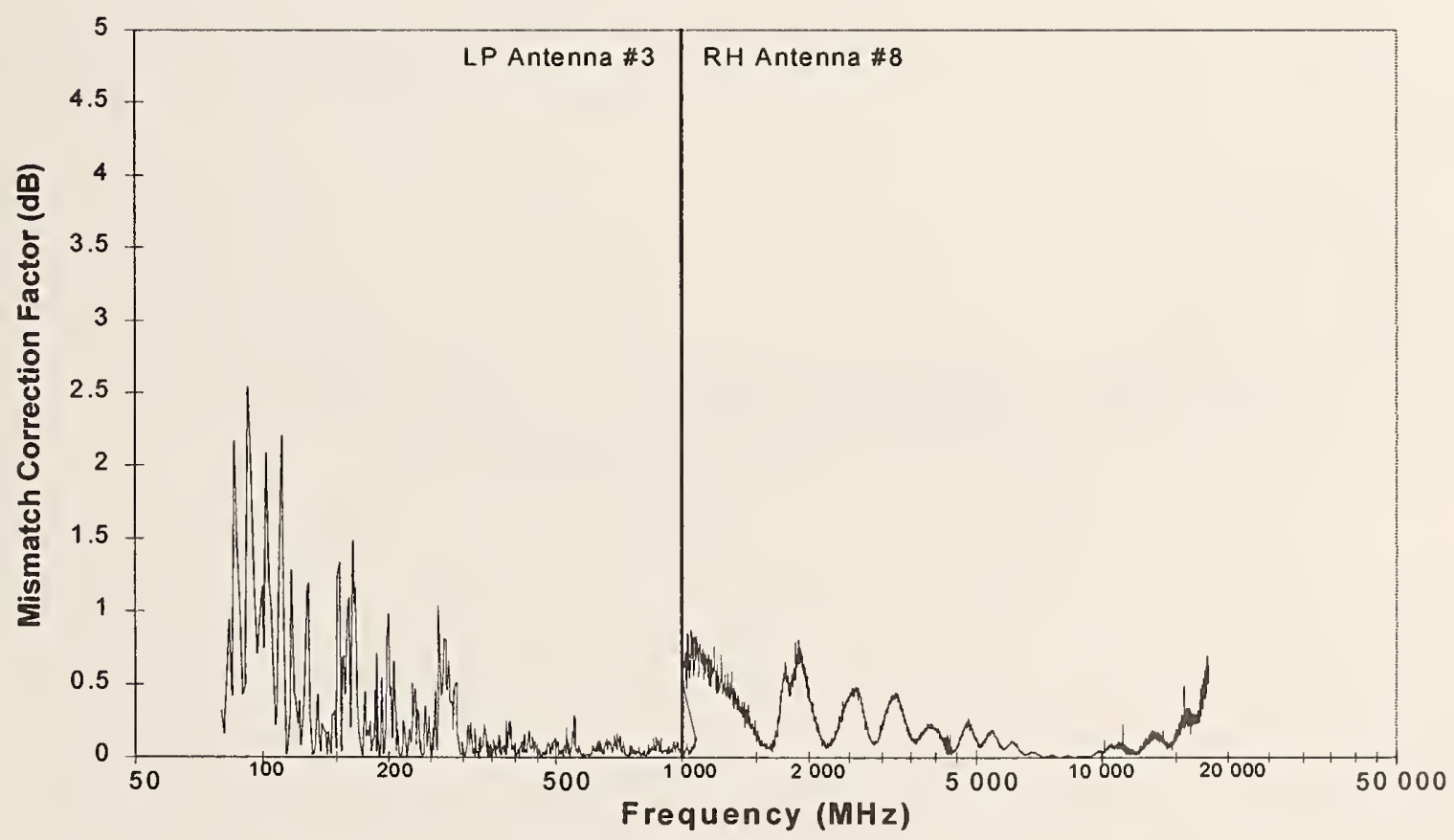

(b)

Figure 40. Mismatch correction factor in chamber A. (a) Port 1 transmitting (forward). Log periodic antenna 2, $80 \mathrm{MHz}$ to $1.08 \mathrm{GHz}$. Ridged horn antenna 7, $1 \mathrm{GHz}$ to $18 \mathrm{GHz}$. (b) Port 2 transmitting (reverse). Log periodic antenna 3,80 MHz to $1.08 \mathrm{GHz}$. Ridged horn antenna $8,1 \mathrm{GHz}$ to $18 \mathrm{GHz}$. 
The chamber gain corrected for the mismatch of the transmitting antenna is plotted in Figure 41a, and the residuals are plotted in Figure 41b. (Each time a correction is applied to the data, the estimated curve fit parameters are recalculated to give the best estimate possible.) The 1 $\mathrm{dB}$ step which we saw in Figure 39 when we normalized to a constant net input power is now present in Figure 41 when we normalize to a constant incident power and correct for reflections in the transmitting antenna. In fact, the residuals shown in Figure $41 \mathrm{~b}$ are now indistinguishable from the residuals shown in Figure 39b, even though the estimated curve fit parameters (specifically the value of $a$ ) are significantly different. The difference between the residuals is less than $0.4 \mathrm{~dB}$ for frequencies greater than $100 \mathrm{MHz}$, and less than $0.05 \mathrm{~dB}$ for frequencies greater than $500 \mathrm{MHz}$.

At this point in the processing, the two different measures of received power (received power based on a constant incident power and corrected for the mismatch of the transmitting antenna, and the received power based on a constant net input power) will be treated identically. These values will be called jointly the received power for a constant input power of $1 \mathrm{~W}$, corrected for the mismatch of the transmitting antenna. Any differences between the measures of received power will be indicated in the accompanying figures.

We now apply a correction for the mismatch of the receiving antenna by multiplying the received power for a constant input of $1 \mathrm{~W}$, corrected for the mismatch of the transmitting antenna by $1 /\left(1-\left|<S_{22}>\right|^{2}\right)$. The average received power for a constant input power of $1 \mathrm{~W}$, corrected for the mismatch of both the transmitting and receiving antennas, is given in Figure 42a. The difference between the data measured with the two normalization methods is negligible above $300 \mathrm{MHz}$, and the differences below $300 \mathrm{MHz}$ are more noticeable in the estimated curve fit than in the actual measured data. The residuals of measured power are given in Figure $42 \mathrm{~b}$.

The corrections we have applied to the data thus far appear to have removed some of the fluctuations in the plots of the residuals, especially in the vicinity of $1.7 \mathrm{GHz}$ and $2.5 \mathrm{GHz}$ (compare to Figure 41). Unfortunately, the step at $1 \mathrm{GHz}$ has actually increased, and the notch at 8.5 GHz is unchanged. Since we have corrected for all antenna mismatch terms to the best of our abilities, we thought that this helped verify that the step at $1 \mathrm{GHz}$ was indeed caused by inefficient log-periodic antennas, but the evidence was not conclusive.

Assuming that antenna efficiency is causing the observed difference, we want to correct for it since it is causing a substantial bias in our estimates of received power. Ideally, we could evaluate the efficiencies of the antennas in question in some other facility and use these data to correct the data we measured in the mode-stirred chambers. Unfortunately, measuring the efficiency requires measuring the total power radiated by the antenna. This is a difficult task and can require time-consuming or inaccurate measurement techniques [26]. Instead, we tried a different approach. If the step at $1 \mathrm{GHz}$ is caused by a difference in antenna efficiency, then this implies that the relative efficiencies of antennas can in some way be observed and, perhaps, measured in a mode-stirred chamber. Operating under this assumption, we evaluated the NIST chamber using the same antennas we used to evaluate the NASA chambers, and then again using waveguide antennas that should be nearly perfectly efficient. We did not have waveguide antennas available that covered the entire frequency range measured in the NASA chambers, so we were forced to make do with the equipment we had available. Below $1 \mathrm{GHz}$, this consisted of two pairs of "coax-to-waveguide" adapters, one designed to operate between $400 \mathrm{MHz}$ and 


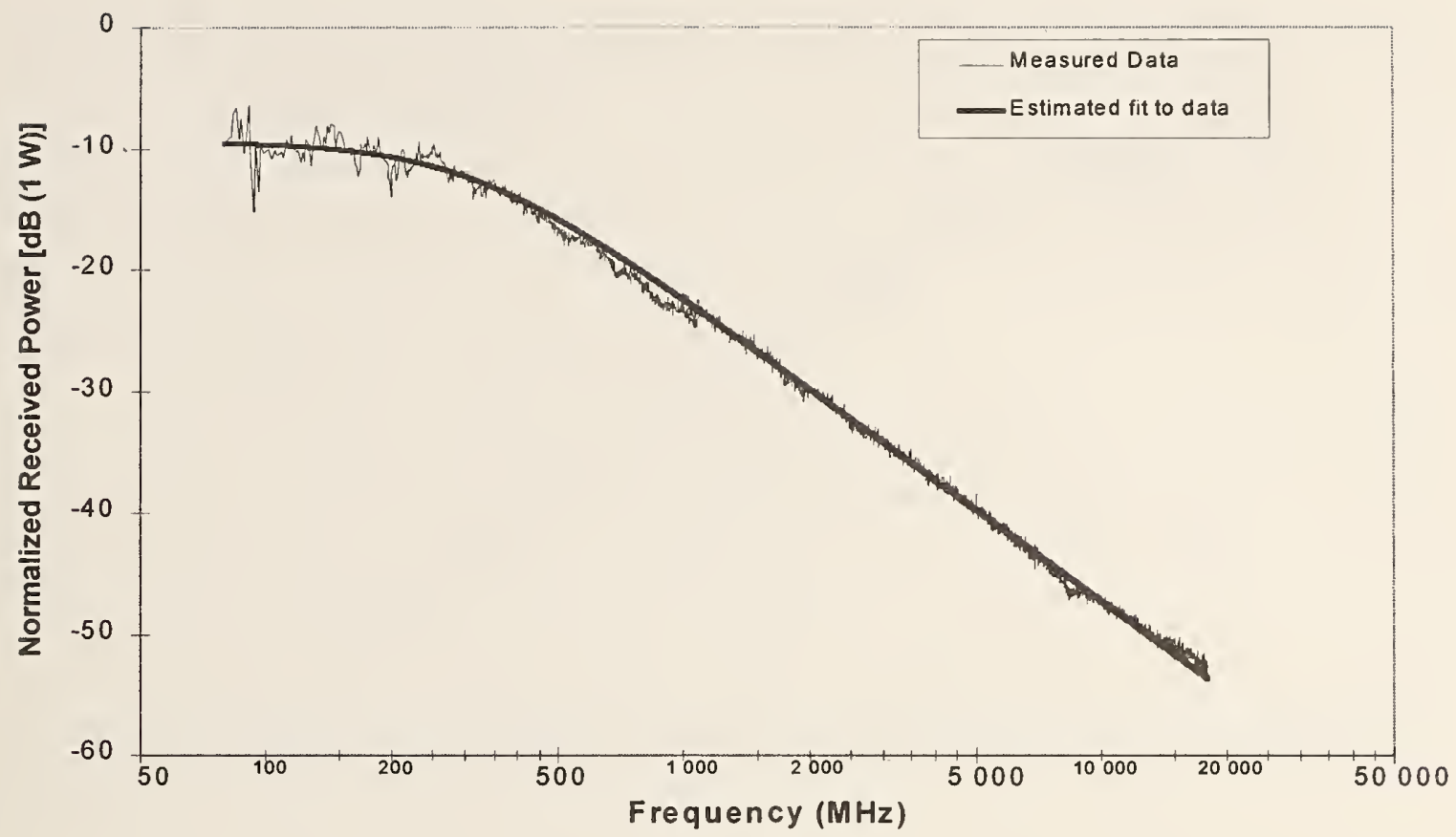

(a)

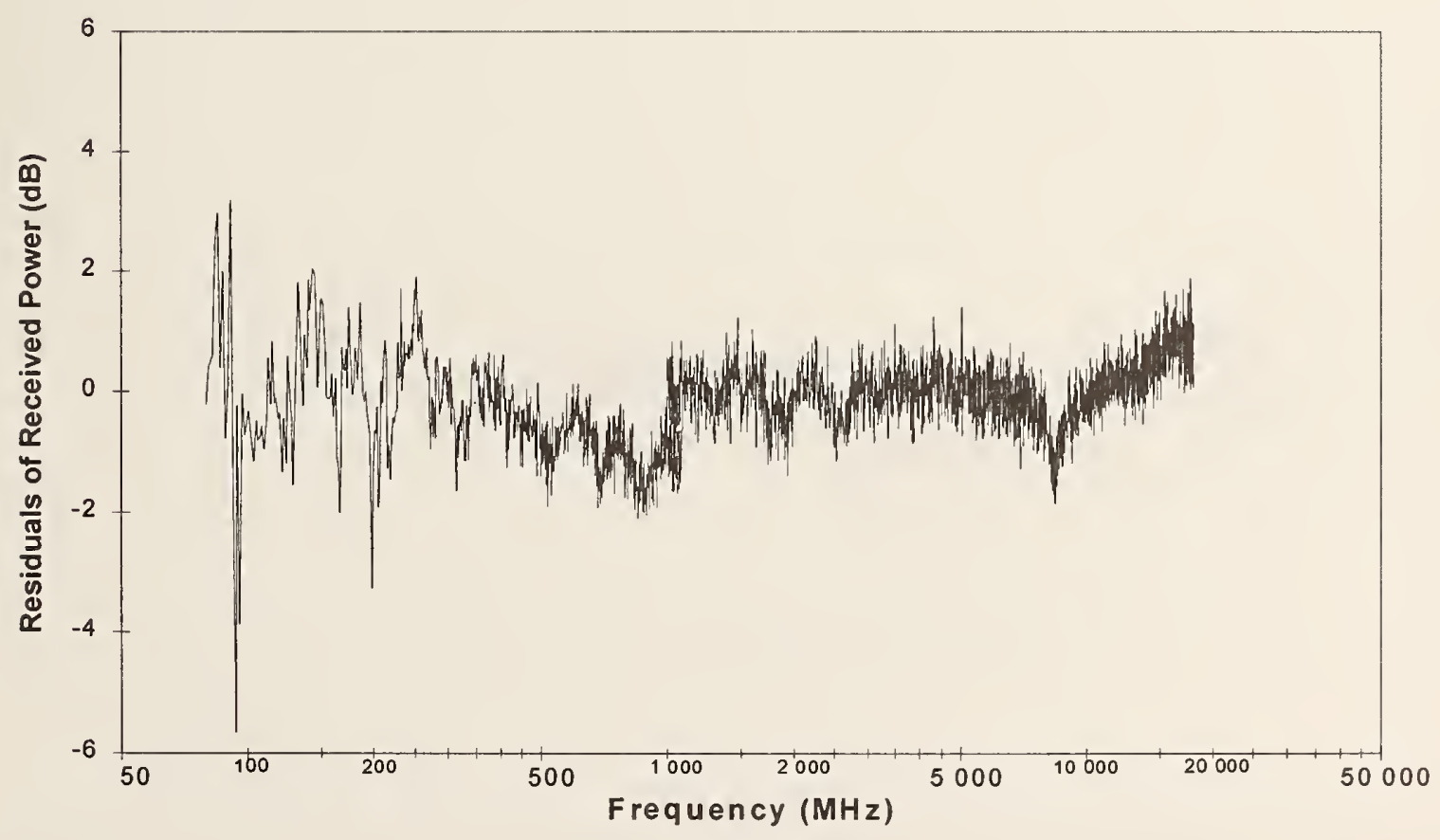

(b)

Figure 41. Average received power for a constant incident power of $1 \mathrm{~W}$, corrected for mismatch of transmitting antenna. (a) Received power. (b) Residuals of received power. 


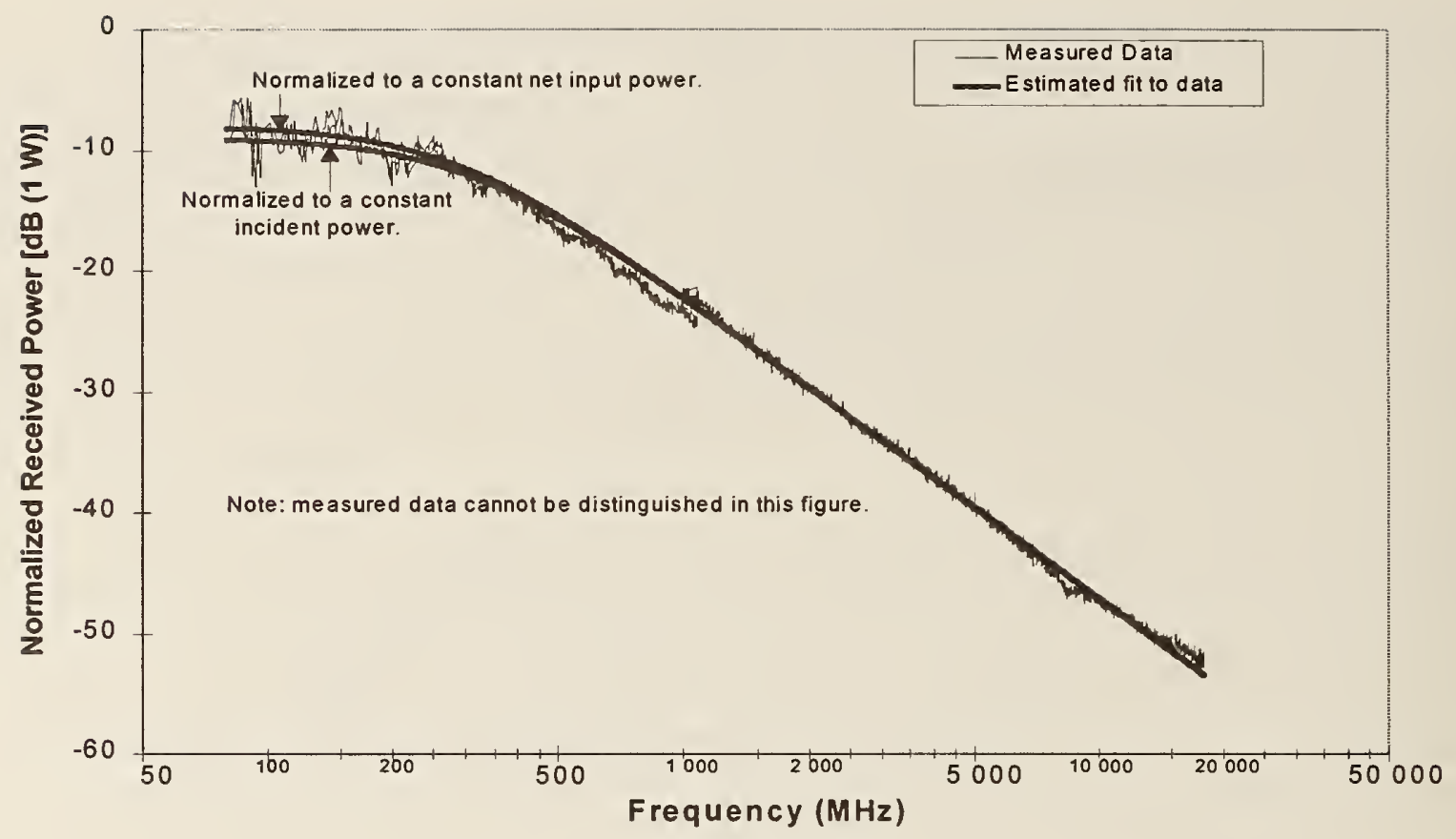

(a)

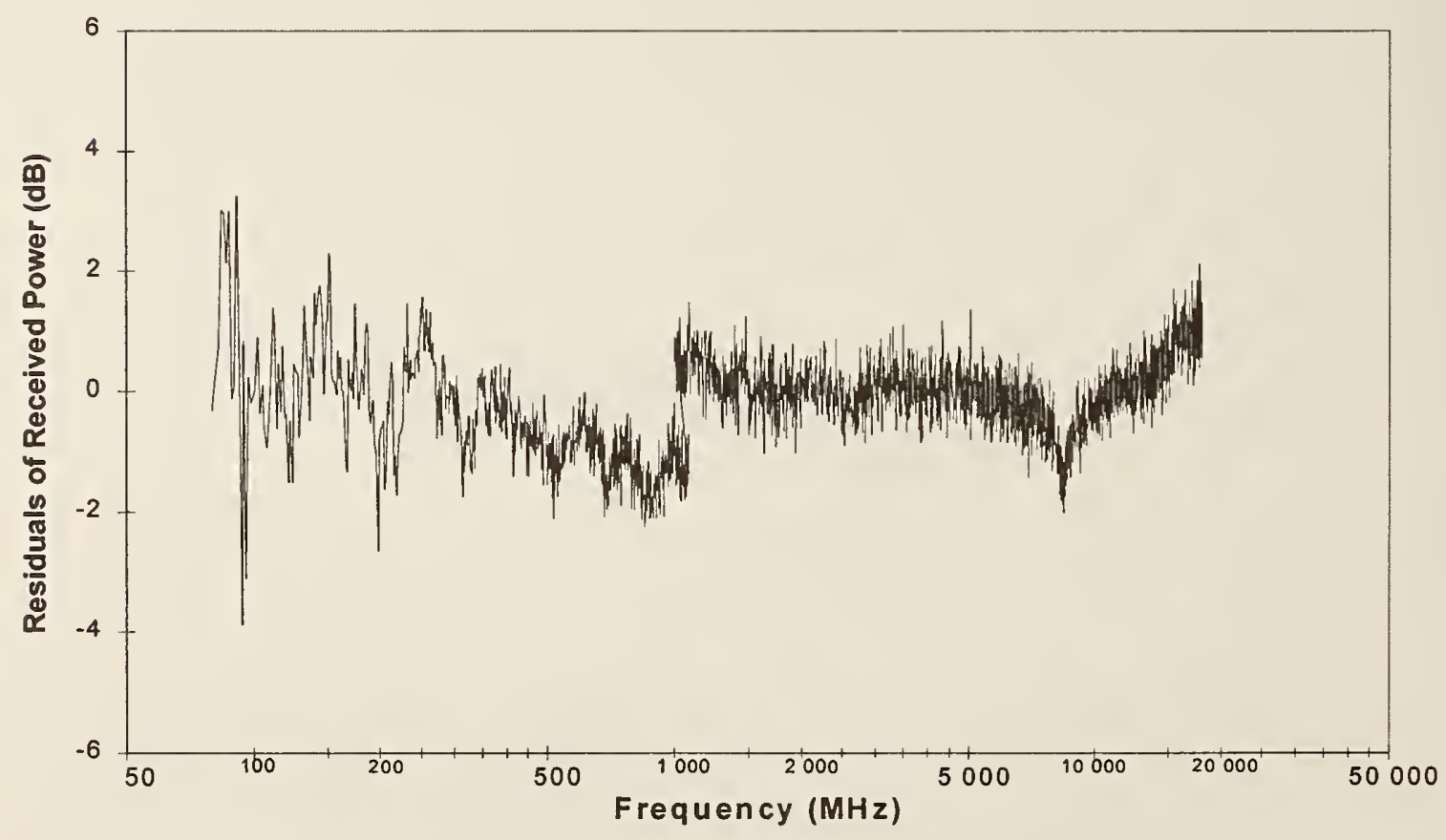

(b)

Figure 42. Average received power for a constant input power of $1 \mathrm{~W}$, corrected for mismatch of transmitting antenna and receiving antenna. (a) Received power. (b) Residuals of received power. 
$600 \mathrm{MHz}$, and the other designed to operate between $500 \mathrm{MHz}$ and $750 \mathrm{MHz}$. No transition sections of waveguide were used to attenuate unwanted modes, because higher order modes in the adapters did not cause substantial problems as long as they did not result in excessive loss (poor efficiency) or large reflections (poor match). The adapters appeared to meet these criteria over their recommended range of operation and even to somewhat higher frequencies. We therefore used the first pair to evaluate the NIST chamber between $400 \mathrm{MHz}$ and $600 \mathrm{MHz}$, and the second pair between $600 \mathrm{MHz}$ and $800 \mathrm{MHz}$. No data were taken below $400 \mathrm{MHz}$, and no data were taken between $800 \mathrm{MHz}$ and $2 \mathrm{GHz}$.

As expected, the estimated power received by the adapters was substantially greater than that received by the log-periodic antennas. The difference (in decibels) between the power received using log-periodic Antennas 2 and 3 (the antennas used to evaluate Chambers A and B) and the coax-to-waveguide adapters is shown in Figure 43a. This figure shows the measured difference between the two measures of received power, a filtered (21-point moving average) version of the measured difference, and a very gross approximation. At this point, we were interested only in gross corrections, since any fine corrections would require a large number of measurements before the noise could be reduced to an acceptable level. The concept of measuring relative antenna efficiency in a mode-stirred chamber is new, and the work presented here should be considered preliminary.

On average, the received power was $2.4 \mathrm{~dB}$ (approximately $70 \%$ ) greater using the adapters compared to the log-periodic antennas. Using the techniques outlined here, we cannot measure the efficiencies of the individual antennas. Instead, we are forced to assume that both antennas have similar efficiency characteristics. Thus, approximately half of the total loss of 2.4 $\mathrm{dB}(1.2 \mathrm{~dB})$ is attributable to the transmitting antenna, and the remainder is attributable to the receiving antenna. The corrections generated using this technique are intended as rough, firstorder approximations only. The results are extremely noisy and the majority of the overall uncertainty of the measurement will be caused by the uncertainty in this estimate of the efficiencies of the transmitting and receiving antennas. Even so, this correction is better than no correction at all. In the absence of any measured data on the efficiencies of the log-periodic antennas below $400 \mathrm{MHz}$, and between $800 \mathrm{MHz}$ and $1.08 \mathrm{GHz}$, we are forced to assume that the efficiency of these antennas is approximately constant over their entire operational frequency range, with the efficiencies of each antenna equal to approximately $76 \%$. Even if waveguide antennas were available below $400 \mathrm{MHz}$, it would be difficult to use them to evaluate the logperiodic antennas below $200 \mathrm{MHz}$, which is the approximate lowest usable frequency of the NIST chamber. Thus, the efficiency of the antennas used to characterize the largest of the NASA chambers, Chamber A, must be evaluated in a chamber that is at least as large as that chamber, and preferably larger.

Similarly, the combined efficiencies of log-periodic Antennas 4 and 5 (used to evaluate NASA Chamber C) is plotted in Figure 43b. These antennas received approximately half (a difference of $3 \mathrm{~dB}$ ) of the power received by the adapters. These antennas are estimated to be $71 \%$ efficient. 


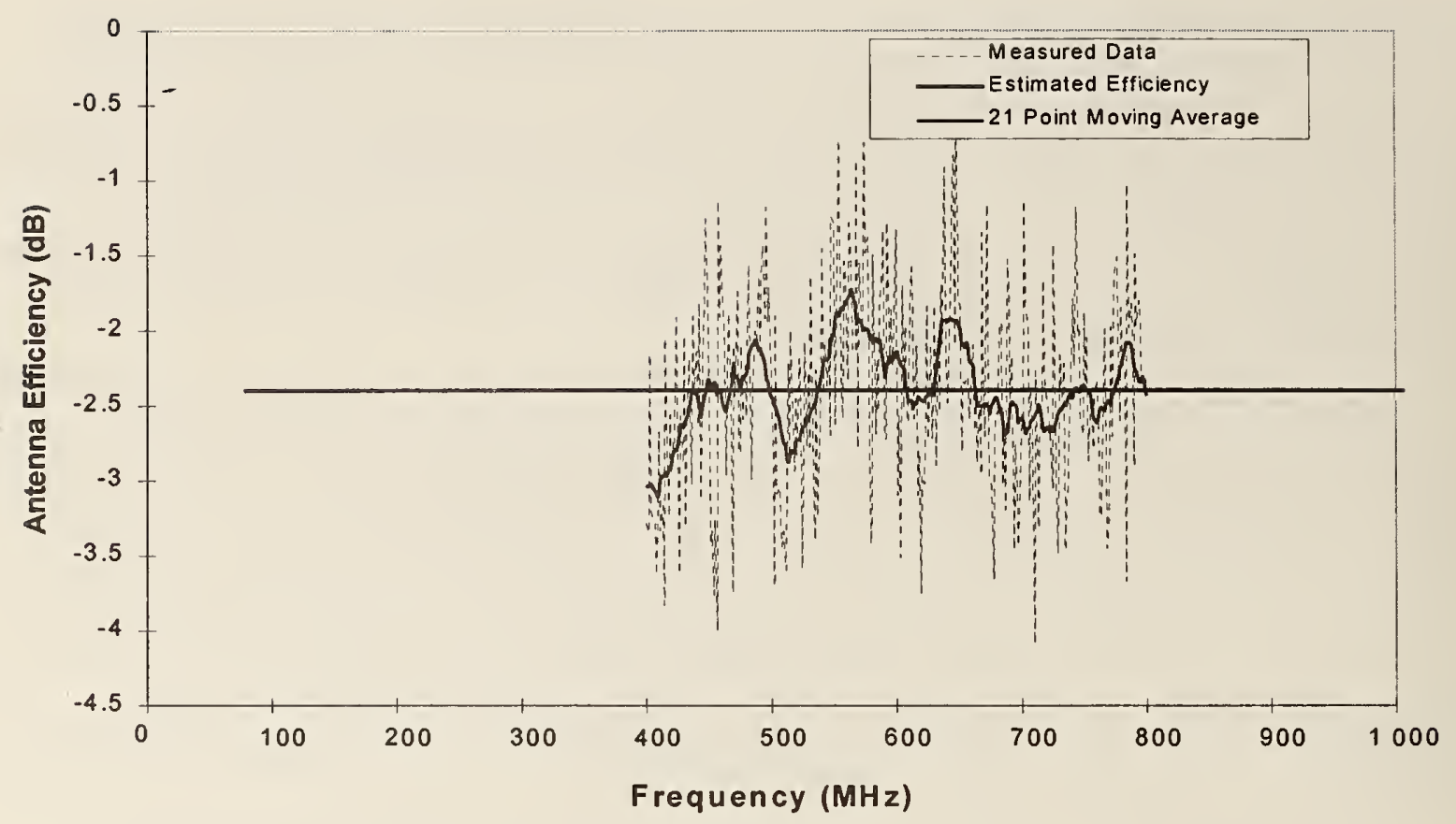

(a)

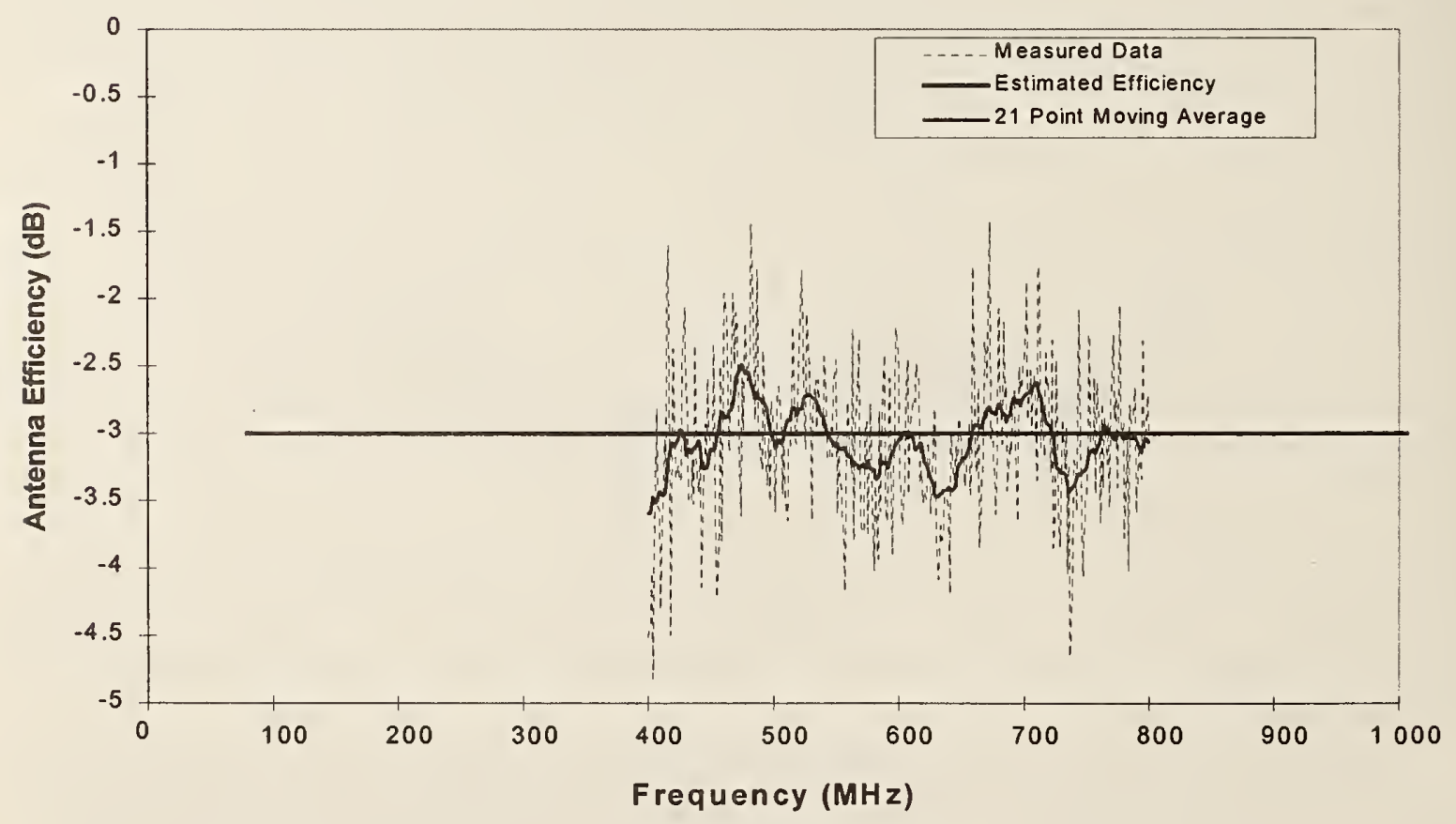

(b)

Figure 43. Estimated combined efficiencies of log-periodic antennas as measured in NIST chamber. (a) Combined efficiency of log-periodic antenna 2 and log-periodic antenna 3. (b) Combined efficiency of log-periodic antenna 4 and log-periodic antenna 5. 


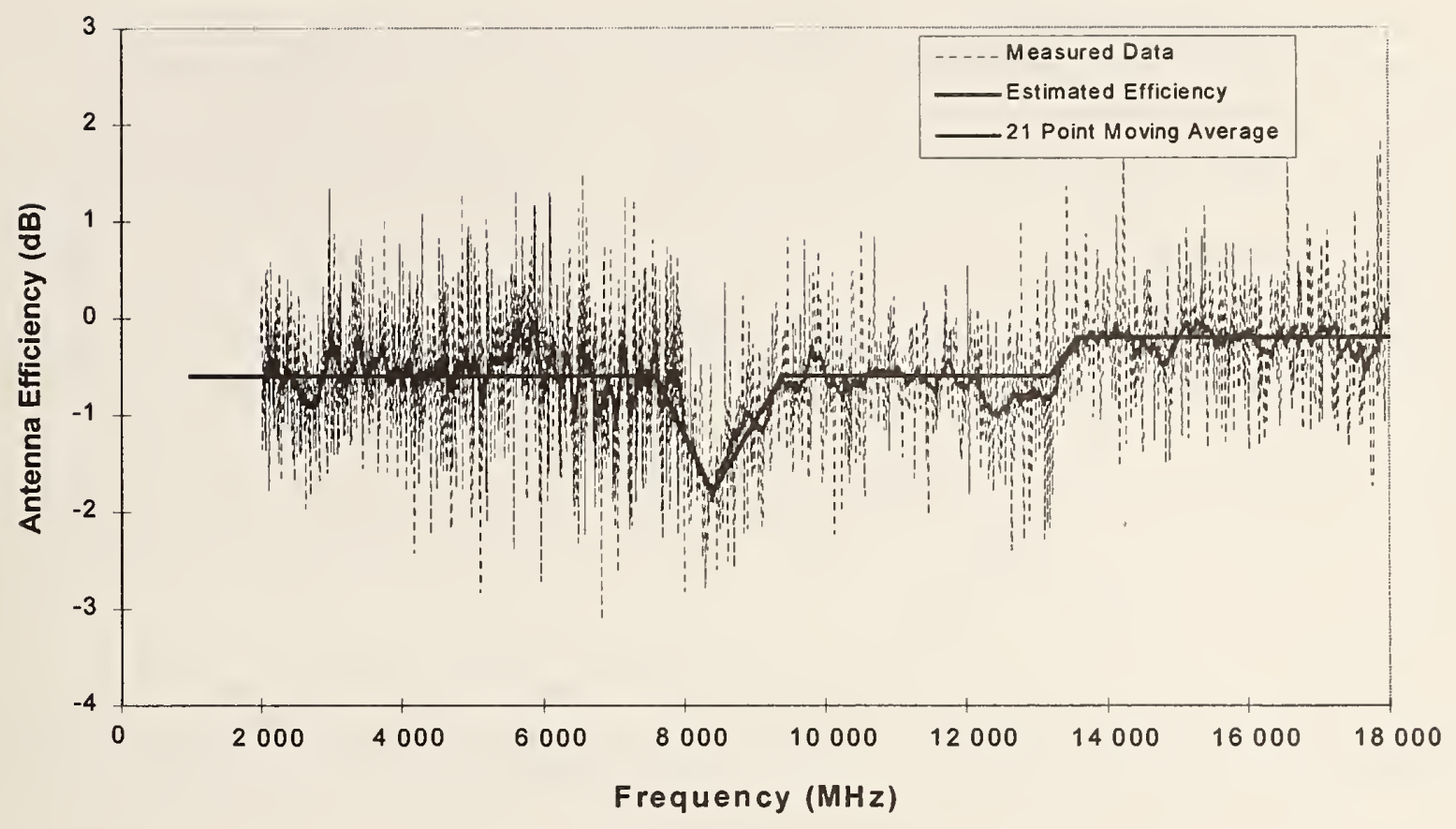

(a)

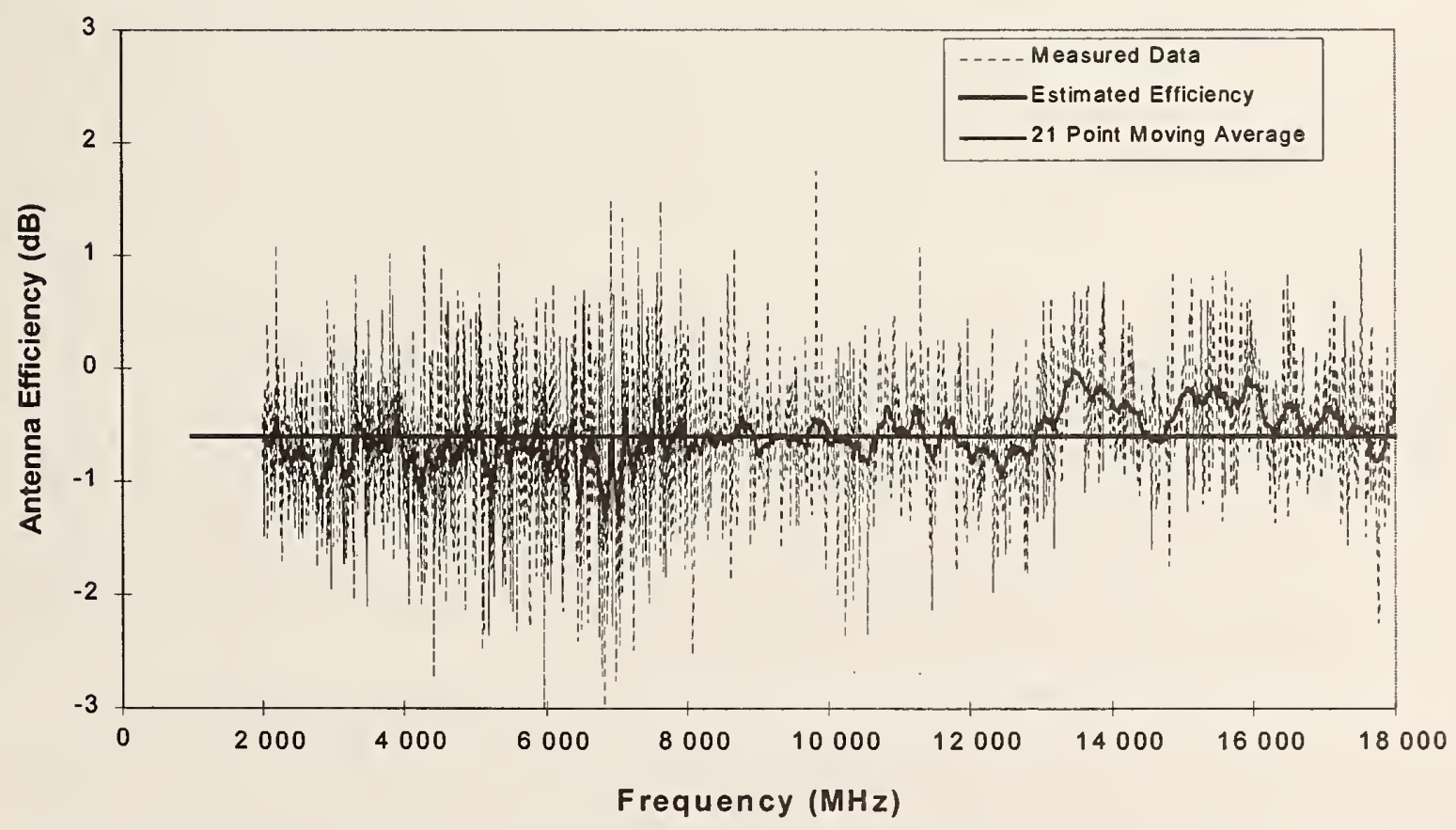

(b)

Figure 44. Estimated combined efficiencies of ridged horn antennas as measured in NIST chamber. (a) Combined efficiency of ridged horn antenna 7 and ridged-horn antenna 8. (b) Combined efficiency of ridged-horn antenna 10 and ridged-horn antenna 11. 
We used three pairs of ridged waveguide horns to evaluate the NIST chamber between 2 $\mathrm{GHz}$ and $18 \mathrm{GHz}$. These three pairs of antennas had operational frequency ranges between 2 $\mathrm{GHz}$ and $4.4 \mathrm{GHz}, 3.5 \mathrm{GHz}$ to $8.2 \mathrm{GHz}$, and $7.5 \mathrm{GHz}$ to $18 \mathrm{GHz}$. The difference (in decibels) between the chamber gain measured using these antennas and ridged-horn Antennas 7 and 8 (which were used to evaluate Chamber $\mathrm{A}$, and one of the measurements in Chamber $\mathrm{C}$ ) is shown in Figure 44a. Once again this plot shows the measured difference, a filtered (21-point moving average) version of the measured data, and a gross approximation of the efficiency of the antenna. Below $13 \mathrm{GHz}$, the broadband ridged-horn antennas received approximately $0.5 \mathrm{~dB}$ less power than the waveguide ridged-horn antennas, with the exception of a large notch at $8.5 \mathrm{GHz}$. Above $13 \mathrm{GHz}$, the broadband ridged-horn antennas appear to be almost as efficient as the waveguide ridged-horns. The broadband horns have an estimated efficiency of $94 \%$ below 13 $\mathrm{GHz}$, and an estimated efficiency of $98 \%$ above $13 \mathrm{GHz}$. Since no waveguide antennas were available between $1 \mathrm{GHz}$ and $2 \mathrm{GHz}$, we were forced to extrapolate an estimate of the efficiency of the broadband horns down to $1 \mathrm{GHz}$.

Ridged-horn Antennas 10 and 11 (used in all measurements in Chamber B and one measurement in Chamber C) were evaluated in a similar fashion, and the estimated combined efficiency is given in Figure 44b. These antennas received approximately $0.5 \mathrm{~dB}$ less power than the waveguide ridged-horn antennas over the entire frequency range. The broadband antennas have an estimated efficiency of $94 \%$. Once again, the estimated efficiency was extrapolated down to $1 \mathrm{GHz}$.

The extrapolation of the estimated efficiency below $2 \mathrm{GHz}$ and above $800 \mathrm{MHz}$ means that the uncertainty about the estimates of the efficiency will be worst at $1 \mathrm{GHz}$, where the step in the chamber gain occurs. As a cross check, we note that the efficiencies of the ridged-horn antennas biases the received power by $0.5 \mathrm{~dB}$ at $1 \mathrm{GHz}$, and the efficiencies of the log-periodic antennas biases the received power by $2.4 \mathrm{~dB}$ at $1 \mathrm{GHz}$, for a difference (and an estimated amplitude of the step in received power) of $1.9 \mathrm{~dB}$, which is consistent with the size of the step shown in Figure 42.

Based on the measurements of the relative combined efficiencies of the measurement antennas described above, the superior efficiency of the waveguide antennas suggests that waveguide antennas should be used in all measurements in a mode-stirred chamber. Unfortunately, these types of antennas are usable over only a narrow frequency range (typically less than an octave). Several sets of reference antennas would be required to do measurements over the same range of frequencies that we are able to cover with only two sets of antennas. Another problem with using waveguide antennas is that low frequency waveguide antennas are large and expensive. If the efficiencies of the measurement antennas can be determined, however, then it is no longer necessary to use waveguide antennas.

The final estimated chamber gain, corrected for all antenna mismatch and efficiency effects, is shown in Figure 45a, and the final residuals of chamber gain are shown in Figure 45b. The step at $1 \mathrm{GHz}$ has been effectively removed, as has the notch at $8.5 \mathrm{GHz}$. This notch caused several hours of aggravated head-scratching. It showed up in every measurement we made in Chamber A, none of the measurements we made in Chamber B, and one of the two measurements we made in Chamber $\mathrm{C}$. As discussed above, every time Antennas 7 and 8 were used in a measurement (this pair was used in every measurement in Chamber $\mathrm{A}$ and one of the measurements in Chamber $\mathrm{C}$ ), the notch was present, and every time Antennas 10 and 11 were used (all measurements in Chamber B, and the other measurement in Chamber C), the notch was missing. None of the mismatch data indicated a problem with any of the antennas, so we expected that this was also an efficiency problem. 


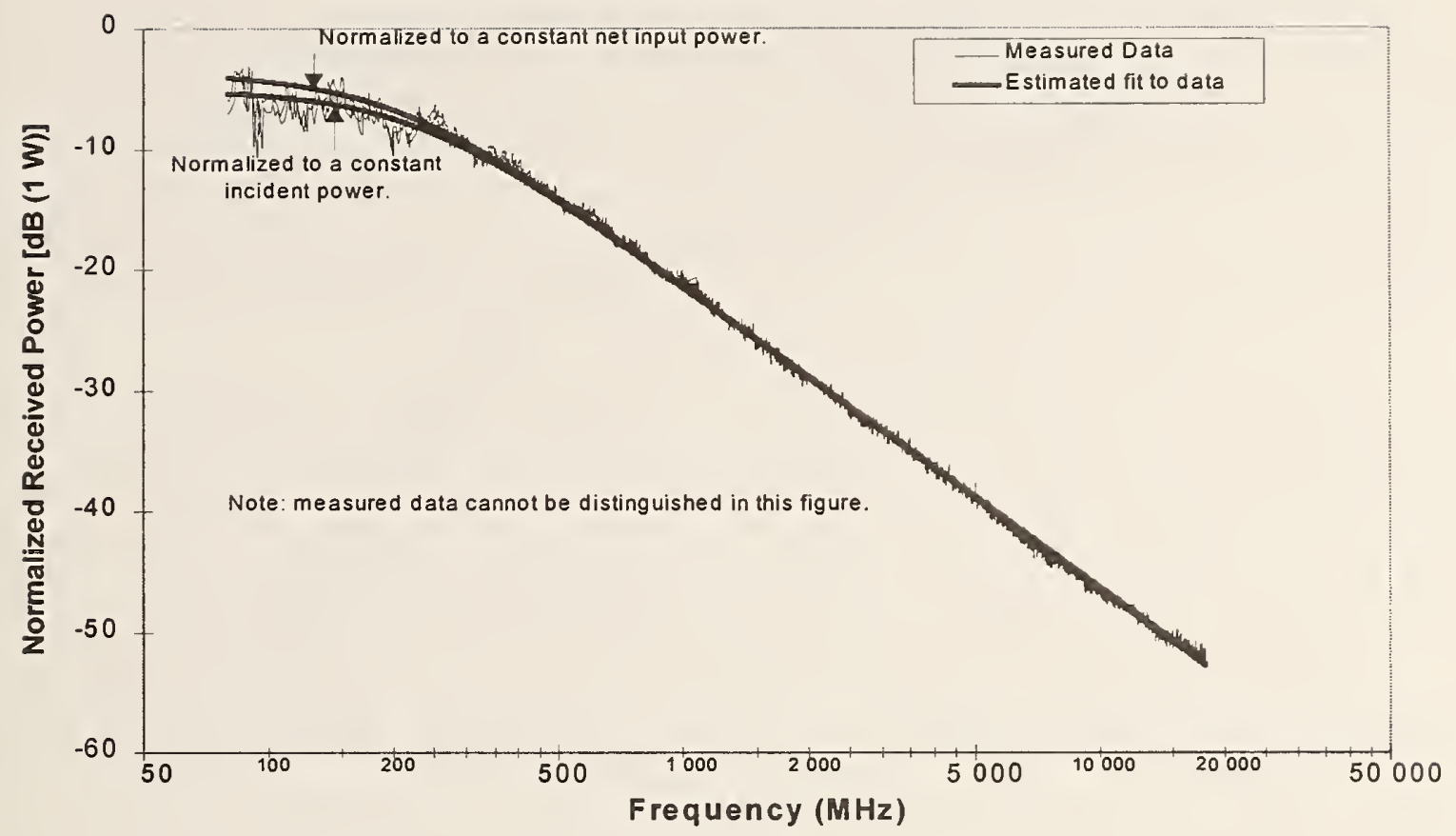

(a)

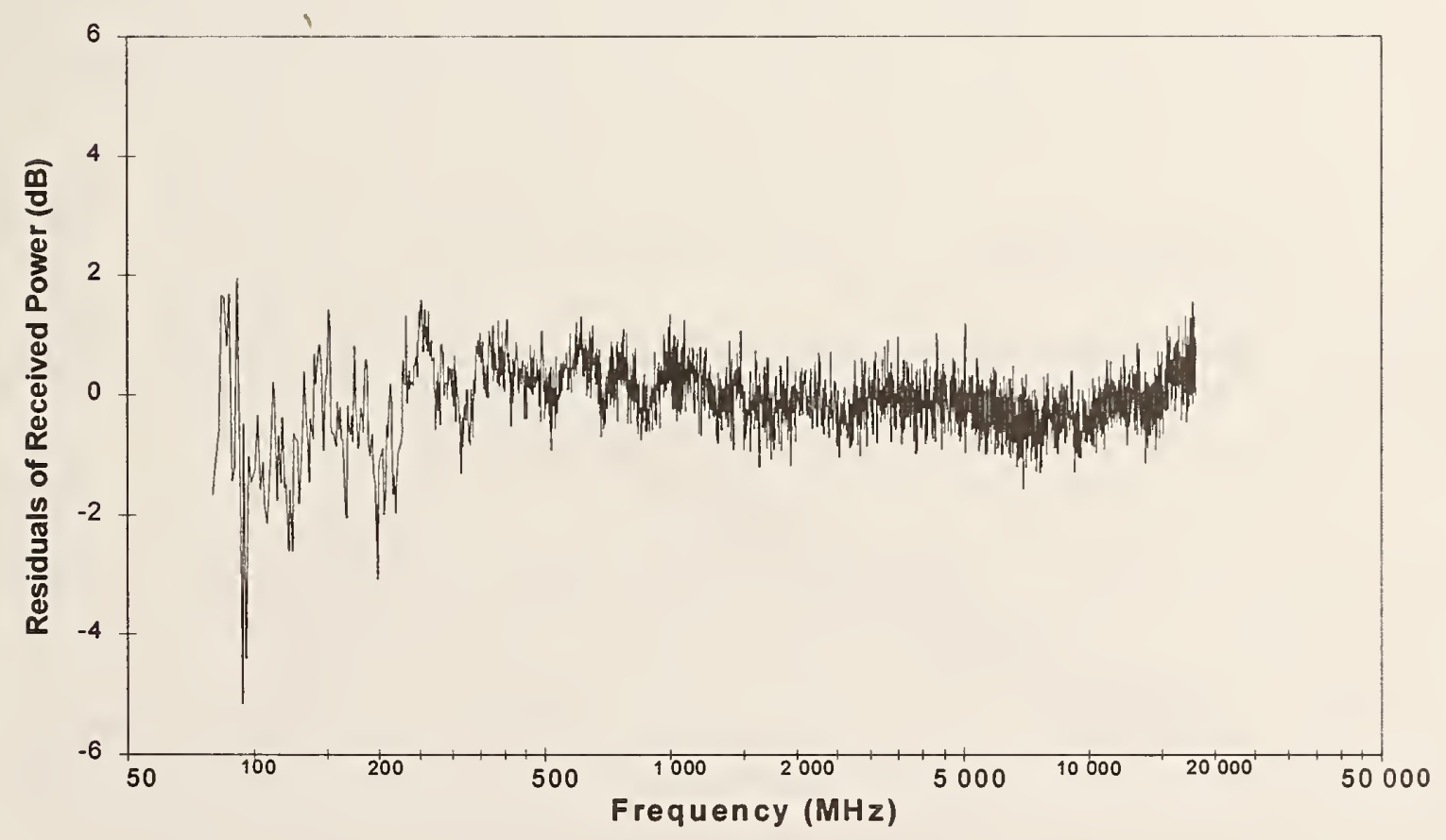

(b)

Figure 45. Average received power for a constant input power of $1 \mathrm{~W}$, corrected for antenna mismatches and efficiencies. (a) Received power. (b) Residuals of received power. 
Given the estimate of the chamber gain shown in Figure 45a, we can now estimate chamber quality factor, scalar power density, magnitude of a rectangular component of the electric field, and magnitude of the total electric field as described in eqs (32), (34), (45), and (53). We do not actually measure any of these quantities using the vector network analyzer measurement system. Instead, we estimate them from measurements of received power. The average quality factor of the chamber is shown in Figure 46. The quality factor is slightly higher when the data have been normalized to a constant net input power versus a constant incident power, as expected.

The average scalar power density (measured in decibels relative to $1 \mathrm{~mW} / \mathrm{cm}^{2}$ ) for a constant input power of $1 \mathrm{~W}$ is shown in Figure 47. Unfortunately, we have no method of actually measuring the scalar power density at each paddle position. As a result, we have no method of verifying the accuracy of the estimates of scalar power density presented here, although we have confidence that the average value of the scalar power density is reasonably close to the average value presented her. We will discuss this in more detail in Section 8.

The average electric field (measured in decibels relative to $1 \mathrm{~V} / \mathrm{m}$ ) for a constant input power of $1 \mathrm{~W}$ is shown in Figure 48. Figure 48a gives the average total electric field, and Figure $48 \mathrm{~b}$ gives the average single-axis electric field or rectangular component of the electric field. This value is simply the total electric field divided by $15 / 8$ (not $\sqrt{3}$, as discussed in Section 3.2.3) or decreased by $5.46 \mathrm{~dB}$. These values are technically not measures of the magnitude of the electric field, but rather a constant times the average of the square root of the received power, which is assumed to be proportional to the magnitude of a rectangular component of the electric field, and the estimated curve fits are based on the curve fits of the chamber gain and a correction factor based on the statistical analysis. If the assumed distributions are incorrect, then the correction factor will also be incorrect. Thus, we need to evaluate the residuals of the electric field, relative to the estimated curve fit. Since the average magnitude of the total electric field is related to the average magnitude of a rectangular component of the electric field by a multiplicative constant, the residuals will be identical. Also the residuals of the electric field normalized to a constant net input power are very similar to the residuals of the electric field normalized to a constant incident power, so we will present only the residuals for a constant incident power. These residuals are presented in Figure 49.

We will now examine how well we can approximate both the maximum and minimum chamber gain. The average chamber gain is described by eq (31), and from Section 3.2 we know that the maximum and minimum chamber gain should be proportional to the average. Therefore, we should be able to estimate the properties of the extrema as a simple constant times the estimate of the average. The maximum should be estimated as $0.577+\ln (N)$ times the estimated average. We need to adjust the estimate of the maximum a little, however. If the estimate of $a$ is smaller than $0.577+\ln (N)$ then simply multiplying the estimated average by $0.577+\ln (N)$ will result in an estimated maximum chamber gain of greater than $0 \mathrm{~dB}$, which is impossible. To rectify this we need two estimates of the maximum chamber gain, depending on the value of $a$. 


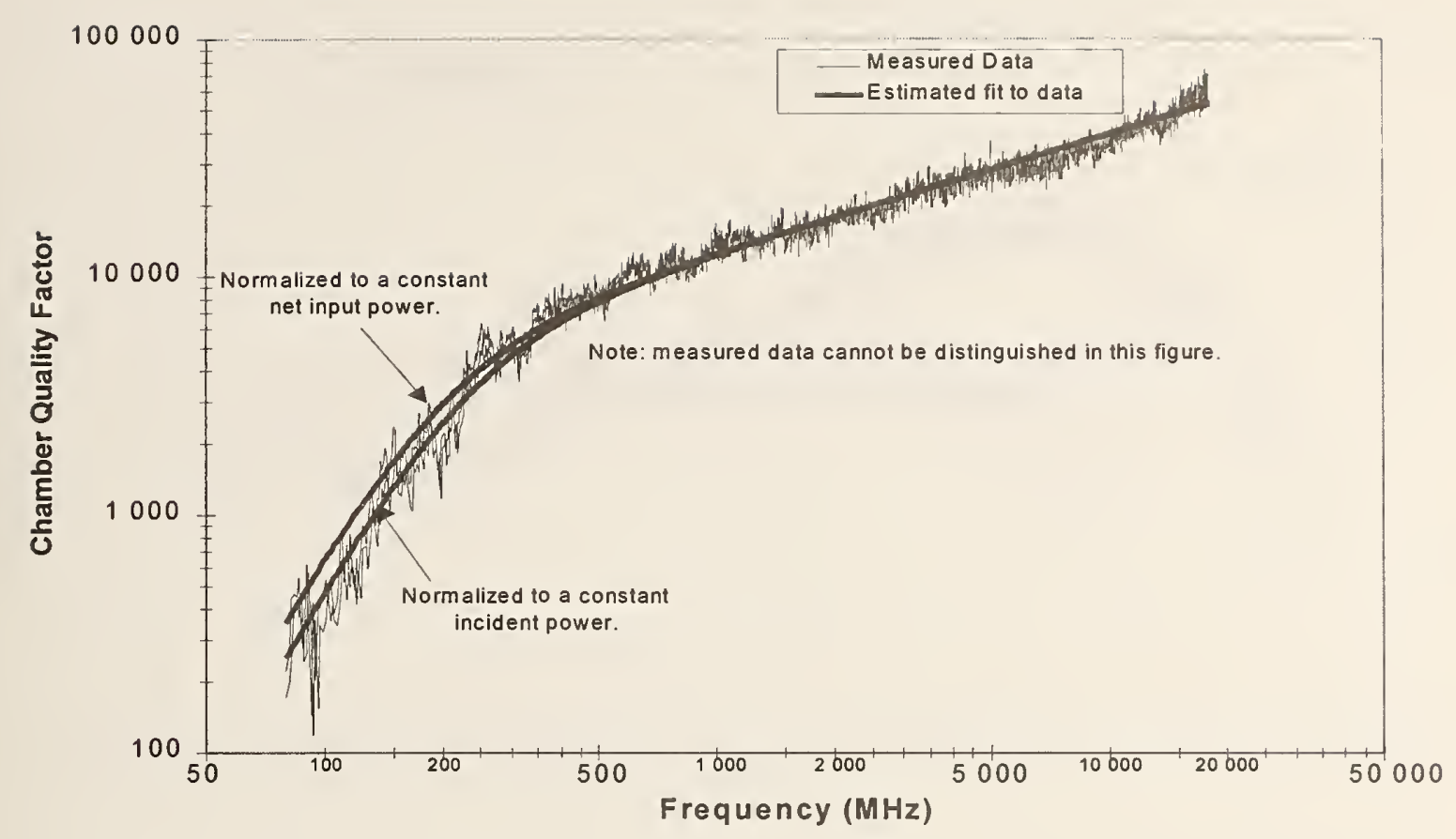

Figure 46. Average quality factor corrected for estimated antenna efficiencies and mismatches.

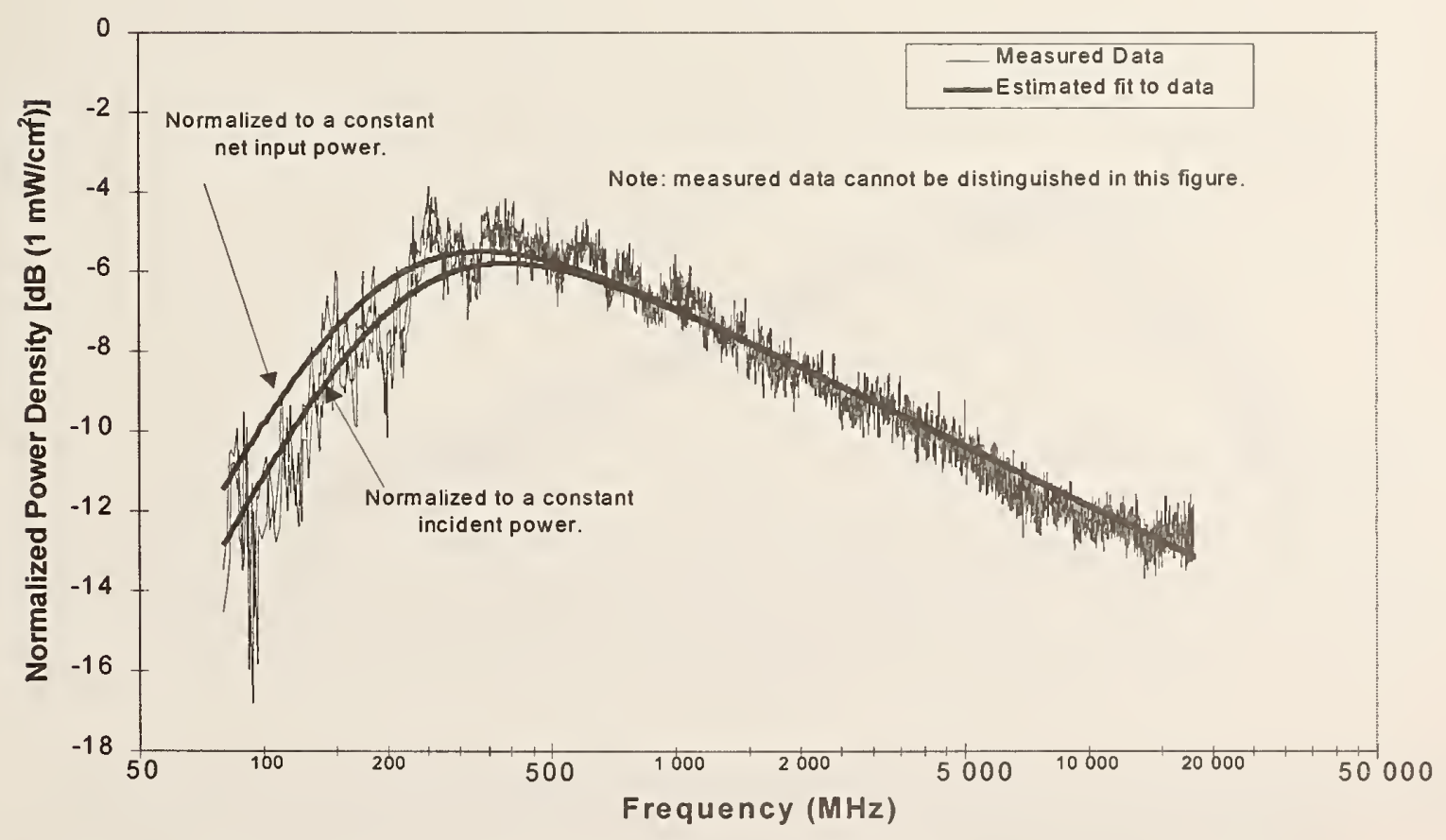

Figure 47. Average power density for a constant input power of $1 \mathrm{~W}$, corrected for estimated antenna efficiencies and mismatches. 


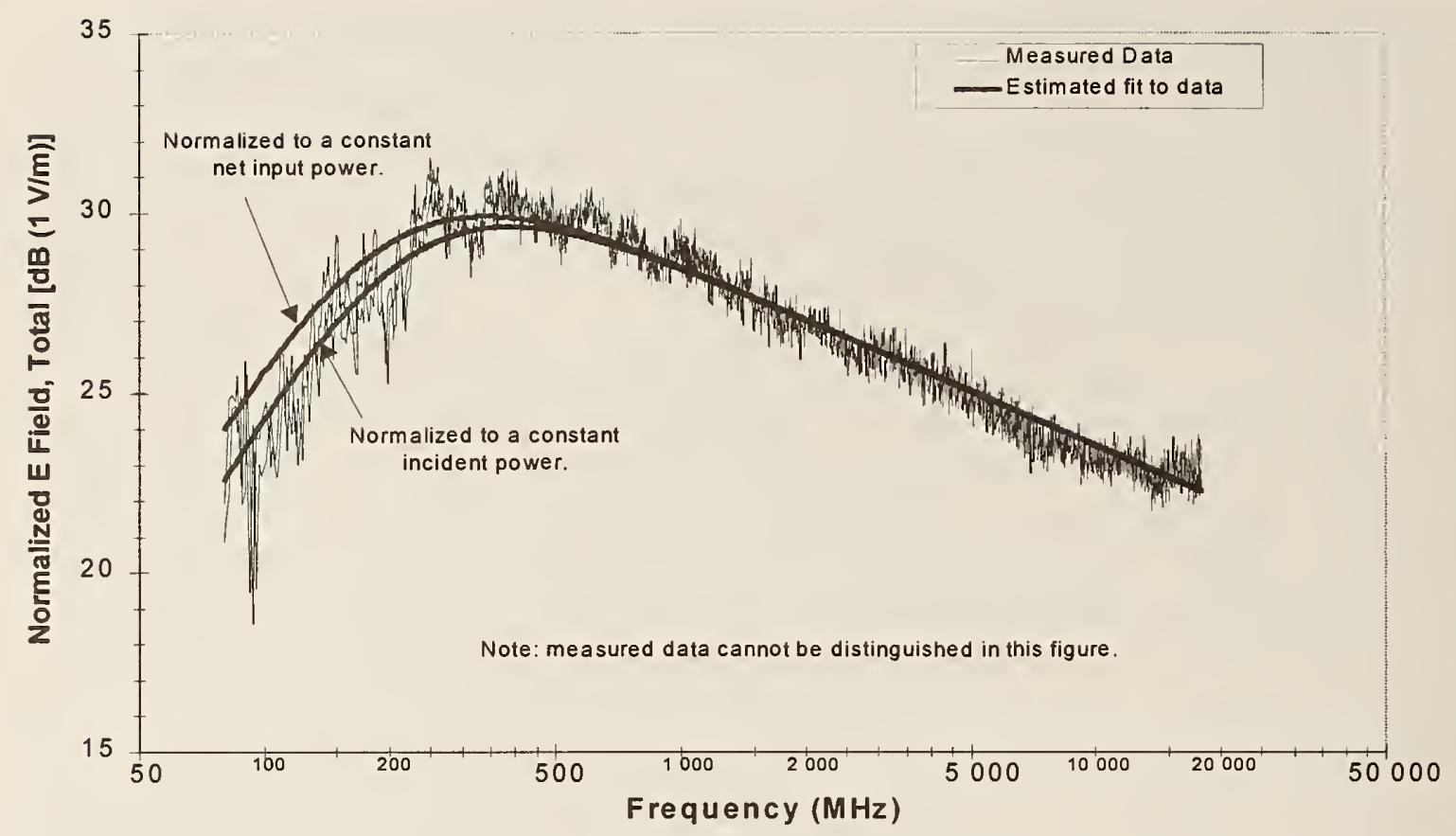

(a)

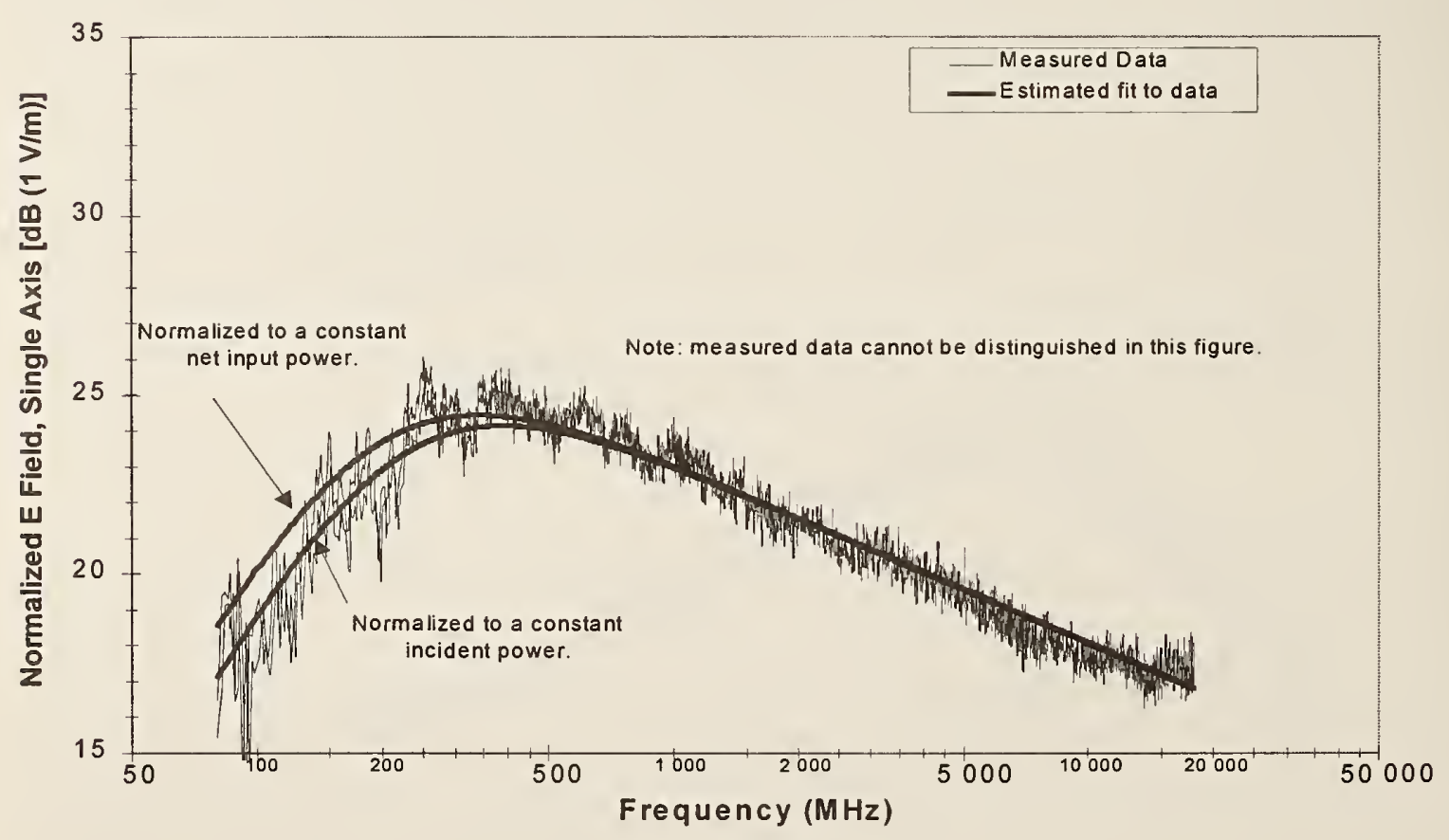

(b)

Figure 48. Calculated average electric field for a constant input power of $1 \mathrm{~W}$, corrected for estimated antenna efficiencies and mismatches. Field based on received power from reference antenna. (a) Total electric field. (b) Single rectangular component of electric field. 


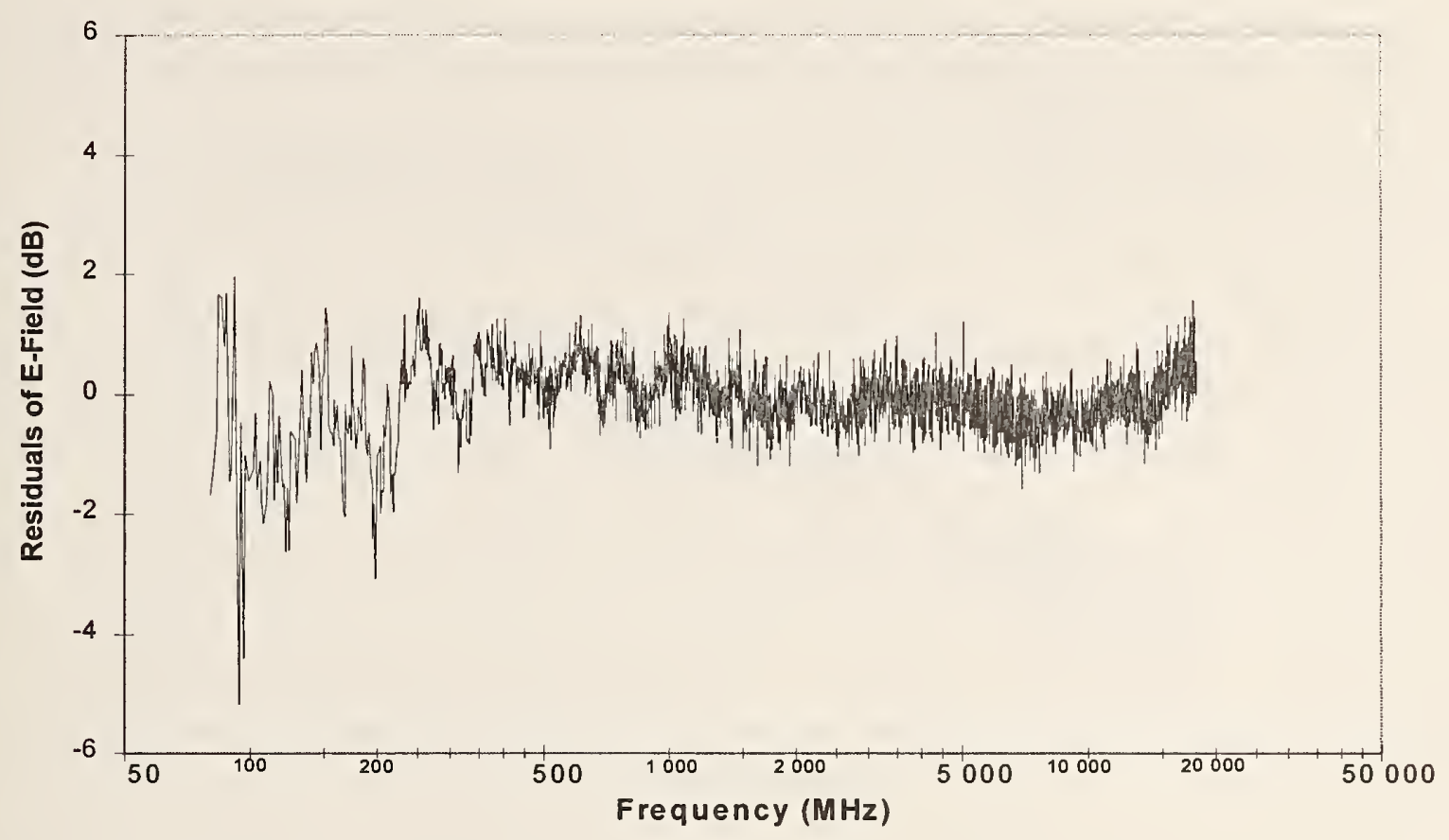

Figure 49. Residuals of calculated average electric field, based on received power from reference antenna. Residuals are identical for rectangular component of electric field and total electric field.

An estimate of the maximum chamber gain given by

$$
\begin{array}{rlrl}
\operatorname{MAX}\left(G_{C}\right) & =\frac{0.577+\ln (N)}{a+b f^{2.5}}, & & a \geq 0.577+\ln (N), \\
& =\frac{1}{1+b f^{2.5} /(0.577+\ln (N))}, & a<0.577+\ln (N)
\end{array}
$$

will force the estimated chamber gain to always be less than or equal to $1(0 \mathrm{~dB})$. The second part of eq (87) has no real theoretical basis. It is simply an estimate that is known to be approximately correct for low frequencies and for high frequencies, but may not be correct in the crossover region. To generate a more accurate estimate would require substantially more research and measurements. Given the limitations of this estimate, the agreement is good, as shown below. 
The minimum chamber gain should be approximately equal to the average chamber gain divided by $N$. This statement assumes that the chamber gain is computed in linear units. If the minimum is estimated on a decibel scale, then we need to decrease the estimate of the minimum by $2.5 \mathrm{~dB}$, as described in Section 3.2.4. The minimum, average, and maximum measured chamber gains are plotted in Figure 50. All correction factors applied to the average chamber gain have been applied to both the minimum and maximum chamber gain. The minimum is plotted simply to demonstrate that the statistical model can be used to describe both the minimum and maximum chamber gain. The minimum chamber gain is used primarily to establish the noise floor of the measurement system or as an approximate gauge of the quality of the paddle used in the chamber, as discussed previously in this section. Given the limited application of the information about the minimum chamber gain, we will not examine this parameter in any additional detail. The maximum chamber gain is of practical importance, as it indicates the maximum power that might be observed by an antenna in the vicinity of any equipment under test. As such, it is indicative of the worst-case test conditions experienced by any test equipment. It is therefore important to investigate our ability to predict the maximum chamber gain, as given in eq (87). To this end, a plot of the residuals of the maximum chamber gain is given in Figure 51. The maximum chamber gain shown in Figure 50 has some values that are greater than $0 \mathrm{~dB}$. This is caused by noise in the measurement system, and also by the fact that we cannot actually measure the power transmitted into the chamber. Instead, we can measure only the power delivered to the antenna. Because of this, we can only estimate the transmitted power, and this estimate is probably good on average, but may be in error at the extremes. For a discussion of the effects of our limited knowledge of the transmitted power, refer to Section 8 .

It is tempting to take the estimate of the maximum chamber gain and use it to estimate the maximum quality factor, scalar power density, and the various measure of the electric field. Before we take this step, we should first evaluate the meanings of these terms. First, by computing the maximum quality factor using estimates of the maximum chamber gain, we are implying that the quality factor changes for different paddle positions in the same manner as the received power. If this were the case, then a transient evaluation of the chamber, which estimates the quality factor by measuring the decay time of a transient transmitted into the chamber, would measure decay times that varied by several orders of magnitude as a function of paddle position. Recent reports have shown that this is not the case for the case of broadband transients [27]. (Narrowband excitation such as pulsed rf methods have extremely high uncertainties because of the inability to separate out decay characteristics from other low frequency effects caused by excitation of closely spaced modes.) In fact, decay times appear to be approximately independent of paddle position. This implies that the quality factor is approximately constant, and therefore only the average quality factor of the chamber has any meaning. It is possible that the quality factor of a mode-stirred chamber varies somewhat with paddle position, since this can influence the ability of an antenna to remove power from a chamber, but the fluctuations should be small. For these reasons, we will not present any estimate of the "maximum" quality factor. 


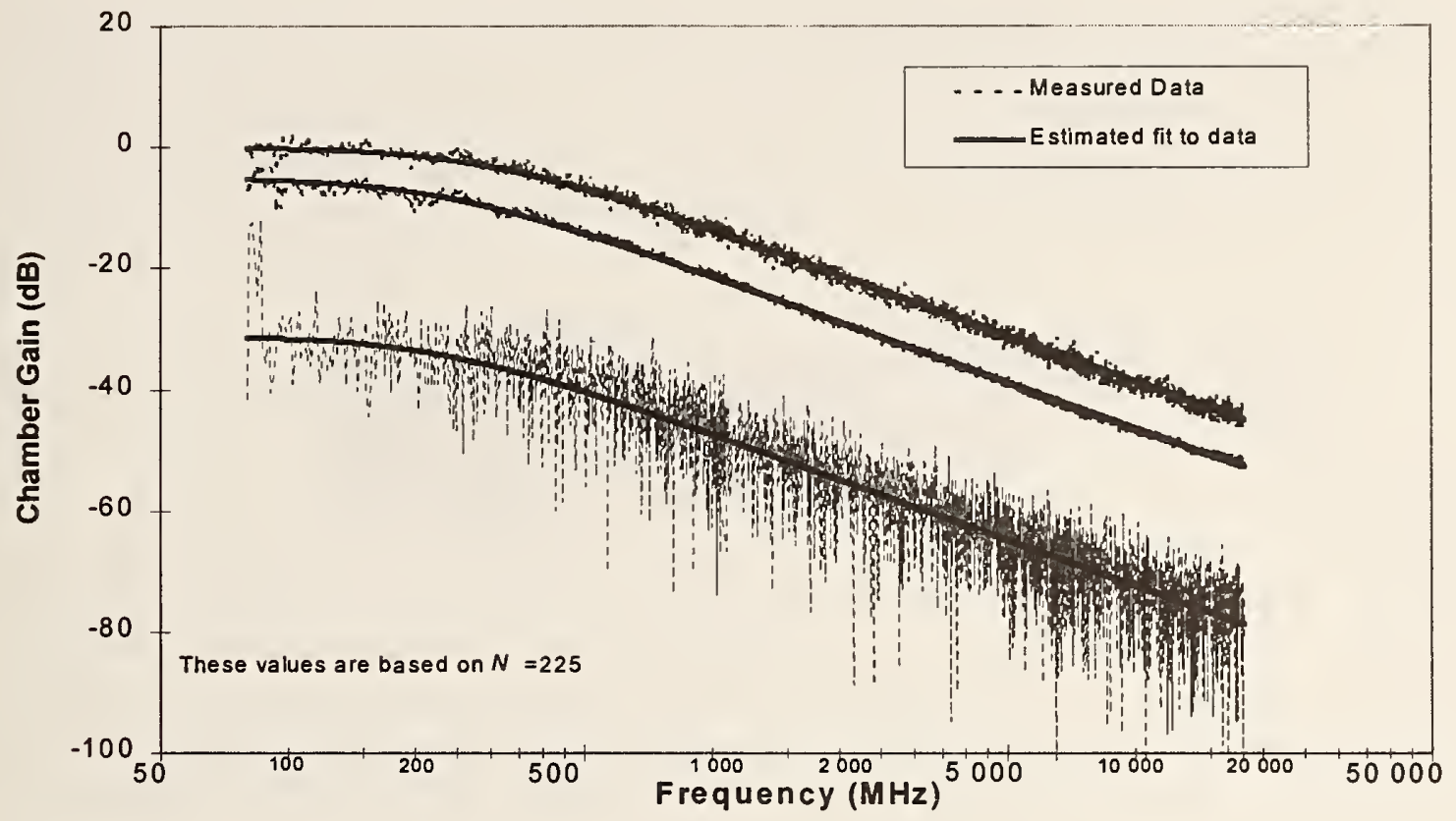

(a)

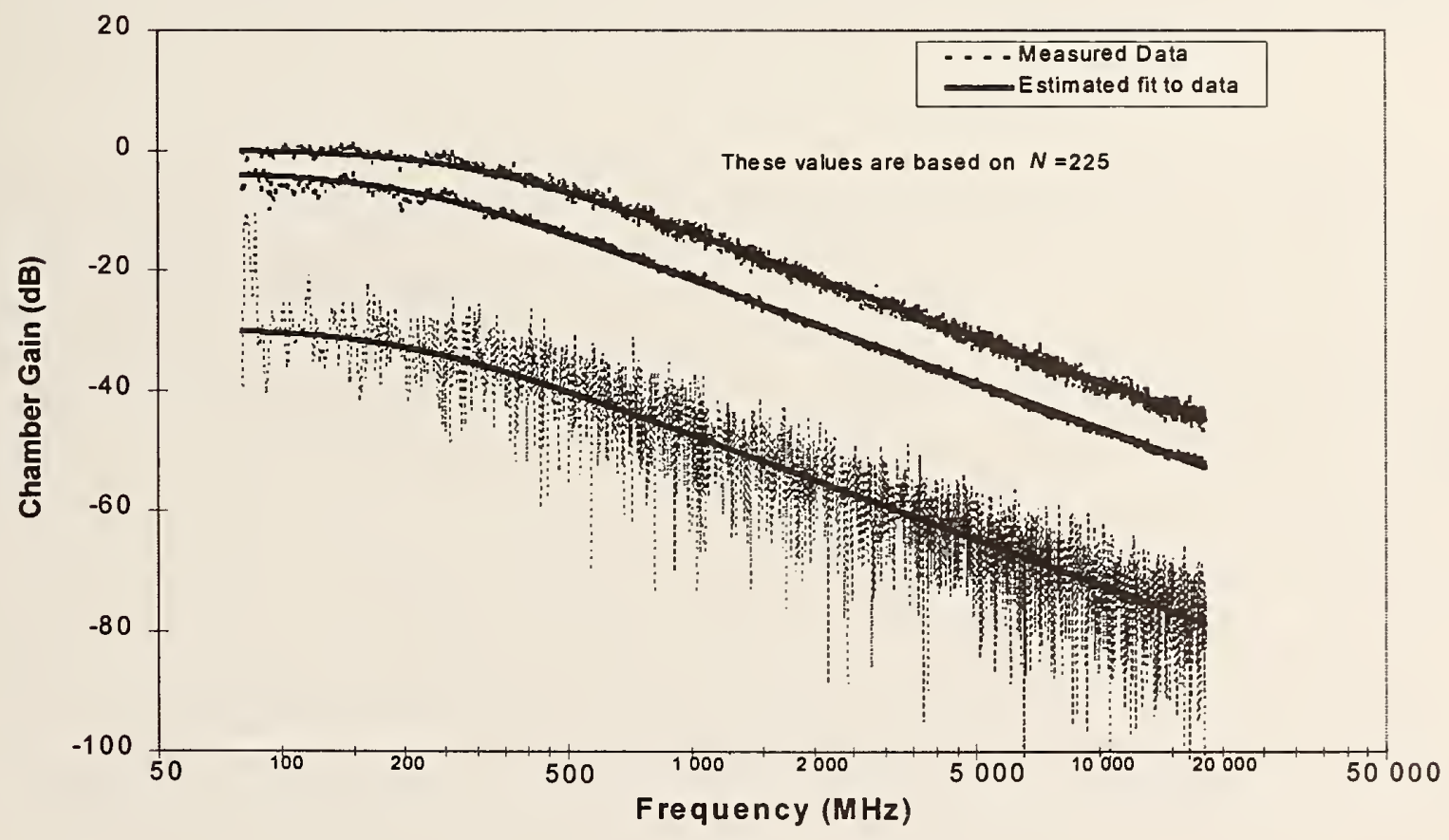

(b)

Figure 50. Minimum, average, and maximum chamber gain and associated estimated curve fits. (a) Constant incident power. (b) Constant net input power. 


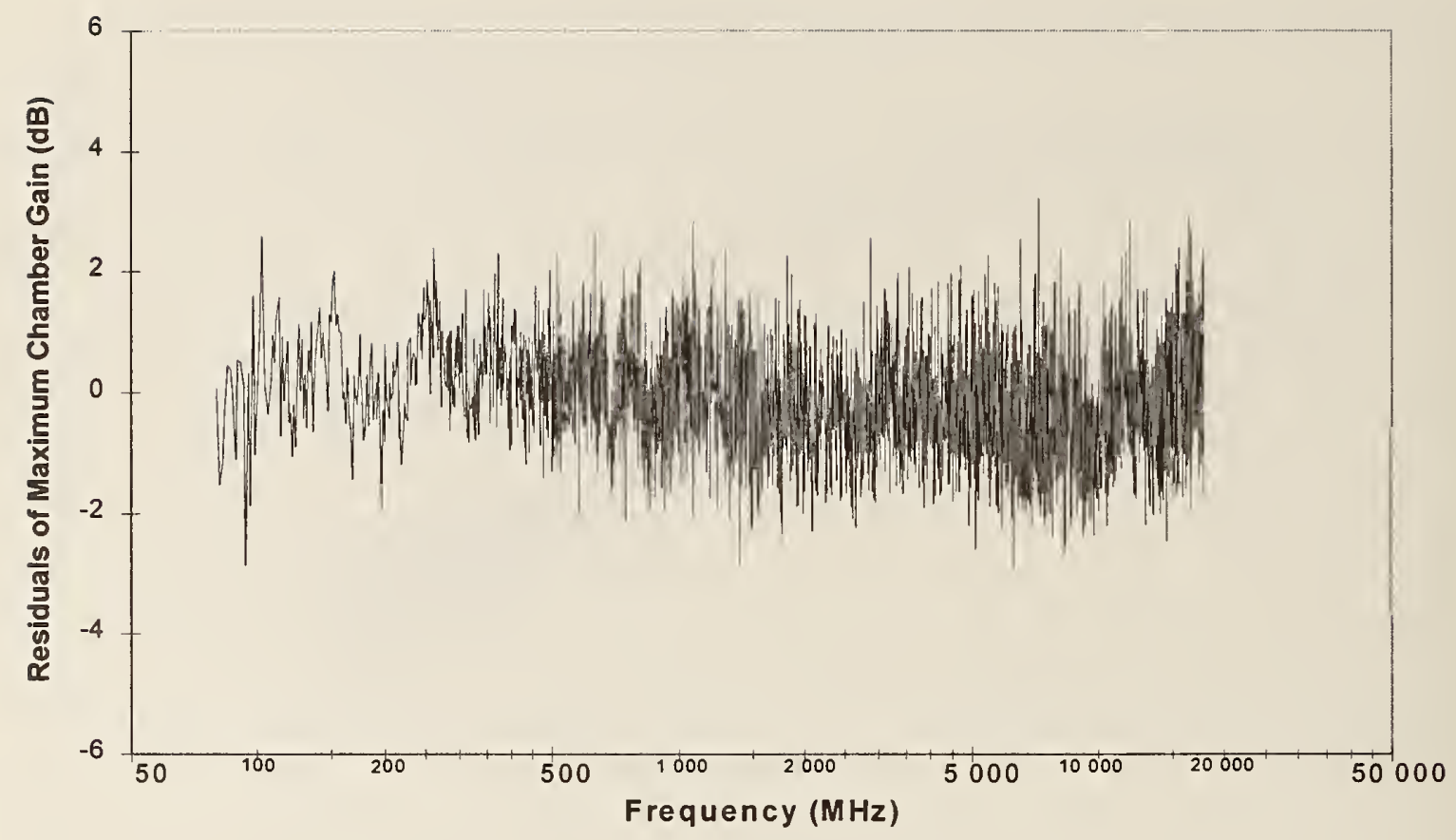

Figure 51. Residuals of maximum chamber gain.

The scalar power density causes us other problems. First, we have no method of measuring the scalar power density at each paddle position and hence no method of verifying any estimates of the maximum scalar power density. Second, we have no theory which provides us a link between any of the parameters we have measured and the maximum scalar power density. All we have is a method of estimating the average scalar power density, as given in eq (34). For these reasons, until a theoretical link between the parameters we measured and the maximum scalar power density is established, we will not attempt to estimate the maximum scalar power density.

Estimates of the maximum electric field are somewhat more manageable, but still cause some problems. As discussed previously in this section, physical limitations can cause the maximum chamber gain to be compressed relative to the average chamber gain, resulting in data that do not match the statistical model. It is possible, although unlikely, that this same compression could occur in measurements of the electric field. If so, then the square root of the power received by an antenna will continue to be proportional to the magnitude of a rectangular component of the electric field, and the maximum measured chamber gain can be translated directly into an estimate of the magnitude of a rectangular component of the electric field. There is no physical reason, however, that the electric field inside a mode-stirred chamber should be compressed in this fashion. If it is not compressed, then maximum magnitude of the electric field (total or rectangular component) cannot be estimated from the maximum measured chamber gain, but it may be possible to predict the maximum electric field based on the average chamber gain and a correction factor based on a statistical analysis of the electric field. Before we do this, however, we must first verify that the maximum electric field is not compressed relative to the 
average electric field. To do this, we need actual measurements of the electric field instead of estimates based on measurements of power received by an obtrusive antenna. This can be accomplished using the NIST probe system. Before we can use the data from the probe system, however, we need to compare the data measured with the probe system with the data measured using the VNA system, and also briefly discuss how the data from the probe system were analyzed.

\subsection{Data Measured Using the Probe System}

The data measured using the probe system were processed in the same way as the data processed using the network analyzer. In fact, the correction factors applied to the probe system data were based on the data measured by the network analyzer. This was done because the network analyzer appeared to give more accurate results, and also gave results that were not obtainable using scalar measurements. Before we blindly attempted to apply the corrections to the probe system data, we performed a set of measurements using the network analyzer with the probes and probe cables inside the chamber. The chamber gain measured both with the probes in the chamber and with the probes absent is presented in Figure 52. From these data, we conclude that the probes and cables load down the chamber and decrease the chamber gain by as much as $4 \mathrm{~dB}$ at low frequencies. (This loading will vary depending on chamber composition and contents. A chamber made with less conductive metals or with lossy objects placed inside would

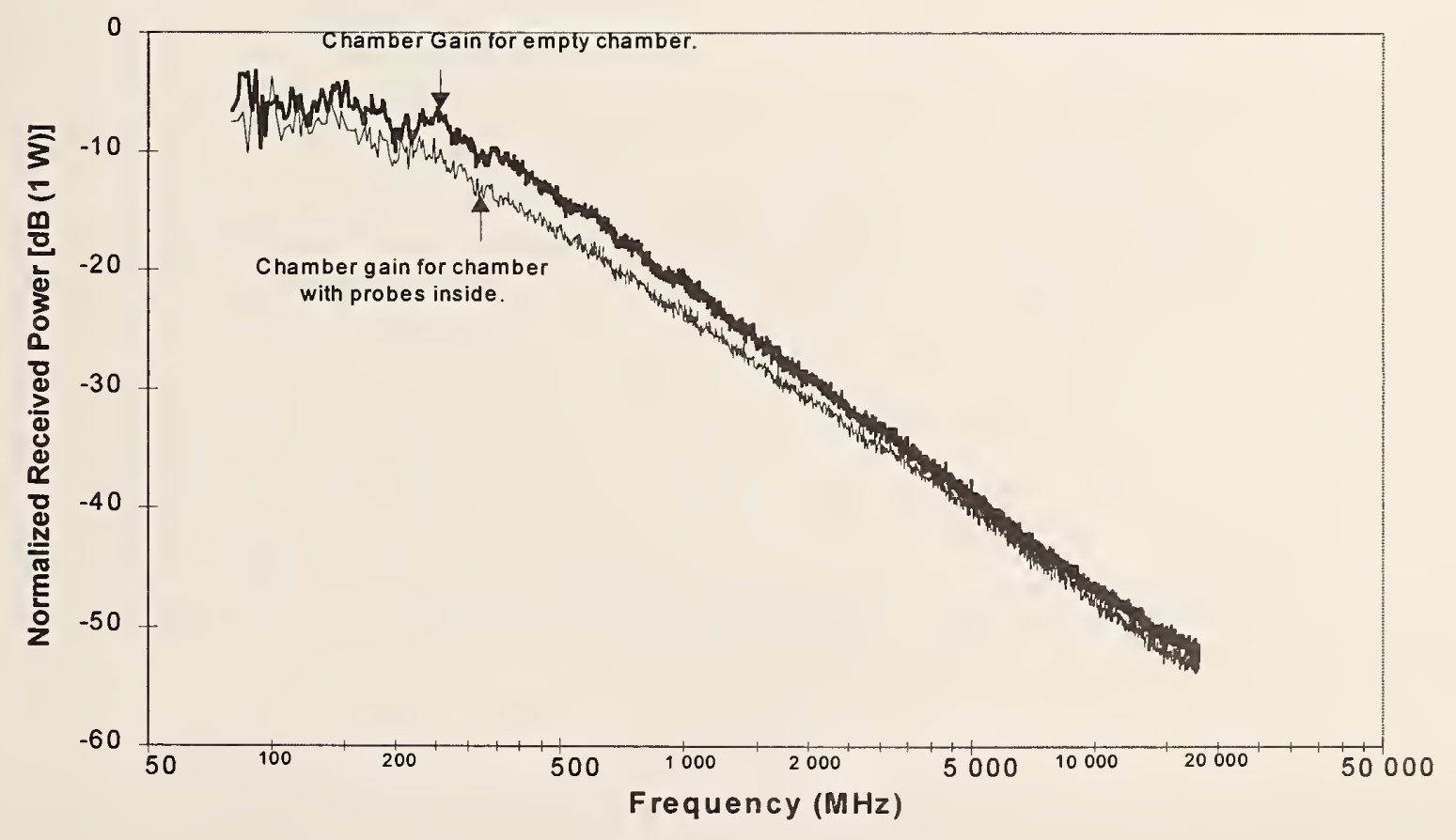

Figure 52. Comparison of chamber gain with and without the probes inside the chamber. Measurements made using a network analyzer. 
show less loading caused by the probes. Conversely, a chamber made of metals with a higher conductivity could show significantly greater loading.)

Because of the loading caused by the placement of the probes in the chamber, any corrections that we made to the probe system data were based on the measurements made with the probes in the chamber. The difference between measurements with and without the probes in the chamber using the VNA measurement system is another reason we chose to use the data measured using the network analyzer instead of the probe system to estimate the characteristics of the chamber. The additional loading caused by the probes makes it very difficult to estimate the characteristics of the chamber alone.

As a cross check, we also compared the maximum and average chamber gain using both the probe system and the network analyzer. These results are shown in Figure 53a. The agreement is good, and the difference between the two measured results is given in Figure 53b. The chamber gain measured using the VNA is generally greater than that measured with the probe system. Assuming that the network analyzer estimate is more accurate than the probe system estimate, it appears that the probe system is either overestimating the transmitted power or underestimating the received power. Even though the difference between the systems is noticeable, the deviation is typically less than $1 \mathrm{~dB}$, so it appears that similar values for the chamber parameters would be calculated regardless of the measurement system.

The 10 probes of the probe system, each consisting of 3 orthogonal dipoles, provide information on the variations in the electric field inside the chamber as a function of position and orientation. Ideally, we want to sample the electric field at all possible locations in the chamber, but this is obviously out of the question. Instead, the locations of the probes were chosen to provide samples throughout the anticipated test volumes in each chamber. The probes were distributed along three orthogonal planes parallel to the walls of each chamber. The intersection of these planes was near the center of the chamber. The probes in Chamber A were evenly spaced over $7 \mathrm{~m}$ in the longitudinal $(\mathrm{Y})$ dimension, $2 \mathrm{~m}$ in the transverse $(\mathrm{Z})$ dimension, and $1.5 \mathrm{~m}$ in the vertical $(X)$ dimension. Chamber $B$, which is a smaller chamber, was evaluated over a smaller test volume. The probes were evenly spaced over $3 \mathrm{~m}$ in the longitudinal (Y) dimension. The spacing in the transverse and vertical dimensions was the same as that used in Chamber A.

The data from the probes can be processed in two different ways, depending on whether we treat the dipoles in each probe as individual sensors, or group them into a single isotropic probe. As discussed in Section 4.1, each individual probe consists of three orthogonal elements. If we treat each dipole as an independent sensor, then each probe will give us three independent estimates of the characteristics of a rectangular component of the electric field. If, on the other hand, we treat a probe as an isotropic sensor, with the resultant estimate of the total electric field given by the square root of the sum of the squared rectangular components (RSS), then each probe gives us a single estimate of the characteristics of the total electric field. Both of these interpretations are useful, so both are presented here. 


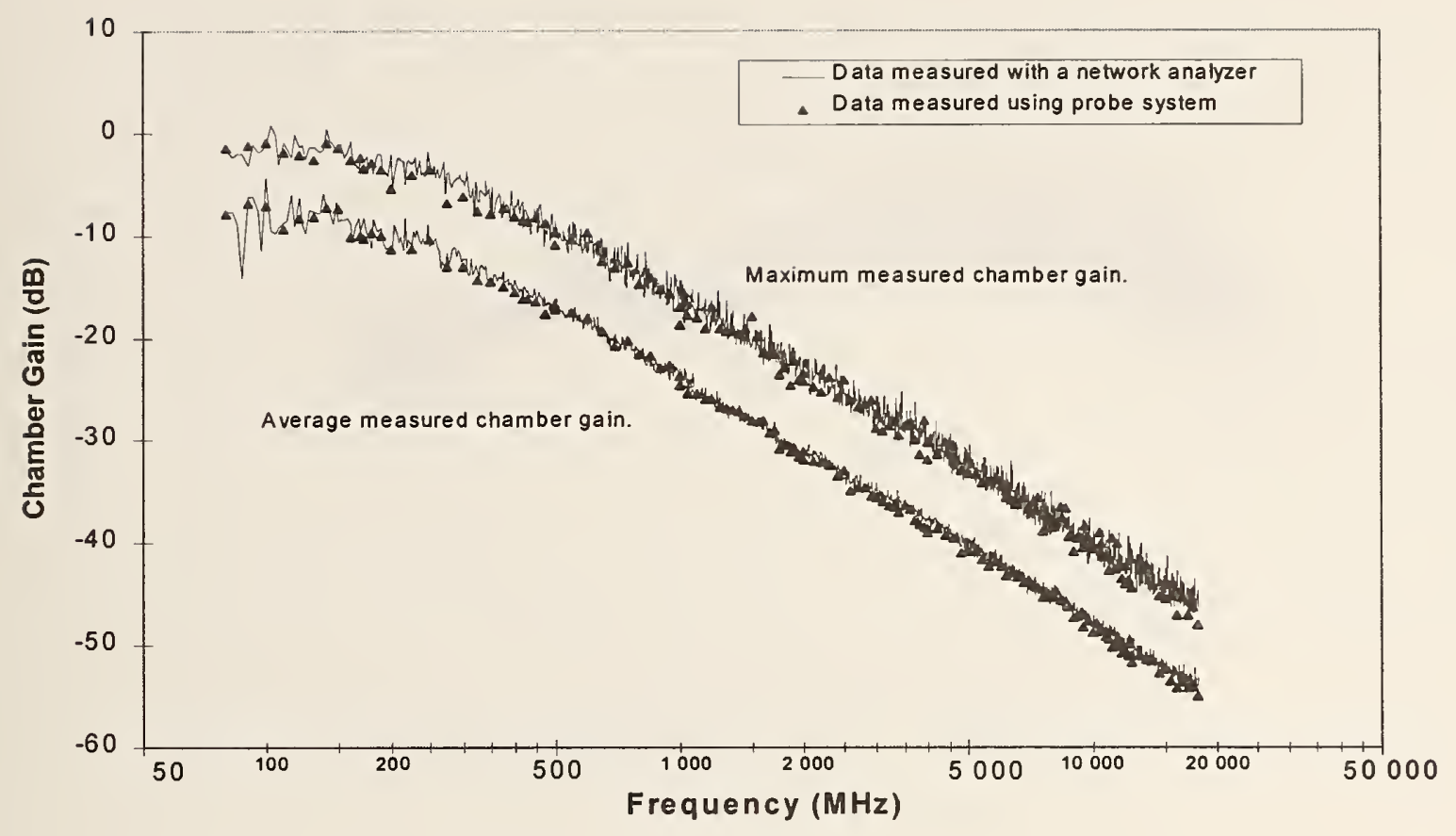

(a)

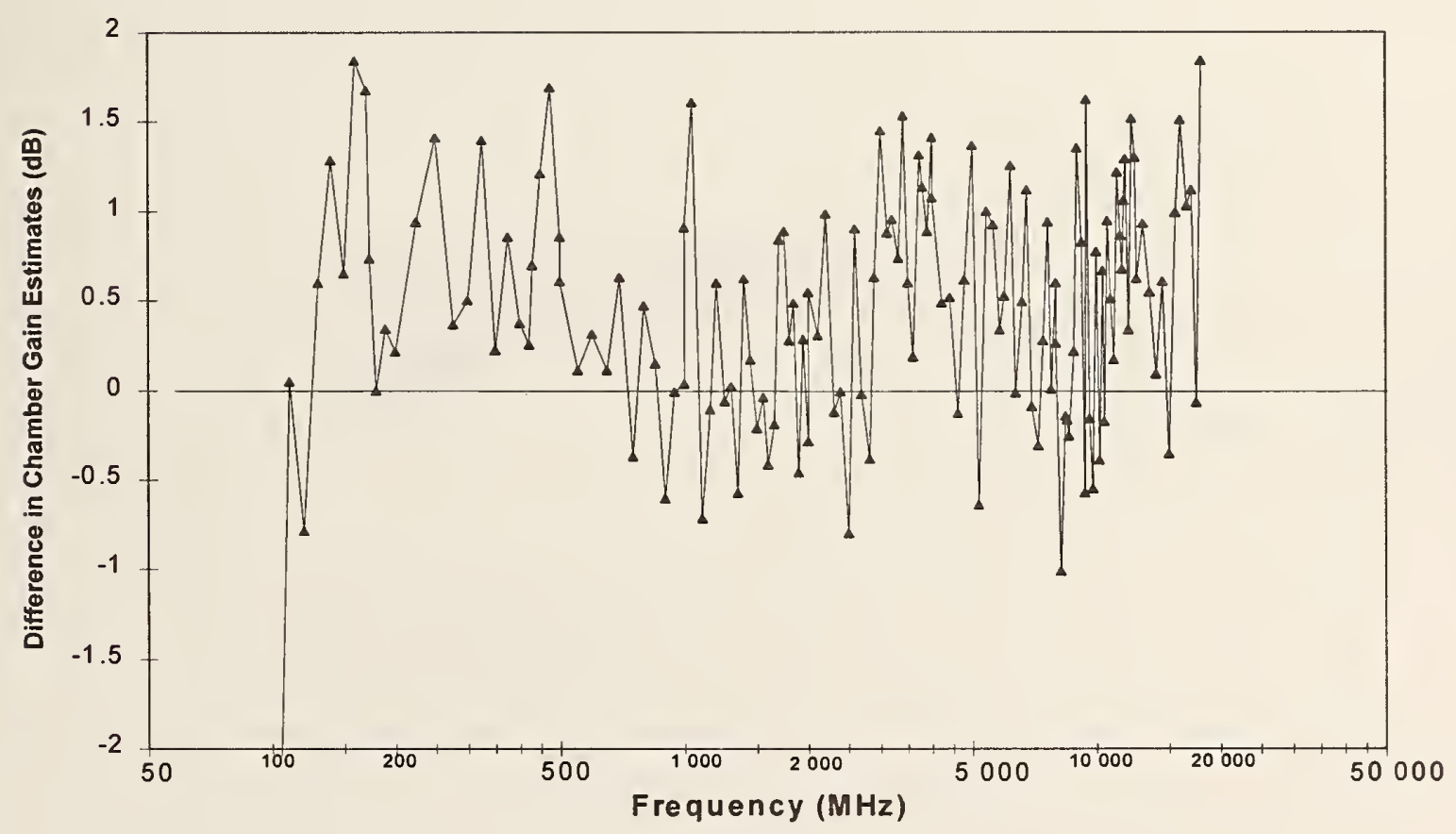

(b)

Figure 53. Comparison of measurement systems. (a) Average chamber gain with probes inside chamber using network analyzer and probe system. (b) Difference between the two estimates of average chamber gain: network analyzer - probe system. 


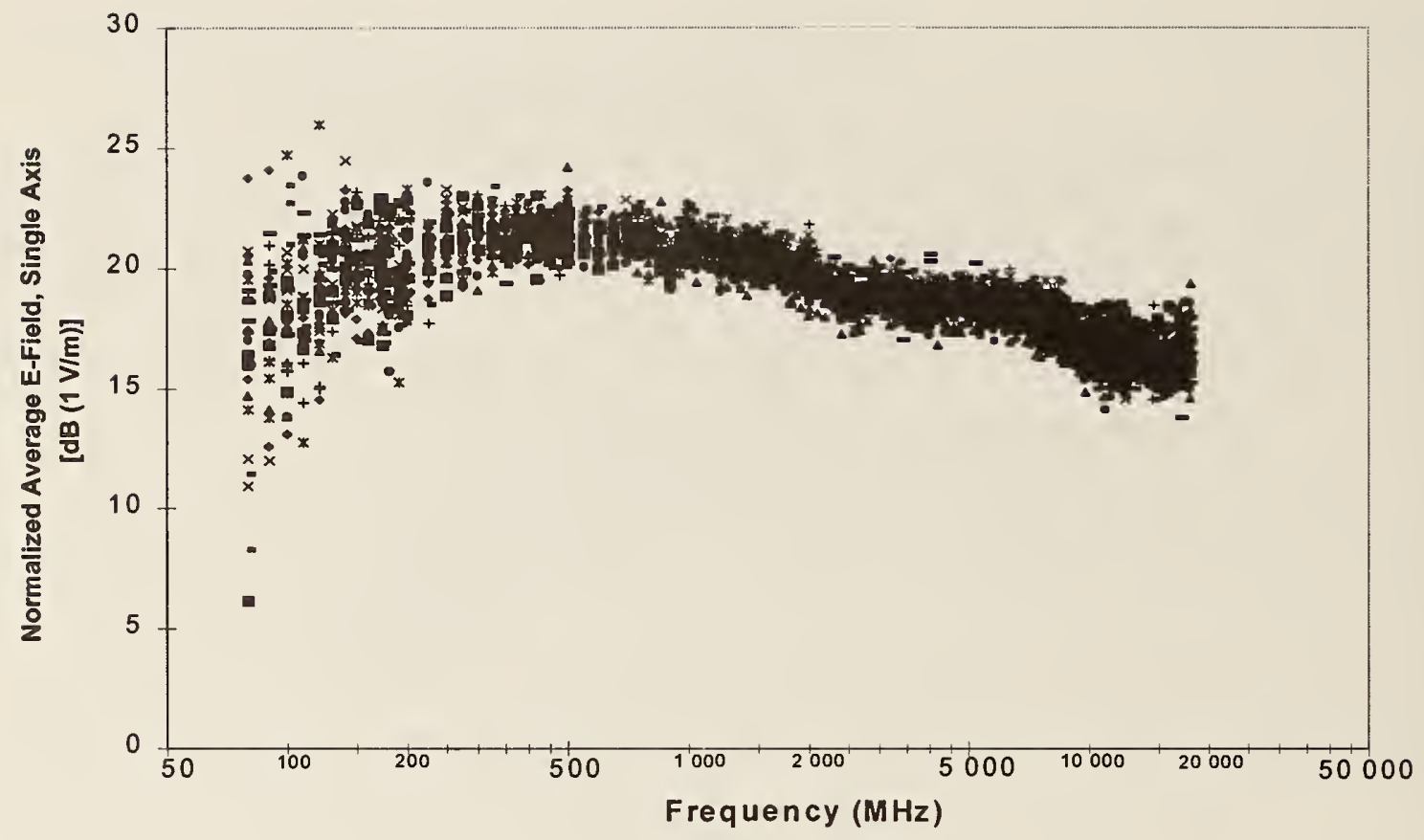

(a)

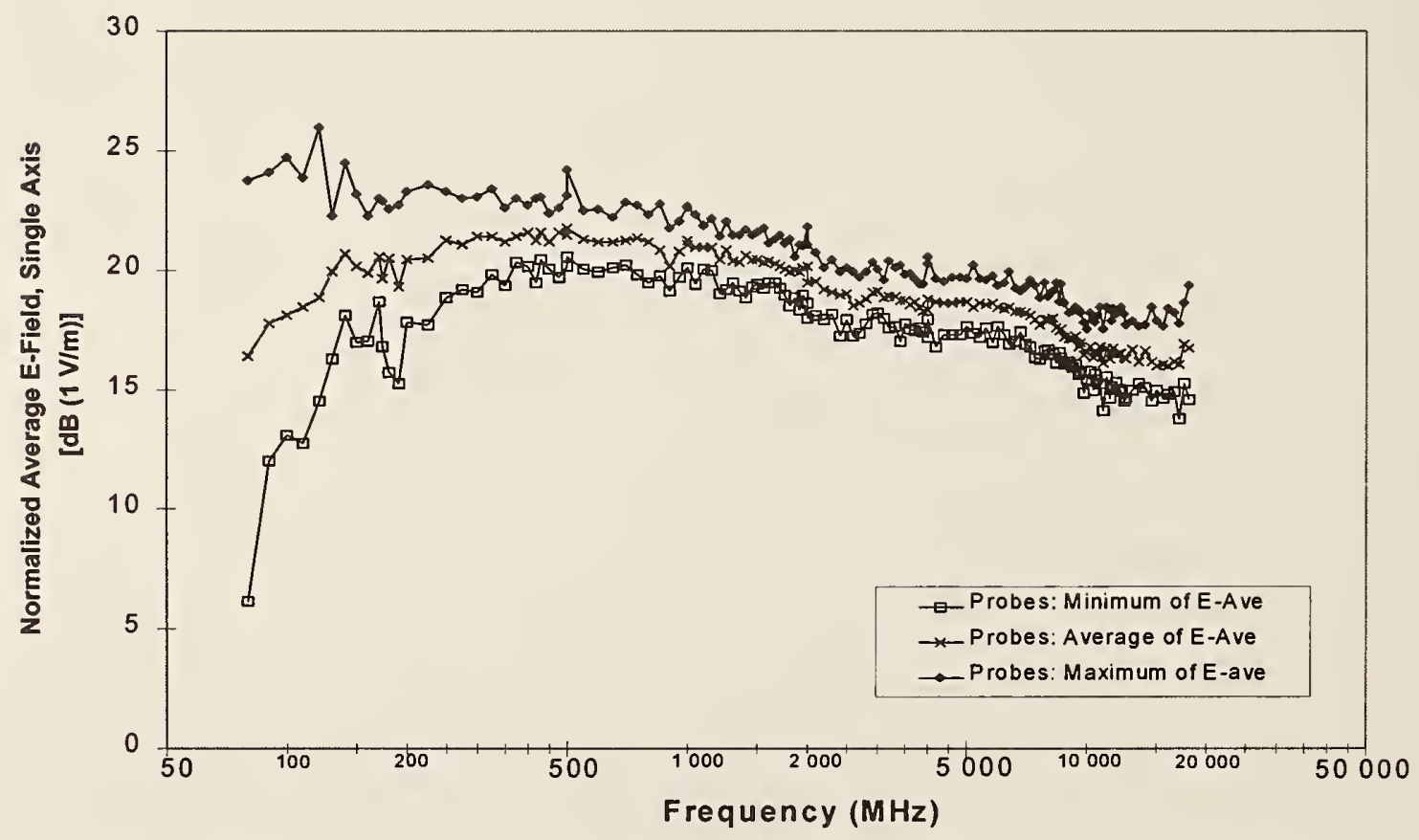

(b)

Figure 54. The average measured electric field (rectangular component) for each of 30 short dipoles in chamber A. Values given are for a constant net input power of $1 \mathrm{~W}$.

(a) Individual measurements. (b) Envelope and average of individual measurements.

(c) Standard deviation of measurements of $E_{R}-A v e$. (d) Comparison of average of individual measurements with reference antenna. 


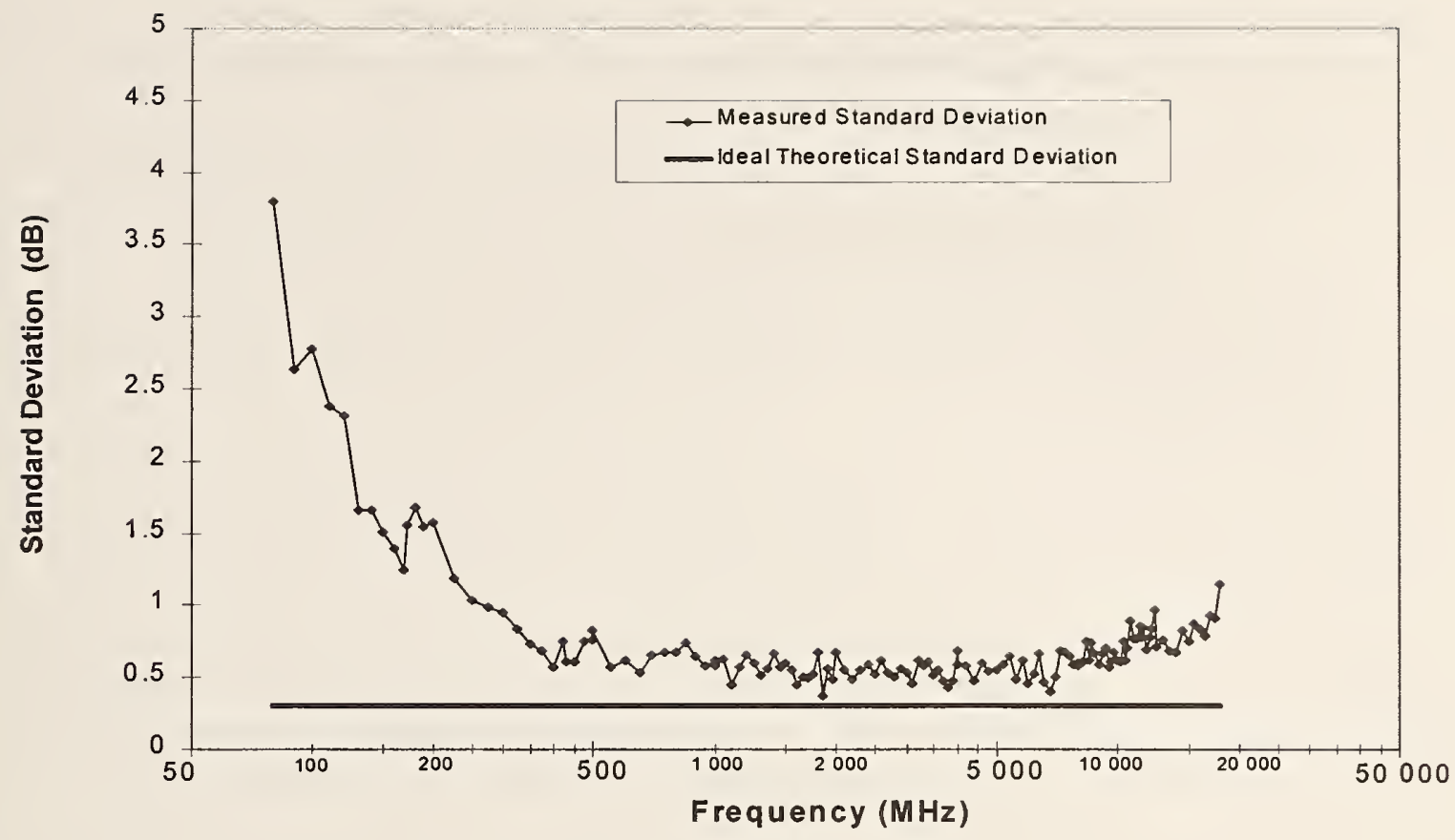

(c)

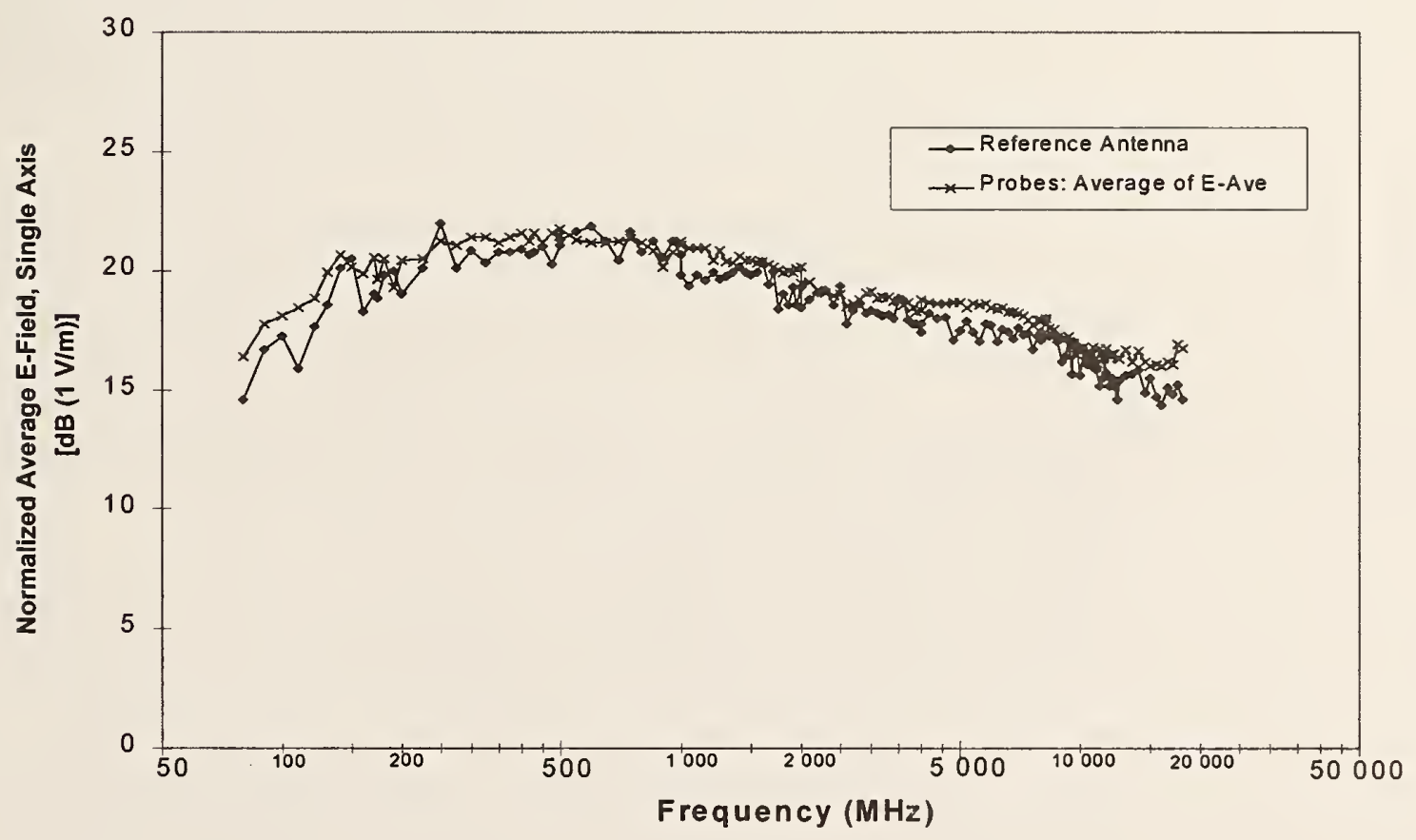

(d) 
First, however, we must present a few words about notation. If we perform $M$ measurements of the electric field (either with $M$ probes, or a single probe measured at $M$ different locations), we will have $M$ different estimates of the average electric field, and $M$ different estimates of the maximum electric field. We can refer to the $M$ measured average electric field values or the $M$ measured maximum electric fields unambiguously. Unfortunately, if we compute the average of any of the $M$ measured quantities we run into problems. Terms such as "the average of the average electric field values" or "the average of the maximum electric field values" is confusing at best, and to make matters worse, we also have the average of the minimum, the maximum of the average, the maximum of the minimum, the maximum of the maximum, the minimum of the average, the minimum of the minimum, and the minimum of the maximum. Worse still, we have two different measures of the electric field: a rectangular component of the electric field and the total electric field. AAARRRRGGGGHHHHH!!!!!!!

There is little we can do to simplify this notation, but we can (hopefully) make it less confusing by using consistent notation. To help matters somewhat, we will ignore the minimum electric field measured by the probe system. Thus, for any given measurement we will have $M$ estimates of the average electric field (which we will call E-Ave in figures), and $M$ estimates of the maximum electric field (which we will call E-Max in figures). The minimum of the $M$ estimates of the average electric field will be called the minimum of E-Ave or MIN(E-Ave). Similarly, we will refer to the average of E-Ave or AVE(E-Ave), the maximum of E-Ave or MAX(E-Ave), the minimum of E-Max or MIN(E-Max), the average of E-Max or AVE(E-Max), and the maximum of MAX(E-Max). (We never said that we would make this easy to understand. We only said that we would (hopefully) make it less confusing.) As for the type of electric field we are referring to (rectangular component or total), we will use subscripts, with $E_{R}-M a x$ referring to the maximum measured rectangular component of the electric field, and $\mathrm{E}_{\mathrm{T}}-\mathrm{Max}$ referring to the maximum measured total electric field. If there appears to be a dependence on orientation of the electric field, we will specifically refer to $E_{X}, E_{Y}$, and $E_{Z}$. In all cases, when we refer to any measure of the electric field, we actually mean the magnitude of the electric field.

Another way to make the data from the probe system somewhat less confusing is to always present the data in a consistent format. We will present some examples of this format here, beginning with the characteristics of the average magnitude of a rectangular component of the electric field. These data will be repeated in Section 7.1, which summarizes the characteristics of Chamber A. We first present the $M$ individual measurements, as shown in Figure 54a. Thus, at each frequency, we will plot $M$ points. We do not intend the values from a specific probe to be selected from such a plot, as there should be nothing special about one probe relative to another. Instead, we provide such a plot simply to give information on the spread of the measured values. We refer to the envelope of the measured values as the range of values between the measured maximum of the $M$ points $\left(\mathrm{MAX}\left(\mathrm{E}_{\mathrm{R}}-\mathrm{Ave}\right)\right)$ and the measured minimum of the $M$ points ( $\mathrm{MIN}\left(\mathrm{E}_{\mathrm{R}}-\mathrm{Ave}\right)$ ). We present the envelope of the $M$ points, as well as the average of the $M$ points ( $\mathrm{AVE}\left(\mathrm{E}_{\mathrm{R}}-\mathrm{Ave}\right)$ ) together in a single plot, as shown in Figure 54b. Since the data are plotted on a decibel scale, we computed the average of the individual measures of the electric field after the data were converted to decibels. While this may be statistically unorthodox, it is sound given the way that these data are typically presented, and the fact that uncertainty and uniformity are generally given in terms of decibels. In Figure 54c, we present the standard deviation of the individual measurements (also computed based on the decibel values) as well as 
an estimate of what the standard deviation would have been had the chamber and all calibrations been ideal. The ideal standard deviation is based on calculations given in Section 3.2.7 and values given in Table 4. Finally, we present a comparison between average of the $M$ measurements and a prediction of what that average should be based on measurements made using the reference antenna, as shown in Figure 54d.

A few characteristics of these data deserve special attention. Over the majority of the frequency range evaluated during these test ( $80 \mathrm{MHz}$ to $18 \mathrm{GHz})$, the worst case difference between the estimates of the electric field is between 2 and $3 \mathrm{~dB}$, or alternatively, the measured values differed from the measured average by less than $\pm 1.5 \mathrm{~dB}$ between $400 \mathrm{MHz}$ and $15 \mathrm{GHz}$ (with some occasional outliers). This is significantly better than anything we had previously measured. Below $400 \mathrm{MHz}$, the spread in the measurements of the electric field (rectangular component) increases as expected. Above $15 \mathrm{GHz}$, the spread also increases. This was not expected and could be due to measurements near the noise floor or increased uncertainty in the calibration of the system. The observed standard deviation (over frequencies where the chamber appears to be operating well) was somewhat greater than would be expected under ideal conditions $(0.6 \mathrm{~dB}$ vs. $0.3 \mathrm{~dB})$. Although we would like to have them agree exactly, this goal is optimistic. The differences tell us that the combination of the chamber and measurement system is not perfect, which in turn implies either that there are small errors in the calibration of the probes (a strong possibility) or that there are small, localized, non-random biases in the chamber fields (another strong possibility). Our measurements do not tell us, however, which of these

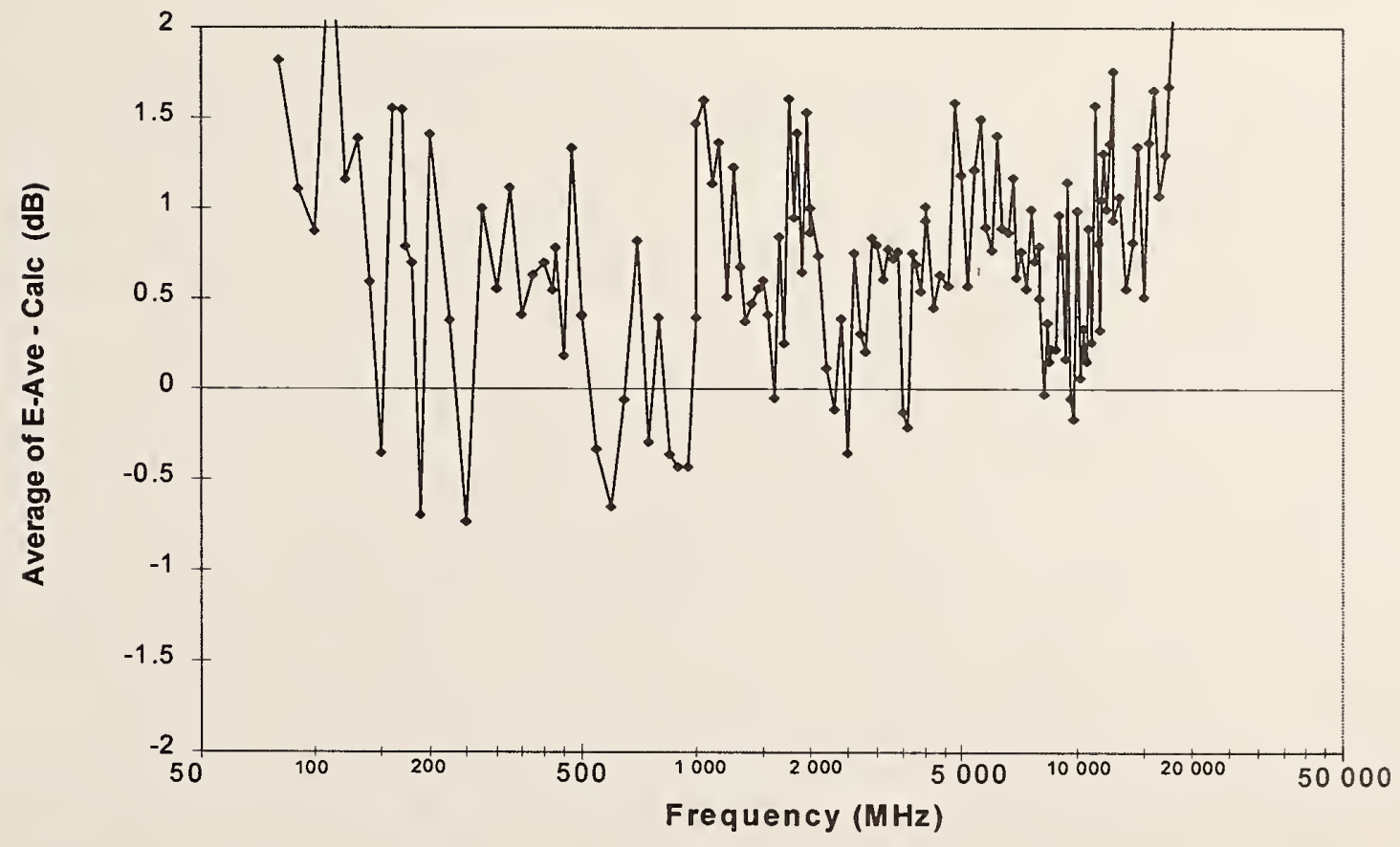

Figure 55. Difference in estimates of average electric field measured in chamber A based on probe readings and power received by the reference antenna. 
possibilities most likely caused the difference. In fact, we have no way to determine the source of the difference given any of the measurements we performed. To determine the source would require a large number of measurements with a single probe. Since this would keep the measurement system constant, any remaining variations would most likely be due to imperfections in chamber. Regardless of the source of the difference, the fact that unknown contributors increase the standard deviation of the measured field by only a small fraction of a decibel is encouraging and is significantly better than has been achieved before.

The comparison between the two methods of estimating the average electric field shown in Figure 54d is also good, although the field measured by the probes is generally greater than that predicted by the average power received by the reference antenna. The difference between these two estimates, plotted in Figure 55, shows that this bias is typically less than $1.5 \mathrm{~dB}$, and is rarely less than $0 \mathrm{~dB}$. Comparing Figure 55 with Figure $53 \mathrm{~b}$ shows that the same type of bias occurs in both measurements and, in fact, the data plotted in these two figures are remarkably similar. This implies that the bias is most likely due to errors in the RF receiving side of the probe system rather than the transmitting side, since an error in the transmitting side would also affect the fields measured by the probes. More research needs to be done to identify and remove this bias.

The fact that the standard deviation of multiple measurements of the electric field is approximately $0.6 \mathrm{~dB}$ has several important implications. If we can remove the apparent biases from the measurement system, uncertainties on the order of $1 \mathrm{~dB}$ are approaching the uncertainties of anechoic chamber measurements. In fact, some of the overall fluctuations and uncertainty may be due to uncertainties in the calibration of the probes in our anechoic chamber (which we estimate to be $\pm 1.5 \mathrm{~dB}$ ). If we can observe errors in the anechoic chamber calibration of the probes using measurements in a mode-stirred chamber, it may be possible to "calibrate" electromagnetic probes inside a mode-stirred chamber. Not only would this take less time than calibrating a probe in an anechoic chamber; it will also allow a large number of probes to be calibrated simultaneously. With additional research, it may be possible to calibrate probes using this technique with uncertainties that are similar to, or perhaps even better than, those associated with anechoic chamber techniques.

Below $500 \mathrm{MHz}$, the fluctuations in the measurements of the electric field measured by the probes and plotted in Figure 54a appear to be greater than those of the estimate of the electric field based on the power received by the reference antenna. Although this is not strictly a valid comparison since we have 30 measurements from the probes and only one from the reference antenna, still, we would expect larger deviations in the received power as a function of frequency than appears to be the case. Our best explanation for this is that, although the local electric field as measured by the probes can vary substantially from point to point at low frequencies, the reference antenna is electrically large and essentially performs some spatial averaging. Thus, the power received by the antenna is "better behaved" than the fields measured by the probes and actually behaves more like the average of a number of probes. The advantage of the spatial averaging is that the received power will have a lower uncertainty associated with it than measurements of the electric field. The disadvantage is that the electric field is varying more than the received power indicates, and this must be remembered when the overall uncertainties of the measurement are estimated. Fortunately, most devices that are tested in mode-stirred chambers 


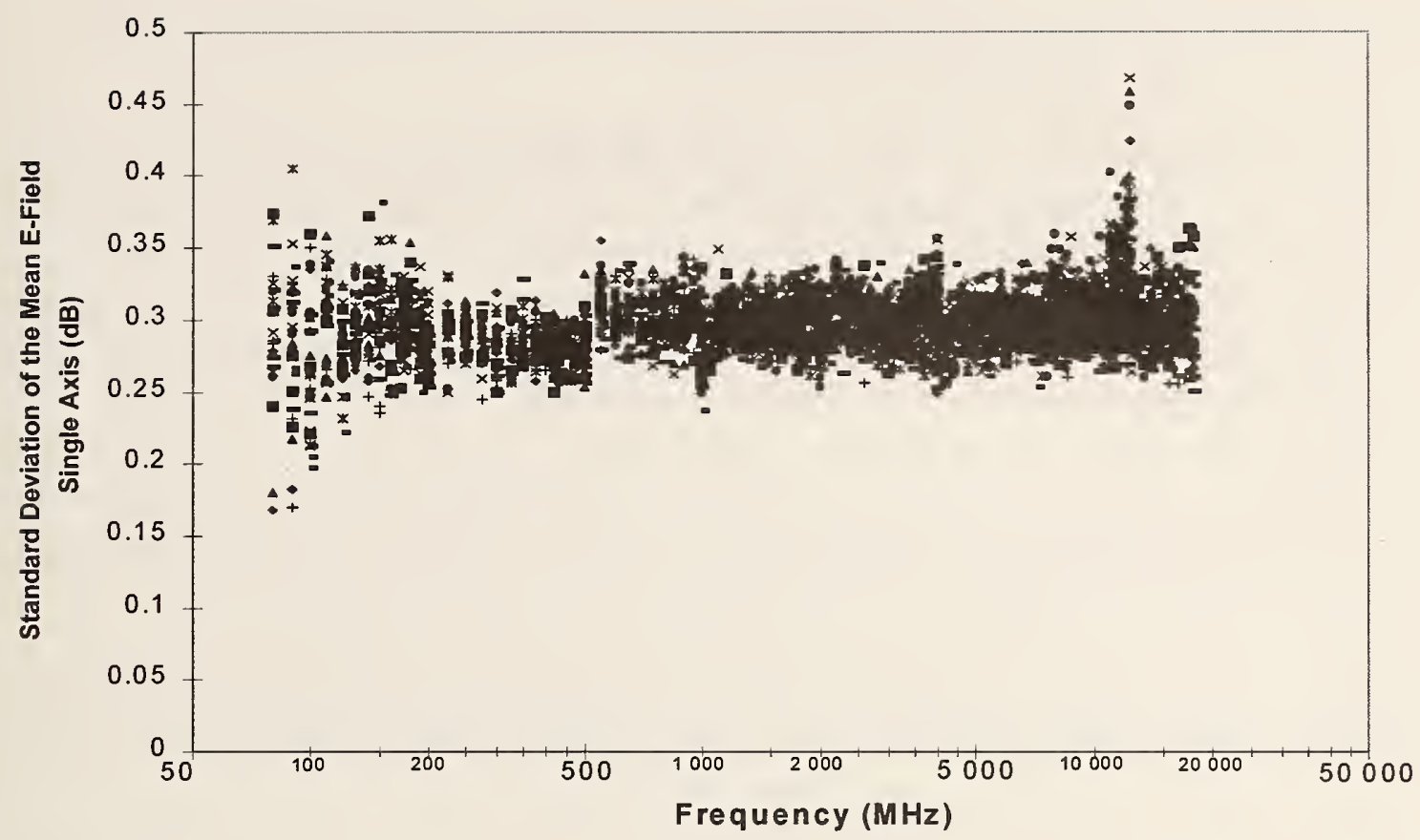

Figure 56. Standard deviation of the mean electric field (rectangular component) for each of 30 short dipoles in chamber A. Values given are for a constant net input power of $1 \mathrm{~W}$.

are also electrically large, so an antenna may be representative of the field a large device is exposed to.

Another way to examine the statistical characteristics of the chamber is to process the measurements from a single probe over a number of paddle positions and calculate the sample mean and standard deviation. From these, we can estimate the standard deviation of the mean (SDOM) by dividing the sample standard deviation by the square root of the number of paddle position. Under ideal conditions, this value will be the same as that given in Figure 54c, which for 225 paddle positions is approximately $0.3 \mathrm{~dB}$. The SDOM can be calculated for each probe at each frequency, and this information is plotted in Figure 56. The estimates of the standard deviation of the mean electric field (rectangular component) is remarkably close to the expected value of $0.3 \mathrm{~dB}$. A few characteristics of the data stand out in this figure. First are the apparent jumps at $500 \mathrm{MHz}, 1 \mathrm{GHz}, 2 \mathrm{GHz}, 4 \mathrm{GHz}$, and $12.4 \mathrm{GHz}$. These indicate either possible errors in the calibrations of the probes, or low signal levels which caused measurements to be near the noise floor (the minimum transmitted power occurred at the highest frequency limits of our amplifiers, which are at $1 \mathrm{GHz}, 2 \mathrm{GHz}, 4 \mathrm{GHz}$, and $12.4 \mathrm{GHz}$ ). In either case, the effect is small, but could be improved with further research. Second, while the range of estimates of the SDOM appears to increase at low frequencies, the increase is not dramatic, remaining between approximately 0.2 and $0.4 \mathrm{~dB}$. The fact that the SDOM is close to the expected value while the standard deviation of the various measurements of the average electric field (as plotted in Figure $54 c$ ) is nearly twice the expected value is consistent with the conclusion that some deterministic bias, unique for each probe, is affecting the measured average. Further, it implies that the 
observed variations in the data shown in Figure 54 will not decrease significantly even if the number of paddle positions is increased dramatically.

Based on the interpretation of the standard deviation of measurements of E-Ave (Figure 54c) and the standard deviation of the mean electric field (Figure 56) given above, we can make recommendations as to the optimal number of paddle positions. This optimum is the number of paddle positions required to reduce the standard deviation due to random fluctuations (based on the statistical model) to approximately the same value as the standard deviation of measurements of E-Ave, which appear to be due to deterministic factors. Beyond this optimal number of paddle positions, only small improvements in measurement uncertainty would be seen, and more significant improvements could probably be seen by repeating the measurement with the transmit antenna or probes in a different location (for emissions or immunity tests, the test device could be moved to another location). For frequencies below $200 \mathrm{MHz}$, where the standard deviation of measurements of E-Ave are greater than $1.5 \mathrm{~dB}$, there is little benefit in taking more than 10 paddle positions, since this gives a standard deviation due to random fluctuations of approximately $1.4 \mathrm{~dB}$. Similarly, 100 paddle positions should reduce the standard deviation due to random fluctuations to less than $0.5 \mathrm{~dB}$, which is lower than we see for almost any frequency in Figure 54c.

Figure 57 shows the same information as Figure 54d and Figure 55, the comparison of the two methods of estimating the average magnitude of a rectangular component of the electric field, but for Chamber B. The actual estimates of the electric field are shown in Figure 57a, and the difference between the estimates is shown in Figure 57b. A similar bias is present in these data above $400 \mathrm{MHz}$, but the higher reflections (as measured by the increased VSWR) caused by the smaller chamber size appears to cause problems at lower frequencies. The performance of the chamber in this low frequency region might be improved with additional research and measurements.

We next present information about the maximum measured magnitude of a rectangular component of the electric field in Figure 58. The format of this figure is identical to that of the average electric field as given in Figure 54, with the exception of a small difference in Figure $58 \mathrm{~d}$, which will be explained below. The various estimates of the maximum electric field typically vary by less than $6 \mathrm{~dB}$ from $150 \mathrm{MHz}$ to $18 \mathrm{GHz}$, or the measurements differ from the average by less than $\pm 3 \mathrm{~dB}$. The measured standard deviation (approximately $1 \mathrm{~dB}$ for frequencies greater than $250 \mathrm{MHz}$ ) is remarkably close to the ideal of $0.9 \mathrm{~dB}$. It would be extremely difficult to improve the performance of the chamber beyond what is presented here. However, since the two estimates of the standard deviation are so close, it may be possible to further reduce the standard deviation by increasing the number of paddle positions, although, as shown in Section 3.2.6, the ideal standard deviation is somewhat insensitive to the number of paddle positions, so the number of paddle positions would need to be increased substantially before any gain would be realized. At low frequencies, however, where the observed standard deviation is substantially greater than the ideal, no improvement can be expected due to increasing the number of paddle positions. In fact, below $200 \mathrm{MHz}$, where the measured standard deviation is typically greater than $1.5 \mathrm{~dB}$, there is little benefit in using more than 50 paddle positions, which gives an ideal standard deviation of approximately $1.2 \mathrm{~dB}$. Thus, measurements of the electric field (rectangular component) with 225 paddle positions or less can be treated as if the chamber were perfect for frequencies greater than approximately $225 \mathrm{MHz}$. 


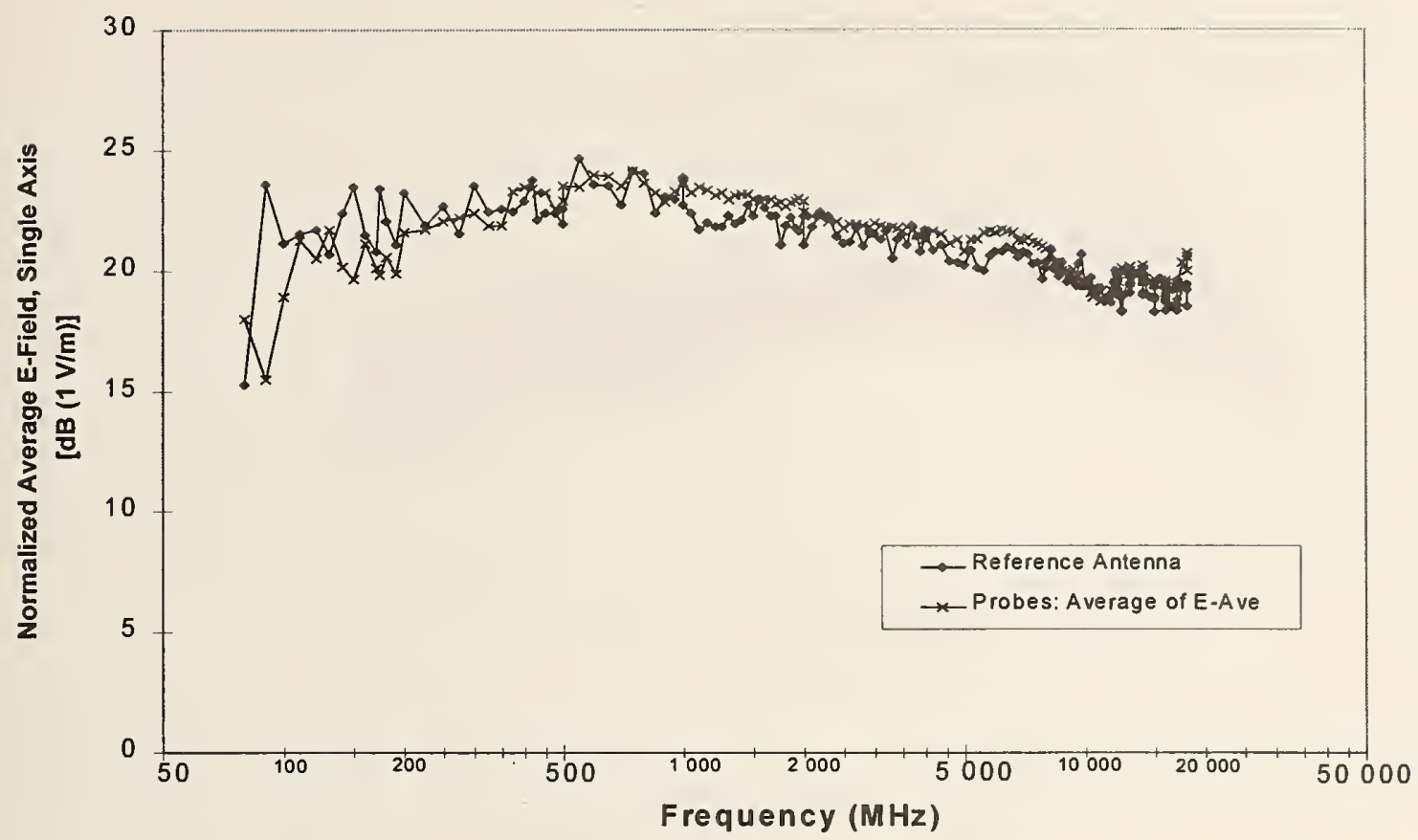

(a)

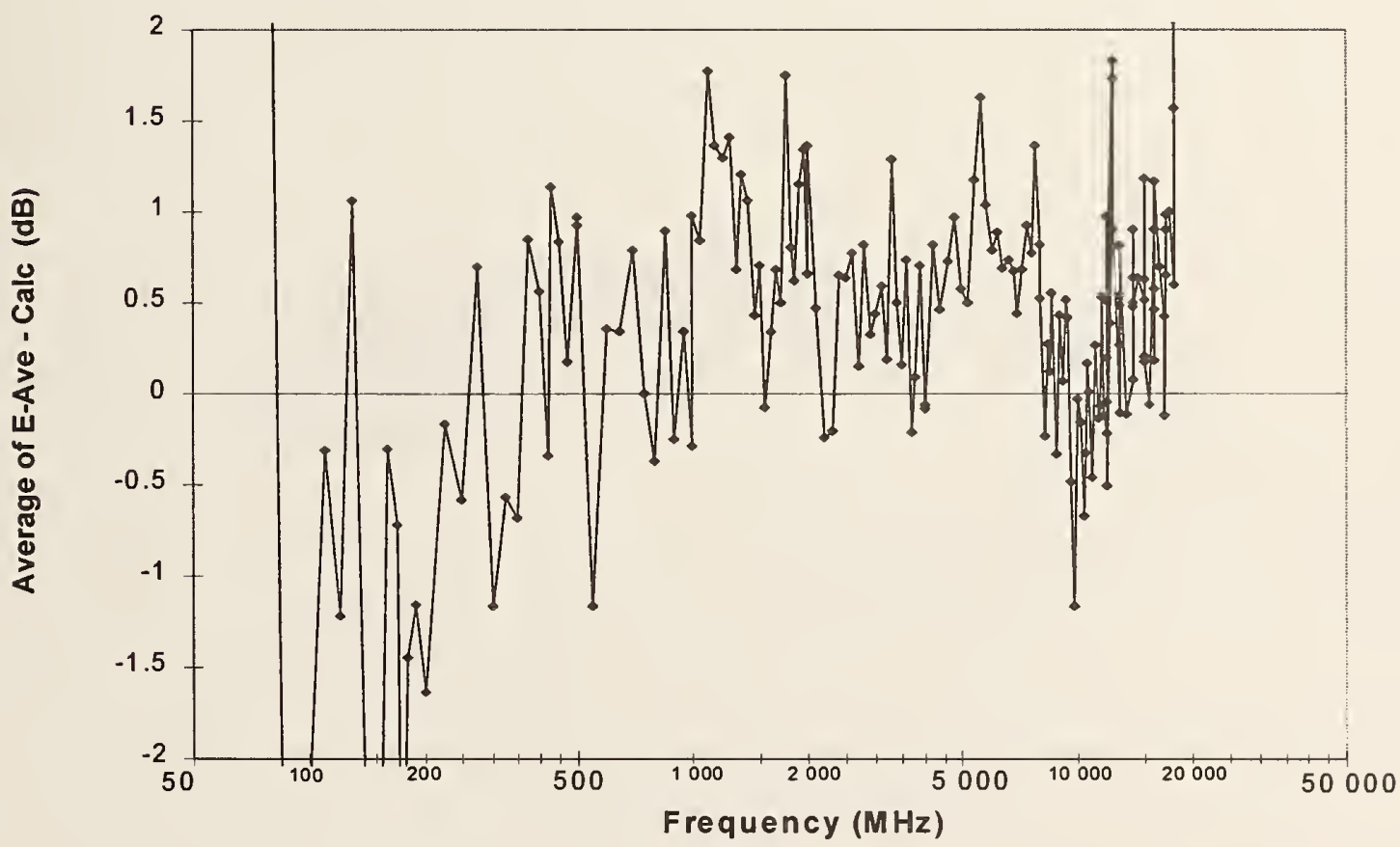

(b)

Figure 57. Comparison of average electric field measured in chamber B using average of probe readings and the reference antenna. (a) Measured values. (b) Difference in measured values. 


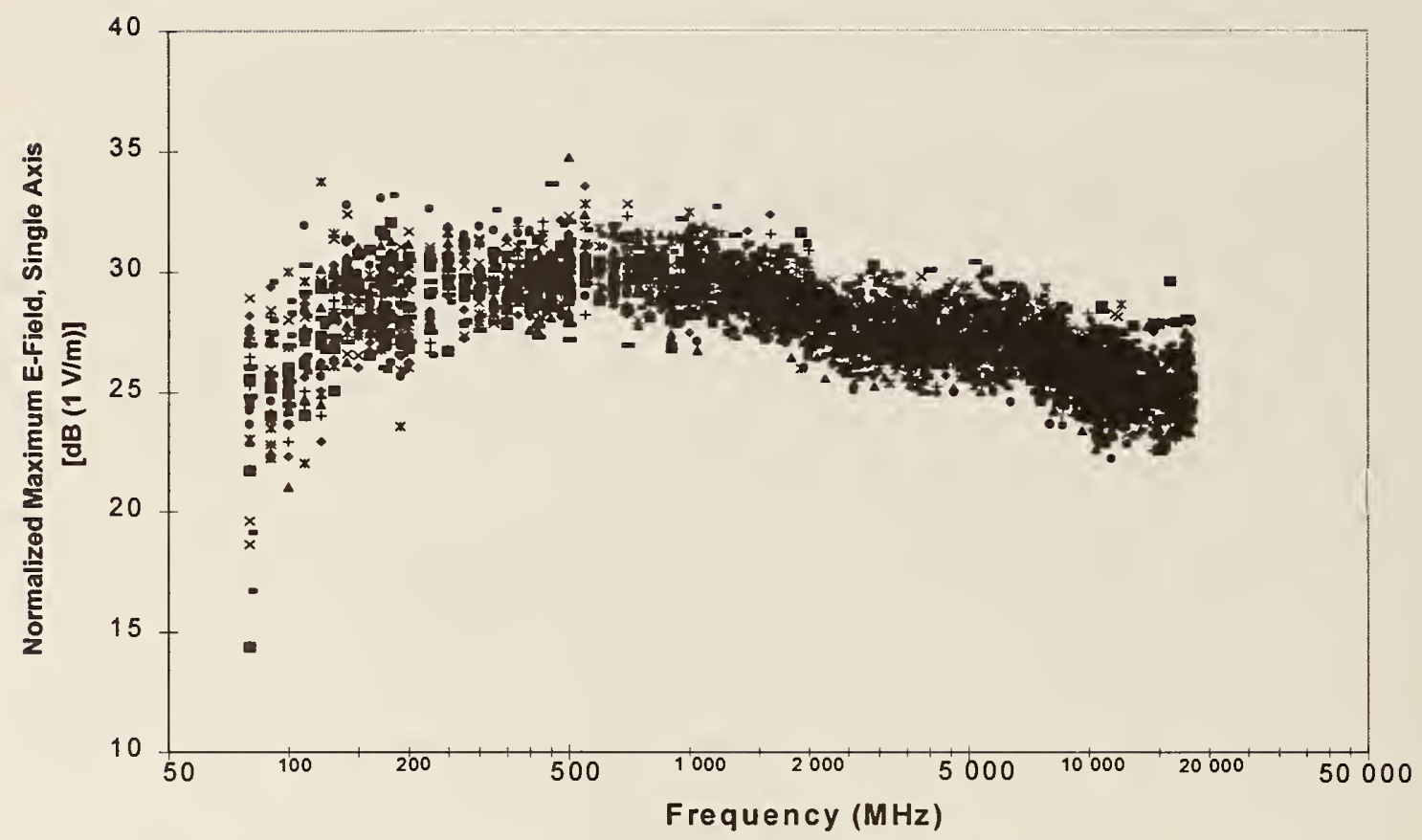

(a)

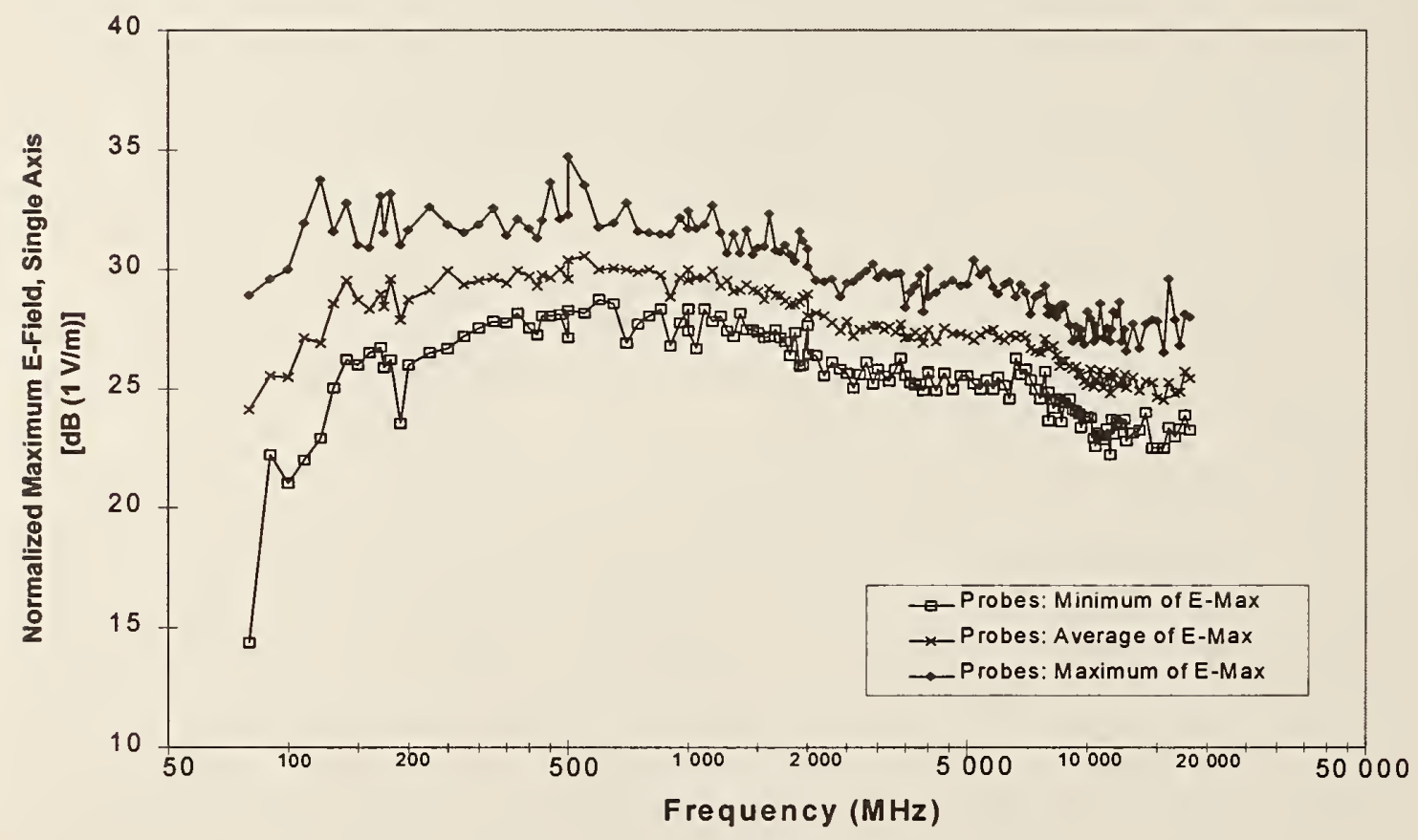

(b)

Figure 58. The maximum measured electric field (single polarization) for each of 30 short dipoles in chamber A. Values given are for a constant net input power of $1 \mathrm{~W}$.

(a) Individual measurements. (b) Envelope and average of individual measurements.

(c) Standard deviation of measurements of $E_{R}-M a x$. (d) Comparison of average of individual measurements with reference antenna. 


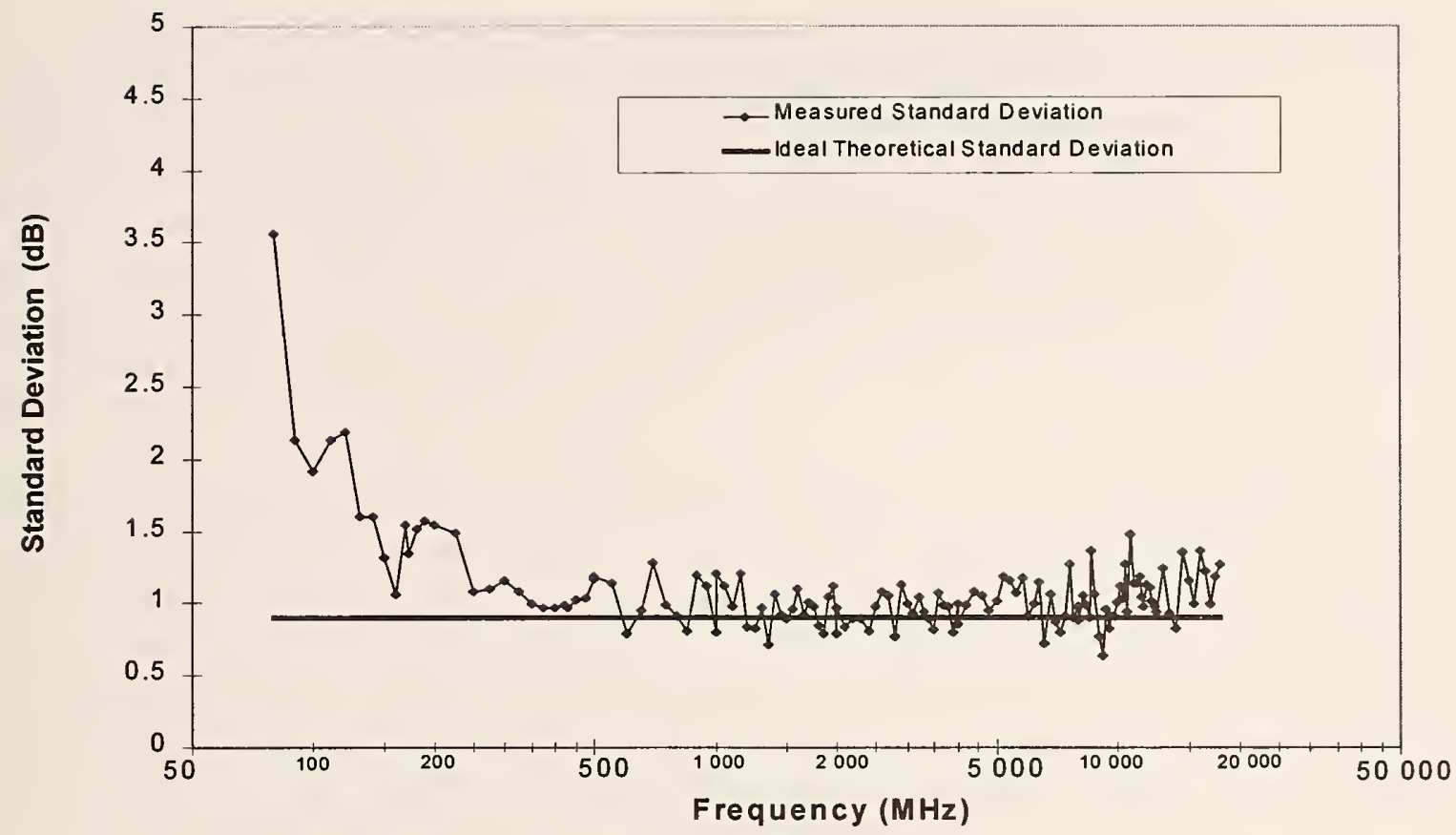

(c)

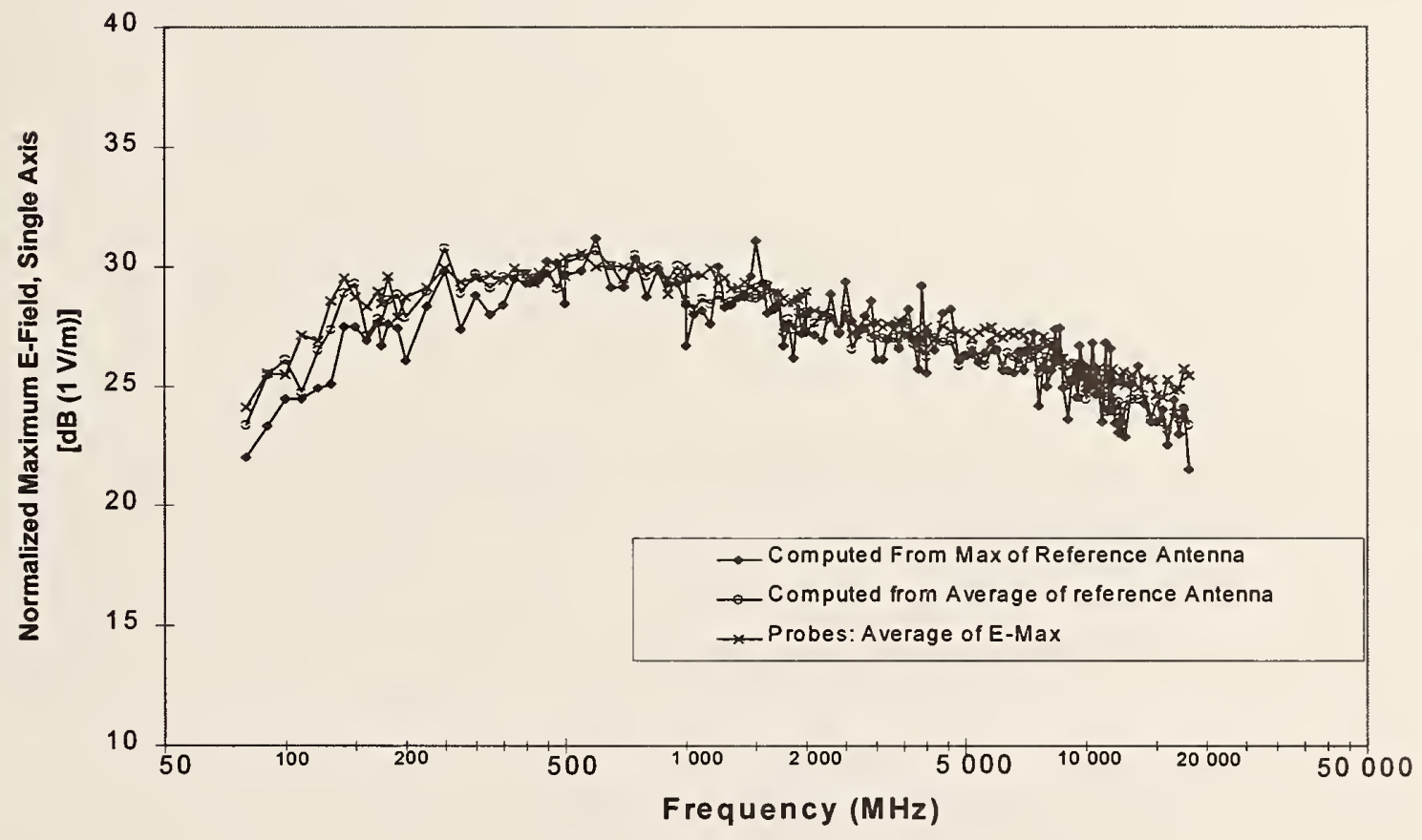

(d) 
The comparison between methods of estimating the maximum electric field shown in Figure 58d has three curves. As noted in Section 3.2.3, the ideal distribution of the square root of the received power is the same as the ideal distribution of the measured electric field (rectangular component). This implies that the maximum electric field can be predicted from the maximum received power using eq (48). Such a prediction has two principal disadvantages, however. First, measurements of the maximum received power are very noisy and have a high degree of uncertainty, resulting in high uncertainties when estimating the maximum electric field. This can be seen in the curve that shows the estimated maximum electric field calculated from the maximum power received on the reference antenna. Second, at low frequencies, the maximum received power is compressed, resulting in a skewed distribution and an estimate of the maximum electric field that is less than the true maximum electric field. This can also be seen in the same curve. Below $500 \mathrm{MHz}$, this curve is consistently below the other two. As an alternative to calculating the maximum electric field from the maximum received power, we can calculate the average electric field as shown in Figure 54d, and multiply by the maximum-to-average ratio for the electric field as calculated from the data in Table 4. For these data, which were measured at 225 paddle positions, the ratio is $3.445 / 1.253=2.75$, or $8.79 \mathrm{~dB}$. This estimate is also plotted in Figure 58d and appears to be smoother and is a better estimate of the maximum electric field as measured by the probes. Thus we recommend estimating the maximum magnitude of a rectangular component of the electric field from the average received power rather than the maximum received power.

Unfortunately, this is not the end of the discussion. Figure 59 shows a similar plot of measurements and estimates of the maximum electric field as measured in Chamber B. This plot shows significantly better agreement between the probes and the estimated maximum based on the maximum received power than the estimate based on the average received power. This contradicts what we said above and does not match our expectations. We have no explanation for this surprising result. It could be a coincidence, but that seems unlikely considering the agreement at a large number of points. Thus we must conclude that there are still some phenomena that we cannot completely explain at this time.

In an effort to identify possible explanations for these unexpected results, we calculated the ratio of the maximum magnitude of the electric field $\left(\mathrm{AVE}\left(\mathrm{E}_{\mathrm{R}}-\mathrm{Max}\right)\right)$ to the average magnitude of the electric field ( $A V E\left(E_{R}-A V E\right)$ ) as measured by the probe system, and the result for Chamber $A$ is given in Figure 60a, and the result for Chamber B is given in Figure 60b. We also include the maximum-to-average ratio of the square root of the received power, which should have a similar distribution to the magnitude of the electric field (rectangular component). As mentioned above, the expected value for this ratio, given 225 samples, is approximately 8.8 $\mathrm{dB}$. This is $1 \mathrm{~dB}$ greater than the expected value for the maximum-to-average ratio of the received power $(7.8 \mathrm{~dB})$. The data measured using the probes are remarkably close to the expected value. In Chamber $\mathrm{A}$, the electric field as measured by the probes does not show any signs of compression at low frequencies, except possibly at the lowest three frequencies $(80,90$, and $100 \mathrm{MHz}$ ). The estimated maximum based on the maximum received power, however, appears to be compressed (fall below the expected value) for frequencies below $500 \mathrm{MHz}$. In Chamber B, there appears to be compression in both estimates of the maximum electric field, although the compression of the received power appears to be more severe than that of the electric field as measured by the probes. 
Given these conflicting results, we are forced to make some assumptions which are not completely justified by the data. First, since Chamber A is larger than Chamber B, and since the data measured in Chamber $\mathrm{A}$ are more consistent with our expectations (a dangerous thing, indeed), we will assume that the data from Chamber A are more representative of a good chamber than the data from Chamber B. Regardless of the behavior at low frequencies, Figure $58 \mathrm{~d}$ and Figure 59 show that the estimated maximum electric field based on the average received power is less noisy than estimates based on the maximum received power, and this is true in both chambers. Therefore, we will assume that the maximum electric field can best be predicted from the average received power.

We also present another way of interpreting the data measured using the probes. In a rectangular chamber, each rectangular component of the electric field $E_{x}, E_{y}$ and $E_{z}$ should be independent; that is, the measured field should be independent of orientation. If we were to group all dipoles that had the same polarization together and compute the average response, we would have an indication of whether the components are independent or not. We computed the average response of all of the copolarized dipoles ( 10 dipoles in each of 3 polarizations) and the average responses are given in Figure 61a. Only data measured at low frequencies are presented here, as any dependence on polarization is most likely to occur at low frequencies. We also computed the maximum response of all of the copolarized dipoles and the average of the maximum responses is given in Figure $61 \mathrm{~b}$. The agreement between the three components is good for both the average and maximum electric field above $200 \mathrm{MHz}$. Below $200 \mathrm{MHz}$, however, there appears to be some dependence on polarization.

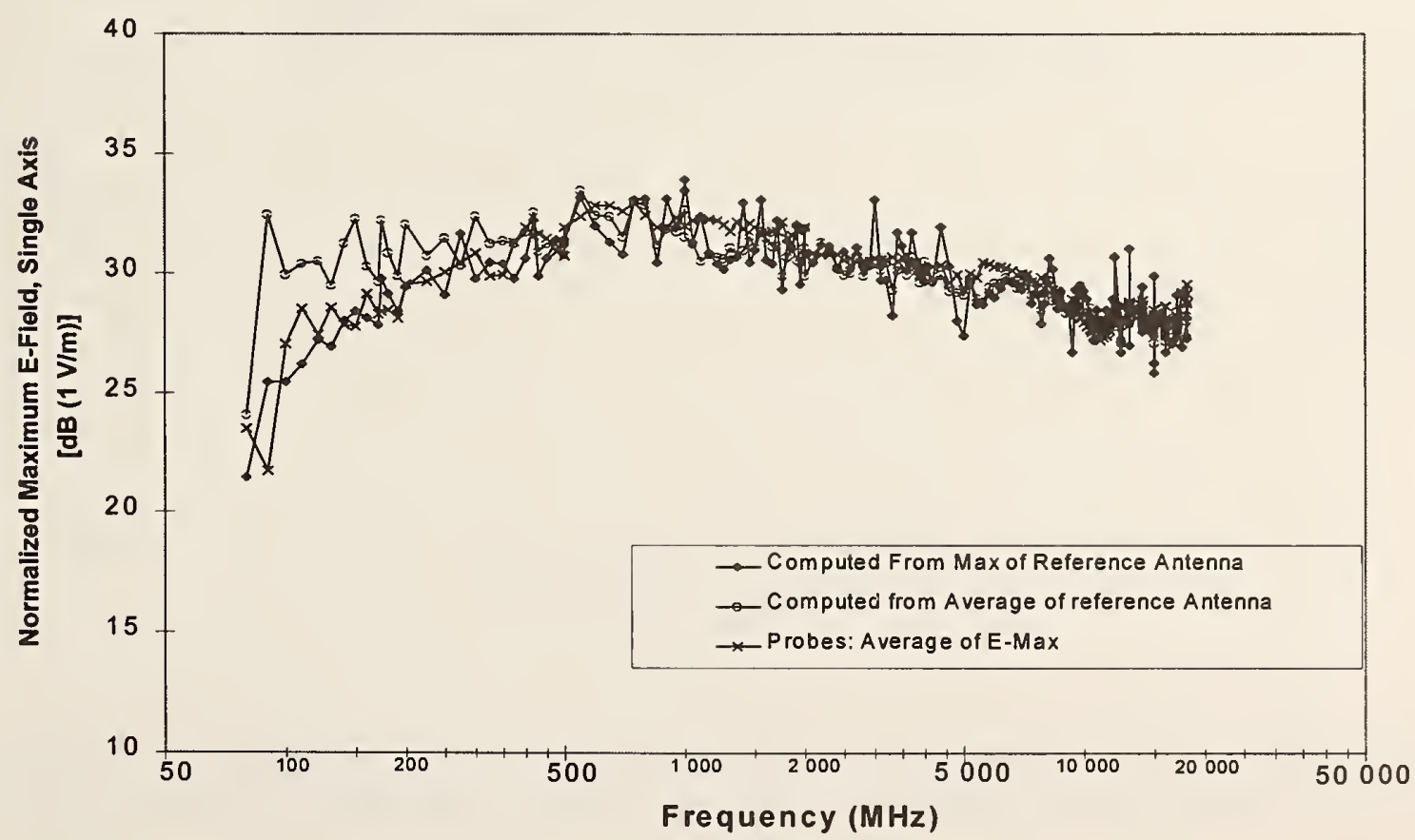

Figure 59. Comparison of maximum electric field measured in chamber B using average of probe readings and the reference antenna. 


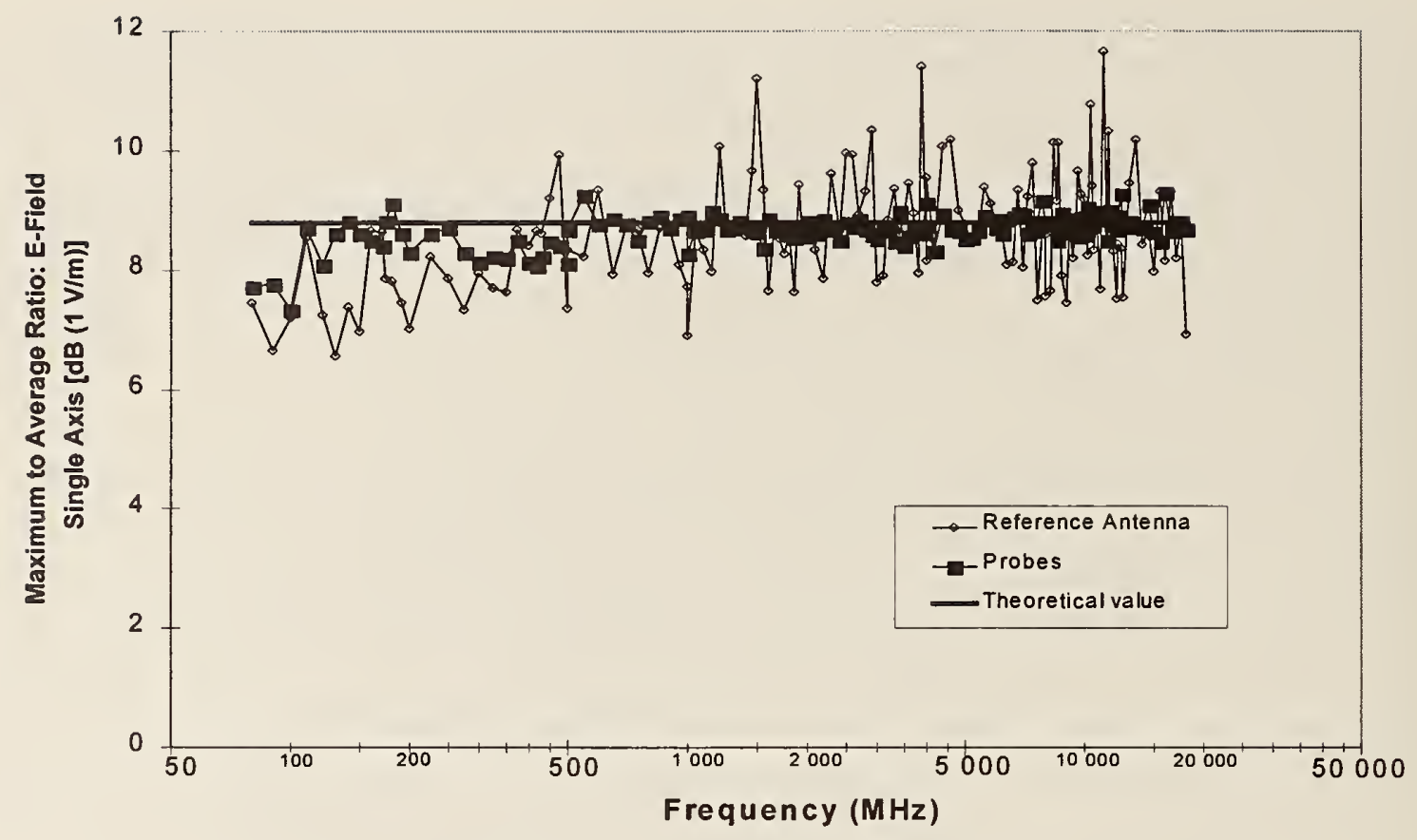

(a)

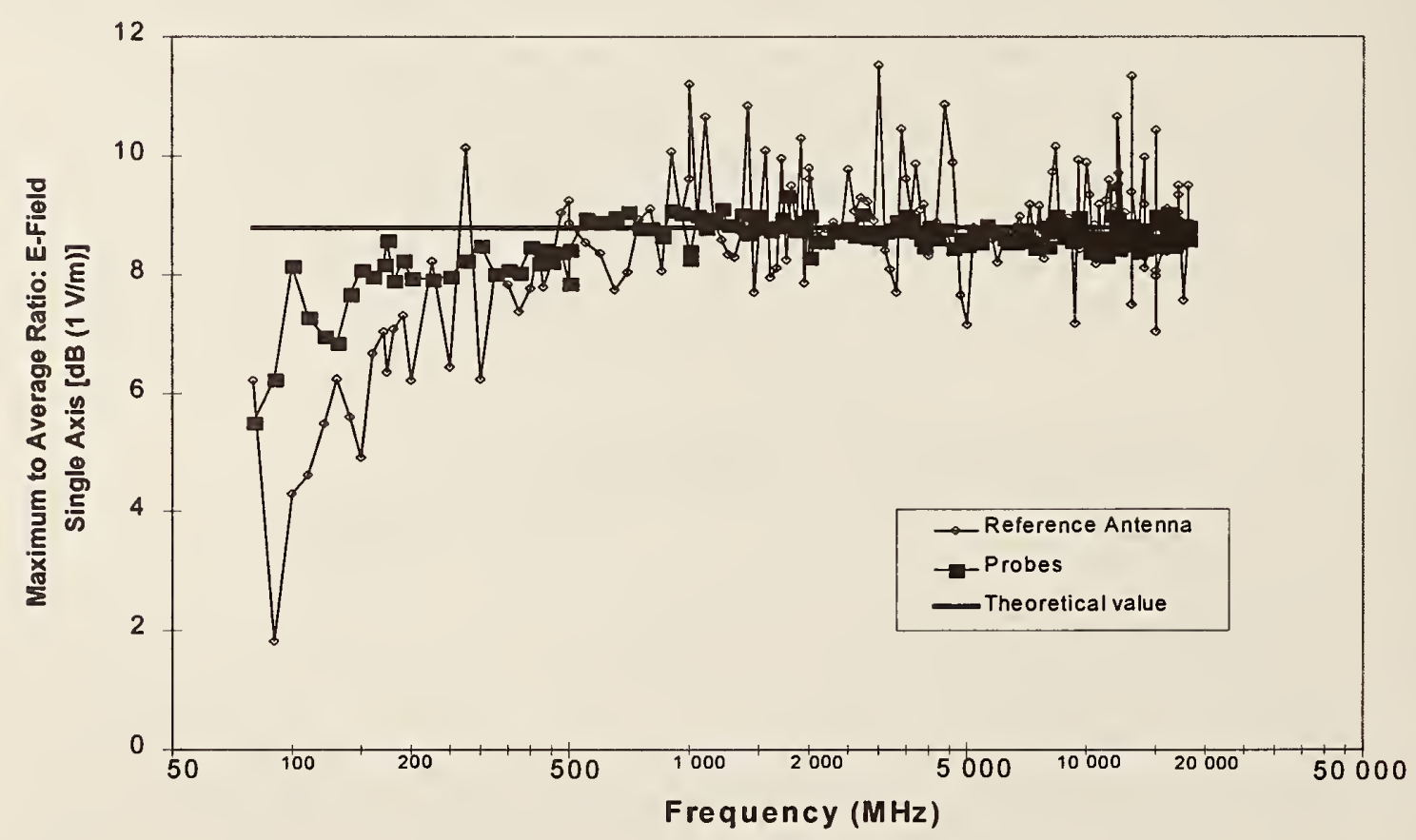

(b)

Figure 60. Max-to-average ratio of electric field measured using probe system, and of the square root of power received by the reference antenna. (a) Chamber A. (b) Chamber B. 


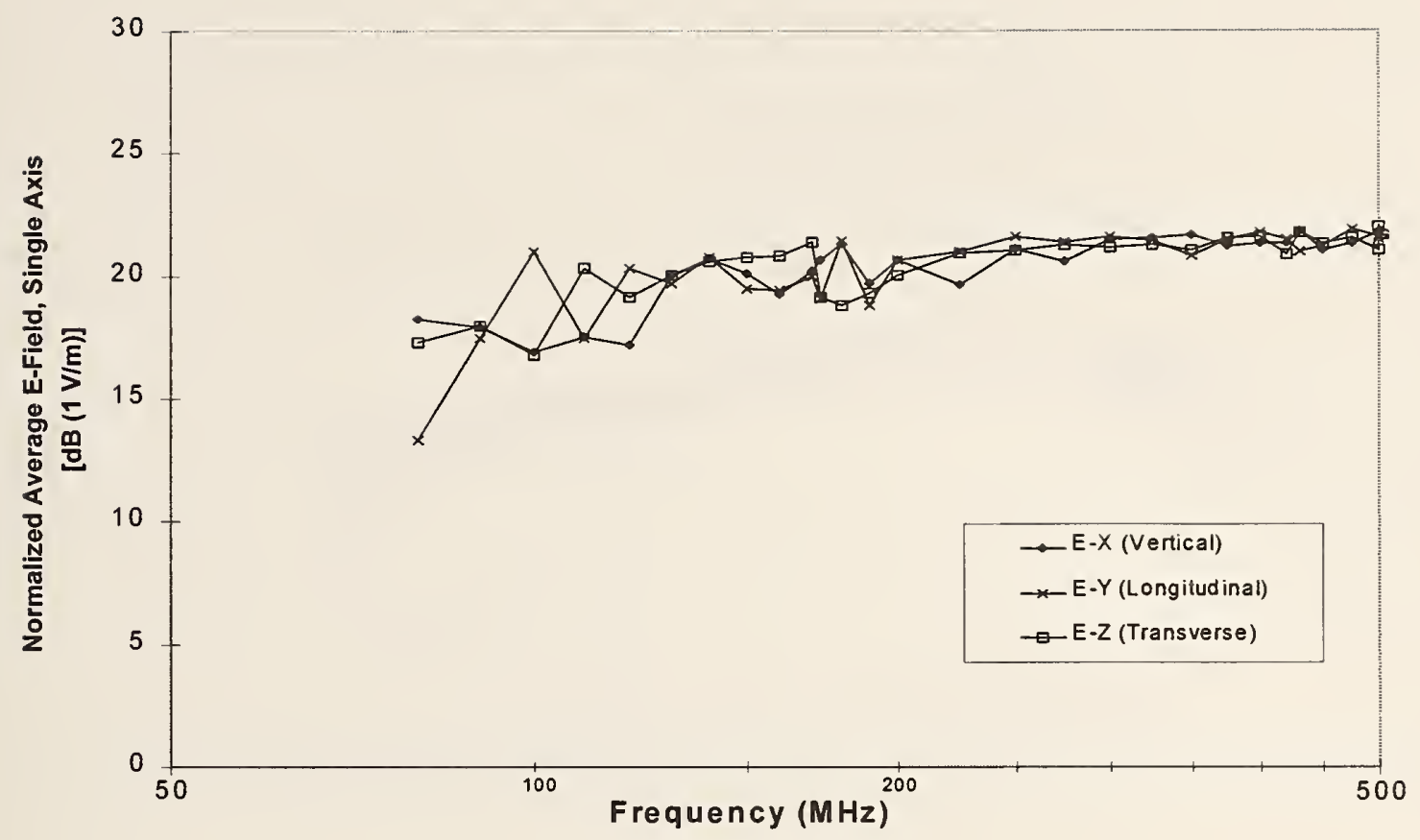

(a)

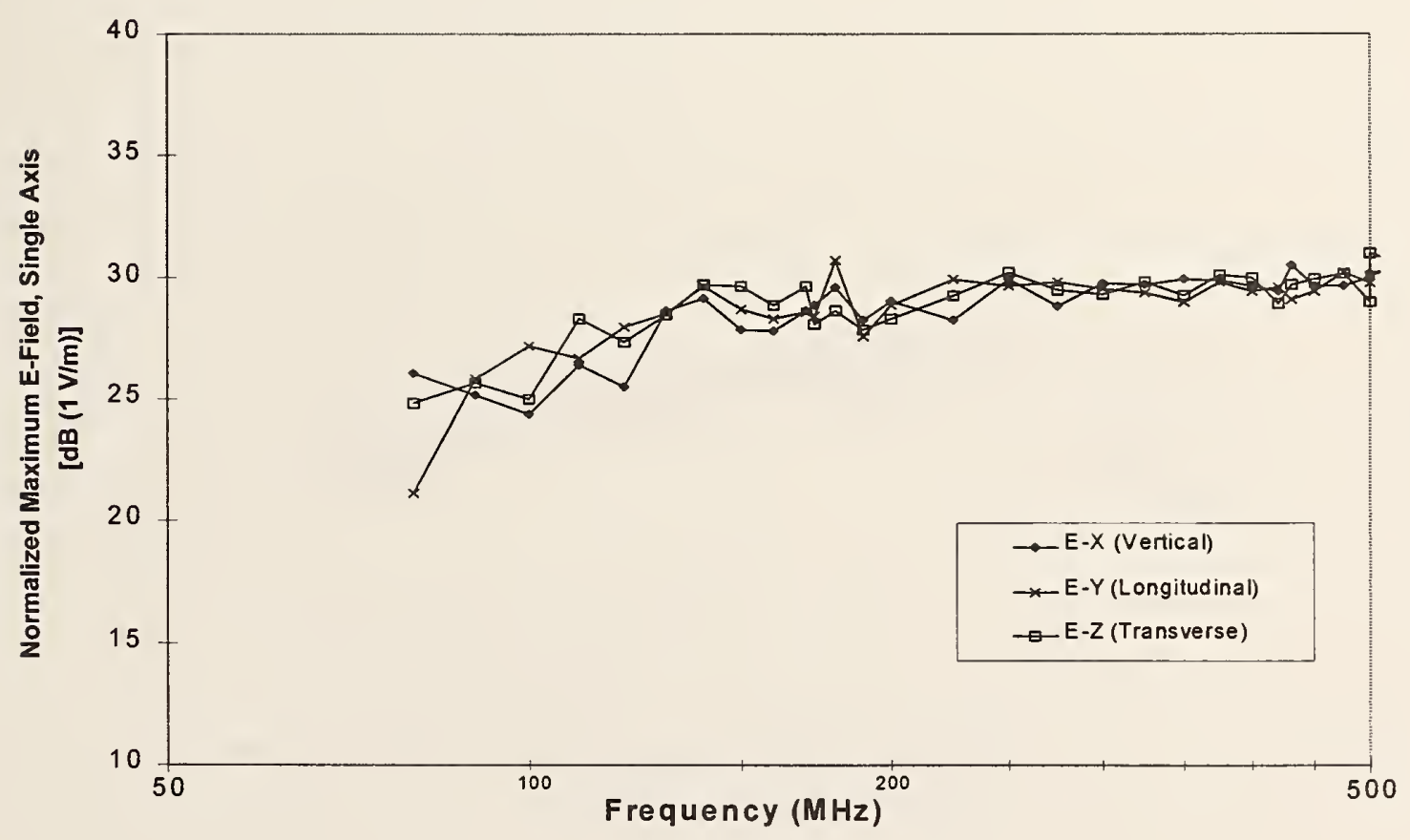

(b)

Figure 61. Comparison of the $\mathrm{x}, \mathrm{y}$, and $\mathrm{z}$ components of the electric field for a constant net input power of $1 \mathrm{~W}$. (a) Average of components. (b) Maximum of components. 


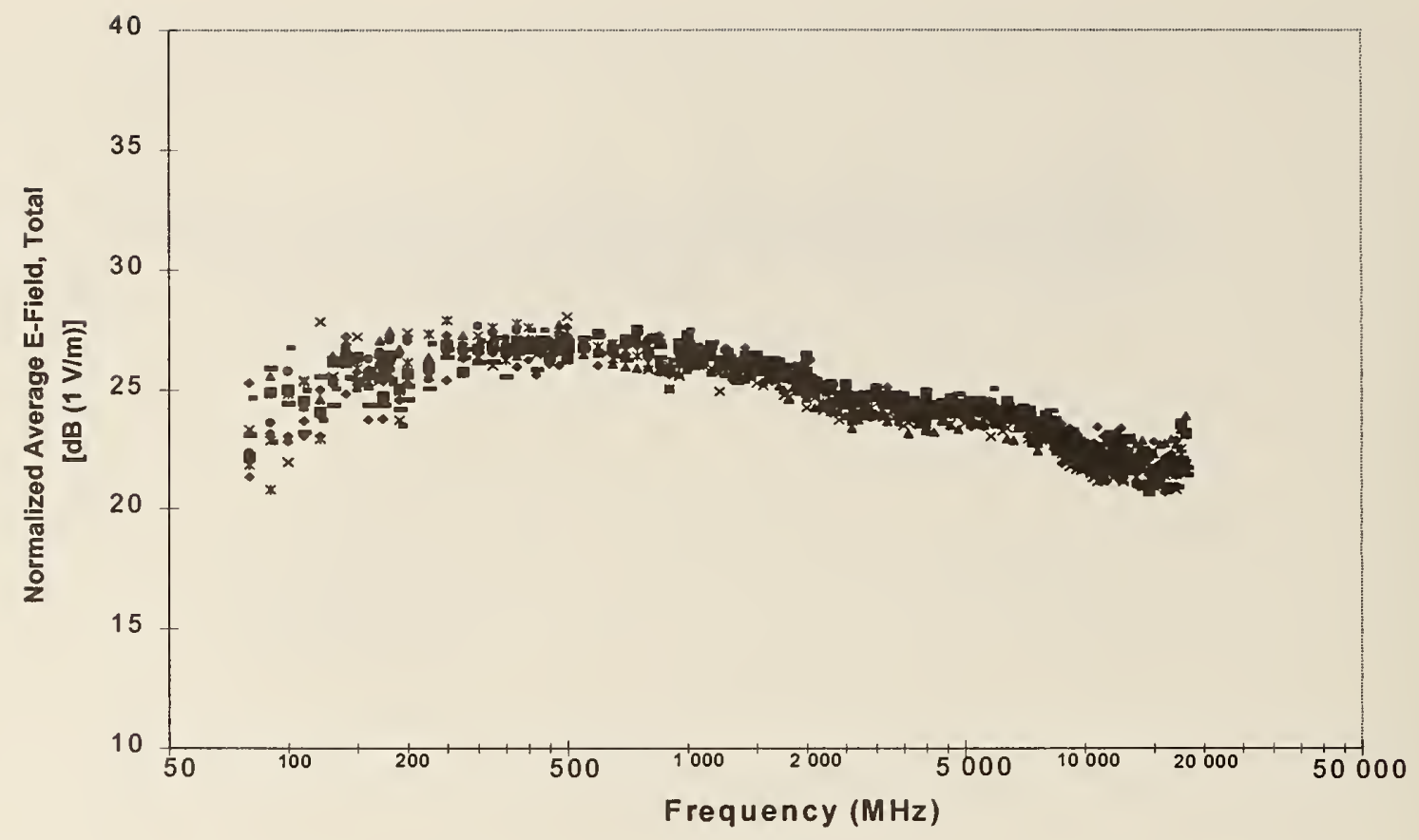

(a)

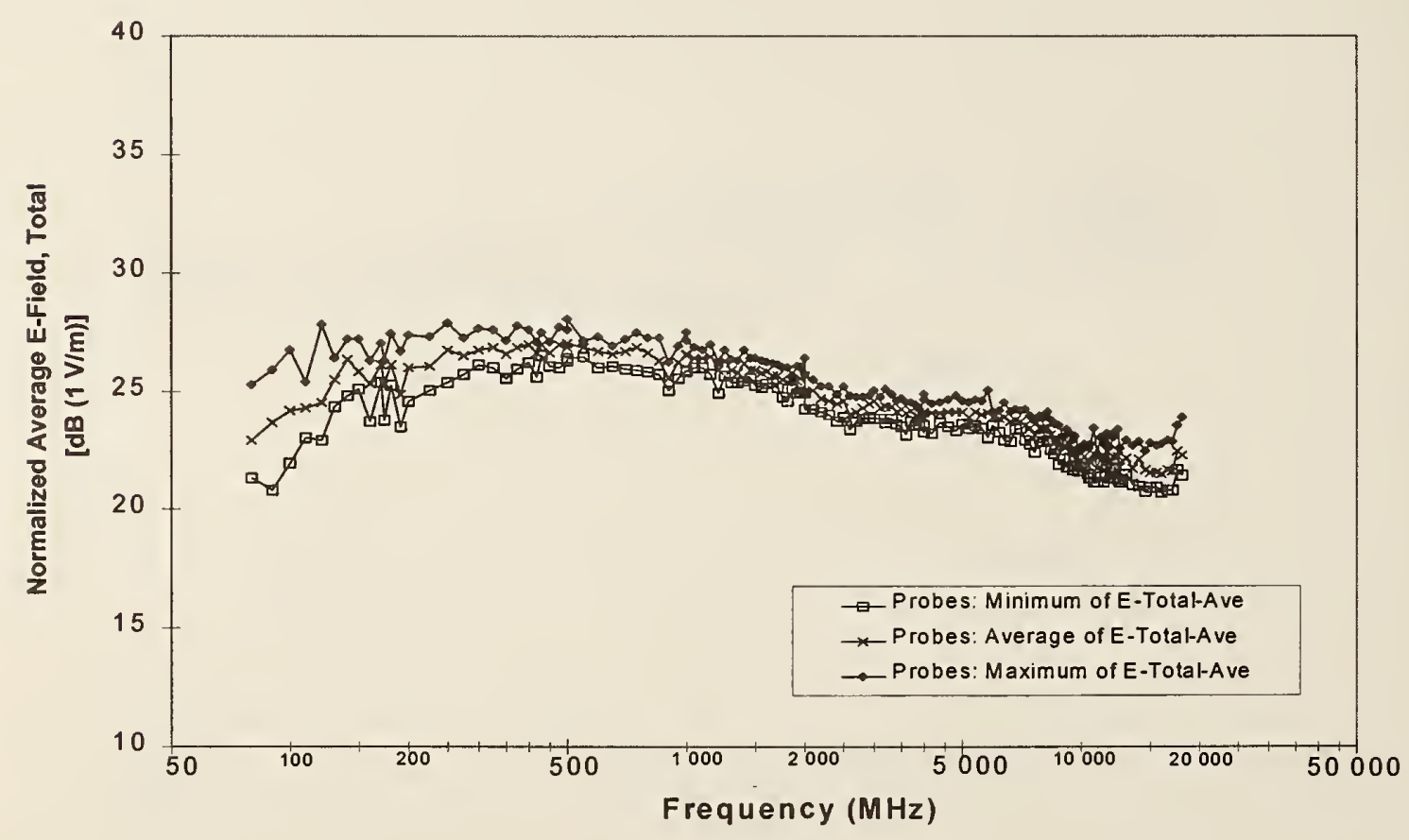

(b)

Figure 62. The average measured total electric field for each of 10 isotropic antennas in chamber A. Values given are for a constant net input power of $1 \mathrm{~W}$. (a) Individual measurements. (b) Envelope and average of individual measurements. (c) Standard deviation of measurements of $E_{T}$-Ave. (d) Comparison of average of individual measurements with reference antenna. 


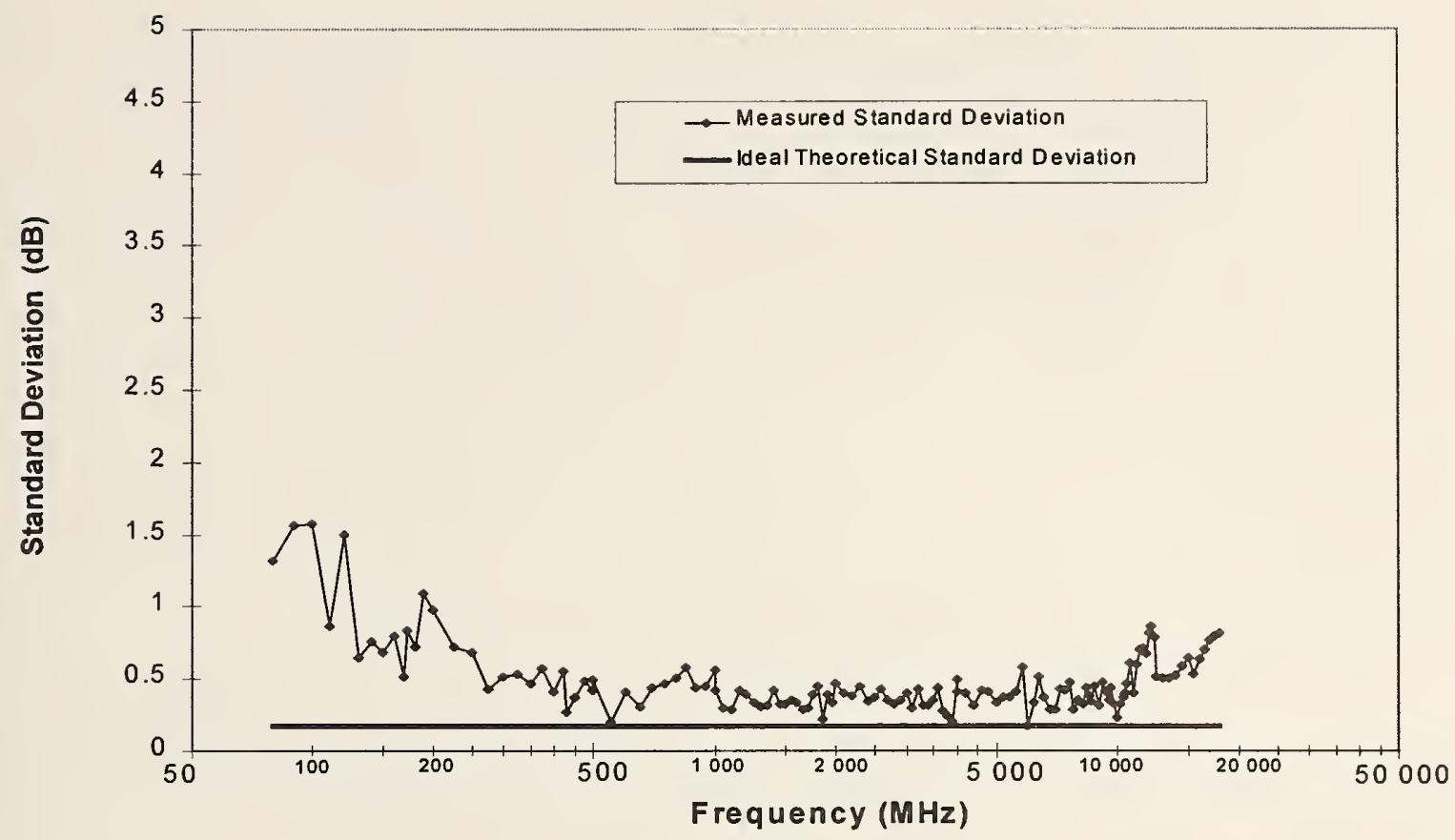

(c)

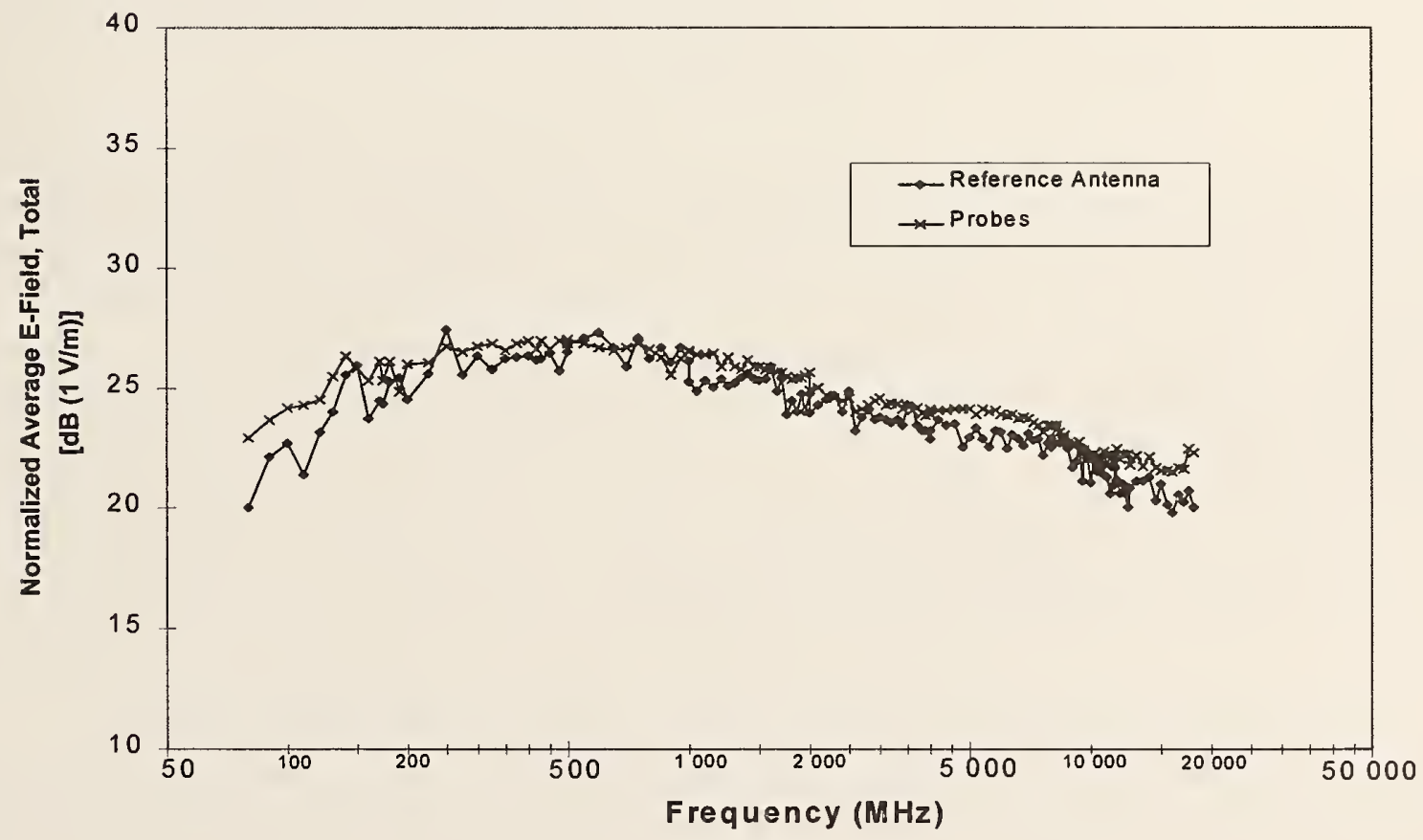

(d) 
The total electric field can be evaluated similarly to the rectangular components of electric field. Figure 62 shows information about the average total electric field presented in the same format as Figure 54. Over the majority of the frequency range evaluated during these test ( $80 \mathrm{MHz}$ to 18 $\mathrm{GHz}$ ), the worst case difference between the estimates of the electric field is less than $2 \mathrm{~dB}$. From another perspective, the measured values differed from the measured average by less than $\pm 1 \mathrm{~dB}$ between $300 \mathrm{MHz}$ and $18 \mathrm{GHz}$ (with some occasional outliers). Below $300 \mathrm{MHz}$, the spread in the measurements of the total electric field increases as expected. The observed standard deviation (over frequencies where the chamber appears to be operating well) was somewhat greater than would be expected under ideal conditions $(0.37 \mathrm{~dB}$ vs. $0.17 \mathrm{~dB})$. The ideal standard deviation is based on calculations given in Section 3.2.7 and values given in Table 7. This leads to two conclusions. First, this chamber is imperfect, and 225 paddle positions are sufficient to demonstrate these imperfections. Second, if 225 paddle positions are sufficient to demonstrate the imperfections, this implies that there should be an optimal number of paddle positions such that taking additional samples does not significantly reduce the uncertainty.

The measured and ideal standard deviations suggest that there is little benefit in using more than 100 paddle positions in this chamber. 100 paddle positions gives an ideal standard deviation of approximately $0.26 \mathrm{~dB}$. Below $200 \mathrm{MHz}$, where the measured standard deviation is greater than $0.75 \mathrm{~dB}, 20$ paddle positions, which gives an ideal standard deviation of approximately $0.57 \mathrm{~dB}$ should be adequate. These recommended numbers of paddle positions are based on the observed characteristics of the chamber. This does not mean that 20 paddle positions will give an acceptably small uncertainty, only that more than 20 positions will not reduce the observed uncertainty by an appreciable amount.

The comparison between the two methods of estimating the average electric field shown in Figure 62d is also good, although the field measured by the probes is generally greater than that predicted by the average power received by the reference antenna. The difference between these two estimates, plotted in Figure 63, is once again very similar to the differences presented in Figure 55 with Figure 53b as expected, since the estimates of the total electric field are based on the same measurements as the rectangular component of the electric field.

The maximum measured magnitude of the total electric field is shown in Figure 64. The format of this figure is identical to that of the maximum rectangular component of the electric field as given in Figure 58. The various estimates of the maximum total electric field typically vary by less than $4 \mathrm{~dB}$ from $150 \mathrm{MHz}$ to $18 \mathrm{GHz}$, or the measurements differ from the average by less than $\pm 2 \mathrm{~dB}$. The measured standard deviation (approximately $0.73 \mathrm{~dB}$ for frequencies greater than $20 \mathrm{MHz}$ ) is very close to the ideal of $0.65 \mathrm{~dB}$. Since the two estimates of the standard deviation are so close, it may be possible to further reduce the standard deviation by increasing the number of paddle positions. However, as shown in Section 3.2.6, the ideal standard deviation is somewhat insensitive to the number of paddle positions, so the number of paddle positions would need to be increased substantially before any gain would be realized. Thus, measurements of the total electric field with 225 paddle positions or less can be treated as if the chamber were perfect at almost all frequencies.

The comparison between methods of estimating the maximum electric field shown in Figure 64d has three curves. As noted in Section 3.2.3, the ideal distribution of the square root of the received power is the same as the ideal distribution of the measured electric field (rectangular component), but not of the total electric field. Thus, attempting to estimate the maximum total 


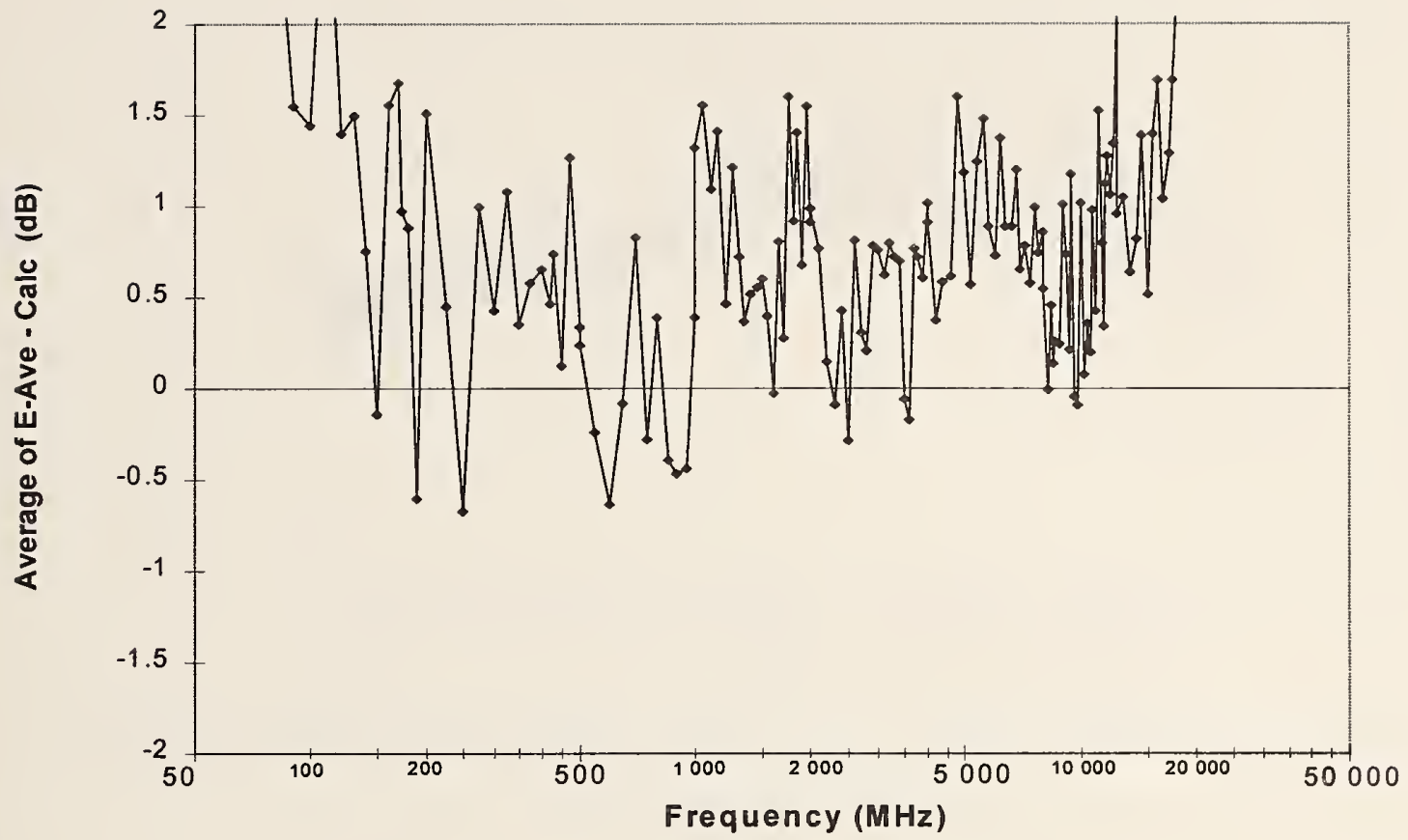

Figure 63. Difference in estimates of average total electric field measured in chamber A based on probe readings and power received by the reference antenna.

electric field based on the maximum received power using eq (53) results in an overestimate of the total electric field. For these measurements using 225 paddle positions, the overestimate is approximately $2.2 \mathrm{~dB}$. Instead, we recommend calculating the average total electric field as given in Figure 62d, and then multiplying the result by the maximum-to-average ratio of the total electric field as computed from Table 7. For these data, which were measured at 225 paddle positions, the ratio is $4.484 / 2.350=1.908$, or $5.612 \mathrm{~dB}$. The estimated total electric field, as calculated from the average received power, is also plotted in Figure 62d, along with the value computed from the measurements of the total electric field using the probes. These last two curved agree quite well, although, once again, the data measured using the probes is consistently greater than the value estimated from the average received power. This is likely caused by the same bias described earlier.

The actual measured maximum-to-average ratio, calculated from the ratio of the maximum magnitude of the total electric field $\left(\mathrm{AVE}\left(\mathrm{E}_{\mathrm{T}}-\mathrm{Max}\right)\right)$ to the average magnitude of the electric field ( $\mathrm{AVE}\left(\mathrm{E}_{\mathrm{T}}-\mathrm{AVE}\right)$ ) as measured by the probe system, is given in Figure 65 , with data from Chamber A given in Figure 65a, and data from Chamber B given in Figure 65b. The measured values are very close to the expected values of $5.6 \mathrm{~dB}$. We also include the maximumto-average ratio of the square root of the received power, which is identical to the curve given in Figure 60 . We do this to show once again why the maximum received power should not be used to estimate the maximum received power. 


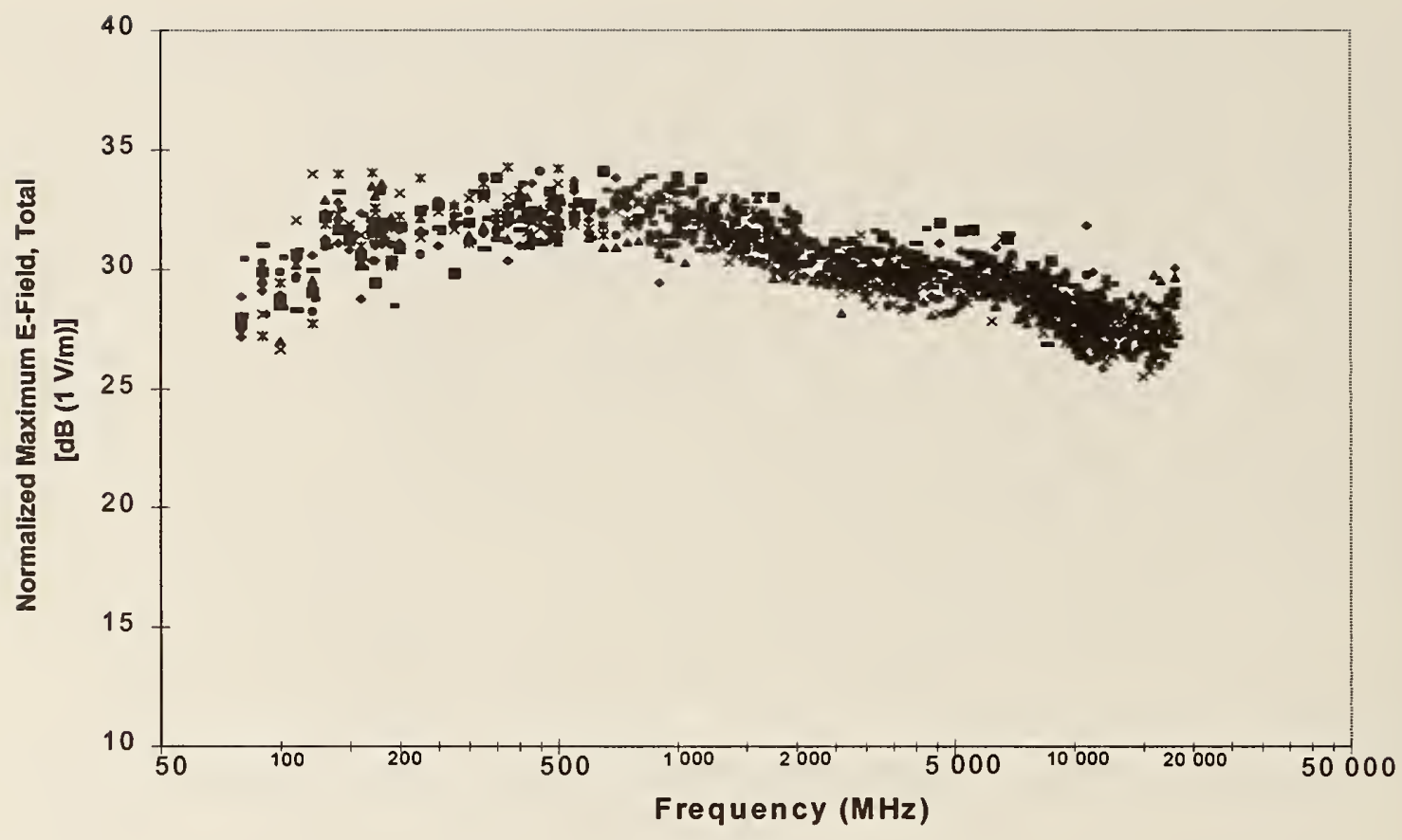

(a)

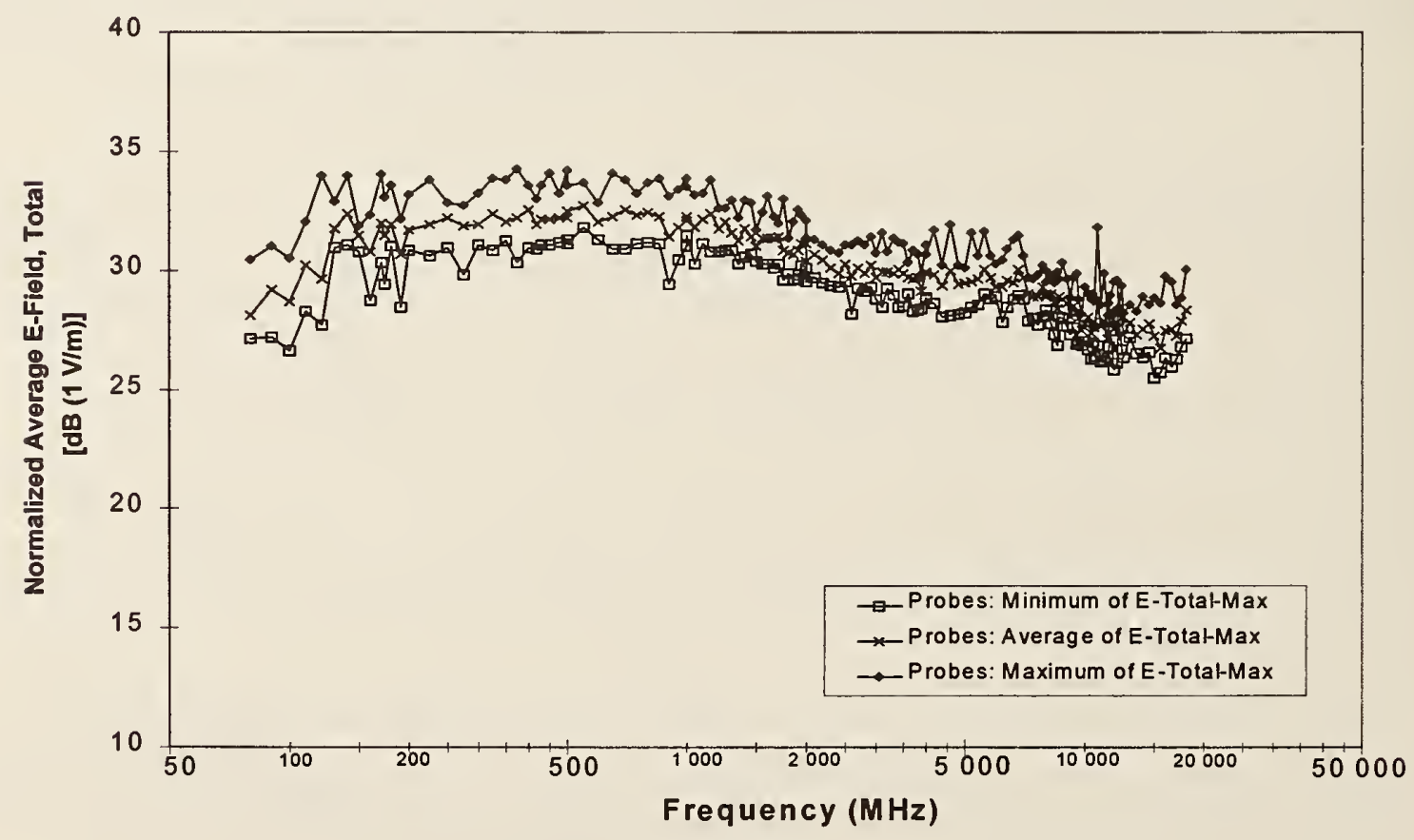

(b)

Figure 64. The maximum measured total electric field for each of 10 isotropic antennas in chamber A. Values given are for a constant net input power of $1 \mathrm{~W}$. (a) Individual measurements. (b) Envelope and average of individual measurements. (c) Standard deviation of measurements of $\mathrm{E}_{\mathrm{T}}$-Max. (d) Comparison of average of individual measurements with reference antenna. 


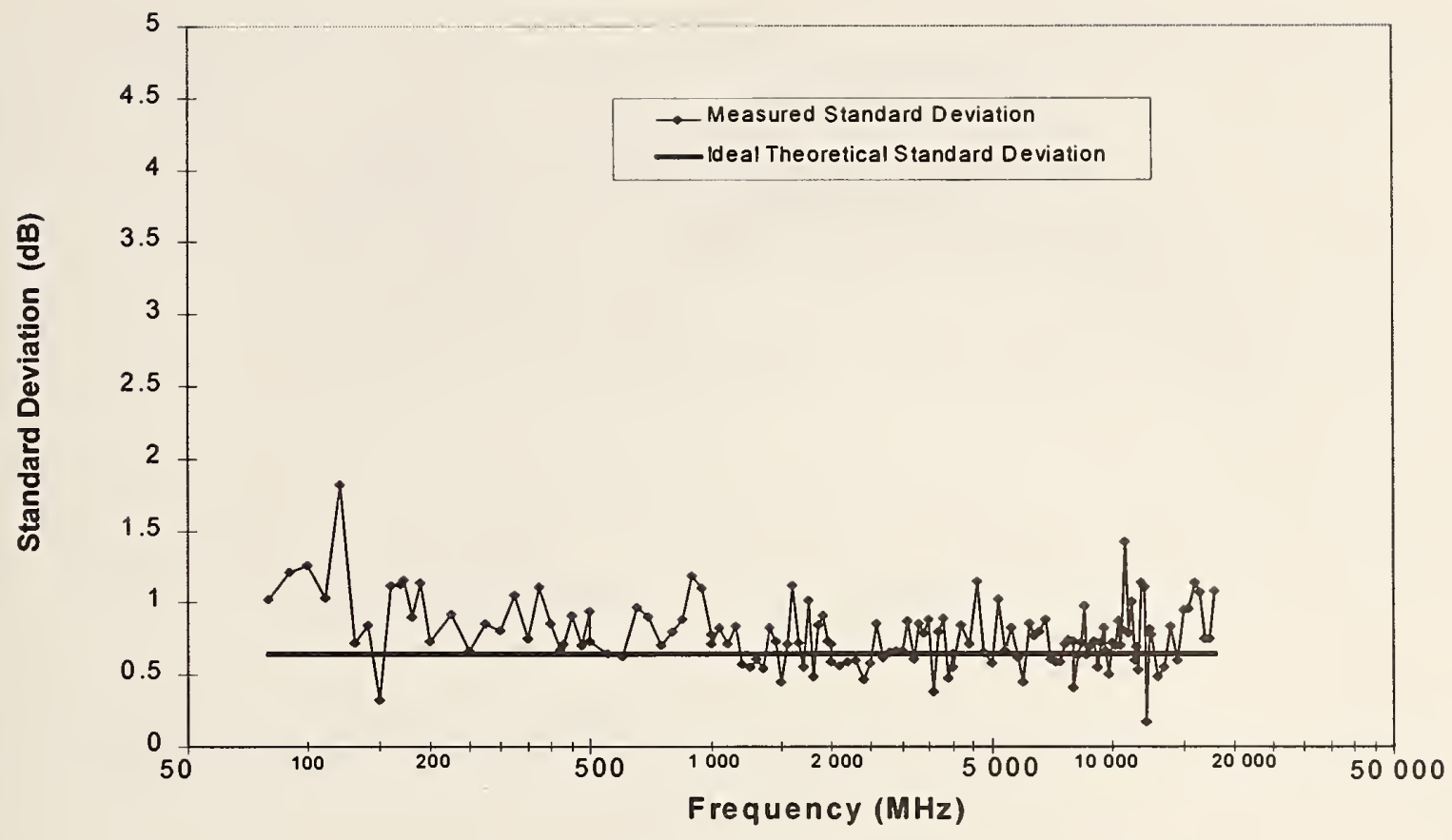

(c)

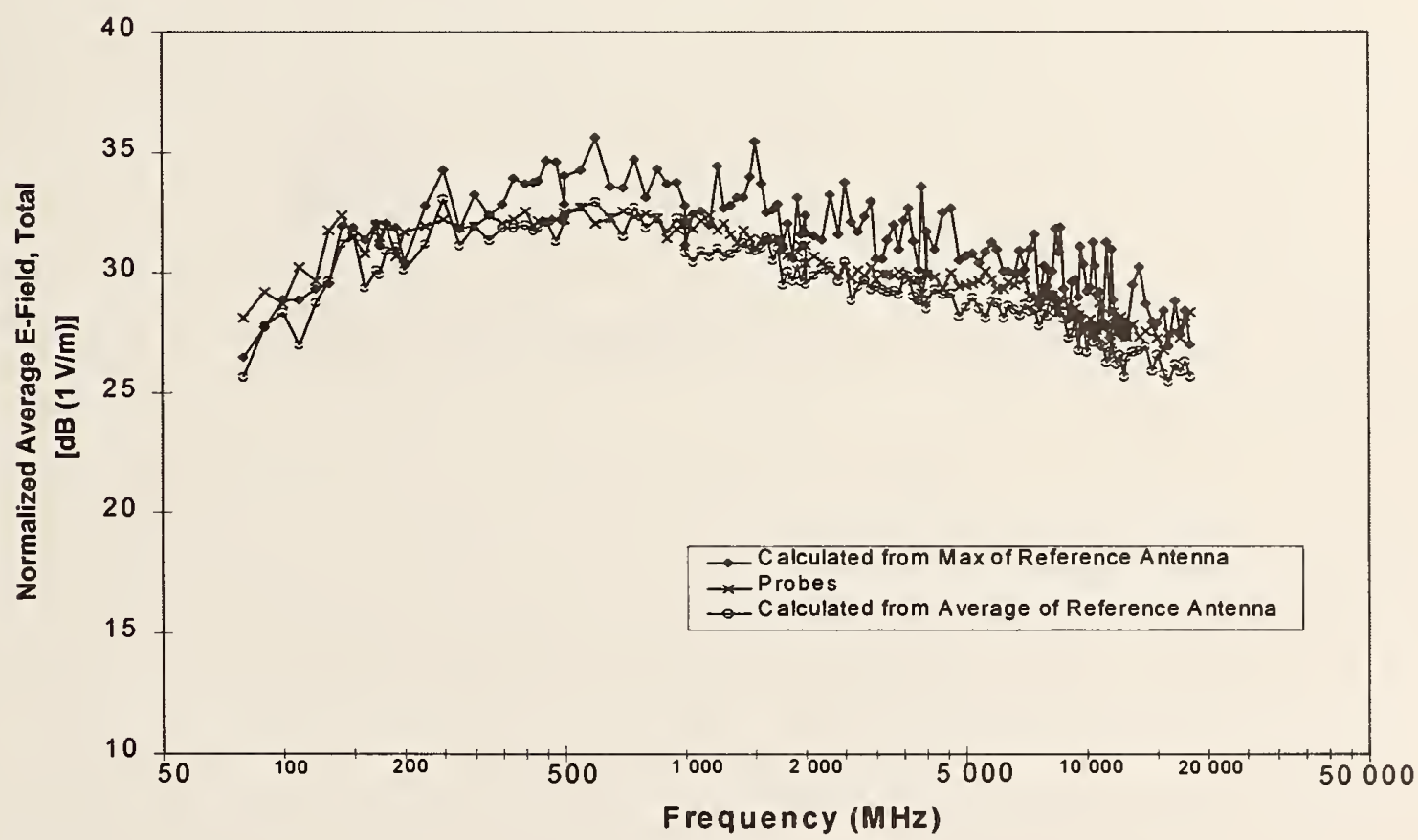

(d) 


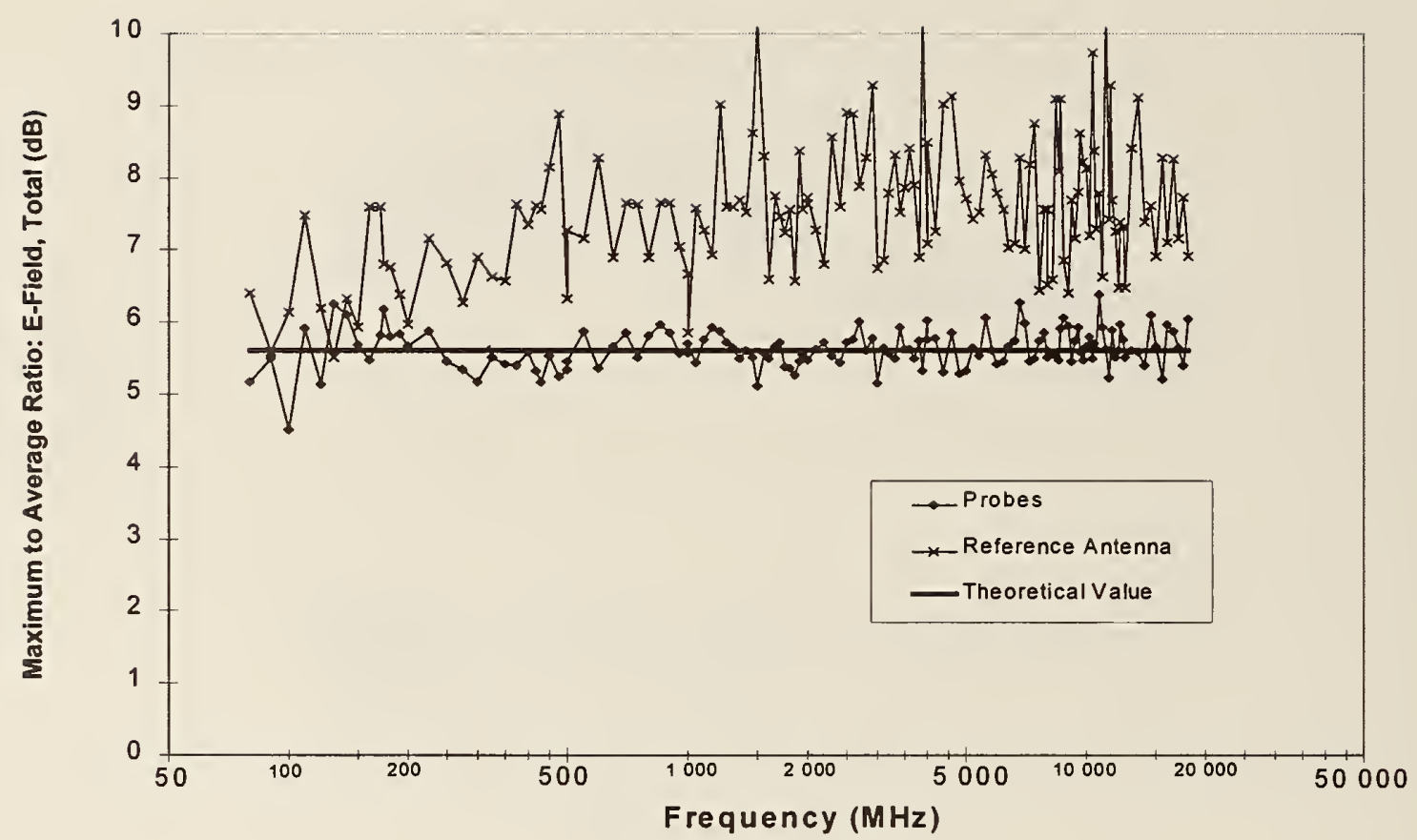

(a)

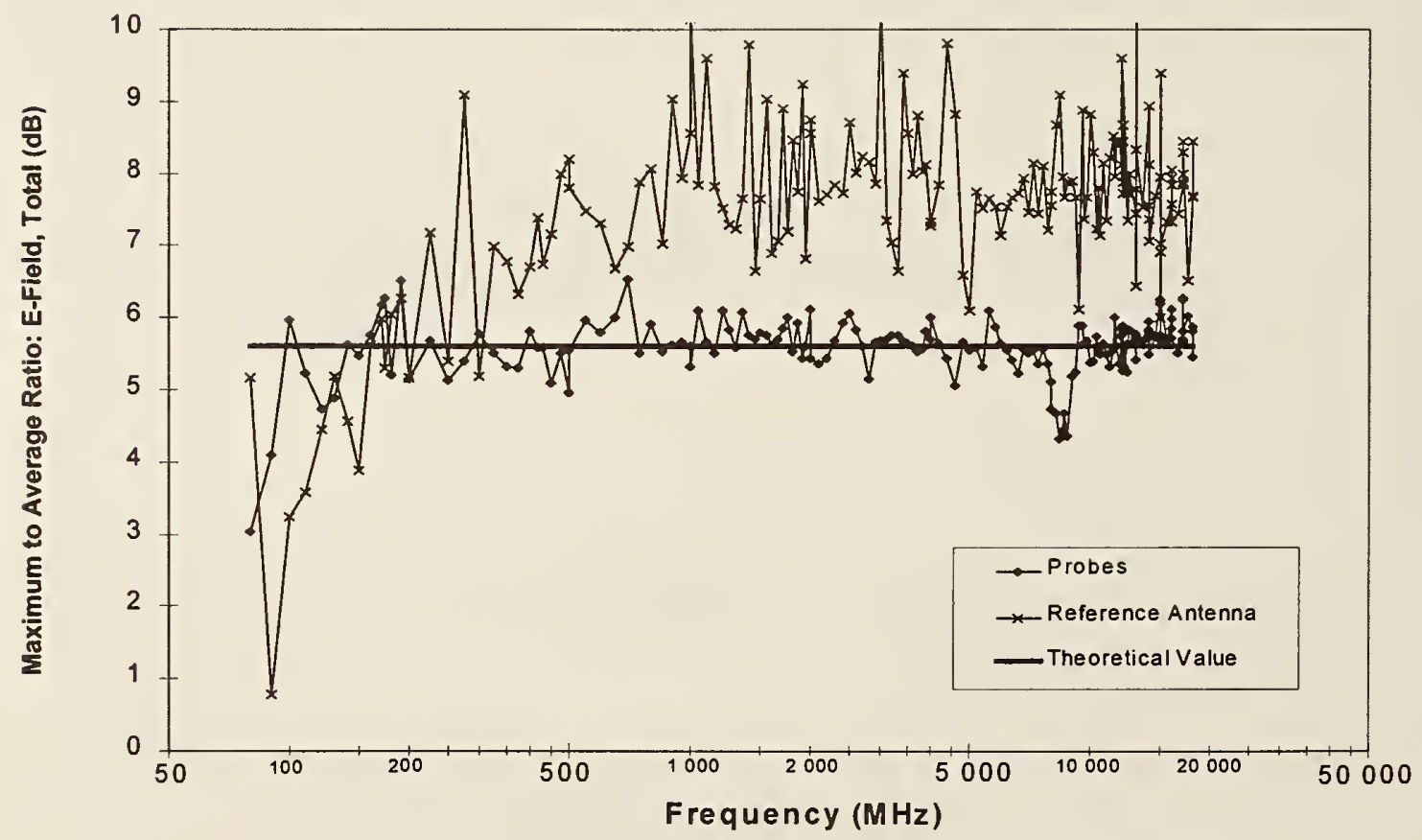

(b)

Figure 65. Max-to-average ratio of total electric field measured using probe system, and of the square root of power received by the reference antenna. (a) Chamber A. (b) Chamber B. 
Finally, we give our estimates of the average and maximum electric field for an empty chamber in Figure 66, with the estimate of the total electric field in Figure 66a, and the estimate of the rectangular component of the electric field in Figure 66b. Since the probes lower the quality factor and chamber gain of the chamber, we must use data taken with the network analyzer for these estimates. Unfortunately, since the network analyzer measures only received power and not electric field, the best we can do is estimate the electric field using the methods outlined above. The plots of the measured data given in these plots are actually estimates based on measurements of received power, and the estimated fits are based on these data as well. Since the link between the maximum received power and the maximum electric field (either form) is questionable due to possible compression problems, we do not include plots of the measured maximum electric field (we have no such data), and any estimates would simply be scaled versions of the average. Thus, we give only estimates of the maximum electric field, although based on our measurements using the probes, these estimates appear to be justified.

\section{Summary of Chamber Characteristics}

The majority of the data presented in this report were generated from measurements made using an automatic network analyzer. Even though the analyzer measured only four complex quantities at each frequency and paddle position, the number of ways that the data can be analyzed is unlimited. We limited our attention to a very small number of parameters, and the amount of final data processed and generated relating to these parameters is still overwhelming. One of our goals in presenting the final evaluations is to provide an efficient and compact format that is easy to use, yet not terribly bulky (we cannot avoid bulk altogether). To this end, we present tables that summarize all the figures associated with the final evaluations of the chambers. These tables include brief descriptions of the figures and the figure numbers for easy reference. A large number of these figures are unremarkable and will therefore be presented without comment. For details on how the data were processed to generate any of these plots, refer to Section 6. Any specific comments about the interpretation of the data that are unique to a particular chamber is will be discussed in their respective sections.

We also present tables of all equations that can be used to estimate the key parameters of each chamber as a function of frequency $f$ and number of paddle positions $N$. These parameters include minimum, average, and maximum chamber gain, average and maximum electric field for a given input power, average scalar power density for a given input power, and average quality factor. Where appropriate, we will give estimates of these parameters for measurements made while maintaining a constant incident power and a constant net input power. All values given in these tables assume that a minimum of 20 paddle positions are used, and the parameters of interest are in linear SI units (values of the average scalar power density expressed in units of $\mathrm{mW} / \mathrm{cm}^{2}$ can be computed from the average scalar power density expressed in SI units by dividing by 10). The equations for the magnitude of the maximum rectangular component of the electric field and the magnitude of the total electric field include functions $R(N)$ and $T(N)$. These functions have not been computed in closed form. Instead, they are based on the tables of the properties of the extreme values of the $\chi_{2}$ and the $\chi_{6}$ distributions given in Section 3.2.6. 


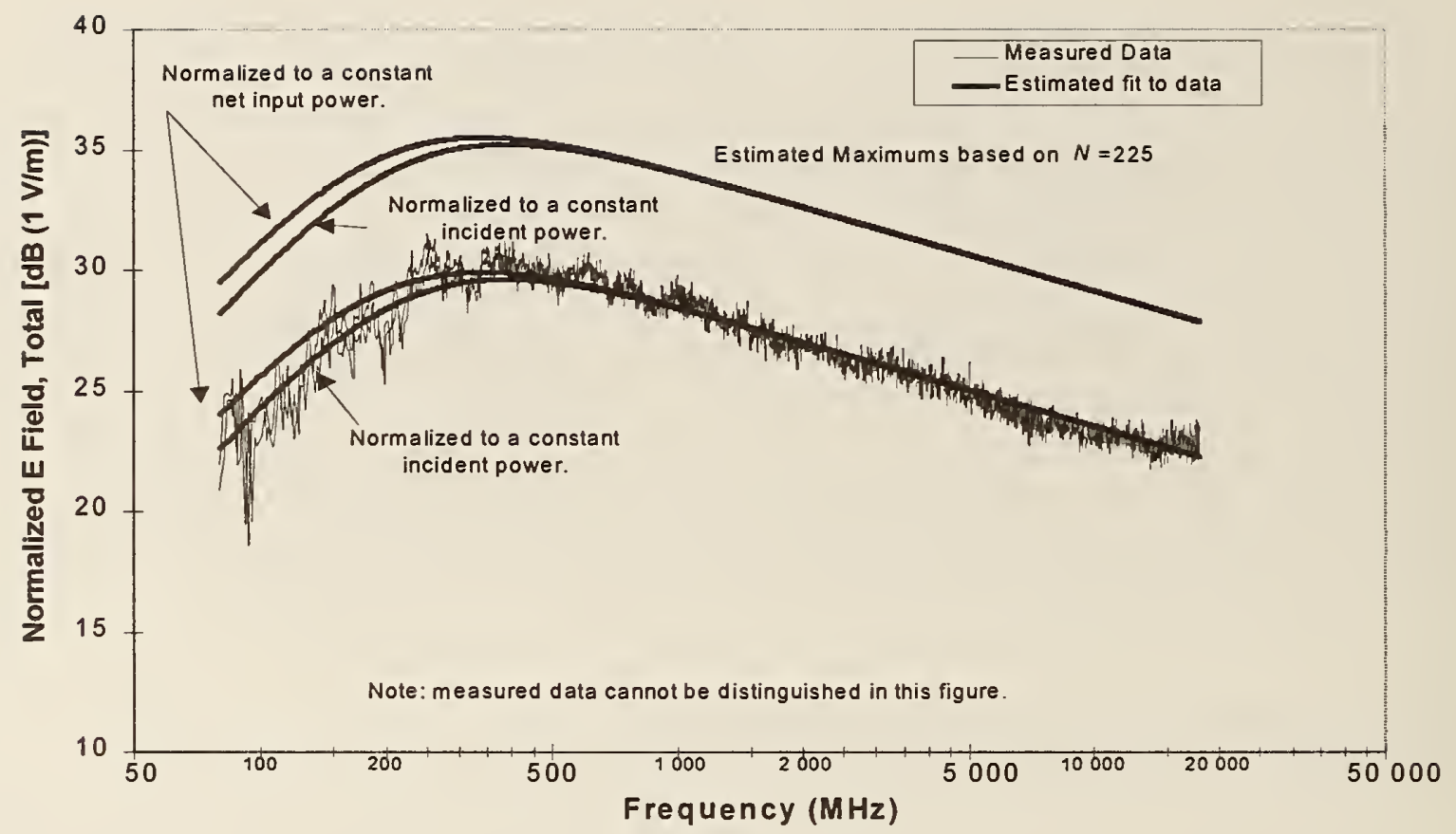

(a)

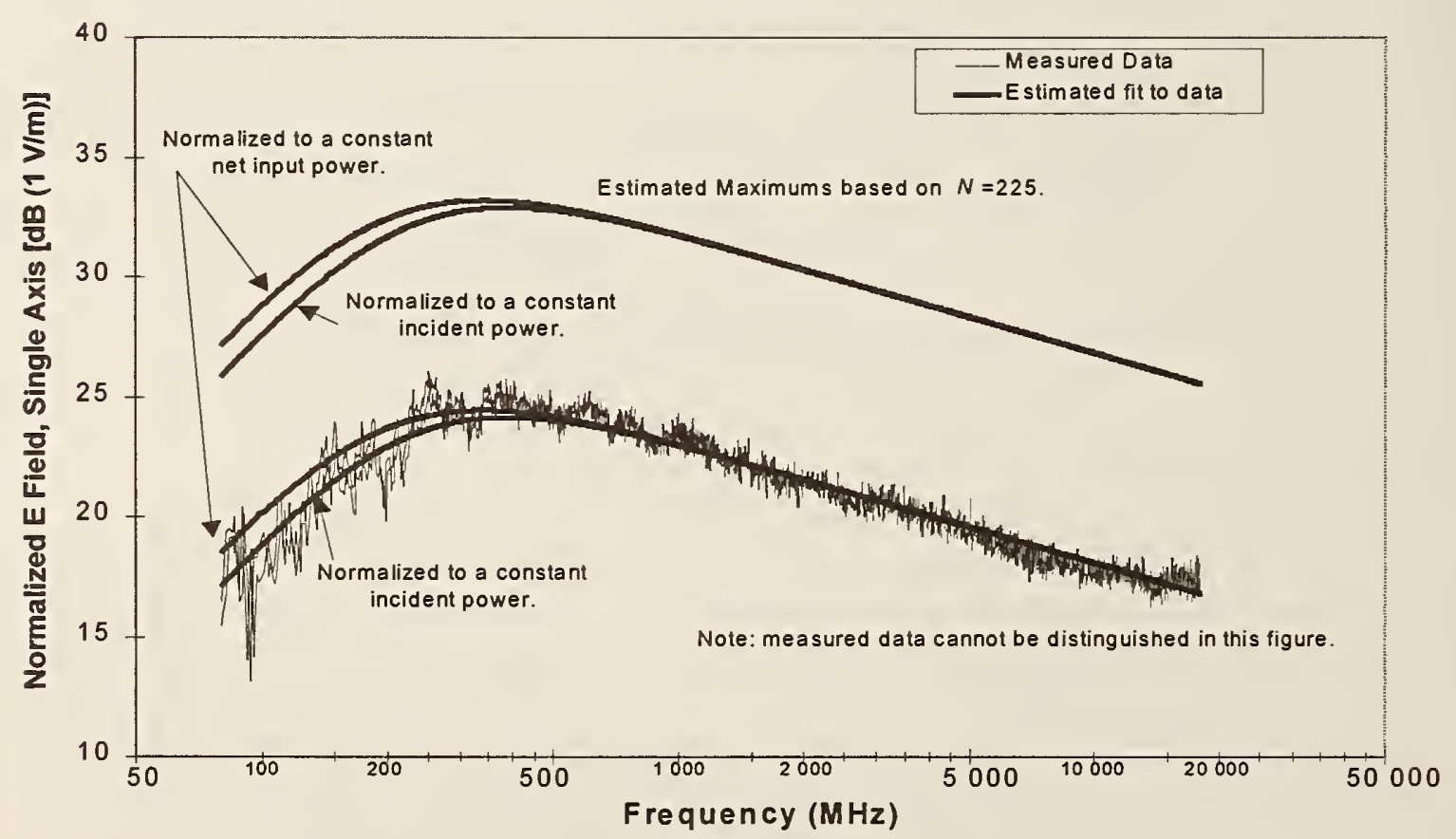

(b)

Figure 66. Calculated average and estimated maximum electric field in chamber A for a constant input power of $1 \mathrm{~W}$, based on received power from reference antenna. (a) Total electric field. (b) Single-axis electric field. 
The values of $R(N)$ are based on entries in Table 4 and the values of $T(N)$ are based on entries in Table 7 . The values of these functions are

$$
R(N) \approx \frac{\left\langle\left\lceil\chi_{2}\right\rceil_{N}\right\rangle}{1.253}
$$

and

$$
T(N) \approx \frac{\left\langle\left[\chi_{6}\right]_{N}\right\rangle}{2.350} .
$$

For values of $N$ other than those included in these tables, the mean for that value of $N$ can be calculated numerically or can be estimated from values in the tables for similar values of $N$.

The estimates of the average chamber quality factor include a term for the volume $V$ of the chamber. We write the variable $V$ instead of the actual estimates of the chamber volume so that any improved estimates of the volume can be easily incorporated into the equation. The actual value of $V$ that was used in the calculation of the chamber quality factor will be presented in the summary of that chamber.

All of the equations given in the tables of summary equations can be converted to decibels directly with minimal error (less than $0.1 \mathrm{~dB}$ for $N \geq 50$ ), with the exception of the minimum chamber gain. The estimates of minimum chamber gain can be expressed in decibels by computing $10 \cdot \log _{10}\left(\mathrm{MIN}\left(G_{C}\right)\right)-2.5$. We will attempt to note any parameter that may be affected when expressed in decibels.

Before presenting the final data, we will discuss some of the simplifying assumptions we have made. The measured data depended on a number of factors. For measurements made using a network analyzer, we had a choice as to which antenna was the transmitting antenna and which antenna was the receiving antenna. In the data presented on the antenna characteristics, both the transmitting and receiving antennas are given, but in the remainder of the figures we assume that data were measured in the forward direction only (the antenna connected to port 1 of the analyzer was the transmitting antenna). We did this to be consistent with the probe measurement system, which was limited to one direction.

We also had a choice as to normalization technique; that is, we could normalize based on a constant incident power or a constant net input power. We present both sets of results here, with the data measured assuming a constant incident power presented first, followed by the data measured assuming a constant net input power. For a given parameter, the plots based on both normalization methods will be presented on the same page, and if possible, the same figure for easy comparison. The data measured using the probe system always assumed a constant net input, so there is no comparison of normalization techniques presented for the probe data.

Following the analysis of the individual NASA chambers, some data taken in the NIST chamber are presented for comparison. Initially, the variability (uncertainty) in the measurements taken in the NIST chamber was so much greater than the variability in the measurements taken in the NASA chambers that we could not do a valid comparison. Because of this, we modified our chamber by increasing the size of the paddle, and this significantly improved the quality of the 
data, but the NASA data still appeared to be "better behaved" than the NIST data. We present data taken in the NIST chamber both before and after the paddle was modified. These data can be used to demonstrate the importance of paddle size related to the uncertainty in the measurement.

Finally, we present some general comments about the differences and similarities of the NASA chambers and the NIST chamber.

\subsection{Summary of Chamber $A$}

A summary of the figures we used to describe the characteristics of Chamber A is given in Table 8. The figures are grouped with antenna characteristics presented first, followed by an evaluation of the mechanical stirrers as described by the unstirred component of $S_{21}$, and then the ratios of the extreme values (maximum-to-average ratio, maximum-to-minimum ratio, average-to-minimum ratio). We then give the average and maximum chamber gain, residuals of chamber gain, followed by all parameters that can be estimated or predicted from measurements of chamber gain (electric field for an input power of $1 \mathrm{~W}$ and the residuals of electric field, average scalar power density, and average quality factor. The estimated volume of Chamber A used to calculate the quality factor of the chamber was $290.8 \mathrm{~m}^{3}$. The characteristics of the electric field as measured by the probe system are presented next, including the average and maximum magnitude of the electric field (both rectangular component and total). Finally, we present data from some of the tools used to verify the statistical characteristics of the power received from an antenna in the chamber. These are plots of the cumulative distribution and the normalized standard deviation.

The equations describing the key parameters are given in Table 9. All equations are based on curve fit parameters for the average chamber gain of $a=3.210$ and $b=4.299 \cdot 10^{-21}$ for data normalized to a constant incident power, and values of $a=2.317$ and $b=4.287 \cdot 10^{-21}$ for data normalized to a constant net input power. 
Table 8. Summary of figures related to Chamber A.

Minimum, average, and maximum VSWR

Minimum, average, and maximum reflected power

Figure 67

Mismatch correction factor

Figure 68

Estimated efficiencies of antennas

Figure 69

Unstirred component of $S_{21}$

Figure 70

Maximum-to-average ratio

Figure 71

Maximum-to-minimum ratio

Figure 72

Average-to-minimum ratio

Figure 73

Average and maximum chamber gain

Figure 74

Residuals of chamber gain

Figure 75

Electric field

Figure 76

Residuals of electric field

Figure 77

Scalar power density

Figure 78

Quality factor

Figure 79

Figure 80

Average magnitude of electric field (rectangular component) using probe system

Average magnitude of electric field (total) using probe system

Figure 81

Maximum magnitude of electric field (rectangular component) using probe system

Maximum magnitude of electric field (total) using probe system

Comparison of average $\mathrm{x}, \mathrm{y}$, and $\mathrm{z}$ components of electric field

Figure 82

Figure 83

Figure 84

Figure 85

Comparison of maximum $\mathrm{x}, \mathrm{y}$, and $\mathrm{z}$ components of electric field

Figure 86

Cumulative distribution of received power at $1 \mathrm{GHz}$

Figure 87

Normalized standard deviation of received power

Figure 88 
Table 9. Estimated equations for descriptive characteristics of Chamber A.

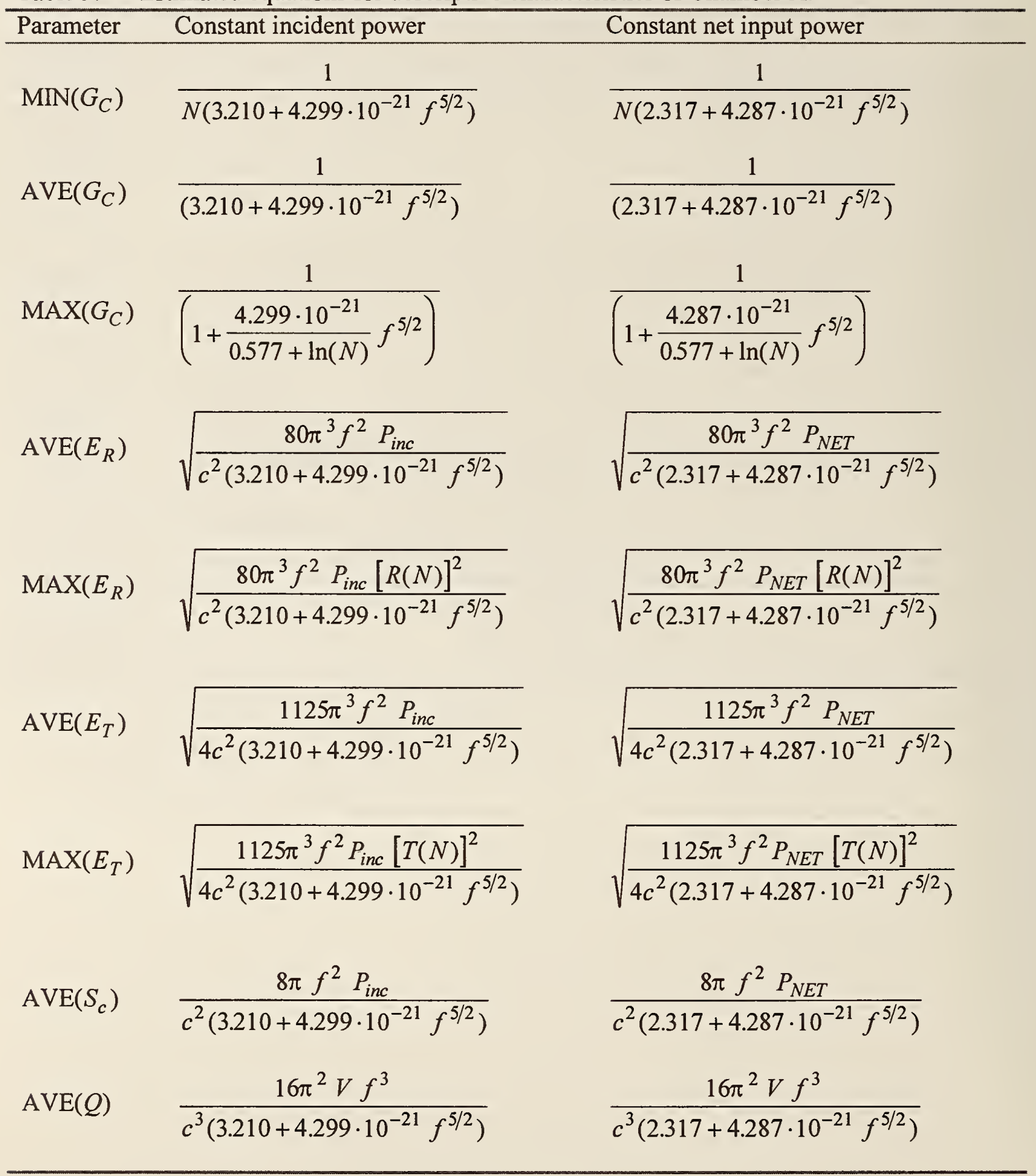

144 


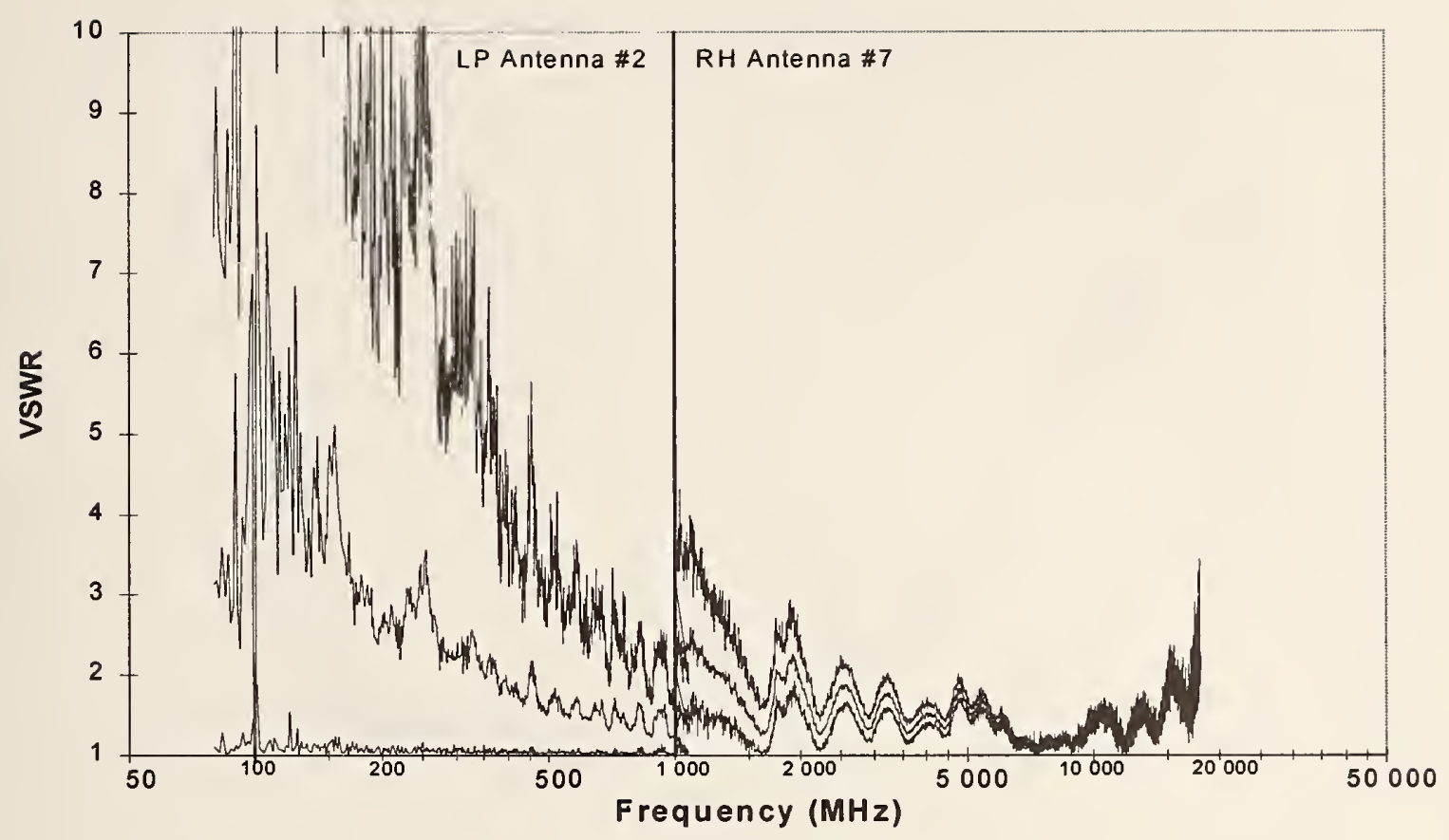

(a)

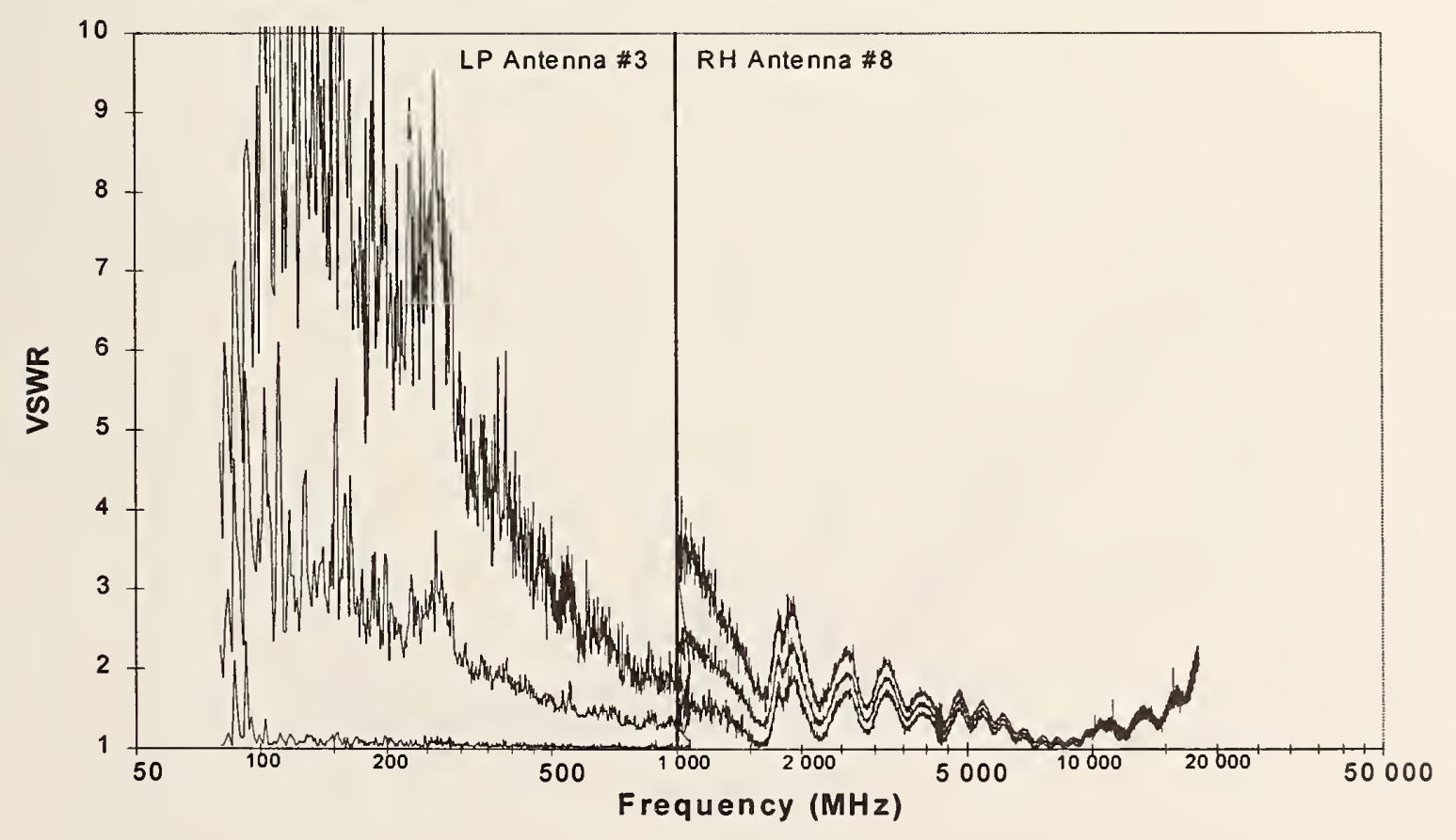

(b)

Figure 67. Minimum, average, and maximum VSWR in chamber A. (a) Port 1 transmitting (forward). Log periodic antenna 2, $80 \mathrm{MHz}$ to $1.08 \mathrm{GHz}$. Ridged horn antenna 7, $1 \mathrm{GHz}$ to $18 \mathrm{GHz}$. (b) Port 2 transmitting (reverse). Log periodic antenna 3, $80 \mathrm{MHz}$ to $1.08 \mathrm{GHz}$. Ridged horn antenna $8,1 \mathrm{GHz}$ to $18 \mathrm{GHz}$. 


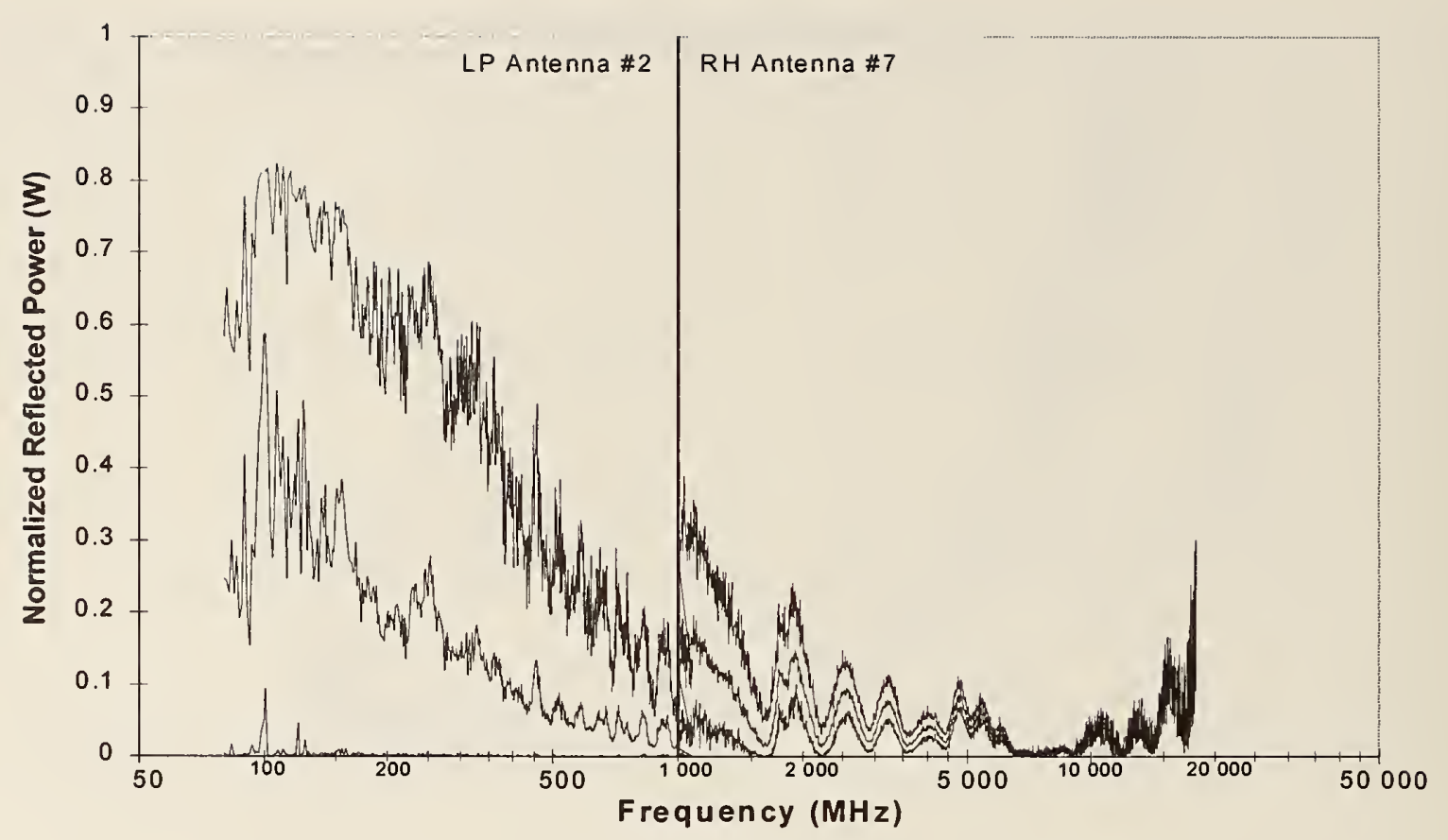

(a)

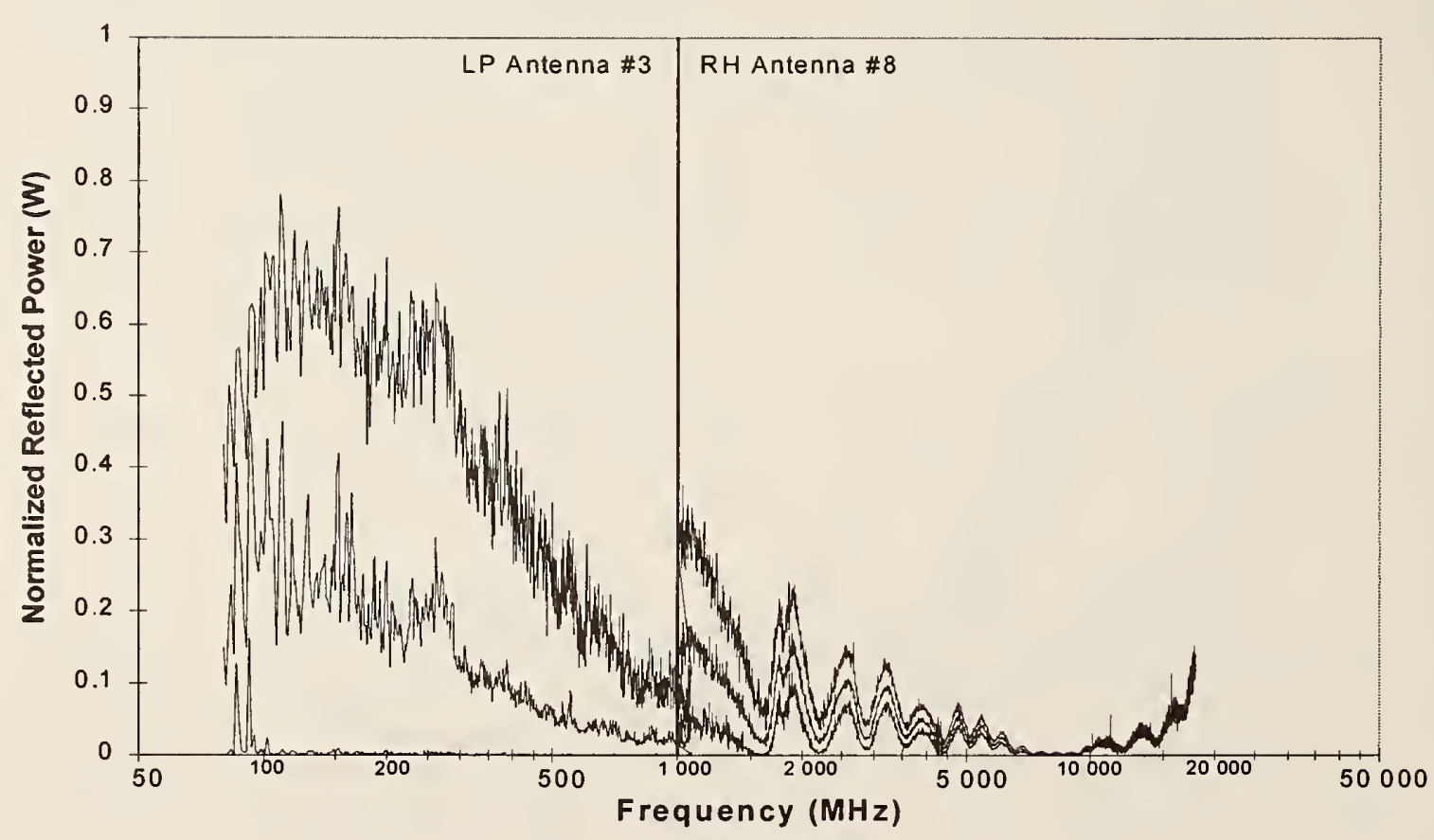

(b)

Figure 68. Minimum, average, and maximum reflected power for an incident power of $1 \mathrm{~W}$ in chamber A. (a) Port 1 transmitting (forward). Log periodic antenna $2,80 \mathrm{MHz}$ to $1.08 \mathrm{GHz}$. Ridged horn antenna 7, $1 \mathrm{GHz}$ to $18 \mathrm{GHz}$. (b) Port 2 transmitting (reverse). Log periodic antenna 3, $80 \mathrm{MHz}$ to $1.08 \mathrm{GHz}$. Ridged horn antenna 8, $1 \mathrm{GHz}$ to $18 \mathrm{GHz}$. 


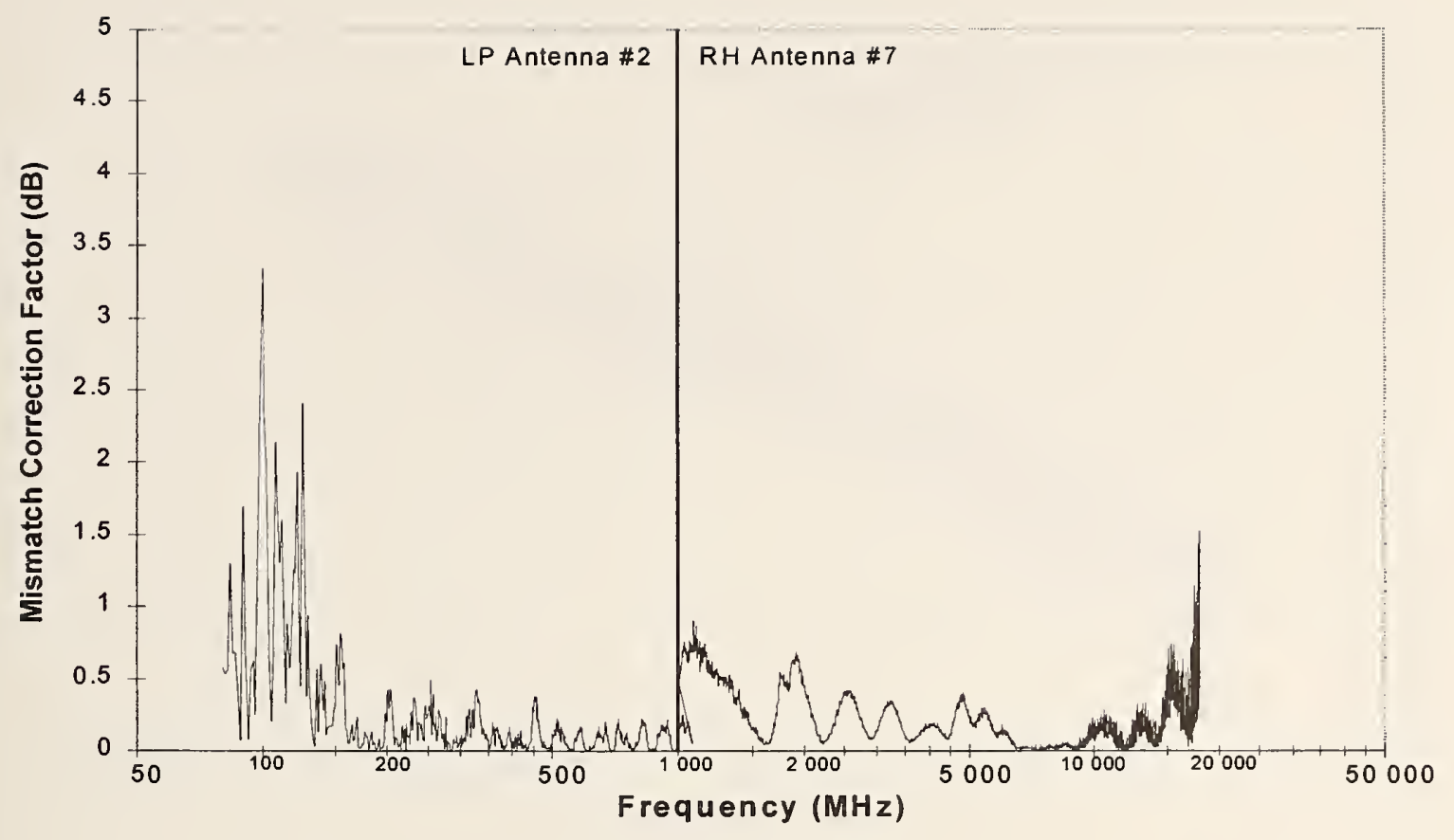

(a)

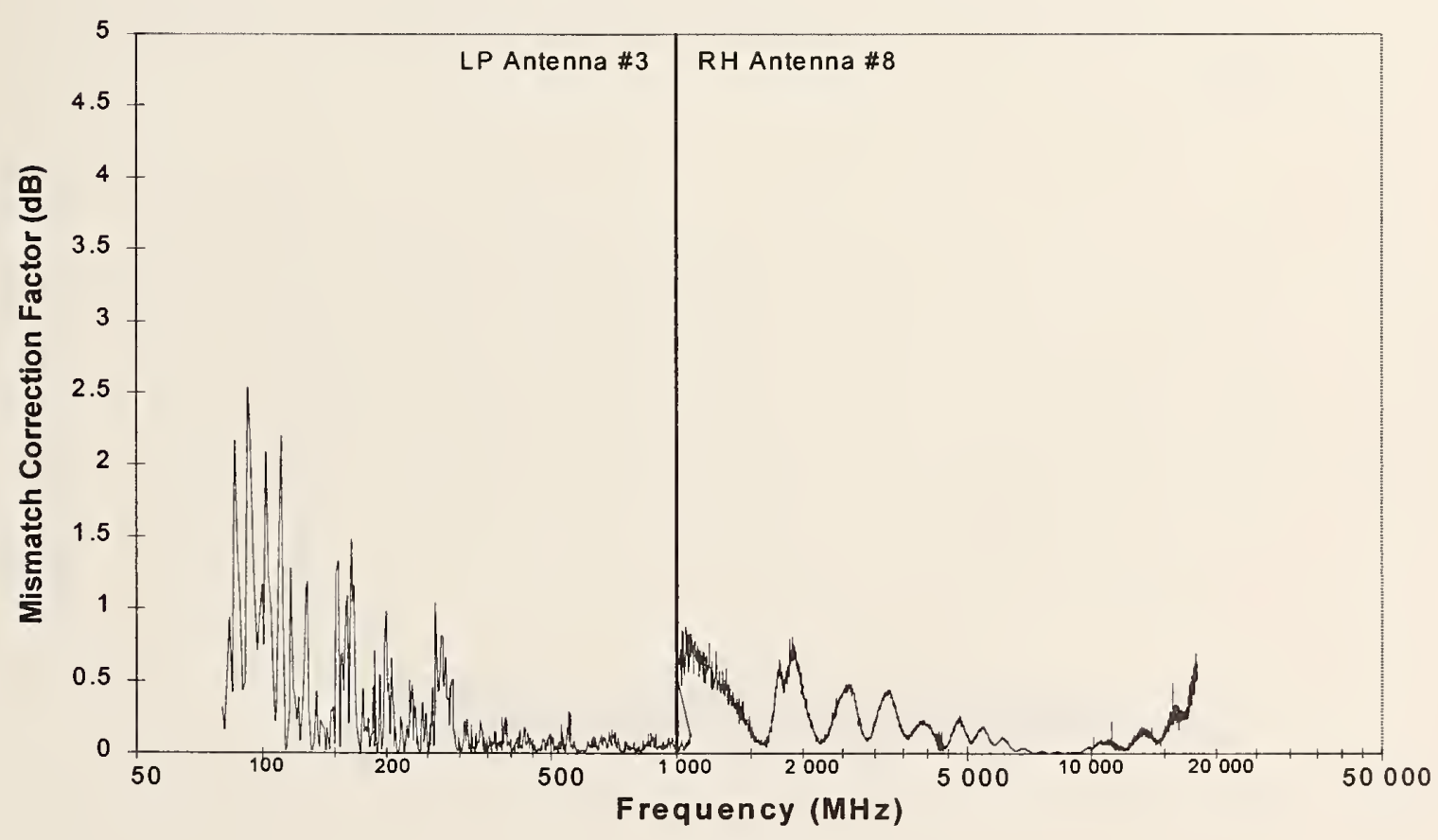

(b)

Figure 69. Mismatch correction factor in chamber A. (a) Port 1 transmitting (forward). Log periodic antenna 2, $80 \mathrm{MHz}$ to $1.08 \mathrm{GHz}$. Ridged horn antenna 7, $1 \mathrm{GHz}$ to $18 \mathrm{GHz}$. (b) Port 2 transmitting (reverse). Log periodic antenna 3, $80 \mathrm{MHz}$ to $1.08 \mathrm{GHz}$. Ridged horn antenna $8,1 \mathrm{GHz}$ to $18 \mathrm{GHz}$. 


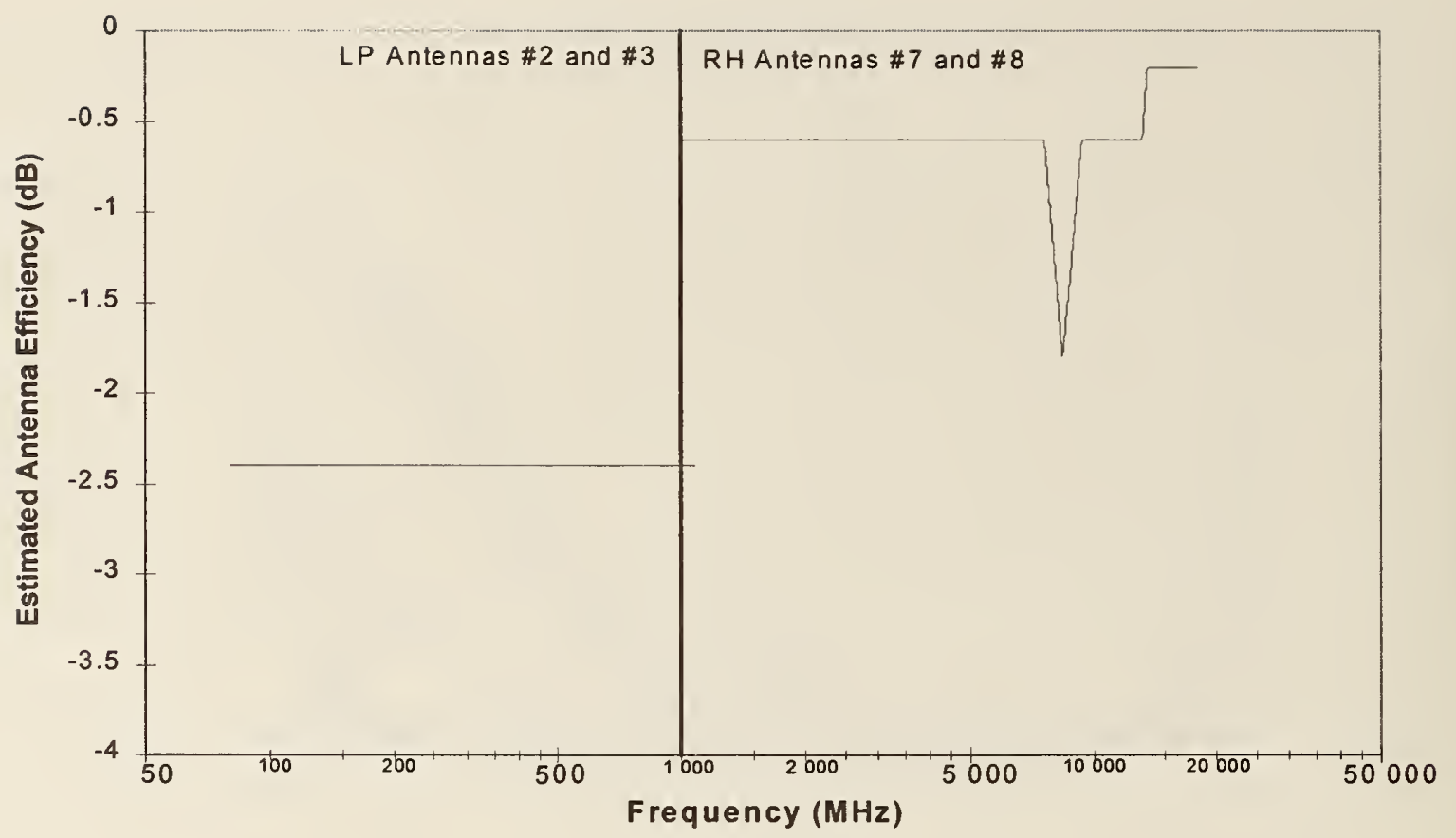

Figure 70. Estimated efficiencies of measurement antennas in chamber A. Log periodic antennas 2 and 3,80 MHz to $1.08 \mathrm{GHz}$. Ridged horn antennas 7 and $8,1 \mathrm{GHz}$ to $18 \mathrm{GHz}$. 


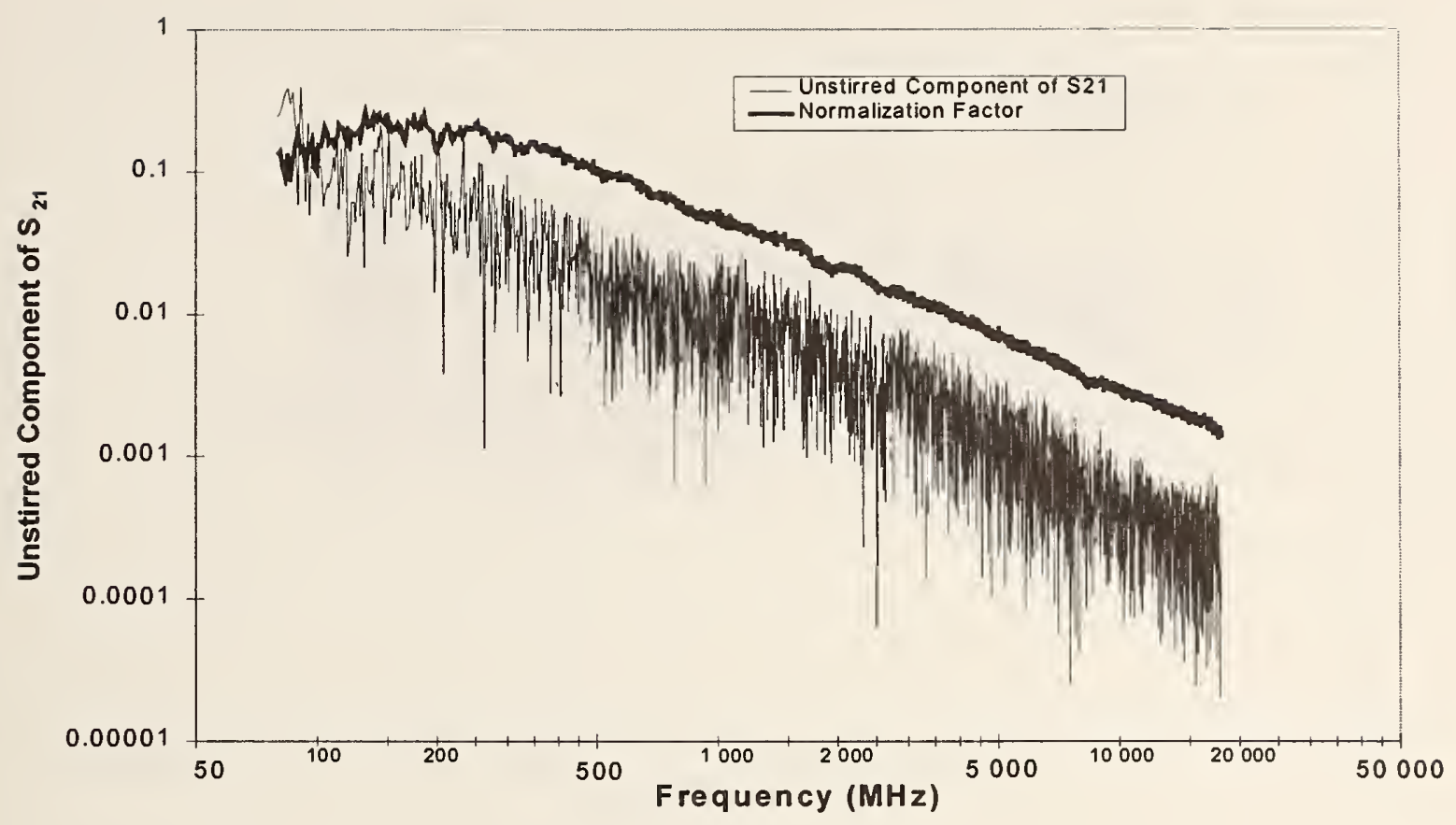

(a)

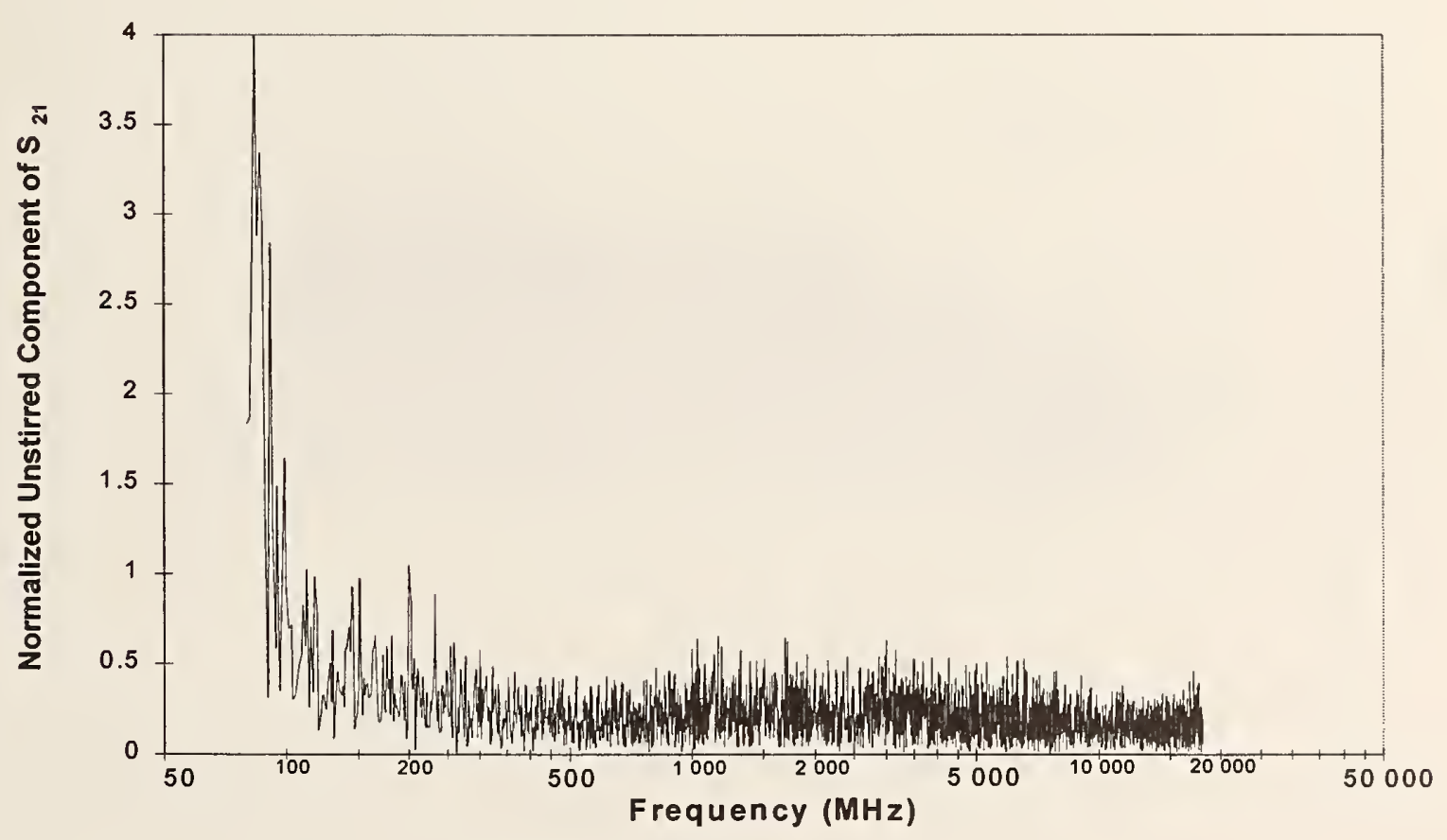

(b)

Figure 71. Evaluation of mechanical stirrers in chamber A. (a) Unstirred component of $S_{21}$. (b) Normalized unstirred component of $S_{21}$. 


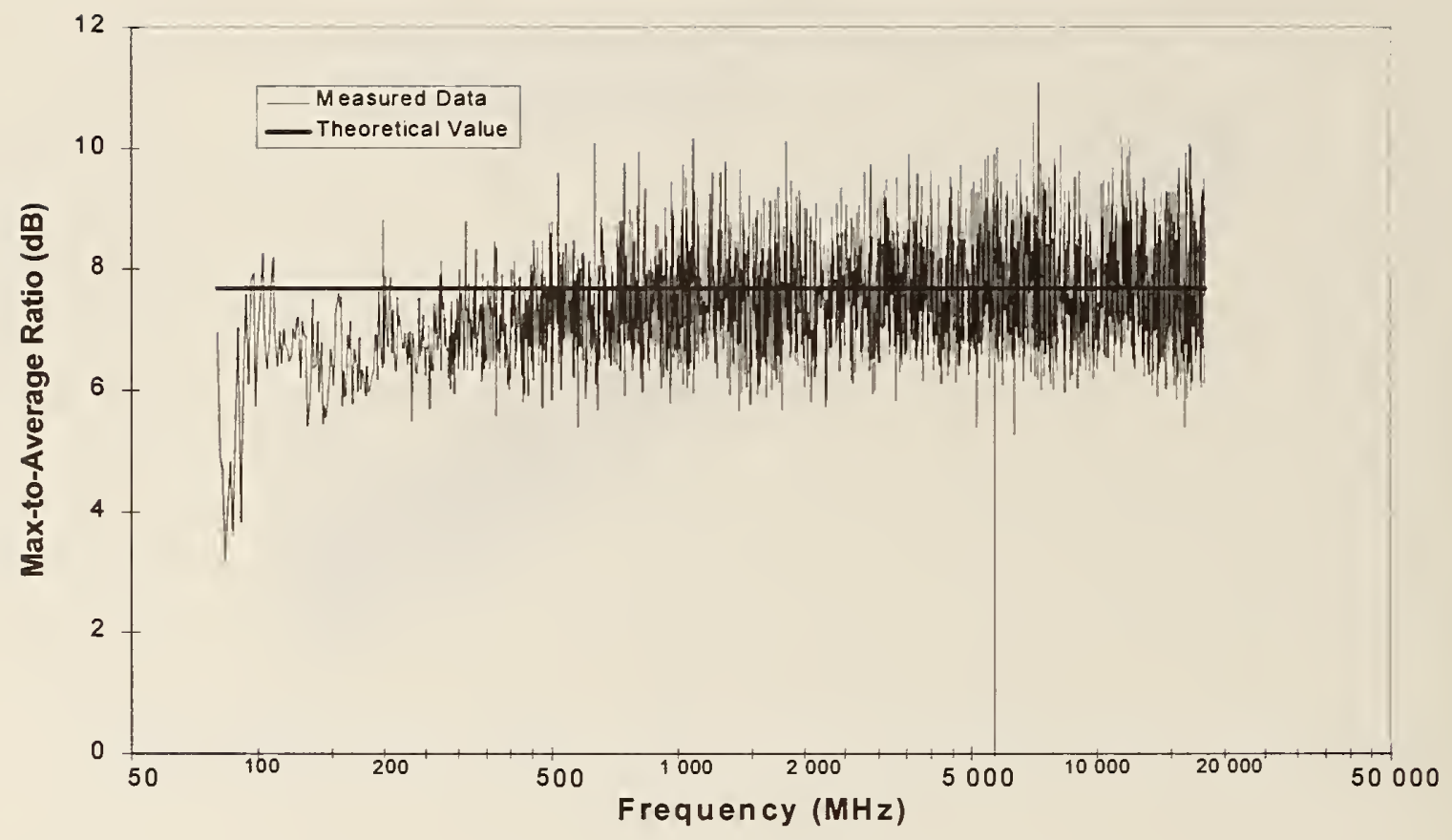

(a)

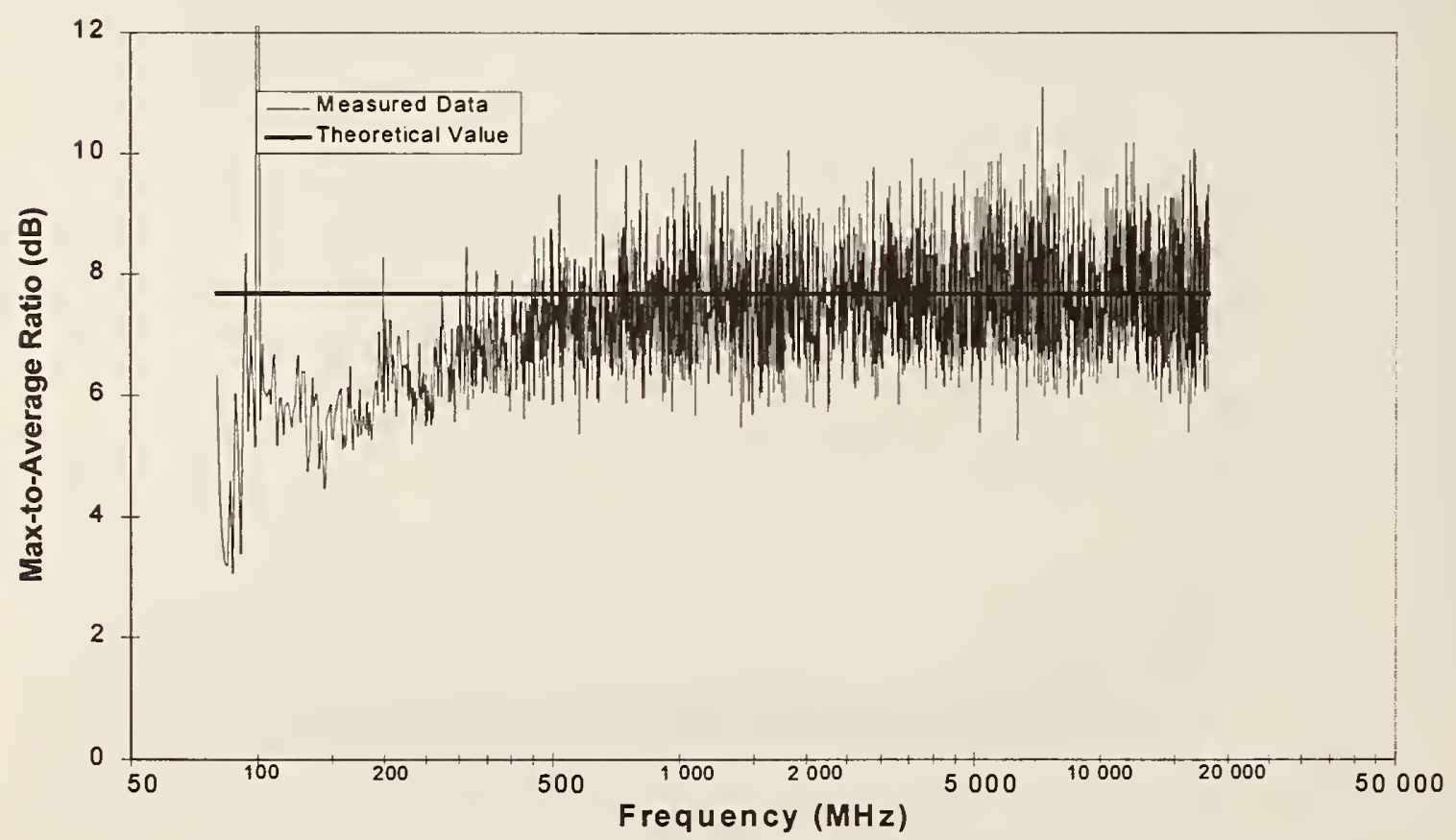

(b)

Figure 72. Max-to-average ratio in chamber A. (a) Constant incident power. (b) Constant net input power. 


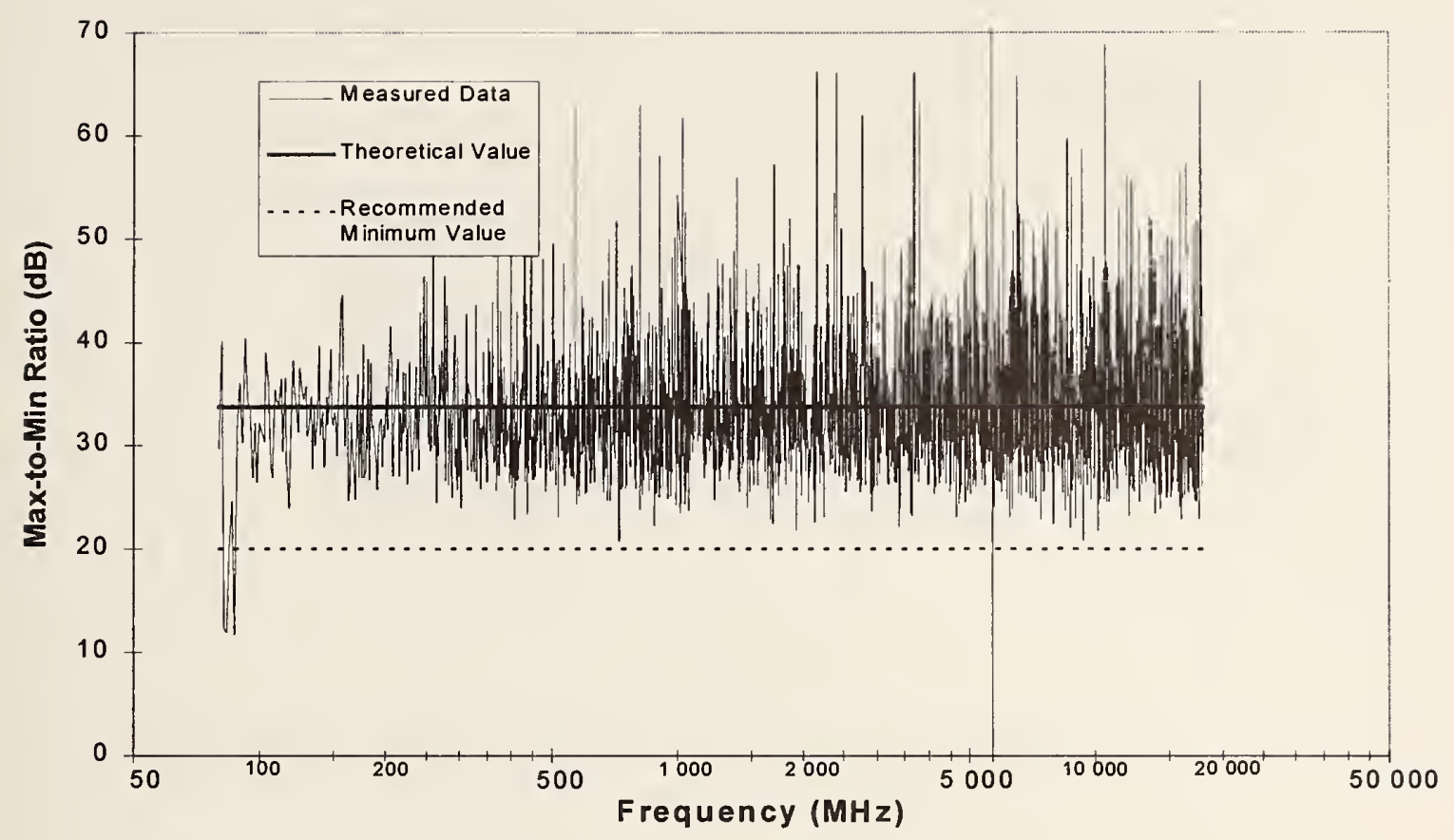

(a)

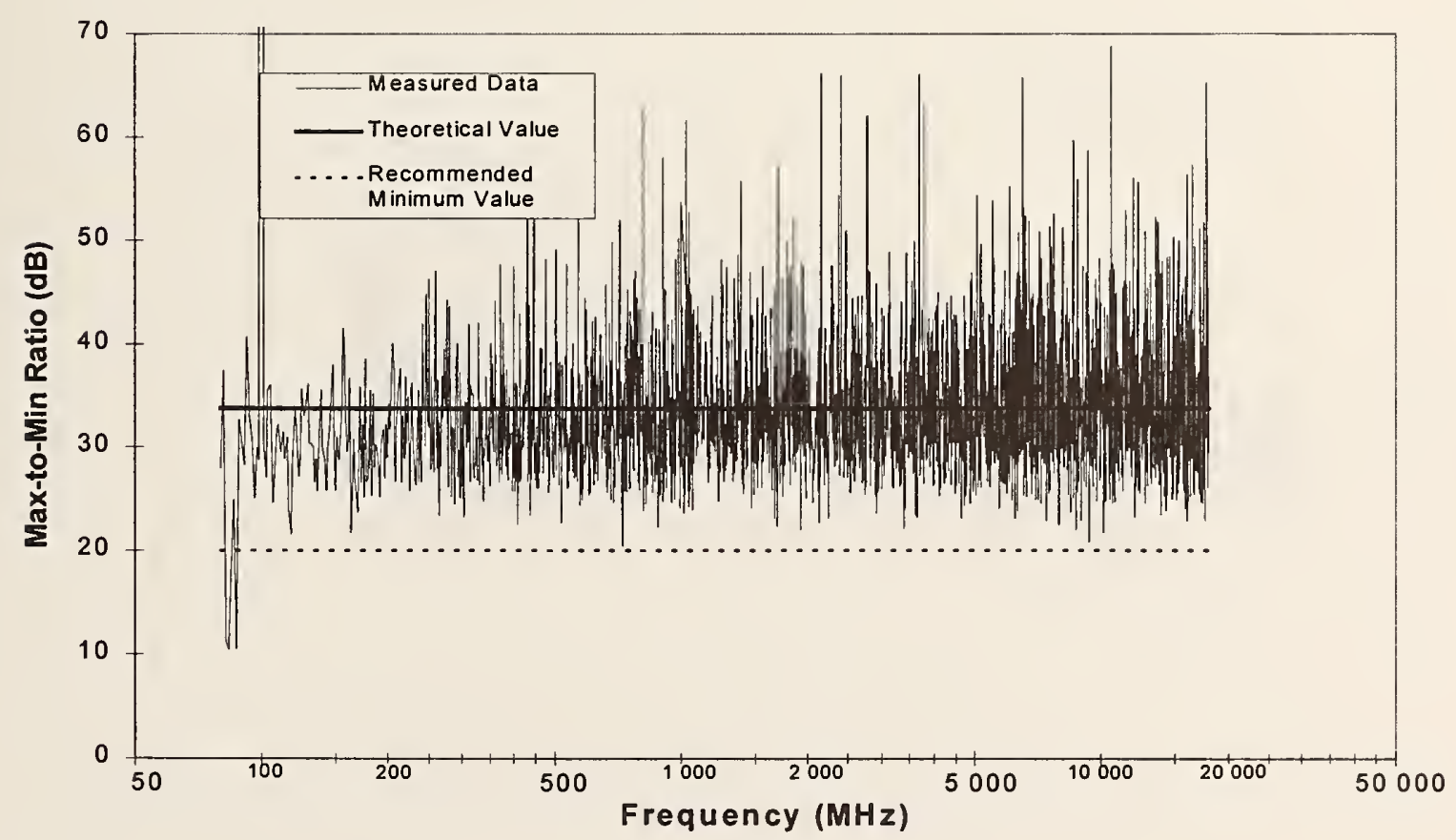

(b)

Figure 73. Max-to-min ratio in chamber A. (a) Constant incident power. (b) Constant net input power. 


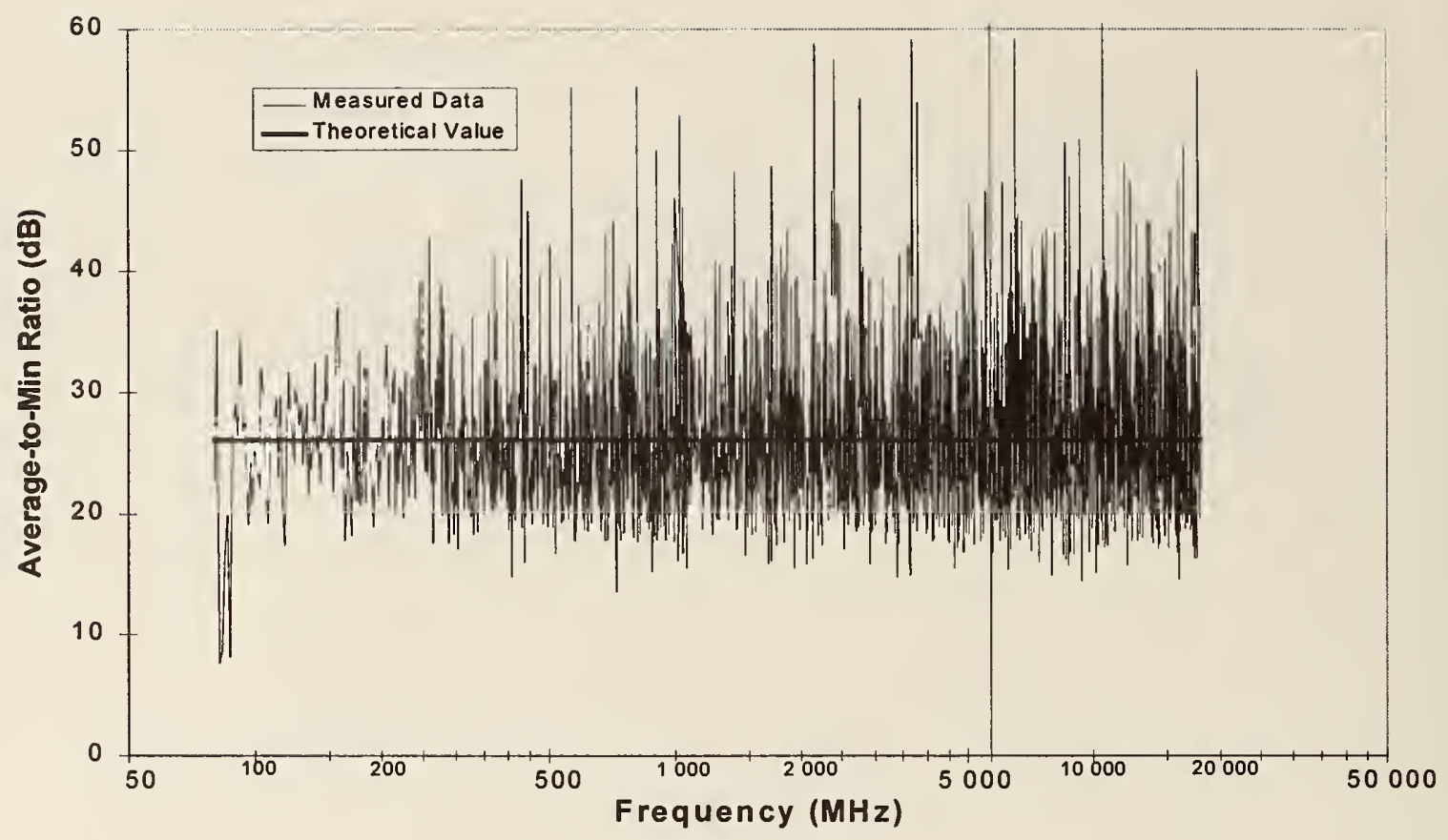

(a)

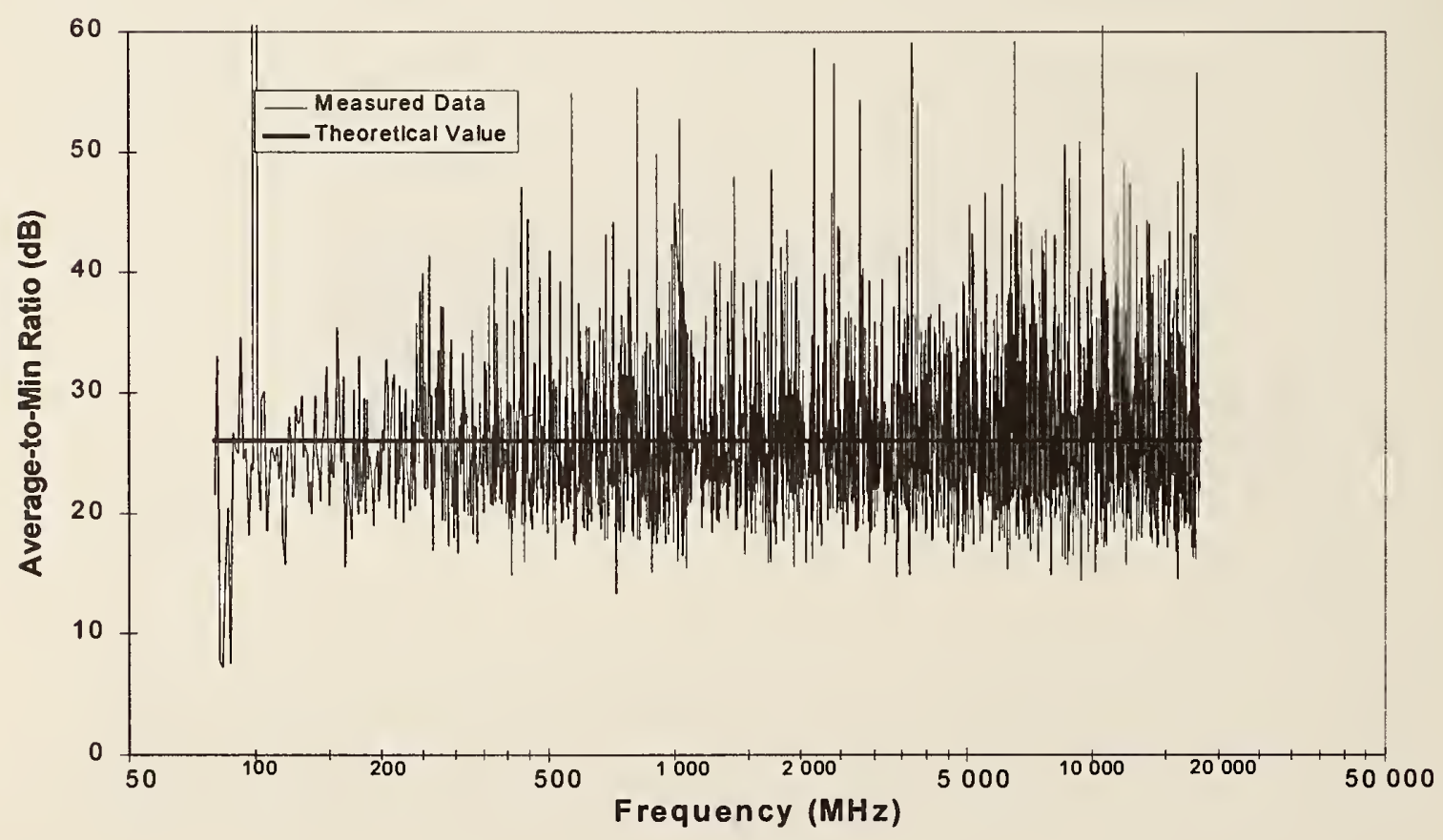

(b)

Figure 74. Average-to-min ratio in chamber A. (a) Constant incident power. (b) Constant net input power. 


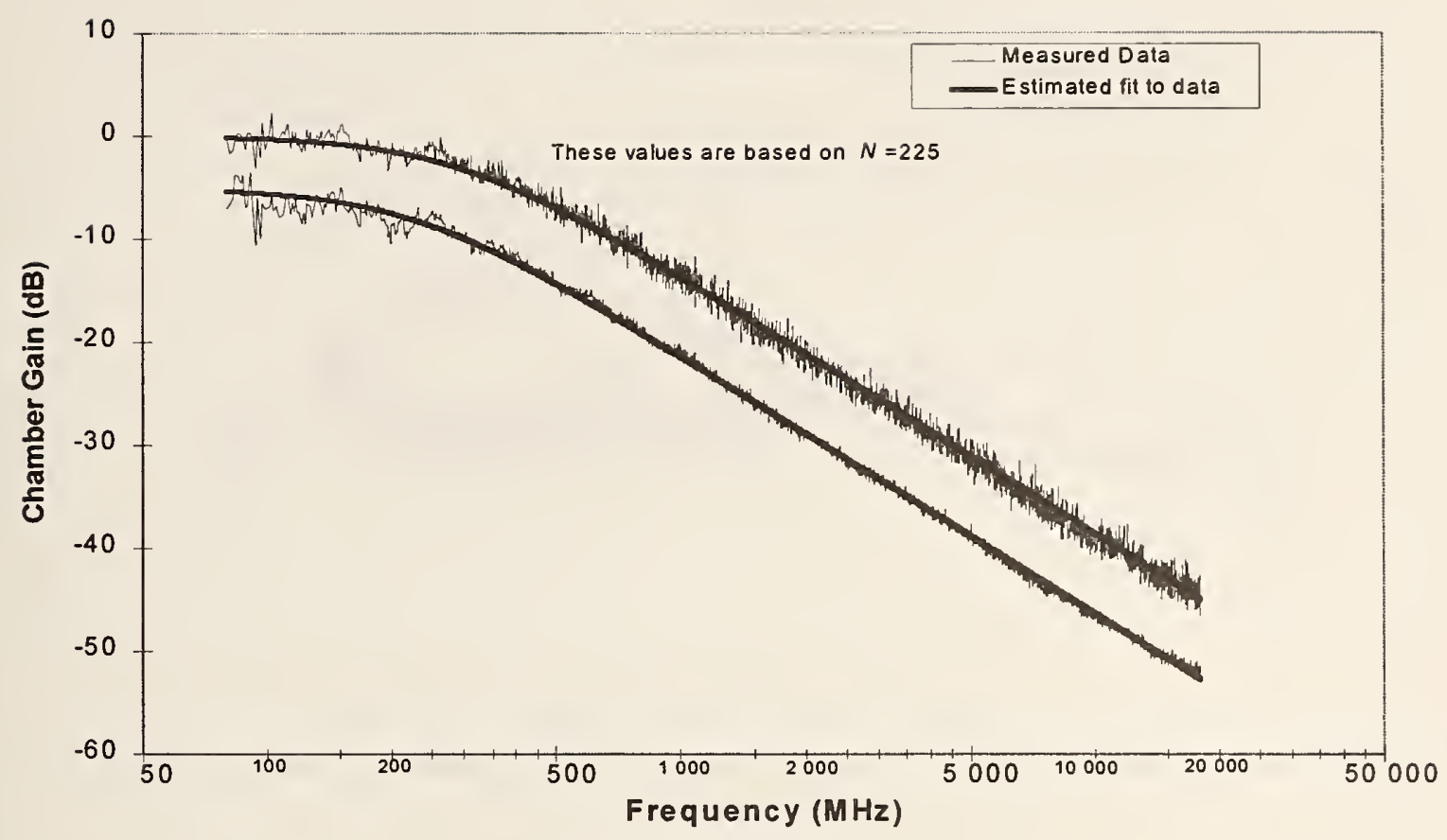

(a)

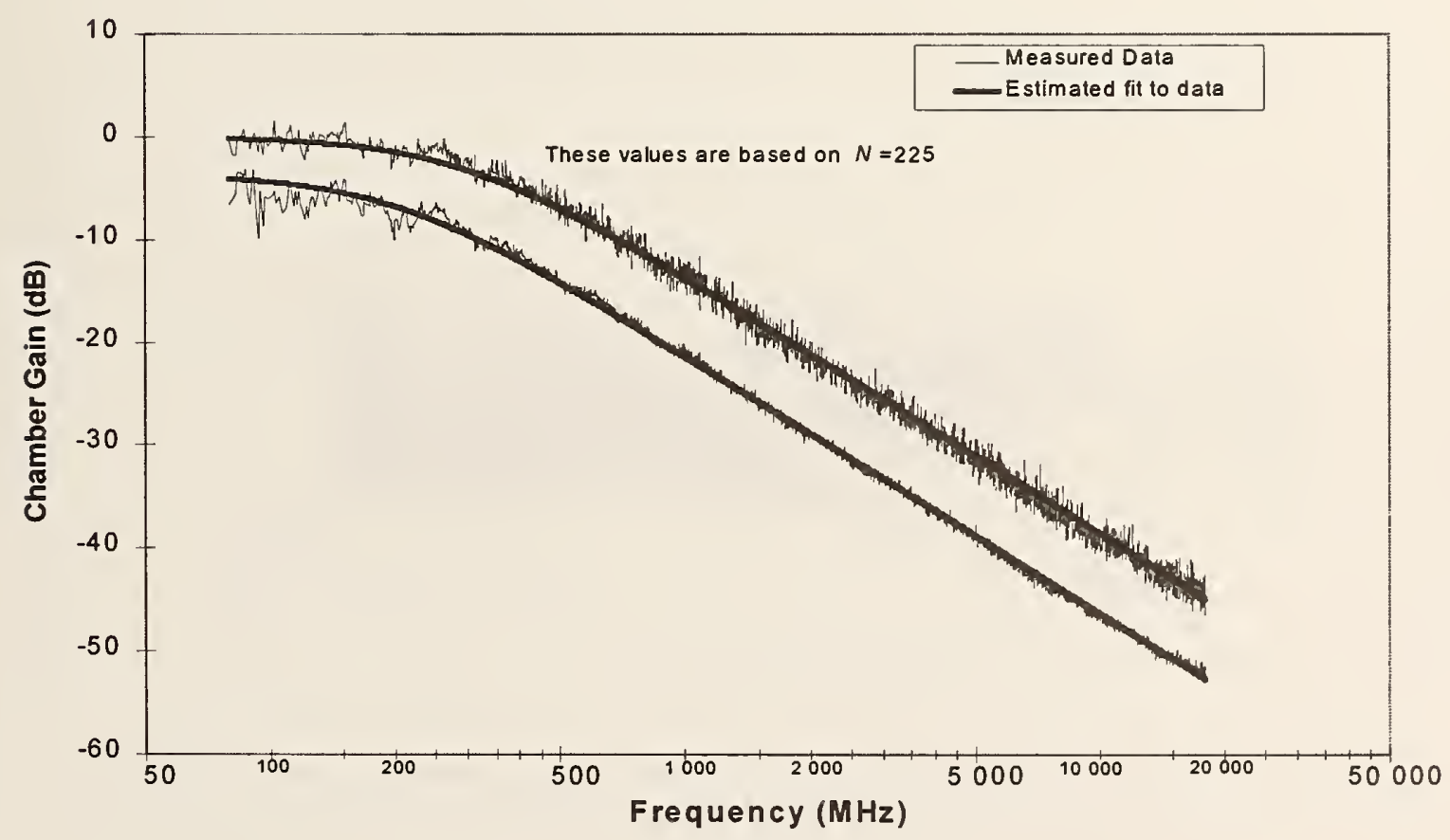

(b)

Figure 75. Average and maximum chamber gain in chamber A. (a) Constant incident power. (b) Constant net input power. 


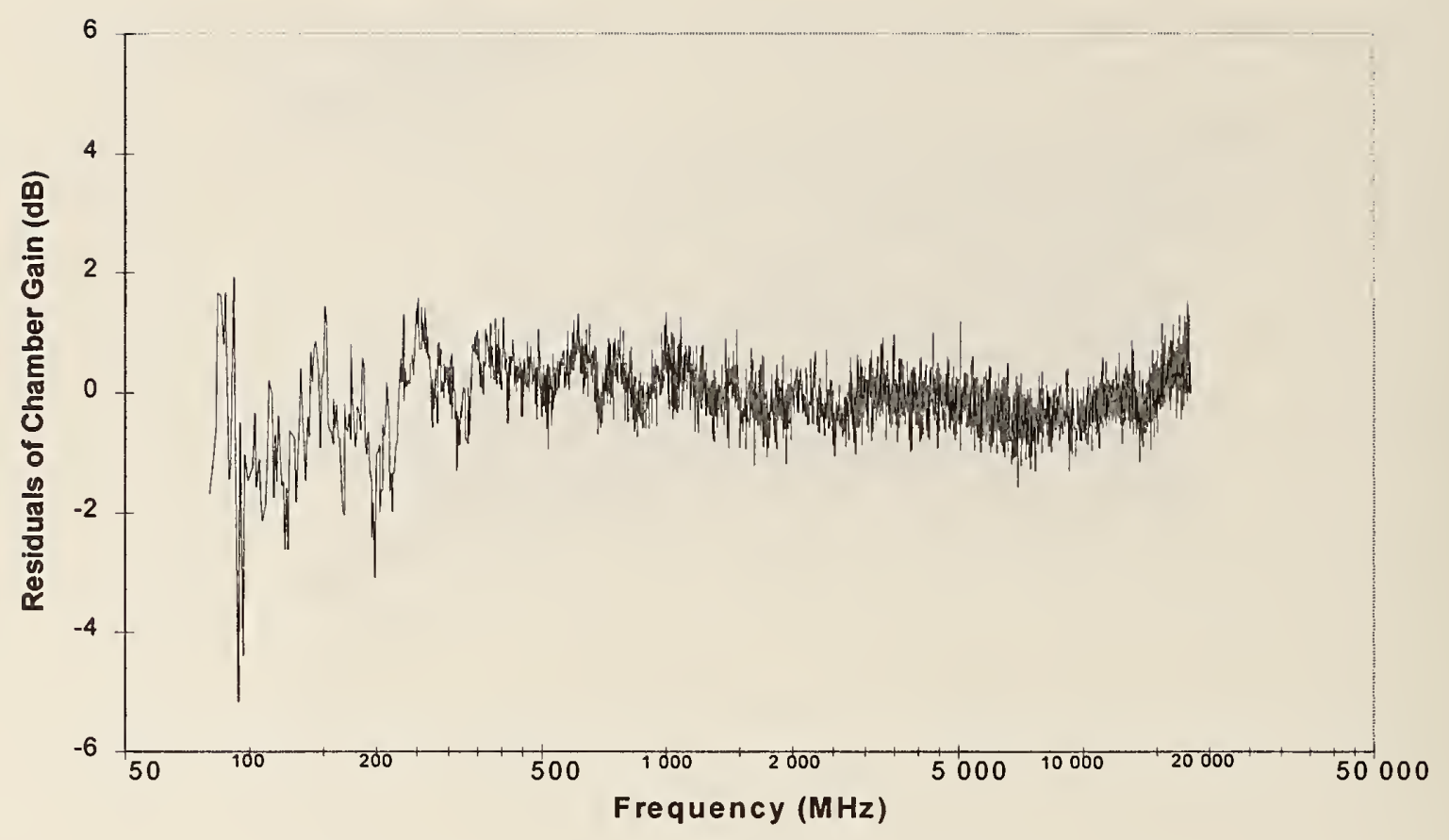

(a)

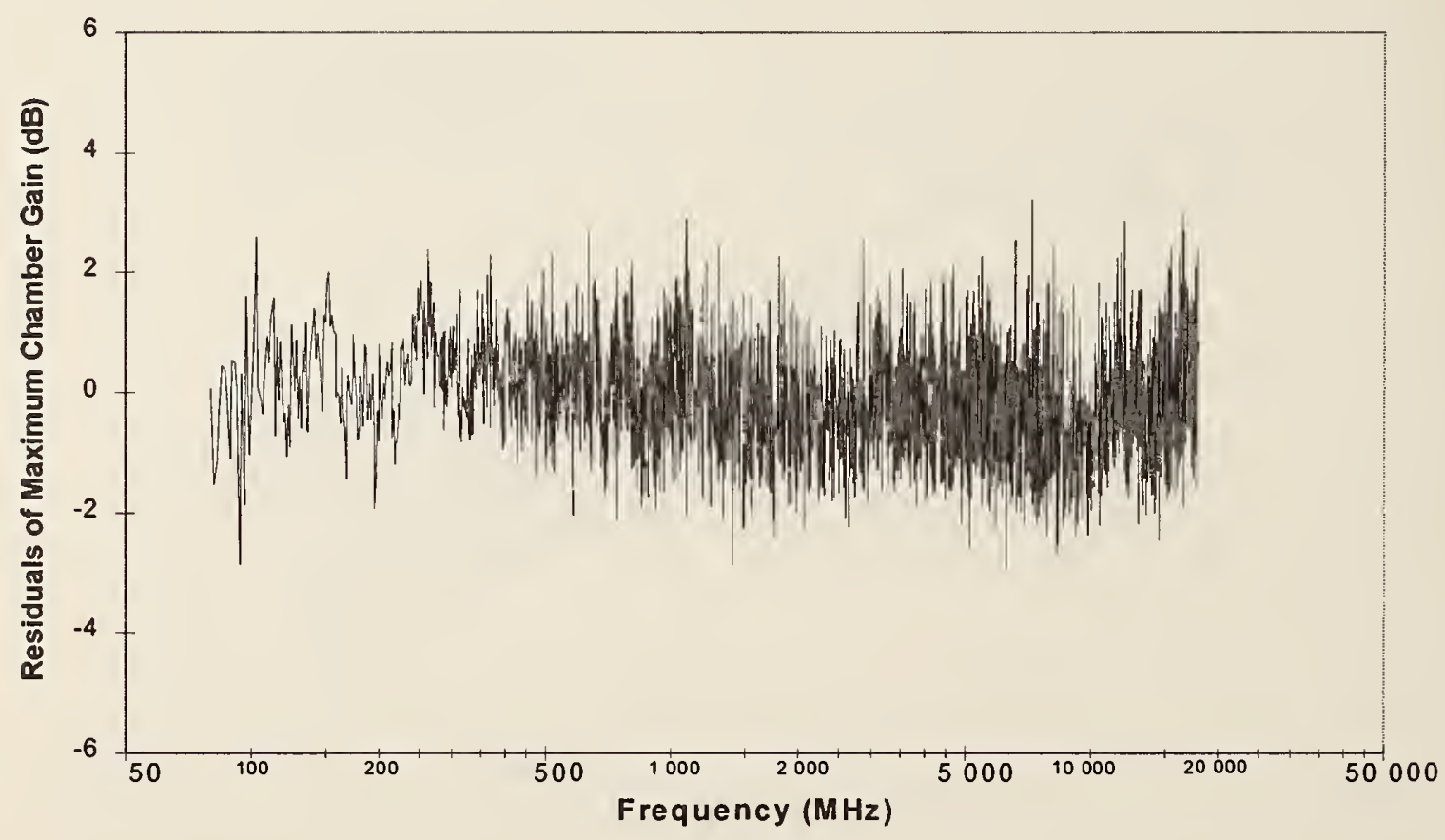

(b)

Figure 76. Residuals of chamber gain in chamber A. (a) Residuals of average chamber gain.

(b) Residuals of maximum chamber gain. 


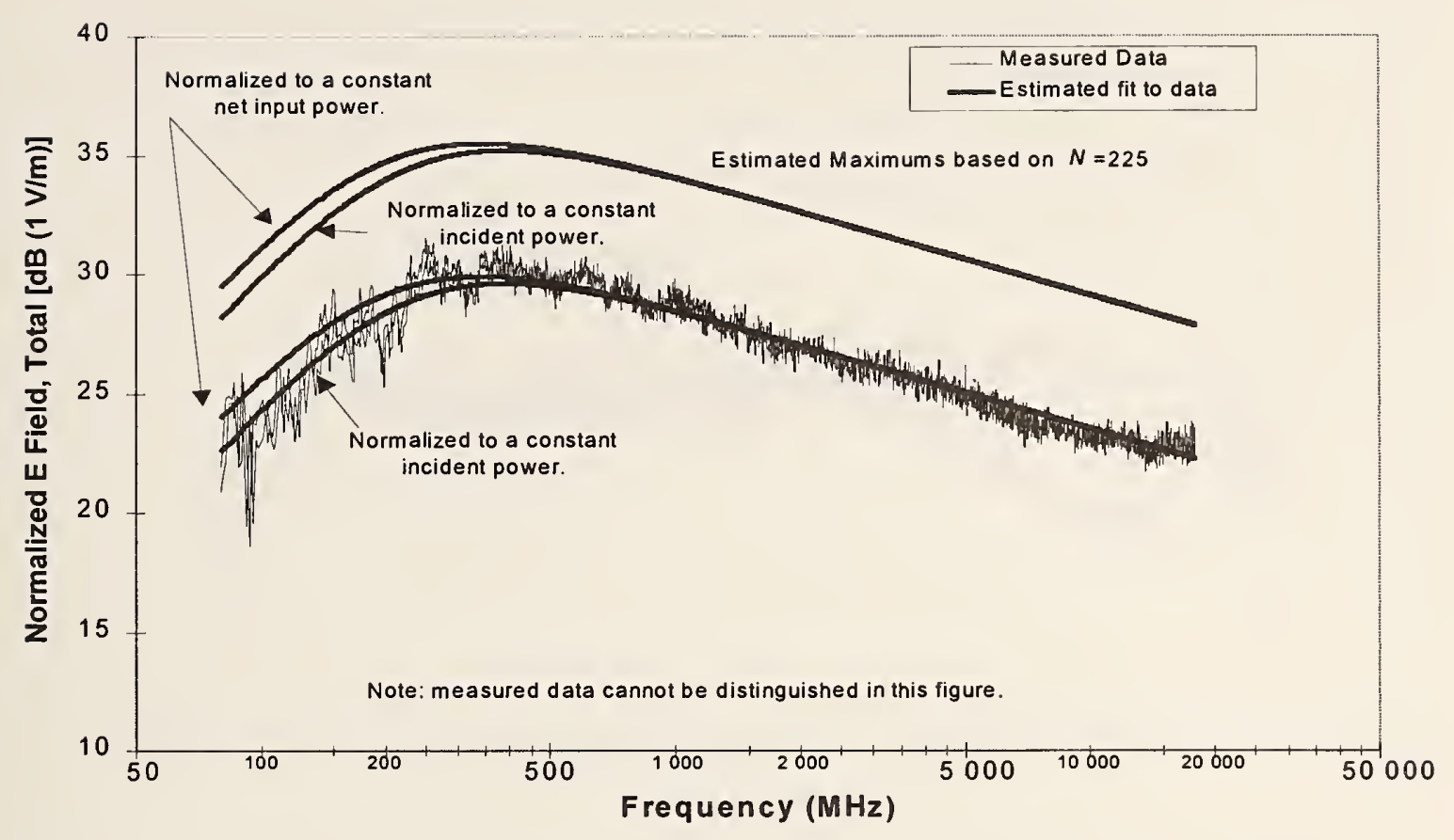

(a)

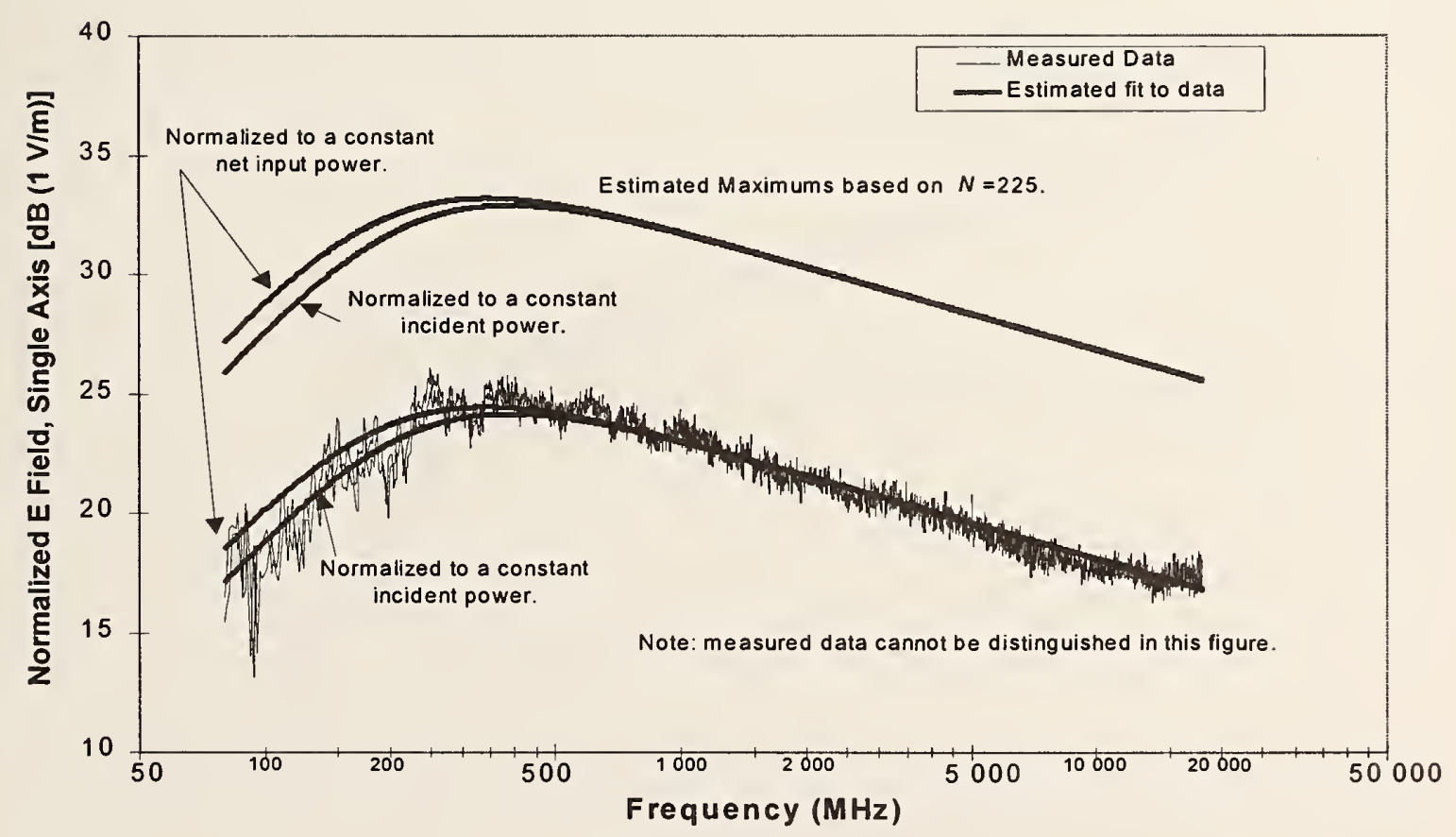

(b)

Figure 77. Calculated average and estimated maximum electric field in chamber A for a constant input power of $1 \mathrm{~W}$, based on received power from reference antenna.

(a) Total electric field. (b) Single-axis electric field. 


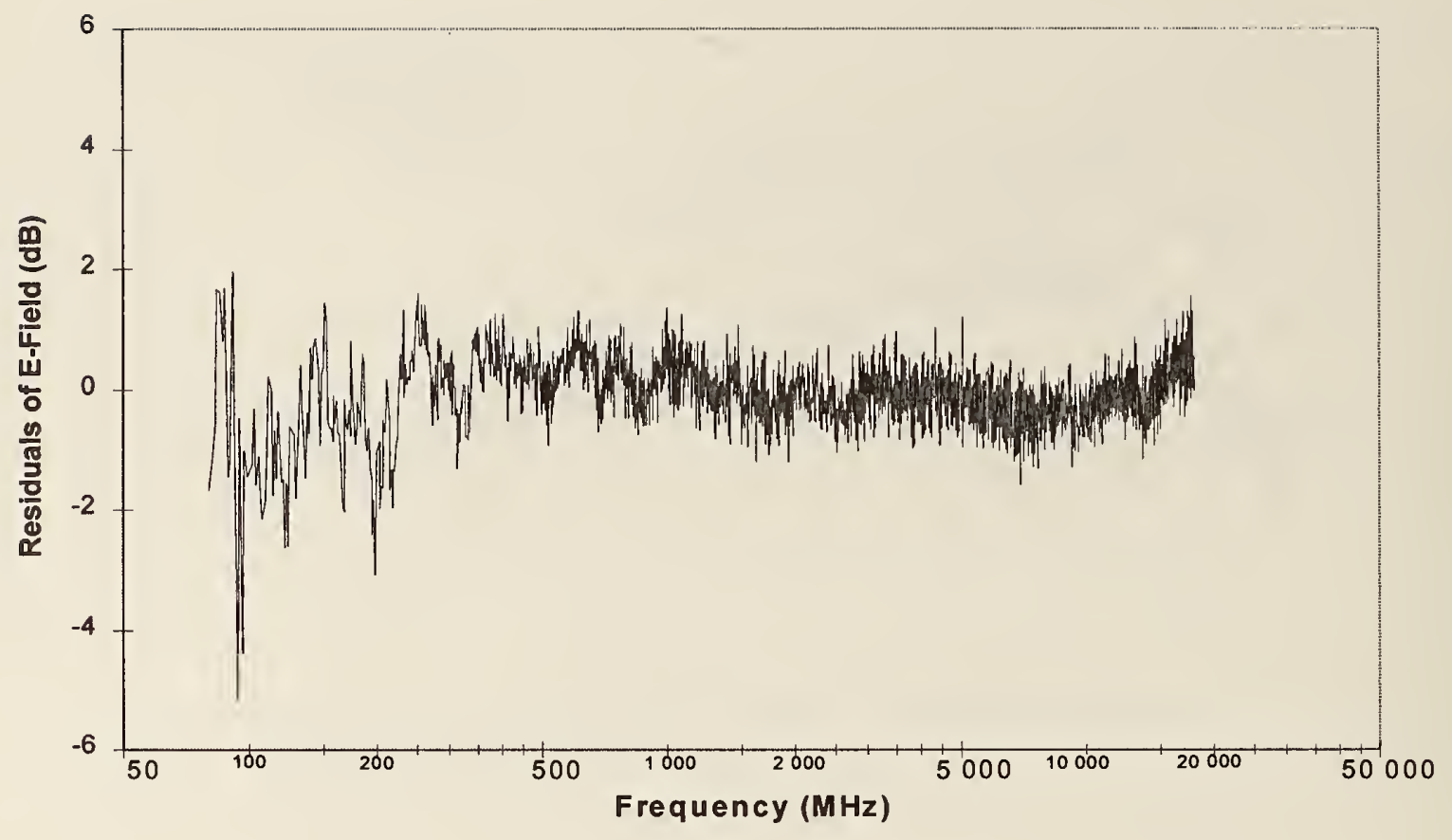

Figure 78. Residuals of calculated average electric field in chamber A, based on received power from reference antenna. 


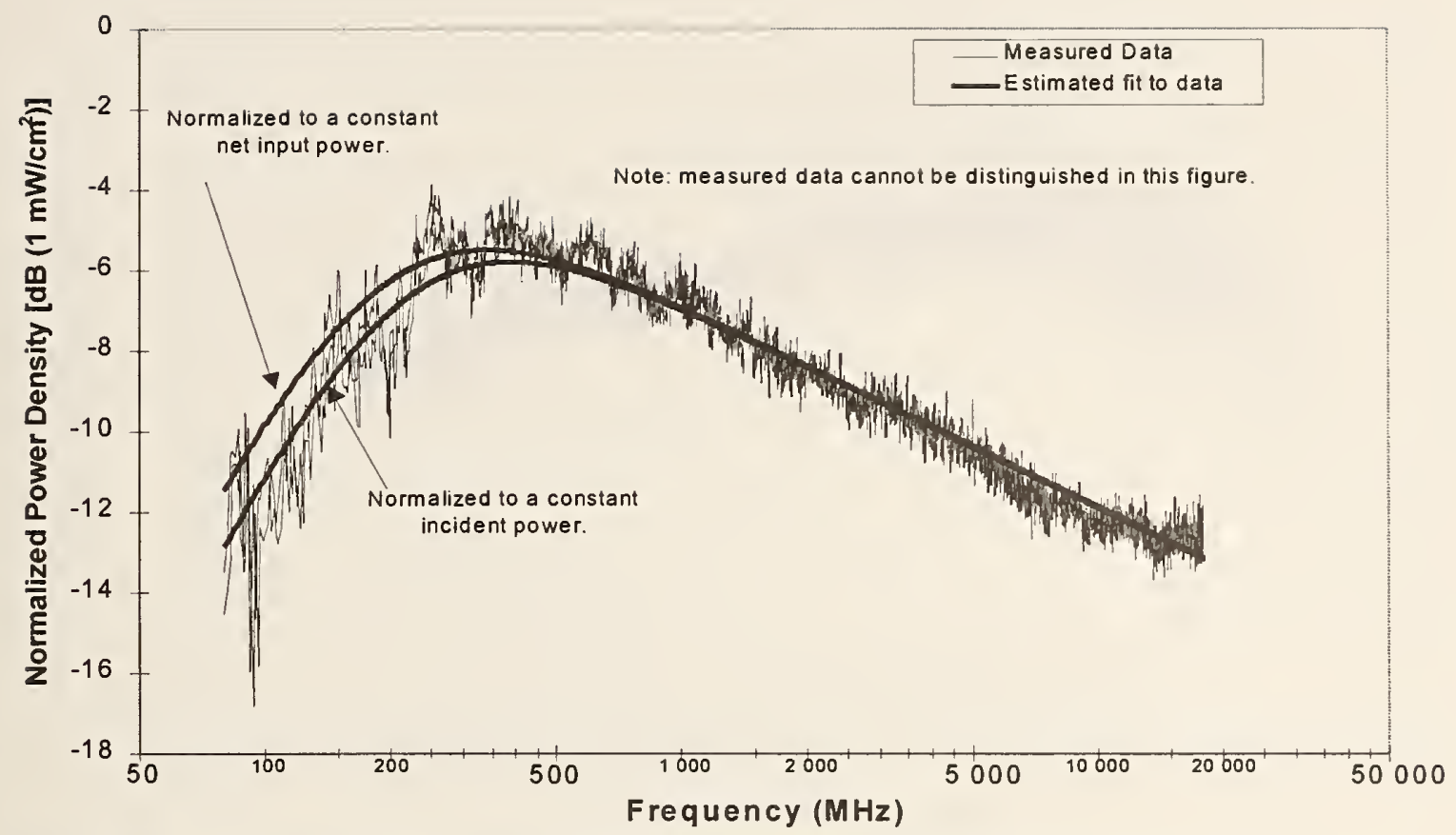

Figure 79. Average power density in chamber $\mathrm{A}$ for a constant input power of $1 \mathrm{~W}$.

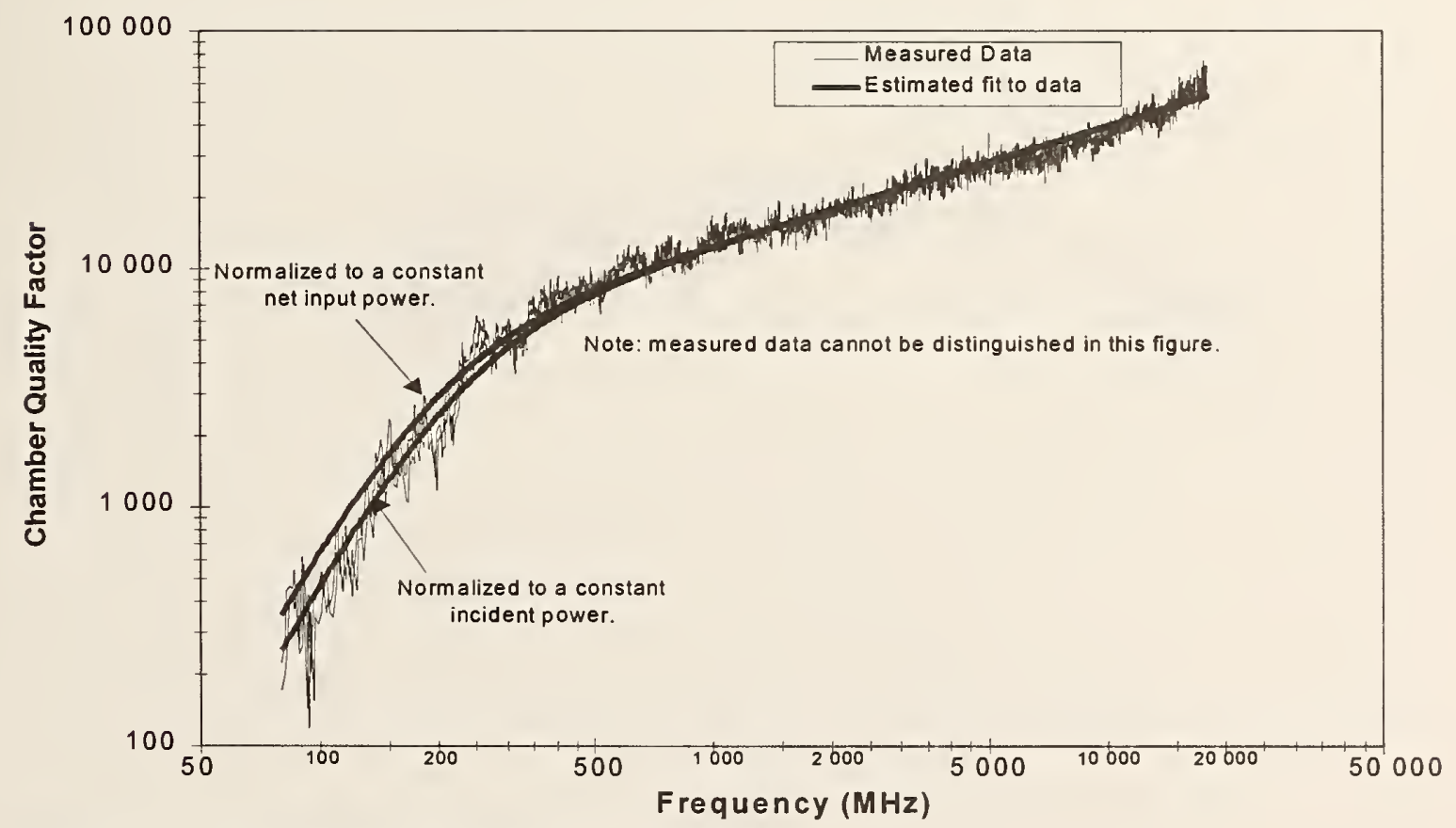

Figure 80. Average quality factor in chamber A. 


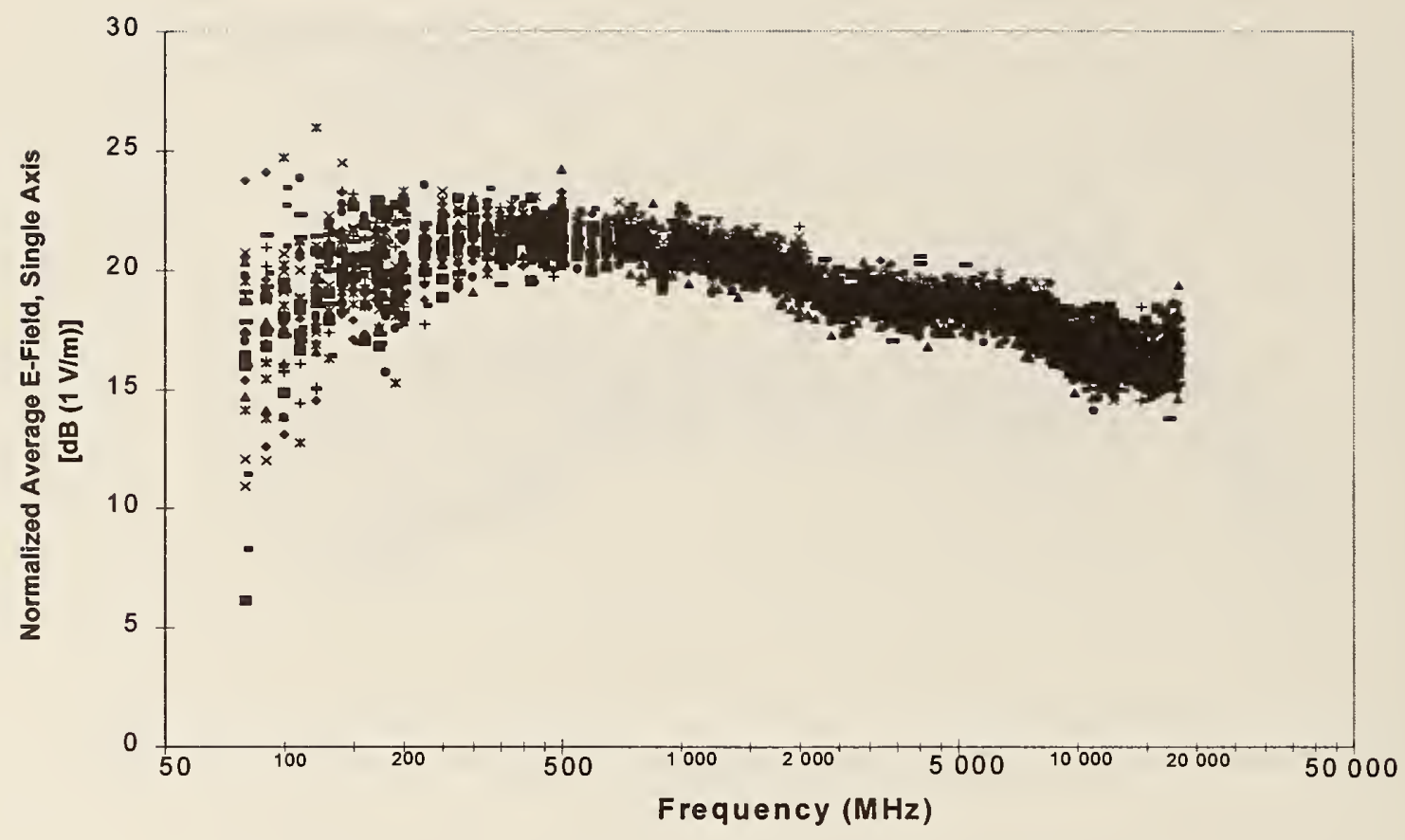

(a)

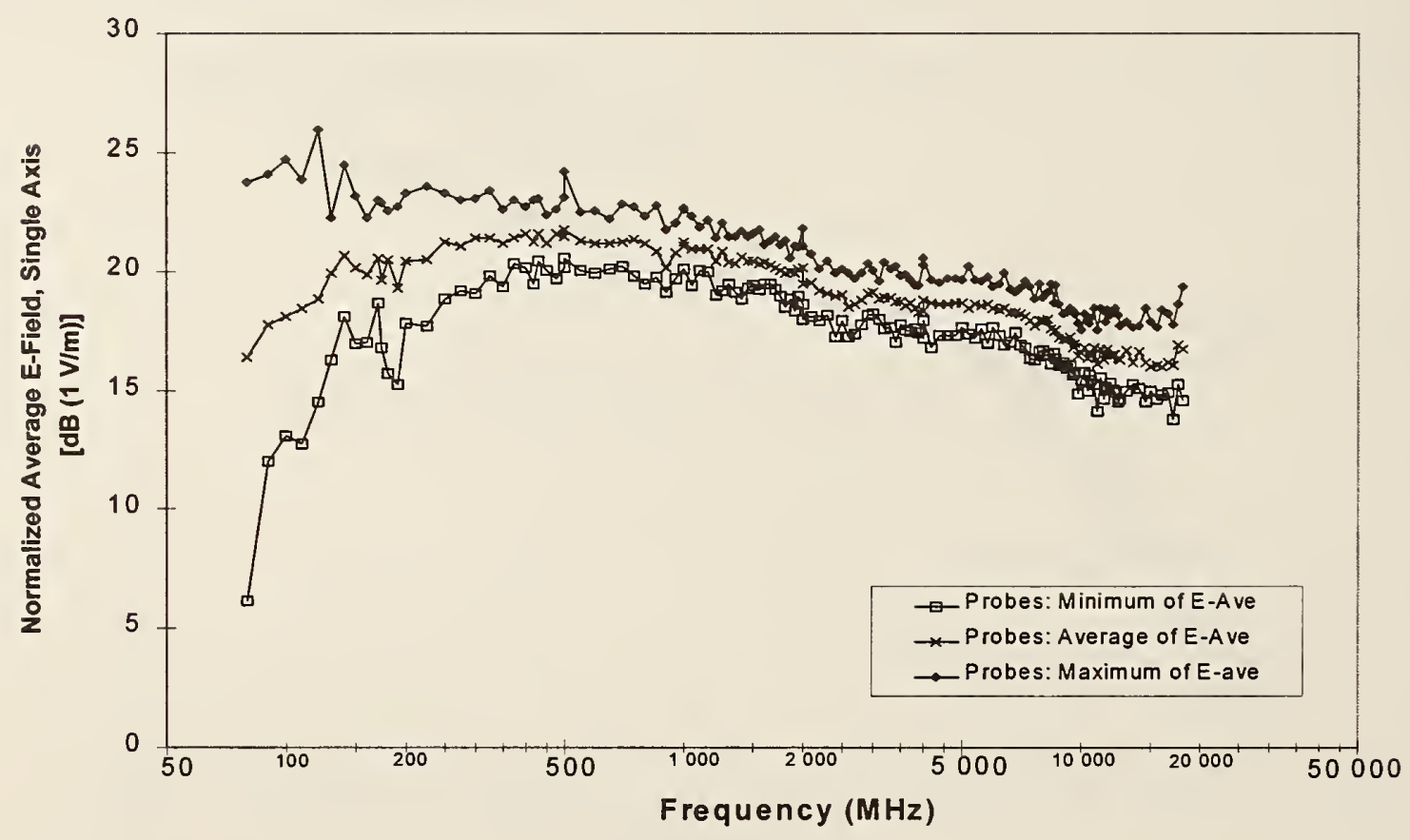

(b)

Figure 81. The average measured electric field (rectangular component) for each of 30 short dipoles in chamber A. Values given are for a constant net input power of $1 \mathrm{~W}$.

(a) Individual measurements. (b) Envelope and average of individual measurements.

(c) Standard deviation of measurements of $E_{R}$-Ave. (d) Comparison of average of individual measurements with reference antenna. 


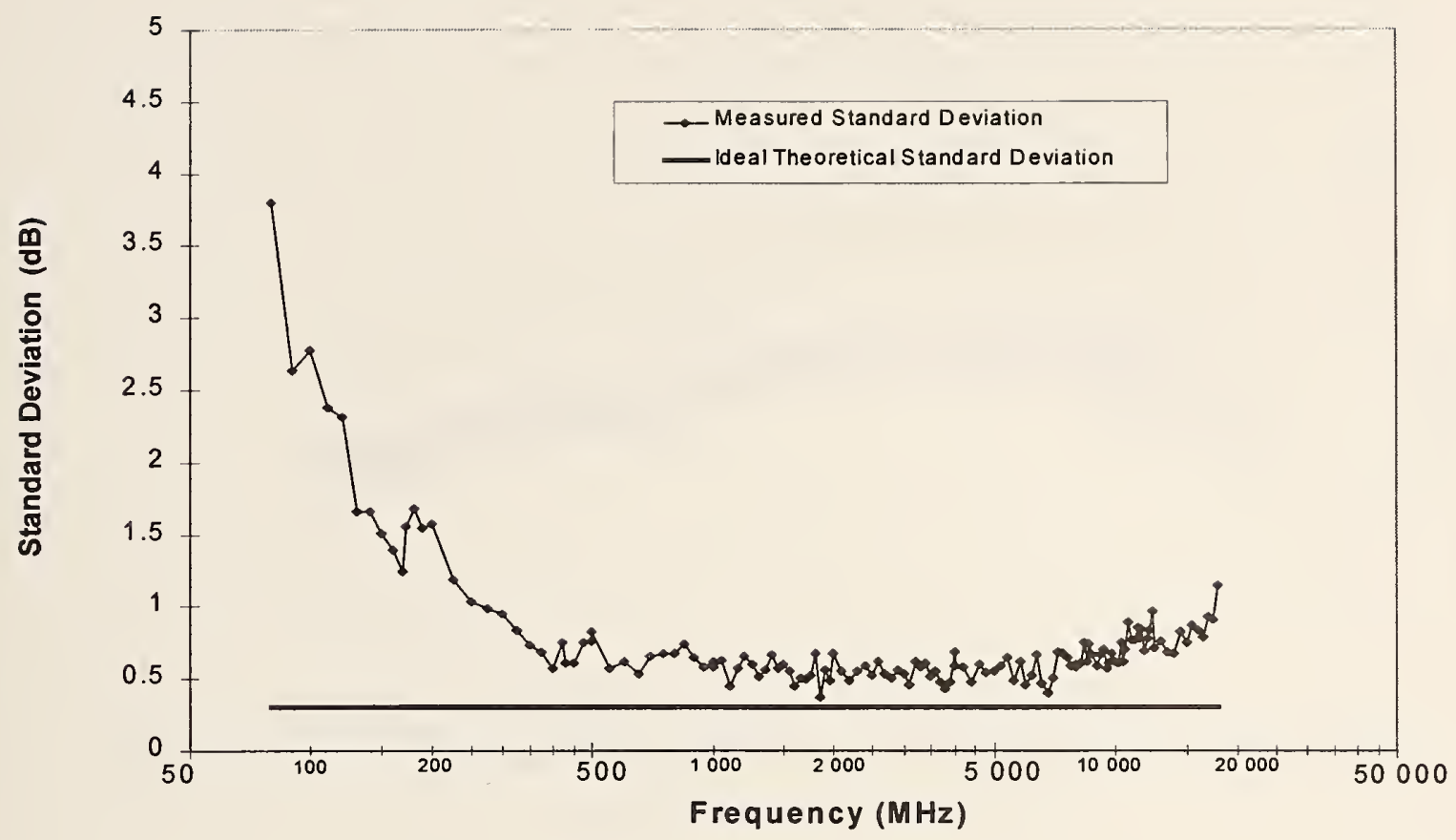

(c)

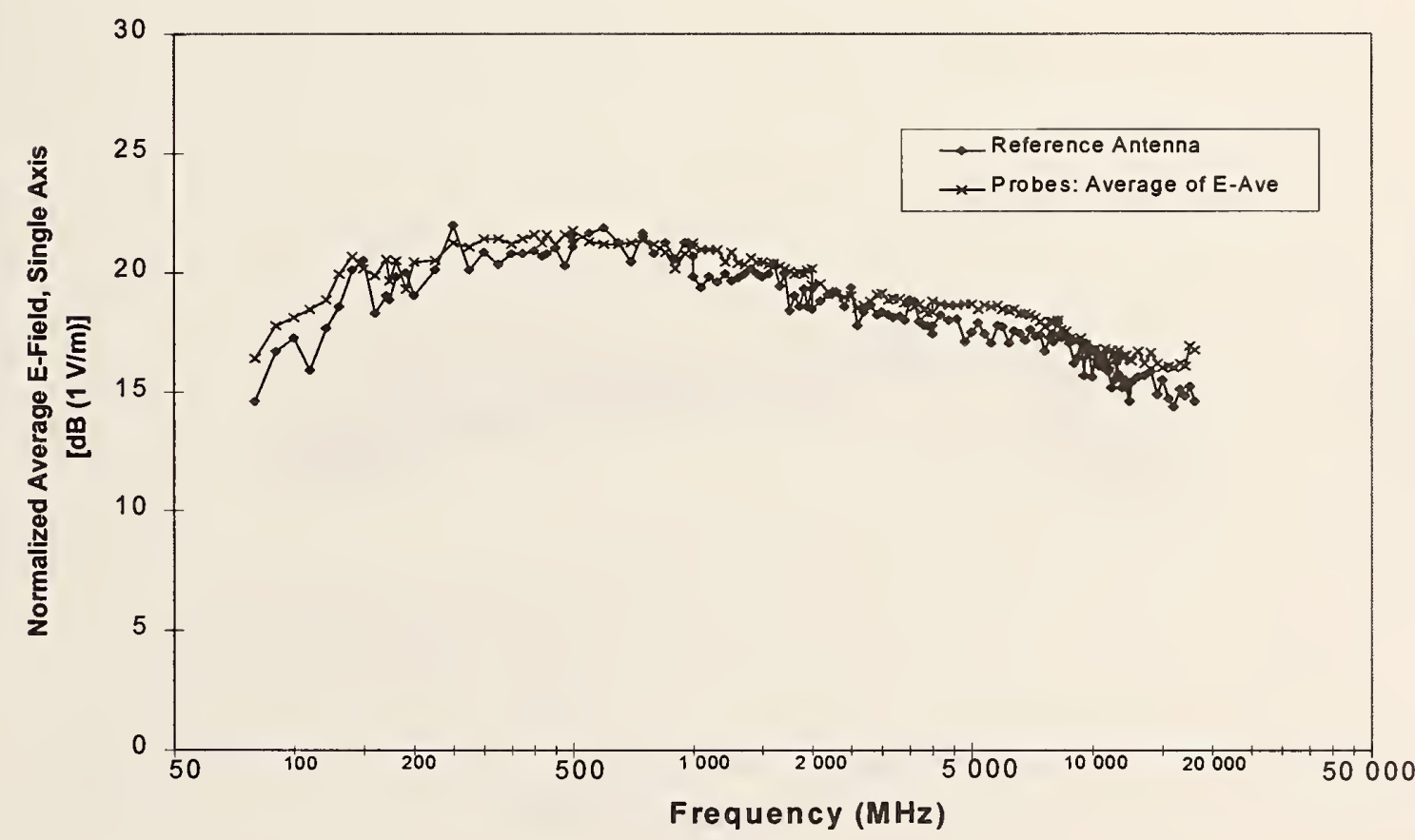

(d) 


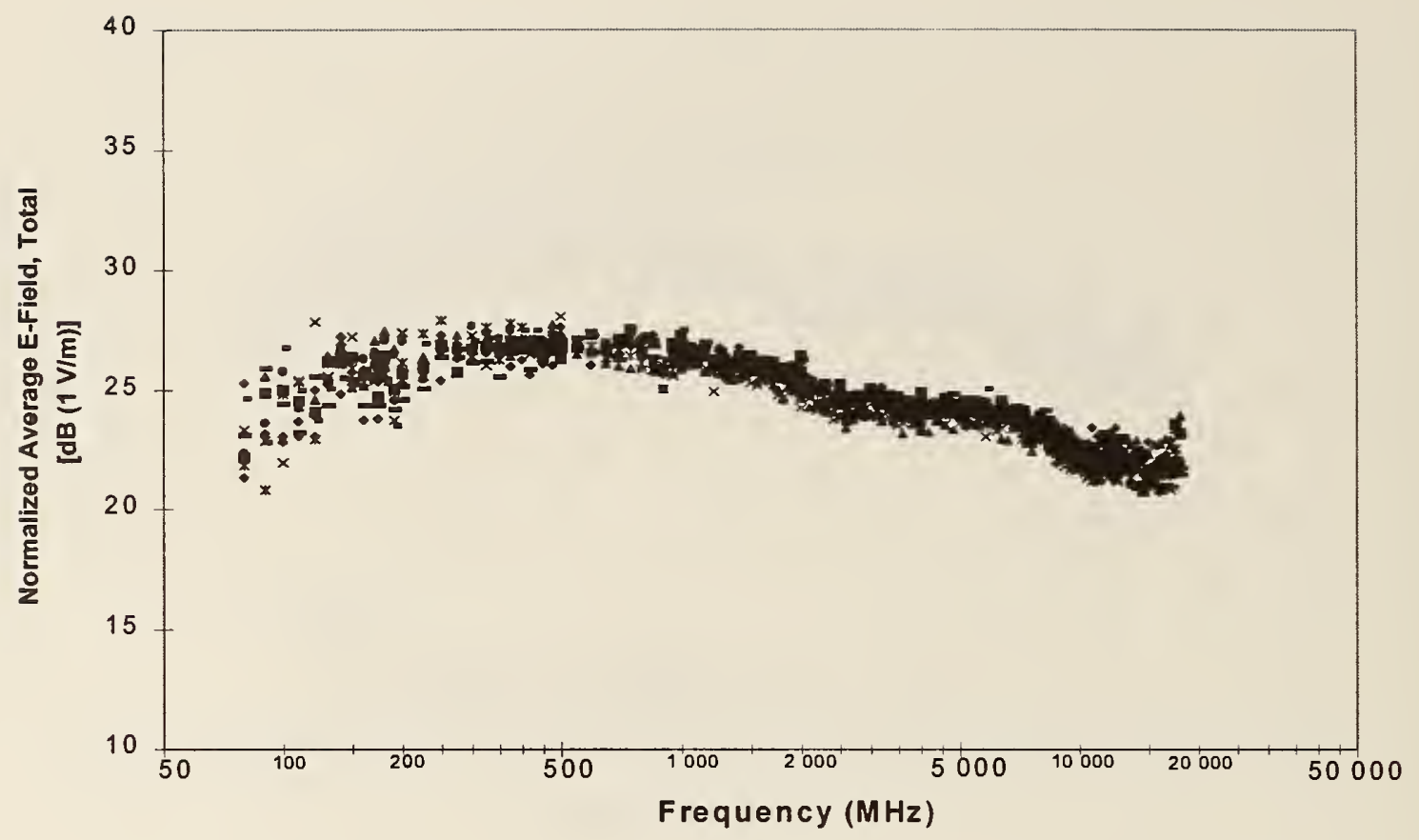

(a)

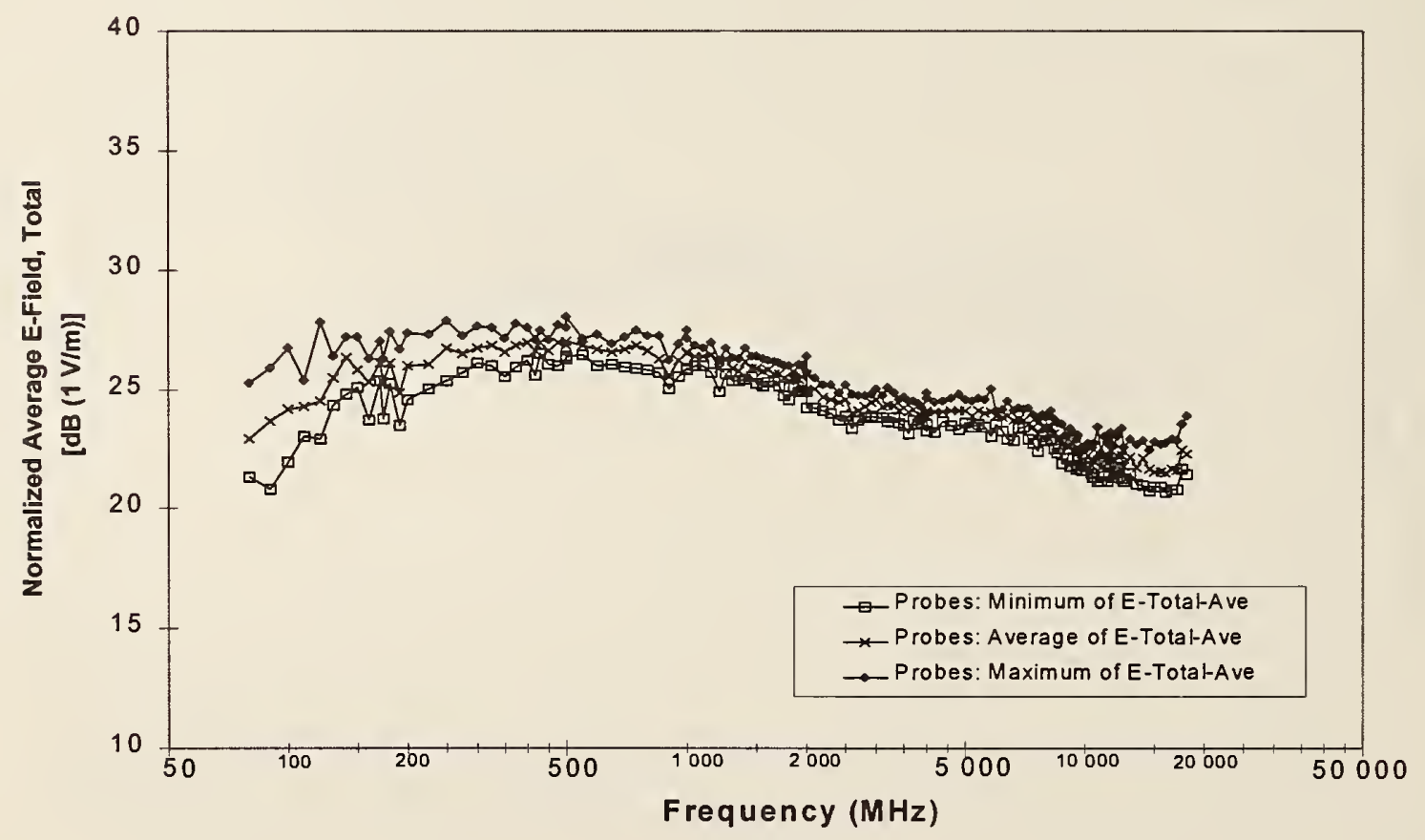

(b)

Figure 82. The average measured total electric field for each of 10 isotropic antennas in chamber A. Values given are for a constant net input power of $1 \mathrm{~W}$. (a) Individual measurements. (b) Envelope and average of individual measurements. (c) Standard deviation of measurements of $E_{\mathrm{T}}$-Ave. (d) Comparison of average of individual measurements with reference antenna. 


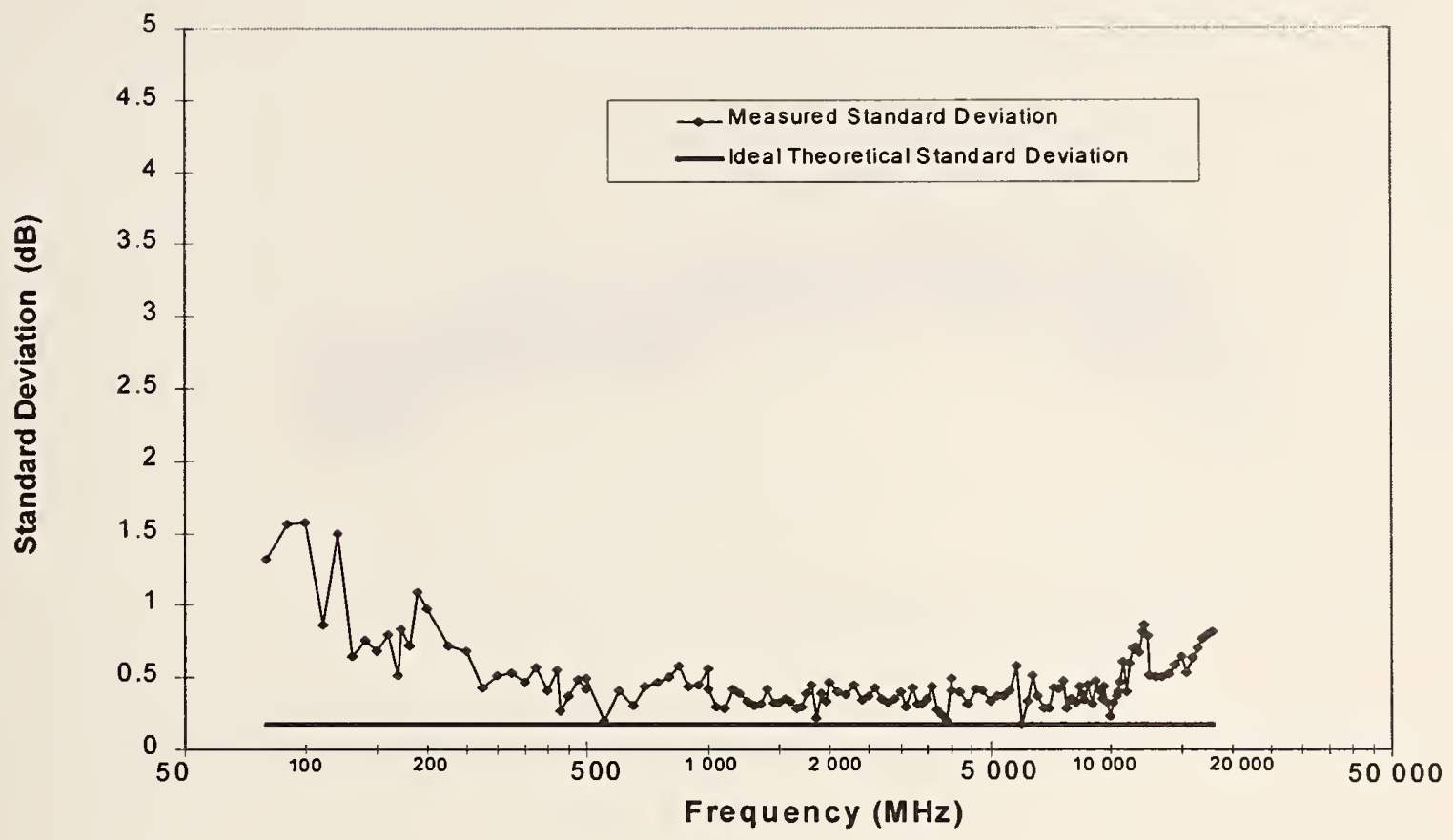

(c)

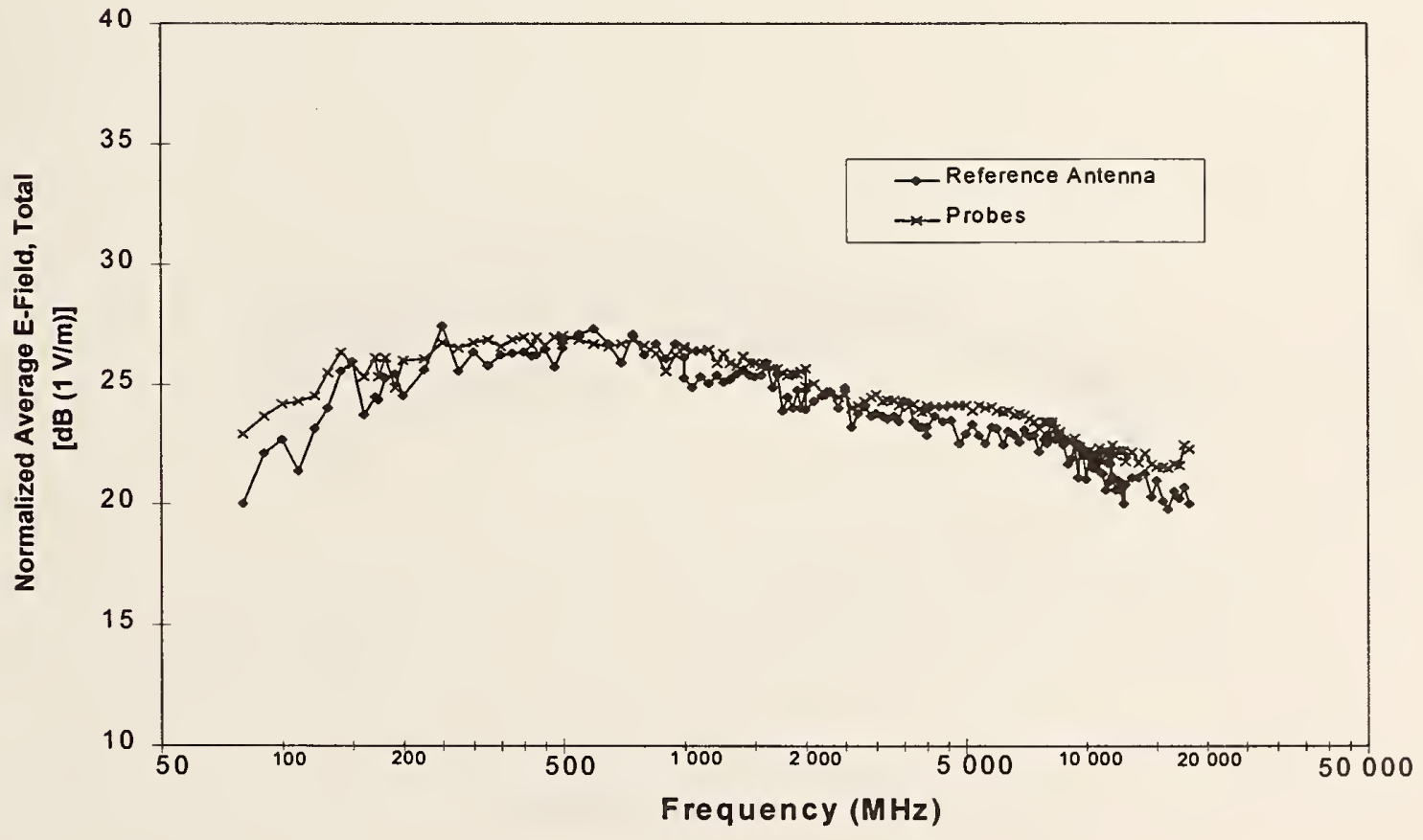

(d) 


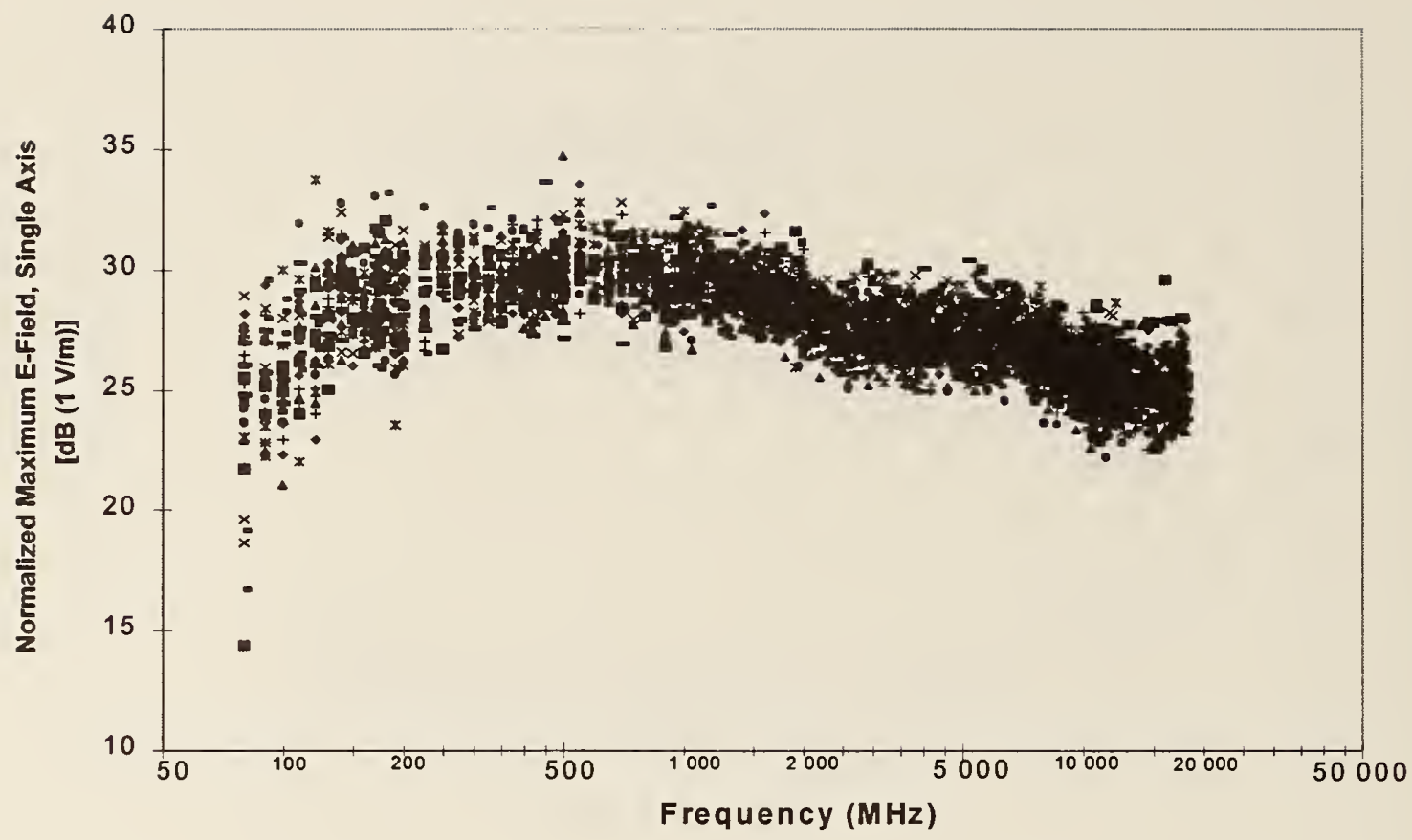

(a)

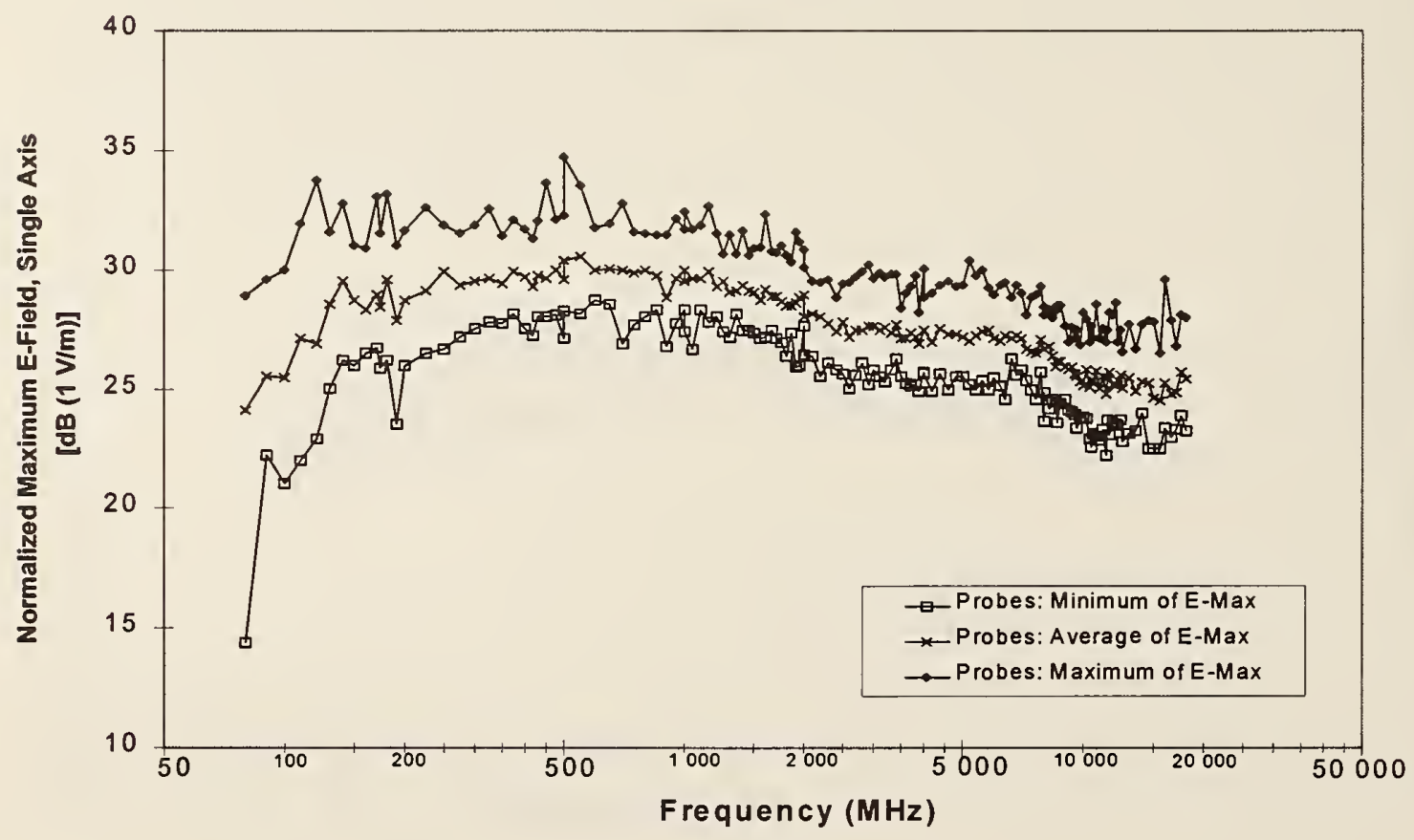

(b)

Figure 83. The maximum measured electric field (single polarization) for each of 30 short dipoles in chamber A. Values given are for a constant net input power of $1 \mathrm{~W}$.

(a) Individual measurements. (b) Envelope and average of individual measurements.

(c) Standard deviation of measurements of $E_{R}-M a x$. (d) Comparison of average of individual measurements with reference antenna. 


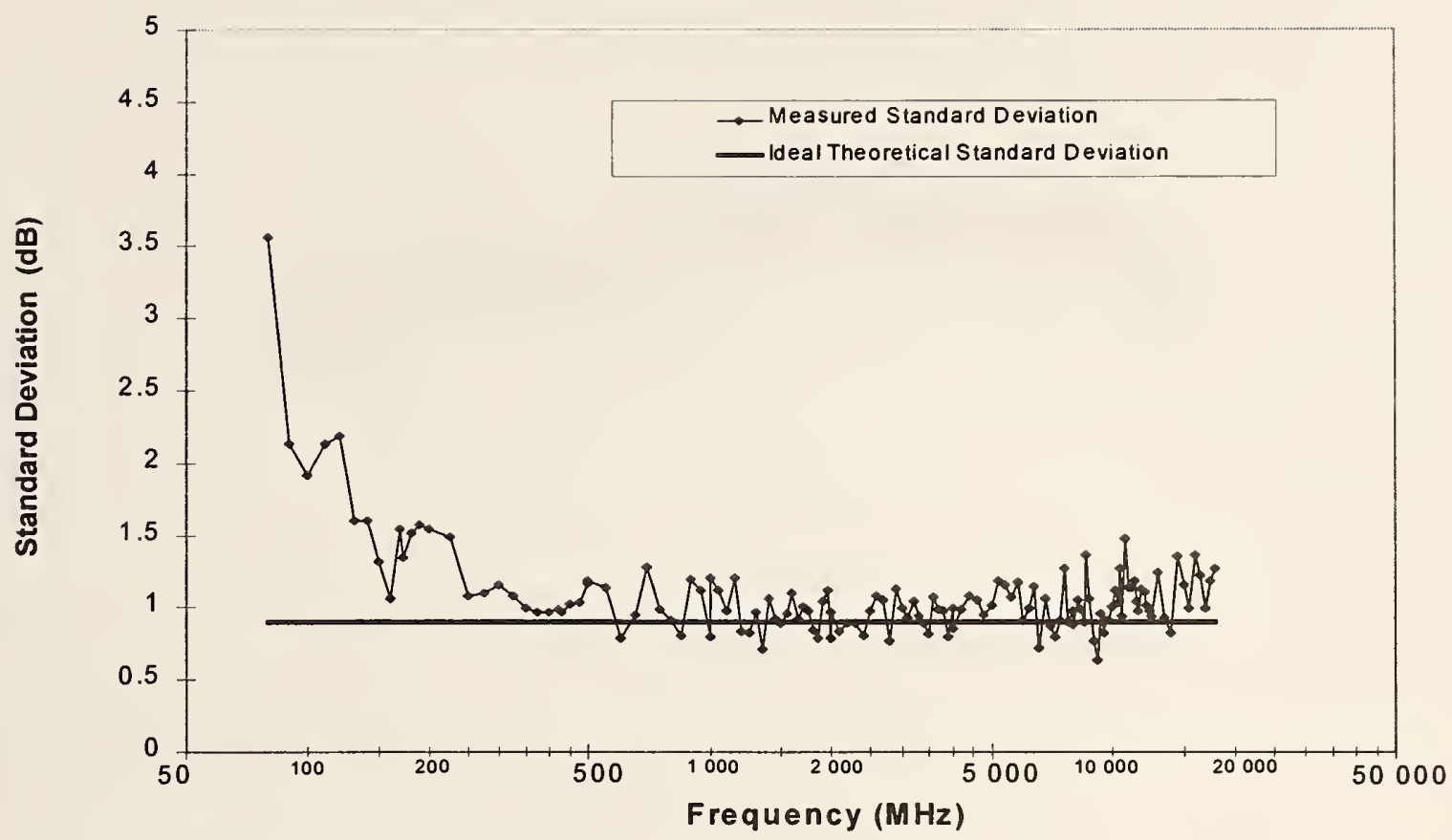

(c)

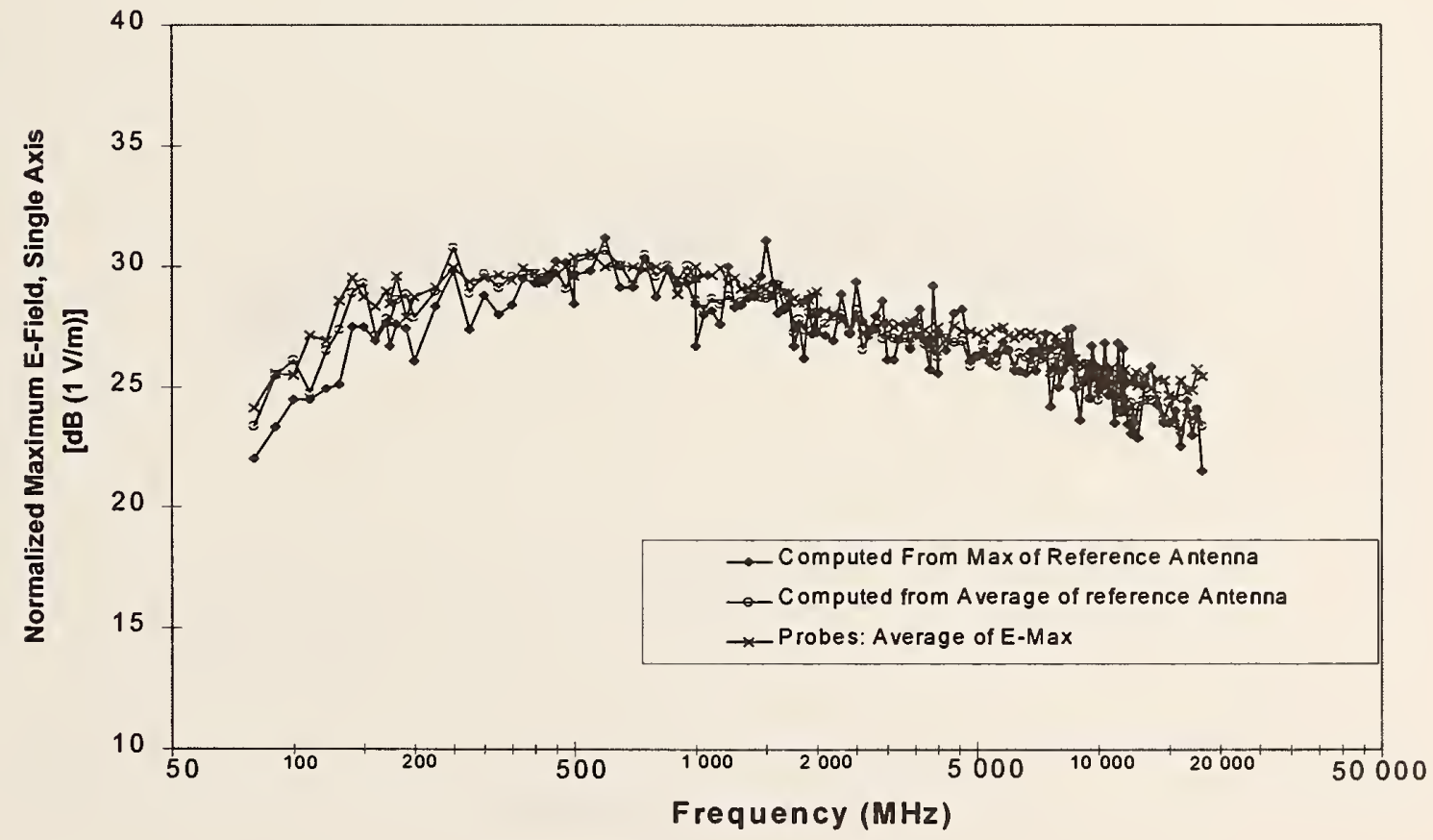

(d) 


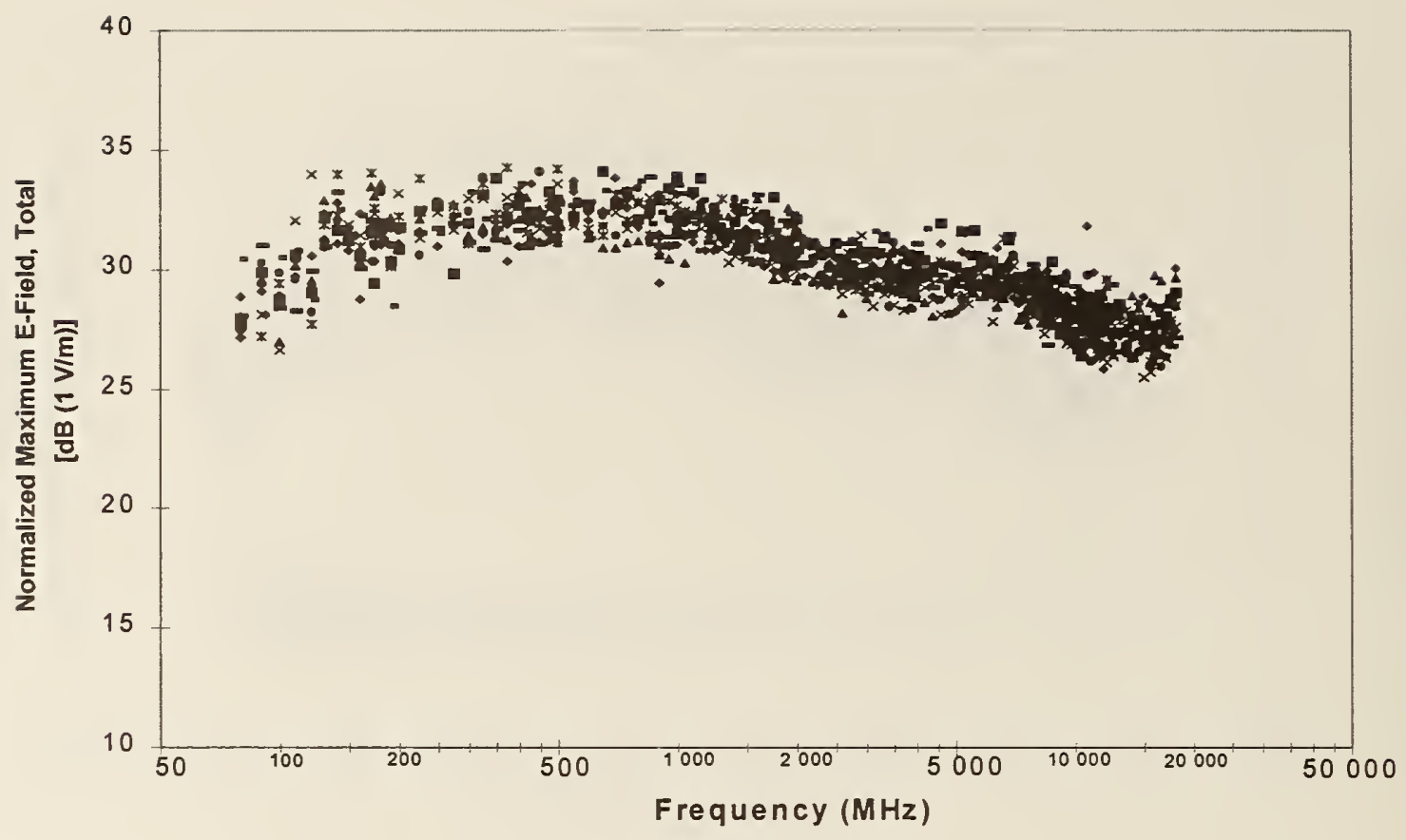

(a)

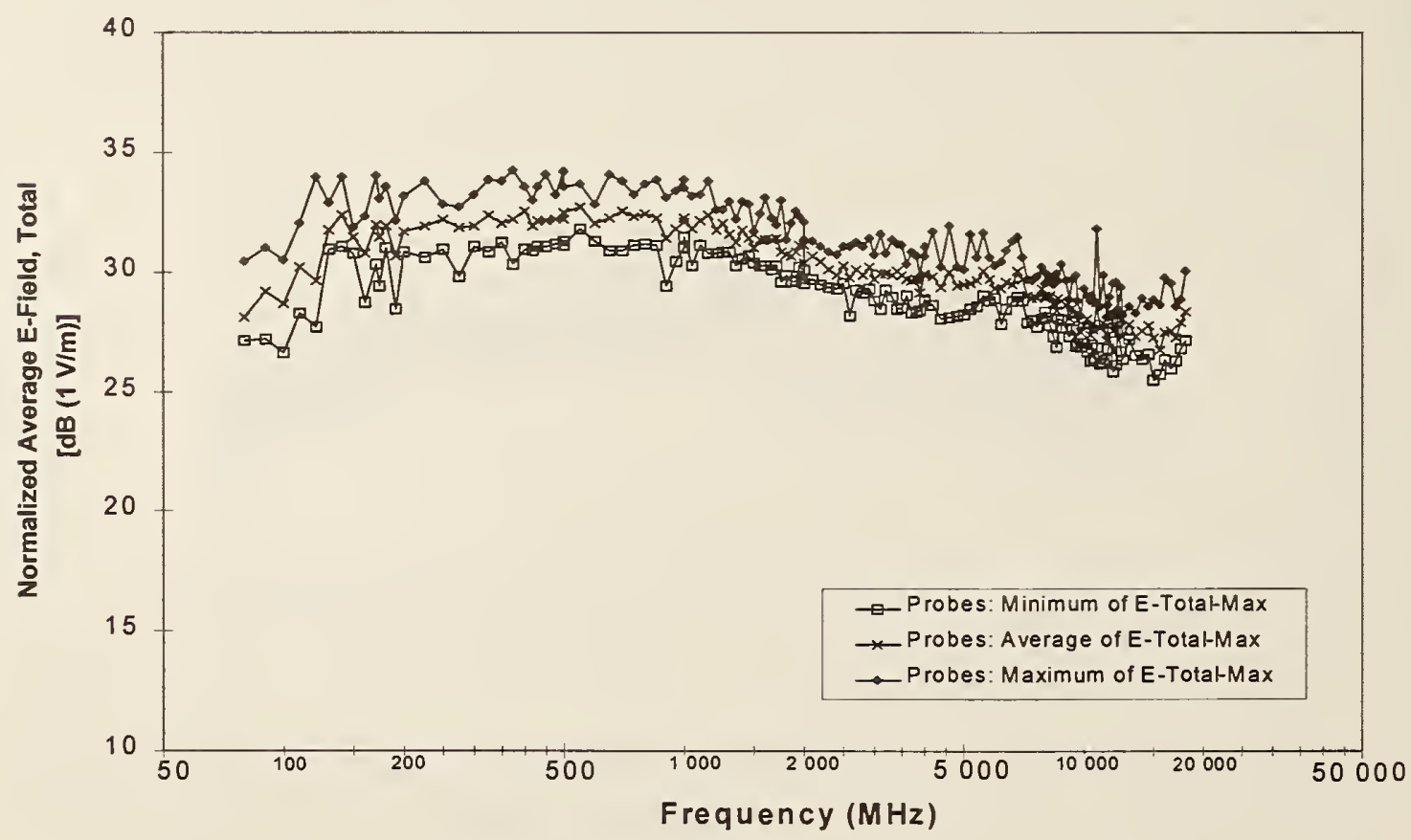

(b)

Figure 84. The maximum measured total electric field for each of 10 isotropic antennas in chamber A. Values given are for a constant net input power of $1 \mathrm{~W}$. (a) Individual measurements. (b) Envelope and average of individual measurements. (c) Standard deviation of measurements of $\mathrm{E}_{\mathrm{T}}$-Max. (d) Comparison of average of individual measurements with reference antenna. 


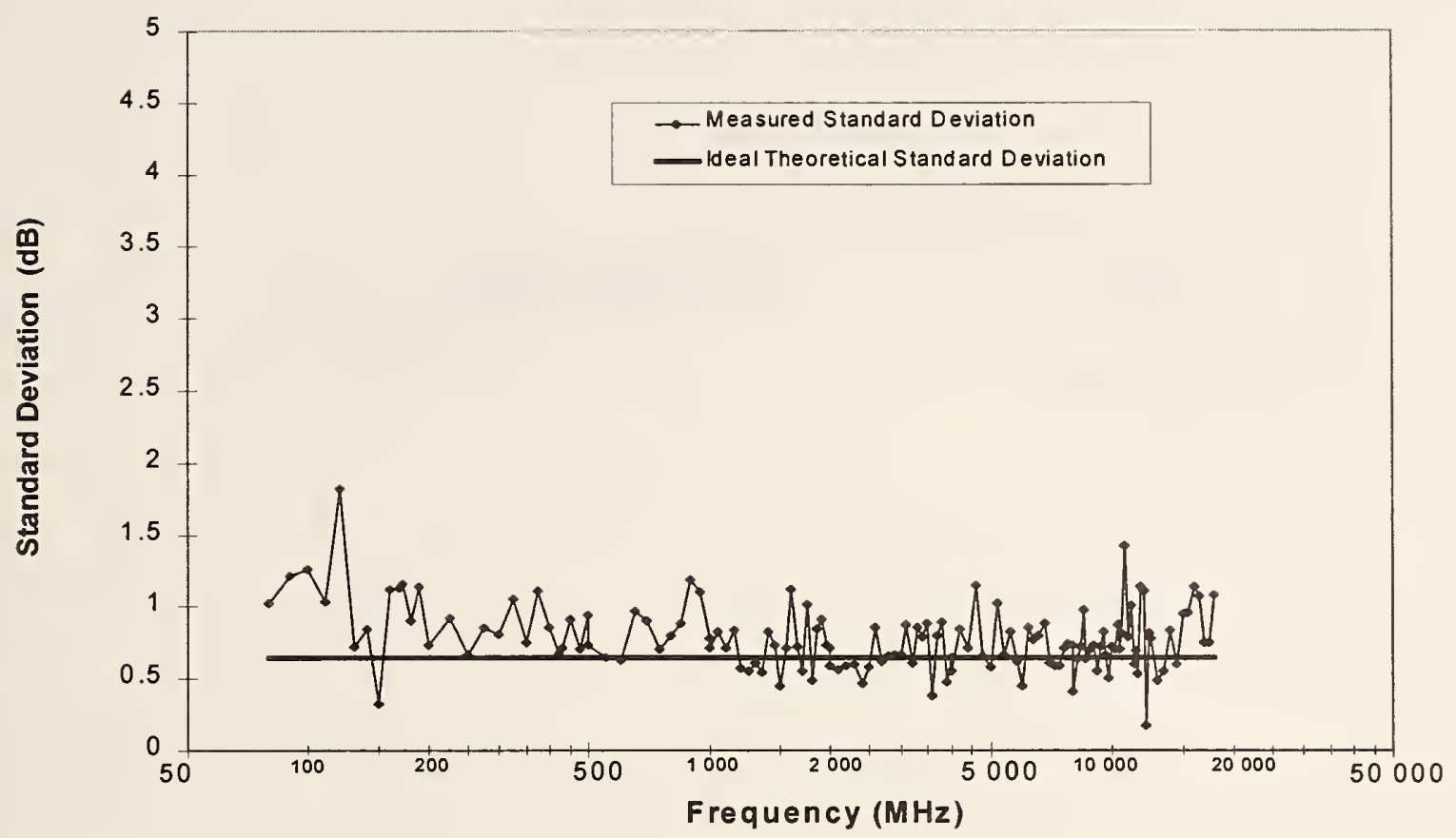

(c)

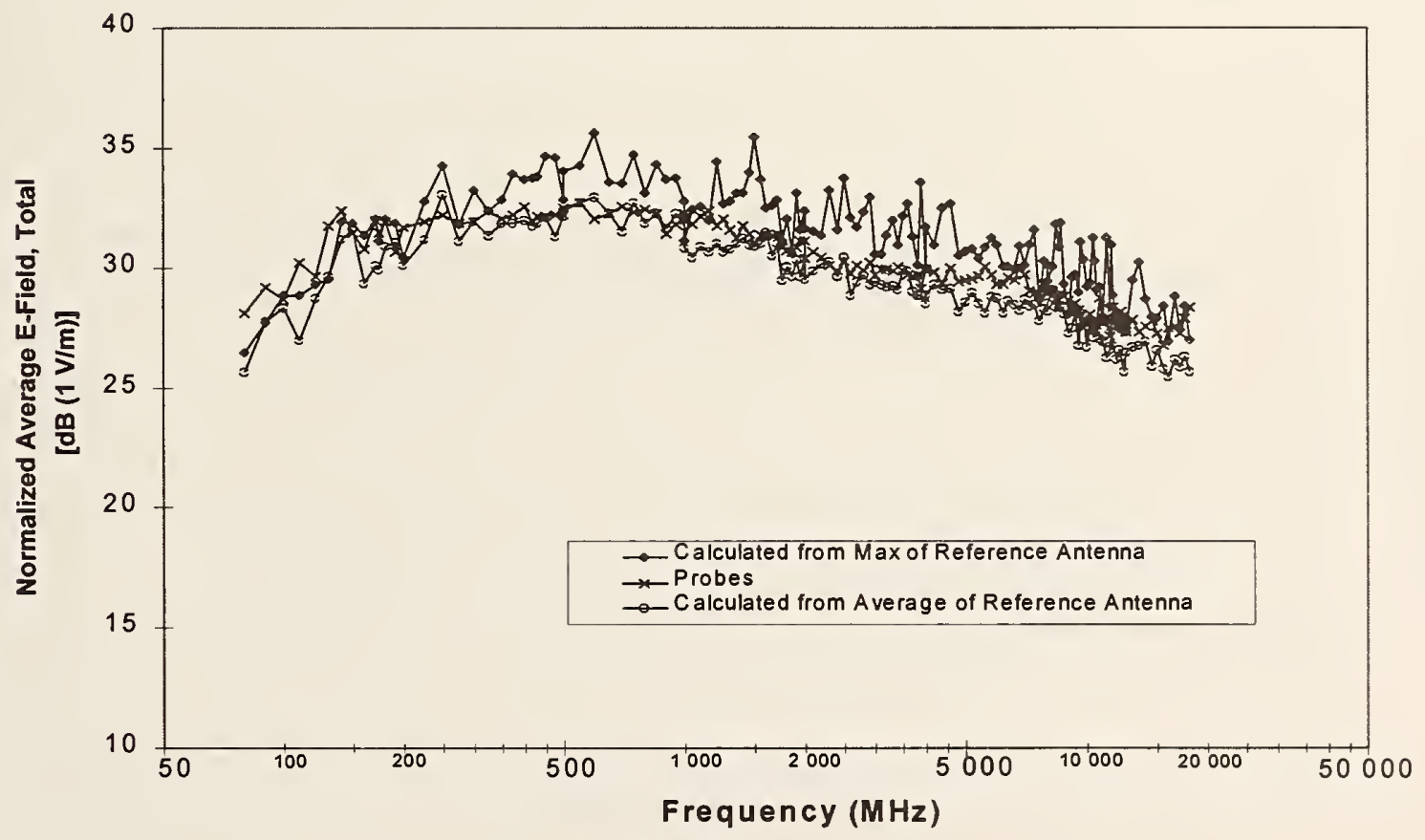

(d) 


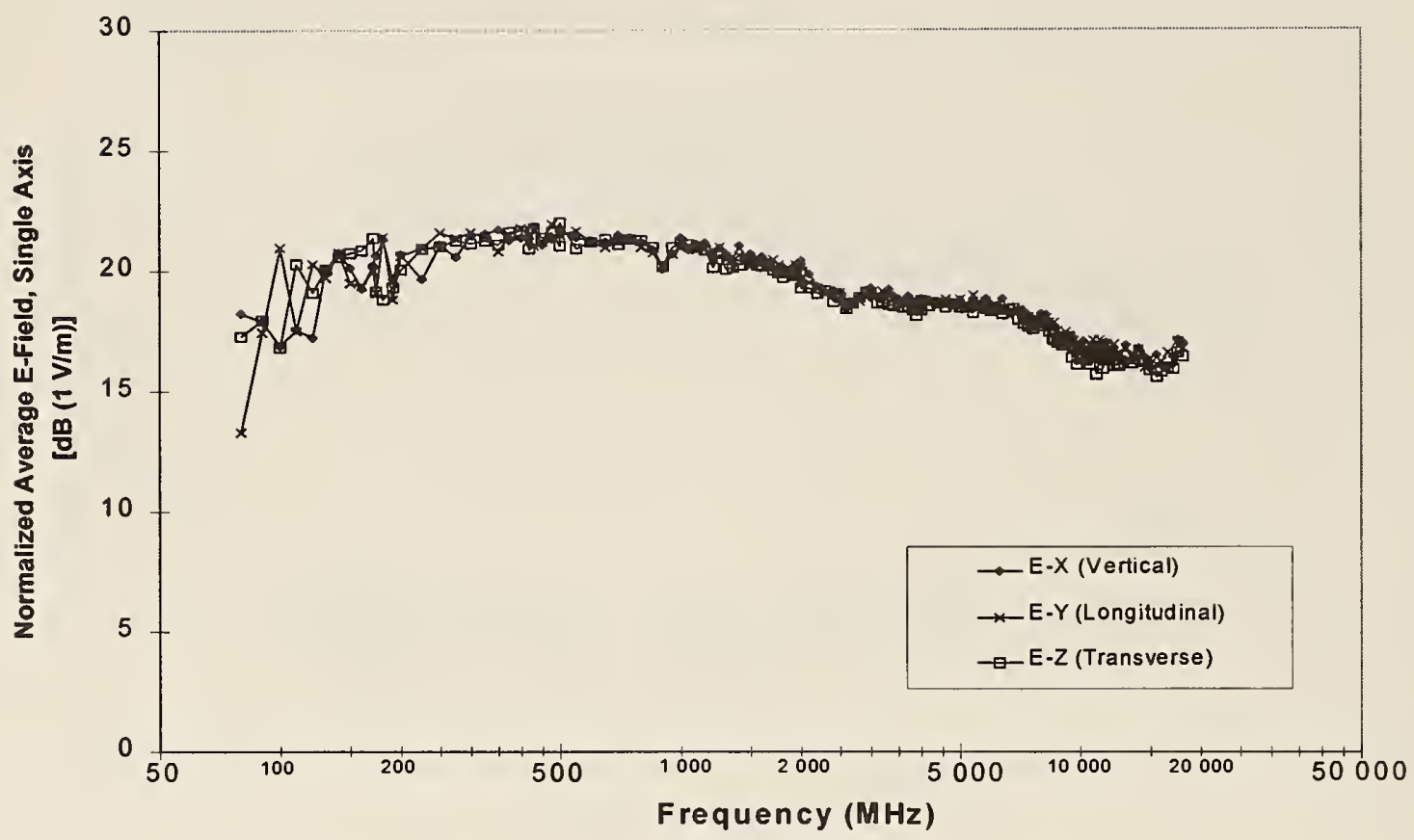

(a)

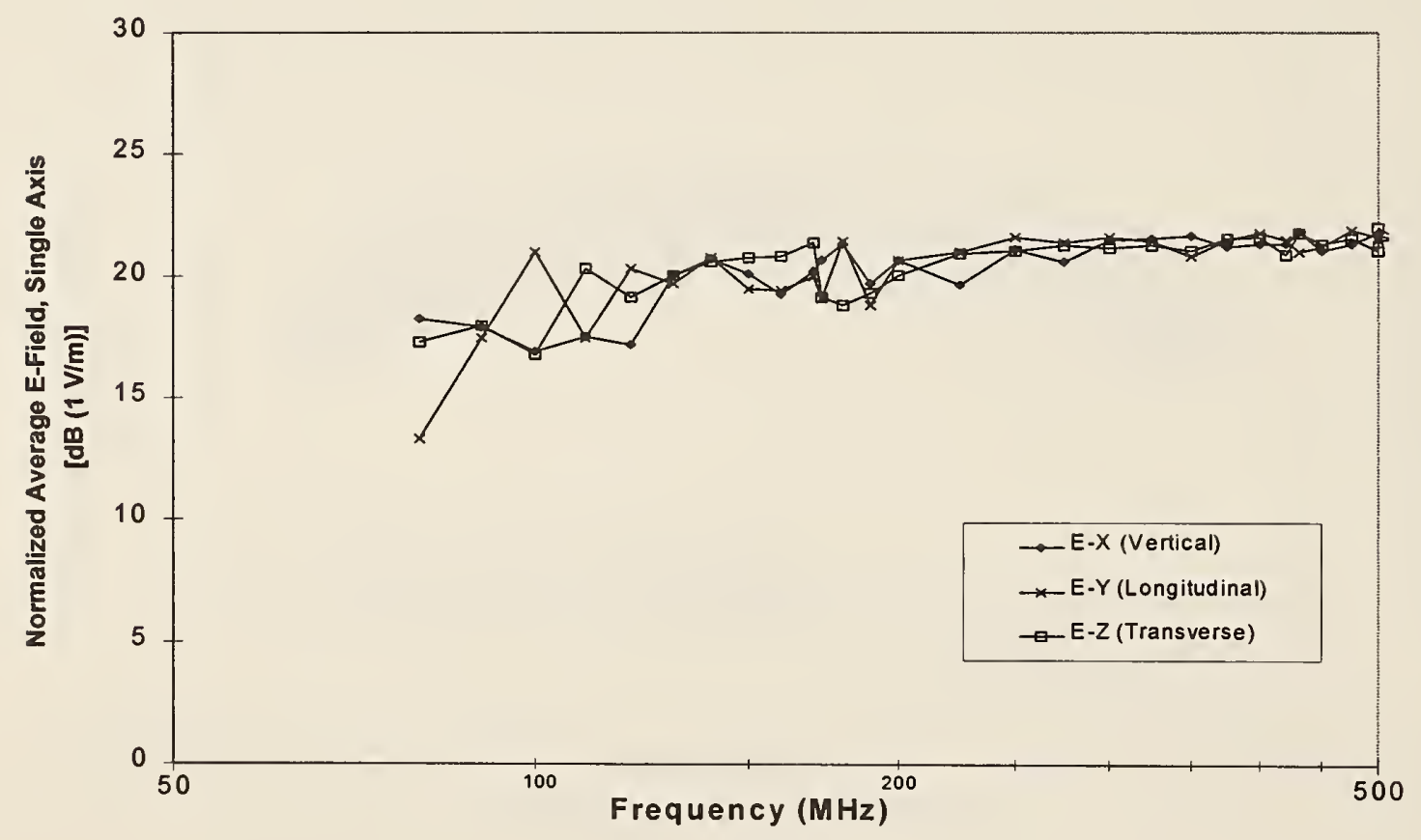

(b)

Figure 85. Comparison of average $\mathrm{x}$ component, $\mathrm{y}$ component, and $\mathrm{z}$ component of electric field in chamber A. Values given are for a constant net input power of $1 \mathrm{~W}$. (a) Full frequency range. (b) Low frequencies only. 


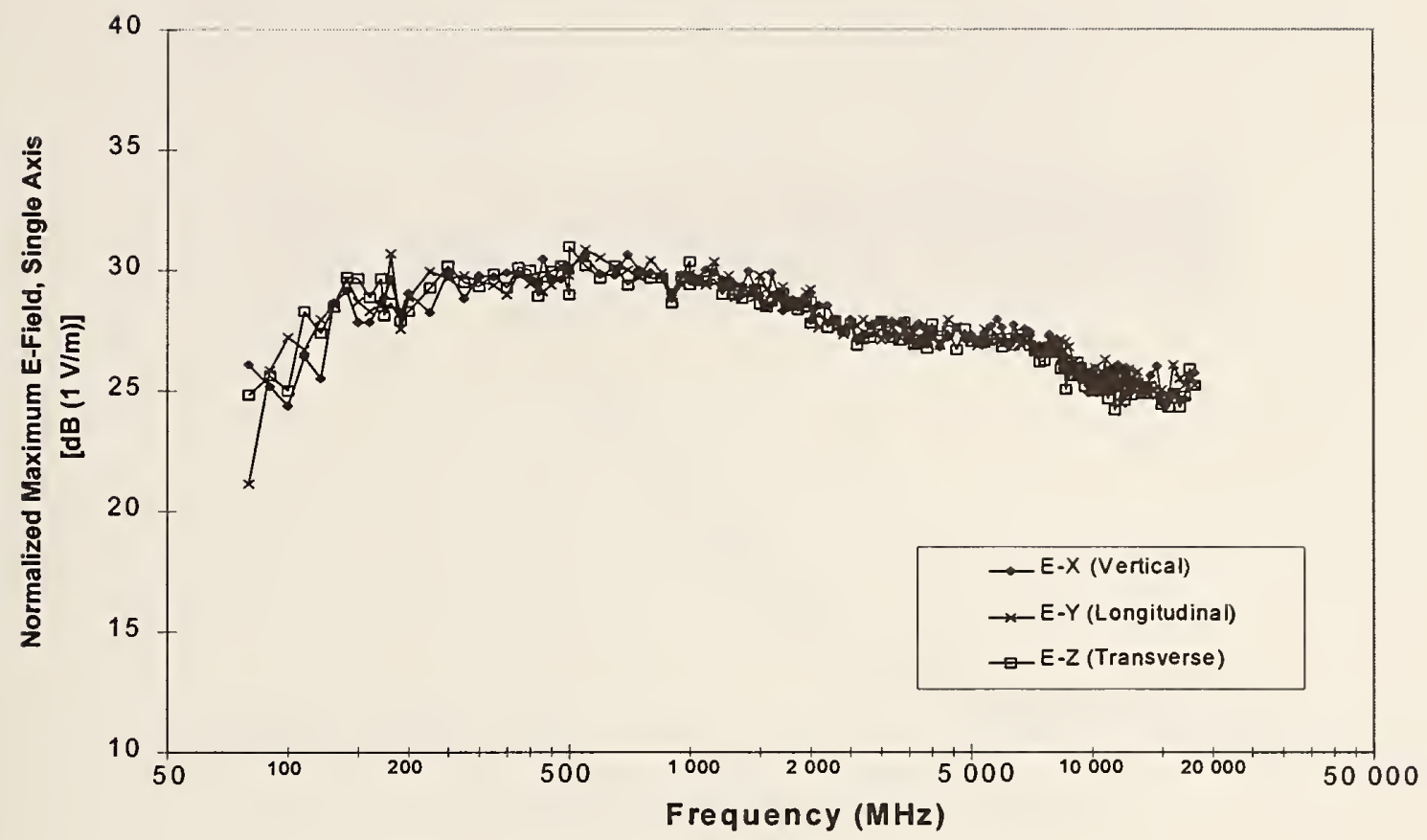

(a)

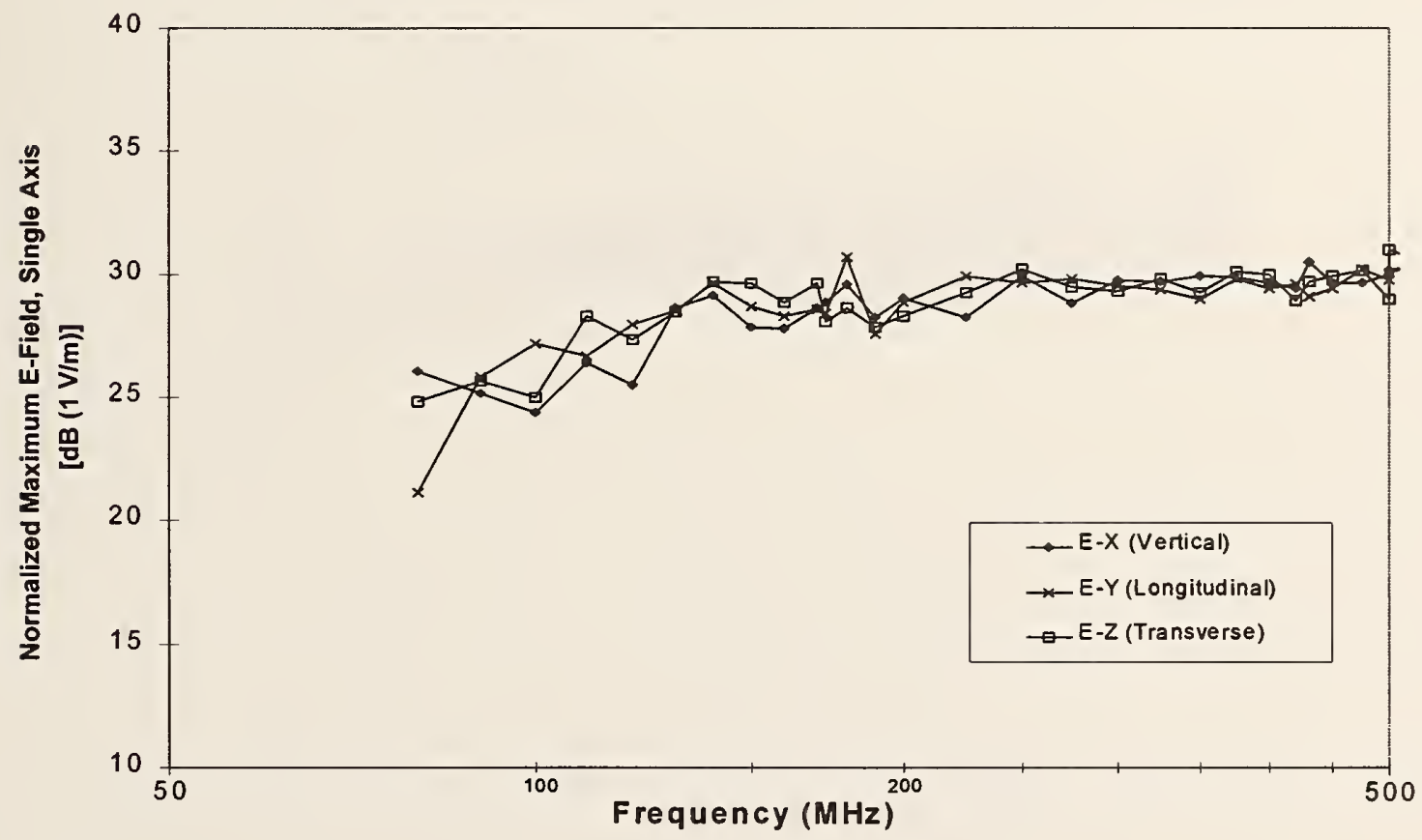

(b)

Figure 86. Comparison of maximum $\mathrm{x}$ component, $\mathrm{y}$ component, and $\mathrm{z}$ component of electric field in chamber A. Values given are for a constant net input power of 1 W. (a) Full frequency range. (b) Low frequencies only. 


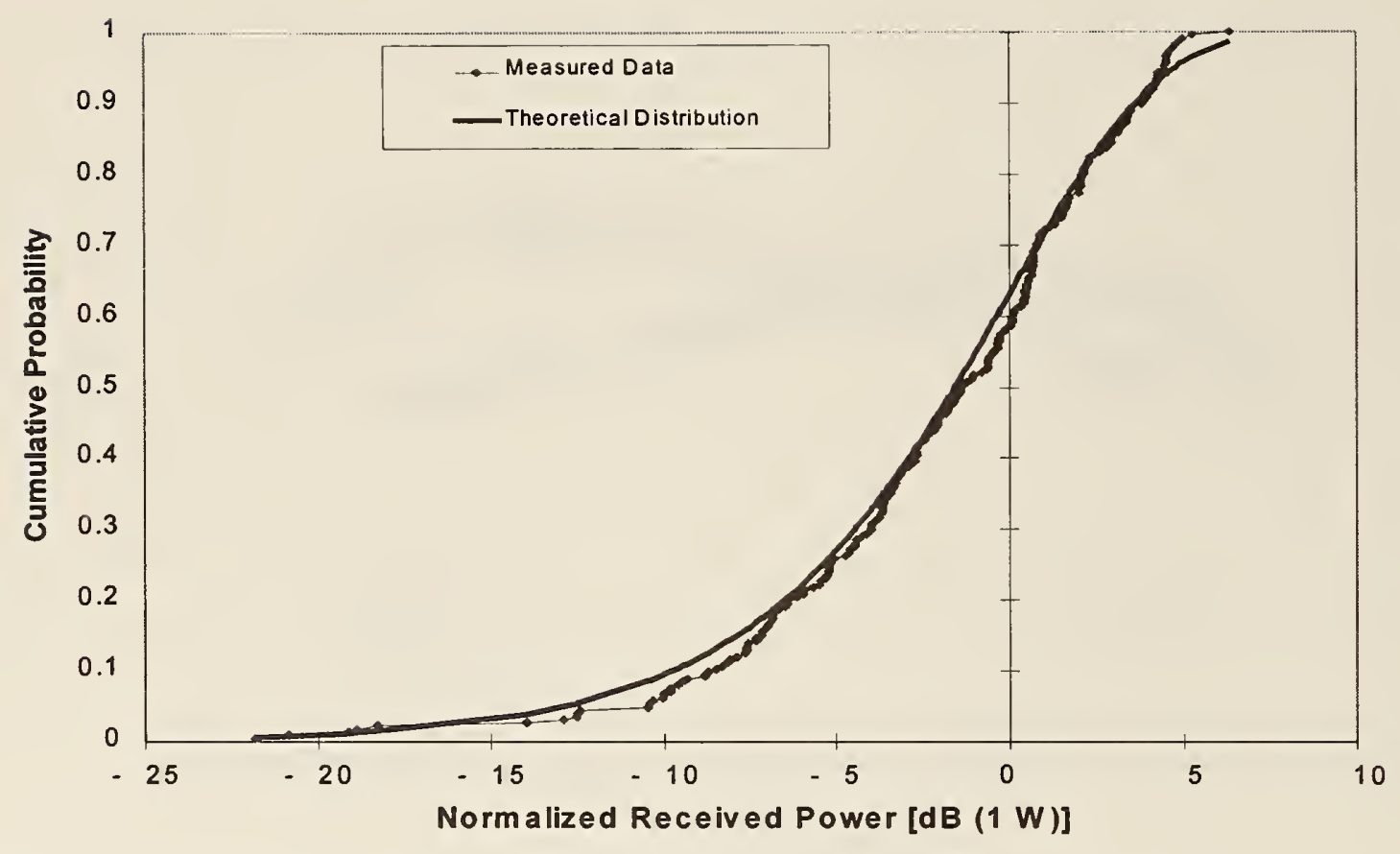

Figure 87. Cumulative distribution of received power at $1 \mathrm{GHz}$ in chamber $\mathrm{A}$. 


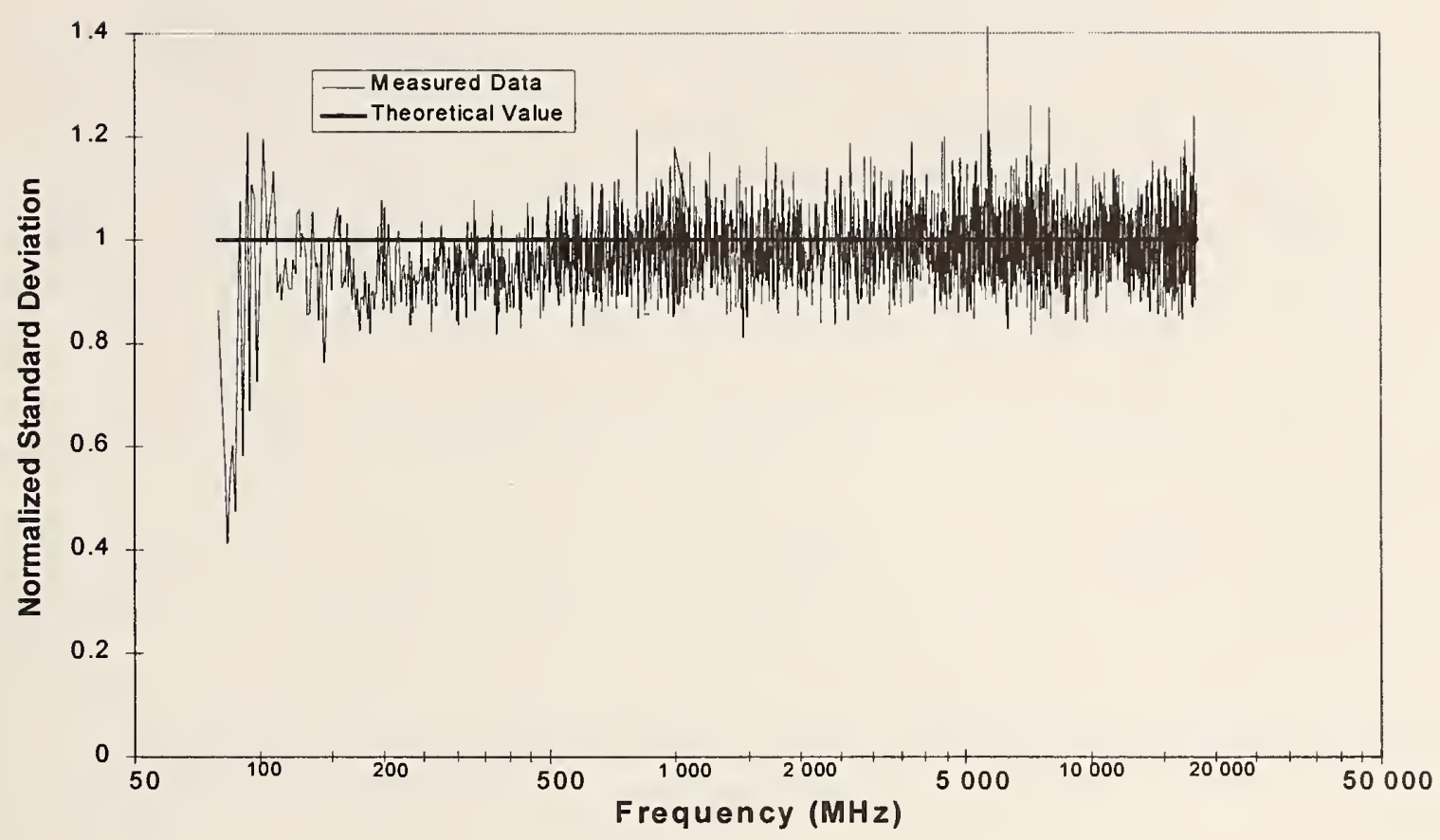

(a)

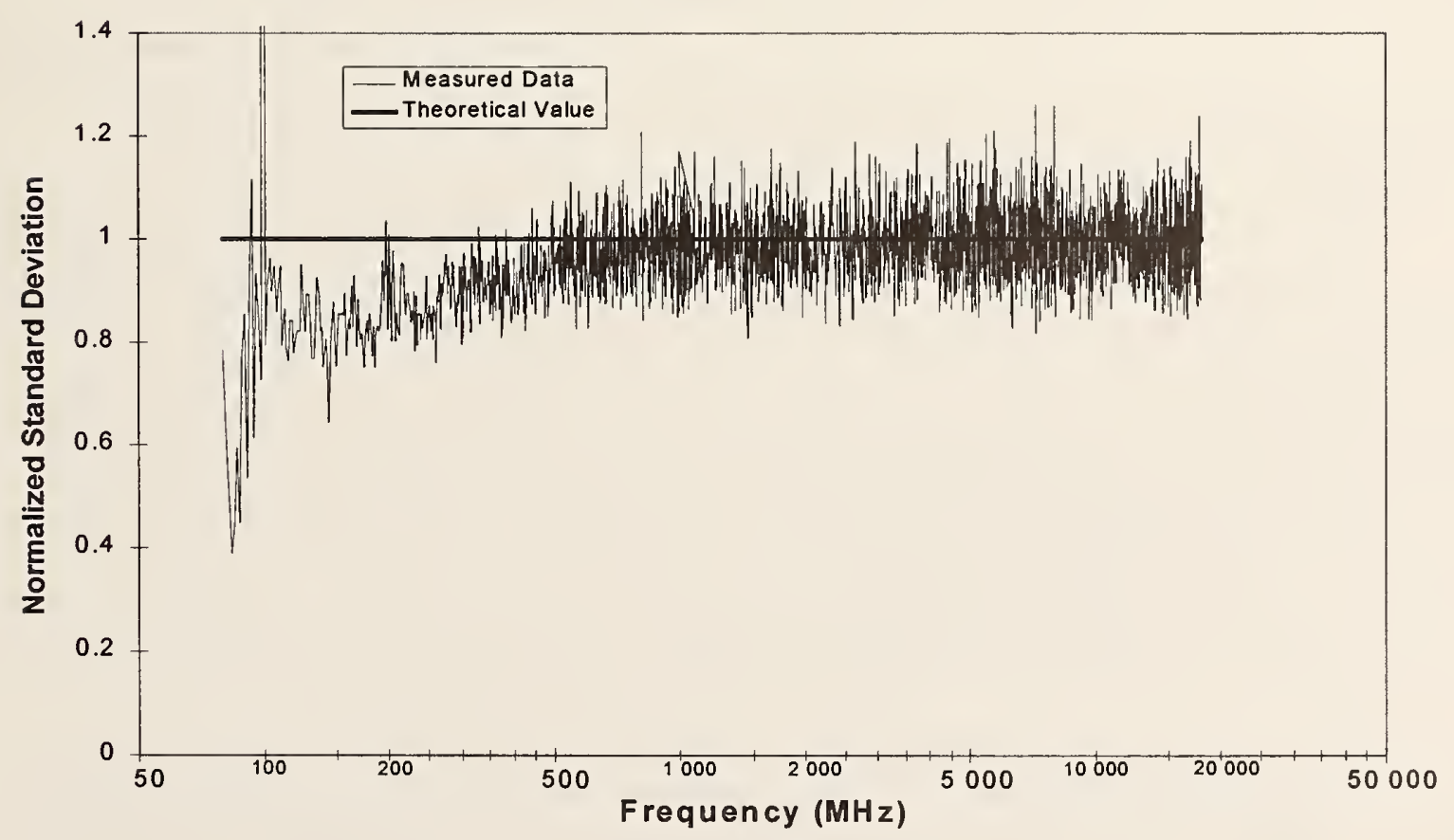

(b)

Figure 88. Normalized standard deviation of received power for a constant input power in chamber A. (a) Constant incident power. (b) Constant net input power. 
The normalized unstirred component of $S_{21}$ given in Figure $71 \mathrm{~b}$ indicates that the paddles are becoming less effective for some frequencies below $250 \mathrm{MHz}$, and show serious problems below $100 \mathrm{MHz}$. Otherwise, the characteristics are good. The measured values are typically less than 0.5 . Based on numerical simulations assuming 225 paddle positions, the measured values should be less than 0.25 , so there appears to be some deterministic component to the data, but it should have a negligible affect on estimates of chamber gain, electric field, etc. The maximum-to-average ratio given in Figure 72 indicates that the idealized statistical model may not accurately describe the power received by an obtrusive (electrically large and well matched) antenna for frequencies below $1 \mathrm{GHz}$, and that this effect is more apparent when the data are normalized to a constant net input power than when the data are normalized to a constant incident power.

The plots of chamber gain and residuals of chamber gain given in Figure 75 and Figure 76 show excellent agreement between the measured data and both the electromagnetic model and the statistical model.

The data taken with the probe system are presented in Figure 81 through Figure 86 . The processing required to generate these figures is discussed in Section 6.4. The optimum performance of the chamber occurs between $400 \mathrm{MHz}$ and $10 \mathrm{GHz}$, with only a slight increase in the spread of the probe readings between $10 \mathrm{GHz}$ and $18 \mathrm{GHz}$. We can think of no reason that the chamber should not continue to behave optimally above $10 \mathrm{GHz}$, so we attribute the apparent increase in uncertainty between $10 \mathrm{GHz}$ and $18 \mathrm{GHz}$ to our measurement system. When examining the data presented here, especially the variation in multiple measurements of a chamber parameter, it is important to remember that we can only observe the combined fluctuations of the parameter inside the chamber and the instrumentation. Therefore, the characteristics of the fields are better behaved than the data indicate.

The measurements of the maximum electric field (both rectangular component and total) shown in Figure 83 and Figure 84 indicate that the chamber is well behaved above $300 \mathrm{MHz}$, and even down to $100 \mathrm{MHz}$ the observed standard deviation of the rectangular component is less than $2.5 \mathrm{~dB}$, and the standard deviation of measured values of the total electric field are less than 2 $\mathrm{dB}$. These figures also show that the data from a reference antenna can be used to predict the maximum electric field, but it is best to estimate the maximum electric field (both rectangular component and total) from the average signal received by the reference antenna rather than the maximum. The difference between estimates of the rectangular component based on the average and maximum of the reference signal is small, but significant, and the difference between estimates of the total electric field based on the average and maximum of the reference signal are substantial.

The plots of the averages of the three orthogonal rectangular components of the electric field given in Figure 85 and Figure 86 show no significant dependence on orientation of the probes for measurements of both the average and maximum electric field above $250 \mathrm{MHz}$, and continues to be well behaved down to almost $100 \mathrm{MHz}$. Use of the chamber below $100 \mathrm{MHz}$ can result in significant biases due to preferred orientation of the dominant modes.

The plot of the cumulative distribution of the received power shown in Figure 87 indicates a reasonable match between the measured data and the ideal distribution, but the match is obviously not perfect. Several factors could be contributing to these imperfections. One problem is the unstirred component of $S_{21}$. The normalized unstirred component of $S_{21}$ for the 
data shown in Figure 87 is 0.4 . This value is small, but large enough to influence the cumulative distribution. Another possibility is that all indications of the validity of the statistical model (maximum-to-average ratio, normalized standard deviation) applied to the power received from a reference antenna show that the model is imperfect below $1 \mathrm{GHz}$ and may also be imperfect at $1 \mathrm{GHz}$. Finally, some measurements we have performed indicate that the data match the model better when a larger number of samples are taken. If we compare the cumulative distribution given in Figure 87 for 225 samples, and compare it with the cumulative distribution given in Figure $7 \mathrm{~b}$ for 1024 samples, the data in Figure $7 \mathrm{~b}$ are a much better match with the theory. Although the agreement between measurement and theory should improve as more samples are taken, the difference presented here is more dramatic than expected.

Figure 88 shows the normalized standard deviation of the power received from the reference antenna, calculated as the standard deviation of the received power divided by the average received power. This ratio is close to the expected value of 1 for frequencies greater than $1 \mathrm{GHz}$, and below $1 \mathrm{GHz}$, this ratio falls below 1 . This effect is more pronounced when the received power is normalized to a constant net input power (Figure 88b) than when the data are normalized to a constant incident power (Figure 88a). The normalized standard deviation indicates that the power received by the reference antenna is not well described by the statistical model below $1 \mathrm{GHz}$. This is most likely caused by the same clipping effect that causes the maximum-to-average ratio to decrease below $1 \mathrm{GHz}$.

\subsection{Summary of Chamber B}

A summary of the figures we used to describe the characteristics of Chamber B is given in Table 10. The parameters presented here are identical to those presented for Chamber A. We will describe them again here so that you will not need to refer to another section to understand this section.

The figures are grouped with antenna characteristics presented first, followed by an evaluation of the mechanical stirrers as described by the unstirred component of $S_{21}$, and then the ratios of the extreme values (maximum-to-average ratio, maximum-to-minimum ratio, average-to-minimum ratio). We then give the average and maximum chamber gain, residuals of chamber gain, followed by all parameters that can be estimated or predicted from measurements of chamber gain (electric field for an input power of $1 \mathrm{~W}$ and the residuals of electric field, average scalar power density, and average quality factor. The estimated volume of Chamber B used to calculate the quality factor of the chamber was $80.434 \mathrm{~m}^{3}$. The characteristics of the electric field as measured by the probe system are presented next, including the average and maximum magnitude of the electric field (both rectangular component and total). Finally, we present data from some of the tools used to verify the statistical characteristics of the power received from an antenna in the chamber. These are plots of the cumulative distribution and the normalized standard deviation.

The equations describing the key parameters are given in Table 11. All equations are based on curve fit parameters for the average chamber gain of $a=3.196$ and $b=1.611 \cdot 10^{-21}$ for data normalized to a constant incident power, and values of $a=2.670$ and $b=1.607 \cdot 10^{-21}$ for data normalized to a constant net input power. 
Table 10. Summary of figures related to Chamber B.

Minimum, average, and maximum VSWR

Figure 89

Minimum, average, and maximum reflected power

Figure 90

Mismatch correction factor

Figure 91

Estimated efficiencies of antennas

Figure 92

Unstirred component of $S_{21}$

Figure 93

Maximum-to-average ratio

Figure 94

Maximum-to-minimum ratio

Figure 95

Average-to-minimum ratio

Figure 96

Average and maximum chamber gain

Figure 97

Residuals of chamber gain

Figure 98

Electric field

Figure 99

Residuals of electric field

Figure 100

Scalar power density

Quality factor

Figure 101

Figure 102

Average magnitude of electric field (rectangular component) using probe system

Average magnitude of electric field (total) using probe system

Figure 103

Figure 104

Maximum magnitude of electric field (rectangular component) using probe system

Maximum magnitude of electric field (total) using probe system

Comparison of average $\mathrm{x}, \mathrm{y}$, and $\mathrm{z}$ components of electric field

Figure 105

Figure 106

Comparison of maximum $\mathrm{x}, \mathrm{y}$, and $\mathrm{z}$ components of electric field

Figure 107

Cumulative distribution of received power at $1 \mathrm{GHz}$

Figure 108

Normalized standard deviation of received power

Figure 109

Figure 110 
Table 11. Estimated equations for descriptive characteristics of Chamber B.

\begin{tabular}{|c|c|c|}
\hline Parameter & Constant incident power & Constant net input power \\
\hline \multirow[b]{2}{*}{$\operatorname{MIN}\left(G_{C}\right)$} & 1 & 1 \\
\hline & $\overline{N\left(3.196+1.611 \cdot 10^{-21} f^{5 / 2}\right)}$ & $\overline{N\left(2.670+1.607 \cdot 10^{-21} f^{5 / 2}\right)}$ \\
\hline \multirow[b]{2}{*}{$\operatorname{AVE}\left(G_{C}\right)$} & 1 & 1 \\
\hline & $\overline{\left(3.196+1.611 \cdot 10^{-21} f^{5 / 2}\right)}$ & $\overline{\left(2.670+1.607 \cdot 10^{-21} f^{5 / 2}\right)}$ \\
\hline \multirow{3}{*}{$\operatorname{MAX}\left(G_{C}\right)$} & 1 & 1 \\
\hline & $\left.1+\frac{1.611 \cdot 10^{-21}}{0.2} f^{5 / 2}\right)$ & $\left.1+\frac{1.607 \cdot 10^{-21}}{} f^{5 / 2}\right)$ \\
\hline & $\left({ }^{1 .} 0.577+\ln (N)\right.$ & $0.577+\ln (N)$ \\
\hline \multirow{2}{*}{$\operatorname{AVE}\left(E_{R}\right)$} & $80 \pi^{3} f^{2} P_{i n c}$ & $80 \pi^{3} f^{2} P_{N E T}$ \\
\hline & $\sqrt{c^{2}\left(3.196+1.611 \cdot 10^{-21} f^{5 / 2}\right)}$ & $\sqrt{c^{2}\left(2.670+1.607 \cdot 10^{-21} f^{5 / 2}\right)}$ \\
\hline \multirow{2}{*}{$\operatorname{MAX}\left(E_{R}\right)$} & $80 \pi^{3} f^{2} P_{i n c}[R(N)]^{2}$ & $80 \pi^{3} f^{2} P_{N E T}[R(N)]^{2}$ \\
\hline & $\sqrt{c^{2}\left(3.196+1.611 \cdot 10^{-21} f^{5 / 2}\right)}$ & $\sqrt{c^{2}\left(2.670+1.607 \cdot 10^{-21} f^{5 / 2}\right)}$ \\
\hline \multirow{2}{*}{$\operatorname{AVE}\left(E_{T}\right)$} & $1125 \pi^{3} f^{2} P_{i n c}$ & $1125 \pi^{3} f^{2} P_{N E T}$ \\
\hline & $\sqrt{4 c^{2}\left(3.196+1.611 \cdot 10^{-21} f^{5 / 2}\right)}$ & $\sqrt{4 c^{2}\left(2.670+1.607 \cdot 10^{-21} f^{5 / 2}\right)}$ \\
\hline \multirow{2}{*}{$\operatorname{MAX}\left(E_{T}\right)$} & $1125 \pi^{3} f^{2} P_{\text {inc }}[T(N)]^{2}$ & $1125 \pi^{3} f^{2} P_{N E T}[T(N)]^{2}$ \\
\hline & $\sqrt{4 c^{2}\left(3.196+1.611 \cdot 10^{-21} f^{5 / 2}\right)}$ & $\sqrt{4 c^{2}\left(2.670+1.607 \cdot 10^{-21} f^{5 / 2}\right)}$ \\
\hline \multirow{2}{*}{$\operatorname{AVE}\left(S_{c}\right)$} & $8 \pi f^{2} P_{i n c}$ & $8 \pi f^{2} P_{N E T}$ \\
\hline & $c^{2}\left(3.196+1.611 \cdot 10^{-21} f^{5 / 2}\right)$ & $c^{2}\left(2.670+1.607 \cdot 10^{-21} f^{5 / 2}\right)$ \\
\hline \multirow{2}{*}{$\operatorname{AVE}(Q)$} & $16 \pi^{2} V f^{3}$ & $16 \pi^{2} V f^{3}$ \\
\hline & $c^{3}\left(3.196+1.611 \cdot 10^{-21} f^{5 / 2}\right)$ & $\overline{c^{3}\left(2.670+1.607 \cdot 10^{-21} f^{5 / 2}\right)}$ \\
\hline
\end{tabular}




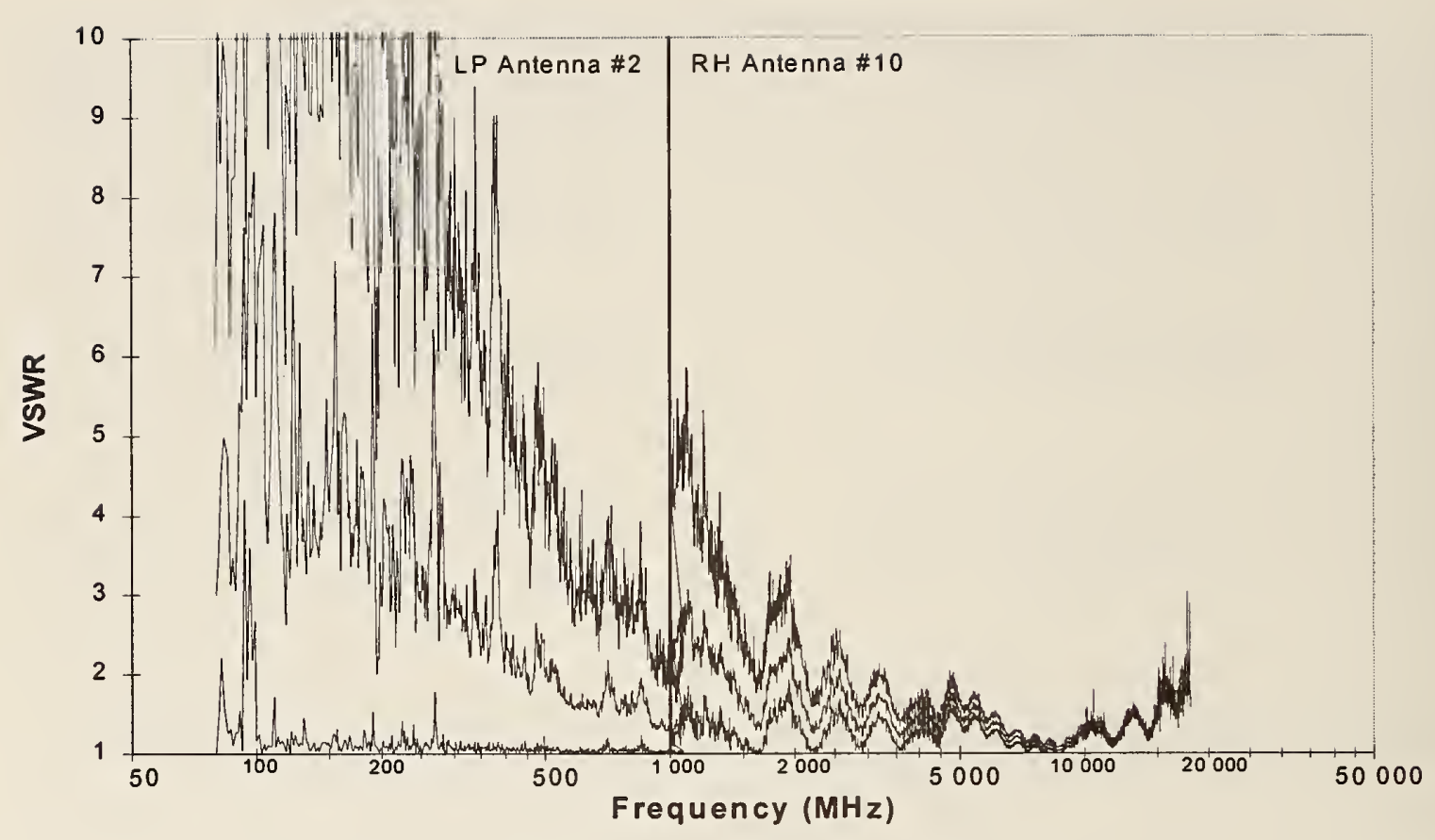

(a)

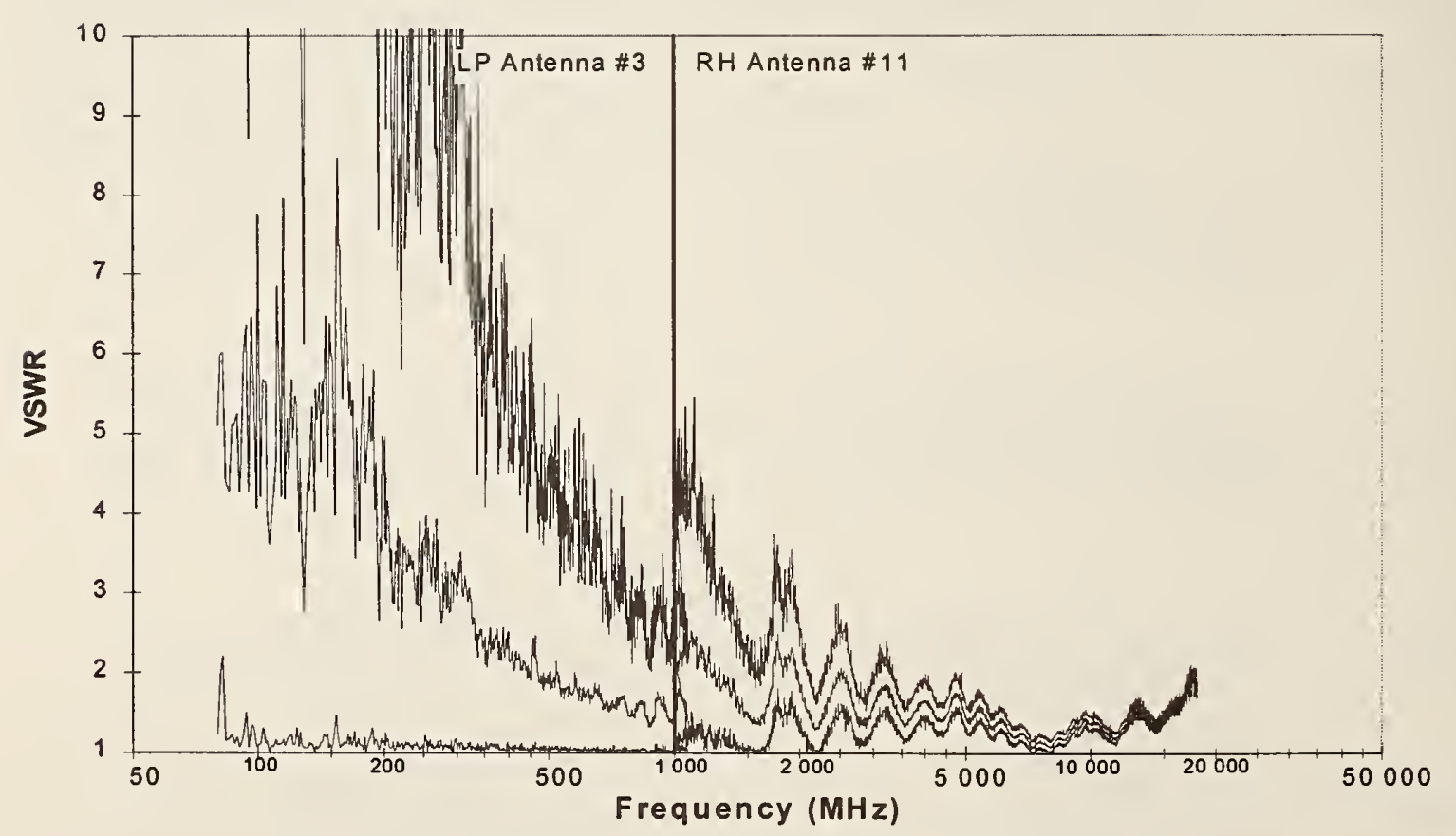

(b)

Figure 89. Minimum, average, and maximum VSWR in chamber B. (a) Port 1 transmitting (forward). Log periodic antenna 2, $80 \mathrm{MHz}$ to $1.08 \mathrm{GHz}$. Ridged horn antenna 10, $1 \mathrm{GHz}$ to $18 \mathrm{GHz}$. (b) Port 2 transmitting (reverse). Log periodic antenna 3, $80 \mathrm{MHz}$ to $1.08 \mathrm{GHz}$. Ridged horn antenna $11,1 \mathrm{GHz}$ to $18 \mathrm{GHz}$. 


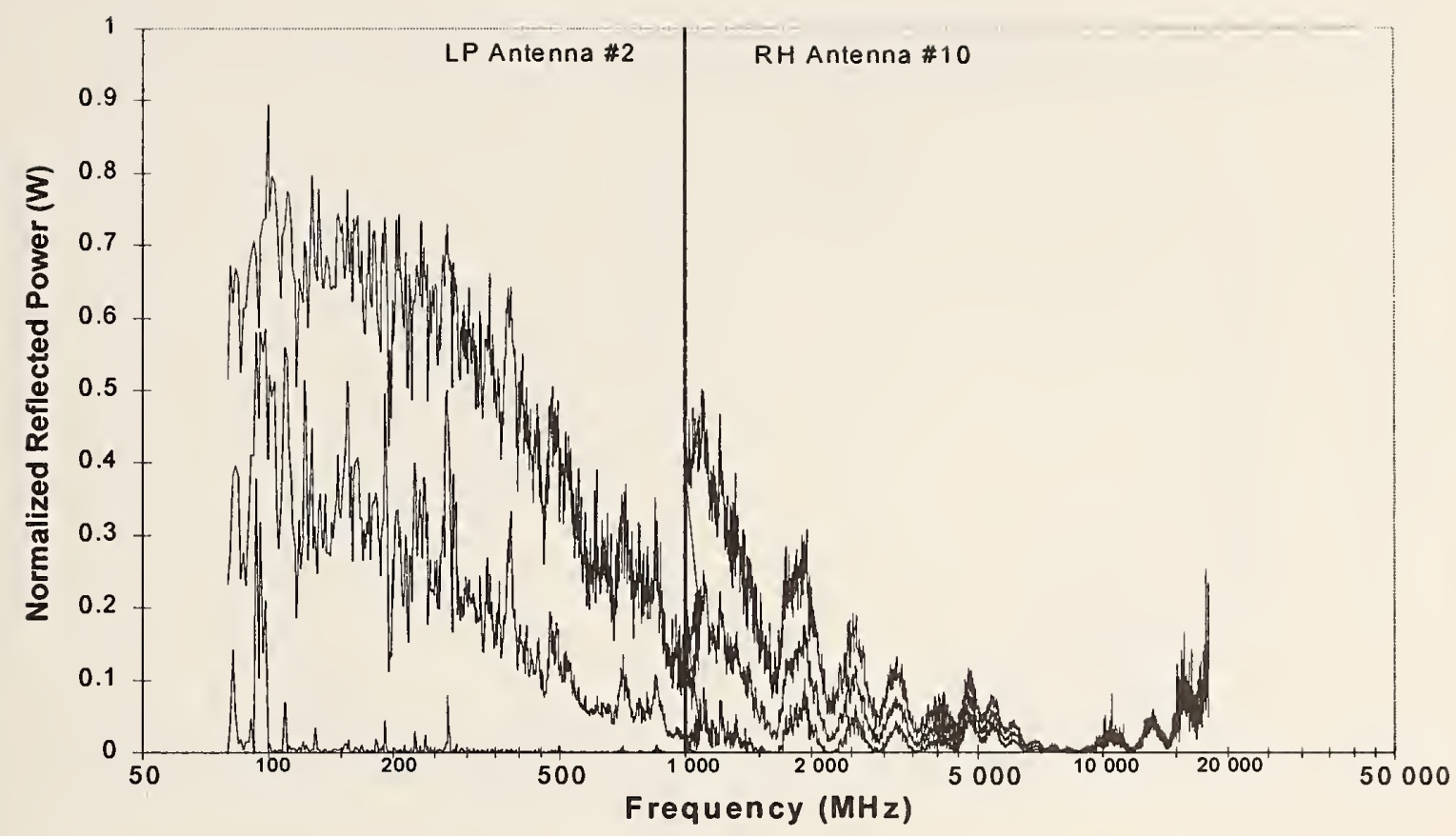

(a)

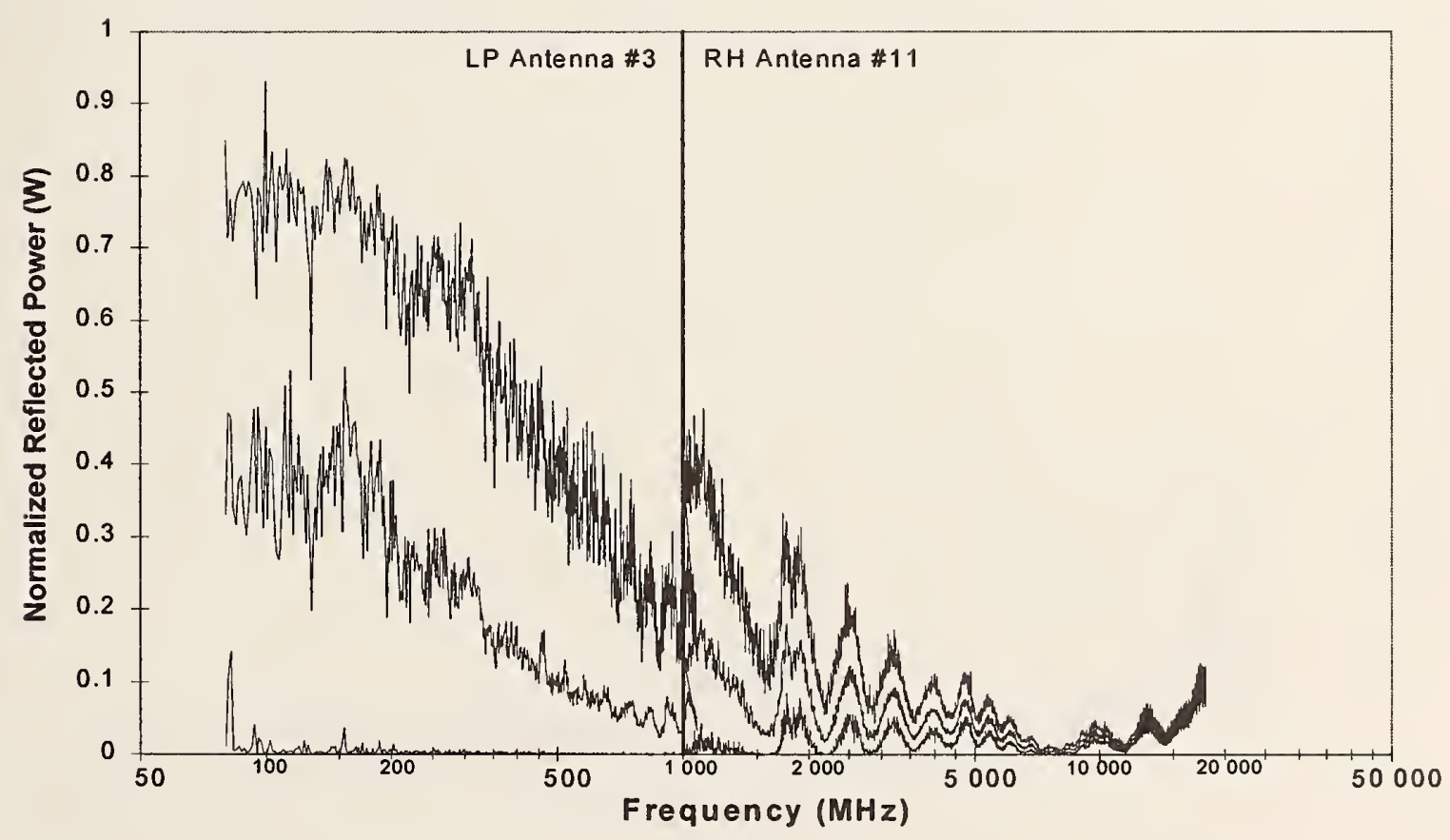

(b)

Figure 90. Minimum, average, and maximum reflected power for an incident power of $1 \mathrm{~W}$ in chamber B. (a) Port 1 transmitting (forward). Log periodic antenna 2, $80 \mathrm{MHz}$ to $1.08 \mathrm{GHz}$. Ridged horn antenna $10,1 \mathrm{GHz}$ to $18 \mathrm{GHz}$. (b) Port 2 transmitting (reverse). Log periodic antenna 3, $80 \mathrm{MHz}$ to $1.08 \mathrm{GHz}$. Ridged horn antenna 11, $1 \mathrm{GHz}$ to $18 \mathrm{GHz}$. 


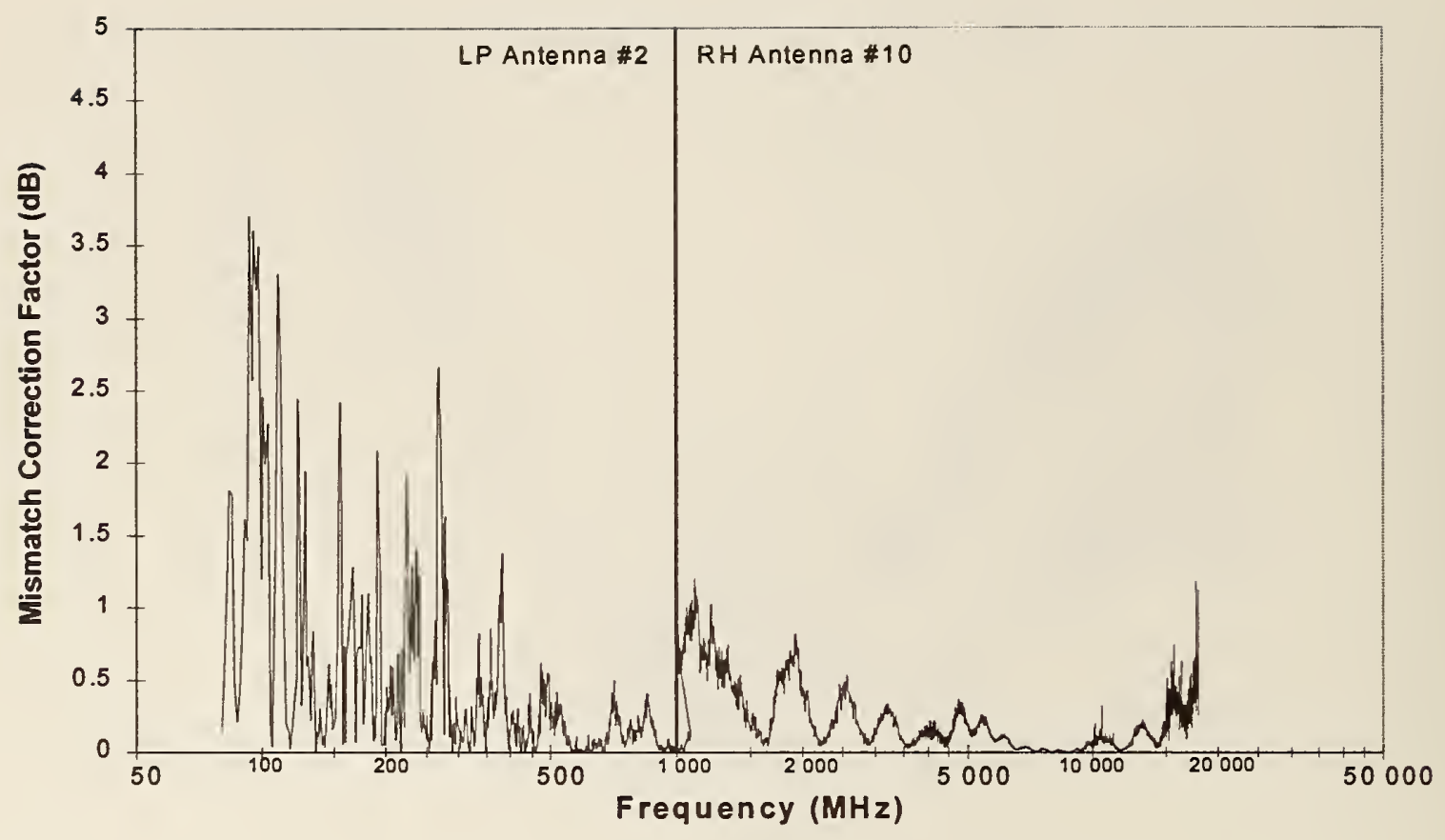

(a)

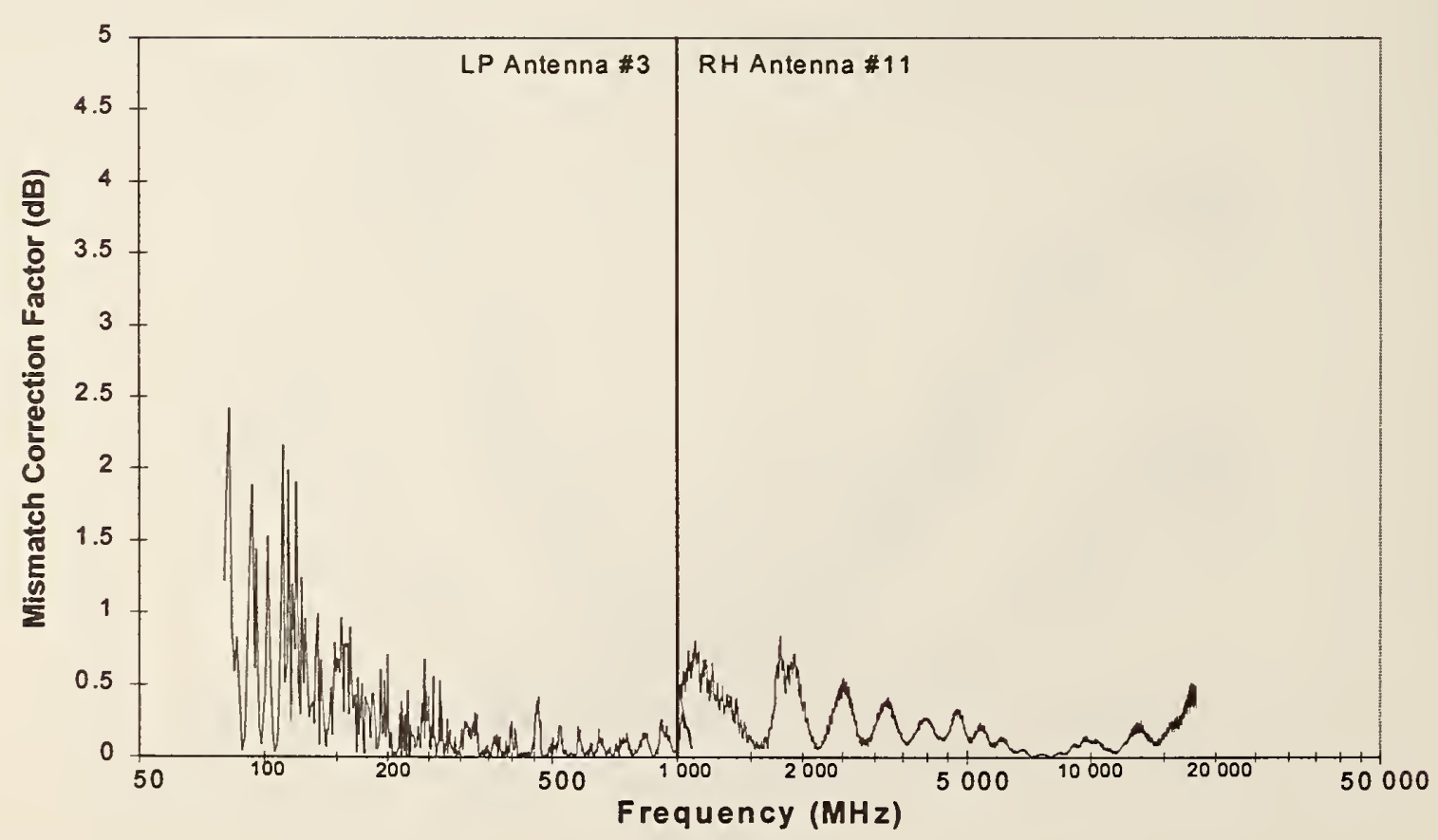

(b)

Figure 91. Mismatch correction factor in chamber B. (a) Port 1 transmitting (forward). Log periodic antenna $2,80 \mathrm{MHz}$ to $1.08 \mathrm{GHz}$. Ridged horn antenna $10,1 \mathrm{GHz}$ to $18 \mathrm{GHz}$. (b) Port 2 transmitting (reverse). Log periodic antenna $3,80 \mathrm{MHz}$ to $1.08 \mathrm{GHz}$. Ridged horn antenna $11,1 \mathrm{GHz}$ to $18 \mathrm{GHz}$. 


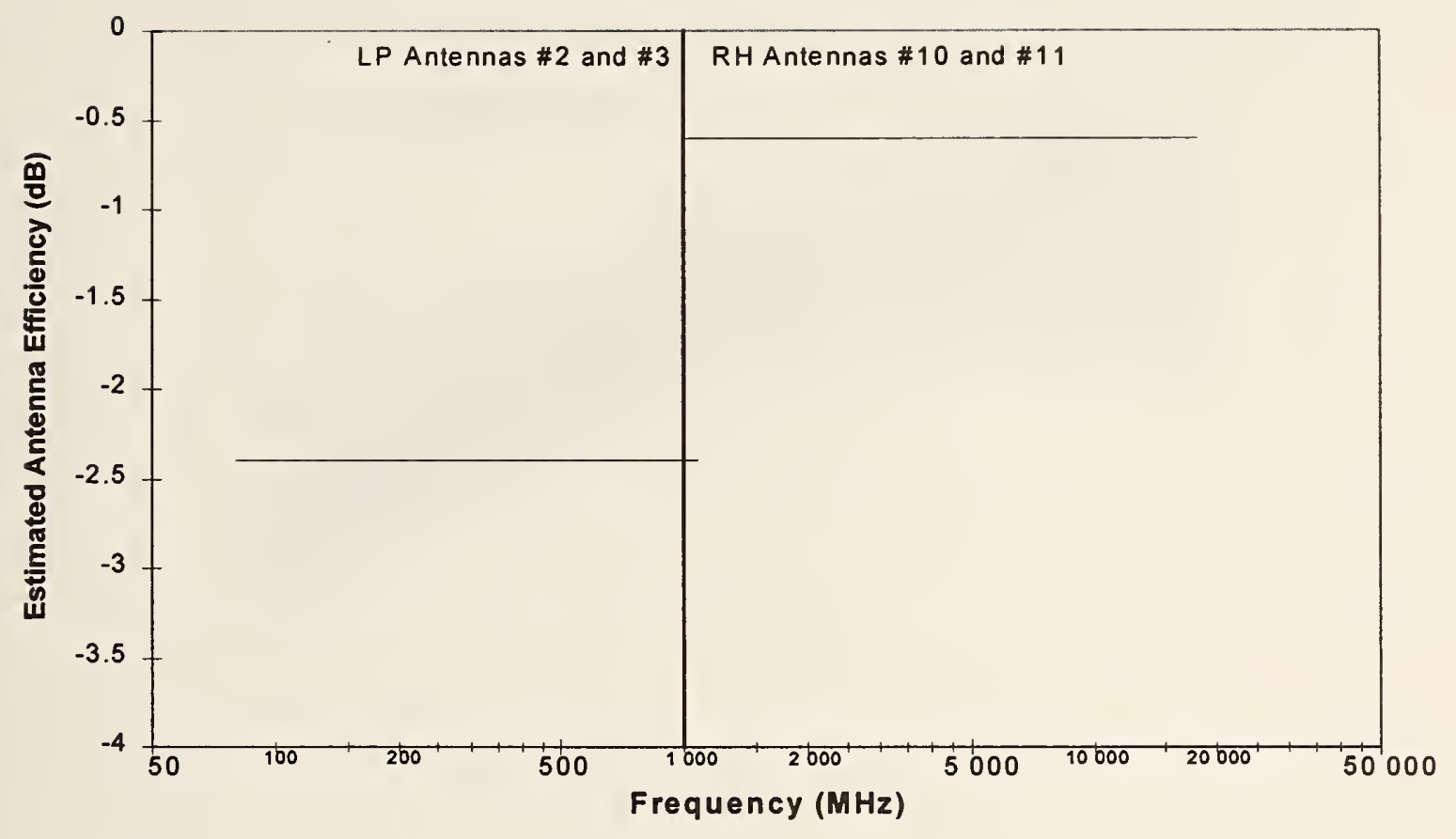

Figure 92. Estimated efficiencies of measurement antennas in chamber B. Log periodic antennas 2 and 3, $80 \mathrm{MHz}$ to $1.08 \mathrm{GHz}$. Ridged horn antennas 10 and $11,1 \mathrm{GHz}$ to $18 \mathrm{GHz}$. 


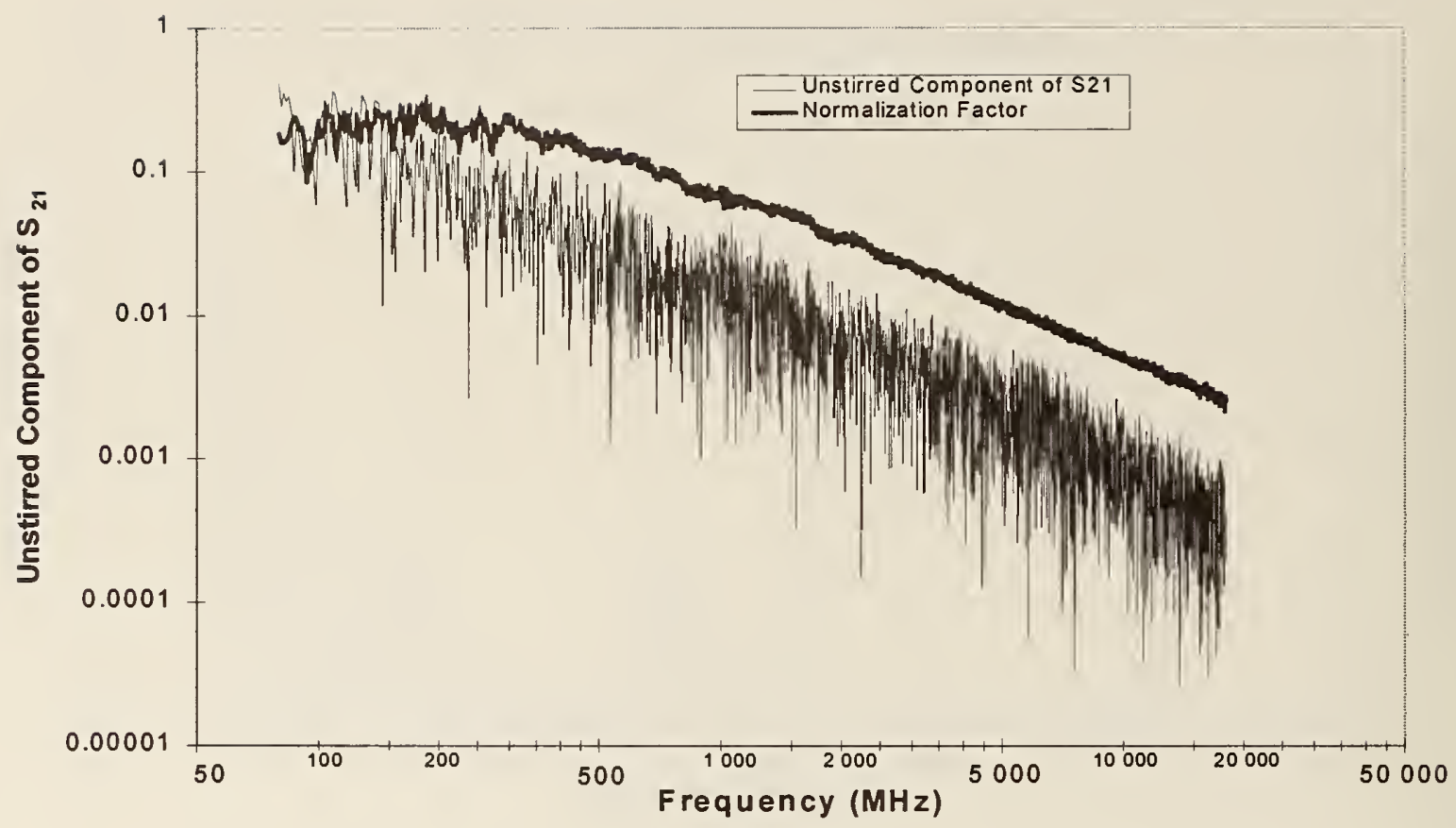

(a)

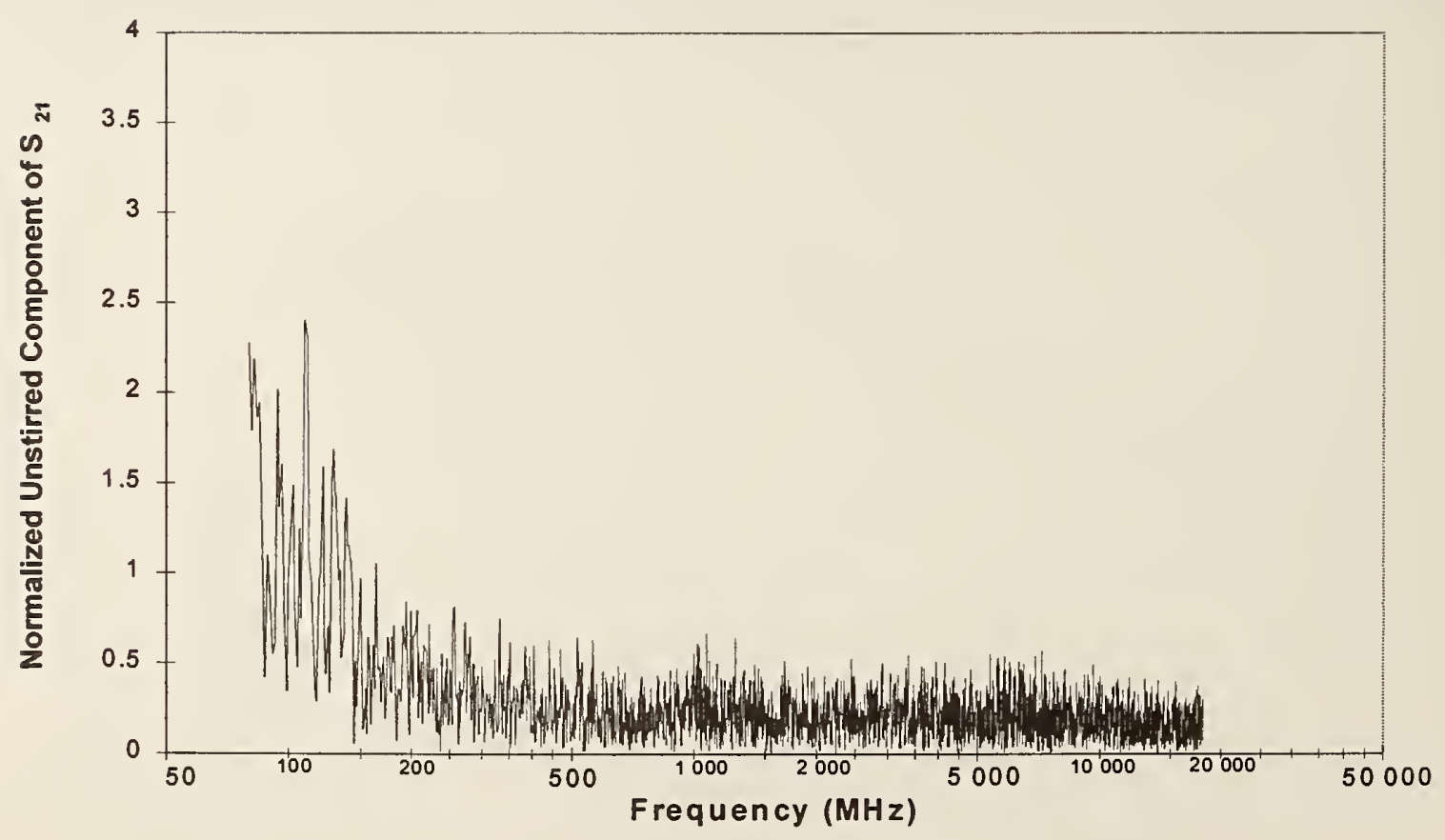

(b)

Figure 93. Evaluation of mechanical stirrers in chamber B. (a) Unstirred component of $S_{21}$. (b) Normalized unstirred component of $S_{21}$. 


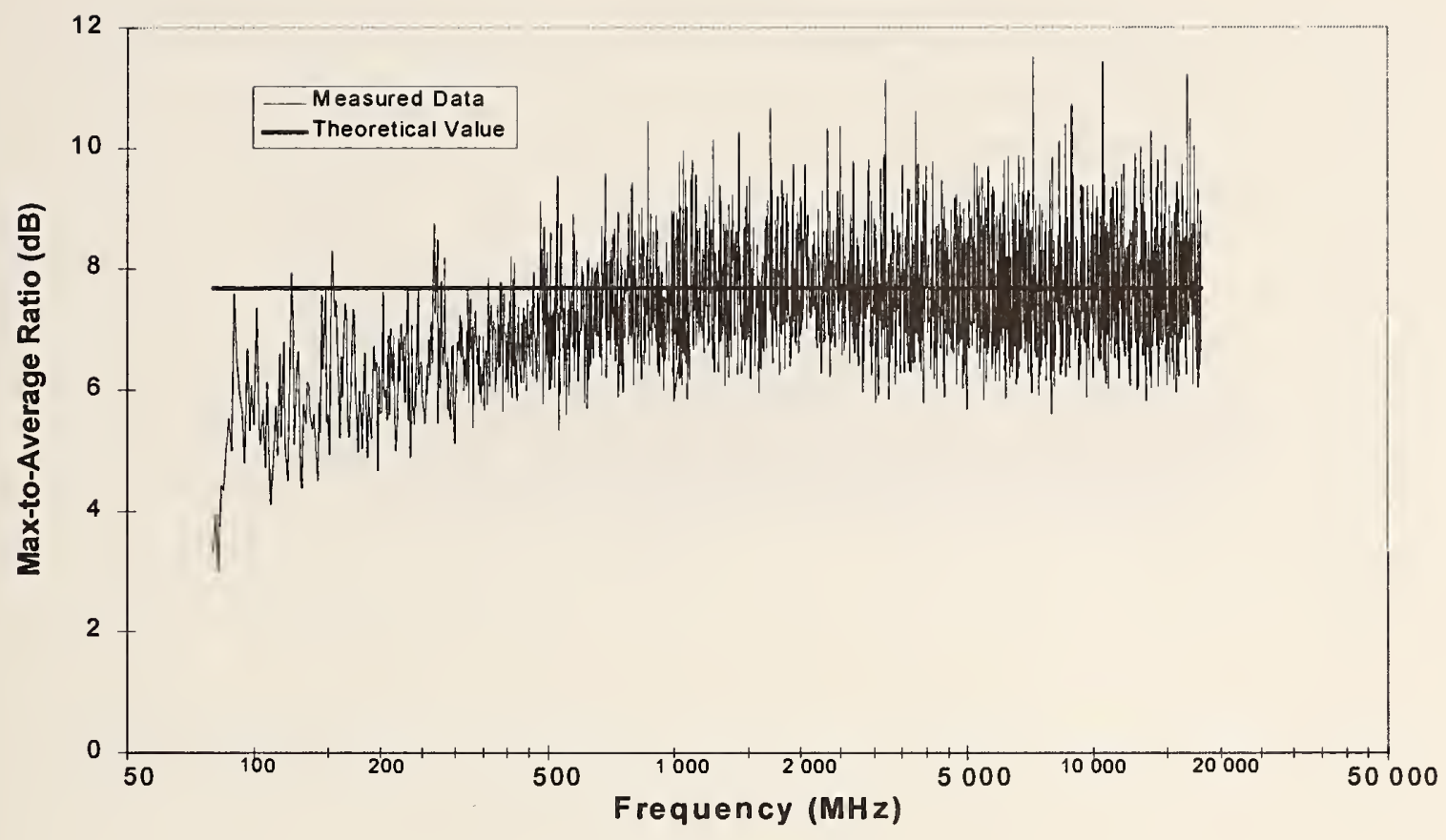

(a)

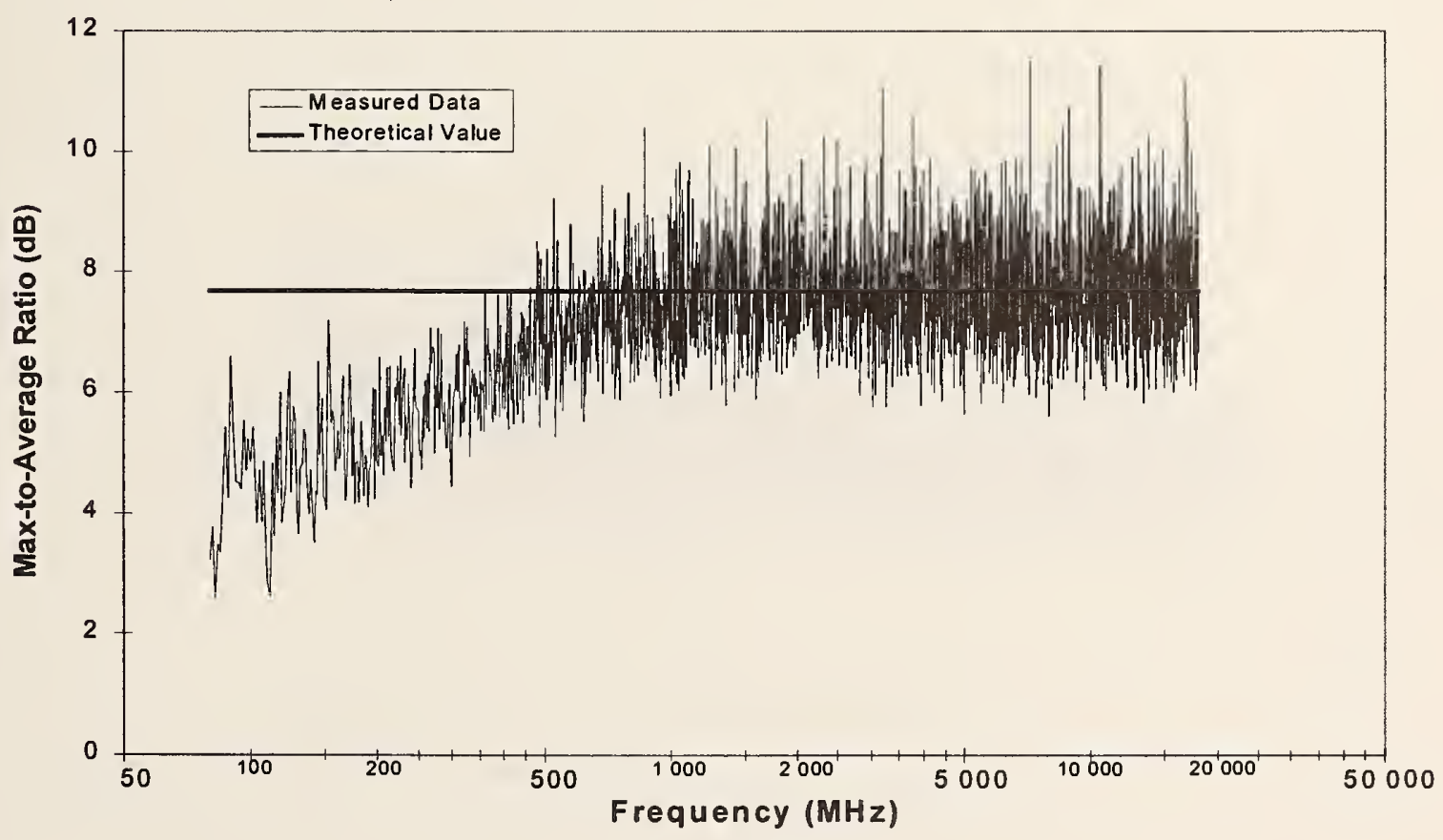

(b)

Figure 94. Max-to-average ratio in chamber B. (a) Constant incident power. (b) Constant net input power. 


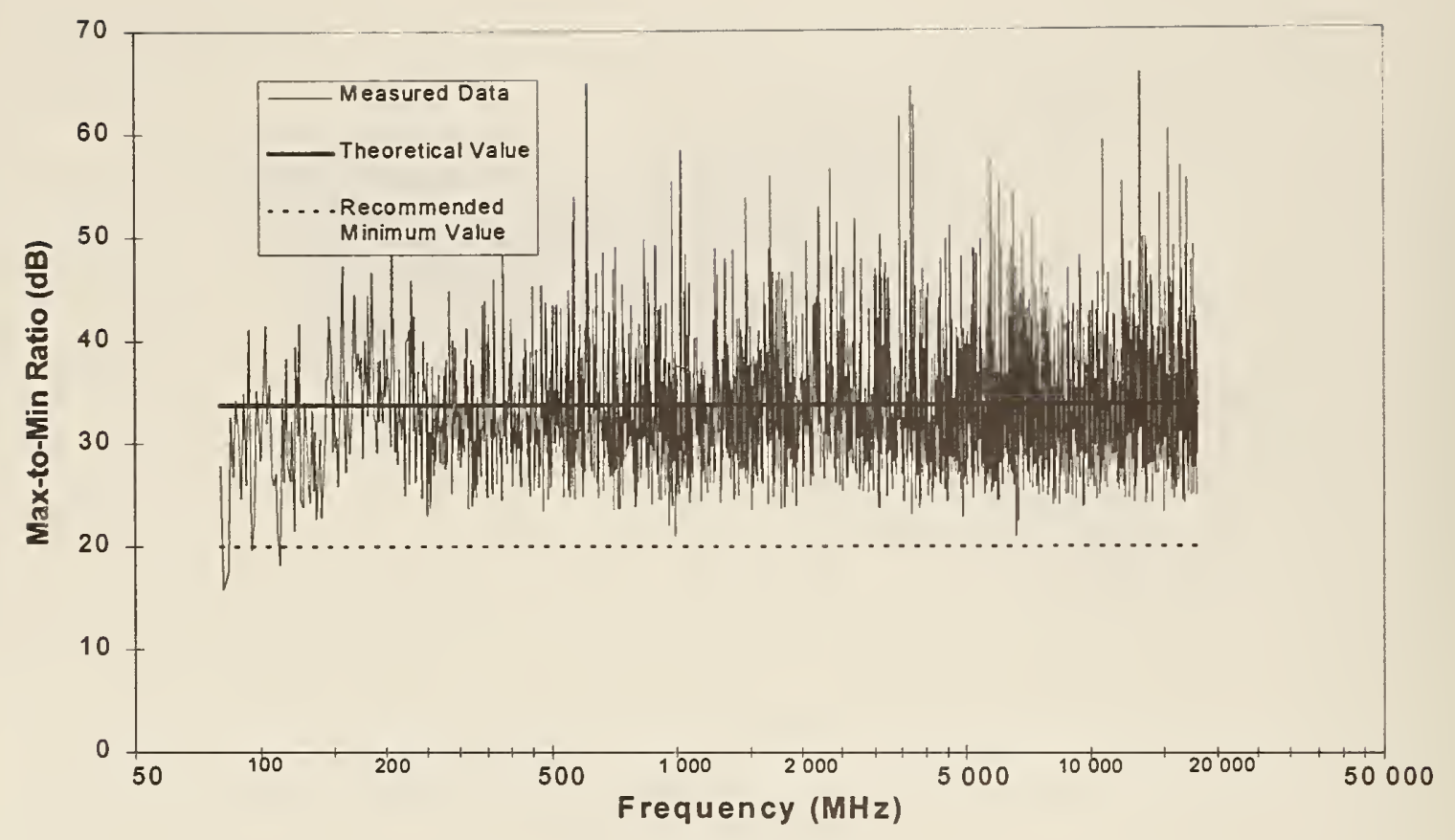

(a)

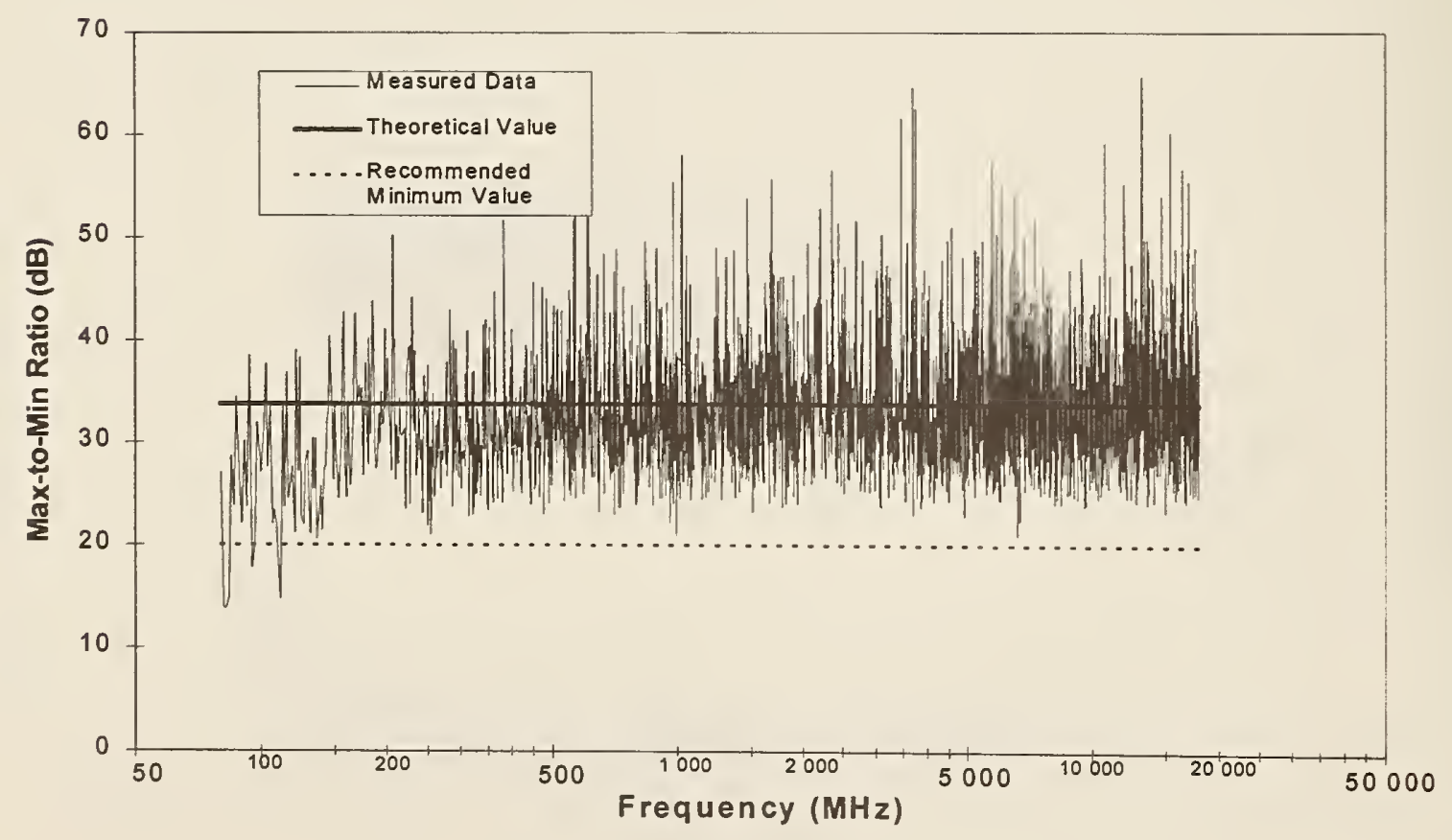

(b)

Figure 95. Max-to-min ratio in chamber B. (a) Constant incident power. (b) Constant net input power. 


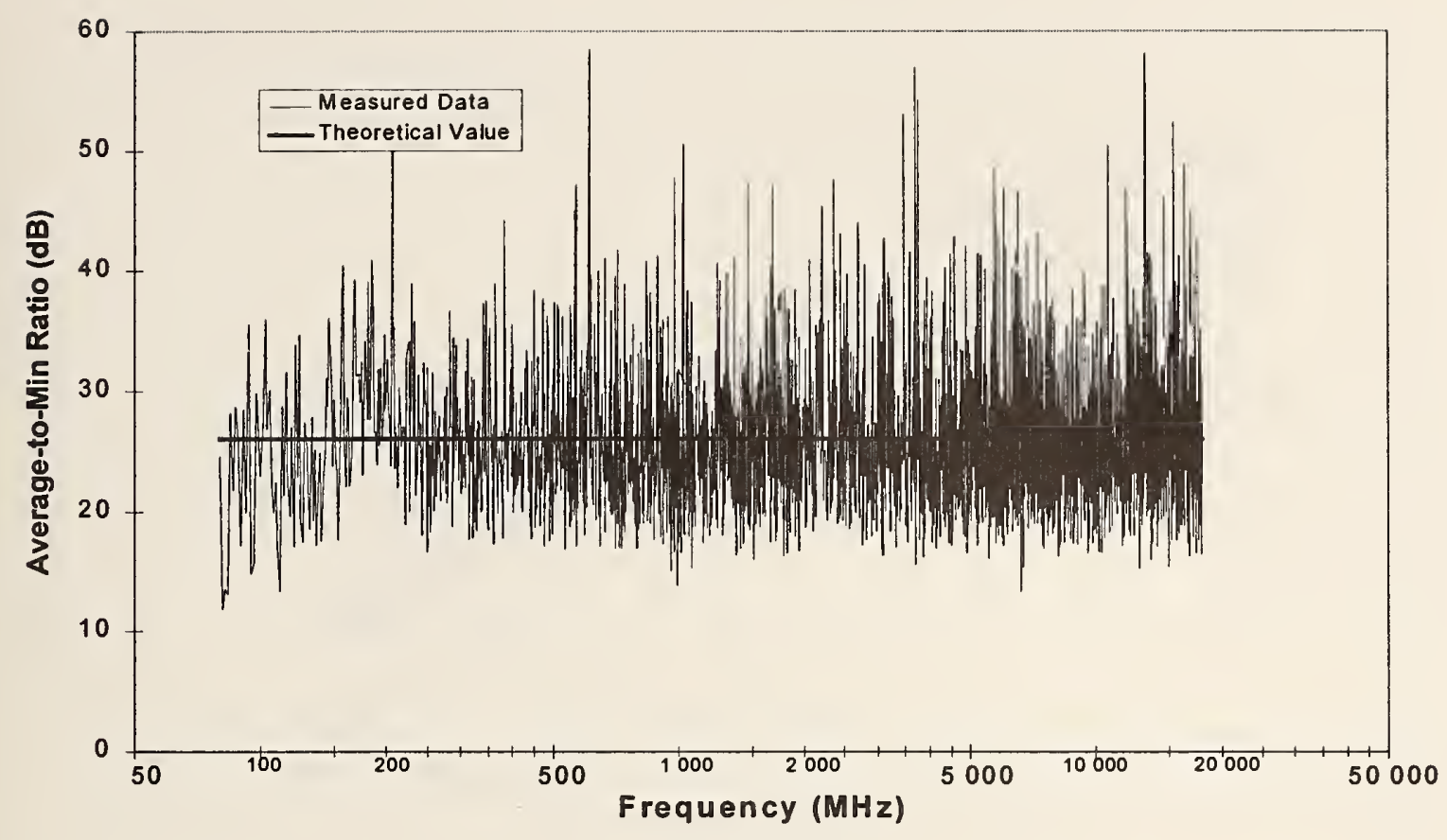

(a)

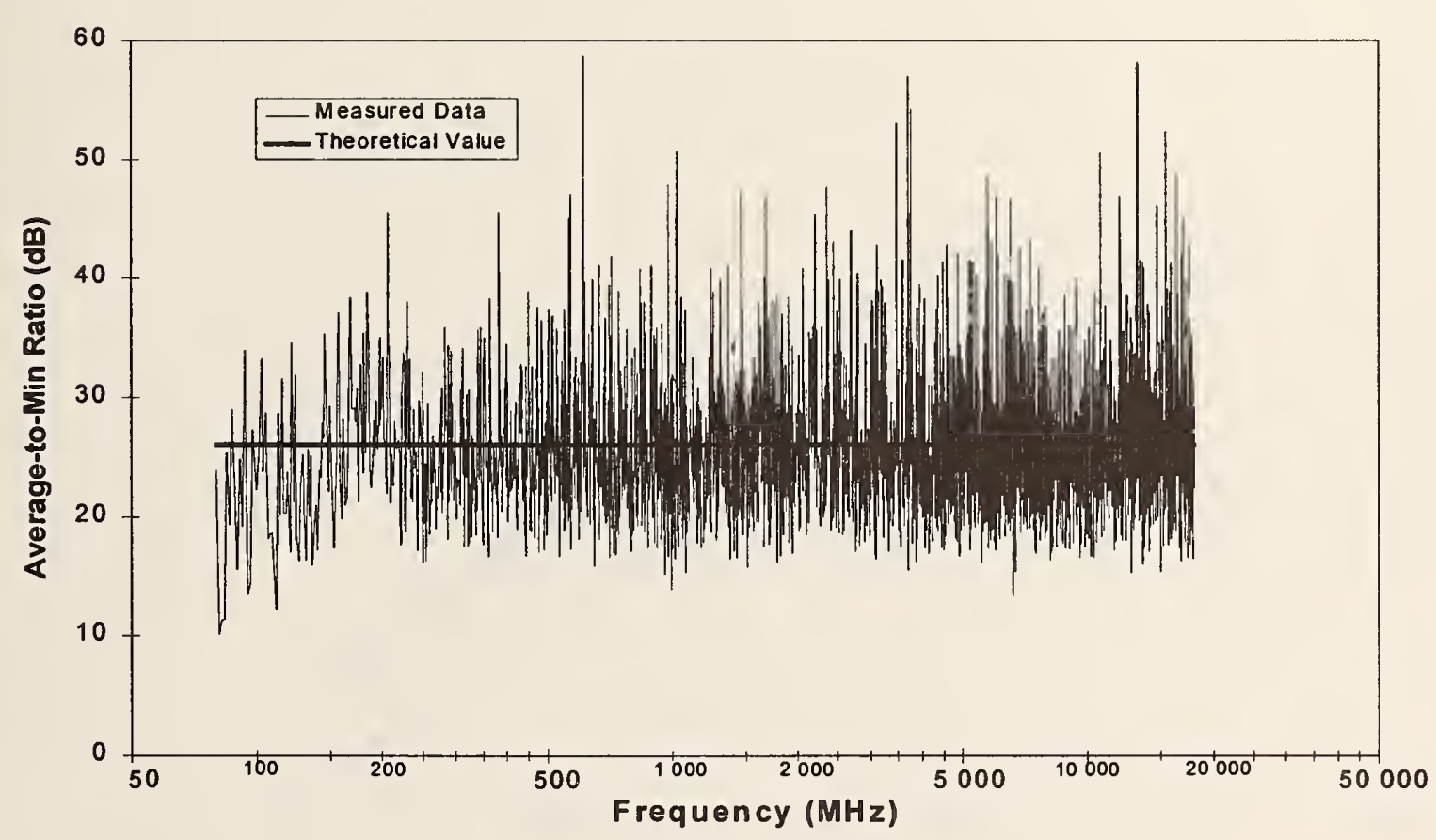

(b)

Figure 96. Average-to-min ratio in chamber B. (a) Constant incident power. (b) Constant net input power. 


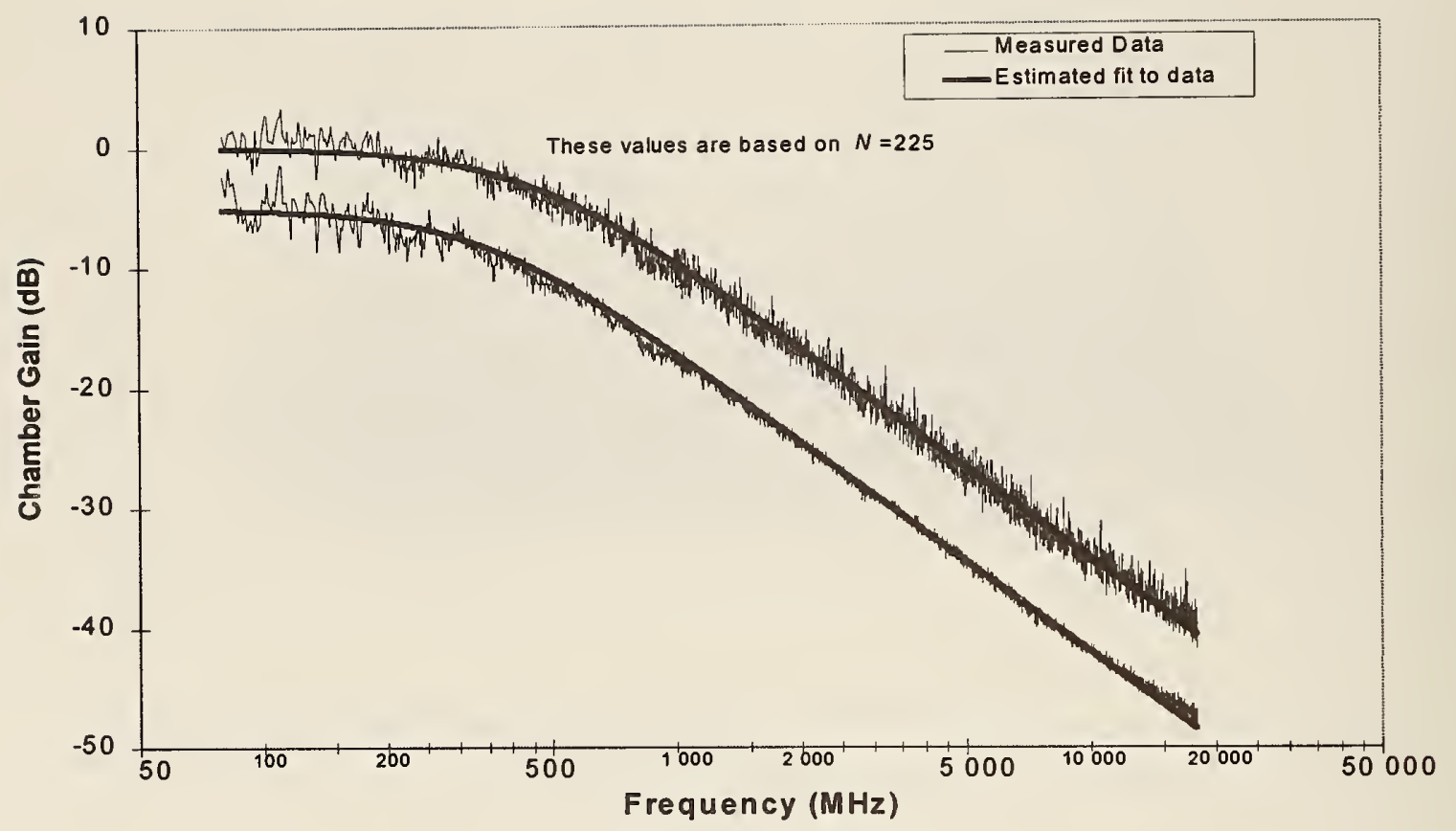

(a)

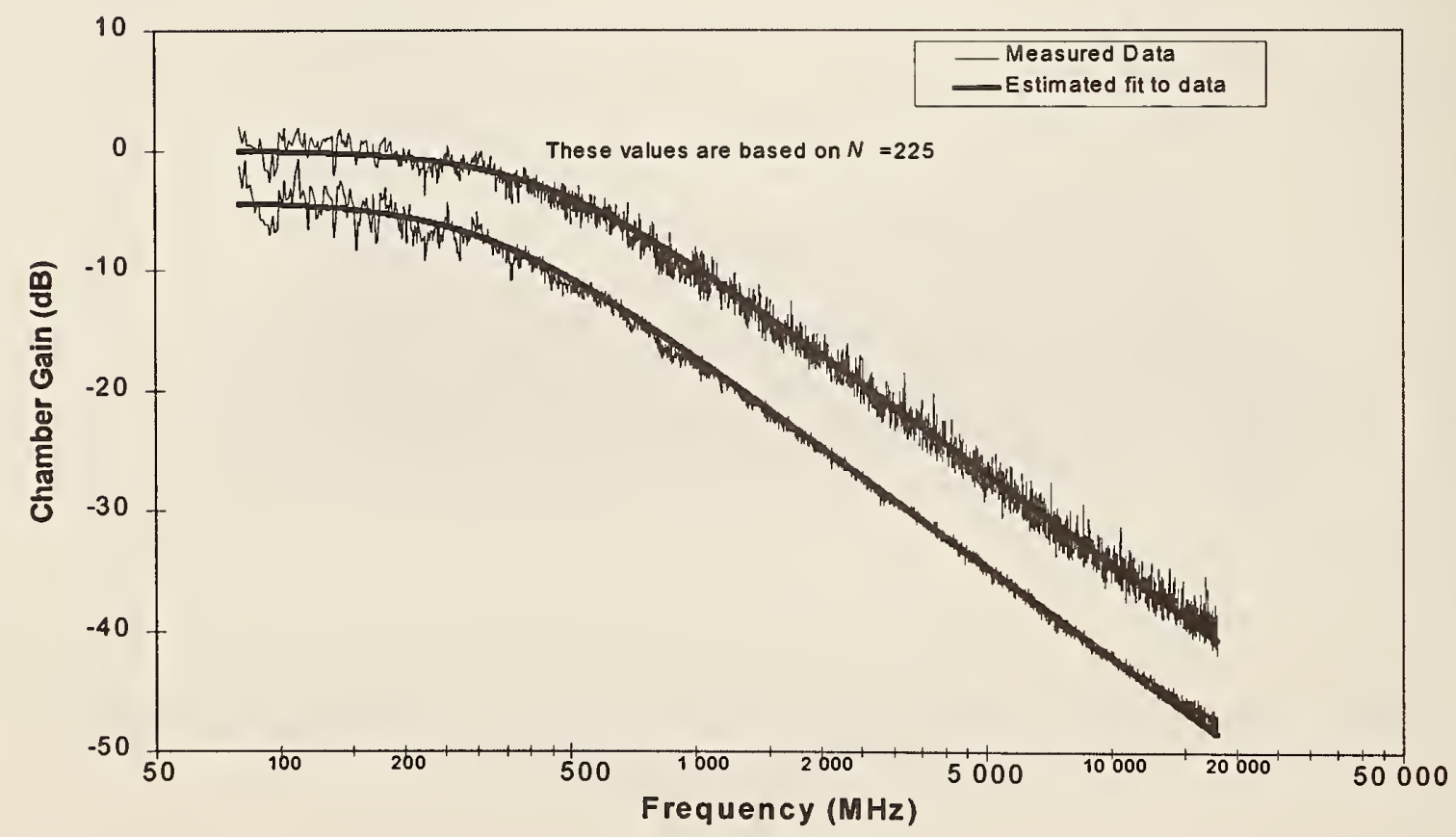

(b)

Figure 97. Average and maximum chamber gain in chamber B. (a) Constant incident power. (b) Constant net input power. 


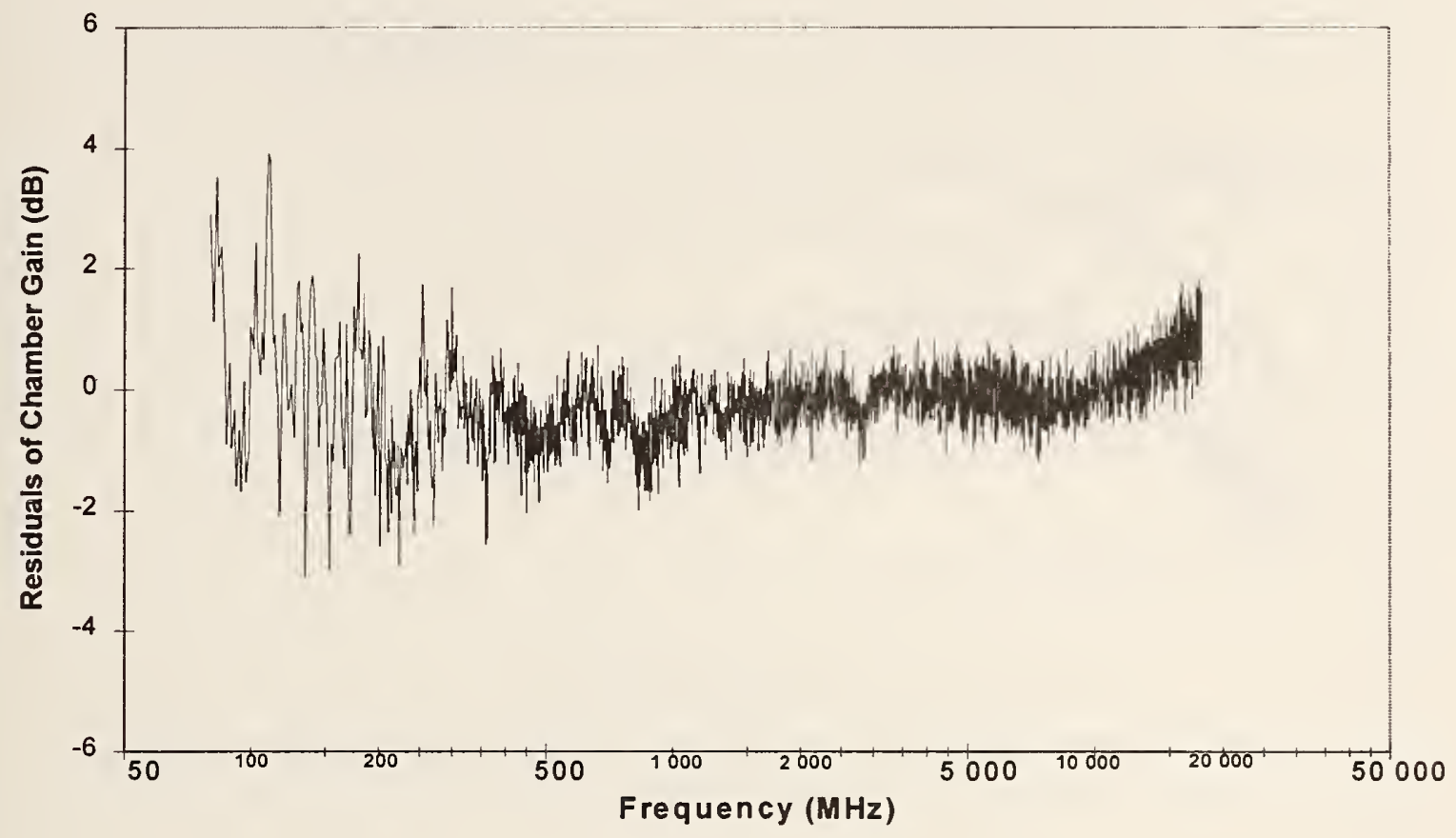

(a)

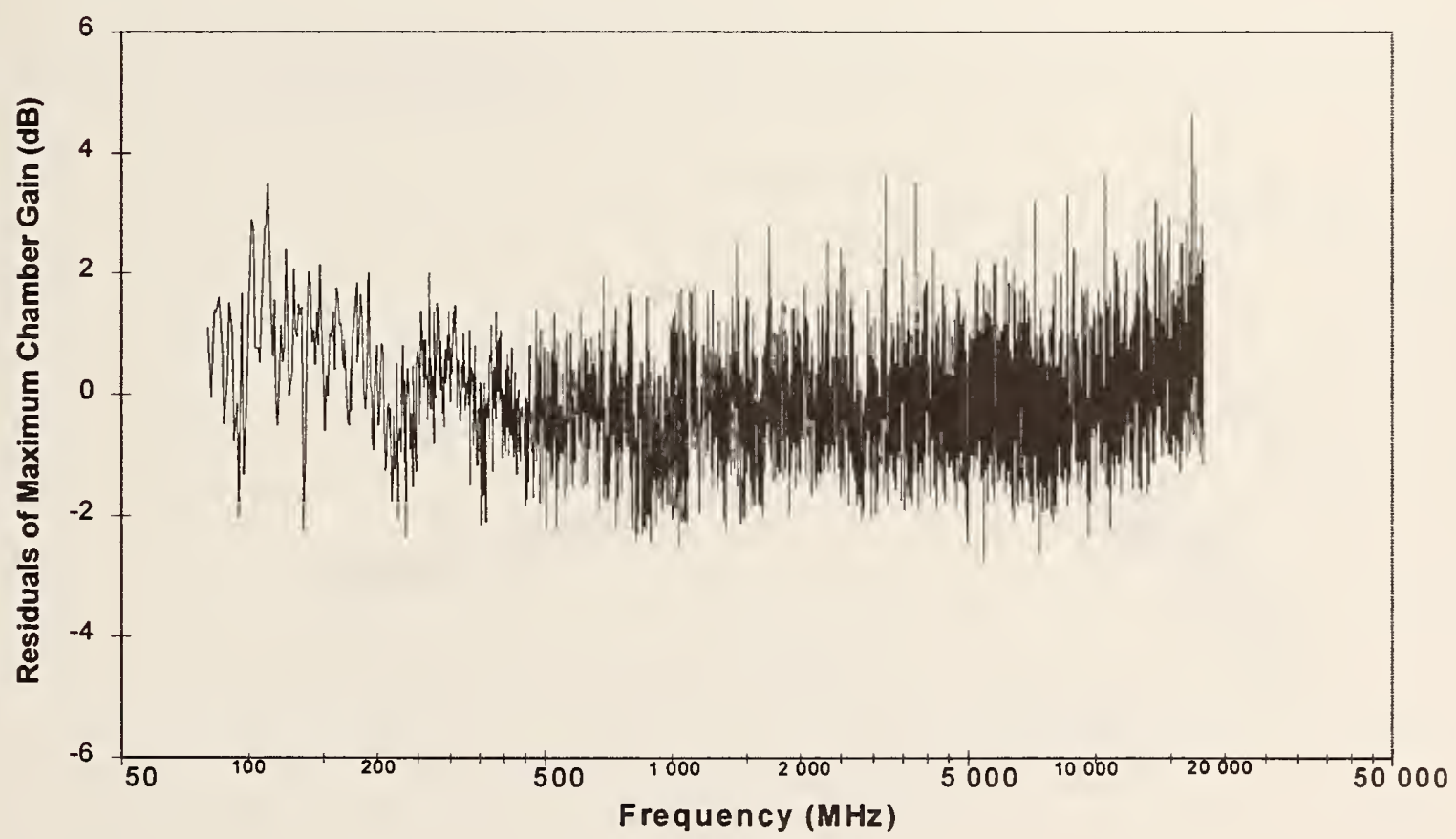

(b)

Figure 98. Residuals of chamber gain in chamber B. (a) Residuals of average chamber gain. (b) Residuals of maximum chamber gain. 


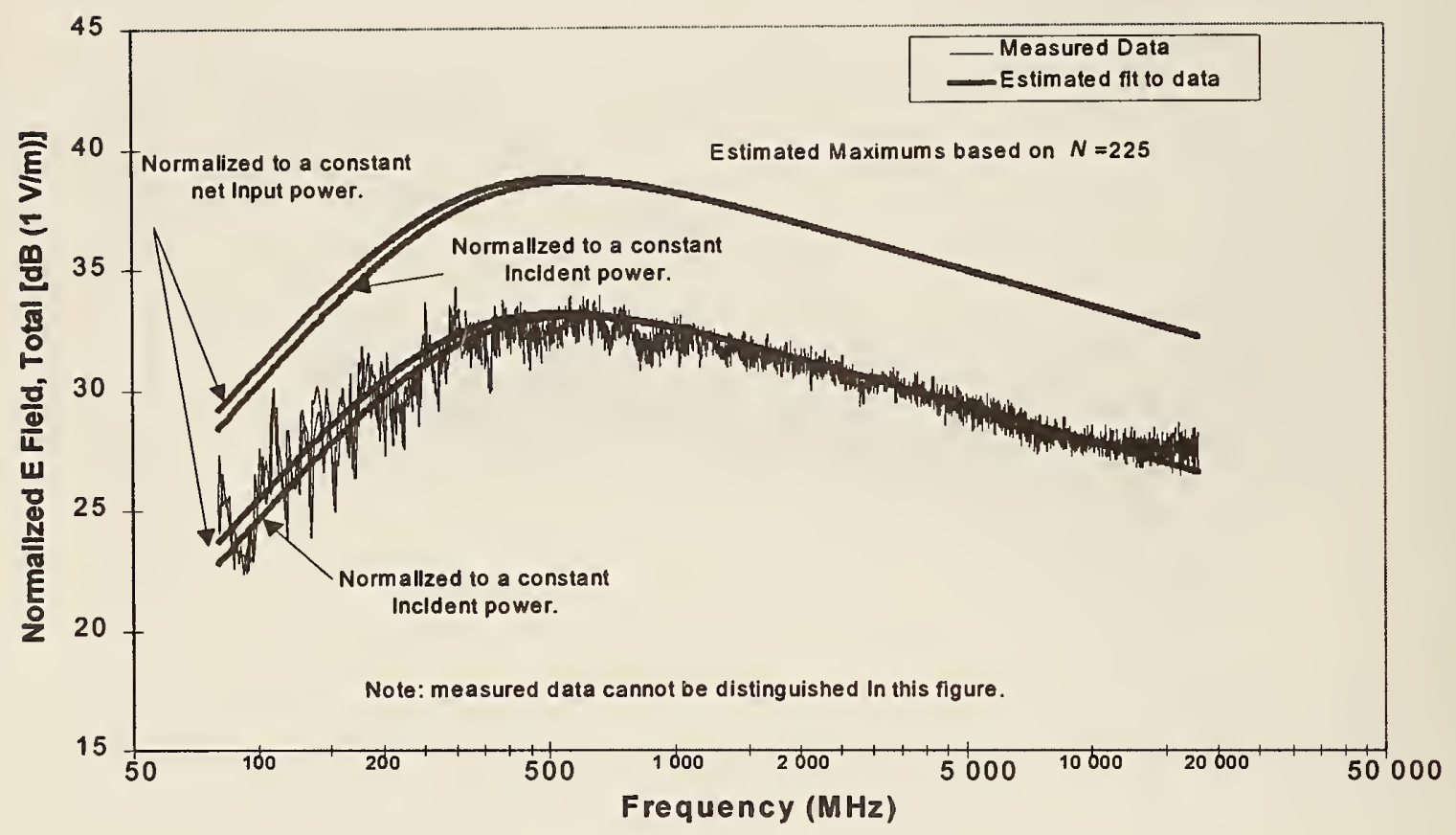

(a)

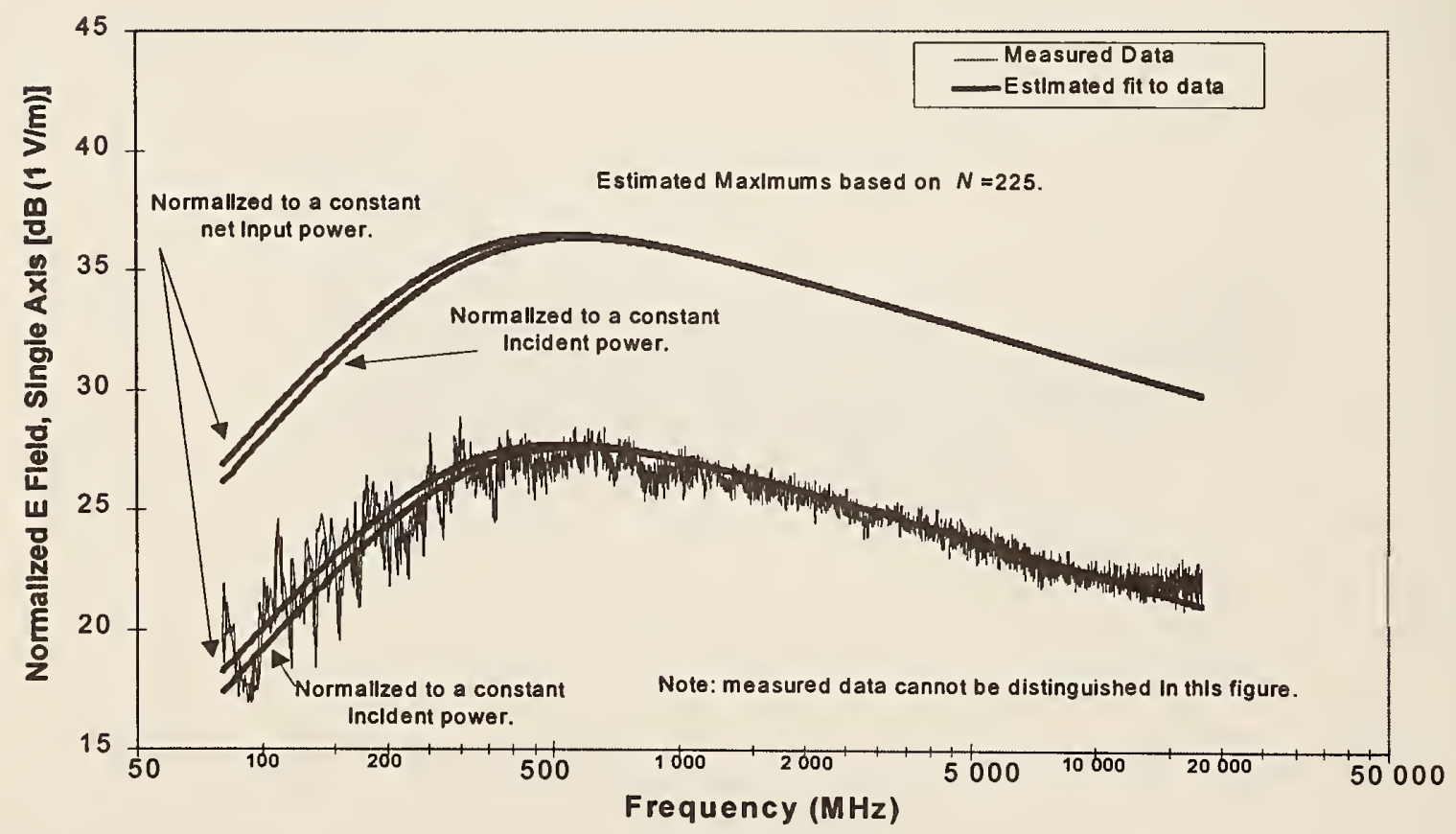

(b)

Figure 99. Calculated average and estimated maximum electric field in chamber B for a constant input power of $1 \mathrm{~W}$, based on received power from reference antenna. (a) Total electric field. (b) Single-axis electric field. 


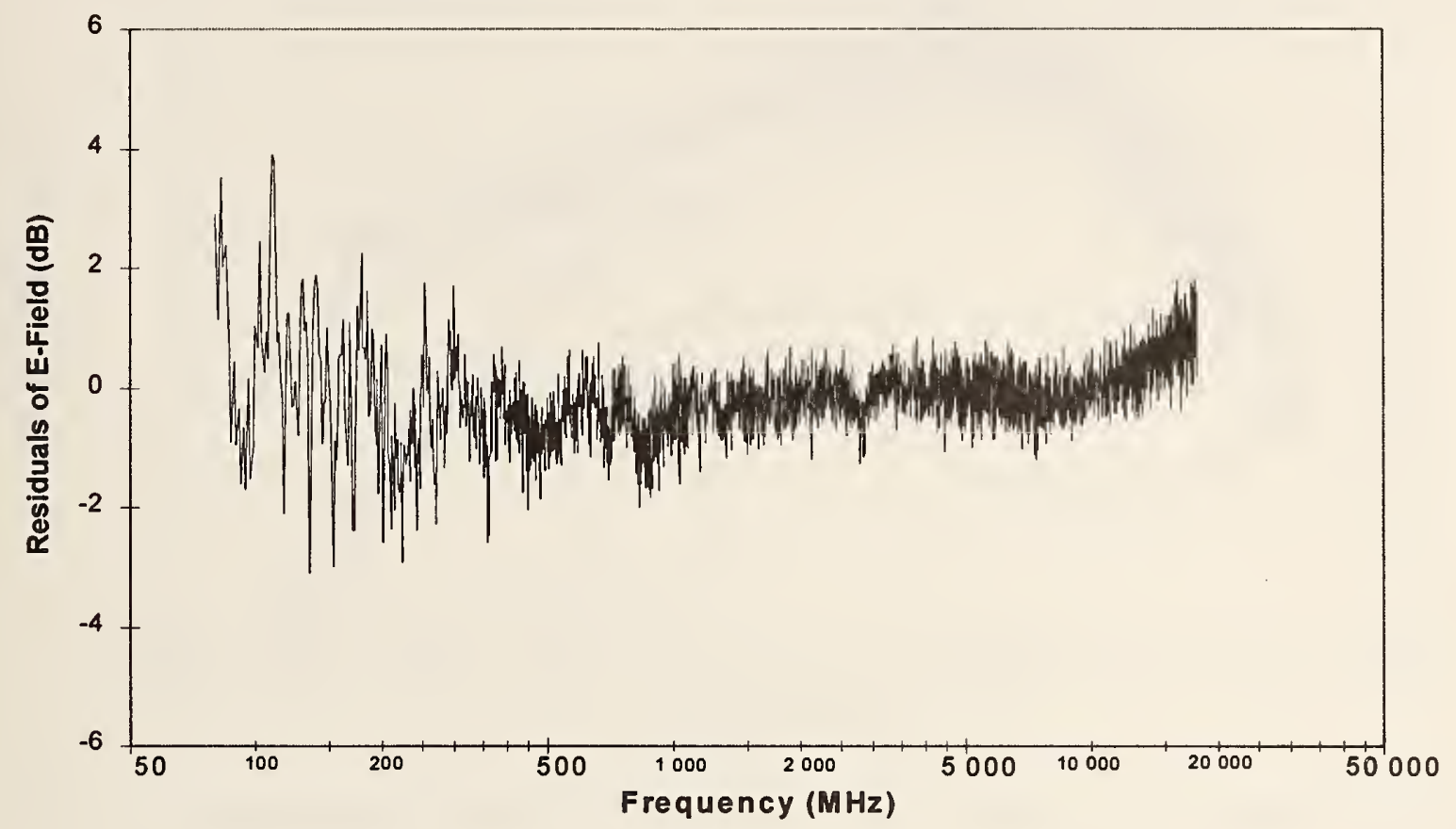

Figure 100. Residuals of calculated average electric field in chamber B, based on received power from reference antenna. 


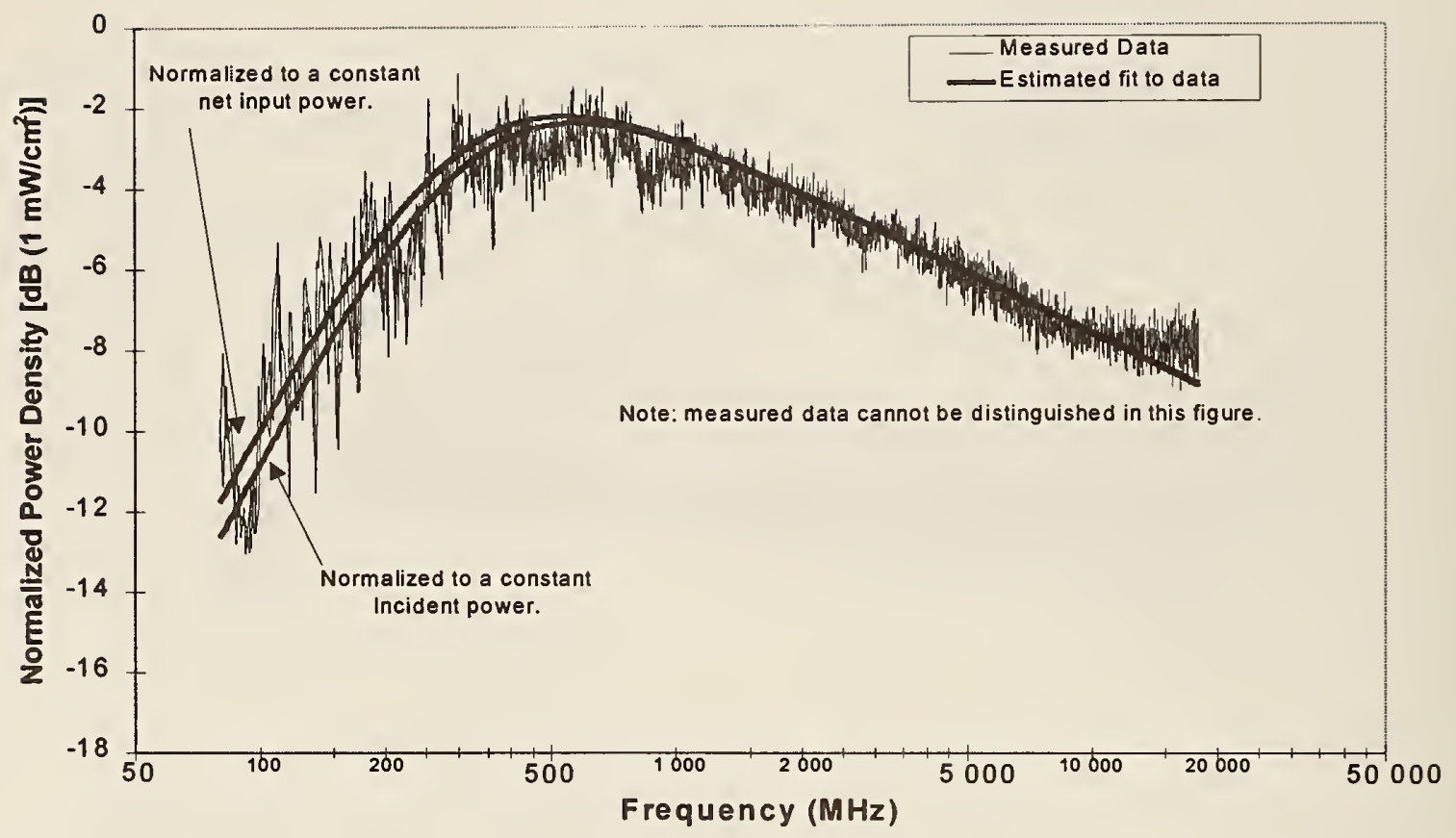

Figure 101. Average power density in chamber B for a constant input power of $1 \mathrm{~W}$. 


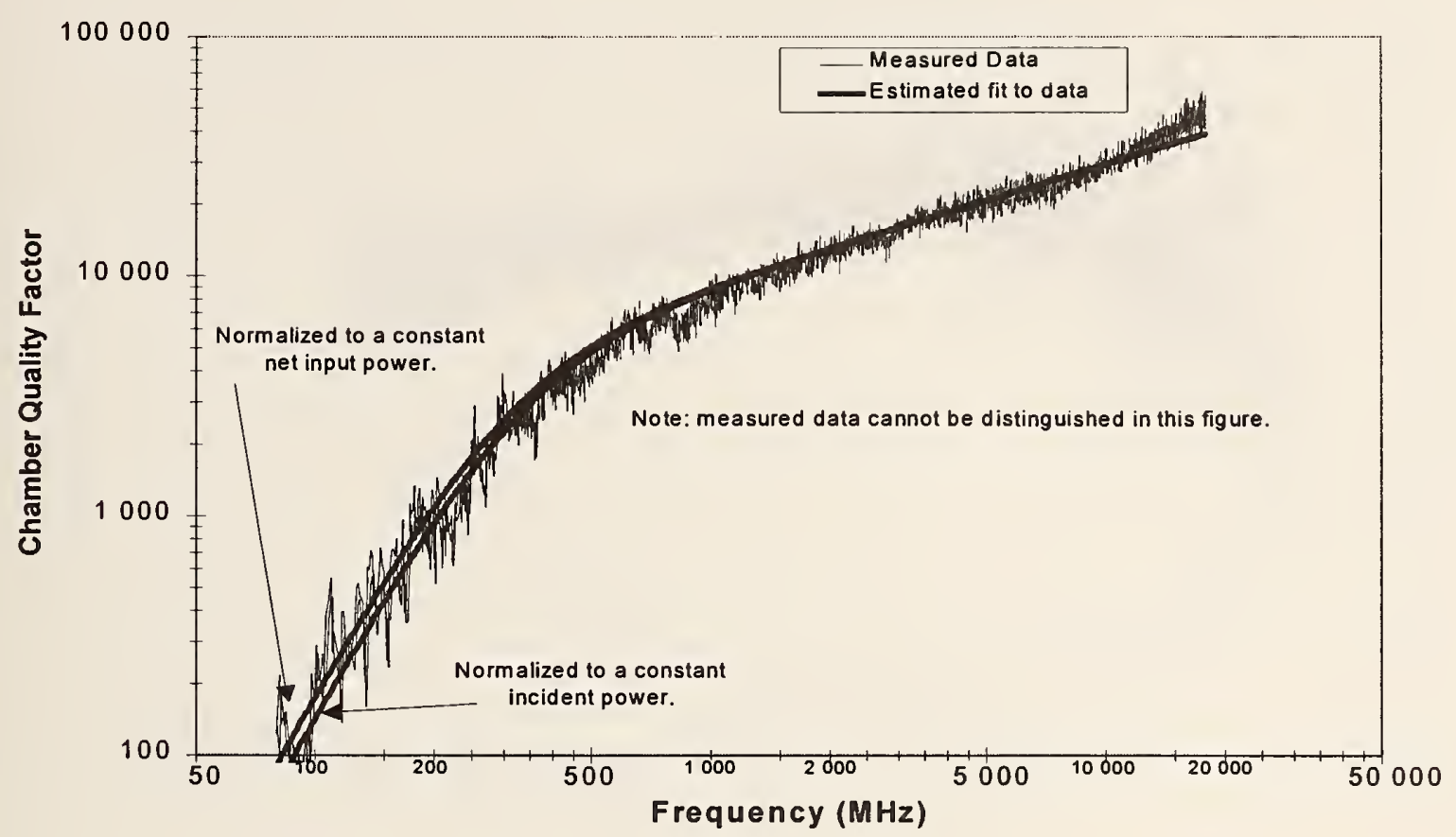

Figure 102. Average quality factor in chamber B.

187 


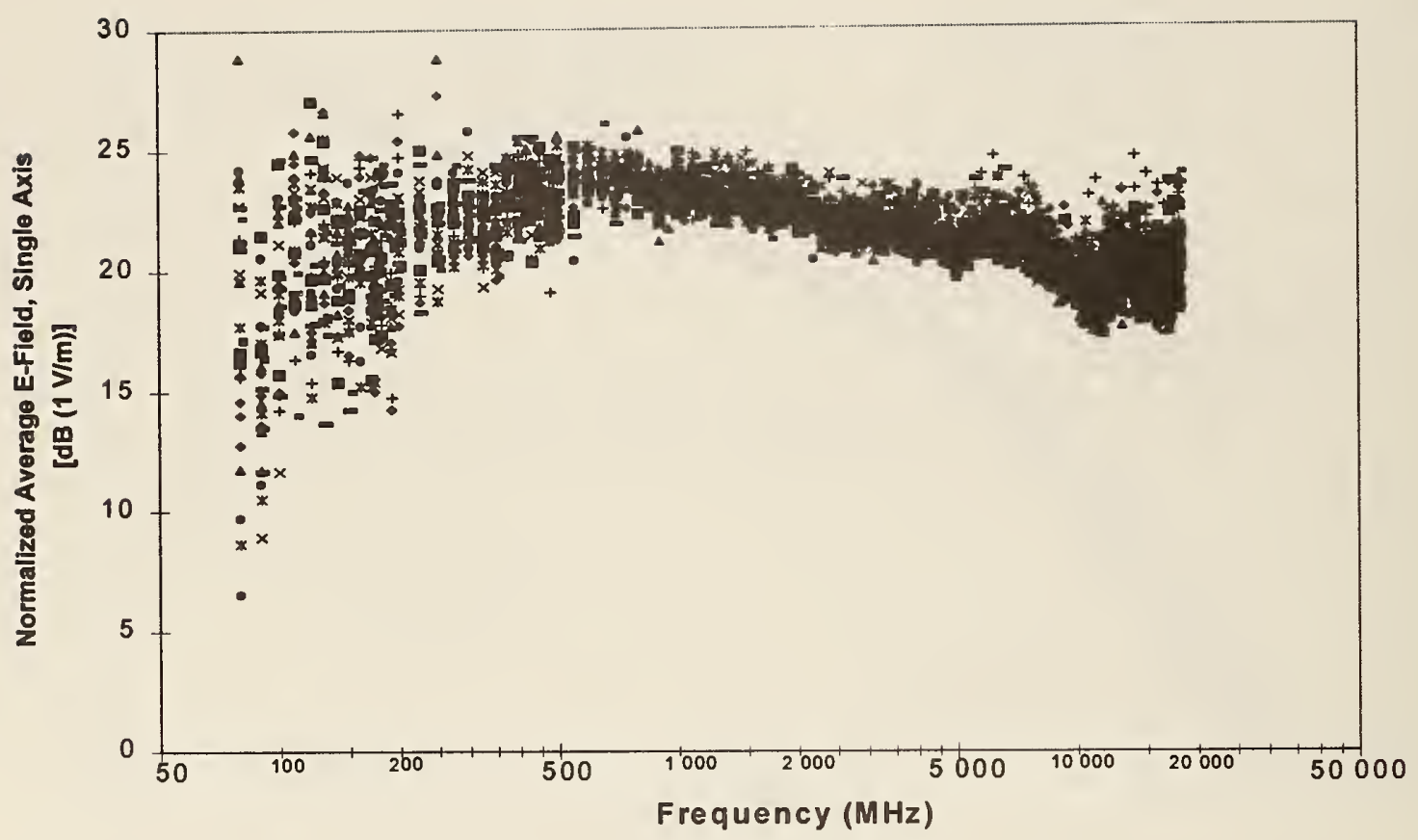

(a)

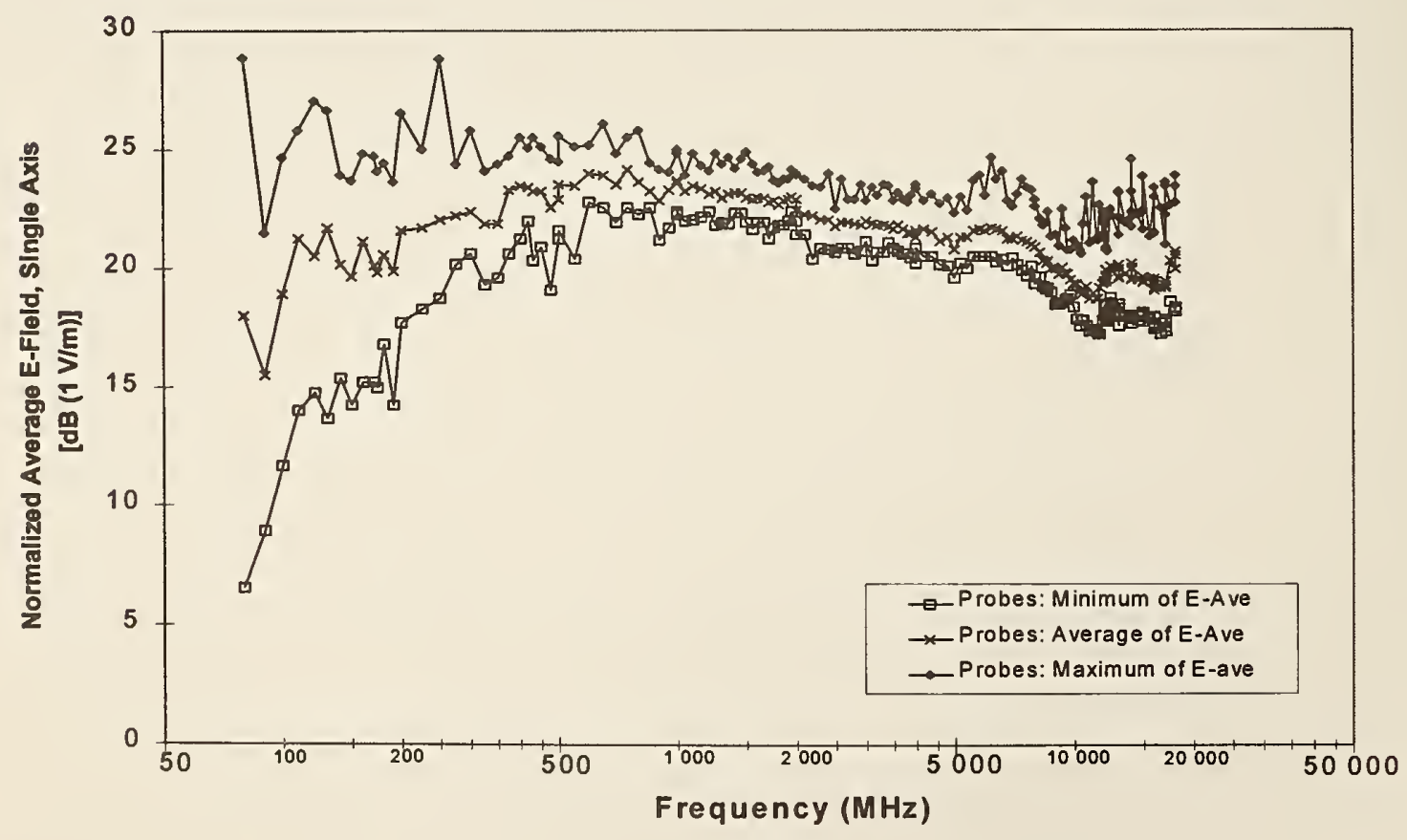

(b)

Figure 103. The average measured electric field (rectangular component) for each of 30 short dipoles in chamber B. Values given are for a constant net input power of $1 \mathrm{~W}$. (a) Individual measurements. (b) Envelope and average of individual measurements. (c) Standard deviation of measurements of $\mathrm{E}_{\mathrm{R}}$-Ave. (d) Comparison of average of individual measurements with reference antenna. 


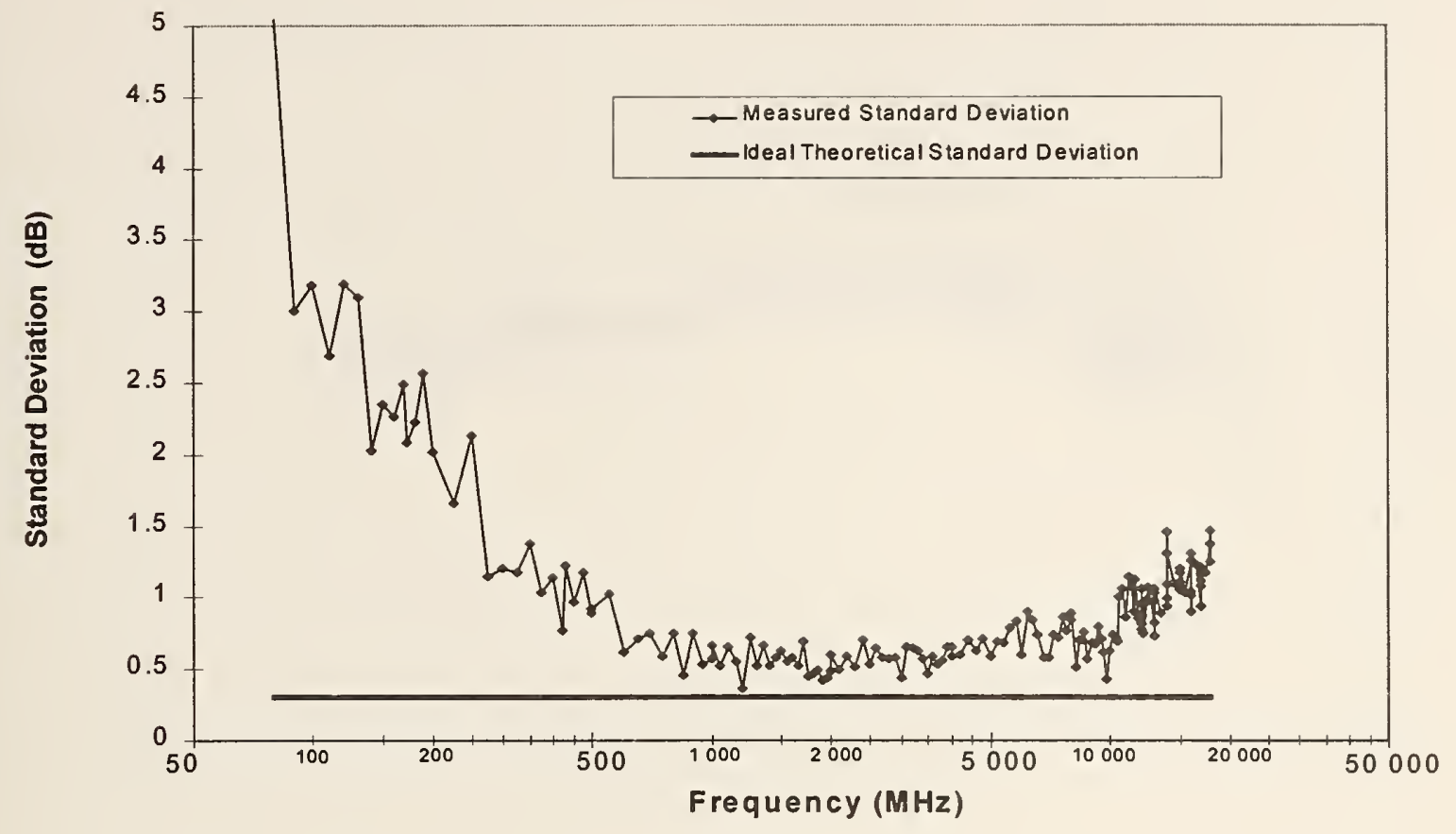

(c)

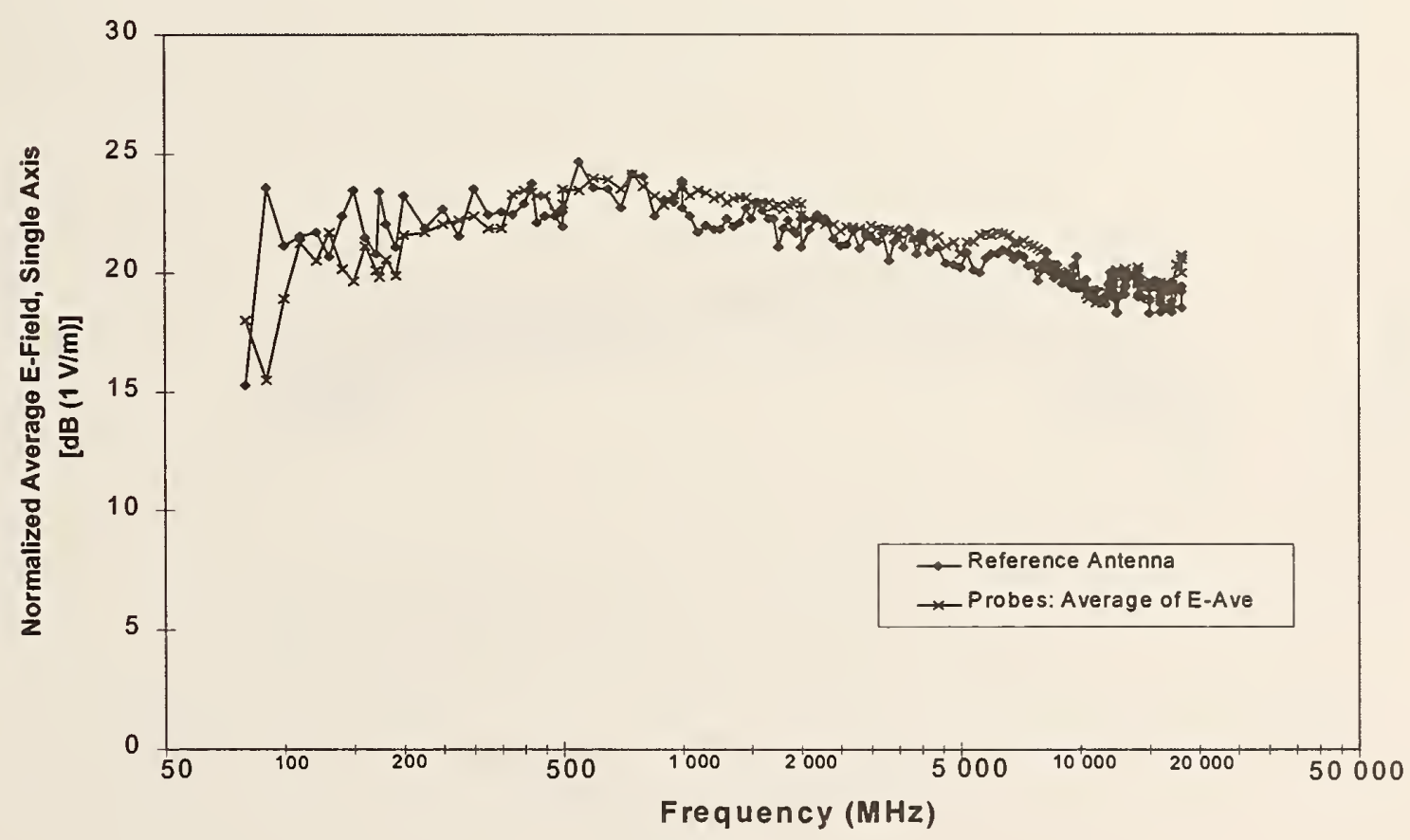

(d) 


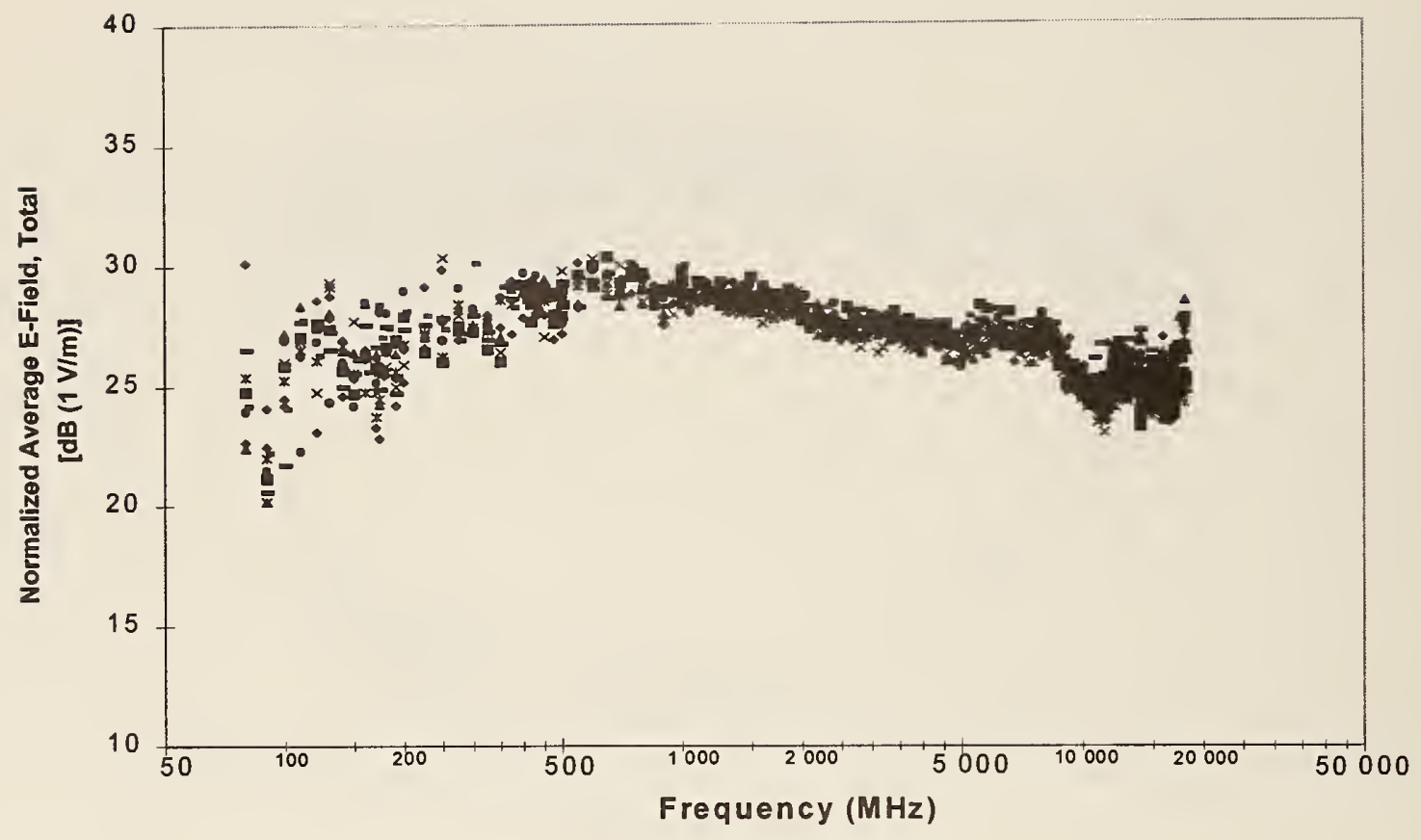

(a)

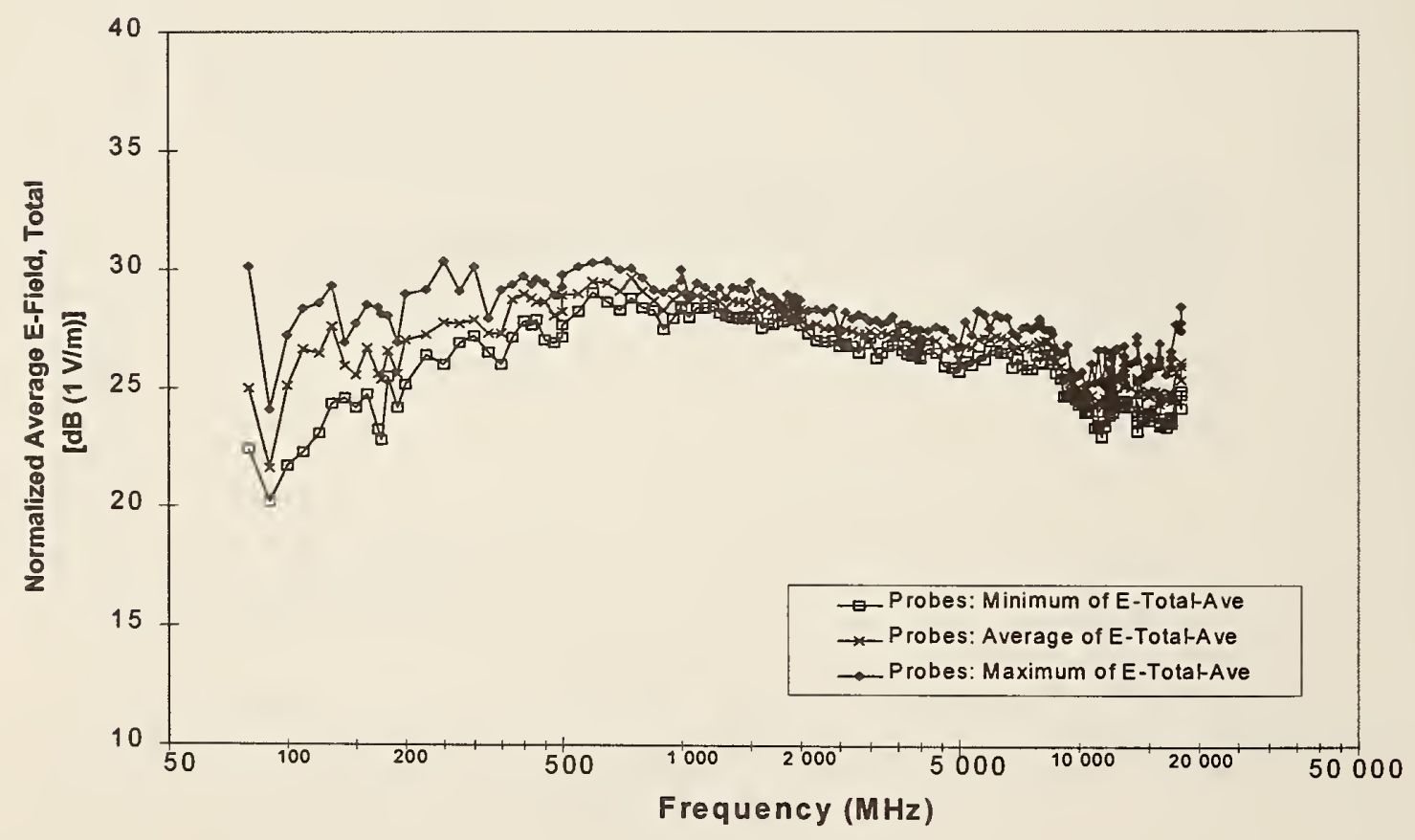

(b)

Figure 104. The average measured total electric field for each of 10 isotropic antennas in chamber B. Values given are for a constant net input power of $1 \mathrm{~W}$. (a) Individual measurements. (b) Envelope and average of individual measurements. (c) Standard deviation of measurements of $\mathrm{E}_{\mathrm{T}}$-Ave. (d) Comparison of average of individual measurements with reference antenna. 


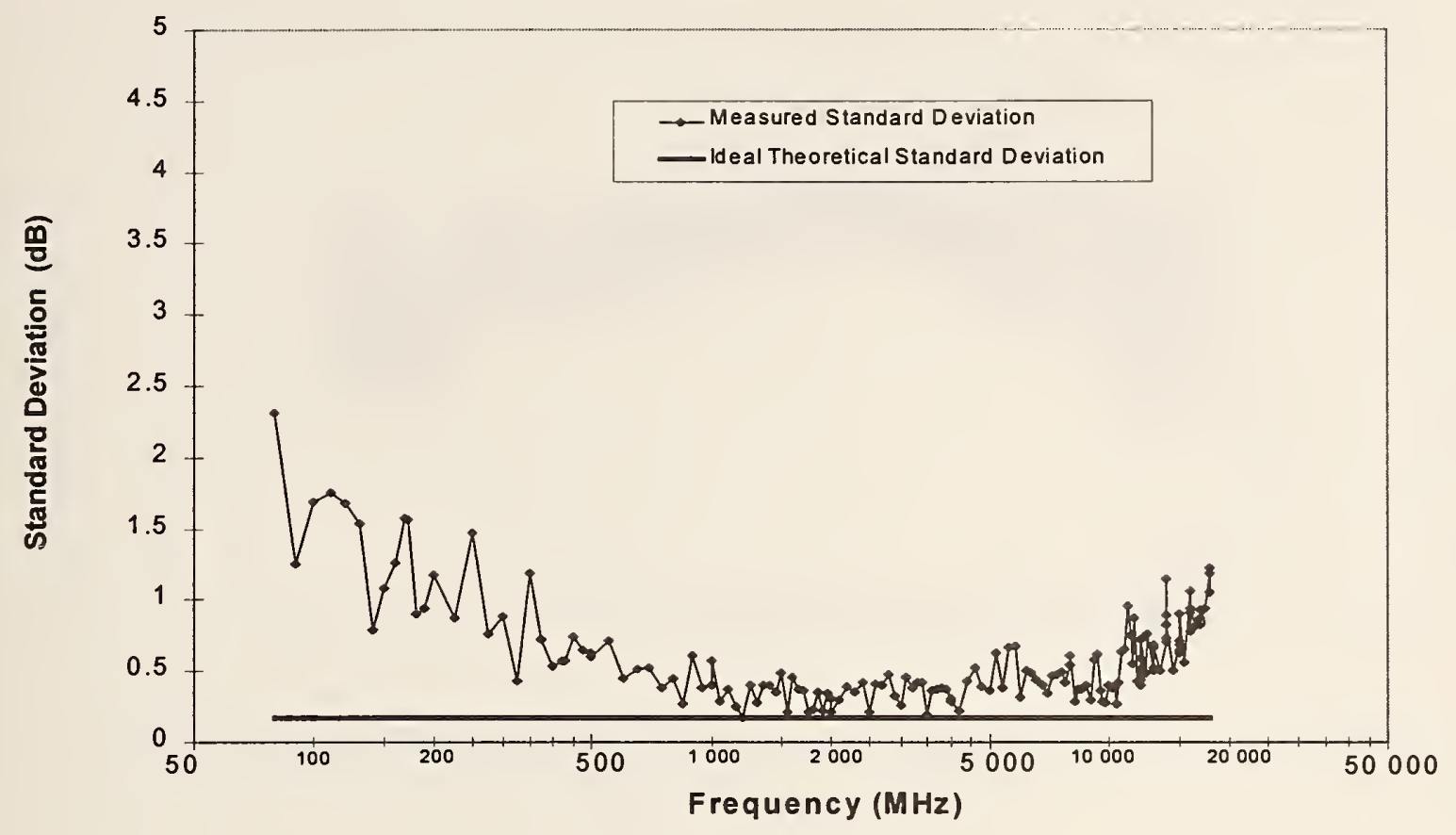

(c)

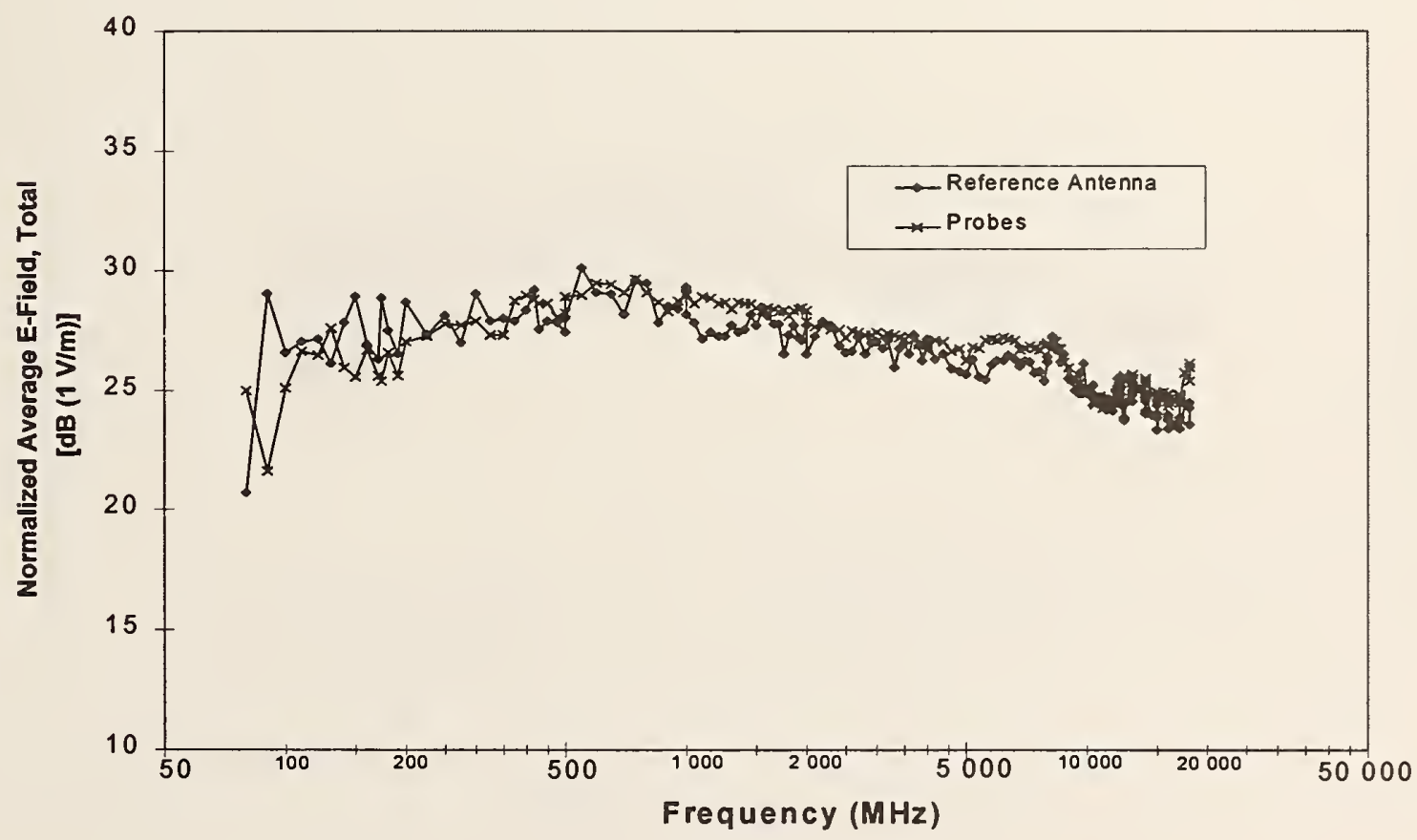

(d) 


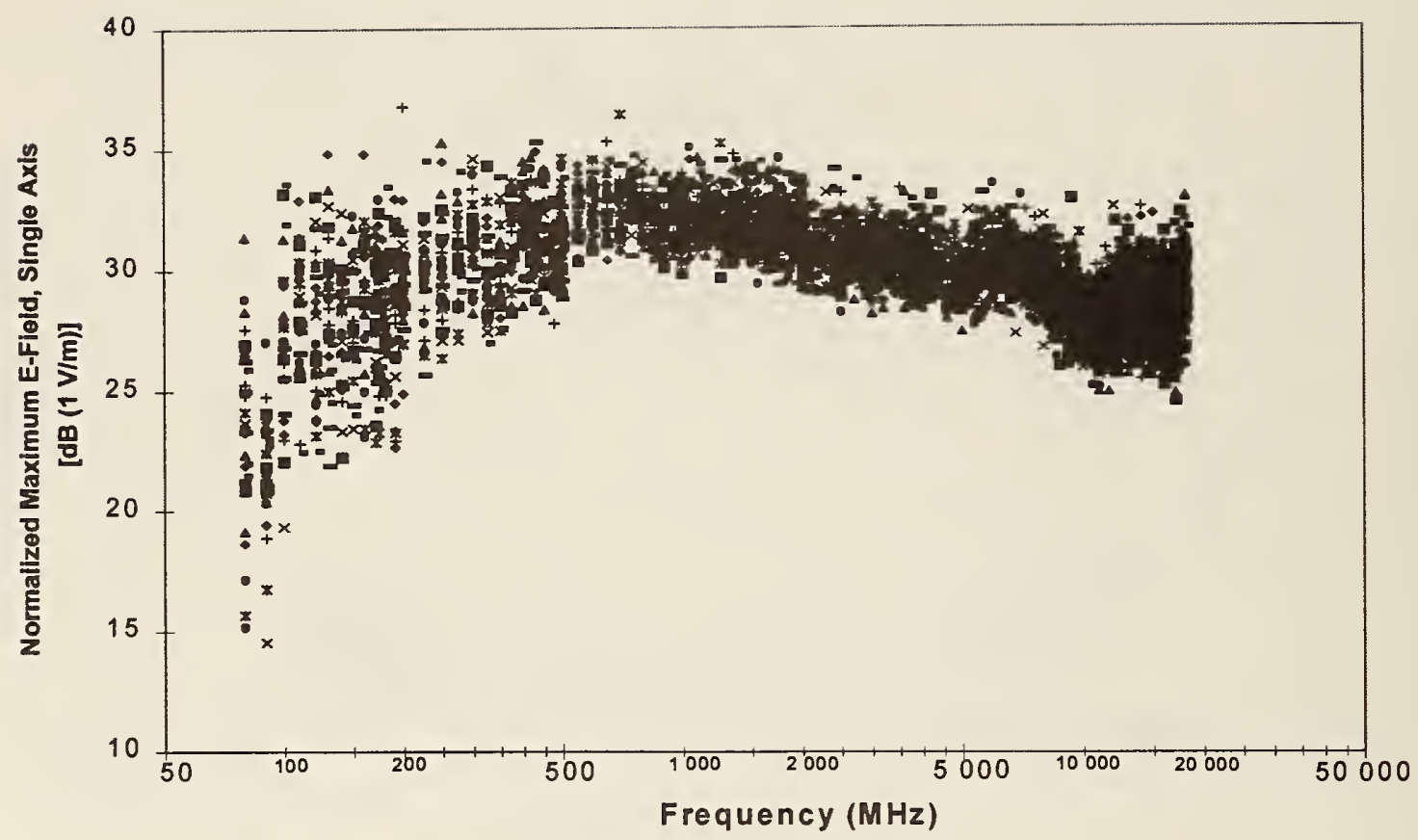

(a)

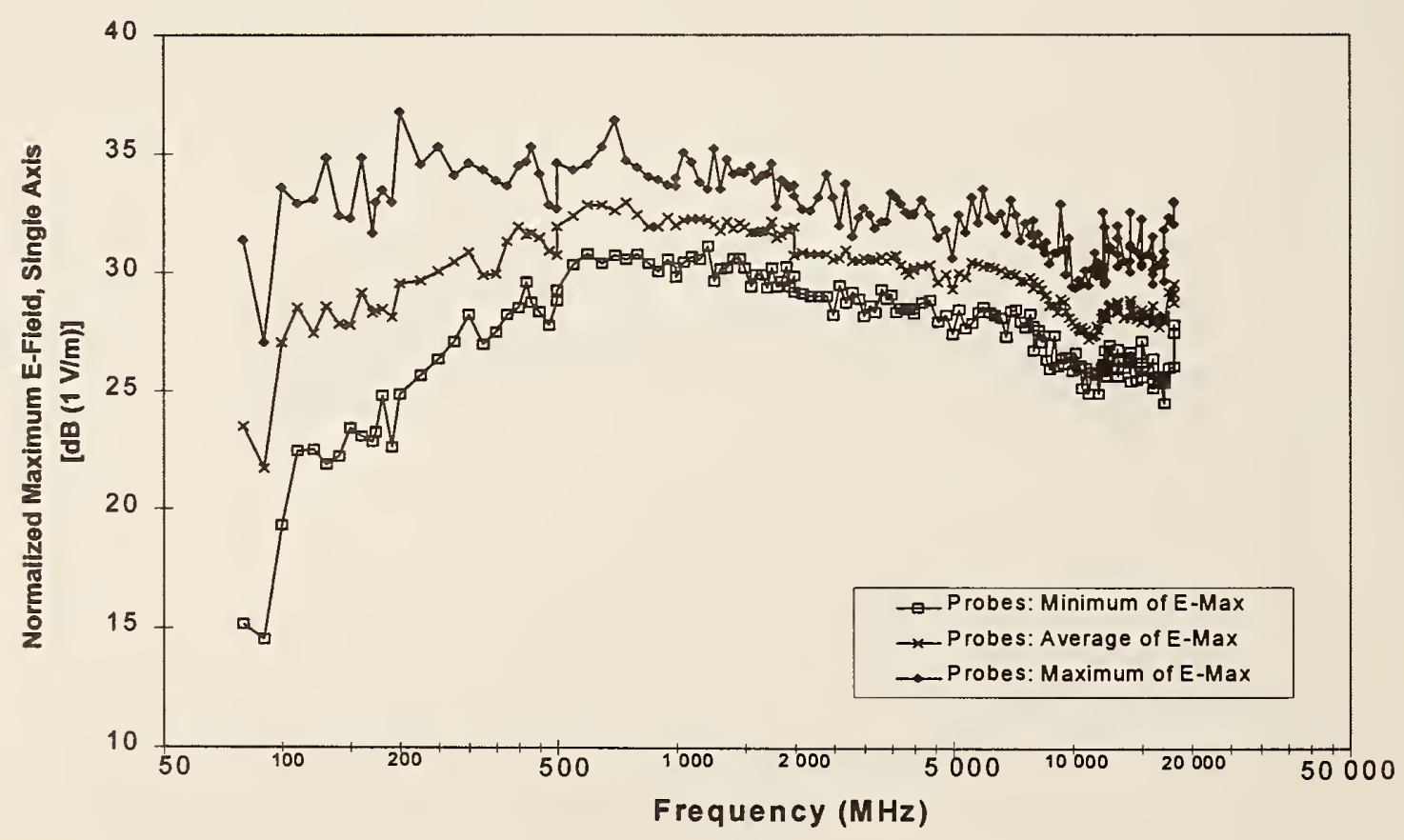

(b)

Figure 105. The maximum measured electric field (single polarization) for each of 30 short dipoles in chamber B. Values given are for a constant net input power of $1 \mathrm{~W}$. (a) Individual measurements. (b) Envelope and average of individual measurements. (c) Standard deviation of measurements of $E_{R}-M a x$. (d) Comparison of average of individual measurements with reference antenna. 


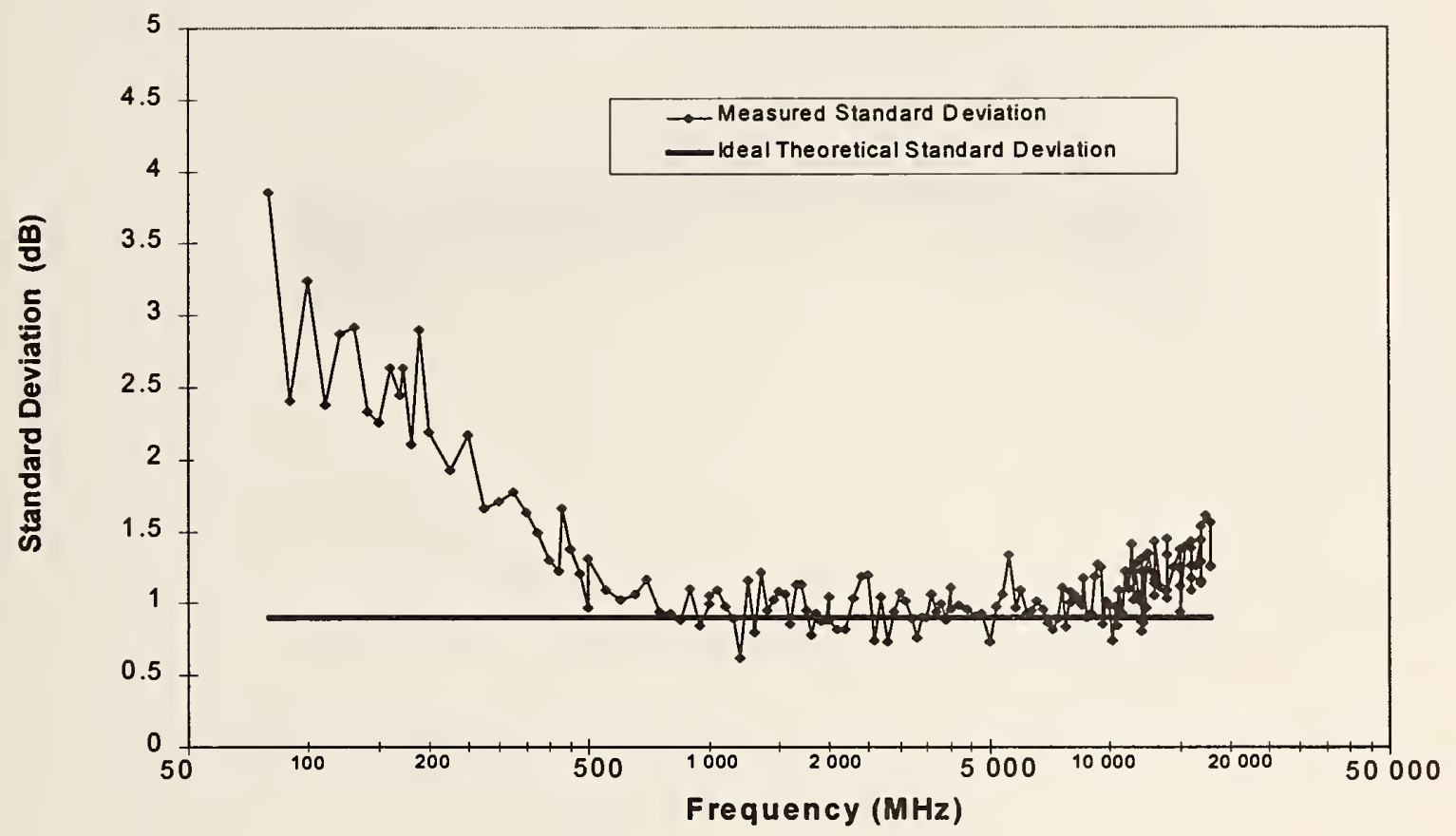

(c)

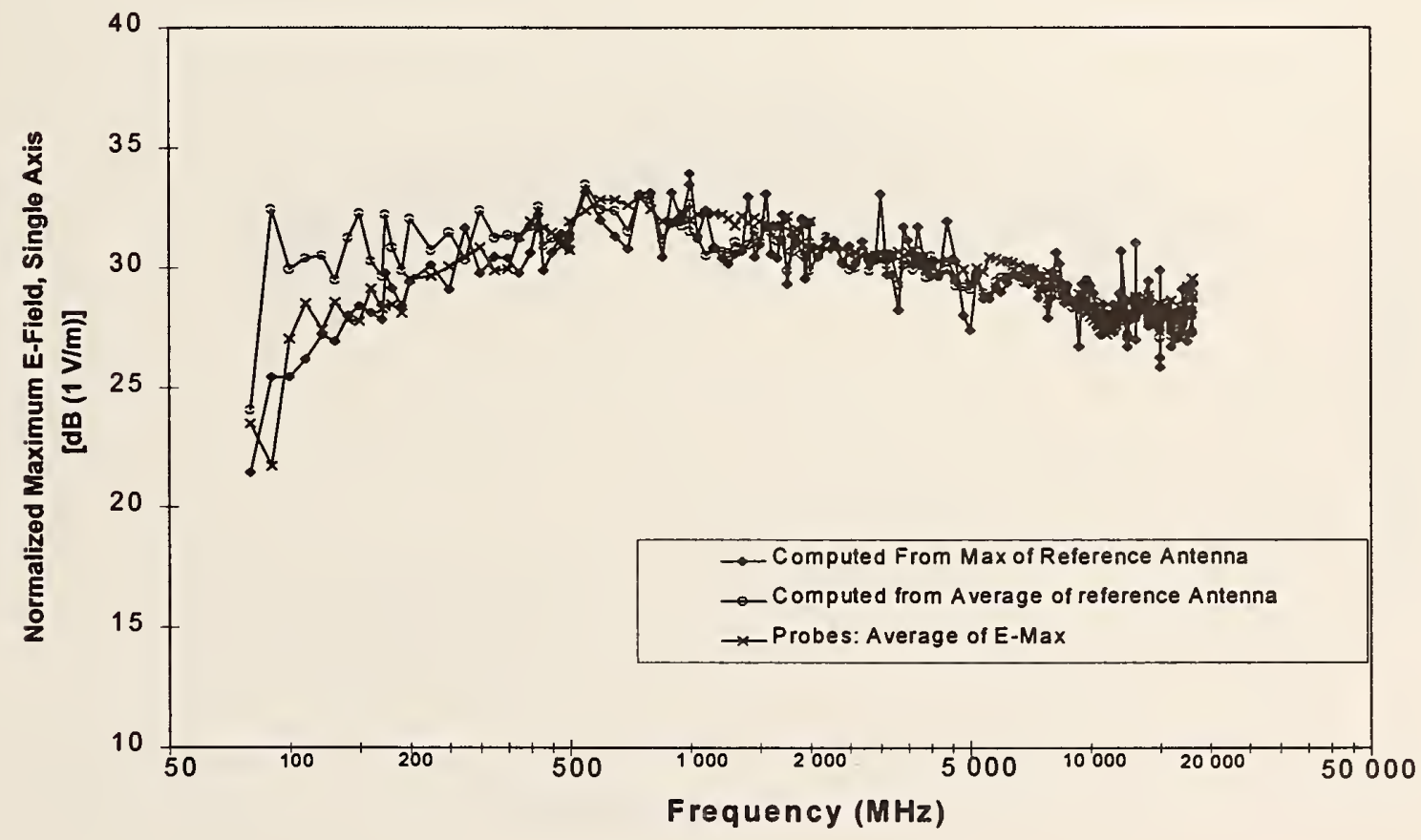

(d) 


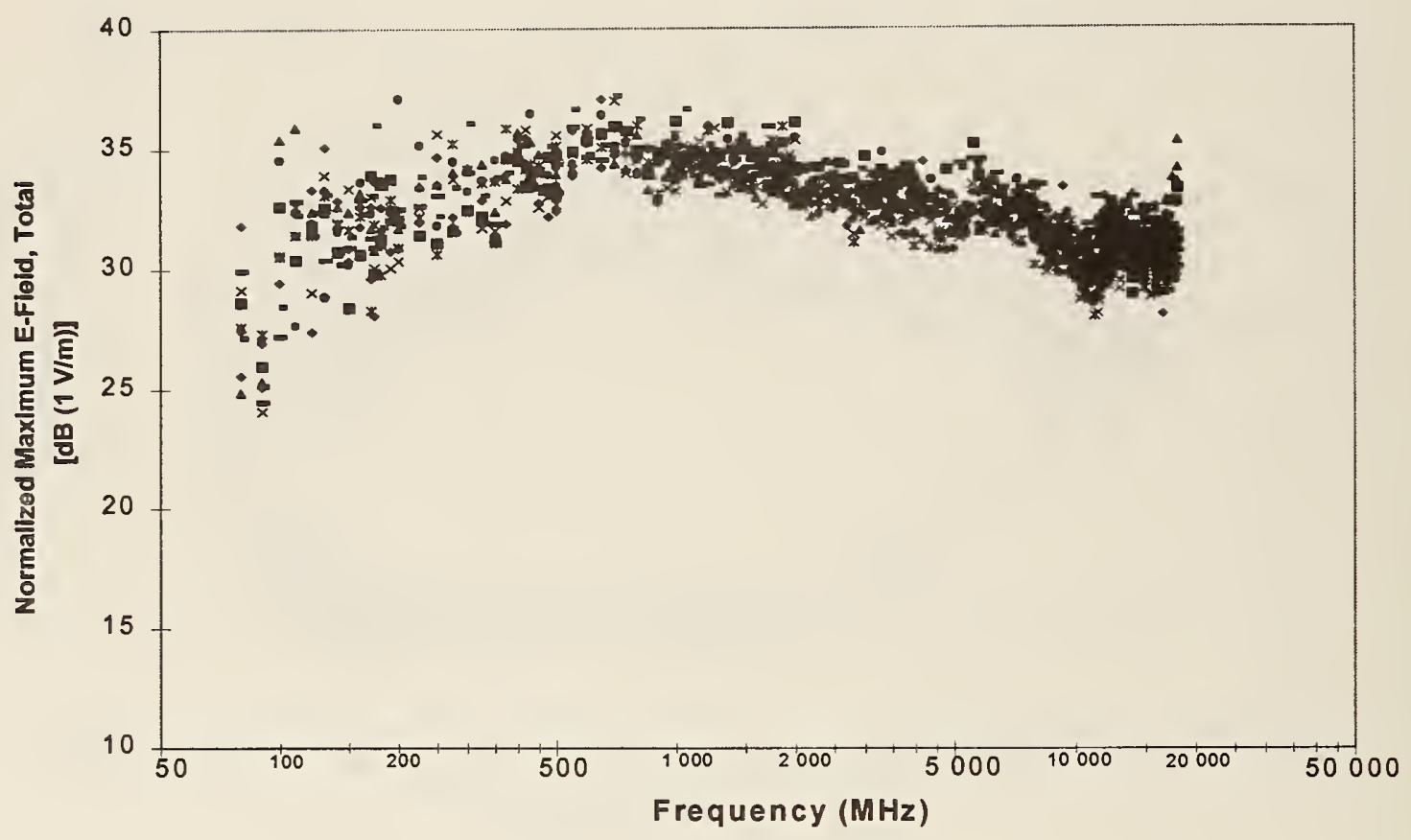

(a)

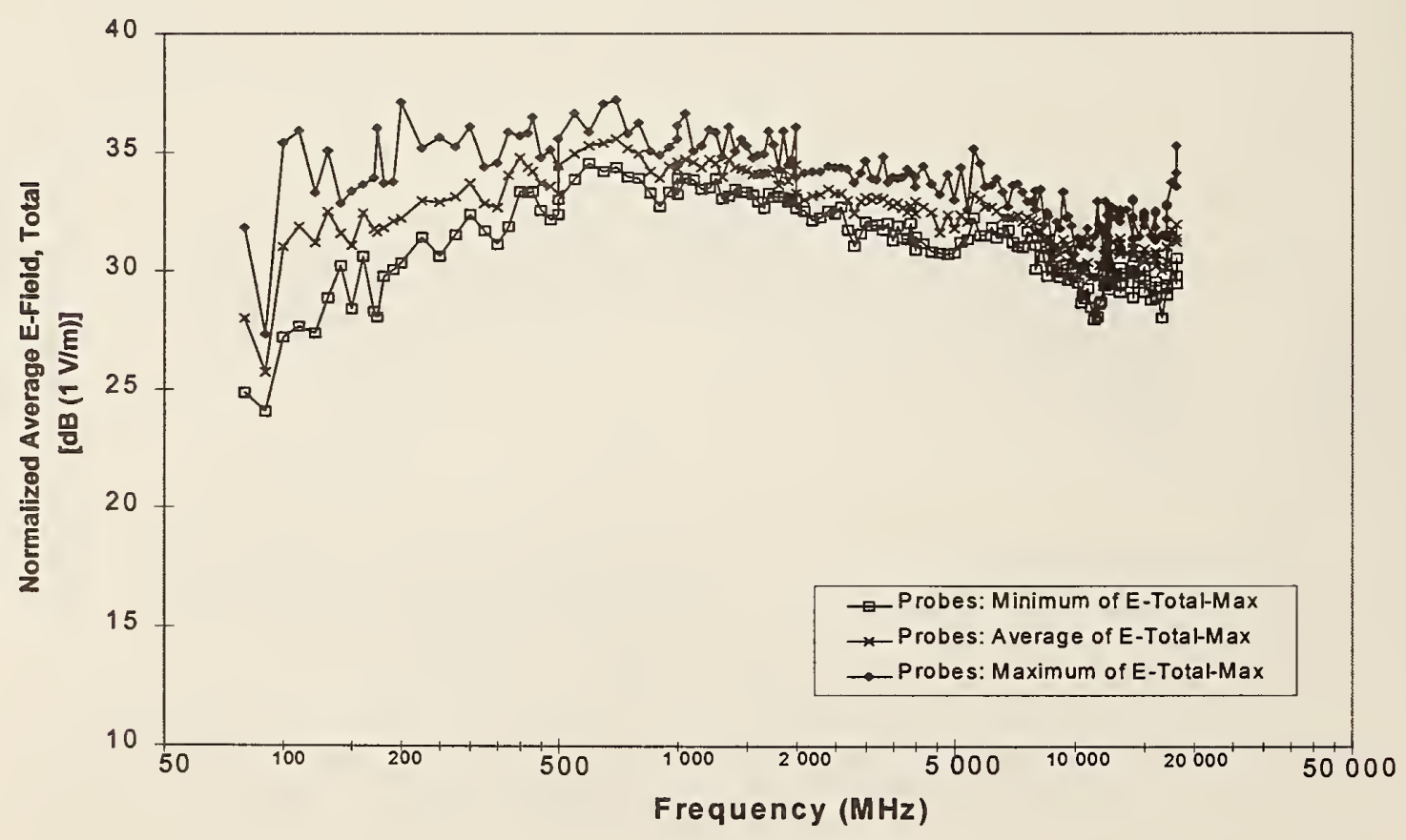

(b)

Figure 106.The maximum measured total electric field for each of 10 isotropic antennas in chamber B. Values given are for a constant net input power of $1 \mathrm{~W}$. (a) Individual measurements. (b) Envelope and average of individual measurements. (c) Standard deviation of measurements of $\mathrm{E}_{\mathrm{T}}-\mathrm{Max}$. (d) Comparison of average of individual measurements with reference antenna. 


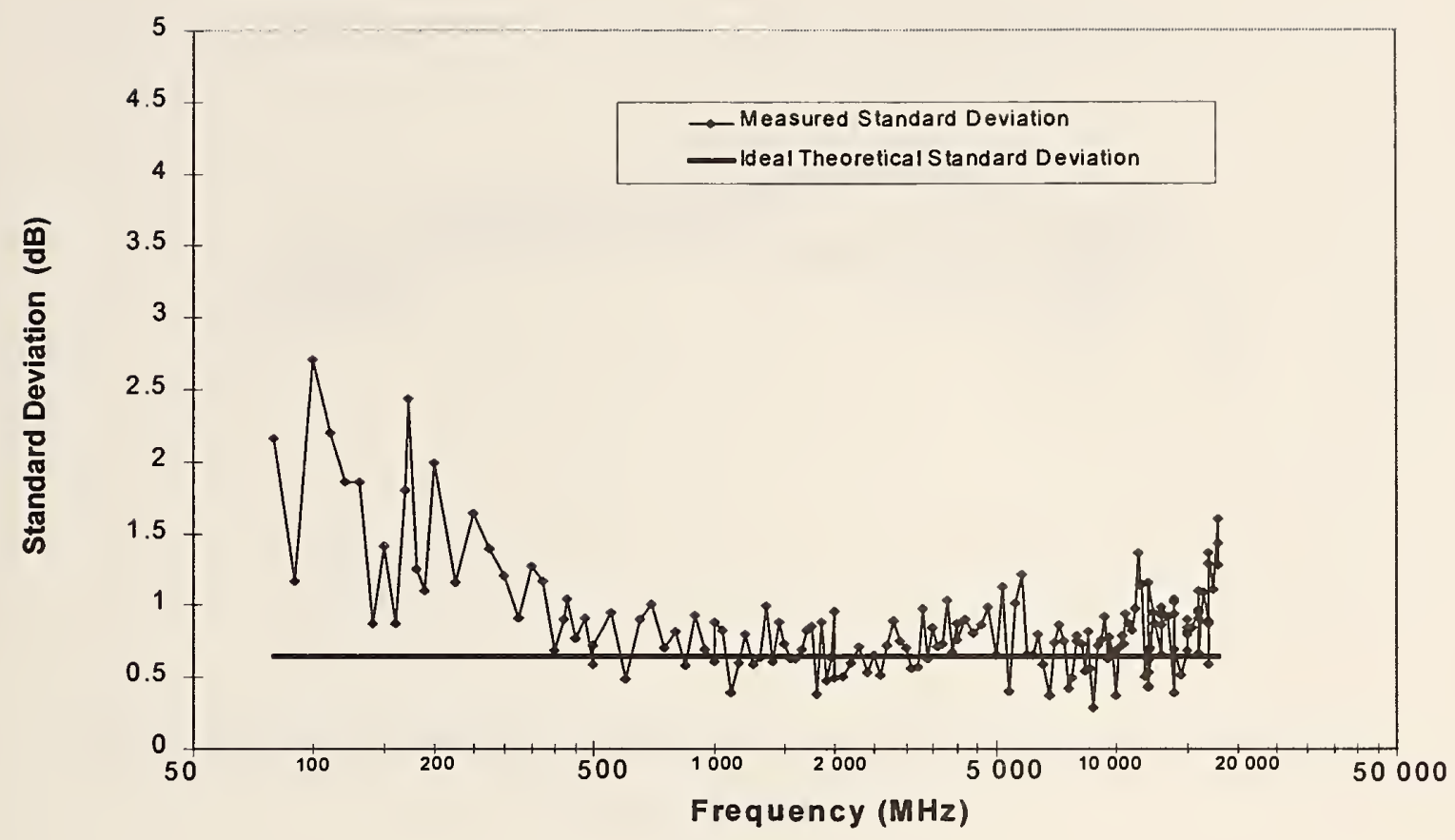

(c)

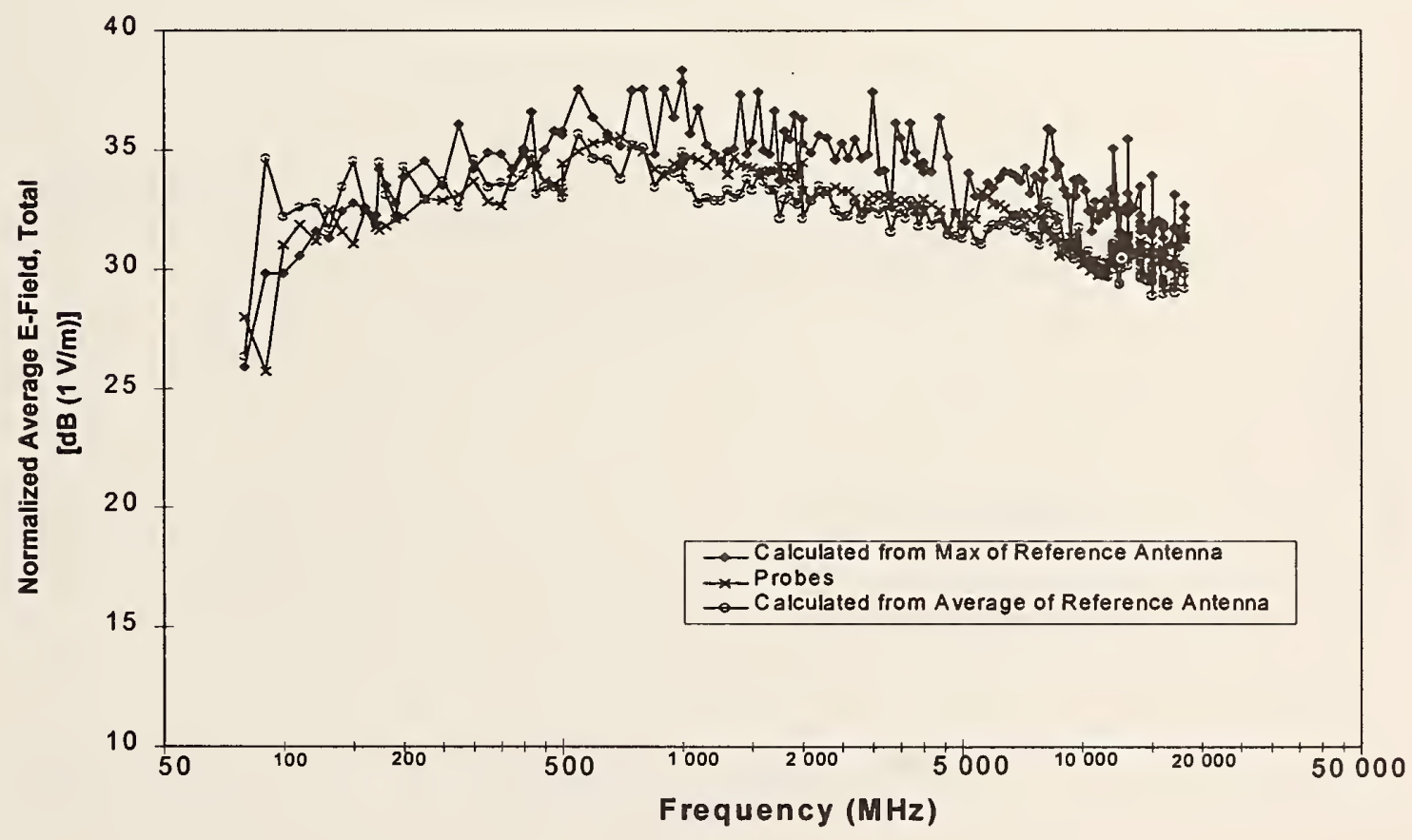

(d) 


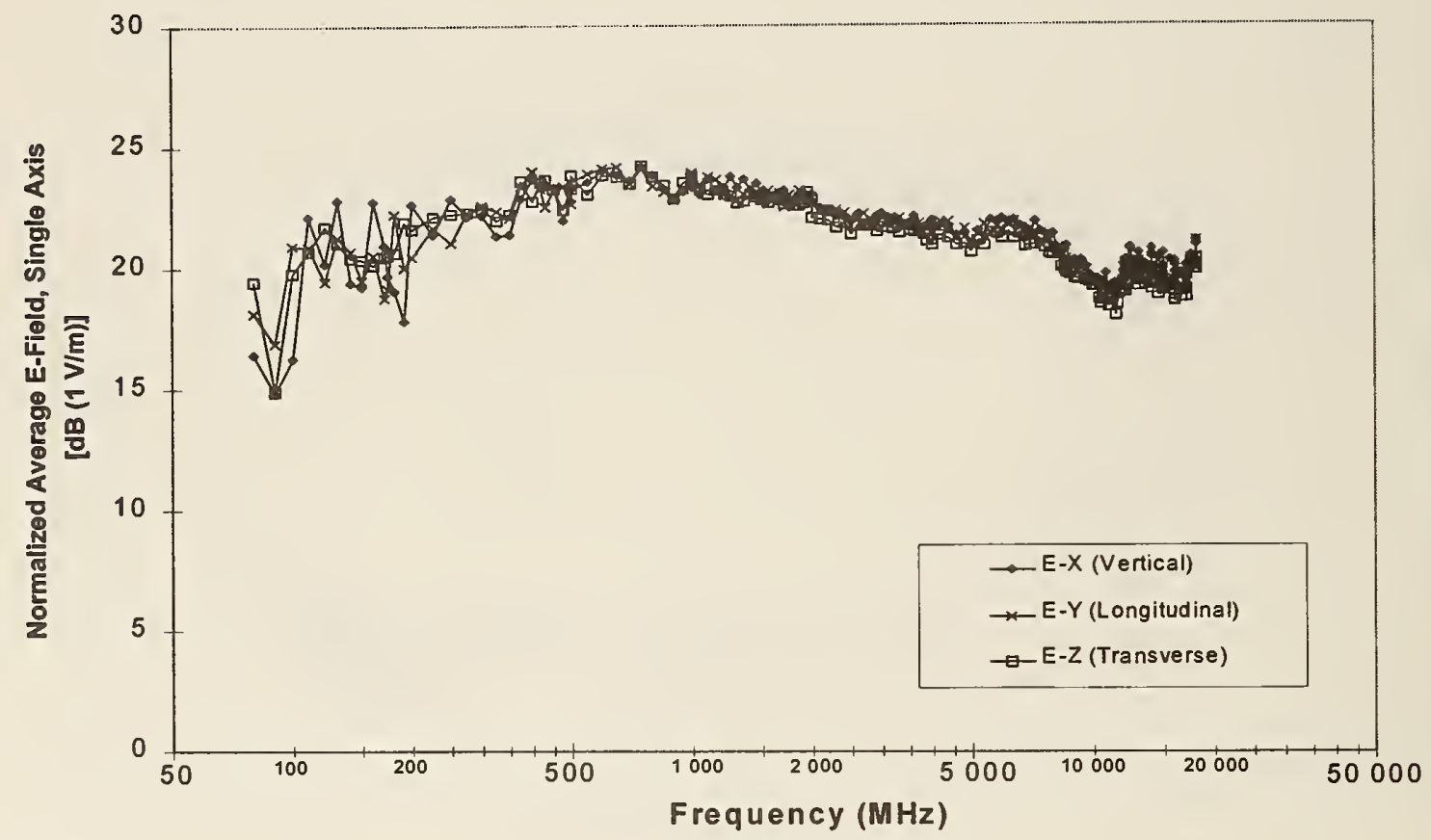

(a)

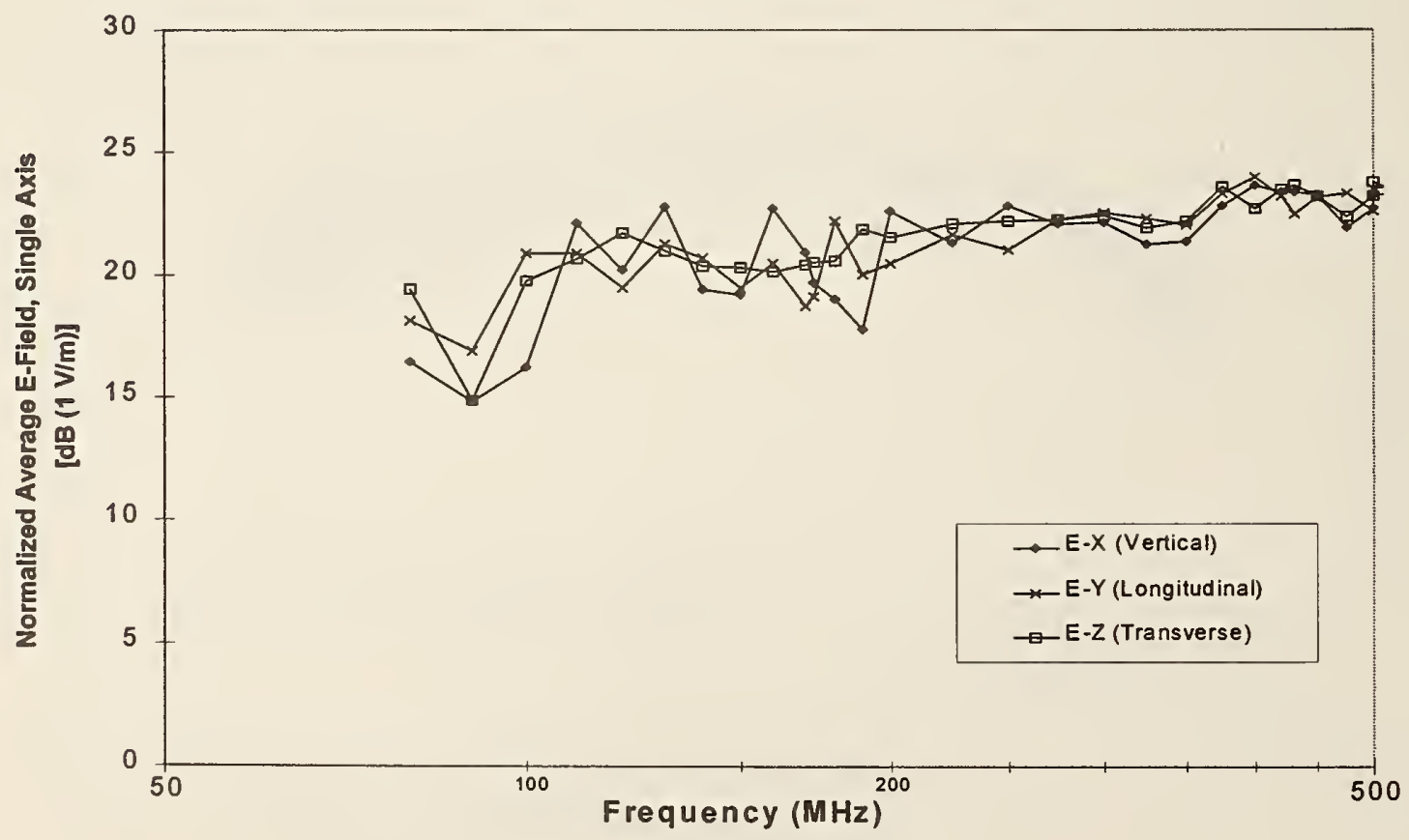

(b)

Figure 107. Comparison of average $\mathrm{x}$ component, $\mathrm{y}$ component, and $\mathrm{z}$ component of electric field in chamber B. Values given are for a constant net input power of $1 \mathrm{~W}$. (a) Full frequency range. (b) Low frequencies only. 


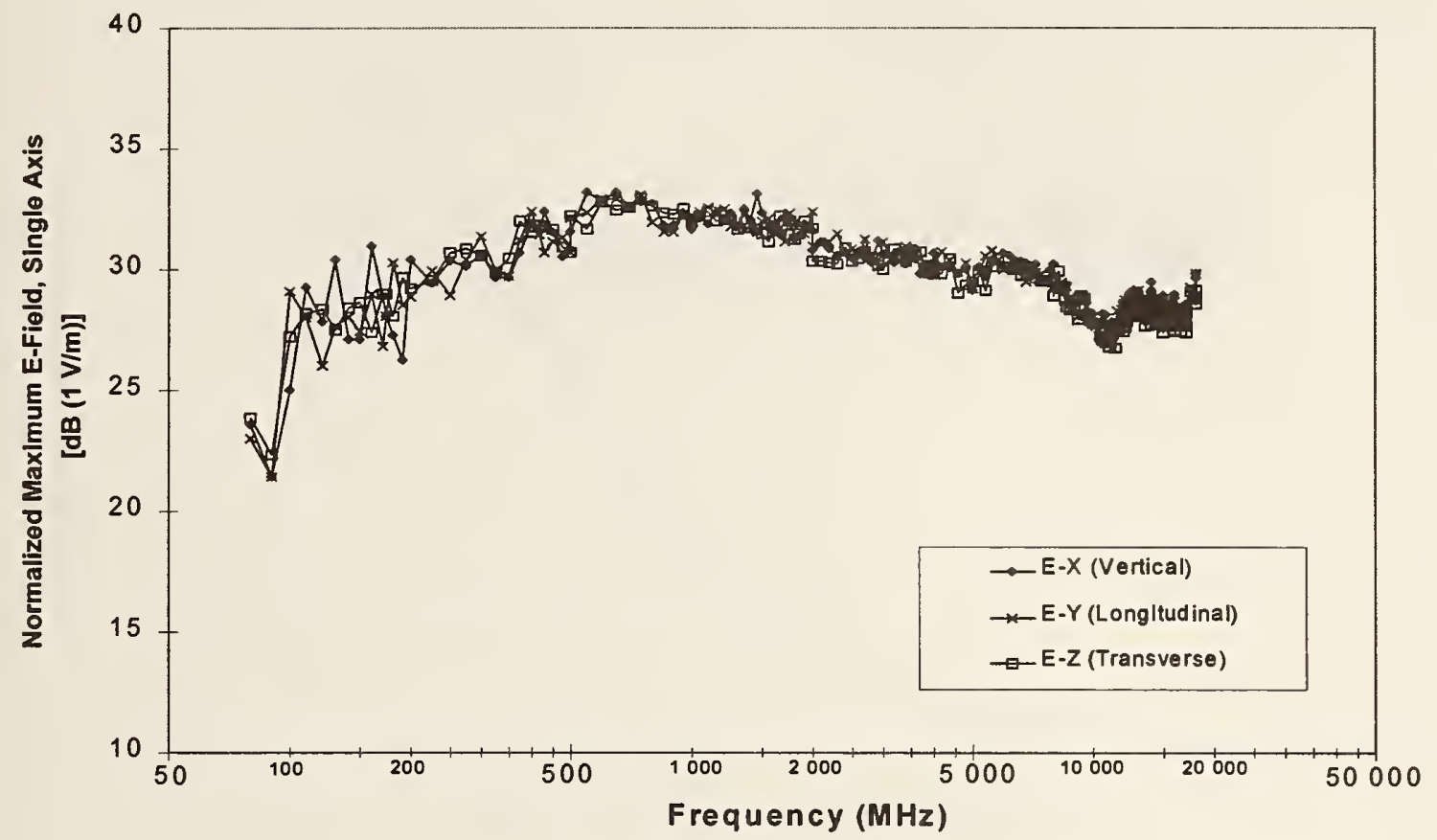

(a)

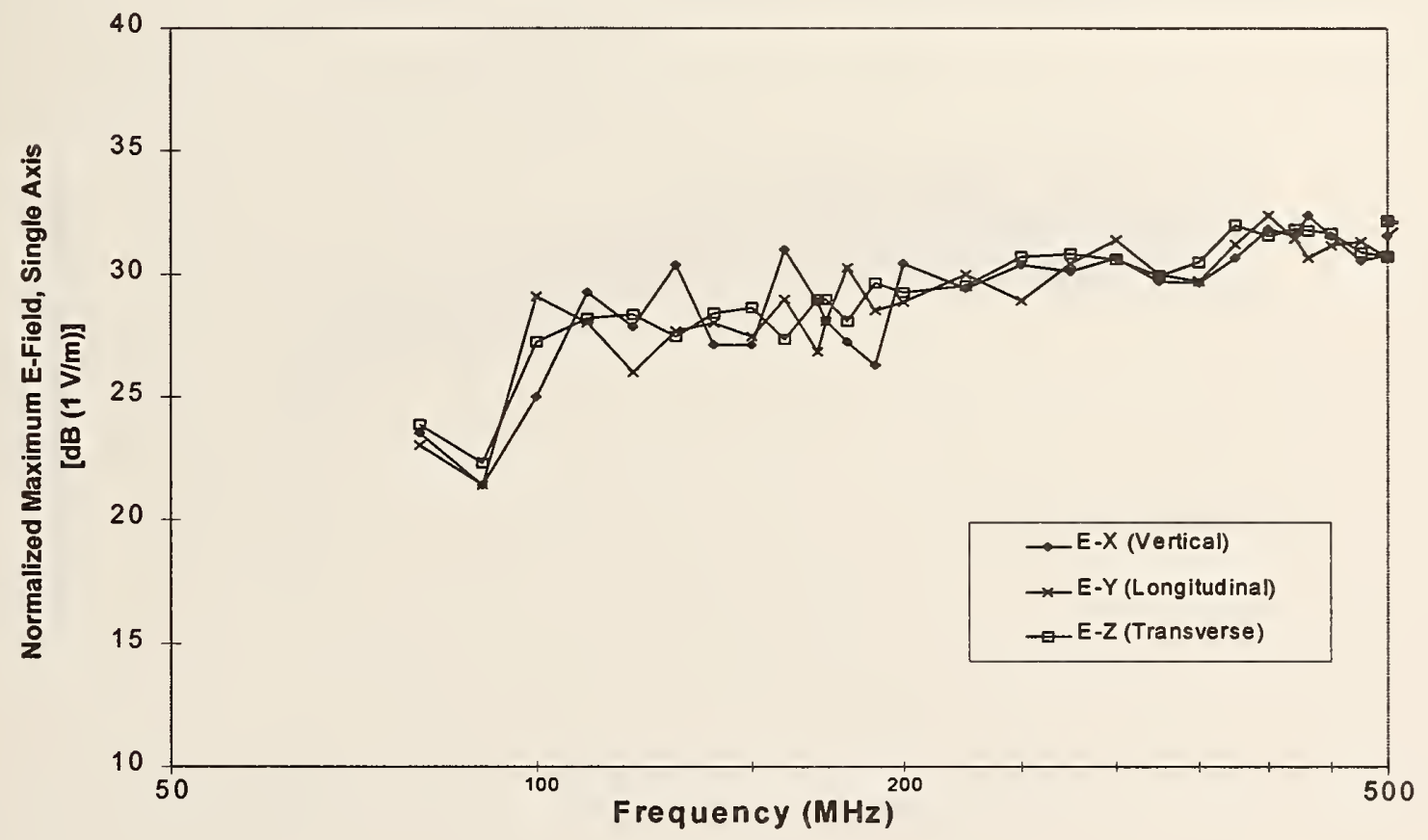

(b)

Figure 108. Comparison of maximum $\mathrm{x}$ component, $\mathrm{y}$ component, and $\mathrm{z}$ component of electric field in chamber B. Values given are for a constant net input power of $1 \mathrm{~W}$. (a) Full frequency range. (b) Low frequencies only. 


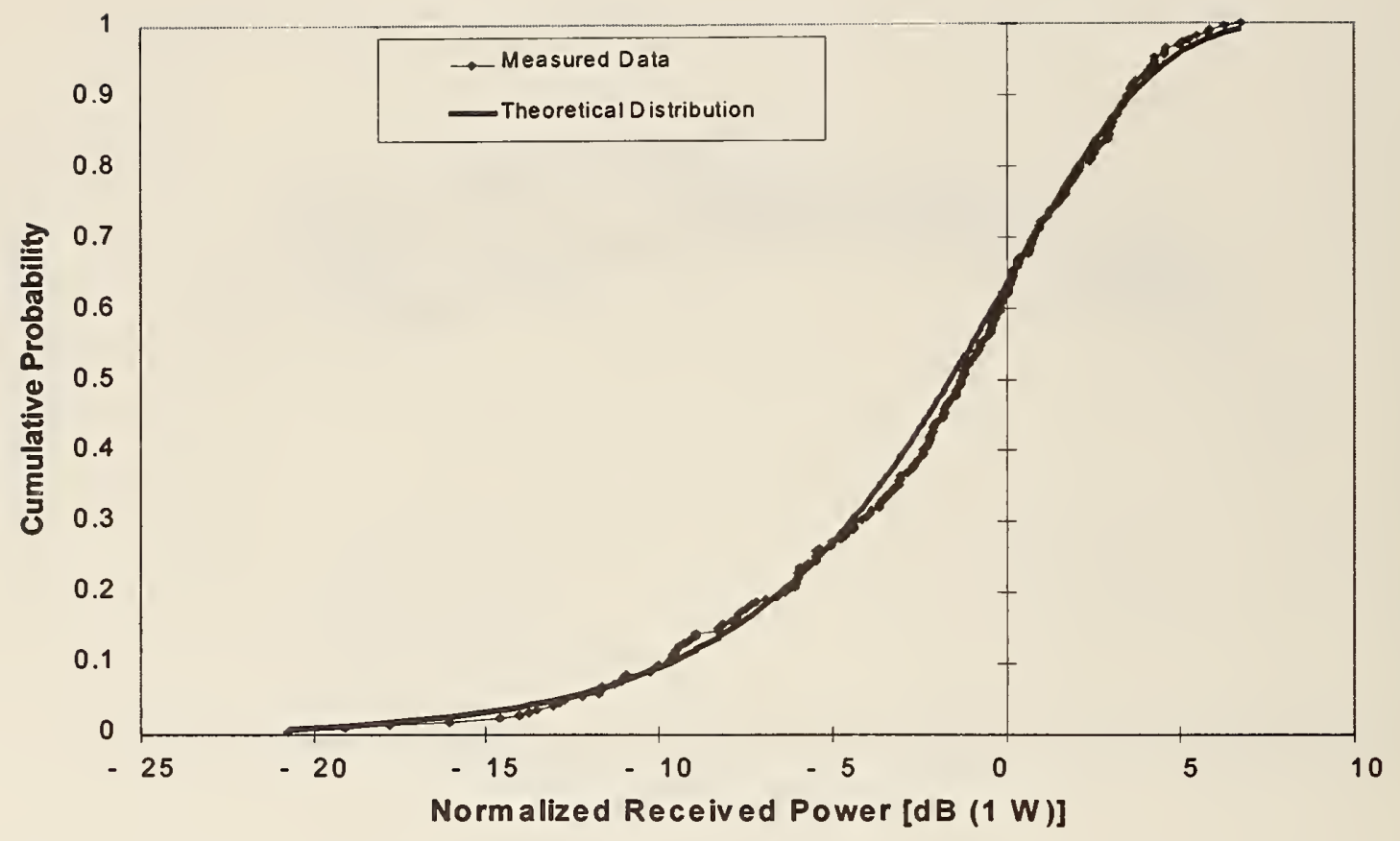

Figure 109. Cumulative distribution of received power at $1 \mathrm{GHz}$ in chamber B. 


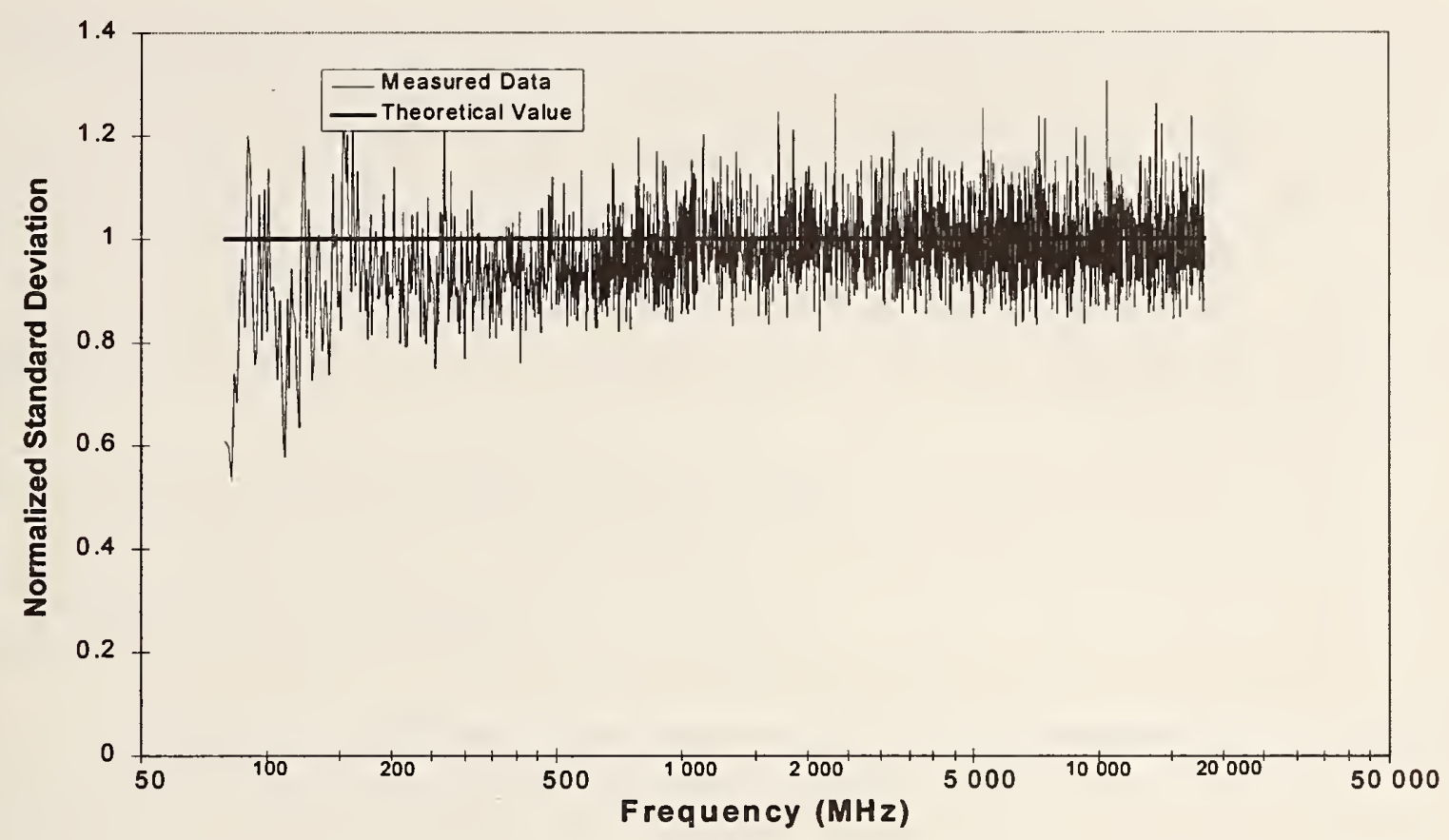

(a)

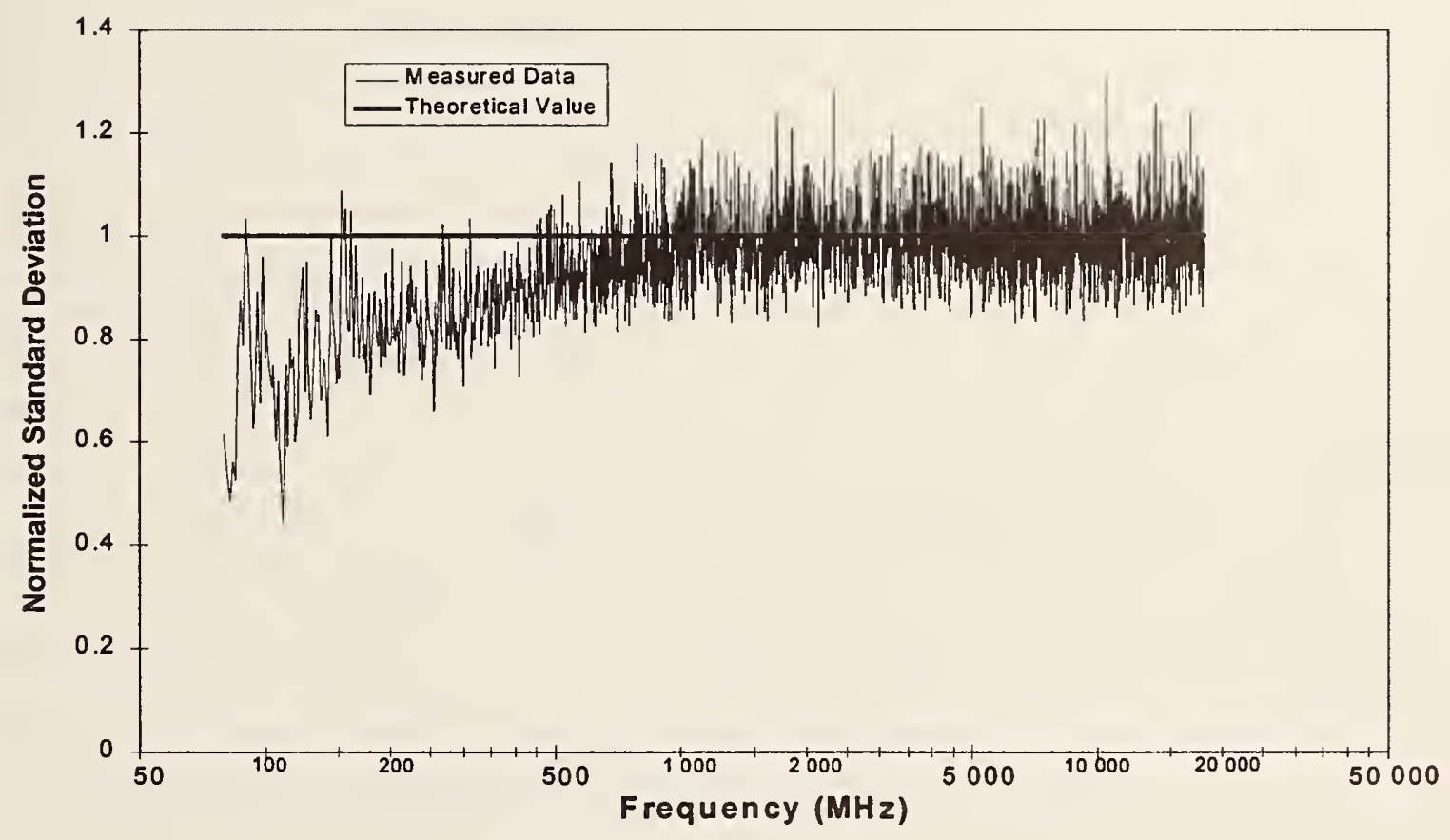

(b)

Figure 110. Normalized standard deviation of received power for a constant input power in chamber B. (a) Constant incident power. (b) Constant net input power. 
The normalized unstirred component of $S_{21}$ given in Figure 93 indicates that the paddle is becoming less effective for some frequencies below $500 \mathrm{MHz}$, and shows serious problems below $175 \mathrm{MHz}$. The maximum-to-average ratio given in Figure 94 indicates that the idealized statistical model may not accurately describe the power received by an obtrusive antenna for frequencies below $1 \mathrm{GHz}$, and that this effect is more apparent when the data are normalized to a constant net input power than when the data are normalized to a constant incident power.

The plots of chamber gain and residuals of chamber gain given in Figure 97 and Figure 98 show excellent agreement between the measured data and both the electromagnetic model and the statistical model.

The data taken with the probe system are presented in Figure 103 through Figure 108. The processing required to generate these figures is discussed in Section 6.4. The optimum performance of the chamber occurs between $1 \mathrm{GHz}$ and $10 \mathrm{GHz}$, with only a slight increase in the spread of the probe readings between $10 \mathrm{GHz}$ and $18 \mathrm{GHz}$. We can think of no reason that the chamber should not continue to behave optimally above $10 \mathrm{GHz}$, so we attribute the apparent increase in uncertainty between $10 \mathrm{GHz}$ and $18 \mathrm{GHz}$ to our measurement system. When examining the data presented here, especially the variation in multiple measurements of a chamber parameter, remember that we can only observe the combined fluctuations of the parameter inside the chamber and the instrumentation. Therefore, the characteristics of the fields are better behaved than the data indicate.

The measurements of the maximum electric field (both rectangular component and total) shown in Figure 105 and Figure 106 indicate that the chamber is well behaved above $1 \mathrm{GHz}$, and even down to $150 \mathrm{MHz}$ the observed standard deviation of the rectangular component is less than $3 \mathrm{~dB}$, and the standard deviation of the total electric field is less than $3 \mathrm{~dB}$ even down to 80 $\mathrm{MHz}$. These figures also show that the data from a reference antenna can be used to predict the maximum electric field, but it is best to estimate the maximum electric field (both rectangular component and total) from the average signal received by the reference antenna rather than the maximum. The difference between estimates of the rectangular component based on the average and maximum of the reference signal is small, but significant, and the difference between estimates of the total electric field based on the average and maximum of the reference signal are substantial.

The plots of the average of the three orthogonal rectangular components of the electric field given in Figure 107 and Figure 108 show no significant dependence on orientation of the probes for measurements of both the average and maximum electric field above $300 \mathrm{MHz}$. Use of the chamber below $300 \mathrm{MHz}$ can result in significant biases due to preferred orientation of the dominant modes.

The plot of the cumulative distribution of the received power shown in Figure 109 shows very good agreement between the measured data and the ideal distribution, but the match is obviously not perfect. The factors that may be contributing to these imperfections are the same as in Chamber A. One problem is the unstirred component of $S_{21}$. The normalized unstirred component of $S_{21}$ for the data shown in Figure 109 is 0.5 . This value is small, but large enough to influence the cumulative distribution. Another possibility is that all indications of the validity of the statistical model (maximum-to-average ratio, normalized standard deviation) applied to the power received from a reference antenna show that the model is imperfect below $1 \mathrm{GHz}$, and may also be imperfect at $1 \mathrm{GHz}$. Finally, as discussed in the previous section, it may be possible 
that the agreement between the measured and theoretical distributions of the data would improve if a larger number of samples were taken.

Figure 110 shows the normalized standard deviation of the power received from the reference antenna, calculated as the standard deviation of the received power divided by the average received power. This ratio is close to the expected value of 1 for frequencies greater than $1 \mathrm{GHz}$, and below $1 \mathrm{GHz}$, this ratio falls below 1 . This effect is more pronounced when the received power is normalized to a constant net input power (Figure 110b) than when the data are normalized to a constant incident power (Figure 110a). The normalized standard deviation indicates that the power received by the reference antenna is not well described by the statistical model below $1 \mathrm{GHz}$. This is most likely caused by the same clipping effect that causes the maximum-to-average ratio to decrease below $1 \mathrm{GHz}$.

\subsection{Summary of Chamber C}

A summary of the figures we used to describe the characteristics of Chamber $\mathrm{C}$ is given in Table 12. The parameters presented here are identical to those presented for Chamber A, except that no probe measurements were performed in Chamber $\mathrm{C}$. We will describe the measured parameters again here so that you will not need to refer to another section to understand this section.

The figures are grouped with antenna characteristics presented first, followed by an evaluation of the mechanical stirrers as described by the unstirred component of $S_{21}$, and then the ratios of the extreme values (maximum-to-average ratio, maximum-to-minimum ratio, average-to-minimum ratio). We then give the average and maximum chamber gain, residuals of chamber gain, followed by all parameters that can be estimated or predicted from measurements of chamber gain (electric field for an input power of $1 \mathrm{~W}$ and the residuals of electric field, average scalar power density, and average quality factor. The estimated volume of Chamber $\mathrm{C}$ used to calculate the quality factor of the chamber was $16.8 \mathrm{~m}^{3}$. The probe system was not used to evaluate Chamber C, so no independent measurements of the electric field are included in this summary. Finally, we present data from some of the tools used to verify the statistical characteristics of the power received from an antenna in the chamber. These are plots of the cumulative distribution and the normalized standard deviation.

The equations describing the key parameters are given in Table 13. All equations are based on curve fit parameters for the average chamber gain of $a=3.237$ and $b=0.56 \cdot 10^{-21}$ for data normalized to a constant incident power, and values of $a=2.749$ and $b=0.559 \cdot 10^{-21}$ for data normalized to a constant net input power. 
Table 12. Summary of figures related to Chamber C.

Minimum, average, and maximum VSWR

Minimum, average, and maximum reflected power

Mismatch correction factor

Estimated efficiencies of antennas

Unstirred component of $S_{21}$

Maximum-to-average ratio

Maximum-to-minimum ratio

Average-to-minimum ratio

Average and maximum chamber gain

Residuals of chamber gain

Electric field

Residuals of electric field

Scalar power density

Quality factor

Cumulative distribution of received power at $1 \mathrm{GHz}$

Normalized standard deviation of received power
Figure 111

Figure 112

Figure 113

Figure 114

Figure 115

Figure 116

Figure 117

Figure 118

Figure 119

Figure 120

Figure 121

Figure 122

Figure 123

Figure 124

Figure 125

Figure 126 
Table 13. Estimated equations for descriptive characteristics of Chamber C

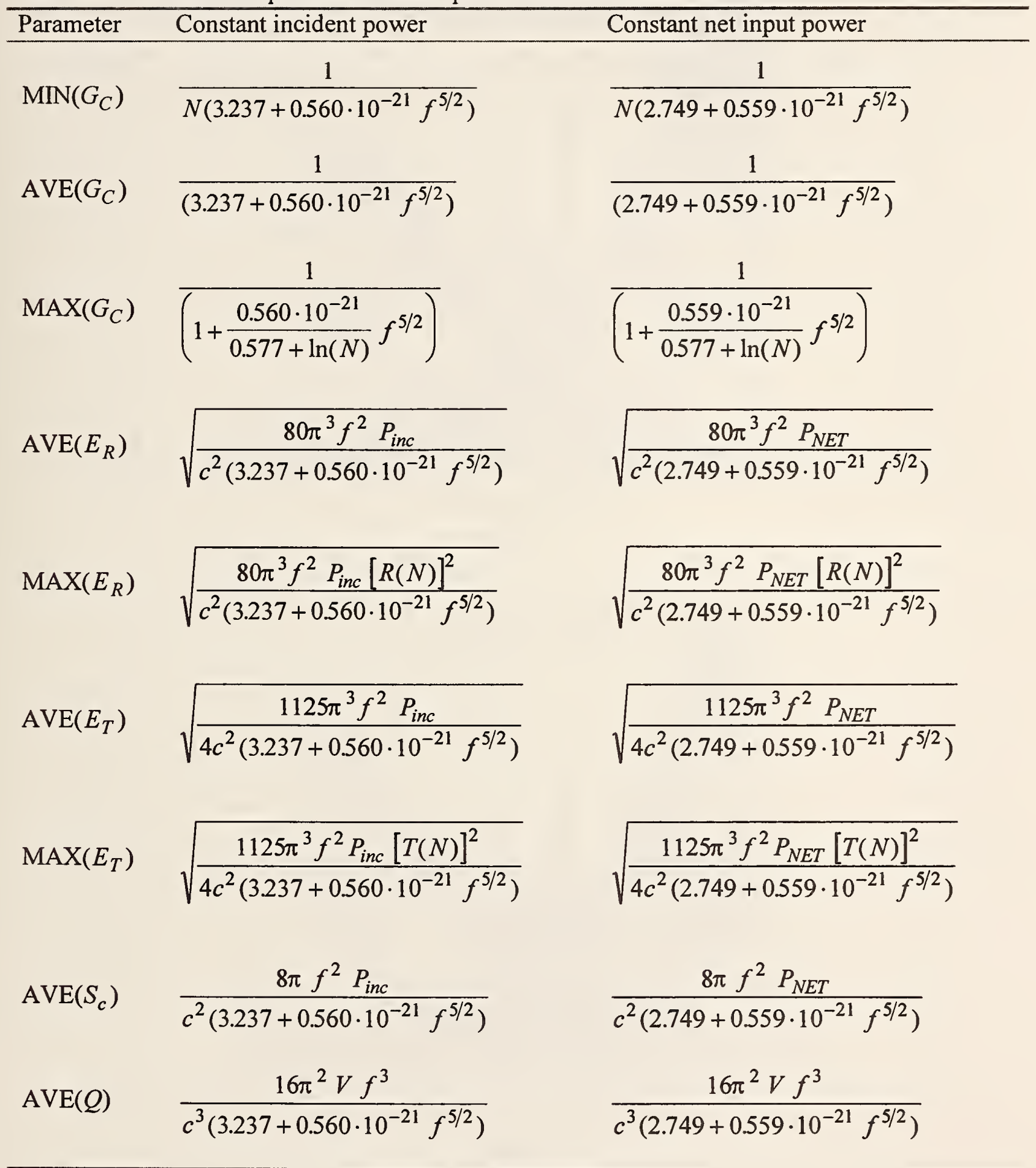




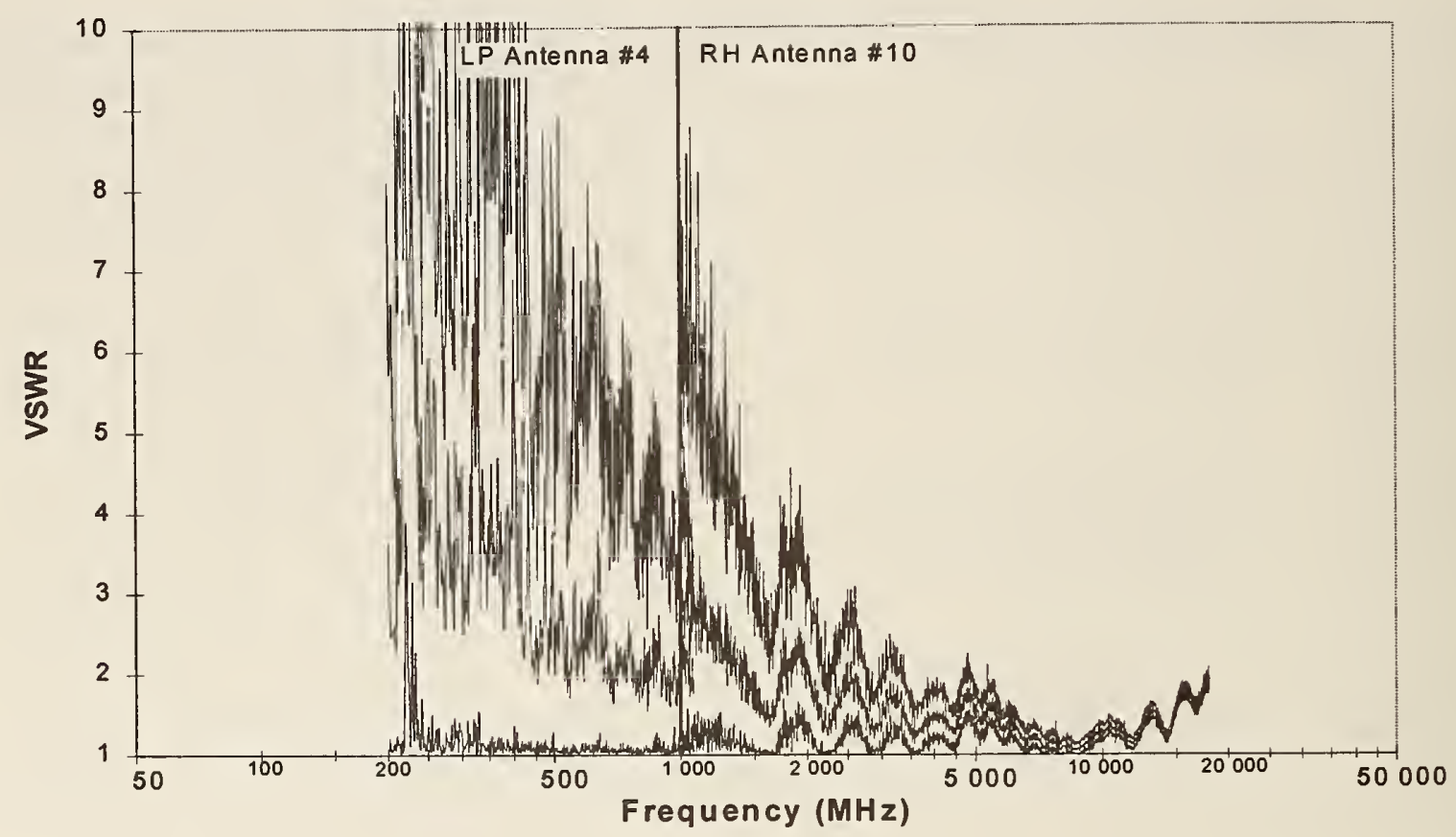

(a)

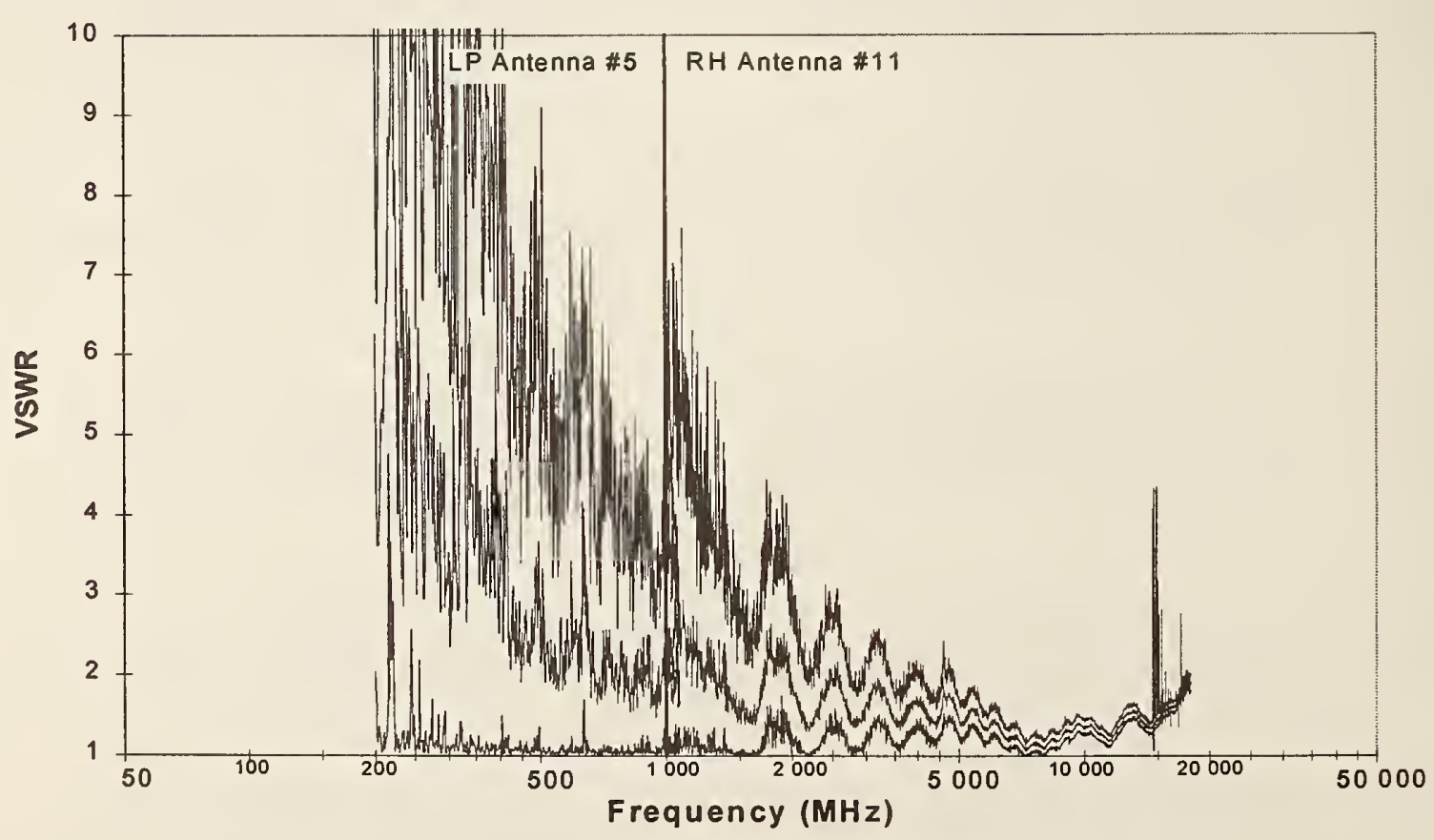

(b)

Figure 111. Minimum, average, and maximum VSWR in chamber C. (a) Port 1 transmitting (forward). Log periodic antenna 4, $200 \mathrm{MHz}$ to $1.08 \mathrm{GHz}$. Ridged horn antenna 10, $1 \mathrm{GHz}$ to $18 \mathrm{GHz}$. (b) Port 2 transmitting (reverse). Log periodic antenna 5, $200 \mathrm{MHz}$ to $1.08 \mathrm{GHz}$. Ridged horn antenna $11,1 \mathrm{GHz}$ to $18 \mathrm{GHz}$. 


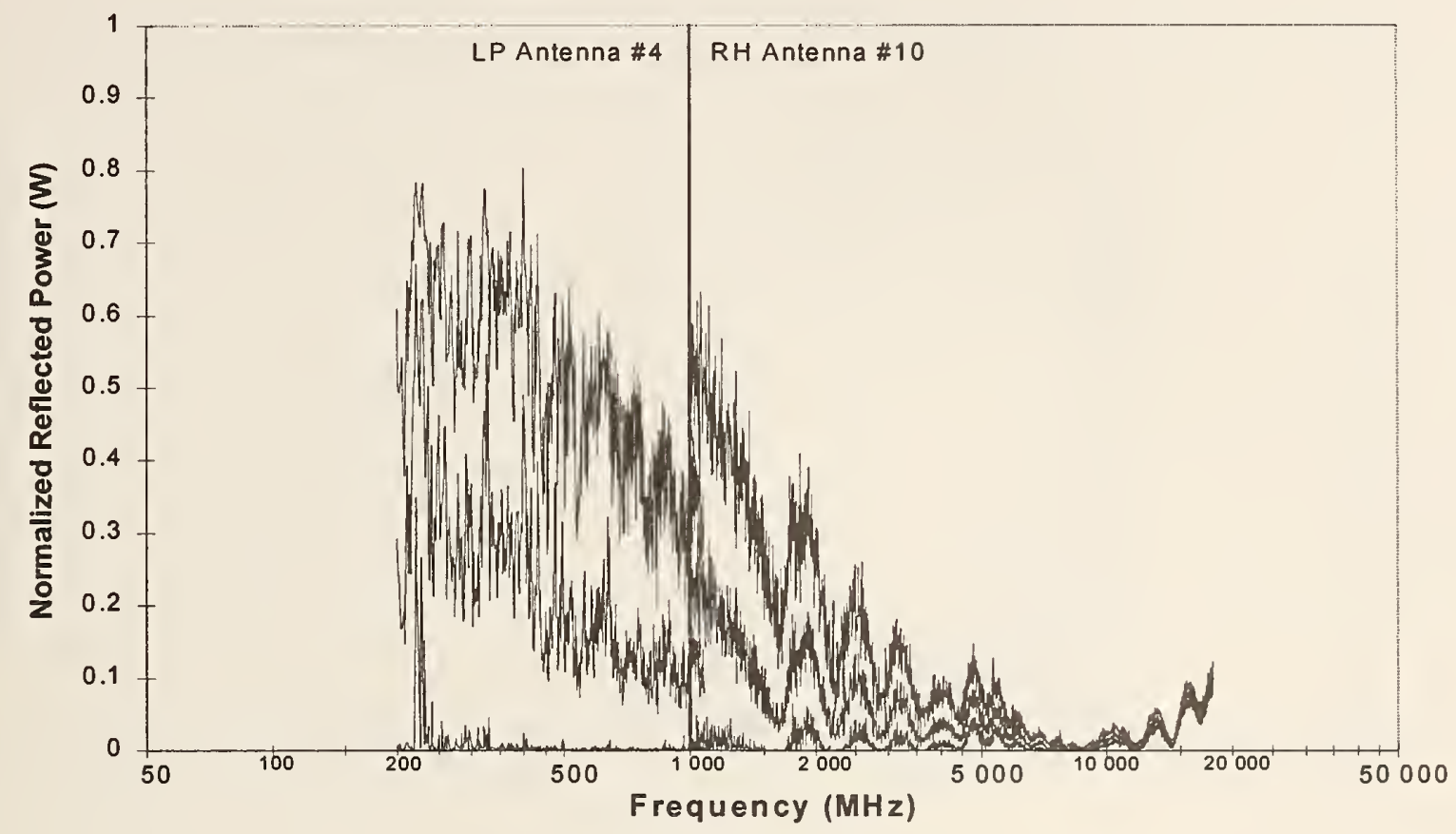

(a)

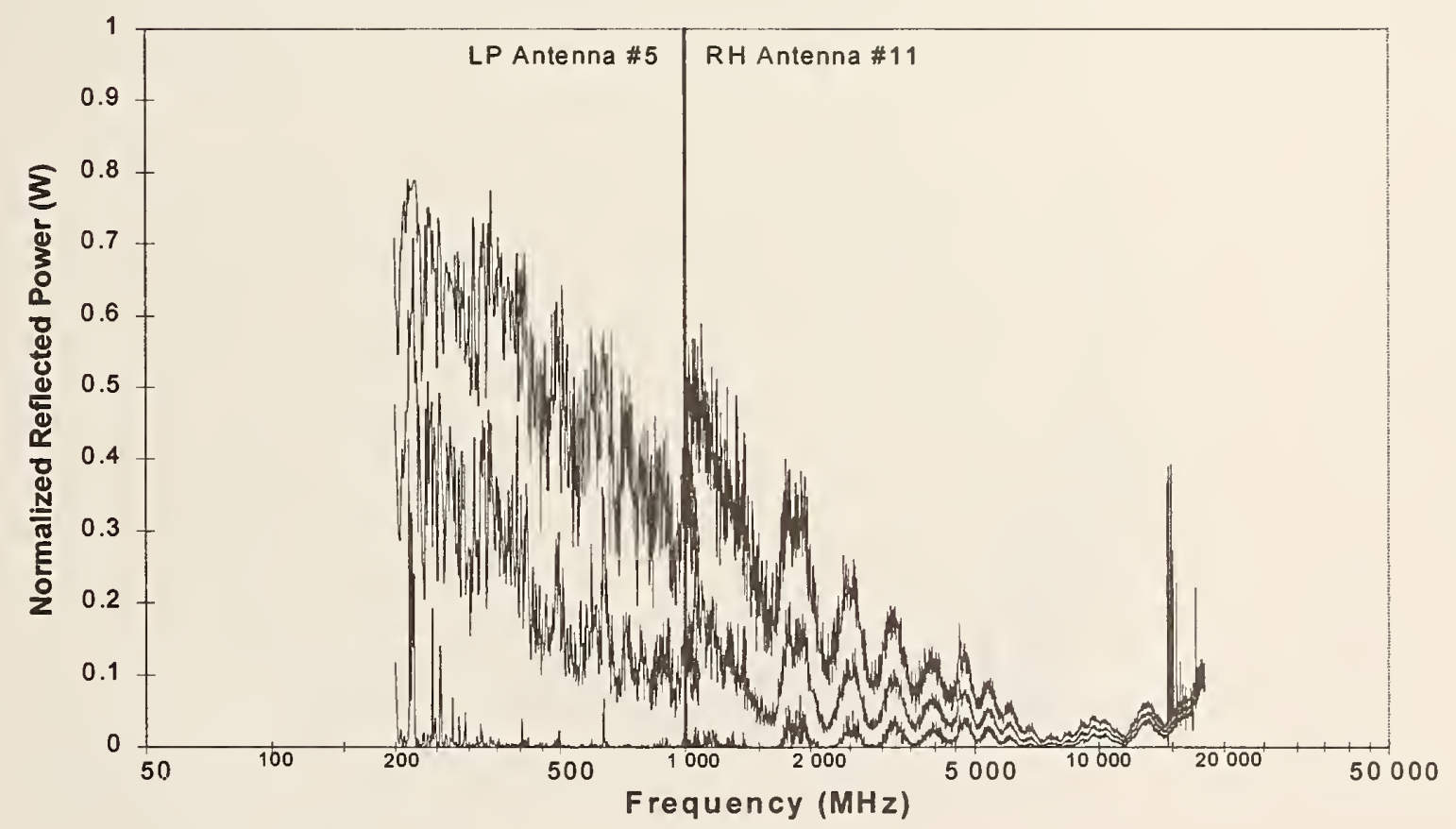

(b)

Figure 112. Minimum, average, and maximum reflected power for an incident power of $1 \mathrm{~W}$ in chamber C. (a) Port 1 transmitting (forward). Log periodic antenna 4, $200 \mathrm{MHz}$ to $1.08 \mathrm{GHz}$. Ridged horn antenna $10,1 \mathrm{GHz}$ to $18 \mathrm{GHz}$. (b) Port 2 transmitting (reverse). Log periodic antenna 5, $200 \mathrm{MHz}$ to $1.08 \mathrm{GHz}$. Ridged horn antenna 11, $1 \mathrm{GHz}$ to $18 \mathrm{GHz}$. 


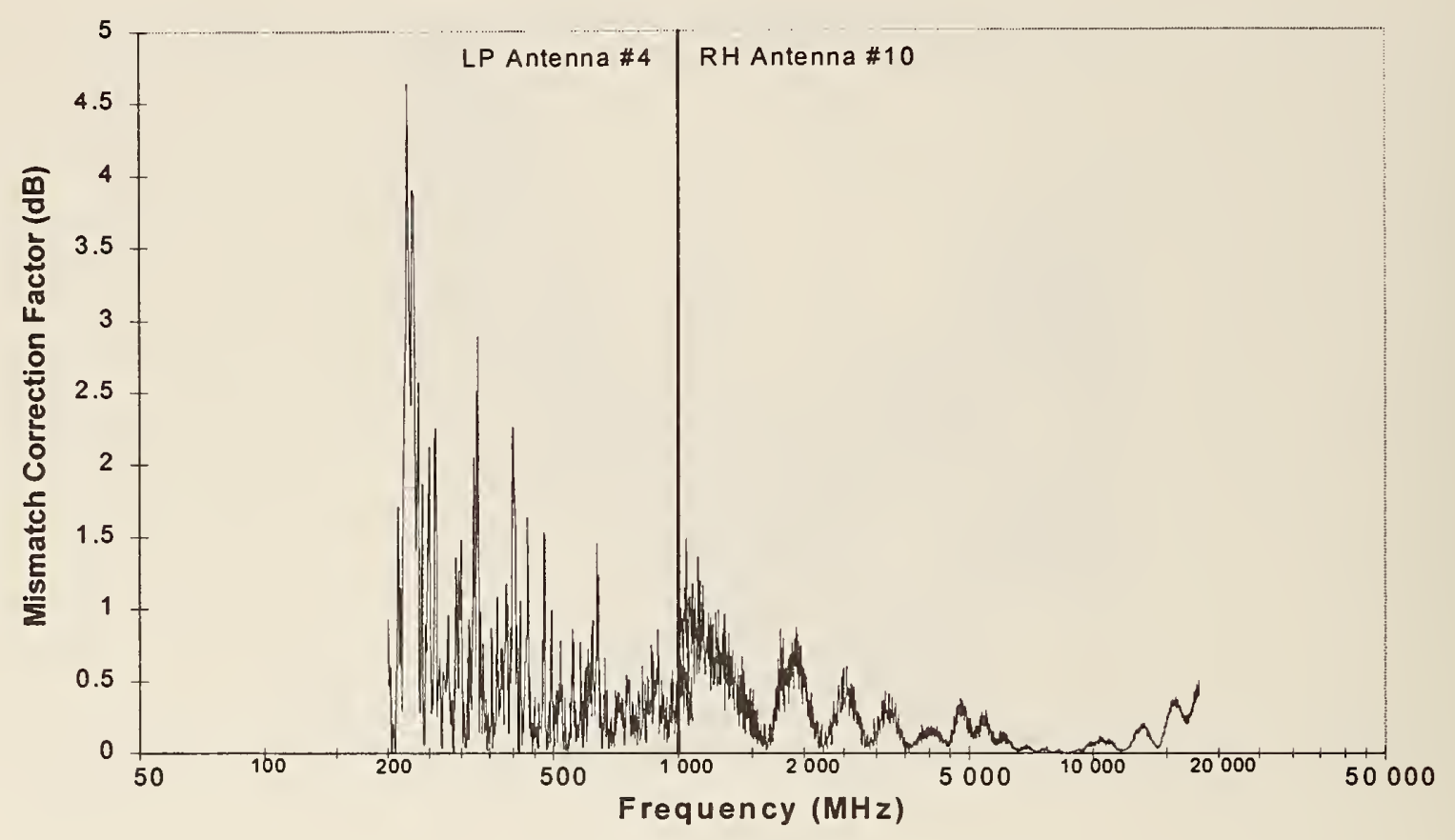

(a)

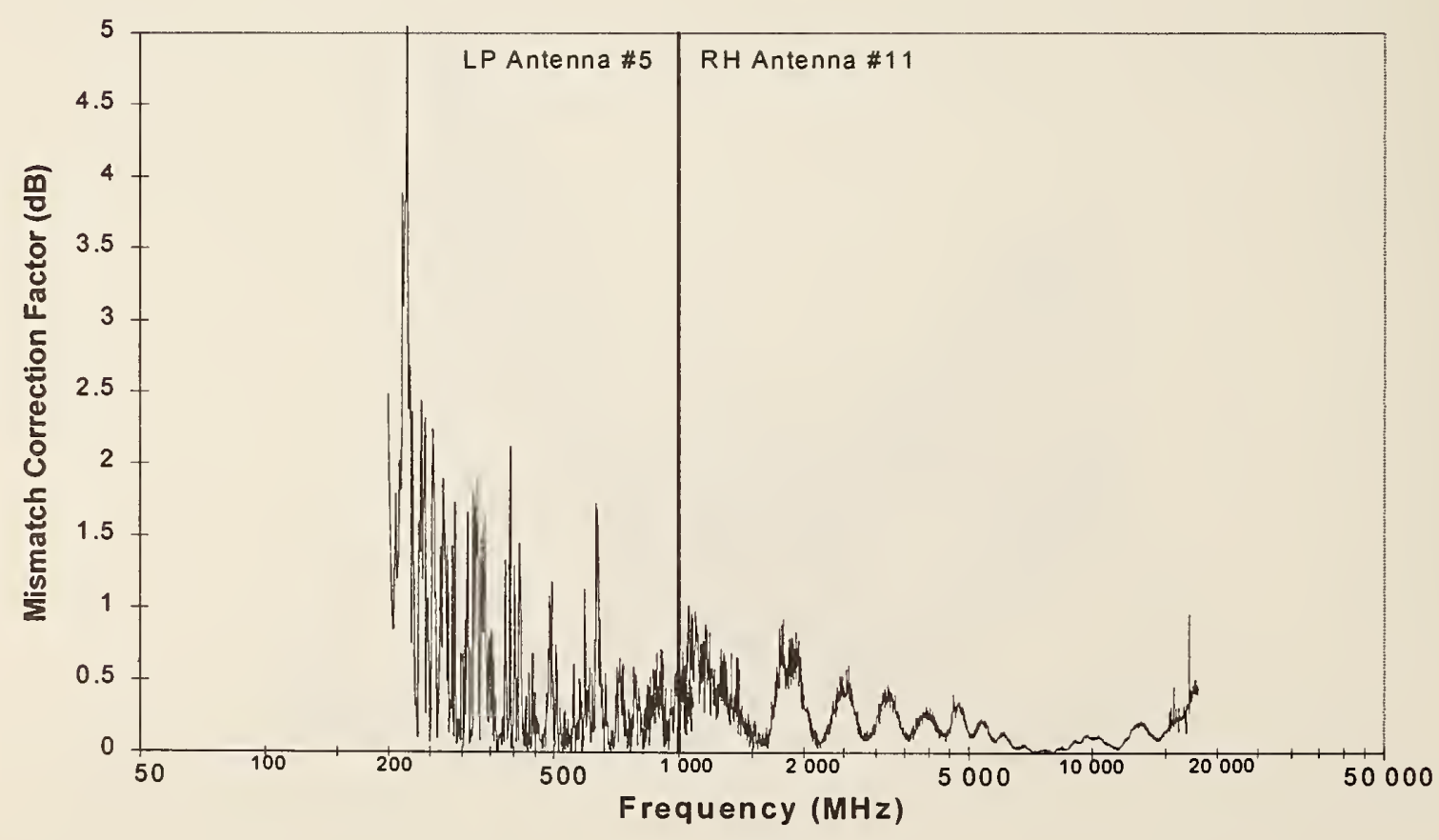

(b)

Figure 113. Mismatch correction factor in chamber C. (a) Port 1 transmitting (forward). Log periodic antenna $4,200 \mathrm{MHz}$ to $1.08 \mathrm{GHz}$. Ridged horn antenna $10,1 \mathrm{GHz}$ to $18 \mathrm{GHz}$. (b) Port 2 transmitting (reverse). Log periodic antenna 5, $200 \mathrm{MHz}$ to $1.08 \mathrm{GHz}$. Ridged horn antenna $11,1 \mathrm{GHz}$ to $18 \mathrm{GHz}$. 


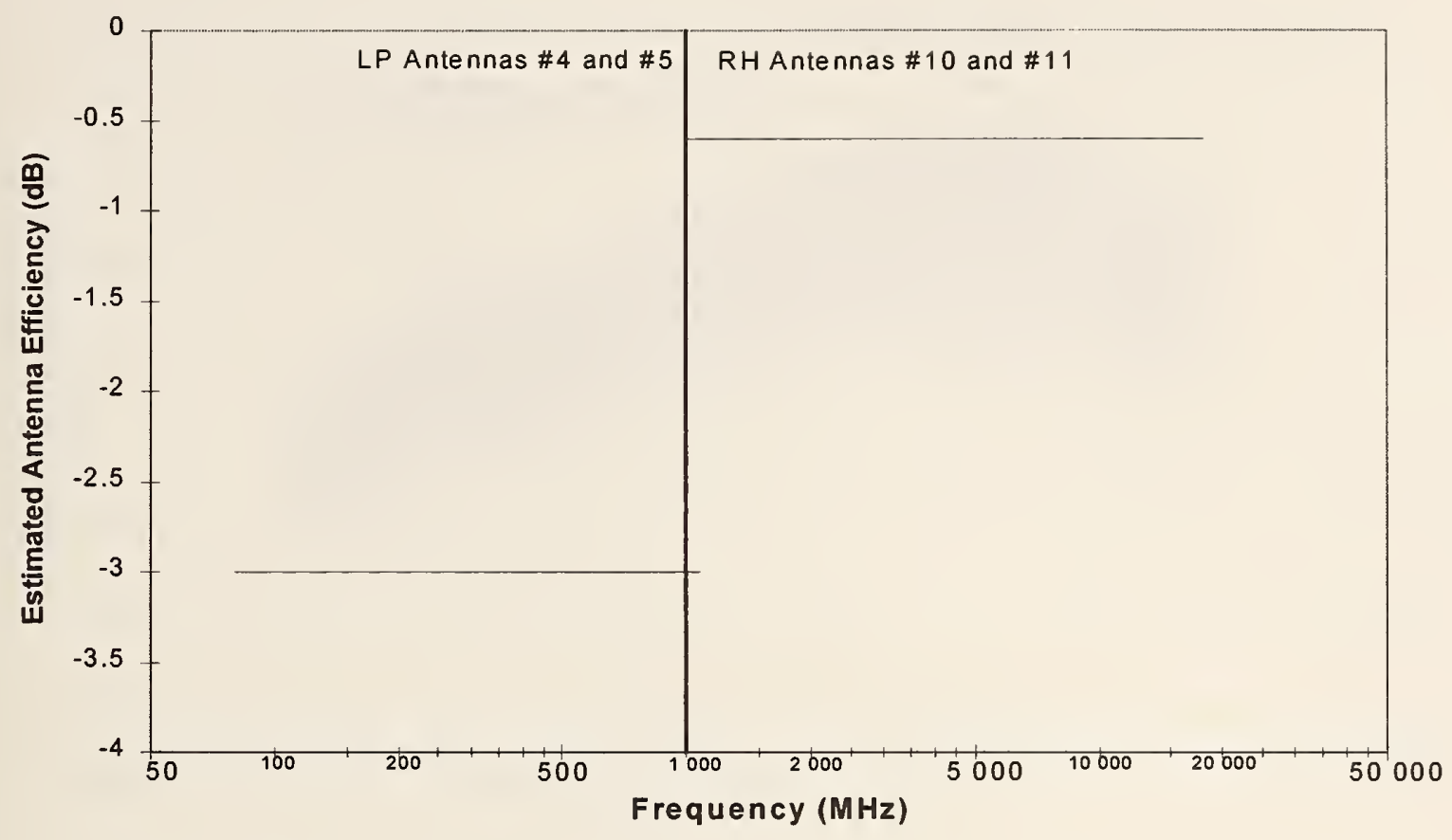

Figure 114. Estimated efficiencies of measurement antennas in chamber C. Log periodic antennas 4 and 5, $200 \mathrm{MHz}$ to $1.08 \mathrm{GHz}$. Ridged horn antennas 10 and $11,1 \mathrm{GHz}$ to $18 \mathrm{GHz}$. 


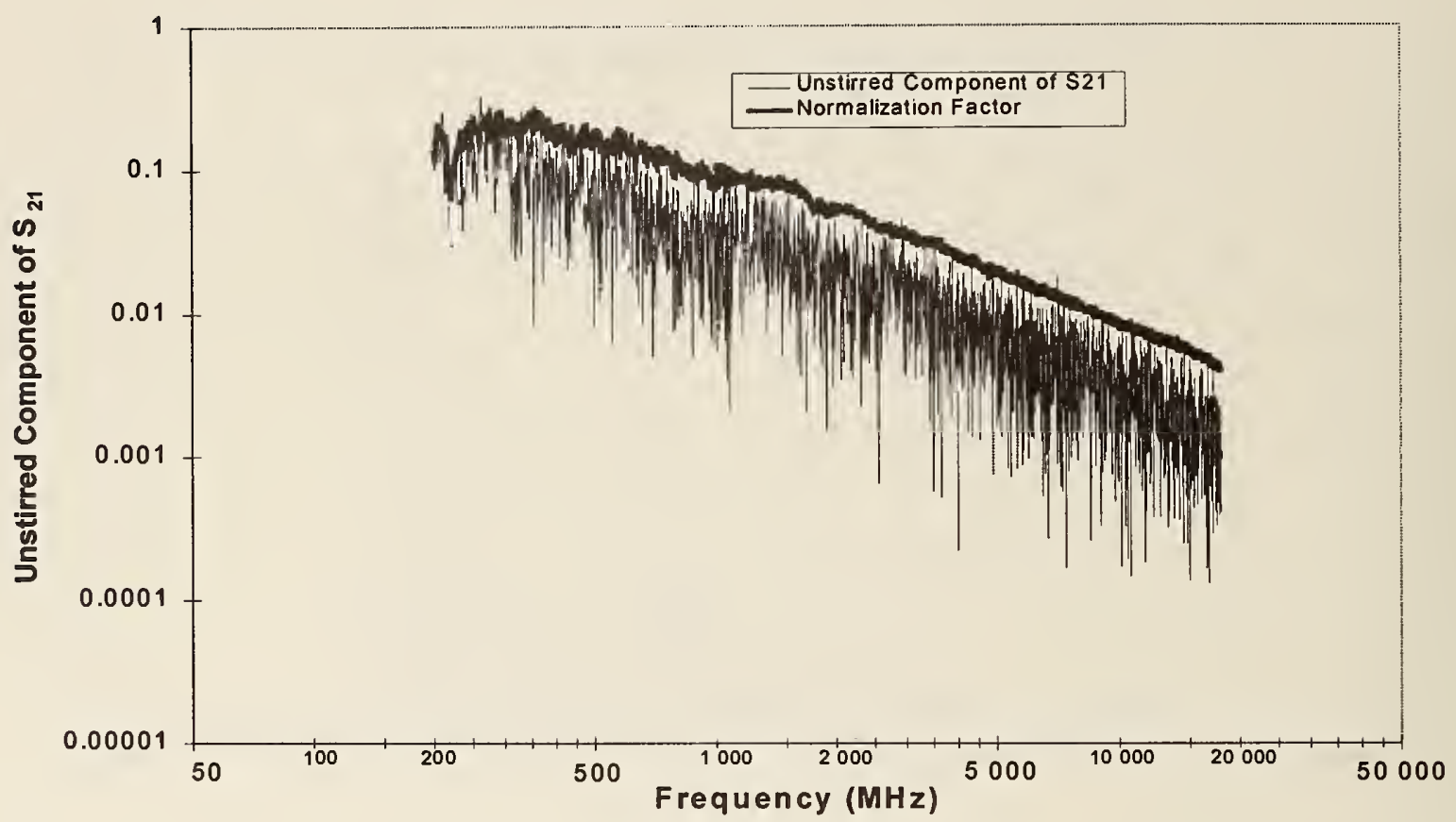

(a)

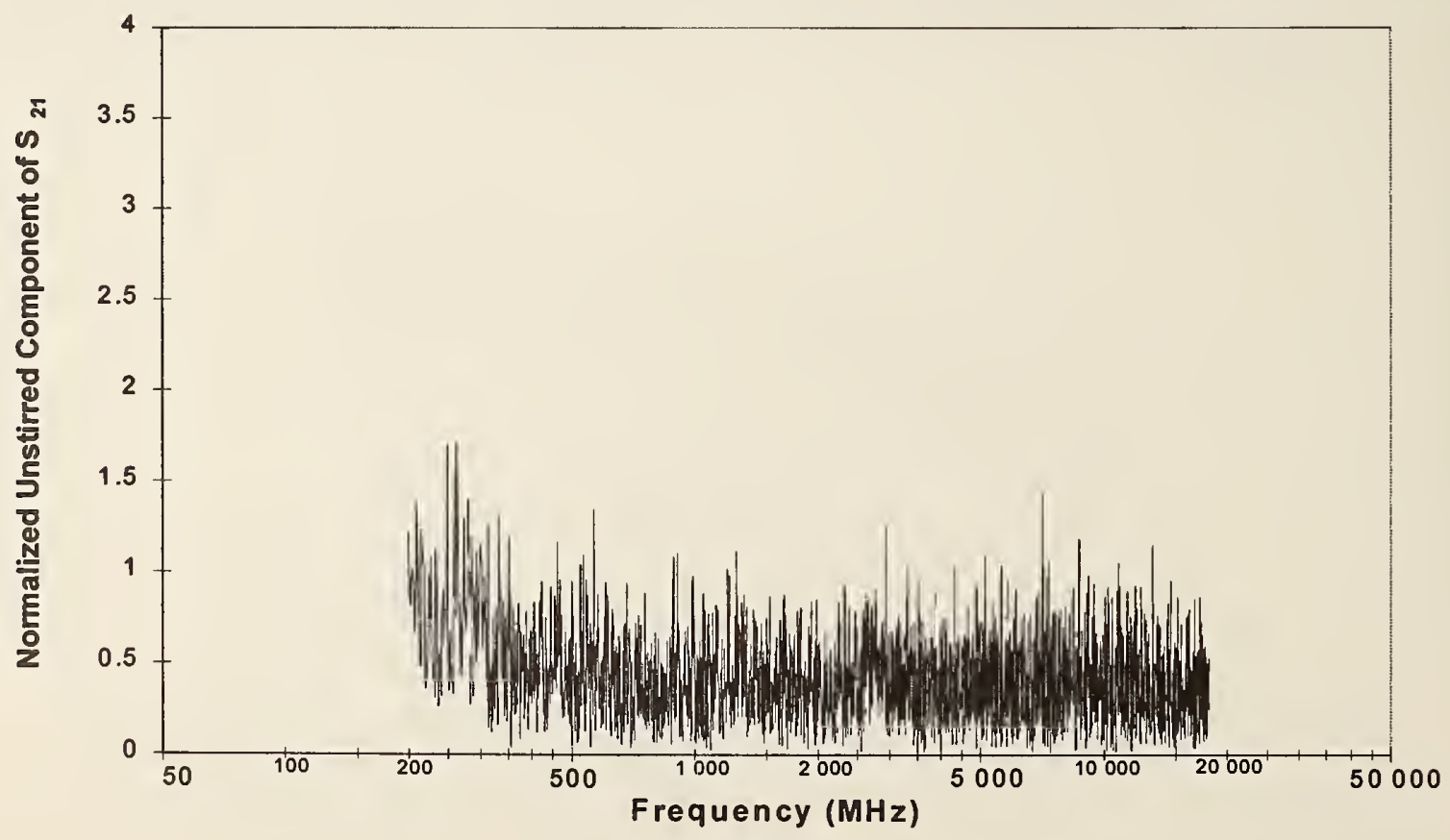

(b)

Figure 115. Evaluation of mechanical stirrers in chamber C. (a) Unstirred component of $S_{21}$. (b) Normalized unstirred component of $S_{21}$. 


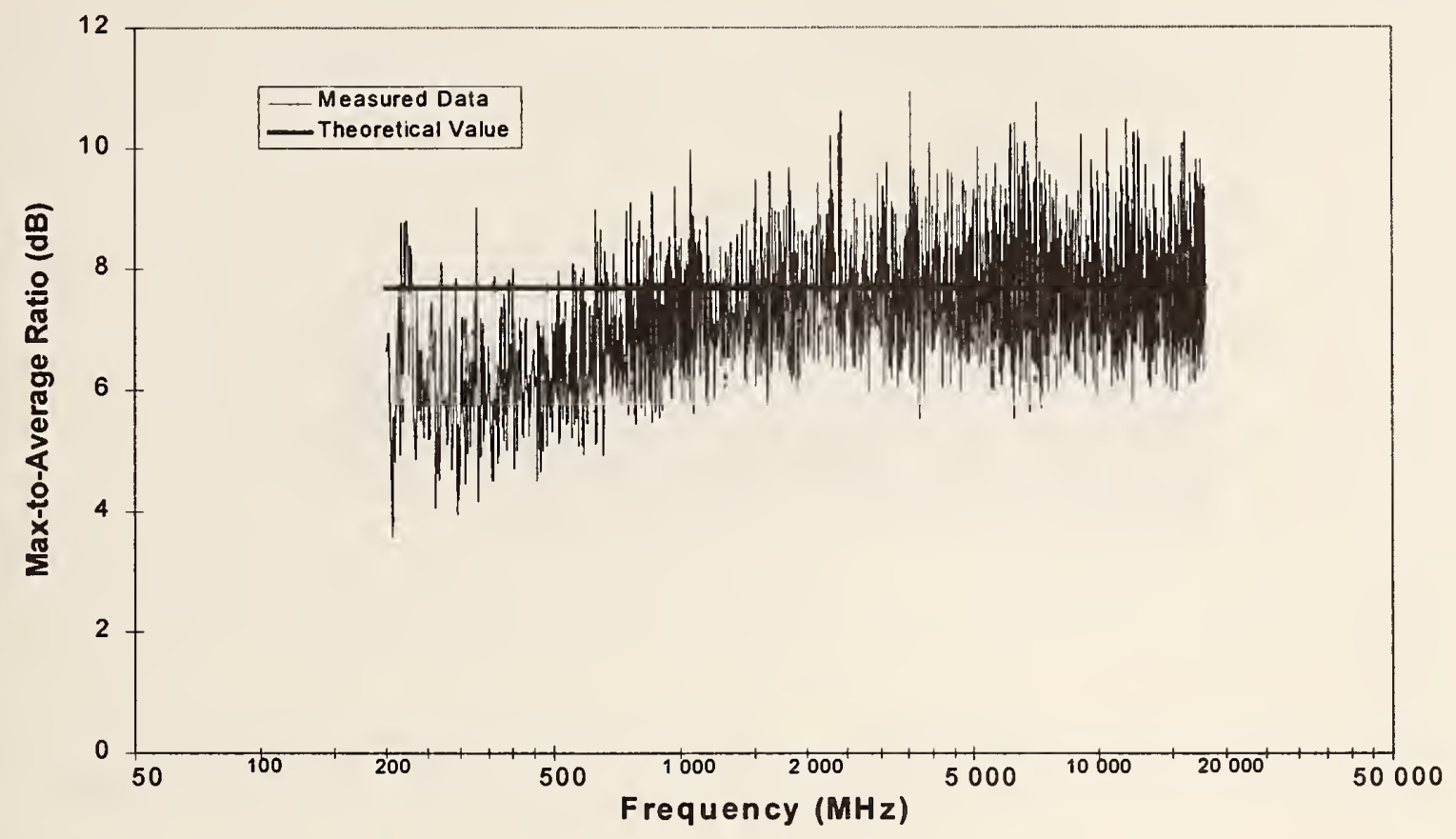

(a)

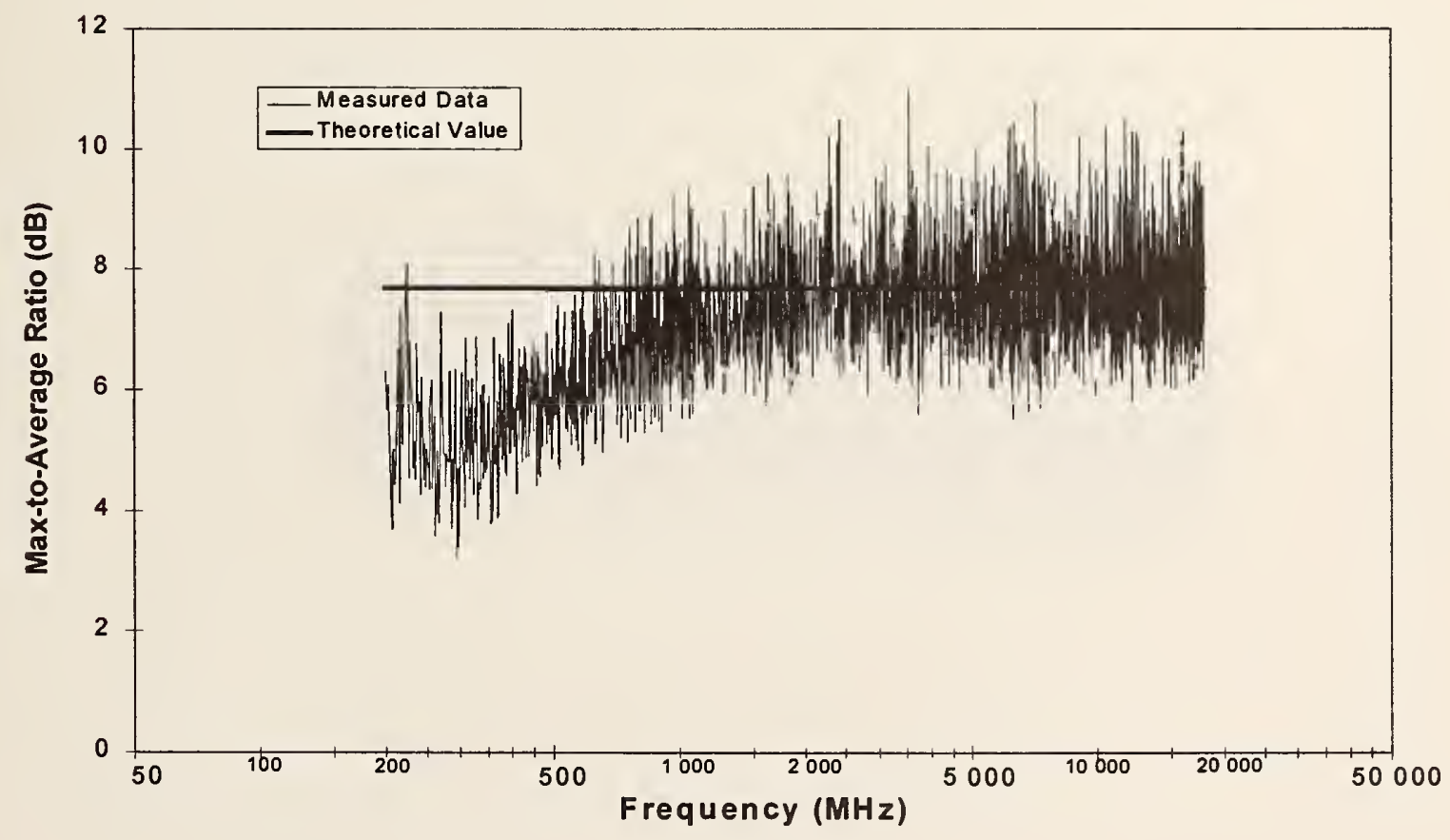

(b)

Figure 116. Max-to-average ratio in chamber C. (a) Constant incident power. (b) Constant net input power. 


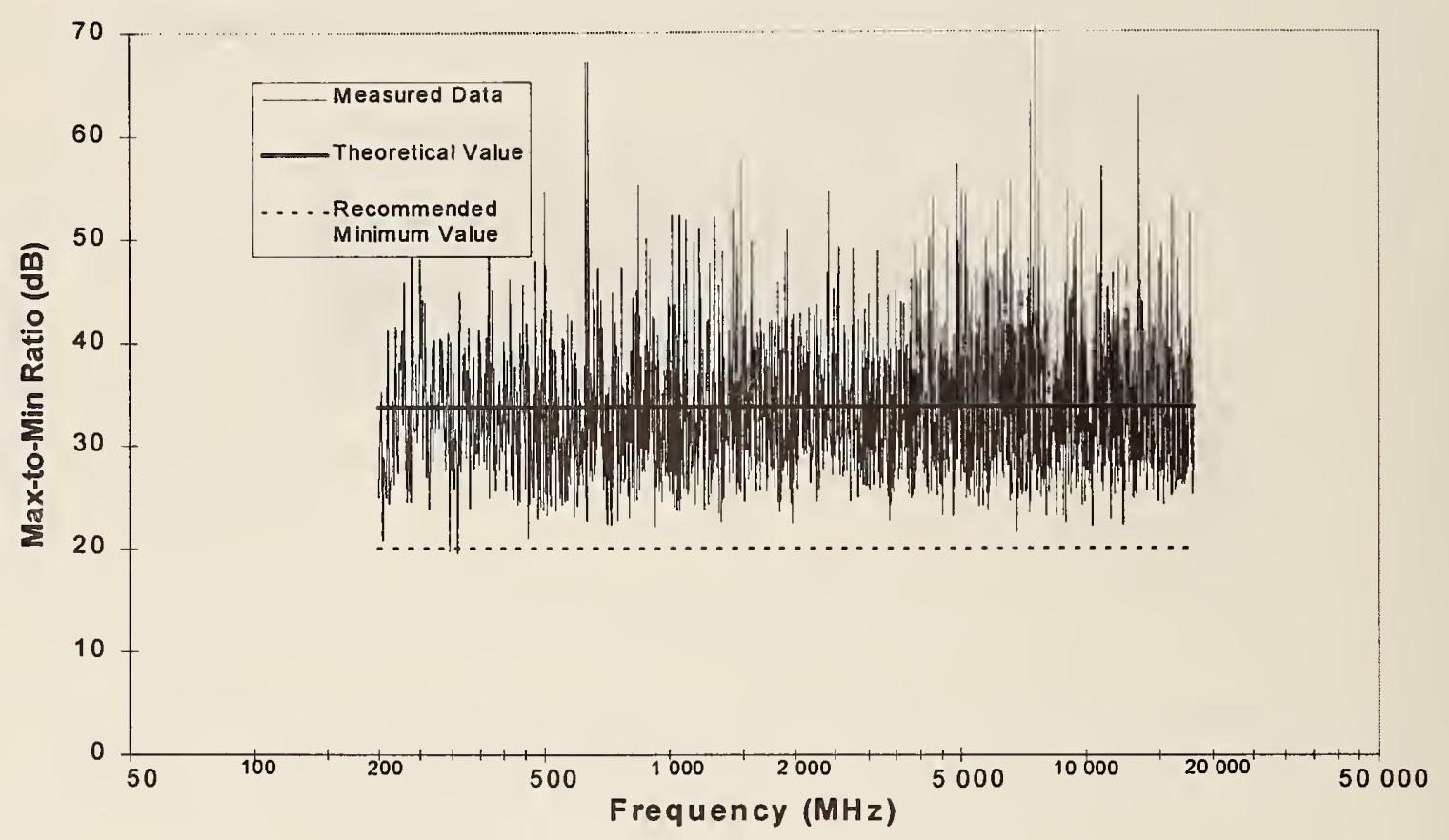

(a)

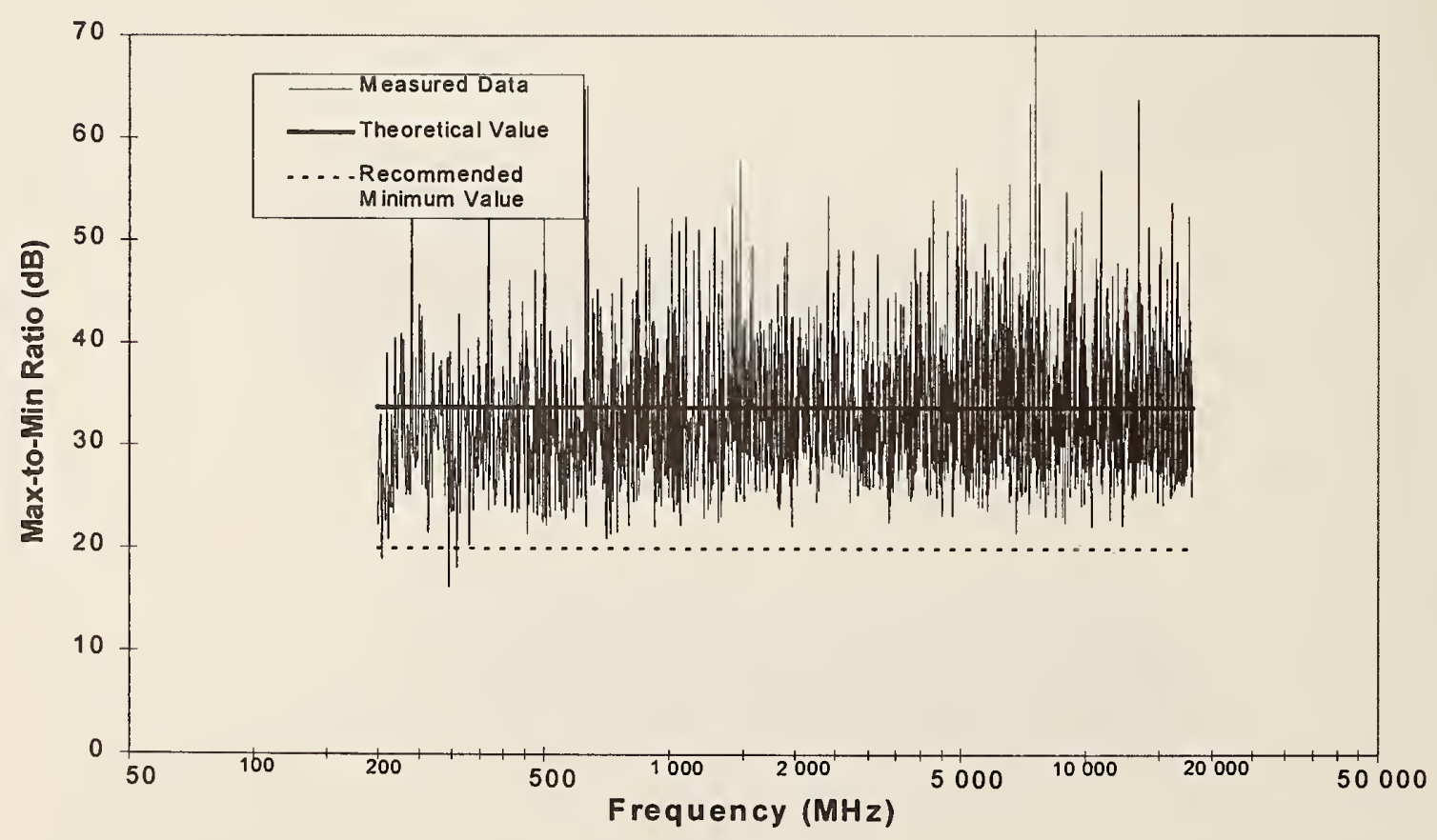

(b)

Figure 117. Max-to-min ratio in chamber C. (a) Constant incident power. (b) Constant net input power. 


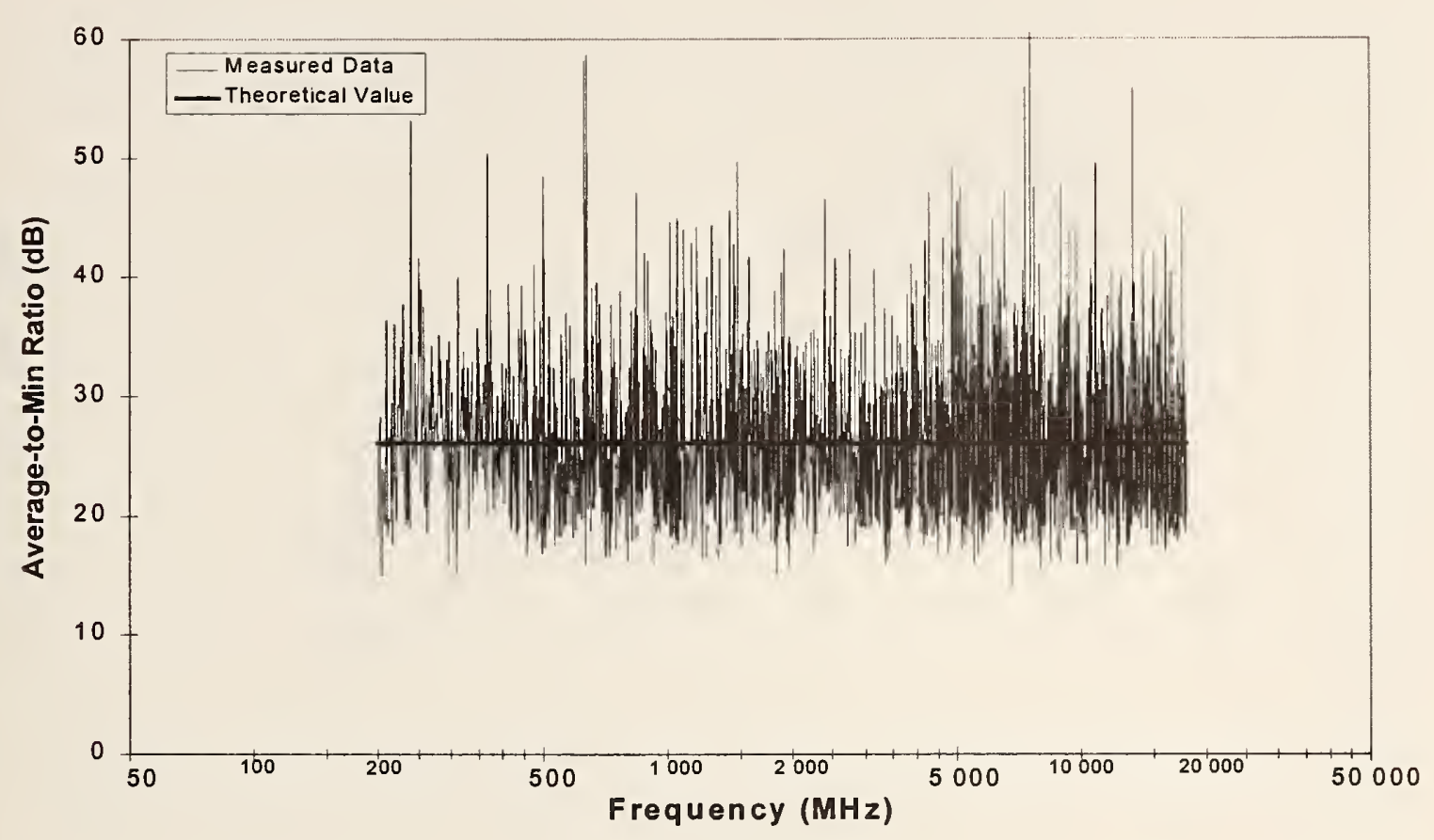

(a)

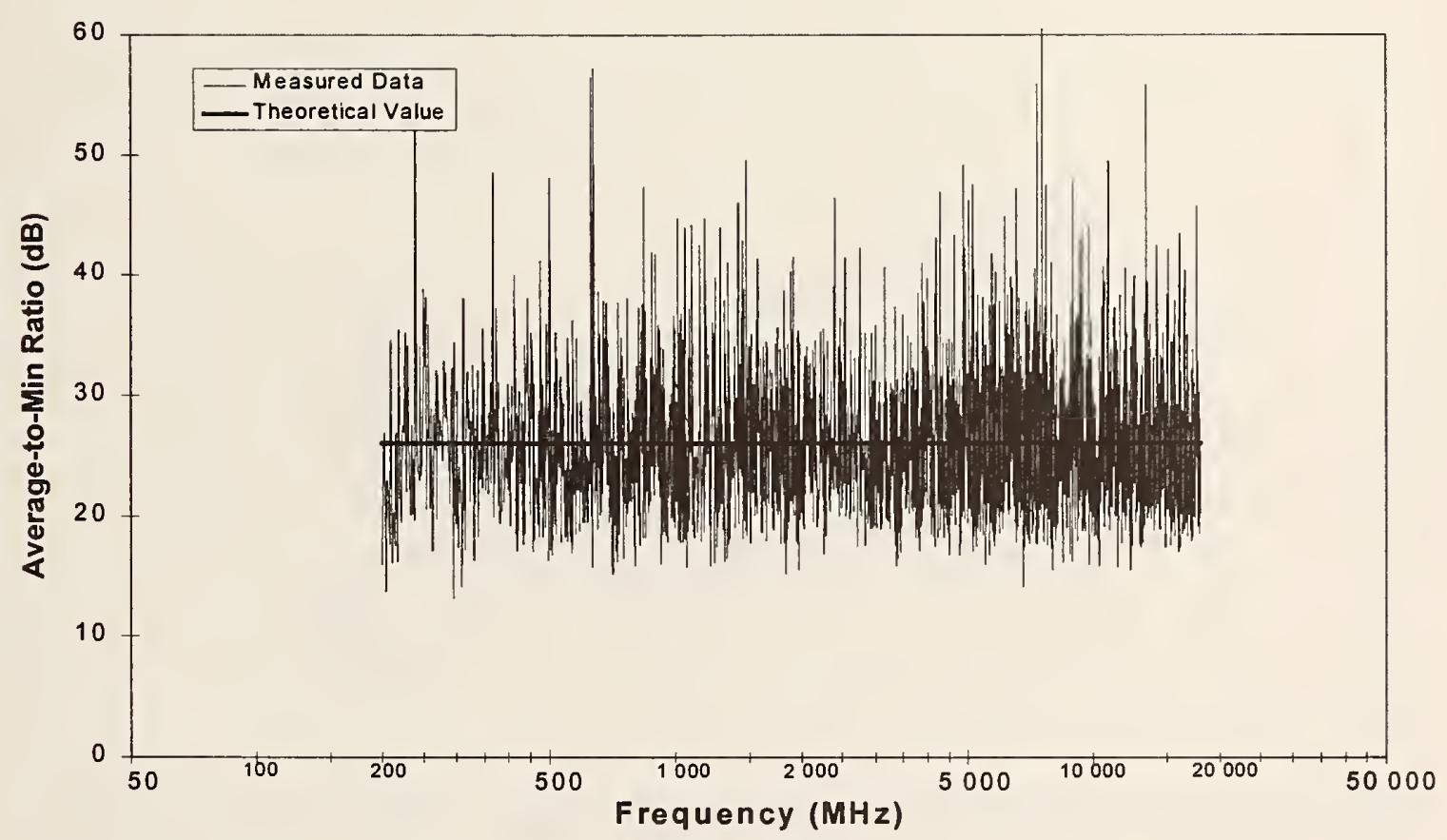

(b)

Figure 118. Average-to-min ratio in chamber C. (a) Constant incident power. (b) Constant net input power. 


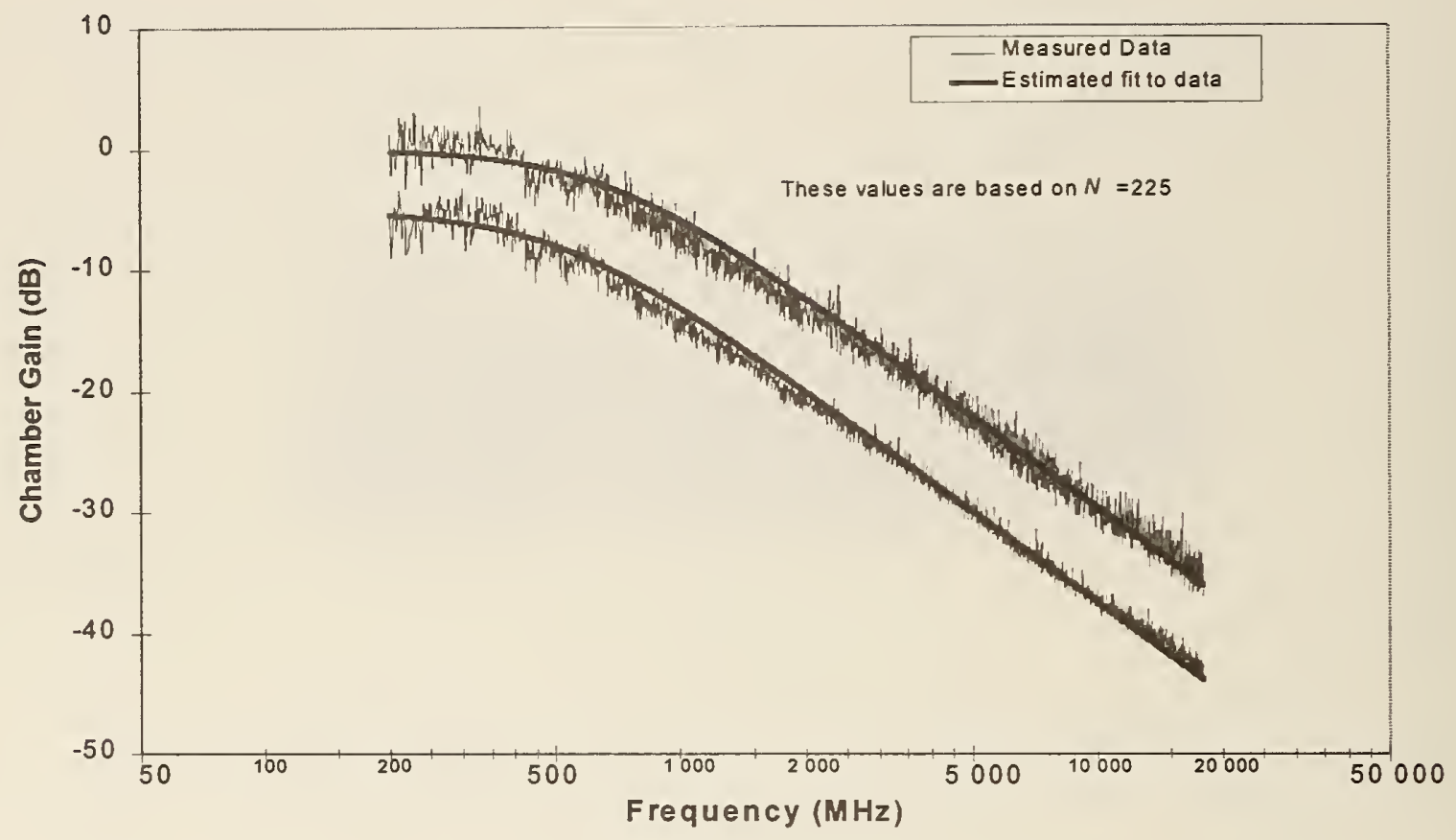

(a)

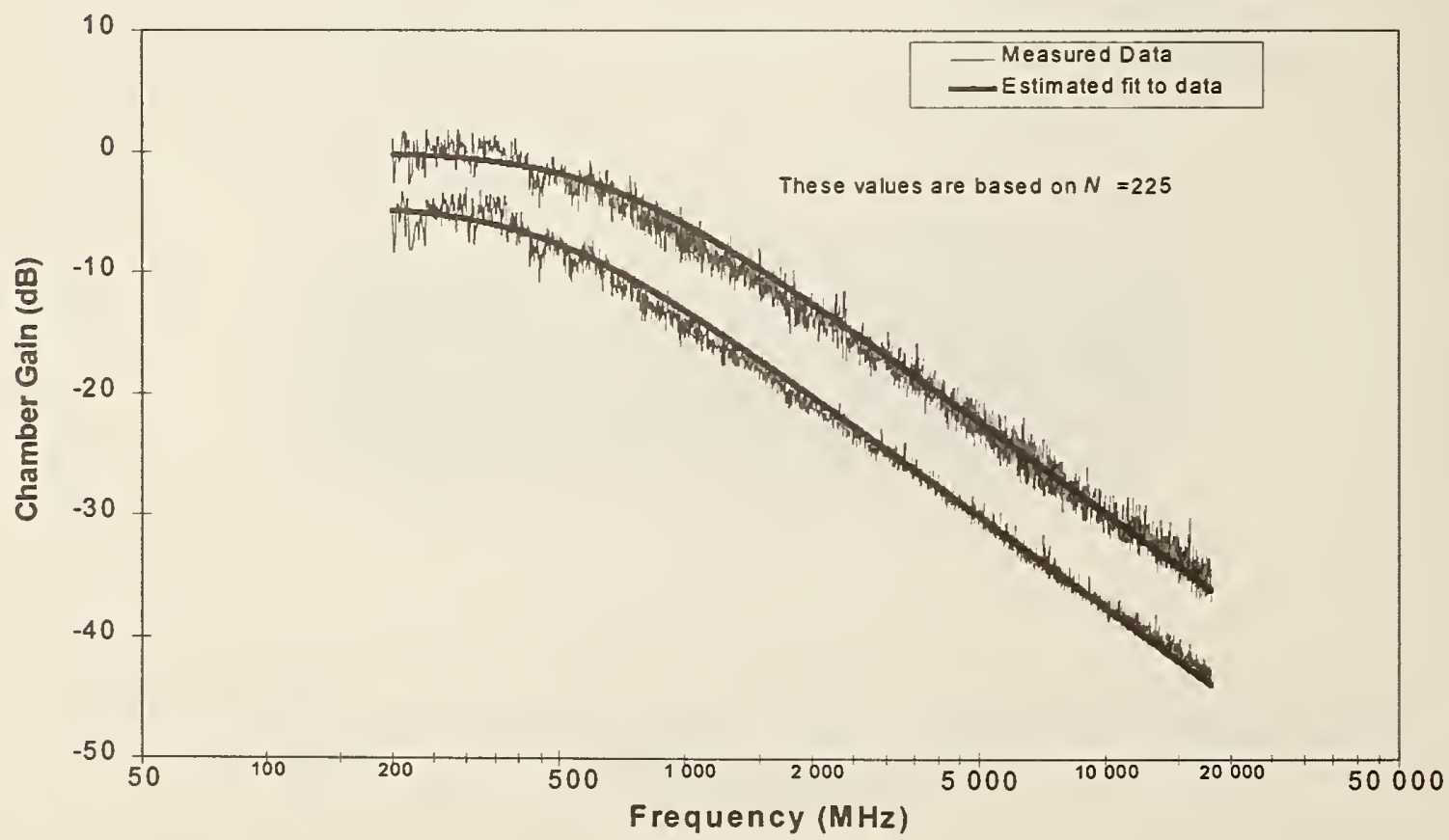

(b)

Figure 119. Average and maximum chamber gain in chamber C. (a) Constant incident power. (b) Constant net input power. 


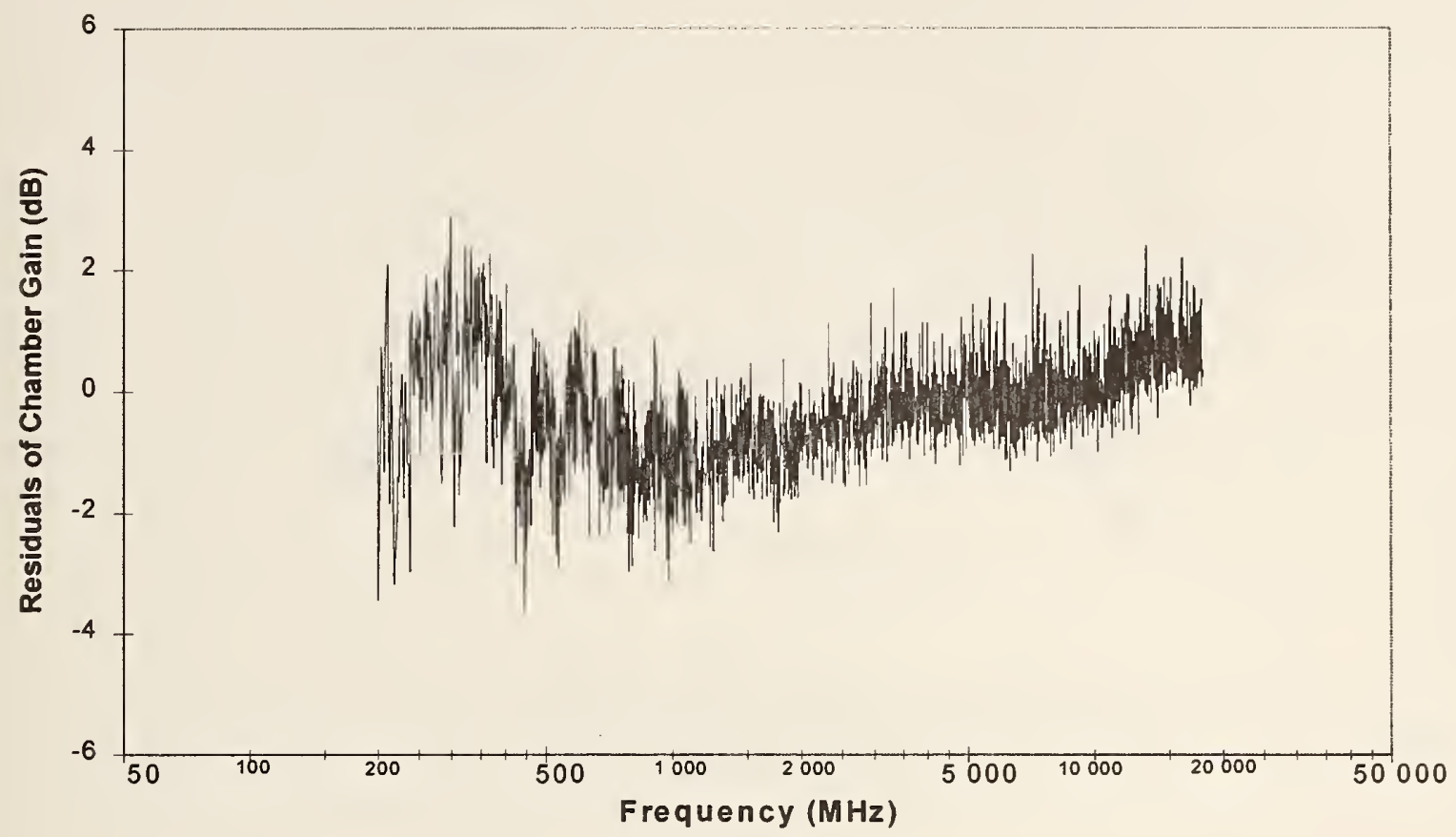

(a)

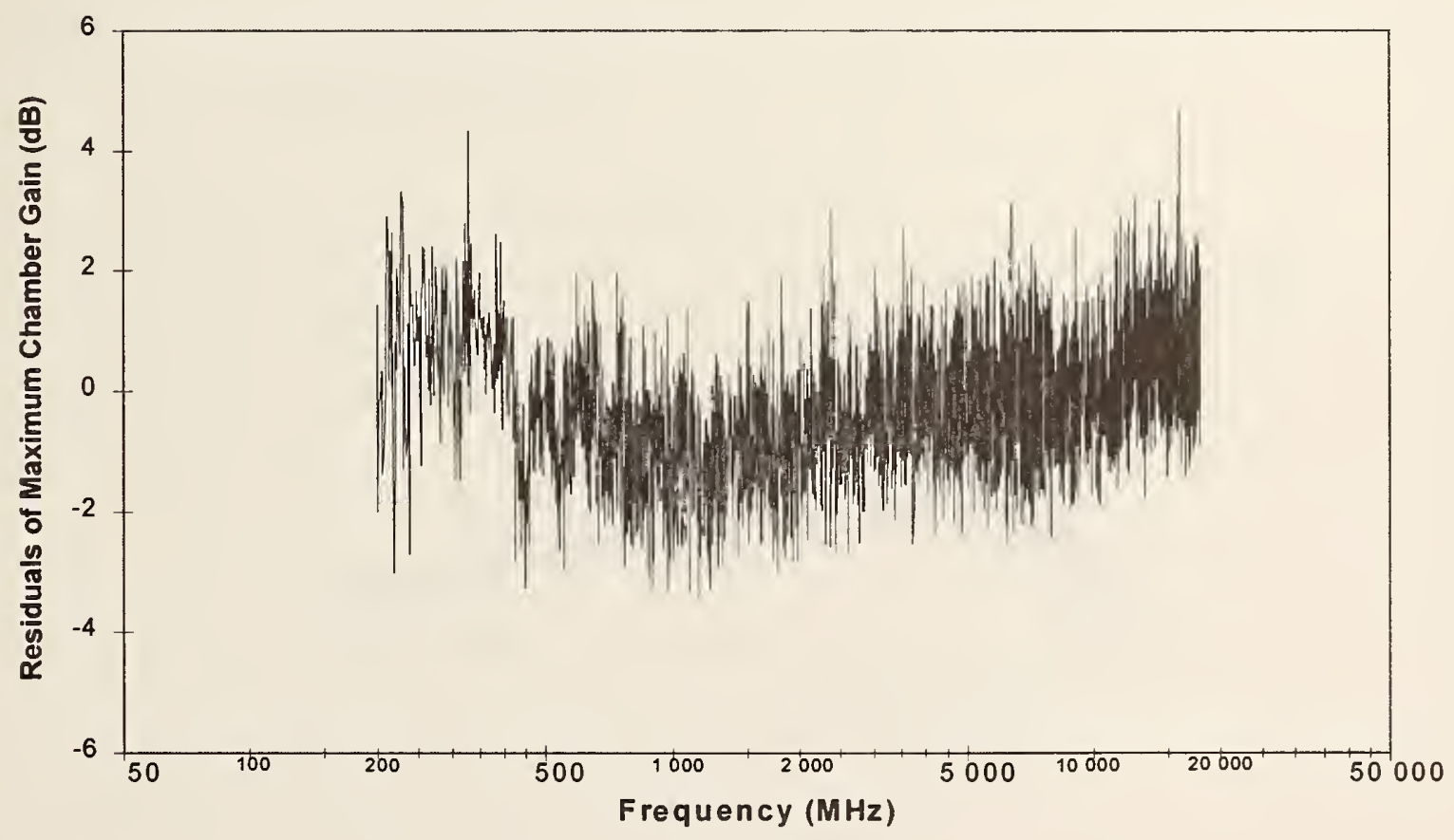

(b)

Figure 120. Residuals of chamber gain in chamber C. (a) Residuals of average chamber gain. (b) Residuals of maximum chamber gain. 


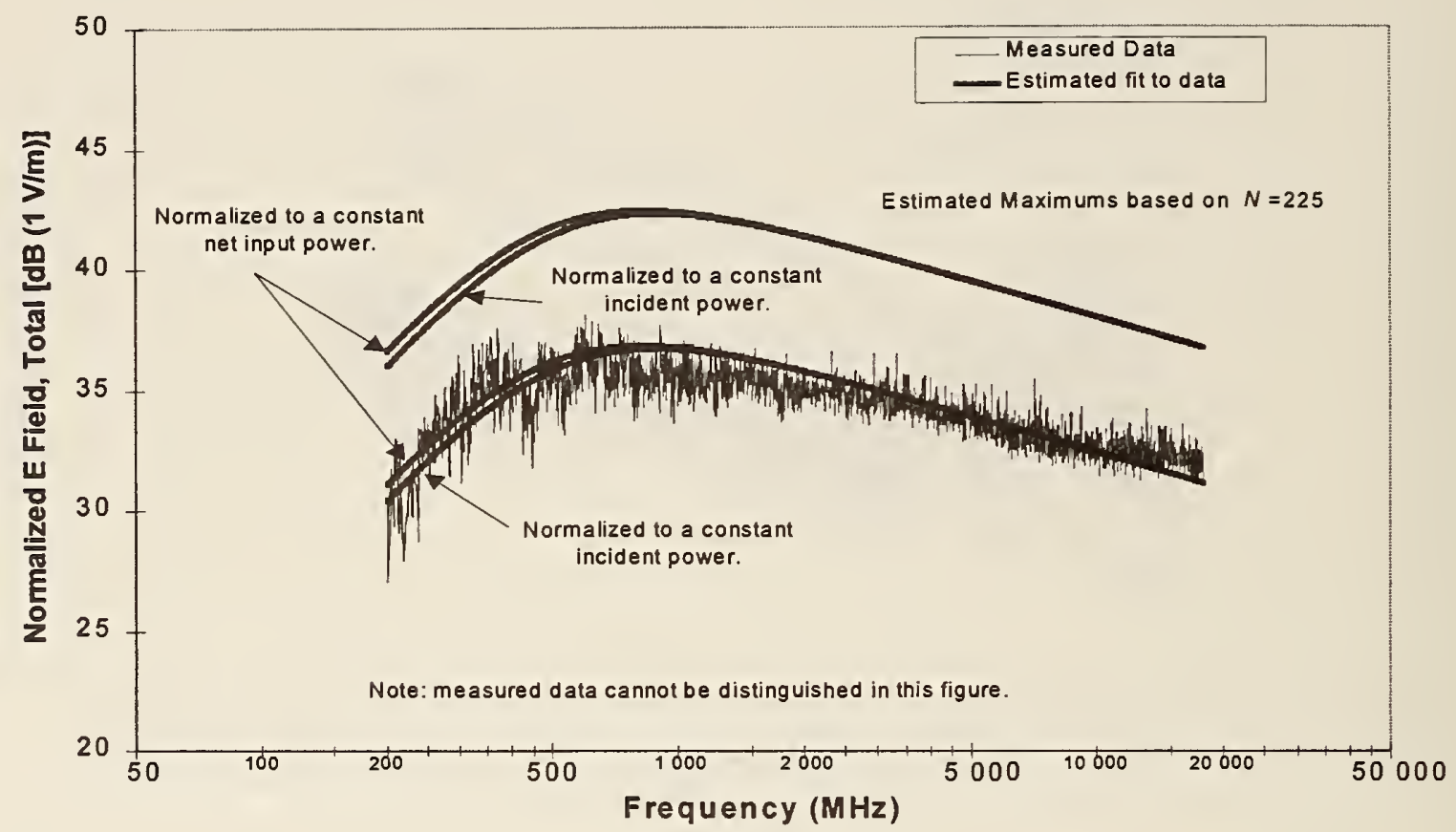

(a)

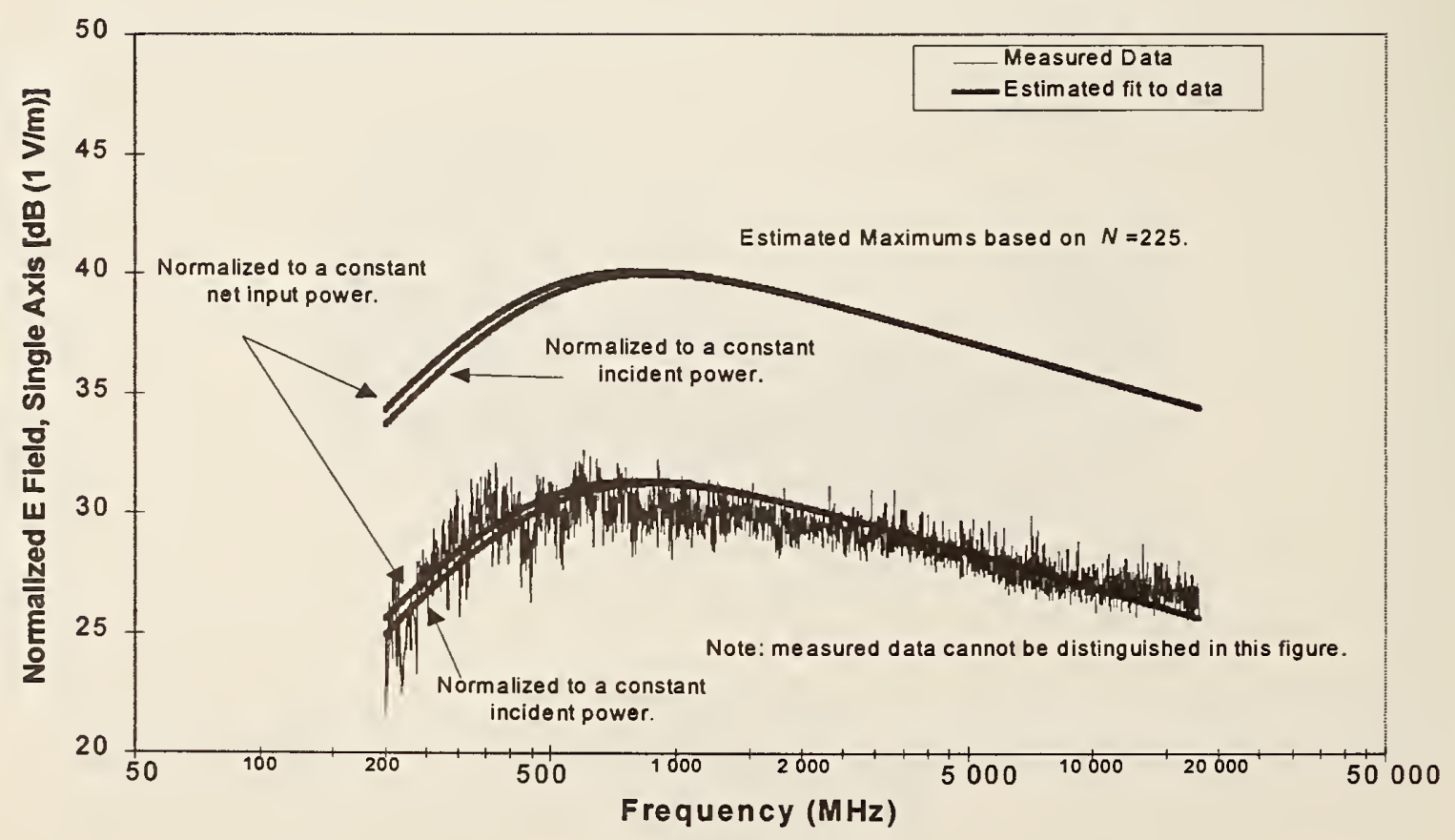

(b)

Figure 121. Calculated average and estimated maximum electric field in chamber $\mathrm{C}$ for a constant input power of $1 \mathrm{~W}$, based on received power from reference antenna. (a) Total electric field. (b) Single-axis electric field. 


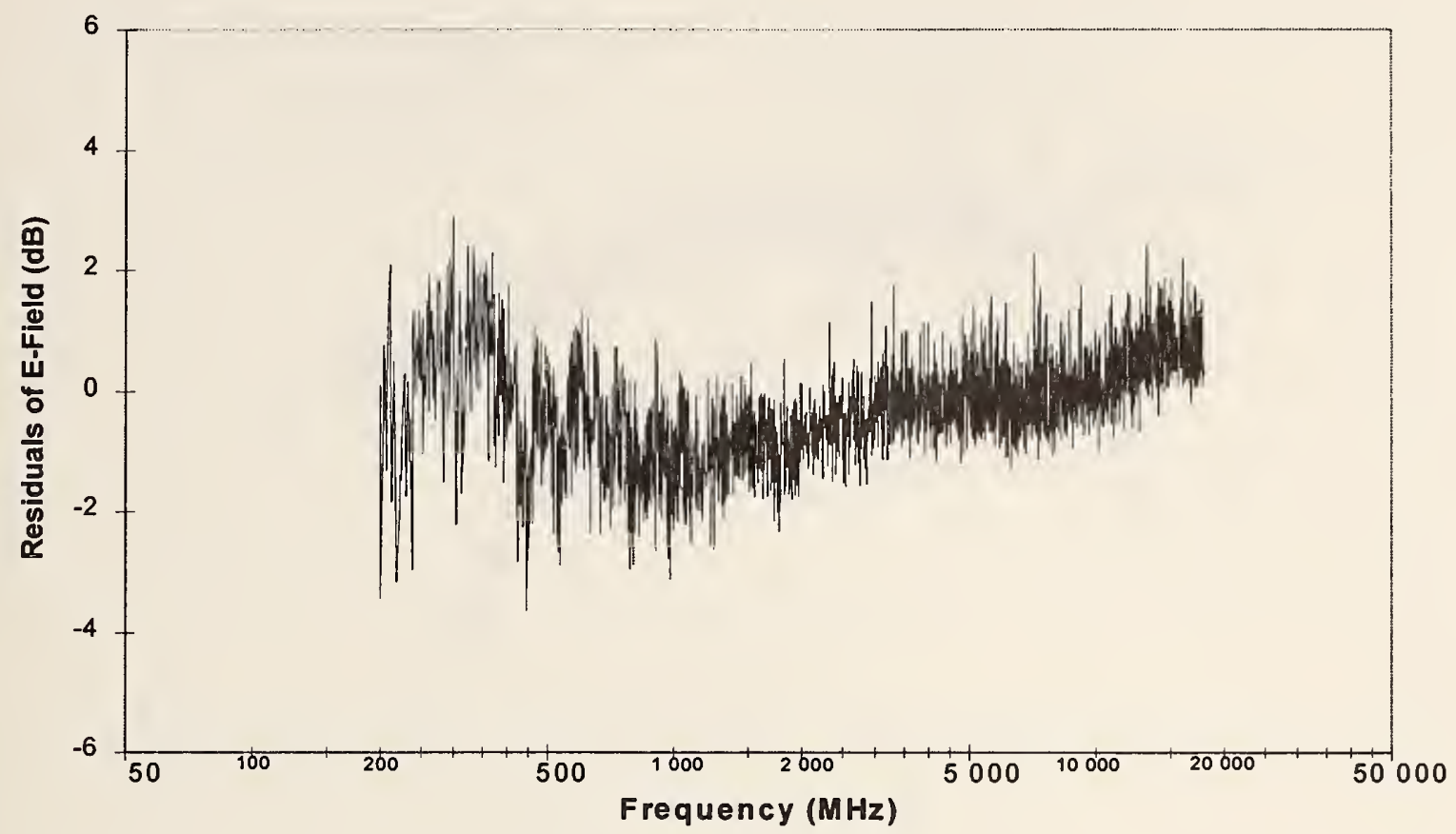

Figure 122. Residuals of calculated average electric field in chamber C, based on received power from reference antenna. 


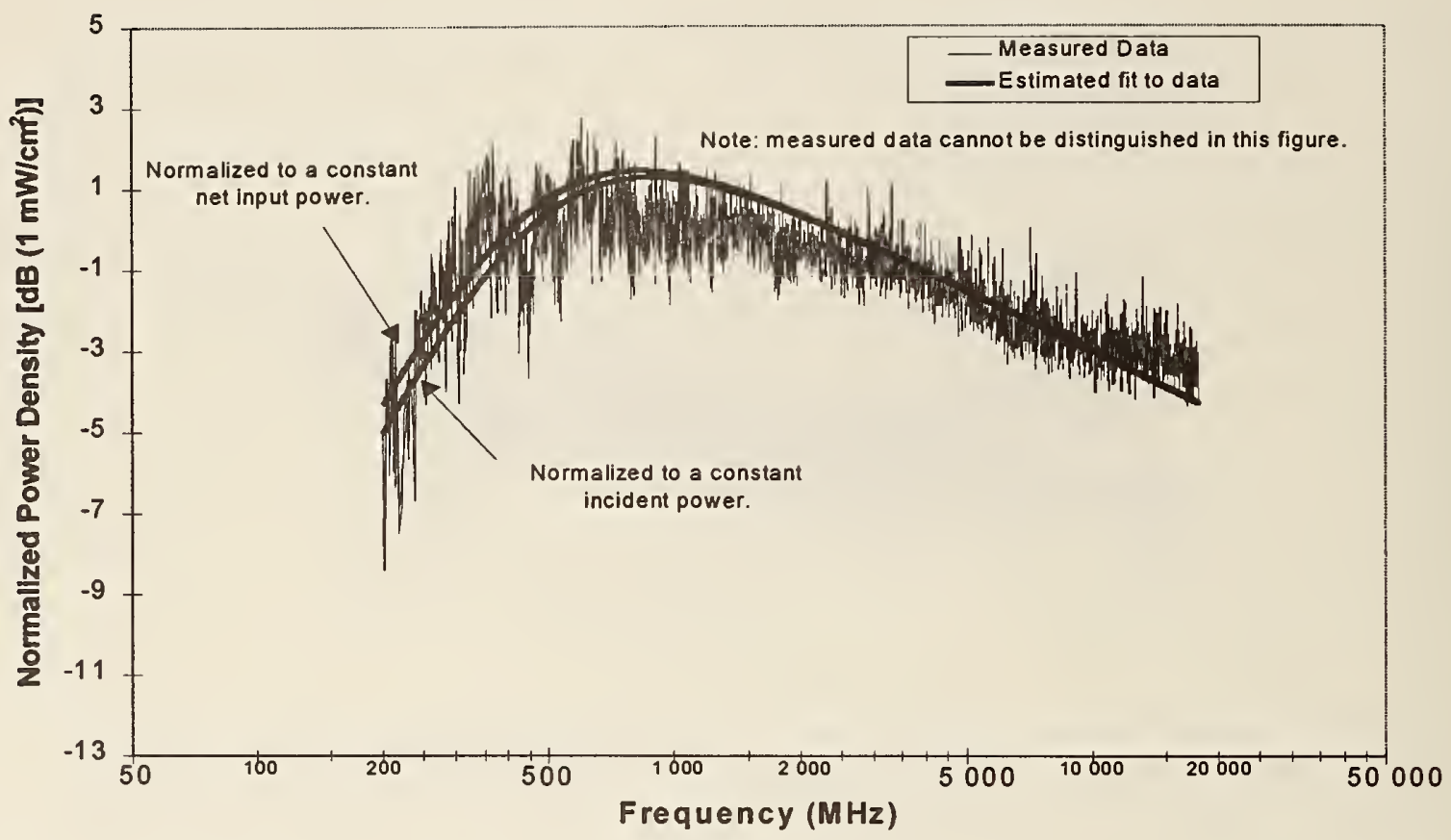

Figure 123. Average power density in chamber $\mathrm{C}$ for a constant input power of $1 \mathrm{~W}$. 


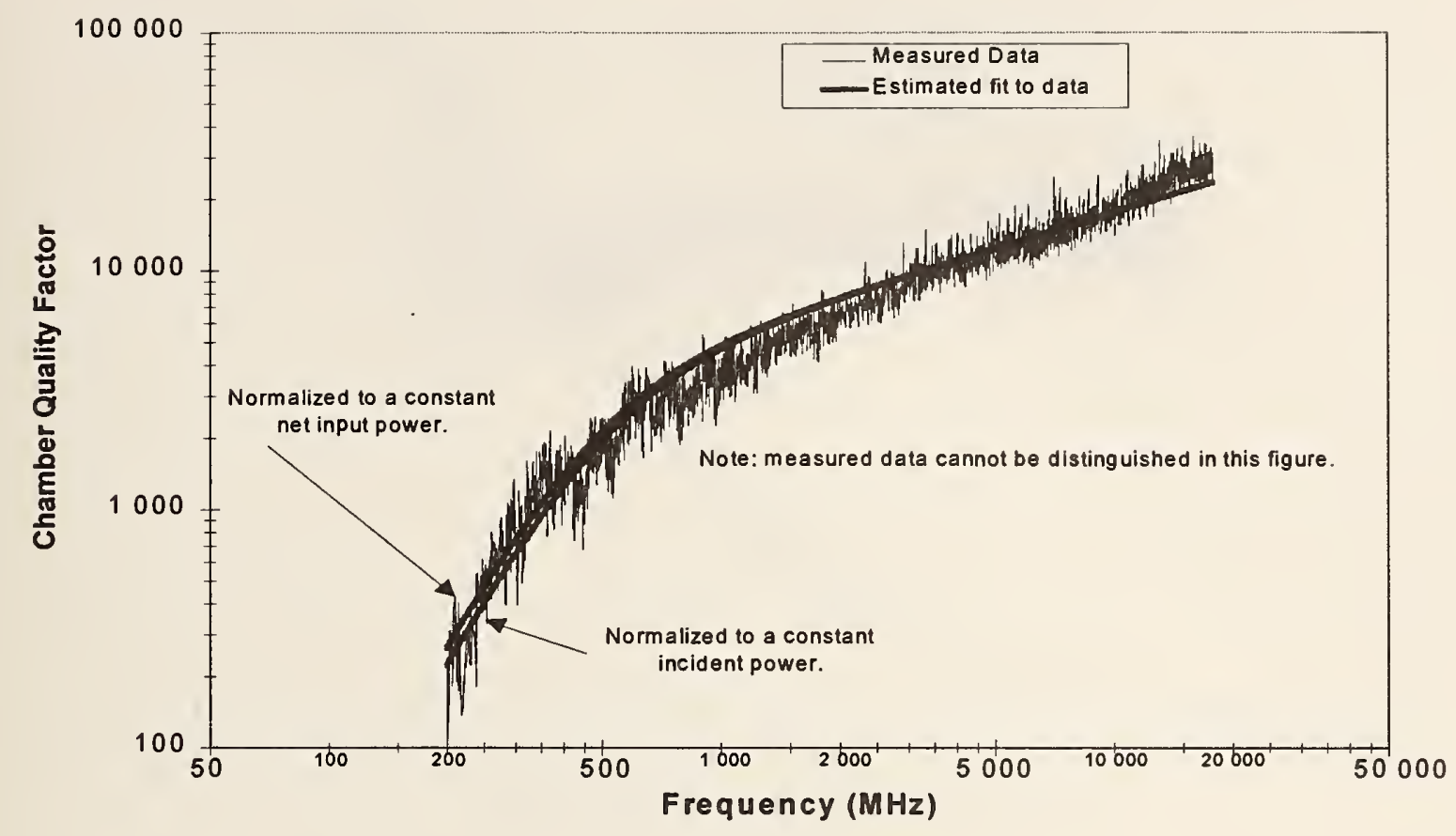

Figure 124. Average quality factor in chamber C. 


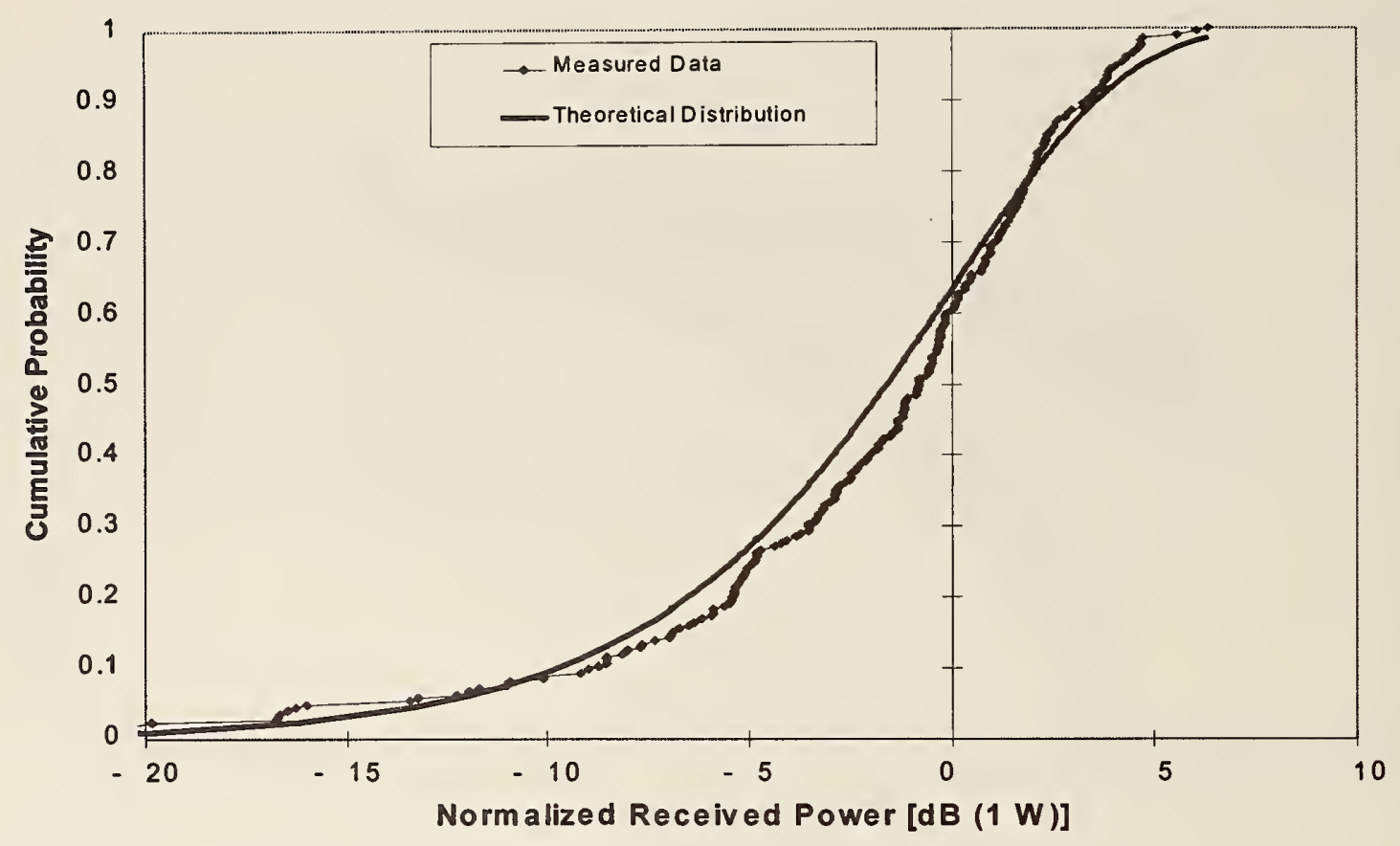

Figure 125. Cumulative distribution of received power at $1 \mathrm{GHz}$ in chamber $\mathrm{C}$. 


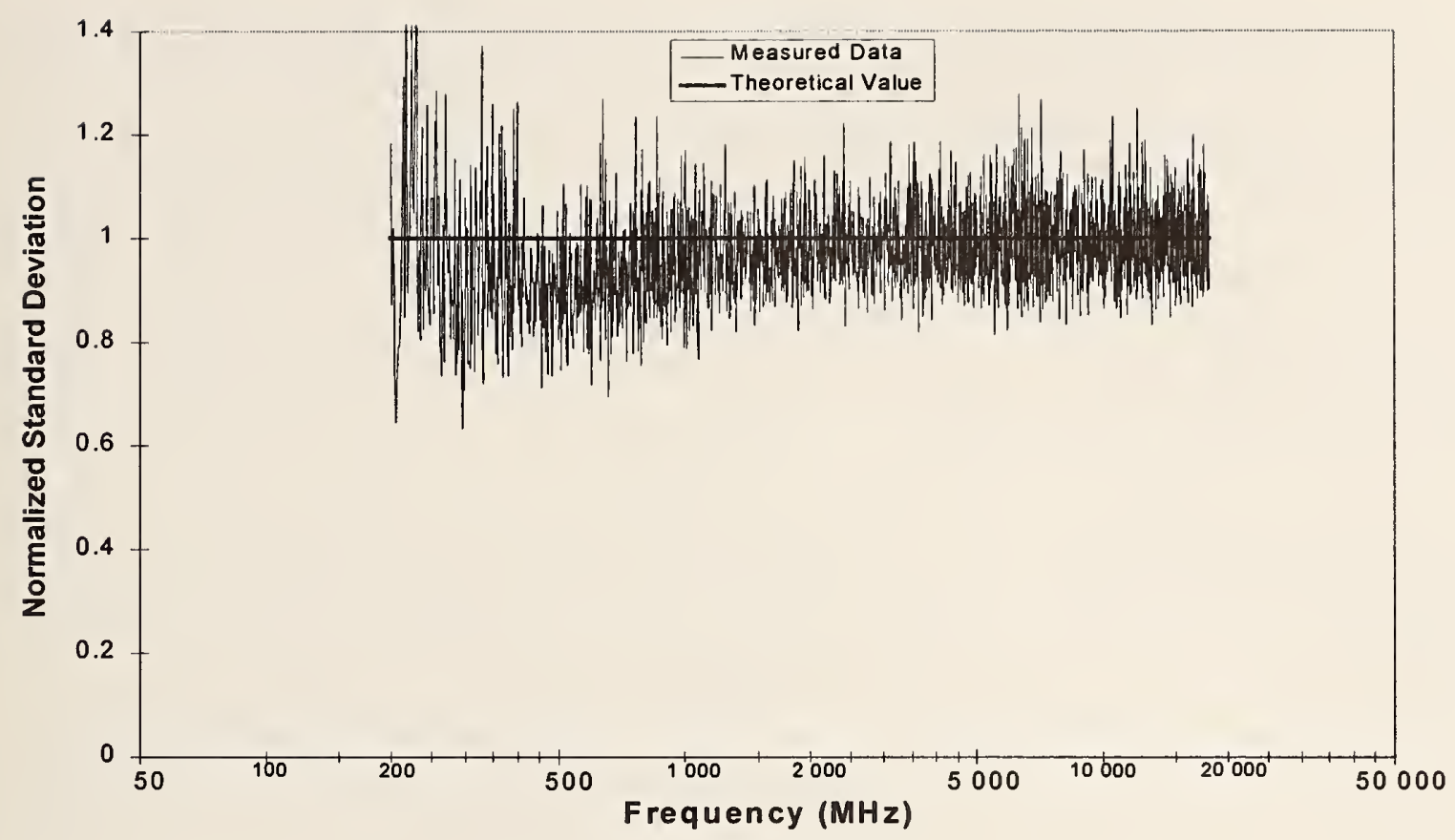

(a)

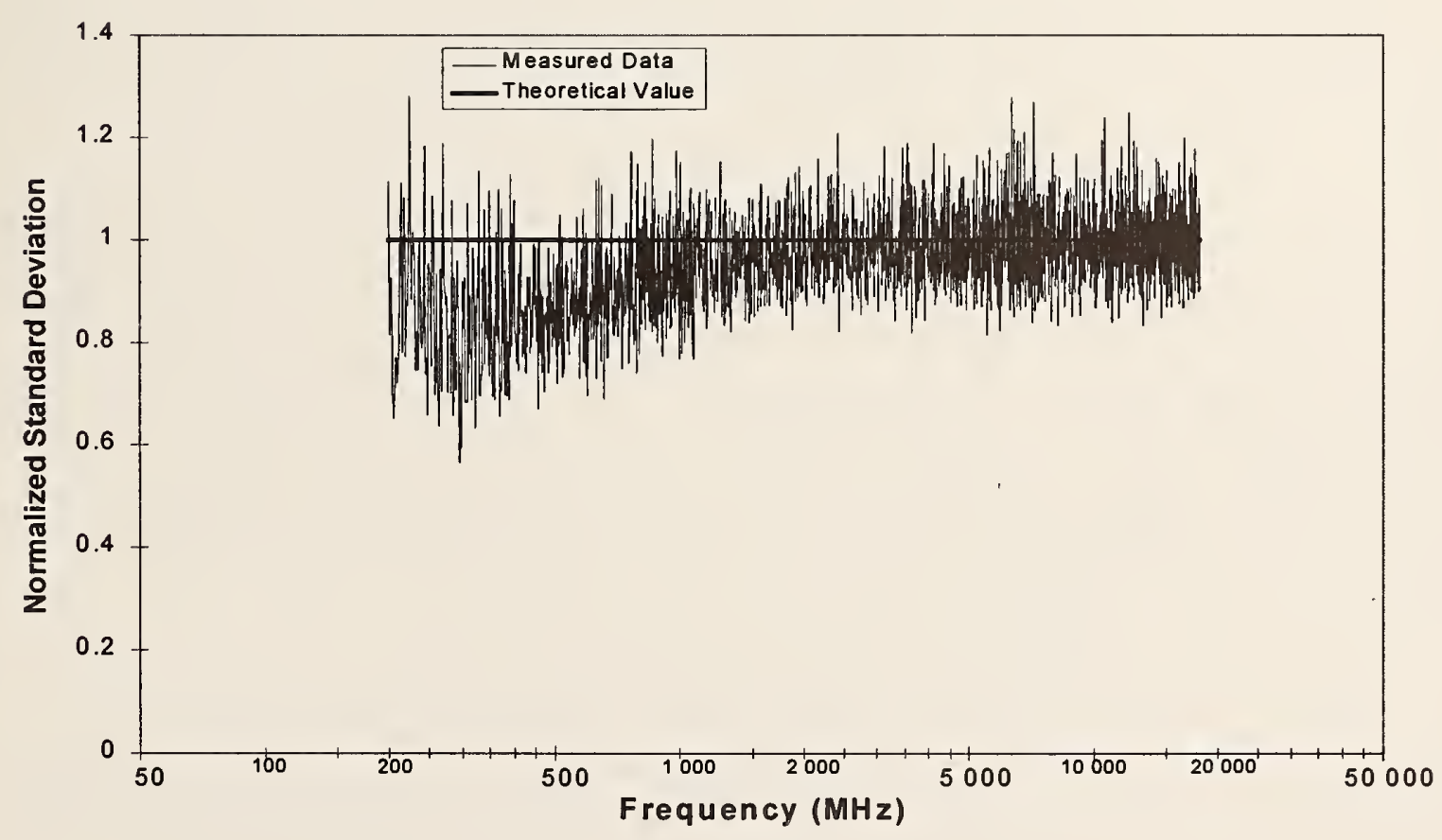

(b)

Figure 126. Normalized standard deviation of received power for a constant input power in chamber C. (a) Constant incident power. (b) Constant net input power. 
The normalized unstirred component of $S_{21}$ given in Figure 115 indicates that the paddle in Chamber $\mathrm{C}$ is less effective than those in Chamber $\mathrm{A}$ or Chamber $\mathrm{B}$. The performance of the paddle is consistent from $300 \mathrm{MHz}$ to $18 \mathrm{GHz}$, but appears to become even less effective below $300 \mathrm{MHz}$. This does not imply that the paddle is bad or that the combination of Chamber $\mathrm{C}$ and the paddle will not function. This merely indicates that the uncertainties will be greater for measurements in Chamber $\mathrm{C}$ than in the other two chambers. The maximum-to-average ratio given in Figure 116 indicates that the idealized statistical model may not accurately describe the power received by an obtrusive antenna for frequencies below $2 \mathrm{GHz}$, and that this effect is more apparent when the data are normalized to a constant net input power than when the data are normalized to a constant incident power.

The plots of chamber gain and residuals of chamber gain given in Figure 119 and Figure 120 show good agreement between the measured data and both the electromagnetic model and the statistical model, although the residuals are less flat than those of Chamber A or Chamber B. This may be due to errors in the estimation of the efficiency or mismatch characteristics of the $\log$-periodic antennas used in the evaluation of Chamber $\mathrm{C}$, or due to less information on the chamber gain at low frequencies, compared to the other two chambers. Another possibility is that, since Chamber $\mathrm{C}$ is smaller than either of the other two chambers, the measurements of the chamber gain at a specific frequency will be noisier in Chamber $\mathrm{C}$ than in the other chambers, and this increased noise could affect the estimates of the parameters $a$ and $b$.

The plot of the cumulative distribution of the received power shown in Figure 125 shows general agreement between the measured data and the ideal distribution, but the match is actually very poor. The factors that may be contributing to these imperfections are the same as in Chamber A. One problem is the unstirred component of $S_{21}$. The normalized unstirred component of $S_{21}$ for the data shown in Figure 125 is 0.4 . This value is small, but large enough to influence the cumulative distribution. Another possibility is that all indications of the validity of the statistical model (maximum-to-average ratio, normalized standard deviation) applied to the power received from a reference antenna show that the model is imperfect below $2 \mathrm{GHz}$, and may also be imperfect at $2 \mathrm{GHz}$. Finally, as discussed in the previous section, it may be possible that the agreement between the measured and theoretical distributions of the data would improve if a larger number of samples were taken. A close examination of the data at the high end of Figure 125 shows a significant gap (greater than $1 \mathrm{~dB}$ ) between the highest three measured values of received power and the next highest value. This type of gap is indicative of an insufficient number of samples.

Figure 126 shows the normalized standard deviation of the power received from the reference antenna, calculated as the standard deviation of the received power divided by the average received power. This ratio is close to the expected value of 1 for frequencies greater than $1 \mathrm{GHz}$; and below $1 \mathrm{GHz}$, it falls below 1 . This effect is more pronounced when the received power is normalized to a constant net input power (Figure 126b) than when the data are normalized to a constant incident power (Figure 126a). The normalized standard deviation indicates that the power received by the reference antenna is not well described by the statistical model below $1 \mathrm{GHz}$. This is most likely caused by the same clipping effect that causes the maximum-to-average ratio to decrease at lower frequencies. 


\subsection{Comparison to NIST Chamber}

We also characterized the NIST chamber with the VNA system, using the same software and instrumentation that was used to characterize the NASA chambers. We did this both to give ourselves a point of reference and to verify the operation of the test method. We will include only a small subset of the data measured in the NIST chamber.

Our initial results were surprising, and many of the surprises can be demonstrated in plots of the average and maximum chamber gain and the respective residual as measured in the NIST chamber. The average and maximum chamber gain are plotted in Figure 127, and the residuals are plotted in Figure 128. Based on the measurements of chamber gain, we estimated parameters of $a=1.768$ and $b=0.804 \cdot 10^{-21}$ for data normalized to a constant incident power, and values of $a=1.143$ and $b=0.799 \cdot 10^{-21}$ for data normalized to a constant net input power. The values of $a$ are close to the expected values of $a=2$ for data normalized to a constant incident power, and $a=1$ for data normalized to a constant net input power. The first surprise is that these values are significantly smaller than those used to describe the characteristics of any of the NASA chambers. The second surprise is that, once we applied corrections for the mismatch and efficiency effects of the antennas used in the measurement, our estimates for the maximum chamber gain were substantially greater than $0 \mathrm{~dB}$. This indicates a problem with our correction factors. Finally, when we compared the residuals of the average chamber gain in the NIST chamber with the same parameter calculated based on data from each of the NASA chambers, the data from the NASA chambers was substantially better behaved, with an apparent range of \pm 1 $\mathrm{dB}$, compared to a range of $\pm 2 \mathrm{~dB}$ for data in the NIST chamber. This broad range in the residuals of the chamber gain suggests an inefficient paddle. If we examine the normalized unstirred component of $S_{21}$ shown in Figure 129b, we see that these values are significantly greater than any of the values observed in the NASA chambers. Even the worst of the NASA chambers, Chamber $\mathrm{C}$, has a normalized unstirred component that is always less than 1.5 and only occasionally greater than 1 for frequencies greater than $300 \mathrm{MHz}$. This compares with a normalized unstirred component in the NIST chamber that is often greater than 1.5. Thus, all indicators showed that all of the NASA chamber were superior to the NIST chamber, with the exception that the NIST chamber had a smaller estimated value for the parameter $a$, which implies that the NASA chambers are lossier and less efficient at low frequencies. The greater loss may be an advantage, however, because low losses cause the statistical model to become invalid at low frequencies when applied to the power received by a reference antenna. Thus higher losses should improve the agreement between the statistical model and the measured data.

Given all of the problems with the data measured in the NIST chamber, we decided to modify the original paddle in the NIST chamber. Since we did so simply to prove to ourselves that the small size of the paddle caused the problems, we made a very simple modification to our paddle: we taped metal rods to the paddle to increase the length from $1.8 \mathrm{~m}$ to 2.7 , and then taped aluminum foil to the rods to increase the overall size of the paddles. Initially we thought that the increased size of the paddle would improve the low frequency response of the chamber but would have negligible effect at high frequencies. This is because several references on the effects of paddle size and shape $[3,28]$ suggested that the only requirement that a paddle must meet is 
that it must be electrically large. This is clearly the case for the original paddle in the NIST chamber for high frequencies, since a $1.8 \mathrm{~m}$ paddle is 6 wavelengths at $1 \mathrm{GHz}$.

The actual average and maximum chamber gain measured in the NIST chamber using the modified paddle are presented in Figure 130. Under these conditions, the parameters for the chamber gain are estimated as $a=2.357$ and $b=0.853 \cdot 10^{-21}$ for data normalized to a constant incident power, and values of $a=1.780$ and $b=0.847 \cdot 10^{-21}$ for data normalized to a constant net input power. These values are larger than those measured with the original paddle, indicating that the chamber gain is slightly lower than previously calculated. This does not mean that the larger paddle decreased the gain of the chamber, although this is possible. Instead, it appears that the smaller paddle caused the chamber gain to have "spikes" at a large number of frequencies, but the general shape of the curves is very similar. These spikes caused a bias in the estimates of the parameters $a$ and $b$. Another effect of the larger paddle is a decrease in the estimated maximum chamber gain. Although this value is still greater than $0 \mathrm{~dB}$ for low frequencies, the effect is not as pronounced as it was with the original paddle.

If we compare the residuals of the average chamber gain measured after the paddle was modified, as shown in Figure 131, with those measured before the paddle was modified and shown in Figure 128, we see a significant improvement in the span of the residuals, and the span is now similar to that observed in the NASA chambers. The residuals show an improvement even at high frequencies, indicating that the size of the paddle may be more important than previously reported.

The increase in the size of the paddle also resulted in an improvement in the normalized unstirred component of $S_{21}$, as shown in Figure 132b. The improvement is greater above $1 \mathrm{GHz}$. This is most likely caused by the fact that we used large log-periodic antennas below $1 \mathrm{GHz}$, and it was difficult to separate them adequately. Also, since the size of the paddle was increased, neither of the antennas could be oriented with a vertical polarization. This resulted in strong direct coupling between the antennas. At higher frequencies, ridged-horn antennas were used, and these could easily be placed in different locations and polarizations, resulting in improved performance.

Finally, we present the average quality factor of the NIST chamber, measured using the modified paddle, in. The estimated volume used to calculate this quality factor is $38.19 \mathrm{~m}^{3}$. Figure 133 is included for comparison with the other chambers. Unfortunately, because of the difference in the volume of the chambers, it is difficult to compare the chambers directly, and we do not attempt to do so here. We simply include this figure for reference purposes only. 


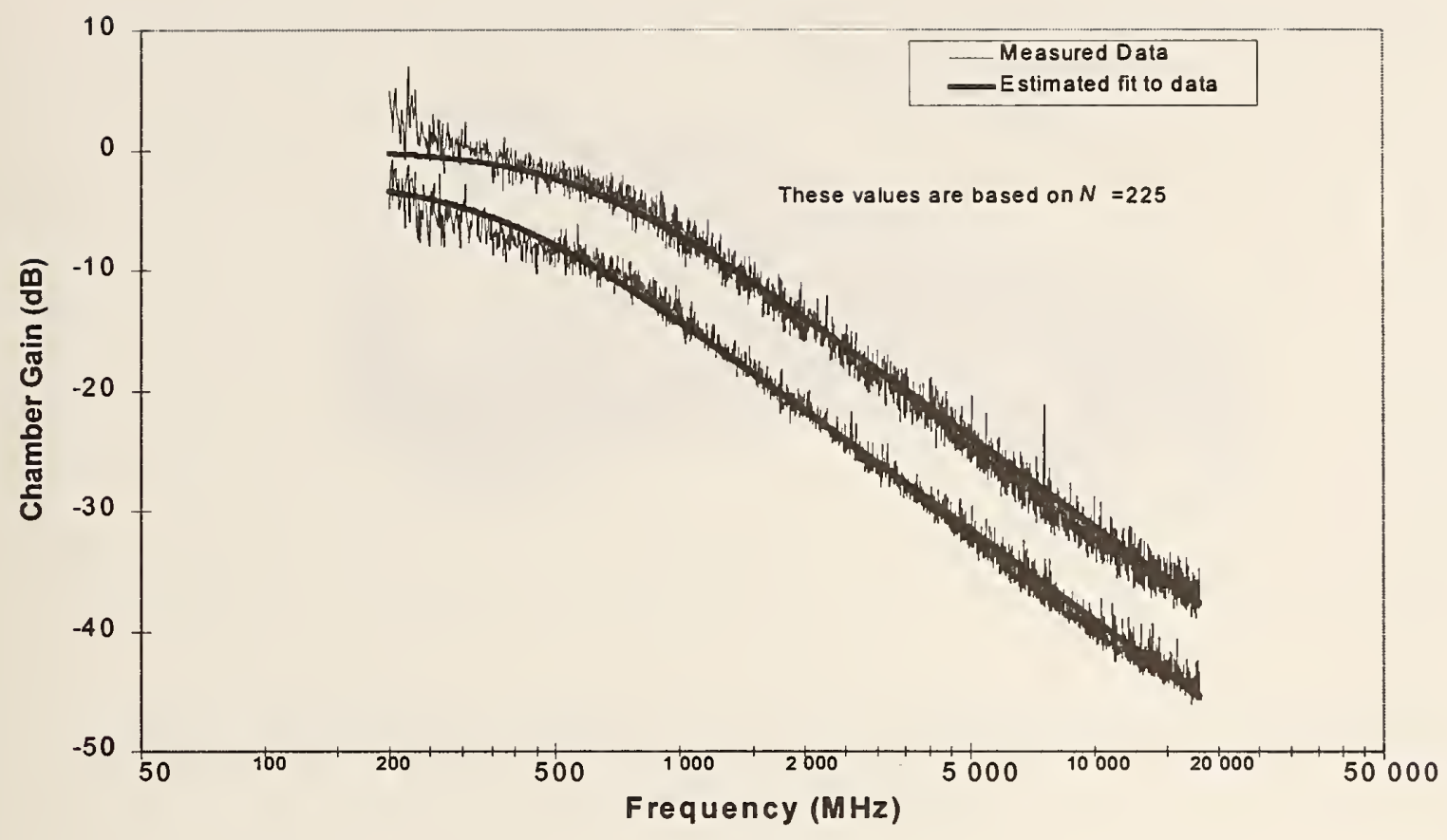

(a)

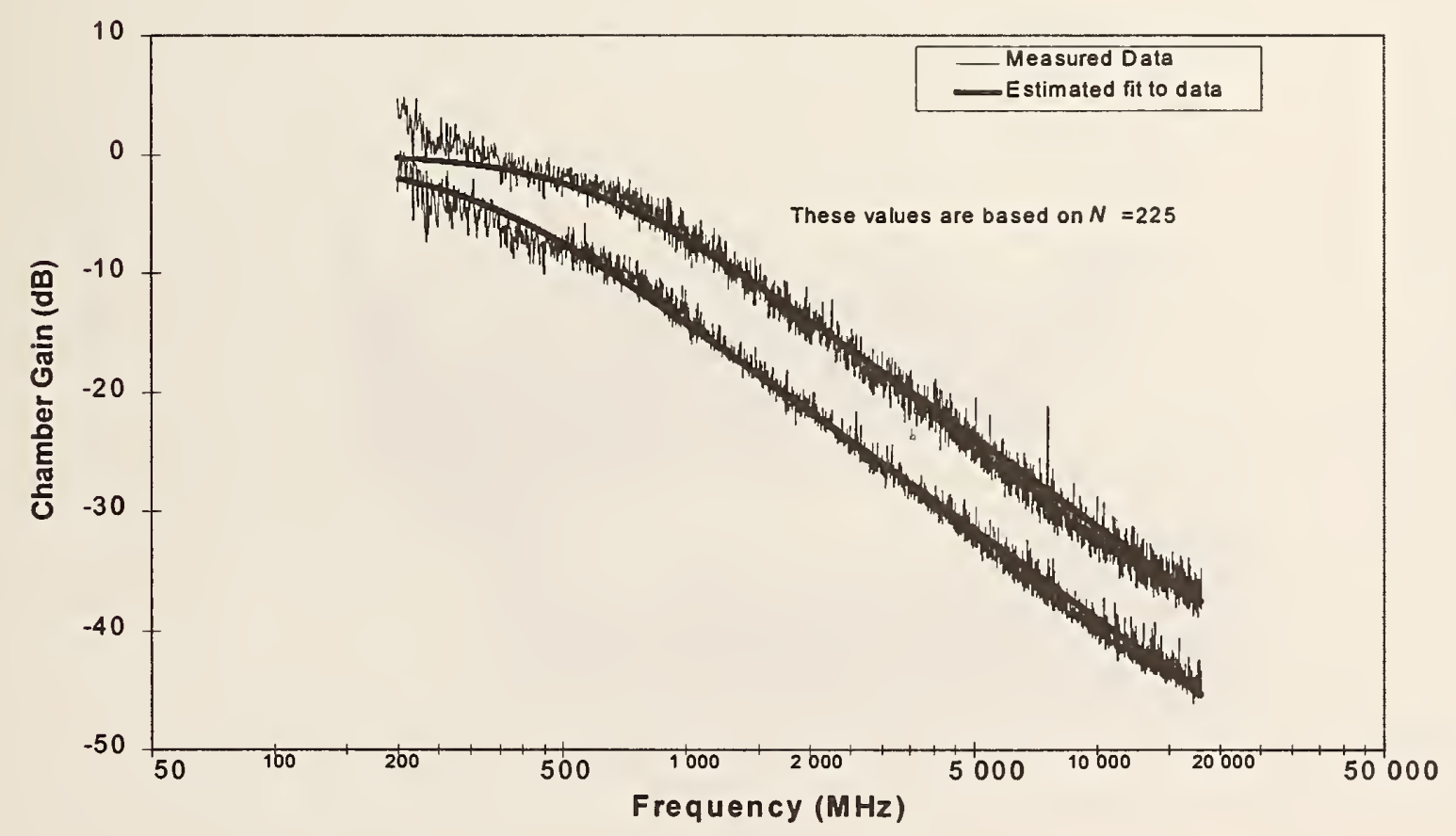

(b)

Figure 127. Original average and maximum chamber gain in NIST chamber. (a) Constant incident power. (b) Constant net input power. 


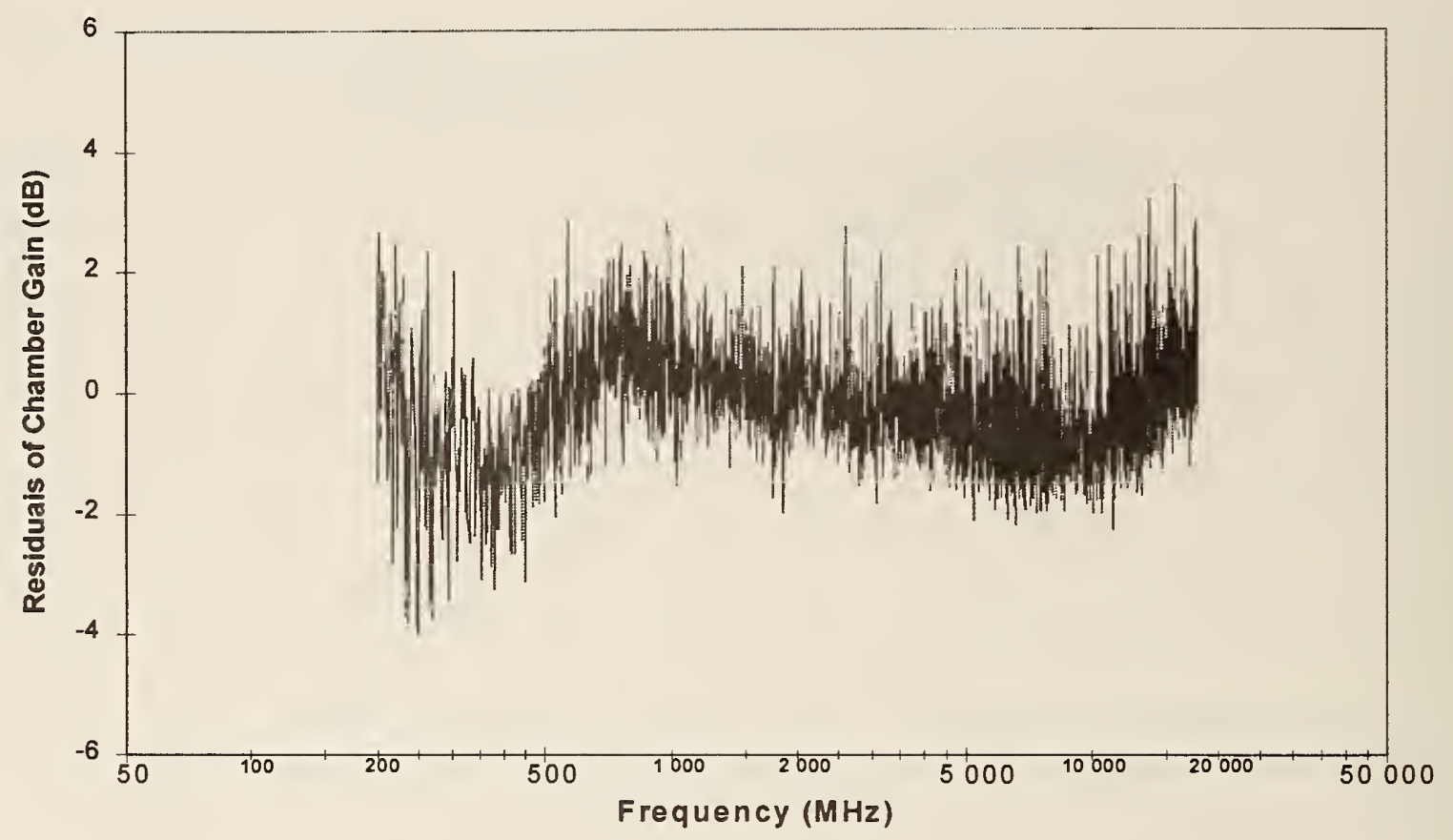

(a)

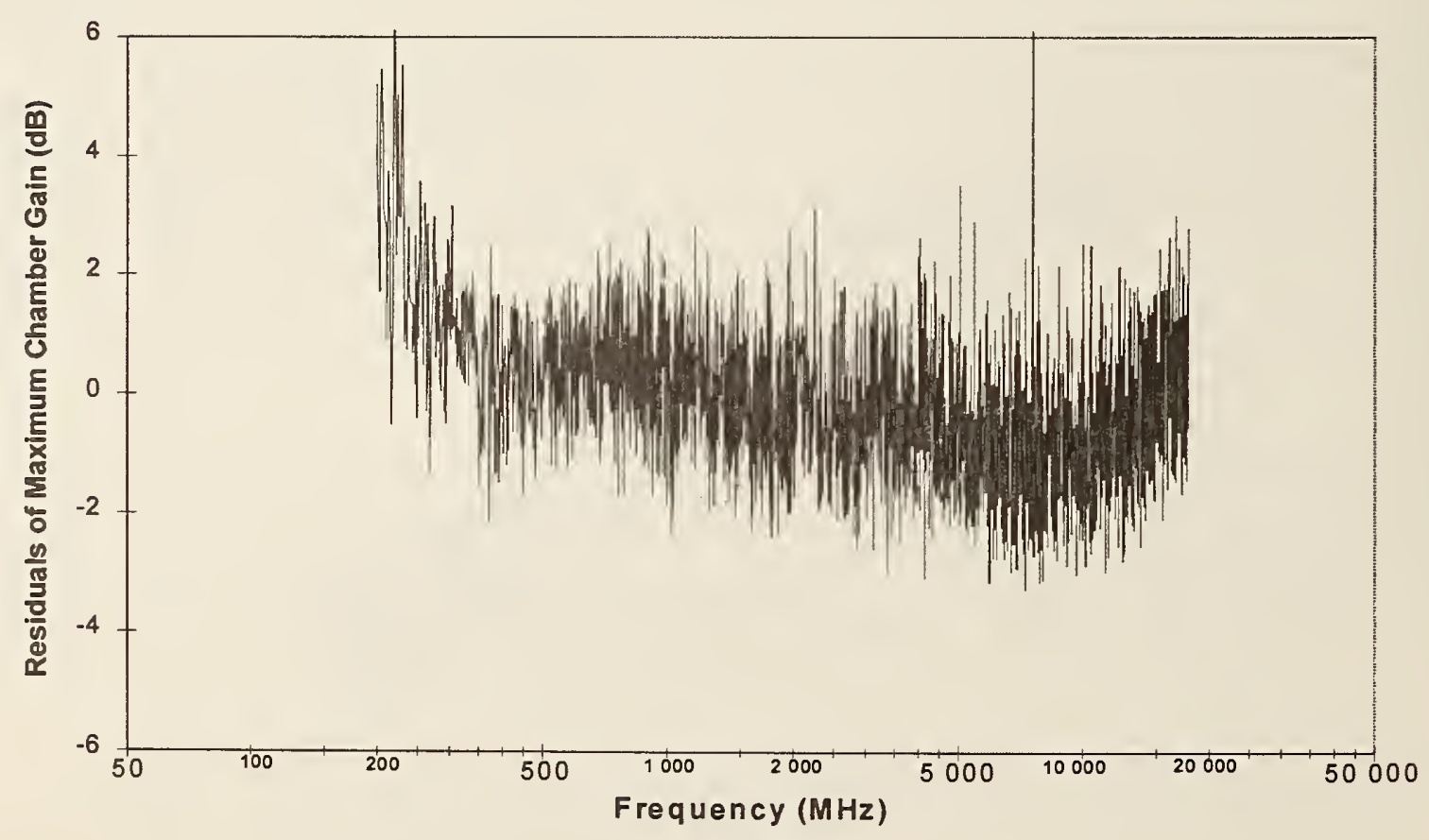

(b)

Figure 128. Residuals of chamber gain in NIST chamber using original paddle. (a) Residuals of average chamber gain. (b) Residuals of maximum chamber gain. 


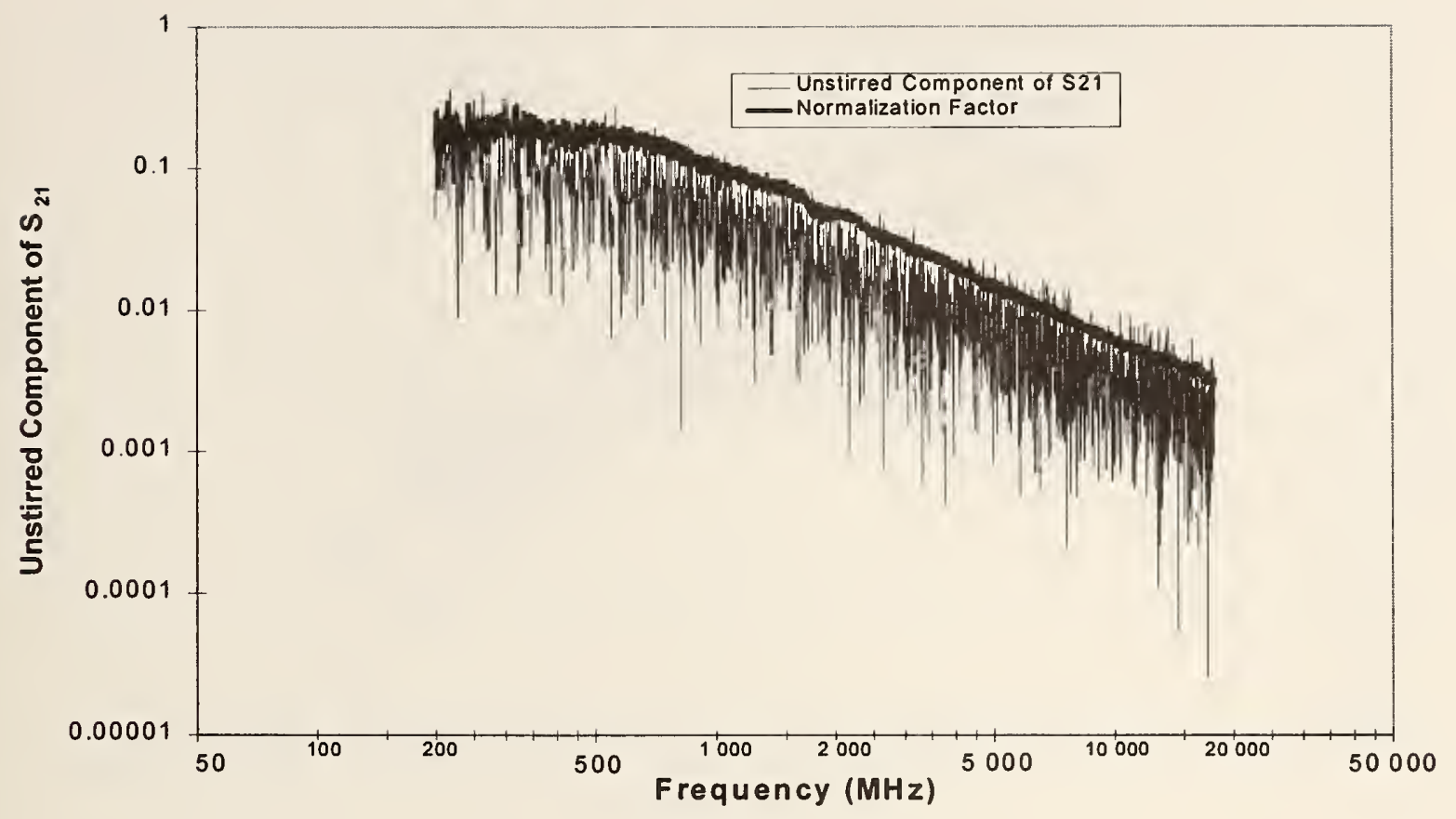

(a)

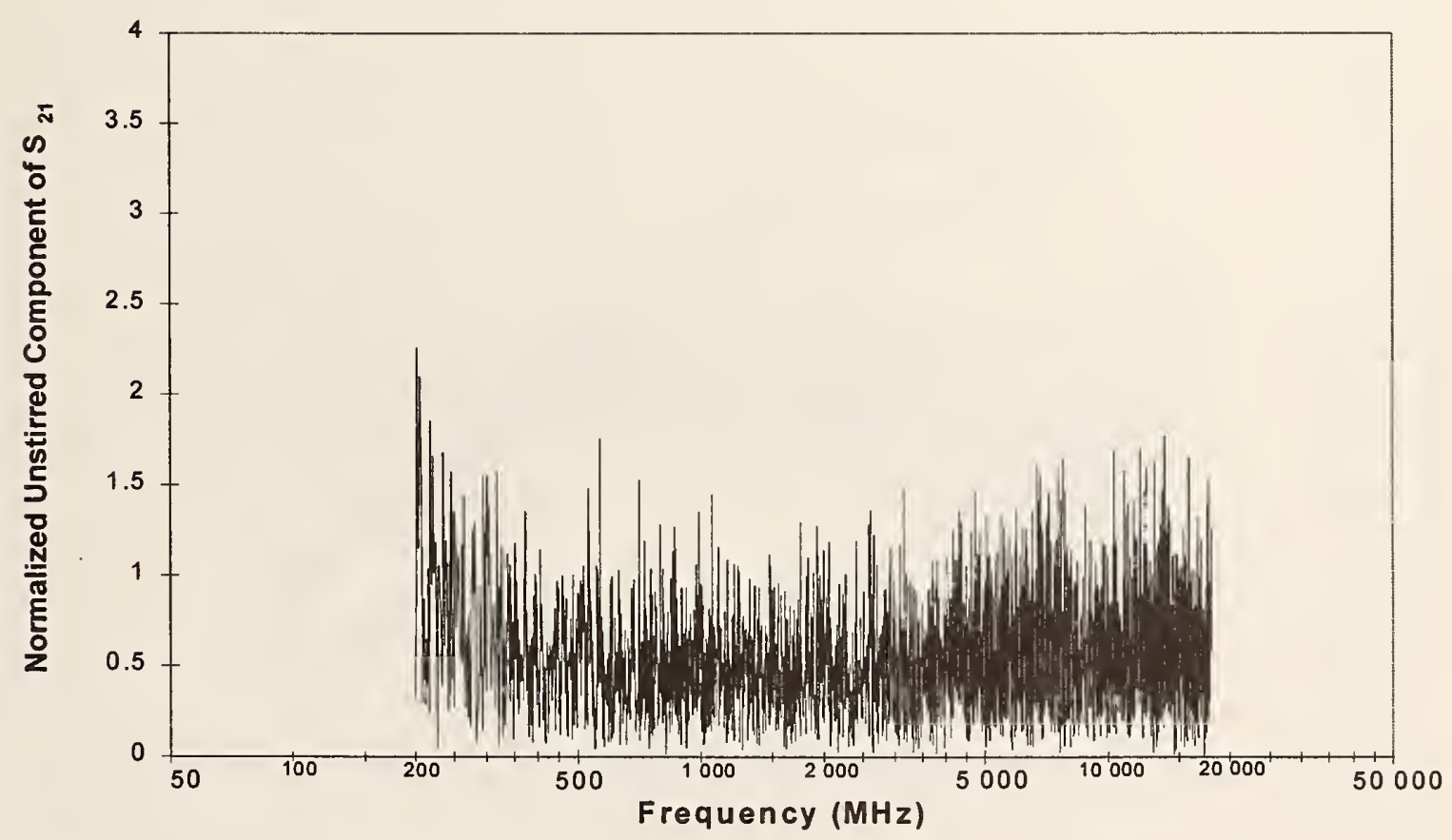

(b)

Figure 129. Evaluation of original mechanical stirrer in NIST chamber. (a) Unstirred component of $S_{21}$. (b) Normalized unstirred component of $S_{21}$. 


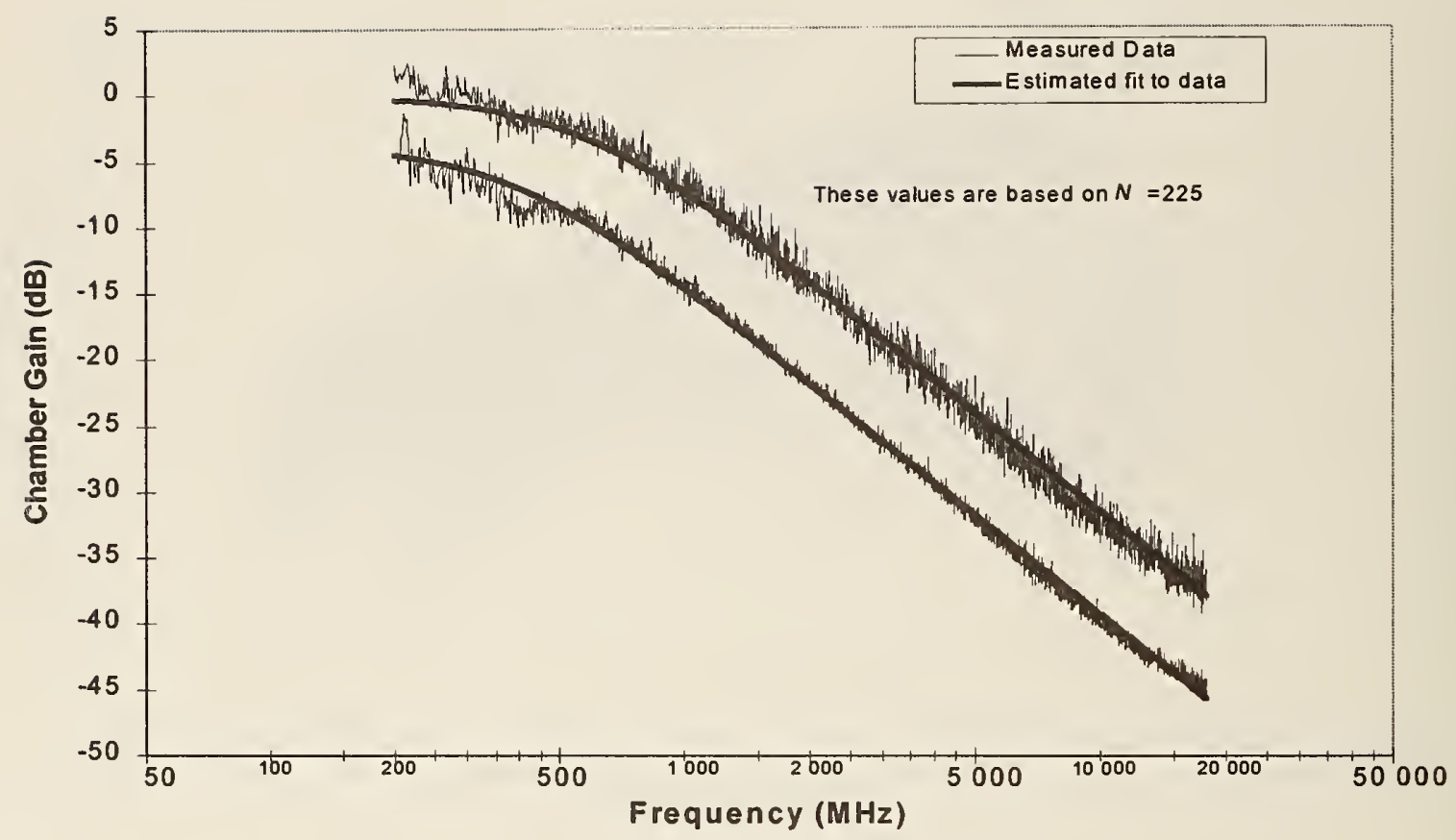

(a)

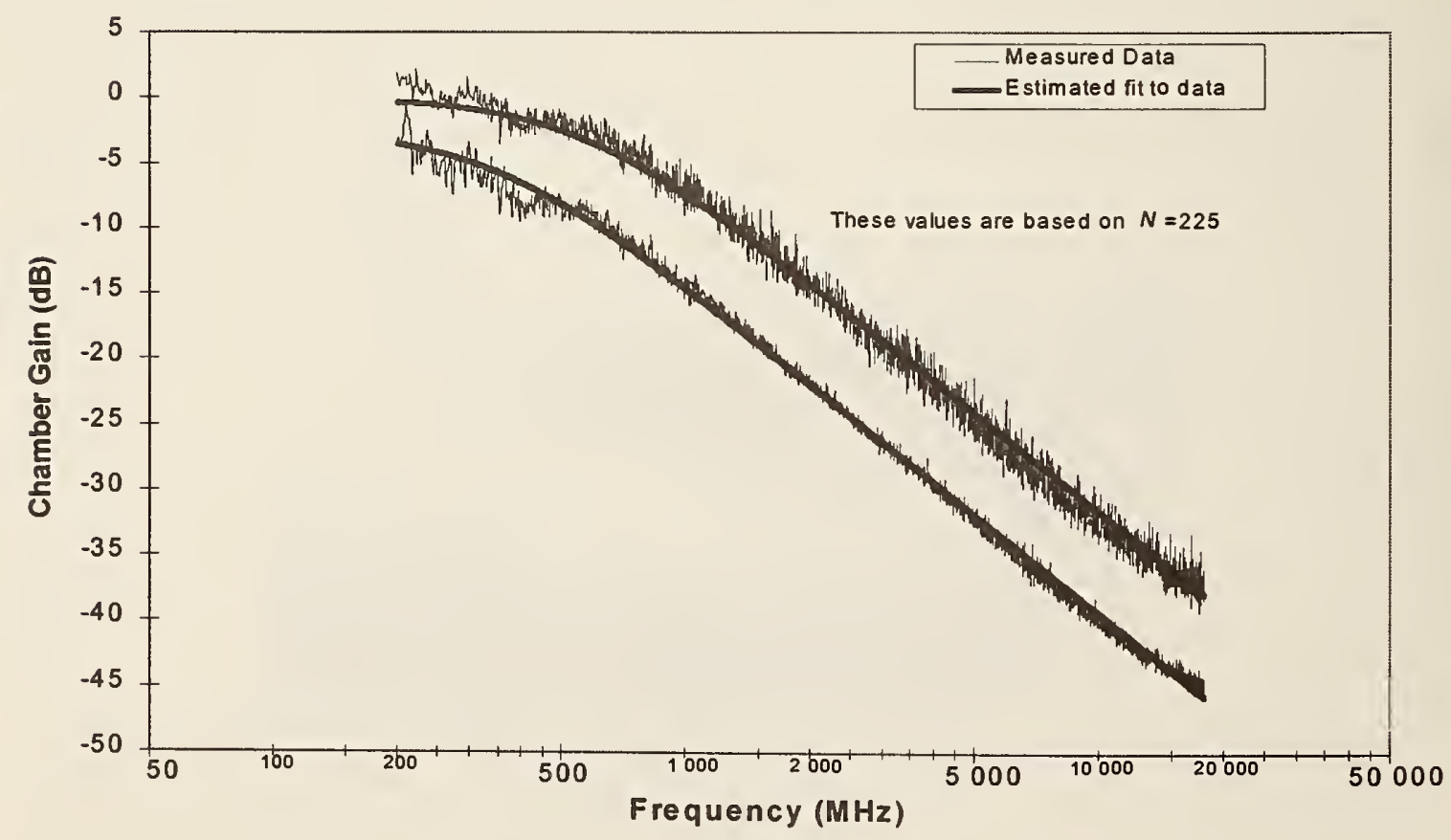

(b)

Figure 130. Average and maximum chamber gain in NIST chamber after paddle was modified. (a) Constant incident power. (b) Constant net input power. 


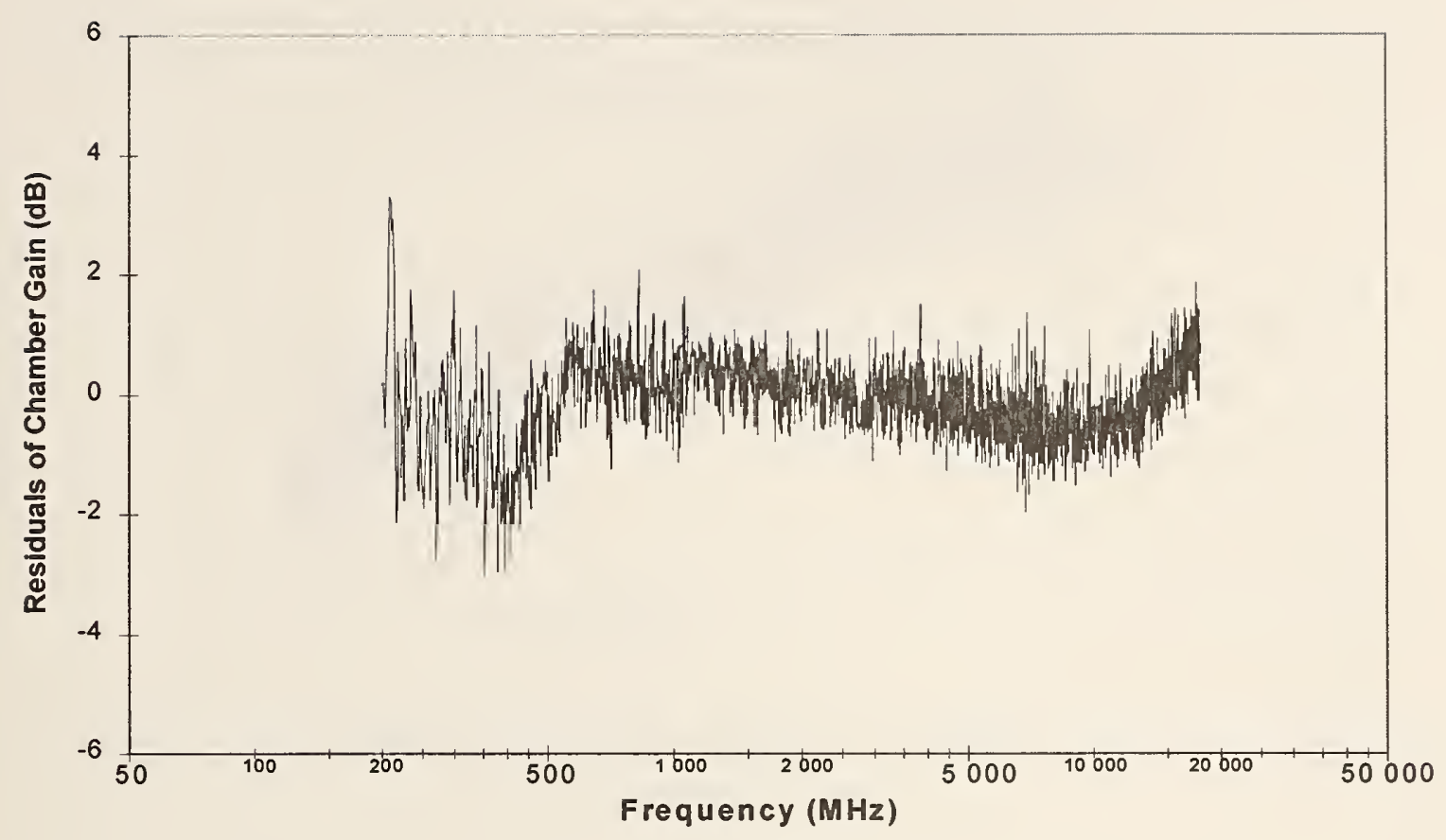

(a)

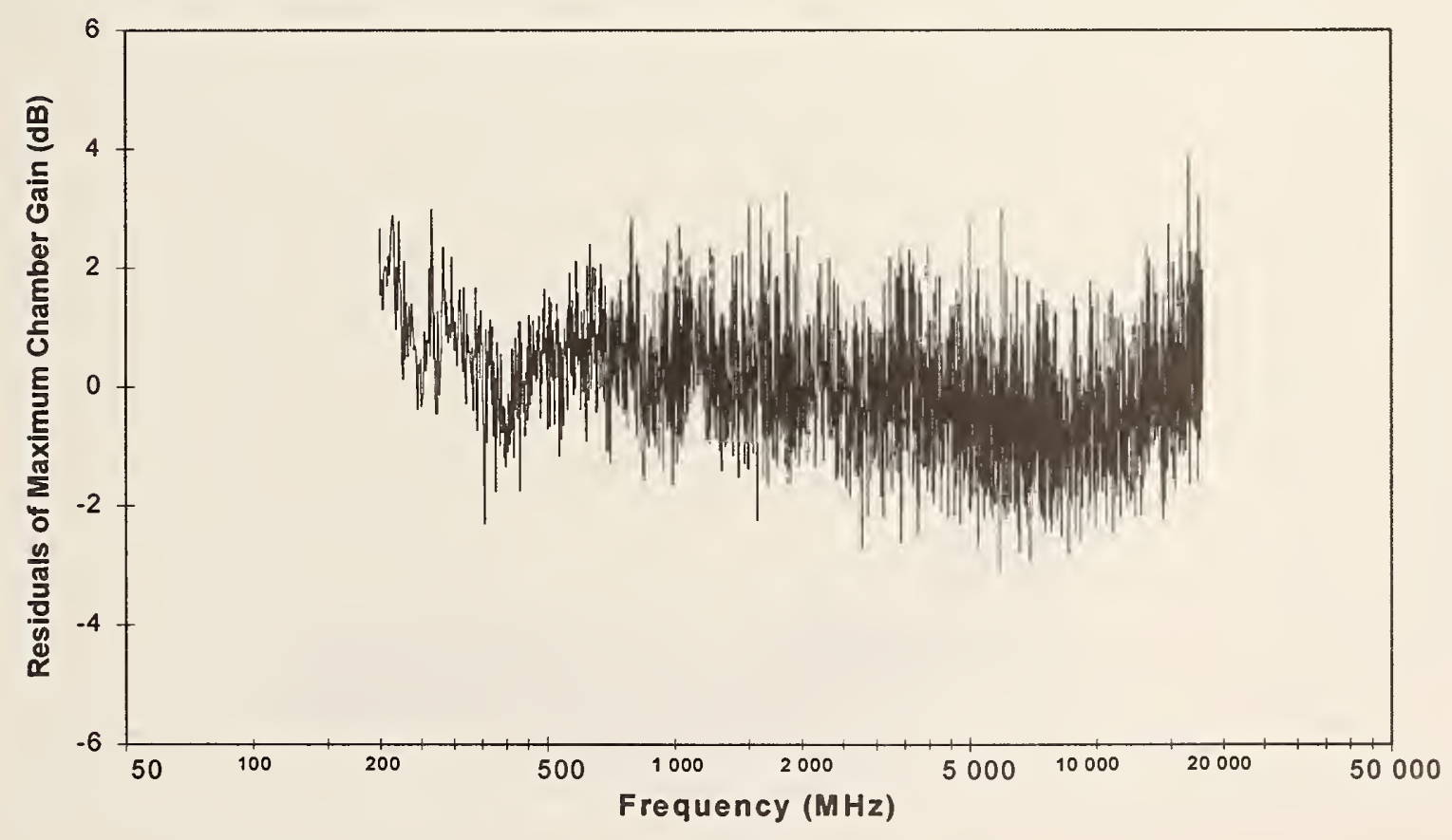

(b)

Figure 131. Residuals of chamber gain in NIST chamber after paddle was modified.

(a) Residuals of average chamber gain. (b) Residuals of maximum chamber gain. 


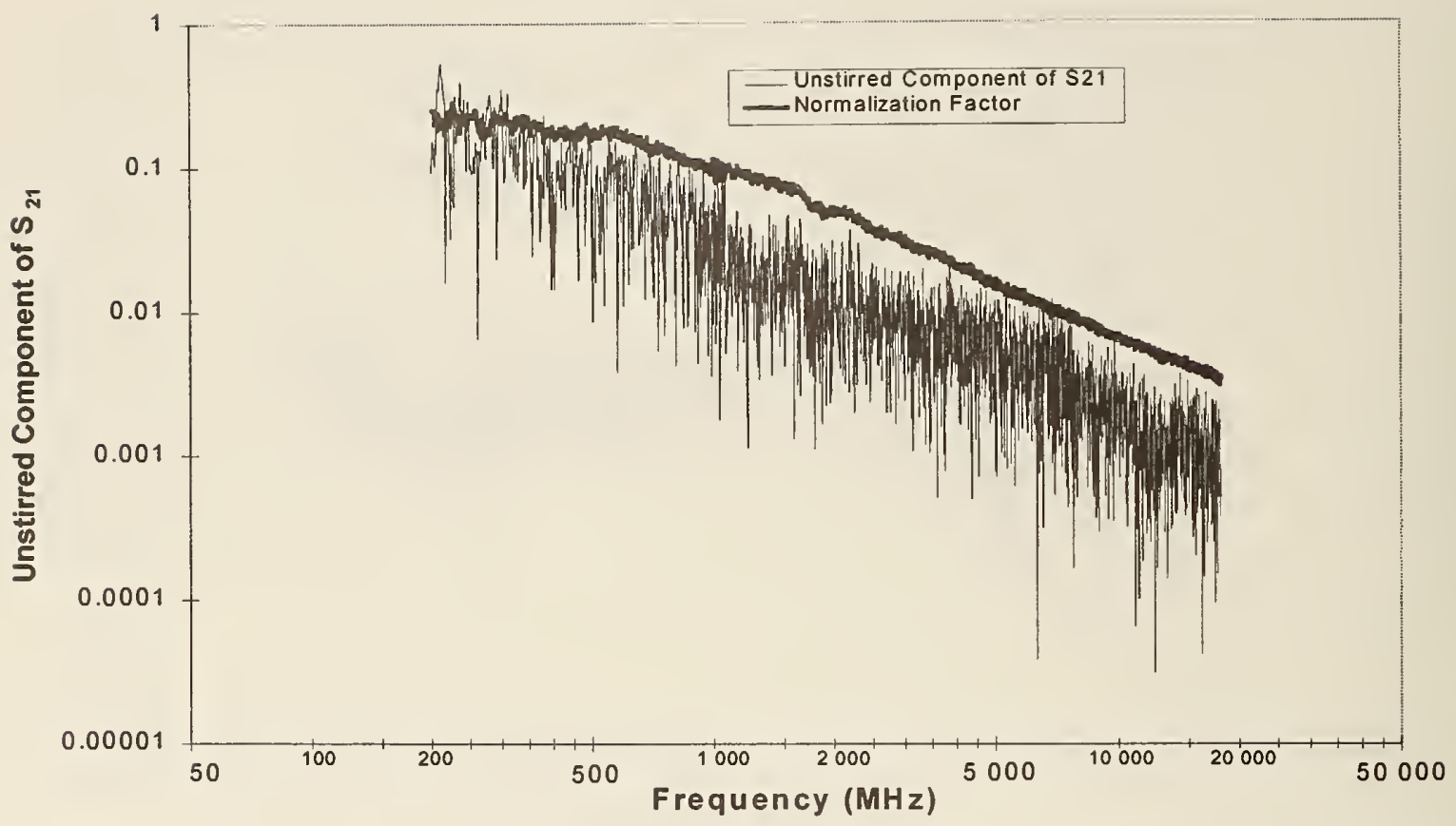

(a)

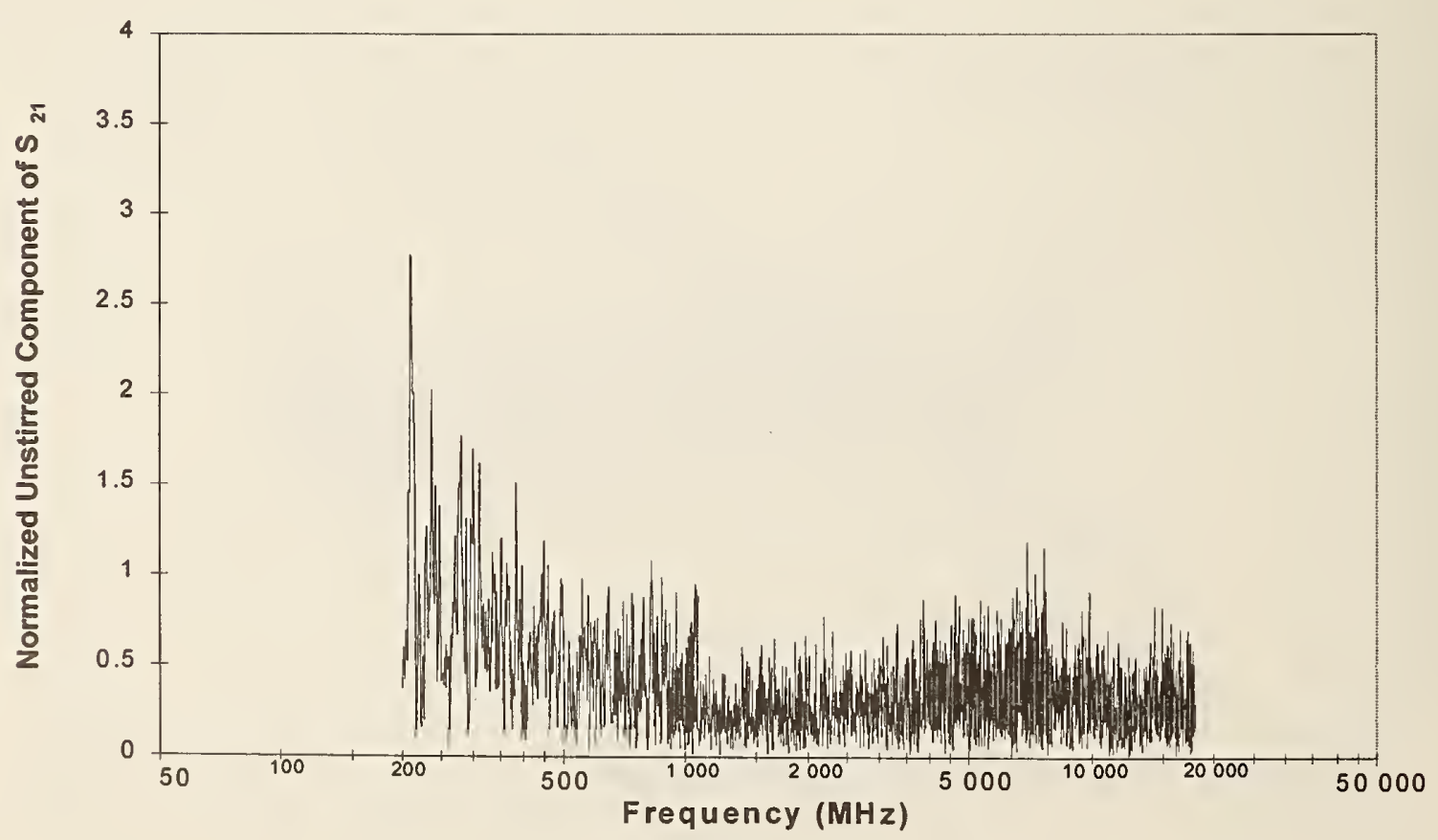

(b)

Figure 132. Evaluation of modified mechanical stirrer in NIST chamber. (a) Unstirred component of $S_{21}$. (b) Normalized unstirred component of $S_{21}$. 


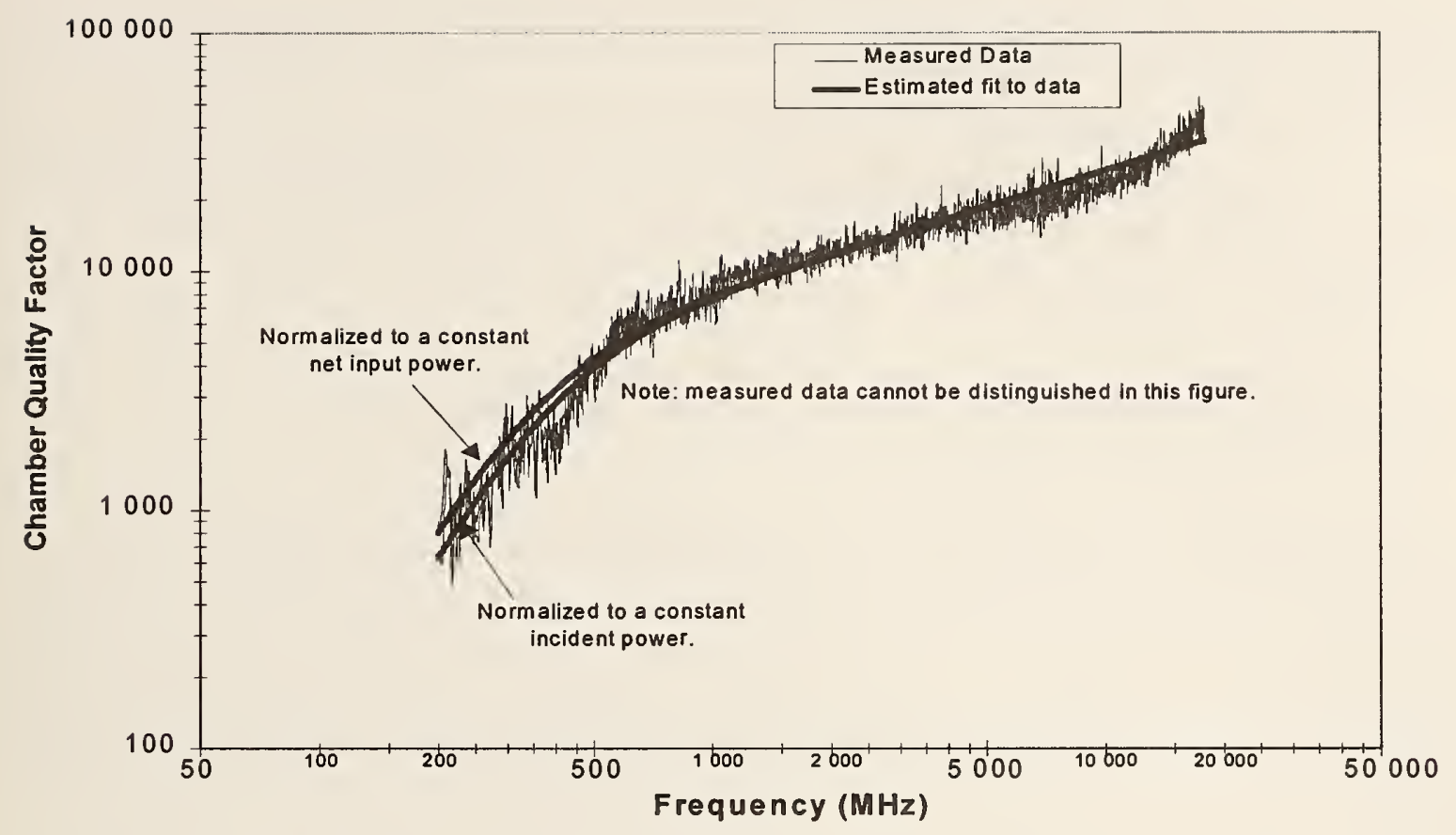

Figure 133. Average quality factor in NIST chamber after paddle was modified. 


\section{Measurement Uncertainties}

The uncertainty in our measurements consists of three individual components, and these three components are then combined to produce a total combined uncertainty. The three components are:

1. Uncertainty due to the random nature of mode-stirred chamber measurements.

2. Residual, unexplained uncertainty (imperfections in chamber).

3. Instrumentation uncertainty.

Uncertainty due to the random nature of mode-stirred chamber measurements will be present in any measurement performed in such a facility. This is essentially the uncertainty that would be observed if the chamber and the associated instrumentation were perfect. As explained in Section 3.2, every measurement will have an ideal distribution associated with it, and therefore an uncertainty associated with it. In general, we can characterize the distributions, and hence the uncertainty, very well. Therefore, we can estimate the uncertainty of any parameter associated with mode-stirred chamber measurements (received power, electric field (total or rectangular component), etc.) and, for each parameter, we can estimate the uncertainty associated with the average or maximum of each parameter as a function of the number of paddle positions. Unfortunately, since each parameter has a different distribution and since the distribution for the maximum of a distribution is different from the distribution for the average, the associated uncertainties must be addressed separately. These uncertainties have already been evaluated in Section 3.2.6, and will be tabulated for our measurements below in Section 8.2.

No chamber is perfect, and there will be an increase in the total uncertainty due to imperfections. We will group all possible imperfections together and call them collectively nonideal spatial uniformity (a sensor will consistently measure different values depending on where it is placed in the chamber). Non-ideal uniformity can be caused by a variety of factors, such as an electrically small paddle, direct coupling between the transmitting antenna and a sensor, or an incorrect statistical model. The terms uniformity and spatial uniformity have been used in the past [3] to describe the performance of a mode-stirred chamber, but the definition of the terms has always been somewhat imprecise or nonexistent. We will suggest a definition of spatial uniformity in Section 8.3, and then evaluate the uncertainties associated with the uniformity as they relate to our measurements.

In Section 8.5, we will evaluate the uncertainty of our measurement system. We will neglect the effect of instrumentation noise on our measurements since, after averaging the results of measurements taken at a large number of paddle positions, the uncertainty due to noise should be negligible relative to other sources of uncertainty. This means that all remaining components of uncertainty due to instrumentation will be deterministic in nature, regardless of the cause of the uncertainty. For example, if a cable is calibrated with a noisy system, the majority of the uncertainty associated with the calibration can be caused by noise in the calibration system. Once it has been calibrated, however, the calibration values are fixed and used throughout all remaining measurements. Thus, even though the source of the uncertainty is noise (random effects), once the calibration values are stored and used, the uncertainty is deterministic, and no 
subsequent averaging during the measurement can remove or reduce the uncertainty due to the noisy calibration.

First, however, before we present our estimated uncertainties, we need to discuss how the uncertainties will be presented.

\subsection{Presentation of Uncertainties and Uncertainties about Uncertainties}

Measurements, specifications, and uncertainties of electromagnetic field parameters are often expressed in decibels, and this is especially true with EMC (electromagnetic compatibility) measurements. As long as the uncertainty of a measurement (as measured by the standard deviation) is small relative to the mean, this poses no significant problems. If the uncertainties are large, however, things can get confusing. To illustrate this, we will present a few different ways to evaluate the uncertainty of a simple ideal mode-stirred chamber measurement.

Initially, let us assume we measure $2 \mathrm{~W}$ received by a reference antenna at a single paddle position (we make no assumptions about the transmitted power), and we want to predict the power that would be received by another antenna at some other location in the chamber. We would of course predict that the same $2 \mathrm{~W}$ will be received by another antenna, but what is the uncertainty in this prediction? (For our purposes here, we will assume standard uncertainty as measured by the theoretical standard deviation of the measurement.) Referring to Table 2, we see that with an average measured power of $2 \mathrm{~W}$ at a single paddle position, the standard deviation is also $2 \mathrm{~W}$. In linear units, we can write this as $2 \pm 2 \mathrm{~W}$, or $2 \mathrm{~W} \pm 100 \%$. Now, an uncertainty of $100 \%$ is not very good, especially when we know that the received power can never be less than $0 \mathrm{~W}$. If we attempt to express an uncertainty of $100 \%$ in decibels, things are even worse.

Calculating the uncertainty as $10 \log (2 \pm 2)$, our uncertainty can be expressed as $3_{-\infty}^{+3} \mathrm{~dB}$ relative to $1 \mathrm{~W}$. (Expressing uncertainty in this way is not entirely accurate, but is sufficient for the analysis presented here. A better approach is to use quantile values in both domains, but such analysis is beyond the scope of this report. Instead, we will use the simplified approach given above. An example of some of the complexities associated with processing uncertainties in the decibel domain is given below).

Alternatively, we could measure the received power on a device that displays power on a decibel scale, such as a power meter of spectrum analyzer. In this case, our instrument would measure $3 \mathrm{~dB}$ relative to $1 \mathrm{~W}$, or $33 \mathrm{~dB}$ relative to $1 \mathrm{~mW}$. (In the subsequent discussion, we will assume that decibel measurements will be made relative to $1 \mathrm{~W}$.) Thus, we would predict a received power from another antenna of $3 \mathrm{~dB}$ relative to $1 \mathrm{~W}$ (once again this is not entirely accurate, but sufficient for our discussion here). Based on Table 3, the standard deviation is $5.569 \mathrm{~dB}$, and we can write the results of our measurement as $3 \pm 5.569 \mathrm{~dB}$ relative to $1 \mathrm{~W}$. Once again, the uncertainty is rather large, but at least all of the bounds are finite.

For those readers who like to be confused (as you must if you have made it this far), we will make one additional observation. The nonlinear transformation for converting linear values to decibels and vice versa is more complicated than presented here. As mentioned above, if we measure a received power of $2 \mathrm{~W}$, we expect a second antenna to pick up a received power of 2 $\mathrm{W}$. But if the second receiver measures power in decibels, we do not actually expect another 
antenna to measure $3 \mathrm{~dB}$ relative to $1 \mathrm{~W}$; instead we expect $0.503 \mathrm{~dB}$ relative to $1 \mathrm{~W}$. However, if we measure a received power of $3 \mathrm{~dB}$ relative to $1 \mathrm{~W}$, we expect another antenna (connected to an instrument that measures power in linear units) to measure $2 \mathrm{~W}$. Further, if we measure a received power of $3 \mathrm{~dB}$ relative to $1 \mathrm{~W}$, we do not actually expect a second antenna to also measure a received power of $3 \mathrm{~dB}$ relative to $1 \mathrm{~W}$, but instead we expect $0.503 \mathrm{~dB}$ relative to 1 W. The proof is left as an exercise for the reader (we've always wanted to say that). Hint: based on the derivation in Section 3.2 and the principle of maximum likelihood, find the most likely value of $\sigma$ for each case presented here.

Ignoring the apparent inconsistencies in predicting the received power, we instead concentrate on the uncertainties in the measurement. As shown above, for a single paddle position, the uncertainty associated with measurements in linear units seems to be greater than that associated with measurements in logarithmic units. (This is an artifact of the severely skewed distribution for the received power and the fact that using standard deviations to evaluate uncertainties is an oversimplification that can have unexpected consequences.) In linear units, it appears that we have no knowledge about the lower uncertainty bound other than the fact that the received power must be nonnegative (which we already knew). This is equivalent to infinite uncertainty in the lower bound. When we express the measurements in decibels, however, we have a fair estimate for the lower uncertainty bound at the expense of a slightly higher estimate for the upper uncertainty bound. Based on this fact, we might perform all measurements and averaging in logarithmic units, in the hope of reducing the estimated uncertainty. Once a second measurement or more is taken and averaged, however, the advantage shifts back to linear measurements. For two measurements, assuming once again an average received power of $2 \mathrm{~W}$, the measurement uncertainty (standard deviation) is reduced by $\sqrt{2}$, so we can write the average received power as $2 \pm \sqrt{2} \mathrm{~W}$, or $2 \mathrm{~W} \pm 71 \%$. We can express the uncertainty in decibels as $10 \log (2 \pm \sqrt{2})=3_{-5.33}^{+2.32} \mathrm{~dB}$ relative to $1 \mathrm{~W}$ (neglecting all of the confusion caused by converting to decibels discussed above). The total range of uncertainty is $2.32+5.33=7.65 \mathrm{~dB}$. Alternatively, if all measurements and calculations are performed in decibels, and assuming an average received power of $3 \mathrm{~dB}$ relative to $1 \mathrm{~W}$, the standard deviation is $5.569 / \sqrt{2} \mathrm{~dB}$, or 3.94 $\mathrm{dB}$, and we can write the results of our measurement as $3 \pm 3.94 \mathrm{~dB}$ relative to $1 \mathrm{~W}$. The total range of uncertainty is $3.94+3.94=7.88 \mathrm{~dB}$. This is slightly greater than the uncertainty of the measurement based on linear units and converted to decibels, and this is true for any number of paddle positions greater than or equal to 2 . This is also true for measurements of electric field (both total and rectangular component), as long as three or more measurements are taken and averaged. For this reason, all of our measurements and averaging were performed in linear units, and subsequently converted to decibels.

When multiple measurements are made, however (as when 30 probes are used to give 30 estimates of the average electric field), we are faced with a similar problem: we can either take the individual measurements (which have already been processed in linear units), evaluate the characteristics (average, standard deviation, etc.), and then convert to decibels, or we can convert to decibels first and then evaluate the characteristics. We based our decision on convenience rather than technical factors (although with a sufficient number of paddle positions the results should be similar regardless of which way the data are processed). Although it may seem 
convoluted, we processed measurements from individual sensors in linear units. The results were then converted to decibels and plotted. Since the data are presented in decibels, all subsequent analysis was also performed in decibels. For example, given 30 measurements of the electric field at each of 225 different paddle positions, we calculated the average electric field measured by each of the probes and then converted the 30 different averages from units of volts per meter to decibels relative to $1 \mathrm{~V} / \mathrm{m}$. We than computed the average and standard deviation of these 30 different estimates of the average to give results that were also in units of decibels relative to 1 $\mathrm{V} / \mathrm{m}$.

This approach to the analysis has some subtle side effects that must be examined in more detail if extremely fine precision is required. These side effects are most apparent in the processing of the maximum of a signal (received power, electric field), but we will first address the effects on the average of a signal.

One problem is a result of the fact that conversion from linear units to logarithmic units can result in asymmetric uncertainty bounds, as shown above. For example, assuming an ideal chamber, ideal instrumentation, and measurements made at 225 paddle positions, the uncertainty in the received power is $\pm 6.67 \%(1 / \sqrt{225})$, which converts to $+0.28 /-0.30 \mathrm{~dB}$. Once a large number of measurements are taken and plotted on a decibel scale, and the average and standard deviation are calculated, the estimated average will have symmetric error bounds. For this reason, we will state the uncertainty as the average of the magnitude of the two bounds $(0.29 \mathrm{~dB}$ in this example).

For the maximum of a set of measurements, we could process the results in a similar fashion. Continuing with the example above, assuming ideal conditions and 225 paddle positions, the uncertainty in the maximum received power (taken from Table 2 ) is $2.562 / 11.992=21.36 \%$, converting to decibels, our uncertainty is $+0.84 /-1.04 \mathrm{~dB}$. If we make these bounds symmetric as above, our uncertainty is $0.94 \mathrm{~dB}$. However, if we examine our procedure closely, we perform the following operations:

1. Take measurements at 225 paddle positions.

2. Select the maximum measured value.

3. Convert to decibels.

4. Plot the result.

5. Repeat until we have a large number of maximums.

Compare this to procedure for taking the measurements in decibels, or equivalently, converting to decibels immediately after the measurement is taken:

1. Take measurements at 225 paddle positions.

2. Convert to decibels.

3. Select the maximum measured value.

4. Plot the result.

5. Repeat until we have a large number of maximums.

Although the procedure is different, the result is exactly the same. This is because the maximum measured signal can be converted from linear units to decibels directly. (This is not true for the 
Table 14. Uncertainty in an ideal chamber for 225 paddle positions.

\begin{tabular}{lcc}
\hline Quantity & \multicolumn{2}{c}{ Standard uncertainty for the } \\
average $(\mathrm{dB})$ & maximum $(\mathrm{dB})$ \\
\hline Received power & 0.29 & 0.88 \\
Electric field & & \\
$\quad$ Rectangular component & 0.30 & 0.88 \\
$\quad$ Total & 0.17 & 0.63 \\
\hline
\end{tabular}

average.) Thus, it might be better to estimate our uncertainties based on the entries in Table 3 instead of Table 2 when evaluating the maximum received power. In this case, the uncertainty is $0.878 \mathrm{~dB}$. Although the difference between these estimates is only $0.062 \mathrm{~dB}$ (yes, we are talking hundredths of a decibel in a mode-stirred chamber report), this difference is apparent in numerical simulations and some measurements performed for this report. The correct estimate of the uncertainty appears to be $0.878 \mathrm{~dB}$.

\subsection{Uncertainty due to Random Nature of Mode-Stirred Chamber Measurements}

The uncertainty associated with ideal measurements in an ideal mode-stirred chamber can be taken directly from the descriptions given in Section 3.2. A summary of these uncertainties is given in Table 14. All uncertainties presented here assume 225 paddle positions were used at each frequency. Also, all uncertainties are given in terms of decibels. All of the uncertainties listed here can be characterized as type A uncertainties: those which are evaluated by statistical methods.

\subsection{Uniformity, or Residual Unexplained Uncertainty}

Once the uncertainties associated with an ideal chamber and ideal conditions have been calculated, we can perform a large number of repeated measurements and compare the observed standard deviation with what we expect for an ideal chamber. If the observed standard deviation is substantially larger than that attributable to an ideal chamber, then the additional uncertainty must be due to imperfections in the instrumentation or imperfections in the chamber. If, instead, the observed standard deviation is similar to the predicted value, we can say that the uncertainty due to imperfections in the chamber are less than or approximately equal to those due to the random nature of chamber measurements. Finally, if the observed standard deviation is consistently less than the predicted value, we can assume that the expected distribution is incorrect. Remember, however, that measurements of the standard deviation are only estimates, and will occasionally be greater than and occasionally be less than the expected values. Thus, unexplained deviations are based on measurements, and will therefore be different depending on 
the chamber being evaluated, the parameter being measured, and the drive frequency of the signal source.

In an effort to better characterize the uncertainties associated with imperfections in a chamber, we offer the following observations.

1. The average of a number of samples is relatively insensitive to the distribution from which the samples are drawn. After a sufficiently large number of samples have been taken and averaged, the only characteristics of the original distribution that affect the sample average are the mean and standard deviation of the parent distribution. (This is a slight oversimplification, but basically correct.)

2. If an infinite number of samples is taken, the average of the samples will be equal to the mean of the parent distribution, the uncertainty about the average will be essentially 0 , and the standard deviation of the parent distribution is unimportant. (Once again this is a slight oversimplification.)

3. Predictions of the maximum of a set of samples based on another set of samples taken from the same distribution can be very sensitive to the original distribution of the individual samples.

4. Predictions of the average of a set of samples based on the average of another set of samples taken from the same distribution will typically have a lower associated uncertainty than predictions of the maximum.

Based on these observations, the only way we can ensure that random variations discussed in Section 8.2 are negligible is to take a large number of measurements and average them. In the limiting case, we can reduce these variations to 0 by taking an infinite number of measurements. This is the only way to separate typical random fluctuations observed at a point from spatial fluctuations. Thus, we propose the following definition:

Spatial uniformity is a measure of the expected variability of a measurement as a function of location within a reverberation chamber. It is numerically equal to the uncertainty associated with the prediction of a parameter at a specific location based on an infinite number of measurements of the same parameter taken at a different and independent location, assuming perfect instrumentation.

The parameter that we are measuring or predicting can be any typical electromagnetic quantity associated with mode-stirred chambers, such as received power, total electric field, or rectangular component of the electric field. In addition, the parameter of interest can be either the average or maximum of the measured electromagnetic quantity. Other characteristics such as the minimum could also be evaluated, but typically only the average and maximum are significant.

We admit that the definition given here is impractical because it requires an infinite number of measurements. In practice, we can take a finite number of measurements and either generate a good estimate of the uniformity or claim that the uniformity is better than can be measured for a given number of measurements.

Another problem with requiring an infinite number of measurements is related to measurements of the maximum of a parameter. Based on the analysis in Section 3.2.6, the 
expected value of the maximum of a measured quantity is a function of the number of measurements, and, assuming ideal distributions, the expected value of these quantities will approach infinity if we take an infinite number of measurements. To solve this problem, we must qualify what we mean by an infinite number of measurements. Generally, measurements will be taken with a preselected number of paddle positions $N$ (200 is often used, and we used 225 for our measurements). Given this value of $N$, we can take a large (infinite) number of sets of measurements, with each set consisting of $N$ measurements. From each set, we select the maximum measured value, and then average the set of measured maximums. Thus, we can only state the uniformity of the maximum of a quantity if we also state the number of paddle positions at which the quantity was measured. We do not have this restriction when we state the uniformity of the average of a quantity. Given that the uncertainty in measurements of the maximum of a parameter can be large compared to the uniformity of the chamber, it is likely that the uniformity will be better than can be measured for a given number of paddle positions. If this is the case, we will assume that the chamber being evaluated is perfectly uniform, and that the component of uncertainty in measurements of the maximum of a parameter due to chamber imperfections is 0 . Although this may be optimistic, it simply means that the uncertainty in measurements performed in a perfect chamber dominate the uncertainties due to chamber imperfections.

Unfortunately, this discussion means that we cannot specify a unique "uniformity" for the chamber. Instead, each parameter has an associated uniformity, and the maximum of a quantity will have a uniformity that is different depending on the number of paddle positions per measurement. Fortunately, the uniformity of the maximum of a quantity is often very similar to the uniformity of the average of that quantity, regardless of the number of positions, but we are still left with different measures of uniformity depending on the parameter measured in the chamber.

Since spatial uniformity is a measure of uncertainty, it will generally be stated with a specific level of confidence. For example, we might say that the uniformity is $\pm 1 \mathrm{~dB}$ with a level of confidence of $68 \%$. This means that, if the average of a parameter is measured at a large number of independent locations, it will be possible to construct a range with an upper and lower limit, separated by $2 \mathrm{~dB}$, such that $68 \%$ of the measurements will be within the range. To simplify the discussion somewhat, we will define the standard uniformity as follows:

Standard uniformity is the spatial uniformity as defined above, where the uncertainty is the standard uncertainty equal to the positive square root of the estimated spatial variance that would be observed given an infinite number of measurements. This is approximately equivalent to a confidence level of $68 \%$.

The spatial uniformity as defined above is slightly different from the conventional usage of spatial uniformity. In reference [3], for example, the uniformity (although not specifically defined) appears to be defined as the total spread in multiple measurements of the average and the maximum electric field. As such, it is more of a characterization of the measurement than of the chamber. The definition we propose depends only on the characteristics of the chamber, not on the characteristics of measurement system. Thus, we think it is a better indication of the performance of the chamber. 


\subsection{Estimation of the Standard Uniformity}

If it were possible to take an infinite number of measurements, we could evaluate the standard uniformity directly. Given a finite number of measurements, however, we must develop a method of estimating the standard uniformity. For this discussion we will once again assume that measurements are performed with perfect instrumentation and that observed fluctuations are due to imperfections in the chamber. Although this is probably not the case, unless we know exactly how much uncertainty is introduced by the instrumentation, we must assume that all variation are due to nonideal spatial uniformity. Thus, all estimates of uniformity will be pessimistic, and we can claim with great confidence that the true uniformity of the chamber is better than the data we present here.

We must base our estimate of the uniformity of the chamber on two pieces of available information: the observed uncertainty $\sigma_{o}$ and the ideal uncertainty $\sigma_{i}$. Given these values, we would like to estimate the unknown uniformity $\sigma_{u}$. If we assume that the ideal uncertainty and the uniformity are independent, then the combined uncertainty $\sigma_{c}$ can be calculated using the RSS (root sum squares) method:

$$
\sigma_{c}=\sqrt{\left(\sigma_{i}\right)^{2}+\left(\sigma_{u}\right)^{2}},
$$

and we can calculate the uniformity as

$$
\sigma_{u}=\sqrt{\left(\sigma_{c}\right)^{2}-\left(\sigma_{i}\right)^{2}} .
$$

Unfortunately, we do not know $\sigma_{c}$; we only have a very noisy estimate given by the observed standard deviation $\sigma_{o}$. Therefore, we can only generate a noisy estimate of the uniformity given by

$$
\sigma_{u} \approx \sqrt{\left(\sigma_{o}\right)^{2}-\left(\sigma_{i}\right)^{2}} .
$$

We will not attempt to estimate the uncertainty of our estimates of the uniformity of any parameters, since the uniformity is itself an estimate of uncertainty, and computation of the uncertainty of an estimated uncertainty is beyond the scope of this report.

One problem with eq (92) occurs if the combined uncertainty is close to the ideal uncertainty. Due to the noisy nature of the measured standard deviation, this can (and probably will) cause the argument of the square root in eq (92) to occasionally be negative, resulting in an imaginary estimate of the uniformity. To avoid this situation, we will assume that the observed (measured) standard deviation is a smooth function of frequency and can be approximated as a piecewise linear function. This assumption should be sufficient for the uncertainty analysis presented here. Thus the task of estimating the chamber uniformity is reduced to estimating the parameters for the linear "pieces." This task is difficult to automate, so the parameters will be 
evaluated visually. Unfortunately, this makes the uncertainty analysis more subjective than we would like, but appears to give acceptable results.

To explain the process of estimating the uncertainties, we give an example based on repeated measurements of received power performed in Chamber A. Using a network analyzer, we performed five repeated measurements in Chamber A (with the transmitting and receiving antennas at a different location for each measurement) at a subset of the frequencies presented earlier in this report. Since these measurements were taken at a subset of the frequencies of another measurement, we can decimate the frequencies of the larger set to give us a sixth repeated measurement. The observed standard deviation of the average received power is plotted in Figure 134a, and the observed standard deviation of the maximum received power is plotted in Figure 134b. We plot the data normalized to both a constant net incident power of $1 \mathrm{~W}$ and a constant incident power of $1 \mathrm{~W}$ to show that both methods yield approximately the same uncertainty. (From this point on we will present only plots for data normalized to a constant net input power of $1 \mathrm{~W}$ since the results in all cases are similar, regardless of the normalization method.) Also plotted in these figures is the standard deviation that would be expected had the chamber and the instrumentation been perfect. In general, the data in both of these charts appear to be approximately constant above $300 \mathrm{MHz}$, so it appears that a piecewise linear approximation will be valid at high frequencies. The challenge now is to decide on a value for the approximating constant.

Since the sample variance of a random variable is an unbiased estimator of the true variance [14], we can average several estimates of the variance to give an improved estimate of the true variance. This is not true of the standard deviation, so estimates of the standard deviation must be computed as the square root of the average variance, not the average of the estimates of the standard deviation. Although the difference is small (on the order of a few percent) it is still significant. Thus, above $300 \mathrm{MHz}$, we can estimate the standard deviation for measurements of the average received power as $0.36 \mathrm{~dB}$ and the standard deviation for measurements of the maximum received power as $0.88 \mathrm{~dB}$ (the standard deviation for a perfect chamber). We do not make an adjustment to these estimates of the standard deviation to account for the small number of samples taken at each frequency (as described in reference [29]) because our estimate is actually based on a much larger number of sample (averaging over location and frequency). If we had taken measurements at only a single frequency, we would be forced to increase our estimated uncertainty by $11 \%$ [29].

Below $300 \mathrm{MHz}$, we do not have estimates of the variance at a large number of frequencies, so we must take a different approach. We will extend the estimated constant described above to as low a frequency as we can and still have a good estimate of the measured standard deviations. For very low frequencies, we will construct a piecewise linear envelope that contains the majority of the measured standard deviations. This will usually be a pessimistic estimate, so again we will not increase our estimated uncertainty to account for the small number of samples. Our estimates for the true standard deviations for the data shown in Figure 134a and Figure 134b are presented in Figure 135a and Figure 135b. We will call this estimate the estimated standard chamber uncertainty, indicating that this is the uncertainty associated with measurements at multiple locations in a chamber. 


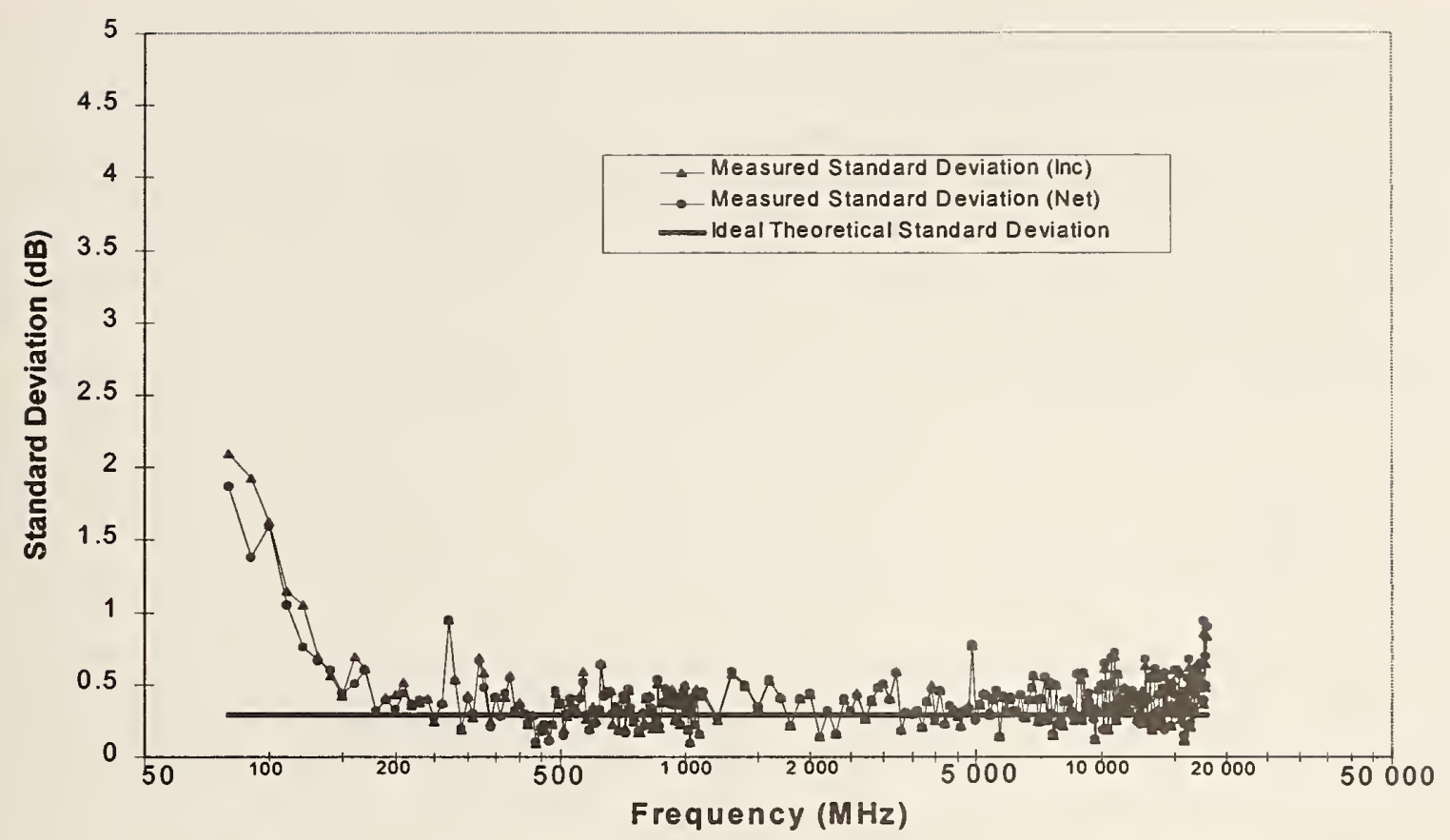

(a)

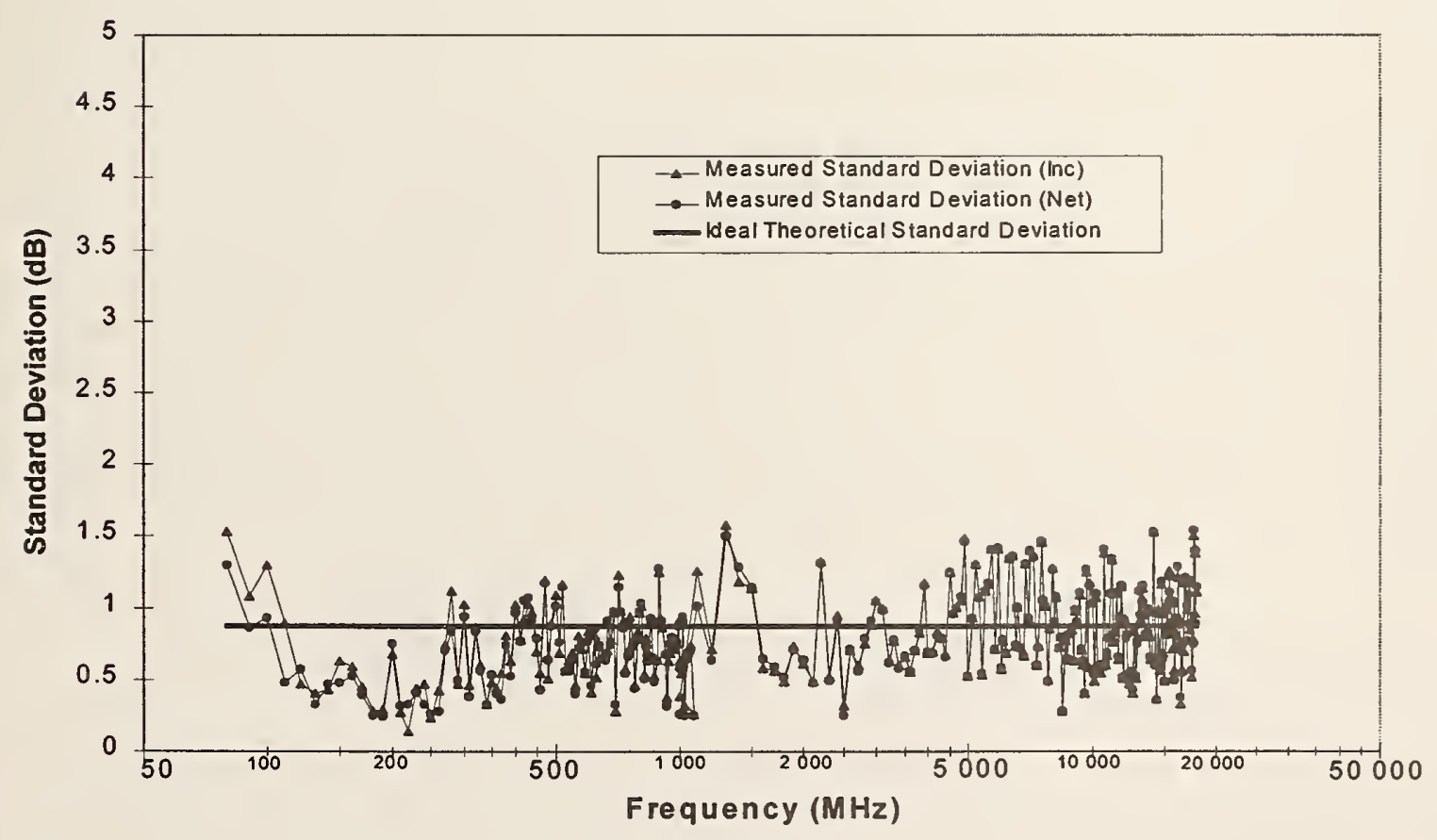

(b)

Figure 134. Sample standard deviation of six measurements of received power in chamber A. Measurements were made at different antenna locations. (a) Standard deviation of average received power. (b) Standard deviation of maximum received power. 


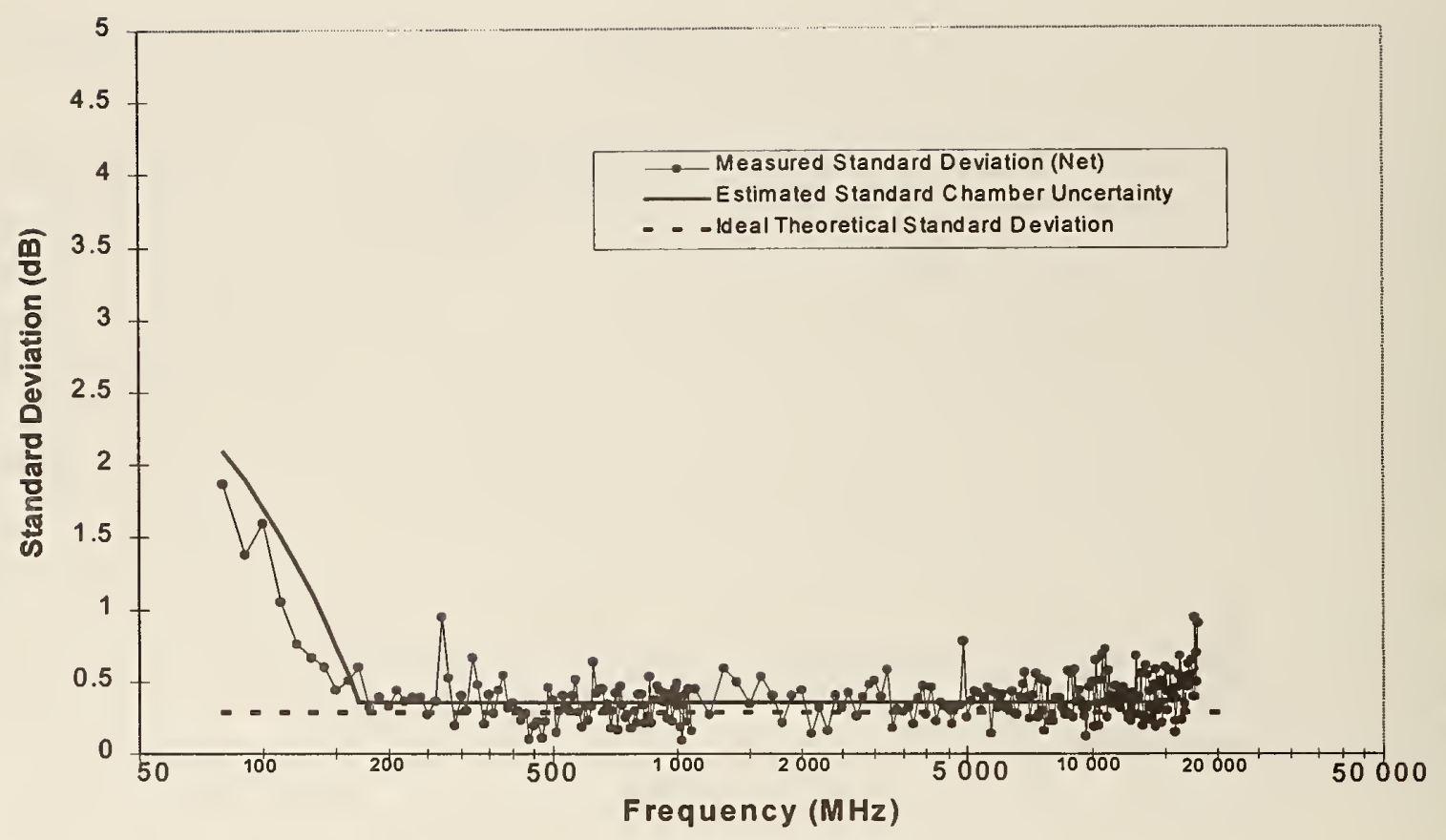

(a)

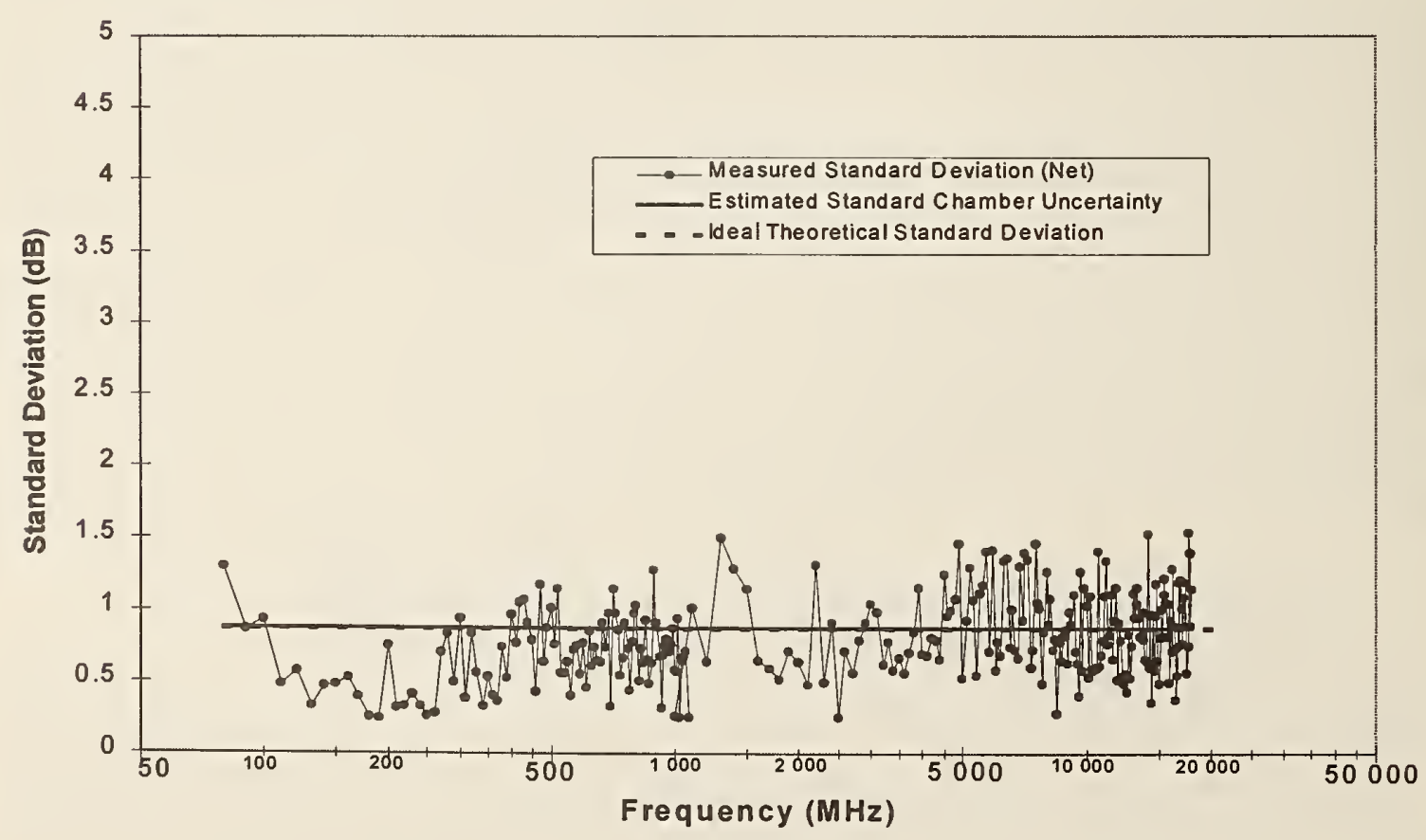

(b)

Figure 135. Sample standard deviation and piecewise linear approximation of six measurements of received power in chamber A. Measurements were made at different antenna locations. (a) Standard deviation of average received power. (b) Standard deviation of maximum received power. 
The standard chamber uncertainty for average received power is good between $170 \mathrm{MHz}$ and $15 \mathrm{GHz}$, and is only slightly greater than the uncertainty associated with an ideal chamber. Above $15 \mathrm{GHz}$, the uncertainty increases somewhat (we think this is due to poor repeatability of the cables), and below $170 \mathrm{MHz}$, the uncertainty increases dramatically. The standard chamber uncertainty for maximum received power is also good. In fact, at some frequencies (specifically between $100 \mathrm{MHz}$ and $300 \mathrm{MHz}$ ), the uncertainty appears to be better than we would expect even if the chamber were ideal. Additionally, between $300 \mathrm{MHz}$ and $1 \mathrm{GHz}$, the uncertainty also appears to be consistently better (on average) than we would expect for an ideal chamber. This does not imply that these chambers are better than perfect. Instead, it implies that the statistical model may be incorrect at low frequencies (as discussed in Section 6.3, there appears to be compression in measurements of received power, and compression will cause measurements of the maximum received power to be better behaved than uncompressed data). This is the most likely cause of the low uncertainty below $300 \mathrm{MHz}$. Another possible explanation of the lower possible uncertainty below $1 \mathrm{GHz}$ is that the spatial samples are not independent, and this is more likely to be the case at low frequencies because the antennas are larger and the wavelengths are longer. This may explain the apparent lower uncertainty between $300 \mathrm{MHz}$ and $1 \mathrm{GHz}$.

Until we have a better statistical model of the received power at low frequencies, we will simply assume that the chamber behaves like an ideal chamber and use the standard deviation associated with such a chamber. Once again this is a pessimistic assumption, but it is the best available alternative. Given this assumption, the uncertainty in measurements of the maximum power received in Chamber $\mathrm{A}$ appears to be indistinguishable from the uncertainty expected in an ideal chamber over the entire tested frequency range of $80 \mathrm{MHz}$ to $18 \mathrm{GHz}$.

Occasionally, it is not possible to take a large number of repeated measurements in a chamber. In this case, we cannot analyze the uncertainties as described above and must find an alternative method. If measurements are performed at a small number of locations and a small number of frequencies, little can be done to give a simple and reliable estimate of the uncertainty. However, if measurements can be made at a large number of frequencies, and once again assuming that the observed (measured) standard deviation is a smooth function of frequency and can be approximated as a piecewise linear function, we can still estimate the spatial variations, even though measurements were taken at only a single location. If we assume that a change in frequency is roughly equivalent to a change in location, we can evaluate the characteristics of measurements at multiple frequencies as if they were taken at multiple locations, and proceed as described above.

Before continuing with this explanation, we must first mention some of the limitations of this approach. Most importantly, this approach will give only an estimate of the spatial variations, and measurements at multiple locations will always be preferable to an approximation based on measurements made at multiple frequencies. This technique should only be used if it is not possible or not feasible to take measurements at multiple locations, or if data have already been taken and it is not possible to take additional data. This is the situation for the measurements we took in Chambers B and C, where there was time to take only three and two repeated measurements, respectively. Fortunately, we can use data from Chamber A to verify this method of estimating uncertainties.

For this approach to be valid, the frequencies must be different enough to give independent measurements (typically $1 \mathrm{MHz}$ is sufficient), but must also be similar enough that 
there will be no significant difference in the expected chamber characteristics at the various frequencies (frequency spacing should probably be significantly less than $100 \mathrm{MHz}$ ). The greatest disadvantage of this approach is that it accentuates imperfections in the chamber, antennas, or instrumentation that cause significant changes in the measured value as a function of frequency.

As an example, we processed the measurements of received power given in Figure 29. At each frequency, we grouped measurements at the specific center frequency and three frequencies on either side of the center frequency, giving us seven samples of received power. We chose seven samples so that the results would be similar to those we obtained using six different antenna positions, and because these calculations are easiest to implement if an odd number of samples are used. We then calculated the standard deviation of these seven samples to give us an estimate of the standard deviation of seven measurements taken at the center frequency, but at different spatial locations. This procedure is similar to calculating a moving average of a set of data, so we call this a "moving standard deviation." By processing measurements of the average and maximum received power in this way, we can obtain estimates of the uncertainty in the measurements of the average and maximum received power. Once we have calculated the moving standard deviation, we can process the results in exactly the same manner as the spatial samples described above. The processed data for Chamber A is given in Figure 136a for measurements of the average received power, and in Figure 136b for measurements of the maximum received power.

The data presented in Figure 136 are comparable to the data presented in Figure 135 with a few minor exceptions. First, repeated measurements indicate that very reliable measurements can be made down to $170 \mathrm{MHz}$, whereas the moving standard deviation technique appears to be accurate down to $300 \mathrm{MHz}$. This is most likely due to the strong frequency dependence of the antennas when placed in a highly conductive cavity. Second, repeated measurements show a small increase in the standard deviation at high frequencies ( $15 \mathrm{GHz}$ to $18 \mathrm{GHz})$, but the moving standard deviation technique shows good results from $300 \mathrm{MHz}$ to $18 \mathrm{GHz}$. This could indicate that changes in antenna location, which in turn result in changes in cable positions, could be sensitive at high frequencies. This is so because reflections are worst at high frequencies, and cable losses are greatest at high frequencies, so equipment calibrations will be most uncertain at high frequencies. This may also explain the fact that the standard deviation is greater at all frequencies when repeated measurements were made as compared to the data from the moving standard deviation. Measurements of the maximum received power continue to show surprisingly low uncertainty below $500 \mathrm{MHz}$, regardless of the method we used to estimate the standard deviation. Also, the observed standard deviation is very similar to the value that we expect for an ideal chamber. Thus, with the few exceptions mentioned above, the moving standard deviation technique seems to be a valid alternative to making measurements at multiple antenna positions, and in both cases, the standard deviations of measurements of received power are small. 


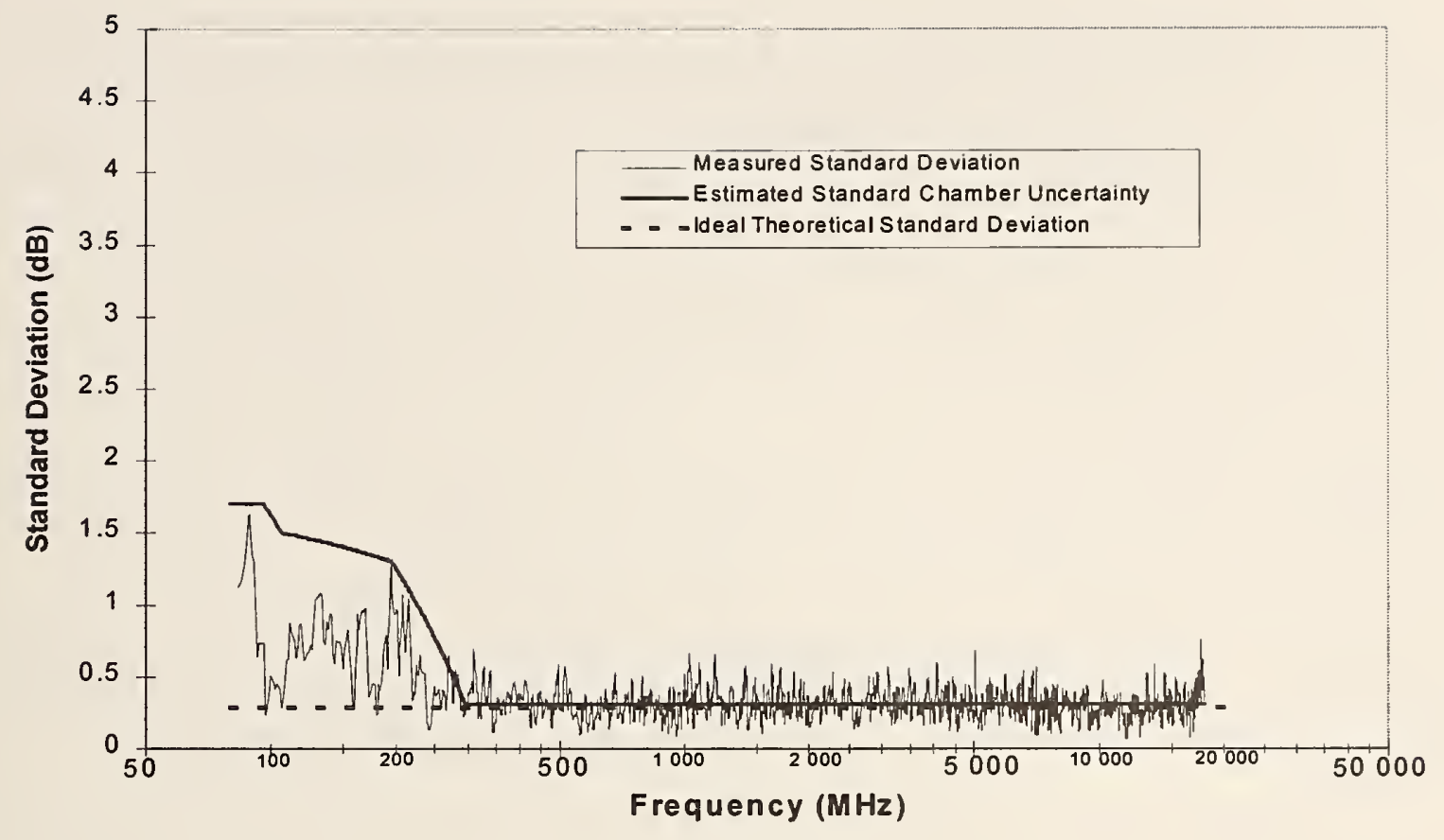

(a)

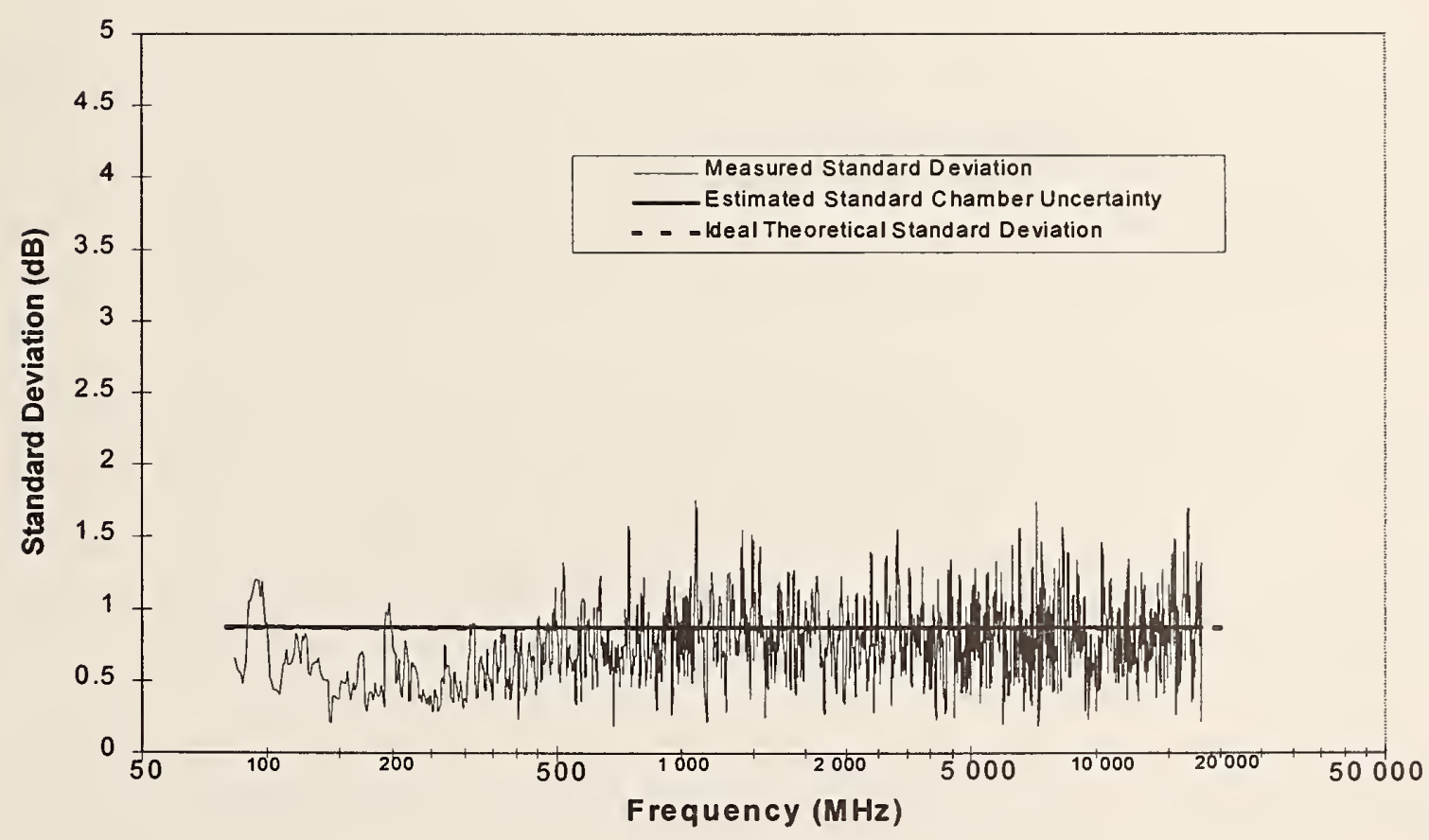

(b)

Figure 136. Sample standard deviation of seven measurements of received power in chamber A using "moving standard deviation" technique. (a) Standard deviation of average received power. (b) Standard deviation of maximum received power. 
Low uncertainty in measurements of received power may not equate to low uncertainty in measurements of electric field. For this reason, we must also analyze the spatial variations as measured by electrically small probes. To this end, we reexamine the data presented in Section 6.4. In an ideal chamber, as shown in Table 14, we expect the standard deviation of the average measured electric field (rectangular component) to be approximately $0.30 \mathrm{~dB}$, assuming 225 paddle positions. By taking the standard deviation of the 30 different estimates of the average electric field, as presented in Figure 81c, and making a piecewise linear estimation of the measured standard deviation as described above, we can estimate the observed uncertainty based on the probe measurements. These results are shown in Figure 137a. The observed standard deviation is greater than expected, but is still low above $350 \mathrm{MHz}$. We cannot determine whether the differences are due to variations in the calibrations of the probes or variations in the chamber environment inside the chamber. Most likely both are contributing to the observed variability. The variability due to the probe calibrations could be removed by using a single probe and repeating tests at multiple locations, or by evaluating a chamber multiple times and removing any statistical biases, essentially "calibrating" the probes in the chamber. We did not have sufficient time for either of these procedures, so we are forced to accept the variability and assume it is due to the chamber.

The standard deviations in the measurements of the maximum electric field are processed in a similar fashion, and the results are given in Figure 137b. The observed standard deviation is much closer to the expected standard deviation of $0.88 \mathrm{~dB}$. Thus, while small imperfections in the uniformity of the chamber (or the calibrations of our probes) may be apparent in measurements of the average electric field, they are significantly less apparent in measurements of the maximum electric field, and therefore immunity measurements (where immunity is assumed to be a function of the maximum electric field) can be performed as if the chamber were nearly perfect. In measurements of the standard deviation of both the average electric field and the maximum electric field that the uniformity of the chamber appears to degrade below 350 $\mathrm{MHz}$.

Measurements of the sample standard deviation of the total electric field can be processed similarly, and the results for the average and maximum total electric field are given in Figures 138a and b, respectively. The standard deviations associated with the total electric field are significantly smaller than those of a rectangular component of the electric field. Also, although the standard deviation of measurements of the average total electric field, although greater than the standard deviation expected from a perfect chamber, are less than $0.5 \mathrm{~dB}$, and the standard deviation of measurements of the maximum total electric field are less than $0.75 \mathrm{~dB}$.

The evaluation of Chamber B is similar to that of Chamber A, except that repeated measurements were not performed using the network analyzer. Because of this, we can only estimate the standard deviation of repeated measurements using the moving standard deviation technique. The results are presented in Figure 139a for measurements of the average received power, and in Figure 139b for measurements of the maximum received power. The piecewise linear estimation of the standard deviation shown in Figure 139a is obviously conservative based on the measured data also presented in this figure. We did this because measurements of received power normalized to a constant incident power had a higher standard deviation than those normalized to a constant net power, and we were interested in establishing an envelope that contained all of the data, regardless of normalization method. 


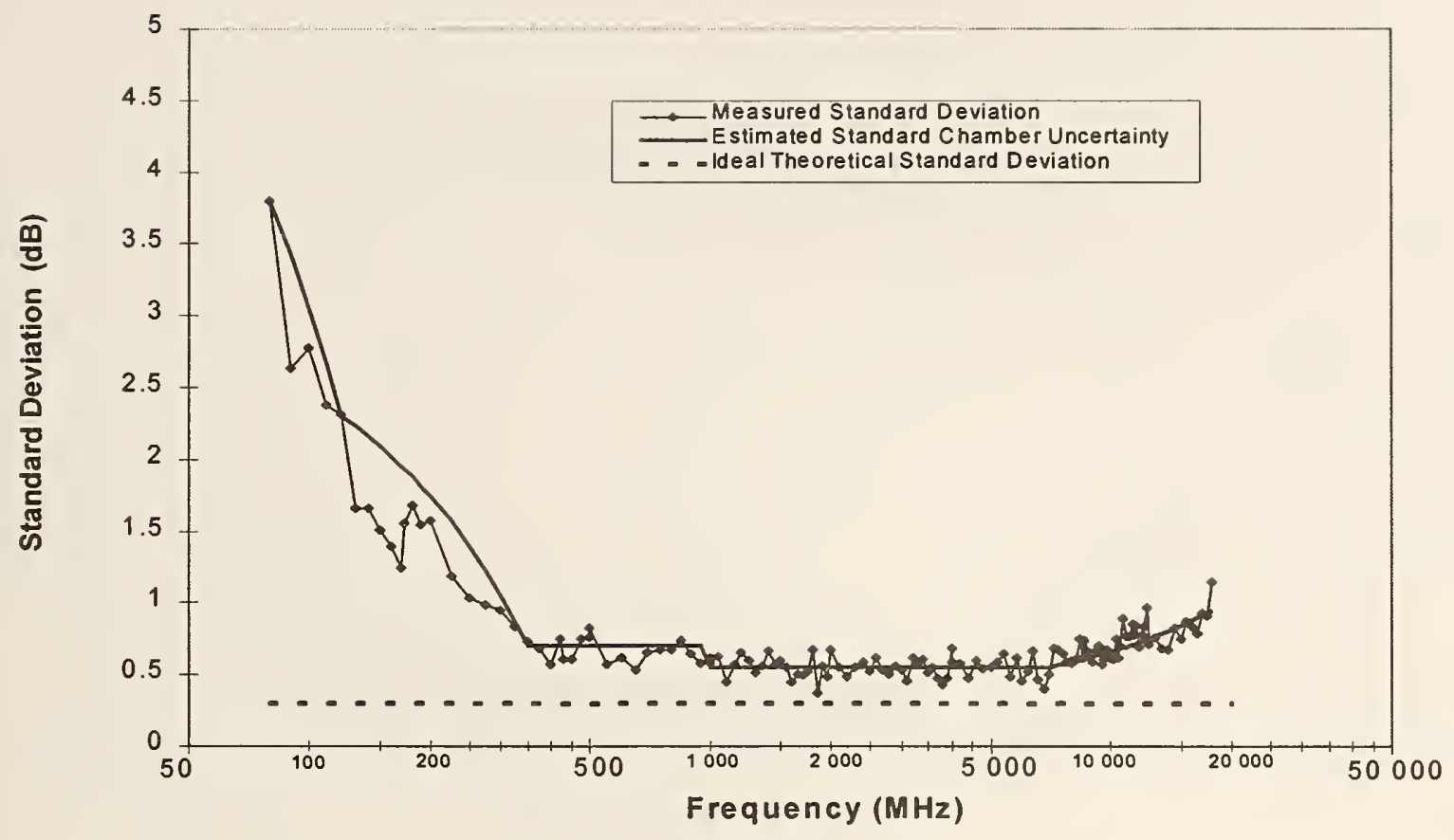

(a)

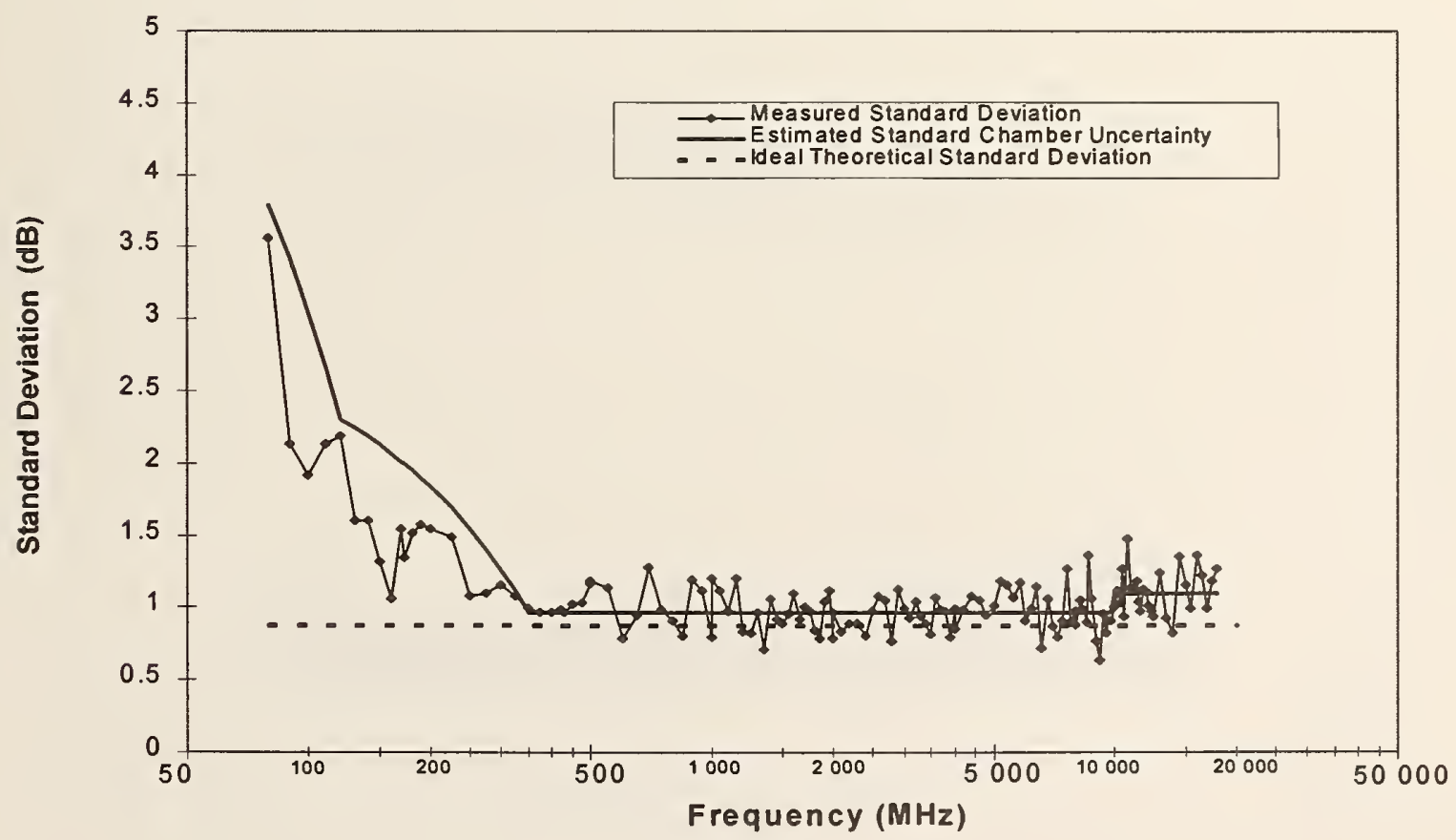

(b)

Figure 137. Sample standard deviation and piecewise linear approximation of 30 measurements of electric field (rectangular component) in chamber A. Measurements were made at different probe locations. (a) Standard deviation of average electric field.

(b) Standard deviation of maximum electric field. 


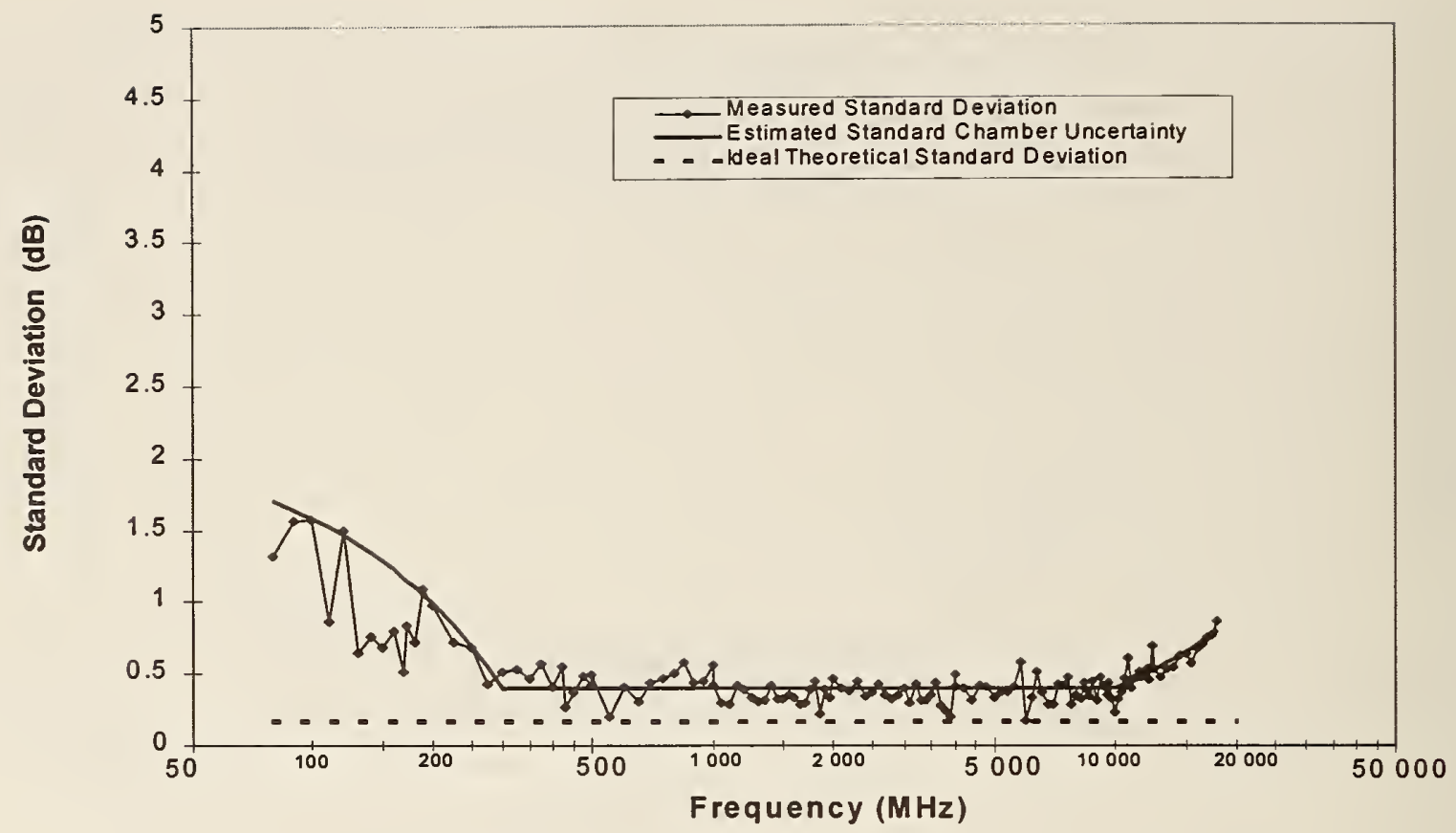

(a)

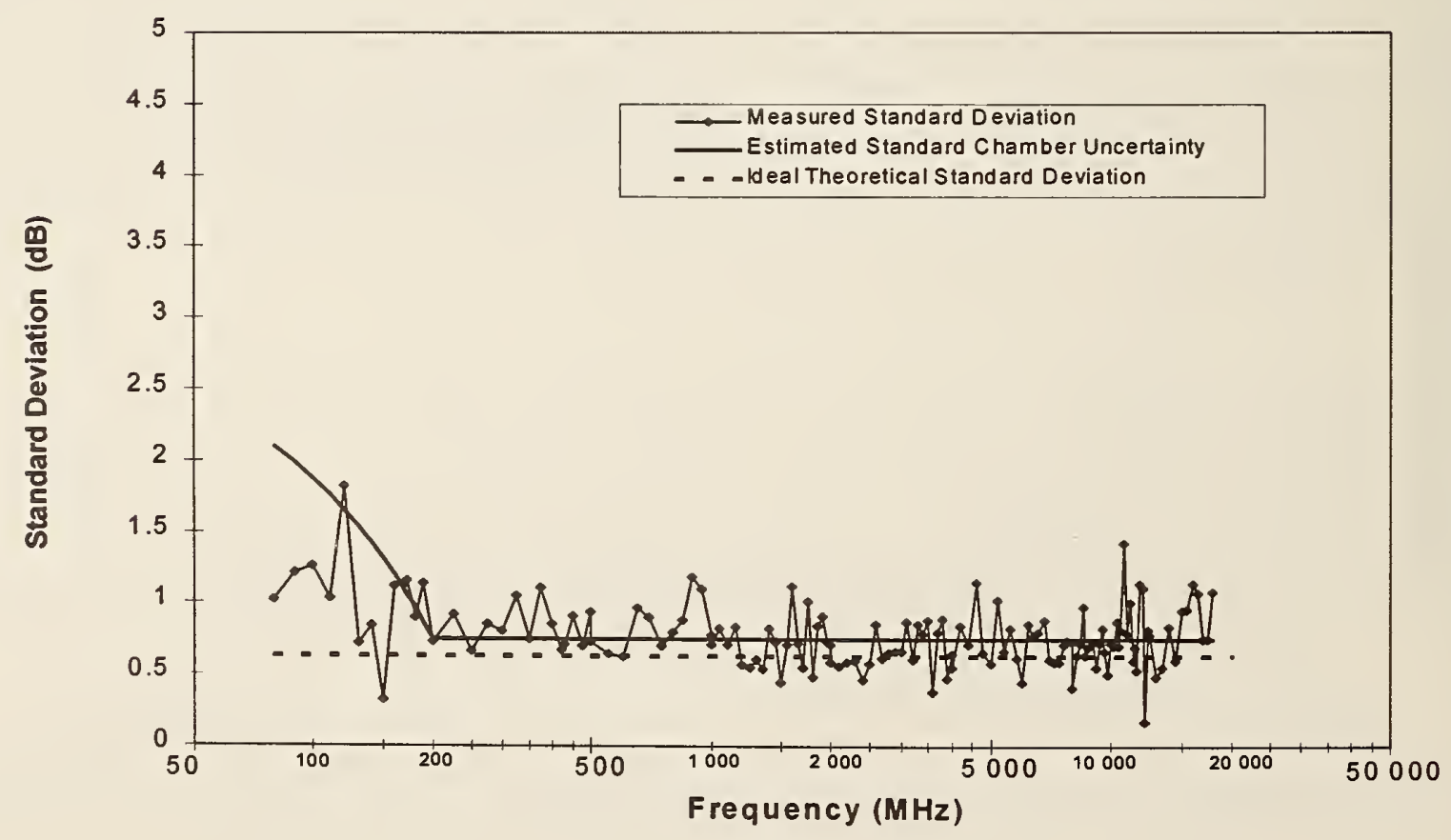

(b)

Figure 138. Sample standard deviation and piecewise linear approximation of ten measurements of total electric field in chamber A. Measurements were made at different antenna locations. (a) Standard deviation of average electric field. (b) Standard deviation of maximum electric field. 


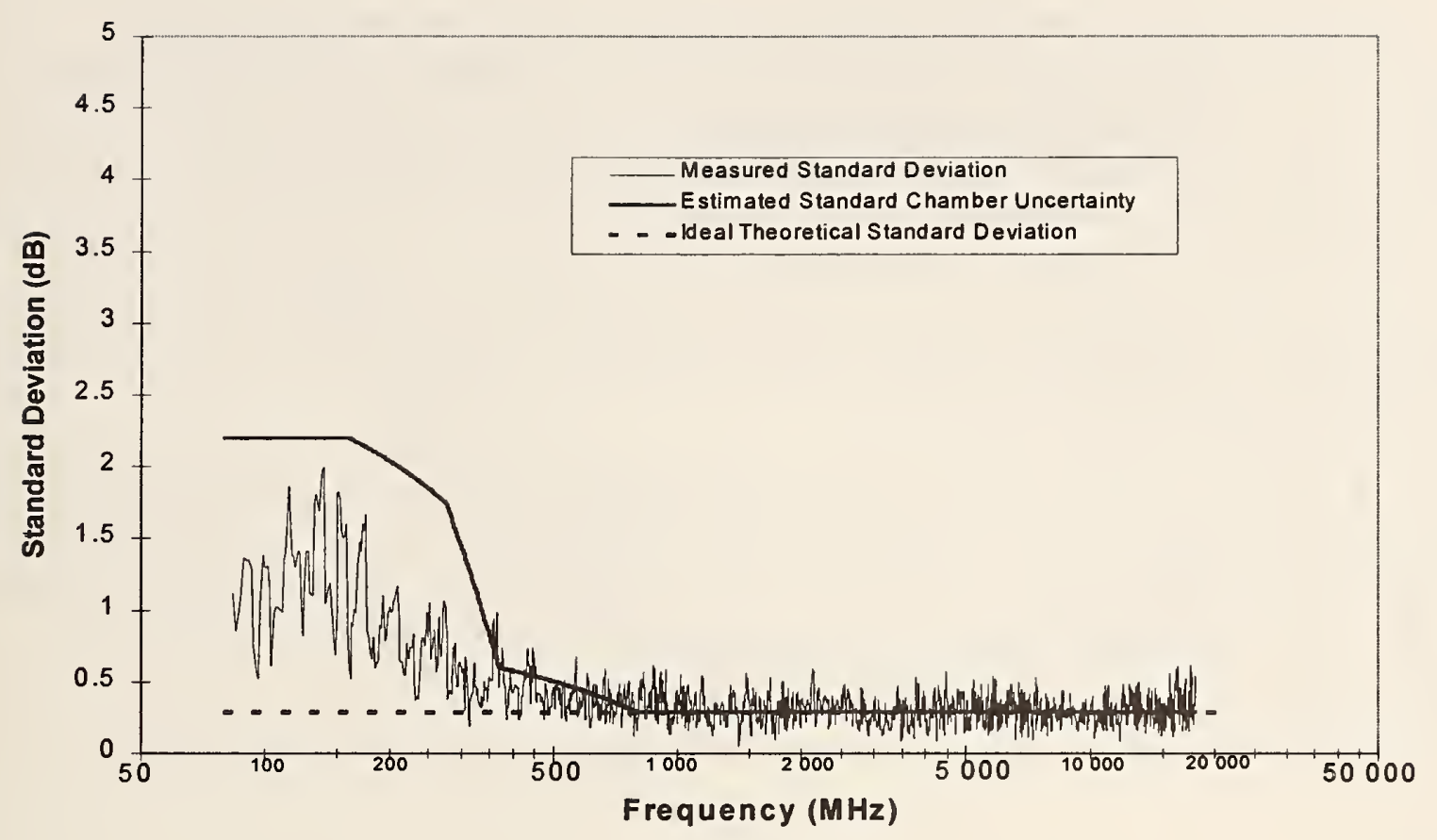

(a)

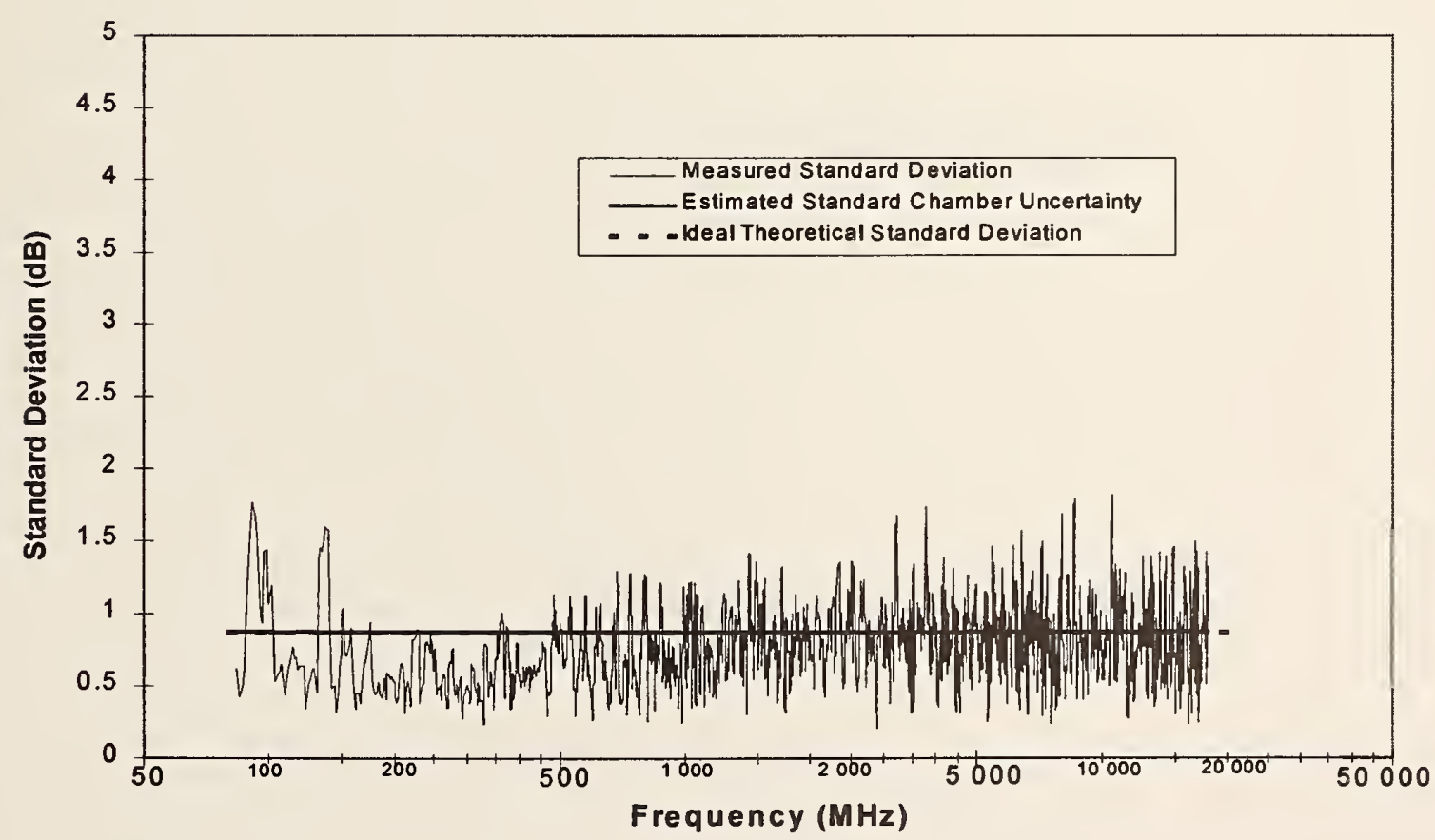

(b)

Figure 139. Sample standard deviation of seven measurements of received power in chamber B using "moving standard deviation" technique. (a) Standard deviation of average received power. (b) Standard deviation of maximum received power. 
The data in Figure 139b show the same unusual result that the observed standard deviation is less than the expected standard deviation below $500 \mathrm{MHz}$. The most likely cause, as described above for Chamber $\mathrm{A}$, is compression of the received power due to low losses in the chamber walls. Based on the data given in Figure 139a, Chamber B appears to give good results above $350 \mathrm{MHz}$, and the maximum received power appears reliable over the entire spectrum.

The standard deviation of measurements of the rectangular components of the electric field given in Figure 140 and the standard deviation of measurements of the total electric field given in Figure 141 show that Chamber B behaves much like Chamber A above $500 \mathrm{MHz}$, but the uniformity degrades below $500 \mathrm{MHz}$.

Finally, since no probe measurements were performed in Chamber $\mathrm{C}$ and a limited number of network analyzer measurements were performed in this chamber, the only available data to evaluate the uniformity of Chamber $\mathrm{C}$ are a moving standard deviation of measurements of received power. These results are shown in Figure 142. Chamber $C$ appears to behave much as Chamber $\mathrm{A}$ and $\mathrm{B}$ do above $700 \mathrm{MHz}$, and the maximum received power again appears to be stable over the entire measured spectrum. 


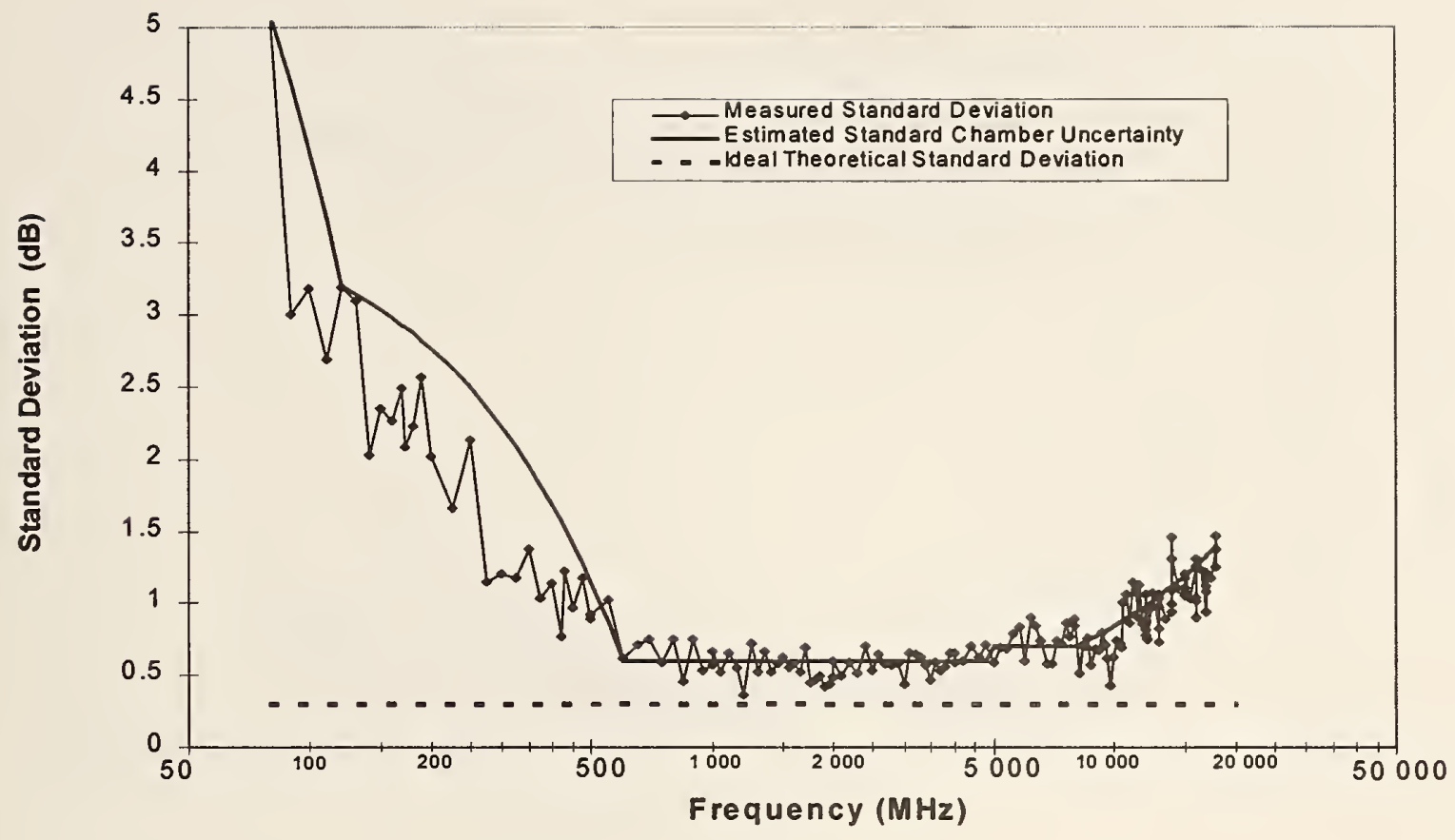

(a)

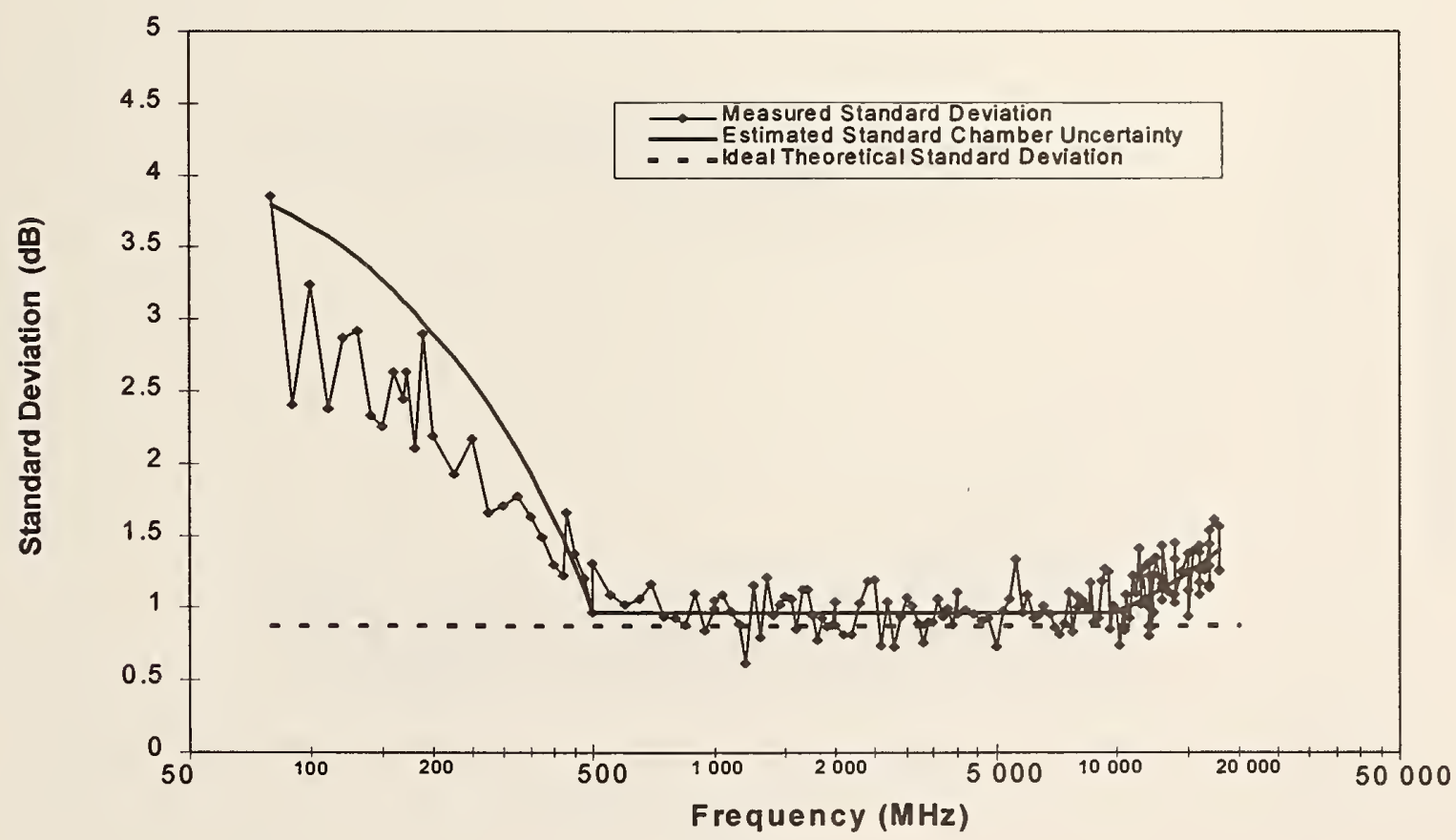

(b)

Figure 140. Sample standard deviation and piecewise linear approximation of 30 measurements of electric field (rectangular component) in chamber B. Measurements were made at different probe locations. (a) Standard deviation of average electric field. (b) Standard deviation of maximum electric field. 


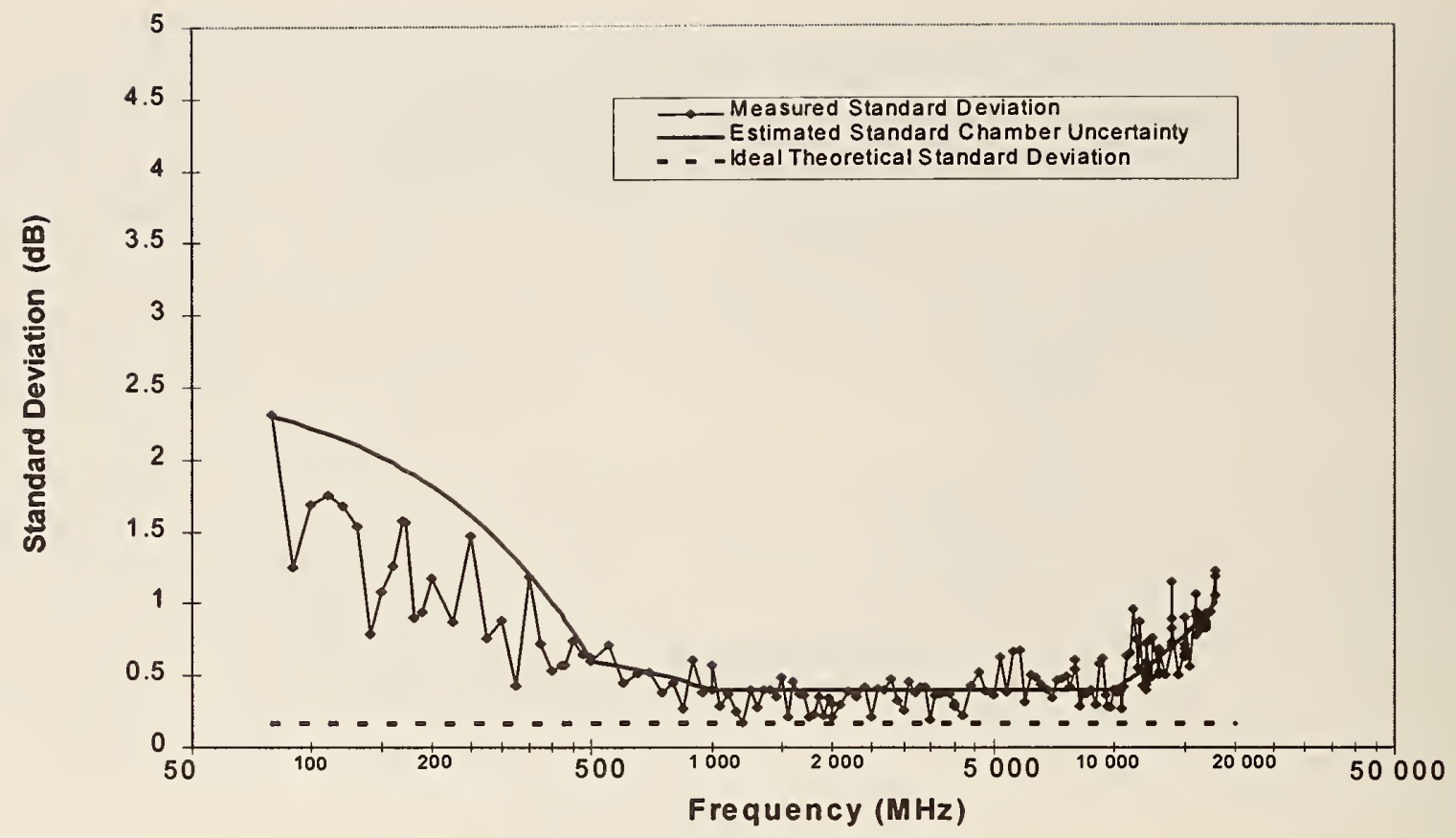

(a)

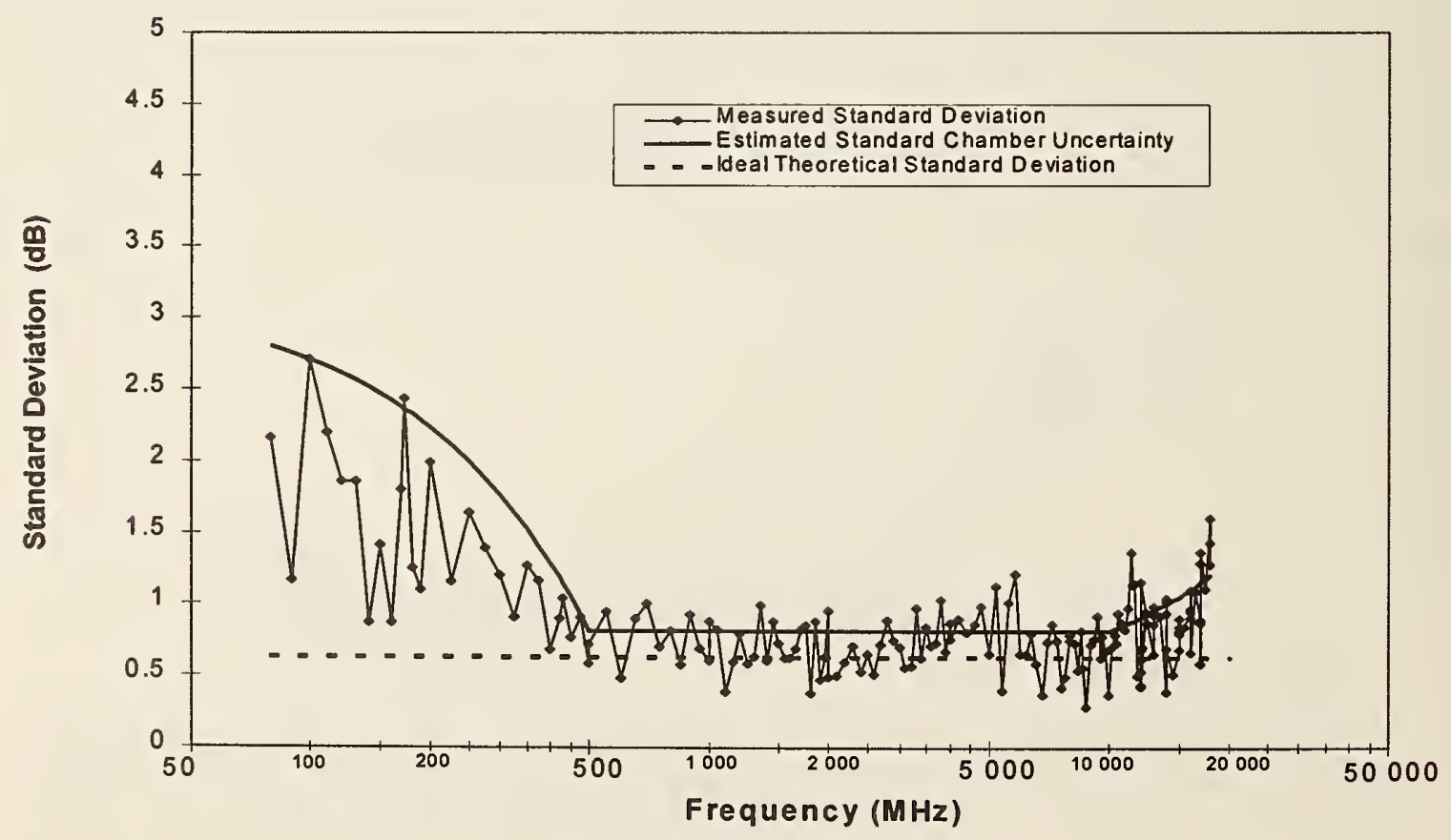

(b)

Figure 141. Sample standard deviation and piecewise linear approximation of ten measurements of total electric field in chamber B. Measurements were made at different probe locations. (a) Standard deviation of average electric field. (b) Standard deviation of maximum electric field. 


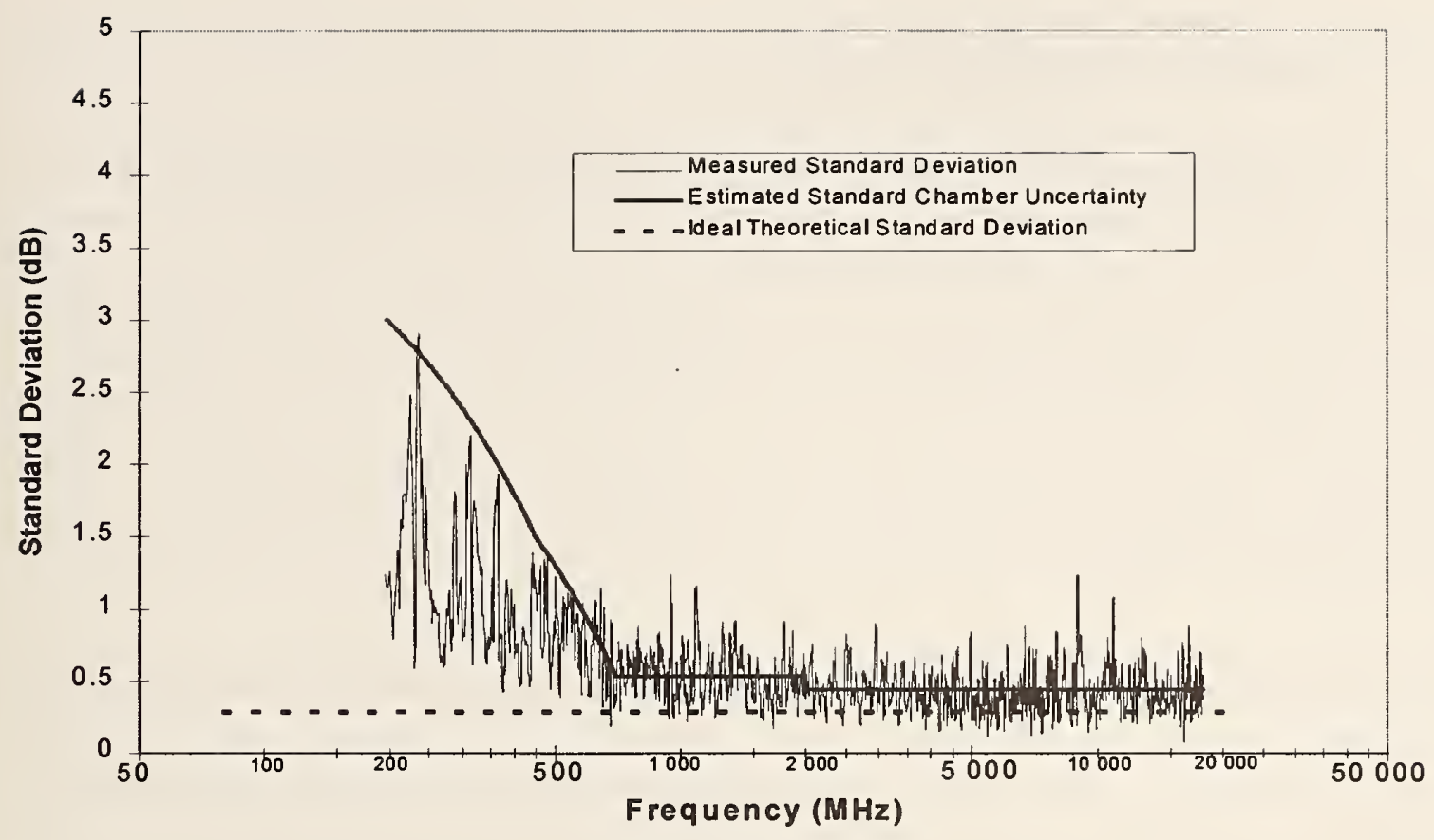

(a)

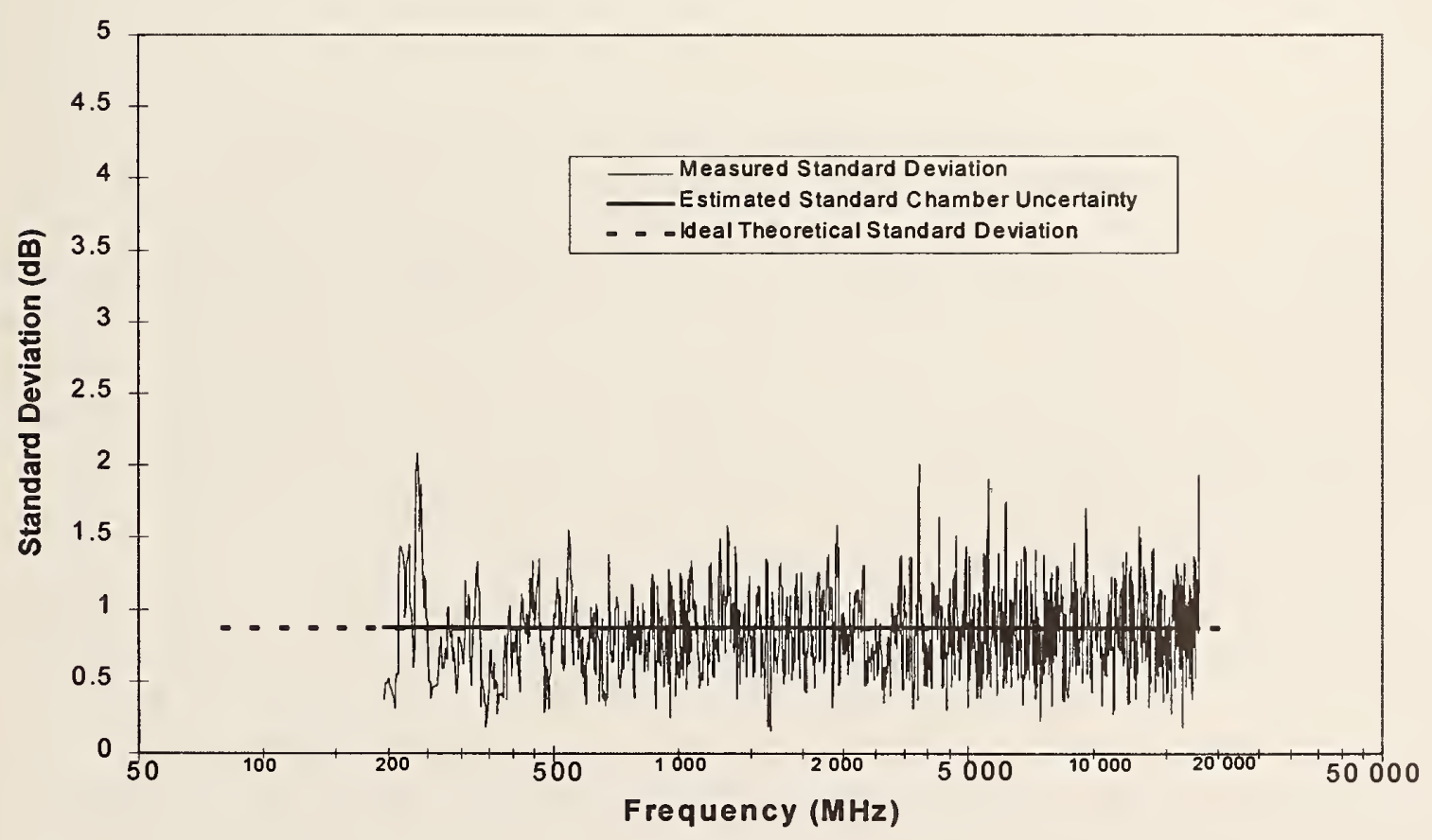

(b)

Figure 142. Sample standard deviation of seven measurements of received power in chamber C using "moving standard deviation" technique. (a) Standard deviation of average received power. (b) Standard deviation of maximum received power. 


\subsection{Uncertainty Due To Instrumentation}

When we first began work on this project, we thought, as did many others, that high uncertainties were expected in all mode-stirred chamber measurements, and these high uncertainties must be tolerated in exchange for the advantages associated with this type of measurement. At that time, had anyone told us that the uncertainties associated with our instrumentation would be significant in, let alone dominate, the uncertainties associated with mode-stirred chamber measurements, we would not have believed them. And yet, after analyzing all of the data we have collected, that is exactly the result we are reporting here: the uncertainty in the majority of the measurements we made in these chambers is dominated by the uncertainties in our equipment. The majority of this instrumentation uncertainty can be traced to a few sources:

1. In measurements of transmitted power, the characteristics of the transmitting antenna are not well known.

2. In measurements of received power, the characteristics of the receiving antenna are also not well known. In addition, the spectrum analyzer used to measure received power in the probe measurement system is not accurate, especially at frequencies greater than $4 \mathrm{GHz}$.

3. In measurements of the electric field, the calibration of the probes has an associated uncertainty of $\pm 1 \mathrm{~dB}$ ( $95 \%$ confidence).

The combination of these uncertainties results in a large overall uncertainty. However, careful measurement procedures and more research should allow us to reduce these factors.

Below, we tabulate the primary contributors to the instrumentation uncertainty. In these tables we make several assumptions. First, we assume that all contributors are independent because there is no obvious connection between any of the contributors. Second, we assume that the uncertainty due to random fluctuations in the measurement system is negligible relative to other contributors. Third, we assume that the uncertainty observed in multiple measurements of a parameter need not be included as instrumentation uncertainty, since it is already included in another part of the uncertainty analysis. For example, in the calibration of the electric field probes, it is possible that positioning of the probes in the test field or subsequent processing of the data will result in different calibrations even if the same probe were calibrated multiple times. Thus the uncertainty in the calibration of the probes could be reported as a combination of the uncertainty in the standard field and the uncertainty in placement and processing. But the uncertainty in placement and processing will also be present in multiple measurements of the electric field, so including the placement and processing uncertainty in the instrumentation uncertainty would mean that some portion of the uncertainty would be accounted for twice. Unfortunately, this means that the uncertainty of the measurement system will be underestimated, and the uncertainty of multiple measurements will be overestimated, but this should give the most conservative estimate for the uncertainty of the measurement.

Our most accurate measurement system, the vector network analyzer, is analyzed first. Vector network analyzers are accurate, especially well above the noise floor. The uncertainty in these measurements is difficult to quantify, but a standard uncertainty of $0.1 \mathrm{~dB}$ is a conservative 
estimate for our ability to calibrate the network analyzer. In addition to the uncertainty in the calibration, we also have some additional uncertainty due to differences in repeated connections to the antennas, as well as the fact that the cables used in these measurements were longer than recommended and could not be held in a fixed configuration. For these imperfections in our measurements, we allot an additional $0.1 \mathrm{~dB}$ for connector repeatability and cable repeatability. These estimates of $0.1 \mathrm{~dB}$ are conservative in all cases. There is little need to improve the estimate, however, since other factors will dominate the total uncertainty, as described below.

The only other source of uncertainty besides actual instrumentation uncertainty is insufficient knowledge about the antennas. As described in Section 6.3, we attempted to correct for antenna mismatch and efficiency by measuring the chamber gain with antennas that were known to be very efficient (assumed to be perfectly efficient) and also with the test antennas. The difference between the two measurements of the chamber gain was then assumed to be the combination of the mismatch characteristics of the antennas and the efficiency characteristics of test antennas. Because of this, the estimates of the efficiency and mismatch correction factors are strongly correlated (we are using three parameters to describe a single quantity), so even if we made a substantial error in estimating the efficiency of the test antennas, the error in the combined correction factor should be small.

One problem with using this technique for estimating a correction factor for the antennas is that we are estimating a parameter from the difference between two very noisy measurements, and so the difference will be noisier still. Thus, estimating the uncertainty based only on the difference in the two measurements implies that we will also be including additional uncertainty due to the chamber, which we have already accounted for elsewhere. This means that an analysis based on the measured data curve in Figure 43 and Figure 44 will be significantly too conservative. Since we assume that the chamber characteristics and the antenna characteristics are smooth functions of frequency, a better approach is to base our analysis on the moving average curves of the same figures. For both sets of data presented in Figure 43, a standard uncertainty of $0.38 \mathrm{~dB}$ ( $95 \%$ of the smoothed data are within $0.76 \mathrm{~dB}$ of the estimated constant) seems to be adequate, and in Figure 44, a standard uncertainty of $0.25 \mathrm{~dB}(95 \%$ of the smoothed data are within $0.5 \mathrm{~dB}$ of the estimated constant) should be sufficient.

The estimated antenna uncertainties describe the antennas from $400 \mathrm{MHz}$ to $800 \mathrm{MHz}$, and from $2 \mathrm{GHz}$ to $18 \mathrm{GHz}$. It should be possible to extend the antenna characteristics and associated uncertainties for the log-periodic antennas from $800 \mathrm{MHz}$ up to $1 \mathrm{GHz}$, and extend the uncertainties for the ridged-horn antennas from $2 \mathrm{GHz}$ down to $1 \mathrm{GHz}$. This assumption is supported by the fact that the offset in the measured chamber gain at $1 \mathrm{GHz}$ and measured using the two antenna configurations is consistent with the extrapolated values. Below $400 \mathrm{MHz}$, however, we run into a problem. We have no data from a reliable reference antenna, so we cannot base our uncertainty estimates directly on measurements. We have additional information that come from an unexpected source: measurements of the maximum received power. At low frequencies, we know that the maximum received power should be approximately equal to the transmitted power, so the maximum chamber gain should be approximately $0 \mathrm{~dB}$. Since the same correction factor is applied to both the average received power and the maximum received power, we can verify that the correction factor is approximately correct by verifying that the maximum chamber gain (as shown in Figure 75, for example) is close to $0 \mathrm{~dB}$. Since this is the case, we are reasonably confident that the correction factor is close to the true correction factor. It 
is difficult to quantify what we mean by "close to," so we must rely on engineering judgment. Obviously, our uncertainty below $400 \mathrm{MHz}$ must be at least as large as our uncertainty between $400 \mathrm{MHz}$ and $800 \mathrm{MHz}$. But, since the maximum chamber gain is close to $0 \mathrm{~dB}$ at low frequencies, the uncertainty should not be too large. For these reasons, we think that our estimate of the antenna characteristics is within $\pm 1 \mathrm{~dB}$ of the true value with $95 \%$ confidence, or our standard uncertainty about the antenna characteristics is $\pm 0.5 \mathrm{~dB}$. Fortunately (depending on your point of view), as shown above, the chamber uncertainty is large at low frequencies, so even if our estimate of the uncertainty in the antenna effects is too small, it will not have a significant effect on the total combined uncertainty.

Our final estimate for the instrumentation uncertainty for repeated measurements using an automatic network analyzer is given below in Table 15. Obviously, we need to improve our antenna characterization procedures so that the instrumentation uncertainty will not be as large as presented here, but this will take substantial research.

We were not able to make repeated measurements in all chambers, so we must also estimate the uncertainty for a single set of measurements using a vector network analyzer in a chamber. The analysis is essentially the same as given above with one exception. We noticed that the standard deviation of multiple measurements increased between $10 \mathrm{GHz}$ and $18 \mathrm{GHz}$, but the standard deviation estimated from a single measurement using the moving standard deviation technique did not show a similar increase. We can only assume that the difference is caused by repositioning the antennas and cables, and therefor must increase the component of uncertainty related to cable repeatability. We base our estimate on the data shown in Figure 135, where the increase in the observed standard deviation is consistent with an uncertainty in the cable repeatability of $0.3 \mathrm{~dB}$.

We next examine the uncertainty in chamber gain measurements using the probe system. Several components are similar to those discussed above. One difference comes from the fact that the system components (cables, attenuators, couplers, etc.) are calibrated separately, and the combined calibration factors are computed from these individual calibrations. Assuming that the network analyzer used to calibrate the components is similar to the one described above, the standard uncertainty in each calibration is $0.1 \mathrm{~dB}$, and the uncertainty in the combined calibration

Table 15. Instrumentation uncertainty for repeated measurements of chamber gain using ANA. All uncertainties are type B.

\begin{tabular}{lccc}
\hline & $\begin{array}{c}80 \mathrm{MHz} \\
\text { to } \\
400 \mathrm{MHz}\end{array}$ & $\begin{array}{c}400 \mathrm{MHz} \\
\text { to } \\
1 \mathrm{GHz}\end{array}$ & $\begin{array}{c}1 \mathrm{GHz} \\
\text { to } \\
18 \mathrm{GHz}\end{array}$ \\
\hline Cal accuracy & 0.10 & 0.10 & 0.10 \\
Connector repeatability & 0.10 & 0.10 & 0.10 \\
Cable repeatability & 0.10 & 0.10 & 0.10 \\
Antenna effects & 0.50 & 0.38 & 0.25 \\
\hline Standard unc. & & & \\
\hline \hline Expanded unc. $\mathrm{K}=2$ & 0.53 & 0.41 & 0.30 \\
\hline
\end{tabular}


Table 16. Instrumentation uncertainty for a single measurement of chamber gain using ANA.

\begin{tabular}{lcccc}
\hline & $\begin{array}{c}80 \mathrm{MHz} \\
\text { to } \\
400 \mathrm{MHz}\end{array}$ & $\begin{array}{c}400 \mathrm{MHz} \\
\text { to } \\
1 \mathrm{GHz}\end{array}$ & $\begin{array}{c}1 \mathrm{GHz} \\
\text { to } \\
10 \mathrm{GHz}\end{array}$ & $\begin{array}{c}10 \mathrm{GHz} \\
\text { to } \\
18 \mathrm{GHz}\end{array}$ \\
\hline Cal accuracy & 0.10 & 0.10 & 0.10 & 0.10 \\
Connector repeatability & 0.10 & 0.10 & 0.10 & 0.10 \\
Cable repeatability & 0.10 & 0.10 & 0.10 & 0.30 \\
Antenna effects & 0.50 & 0.38 & 0.25 & 0.25 \\
\hline Standard unc. & 0.53 & 0.41 & 0.30 & 0.42 \\
\hline \hline Expanded unc. $\mathrm{K}=2$ & 1.06 & 0.83 & 0.61 & 0.83 \\
\hline
\end{tabular}

of $N$ individual components is $0.1 \sqrt{N} \mathrm{~dB}$. The probe system typically used six components, for a component calibration uncertainty of $0.25 \mathrm{~dB}$. The connector and cable repeatability are identical to that presented in Table 16.

In additional to the uncertainty in the components, the uncertainty in the instruments used to measure the characteristics have a significant uncertainty. The power meters used in the measurements are very good, and we assign them a standard uncertainty of $0.1 \mathrm{~dB}$. The spectrum analyzer used in the measurements is not very accurate. Even though we calibrated the spectrum analyzer against a standard power meter, we encountered two problems: the calibrations would change over time (stability), and even though we could remove obvious biases or trends in the calibration as a function of frequency, the calibration remained noisy, even for repeated at short time intervals (cal). Based on repeated evaluations of the spectrum analyzer used in the measurements, the calibrations had a standard deviation of $0.25 \mathrm{~dB}$ below $12 \mathrm{GHz}$, but above 12 $\mathrm{GHz}$, the calibration did not significantly improve the accuracy of the measurements. At these frequencies, the observed standard deviation was $0.5 \mathrm{~dB}$.

The uncertainty in the antenna effects were identical to those given in Table 16, but other effects related to the antennas caused additional uncertainty. Due to the way in which the net transmitted power was calculated, we were forced to assume that all reflected power measured at the directional coupler was due only to reflections at the antenna test port. If any portion of the reflections were actually caused by mismatches in the connecting cables, then estimates of the transmitted power could have significant errors. These errors will be most pronounced if the cables used in the measurement are poorly matched or are very lossy (our cable had good characteristics both before and after these measurements, so this should not be the case), or if the reflections measured at the antenna test port are large (as was the case for measurements below $175 \mathrm{MHz}$ ). Thus we must include the possibility that estimates of transmitted power could be uncertain (standard uncertainty of $0.5 \mathrm{~dB}$ ) below $175 \mathrm{MHz}$. Above $175 \mathrm{MHz}$, a standard uncertainty of $0.25 \mathrm{~dB}$ is sufficient to cover any expected uncertainty. Additionally, reflections in the receiving antenna could result in underestimating the received power. We could have applied corrections based on the network analyzer measurements in the chamber, but since the test conditions were not identical for both measurement systems (antenna positions were different), such a correction may not be valid. We do, however, estimate the uncertainty due to neglecting 
the mismatch characteristics of the receiving antenna by examining the mismatch correction data calculated from network analyzer measurements and presented in Figure 69, and Figure 91. Below $175 \mathrm{MHz}$ in Chamber A and below $400 \mathrm{MHz}$ in Chamber B, the mismatch correction factor can be quite large (greater than $3.5 \mathrm{~dB}$ ). Estimating the uncertainty associated with neglecting the reflection characteristics of the receiving antenna is somewhat difficult. Assuming well matched cables and instrumentation, a poorly matched receiving antenna will cause us to underestimate the power that would have been received by a perfectly matched antenna. Because of this, any errors will be one-sided (uncertainty is not symmetric about some mean value), and it is not fair (or at least overly conservative) to claim that the uncertainties are $\pm 3.5 \mathrm{~dB}$ below 175 $\mathrm{MHz}$. A better approach might be to increase the measured values so that the uncertainty bounds are symmetric, and this would allow us to reduce our estimated uncertainties by a factor of 2 (for example, increase the measured values of received power below $175 \mathrm{MHz}$ by $1.75 \mathrm{~dB}$, and then claim an uncertainty of $\pm 1.75 \mathrm{~dB}$. Unfortunately, this approach would result in discontinuities at the selected break points in measurements of received power, and this is even less appealing than large uncertainties. Therefore, we will accept the large uncertainties, and estimate these uncertainties by constructing a piecewise constant envelope that contains the majority of the data shown in Figure 69 and Figure 91. We will assume that approximately $95 \%$ of the estimated mismatch correction factors fall within this envelope, and we can estimate the standard uncertainty as one half of the uncertainty given by the envelope. For example, we assume that $95 \%$ of the estimated mismatch correction factors below $175 \mathrm{MHz}$ are less than $3.5 \mathrm{~dB}$, and we can assume a standard uncertainty of $\pm 1.75 \mathrm{~dB}$ at these low frequencies. This is an extremely conservative estimate and demonstrates the importance of additional research into characterizing and reducing the uncertainties related to the antennas used in the chamber evaluation. The instrumentation uncertainty associated with measurements of received power are summarized in Table 17.

Table 17. Instrumentation uncertainty for measurements of received power using probe system.

\begin{tabular}{lccccccc}
\hline & $\begin{array}{c}80 \mathrm{MHz} \\
\text { to } \\
175 \mathrm{MHz}\end{array}$ & $\begin{array}{c}175 \mathrm{MHz} \\
\text { to } \\
400 \mathrm{MHz}\end{array}$ & $\begin{array}{c}400 \mathrm{MHz} \\
\text { to } \\
1 \mathrm{GHz}\end{array}$ & $\begin{array}{c}1 \mathrm{GHz} \\
\text { to } \\
5 \mathrm{GHz}\end{array}$ & $\begin{array}{c}5 \mathrm{GHz} \\
\text { to } \\
10 \mathrm{GHz}\end{array}$ & $\begin{array}{c}10 \mathrm{GHz} \\
\text { to }\end{array}$ & $\begin{array}{c}12 \mathrm{GHz} \\
\text { to }\end{array}$ \\
\hline Component cals & 0.25 & 0.25 & 0.25 & 0.25 & 0.25 & 0.25 & 0.25 \\
Connector repeat & 0.10 & 0.10 & 0.10 & 0.10 & 0.10 & 0.10 & 0.10 \\
Cable repeatability & 0.10 & 0.10 & 0.10 & 0.10 & 0.10 & 0.30 & 0.30 \\
Power meter cals & 0.10 & 0.10 & 0.10 & 0.10 & 0.10 & 0.10 & 0.10 \\
Transmit mismatch & 0.50 & 0.25 & 0.25 & 0.25 & 0.25 & 0.25 & 0.25 \\
SA cal & 0.25 & 0.25 & 0.25 & 0.25 & 0.25 & 0.25 & 0.50 \\
SA stability & 0.10 & 0.10 & 0.10 & 0.10 & 0.38 & 0.38 & 0.38 \\
Antenna effects & 0.50 & 0.50 & 0.38 & 0.38 & 0.25 & 0.25 & 0.25 \\
Rcv ant mismatch & 1.75 & 1.25 & 0.25 & 0.50 & 0.15 & 0.15 & 0.50 \\
\hline & & & & & & & \\
Standard unc. & 1.93 & 1.43 & 0.65 & 0.82 & 0.66 & 0.72 & 0.97 \\
\hline Expanded unc. $\mathrm{K}=2$ & 3.86 & 2.85 & 1.31 & 1.63 & 1.33 & 1.44 & 1.93 \\
\hline
\end{tabular}


Table 18. Instrumentation uncertainty for measurements of electric field using probe system.

\begin{tabular}{|c|c|c|c|c|c|c|c|}
\hline & $\begin{array}{c}80 \mathrm{MHz} \\
\text { to } \\
175 \mathrm{MHz}\end{array}$ & $\begin{array}{c}175 \mathrm{MHz} \\
\text { to } \\
400 \mathrm{MHz}\end{array}$ & $\begin{array}{c}400 \mathrm{MHz} \\
\text { to } \\
1 \mathrm{GHz}\end{array}$ & $\begin{array}{c}1 \mathrm{GHz} \\
\text { to } \\
5 \mathrm{GHz}\end{array}$ & $\begin{array}{c}5 \mathrm{GHz} \\
\text { to } \\
10 \mathrm{GHz}\end{array}$ & $\begin{array}{c}10 \mathrm{GHz} \\
\text { to } \\
12 \mathrm{GHz}\end{array}$ & $\begin{array}{c}12 \mathrm{GHz} \\
\text { to } \\
18 \mathrm{GHz}\end{array}$ \\
\hline Component cals & 0.20 & 0.20 & 0.20 & 0.20 & 0.20 & 0.20 & 0.20 \\
\hline Connector repeat & 0.10 & 0.10 & 0.10 & 0.10 & 0.10 & 0.10 & 0.10 \\
\hline Cable repeatability & 0.10 & 0.10 & 0.10 & 0.10 & 0.10 & 0.30 & 0.30 \\
\hline Power meter cals & 0.10 & 0.10 & 0.10 & 0.10 & 0.10 & 0.10 & 0.10 \\
\hline Transmit mismatch & 0.50 & 0.25 & 0.25 & 0.25 & 0.25 & 0.25 & 0.25 \\
\hline Antenna effects & 0.25 & 0.25 & 0.20 & 0.10 & 0.10 & 0.10 & 0.10 \\
\hline Probe cals & 0.50 & 0.50 & 0.50 & 0.50 & 0.50 & 0.50 & 0.50 \\
\hline Standard unc. & 0.80 & 0.67 & 0.65 & 0.63 & 0.63 & 0.69 & 0.69 \\
\hline Expanded unc. $K=2$ & 1.59 & 1.33 & 1.30 & 1.25 & 1.25 & 1.37 & 1.37 \\
\hline
\end{tabular}

The instrumentation uncertainty in measurements of electric field using the probe system are similar to those of received power, with a few minor exceptions. First, since we are not measuring received power, all terms related to the spectrum analyzer, the receiving antenna, and the connecting components (two fewer cables result in a lower uncertainty for the component calibration) will not be present. Instead, the only uncertainty associated with the receiving side of the system is the uncertainty in the calibration of the probes, which is $\pm 1 \mathrm{~dB}$ with $95 \%$ confidence, or a standard uncertainty of $\pm 0.5 \mathrm{~dB}$. This results in a substantially lower expanded uncertainty for measurements using the probes than with the reference antenna. This is especially true at low frequencies. The instrumentation uncertainty associated with measurements of received power are summarized in Table 18.

\subsection{Combined Uncertainty}

Given the estimated uncertainties for each component, we can now estimate the combined uncertainty of each measurement. Although it would have been possible to describe the combined uncertainty with only two components, the observed uncertainty and the instrumentation uncertainty, we thought that splitting the observed uncertainty into its two components was more descriptive. 


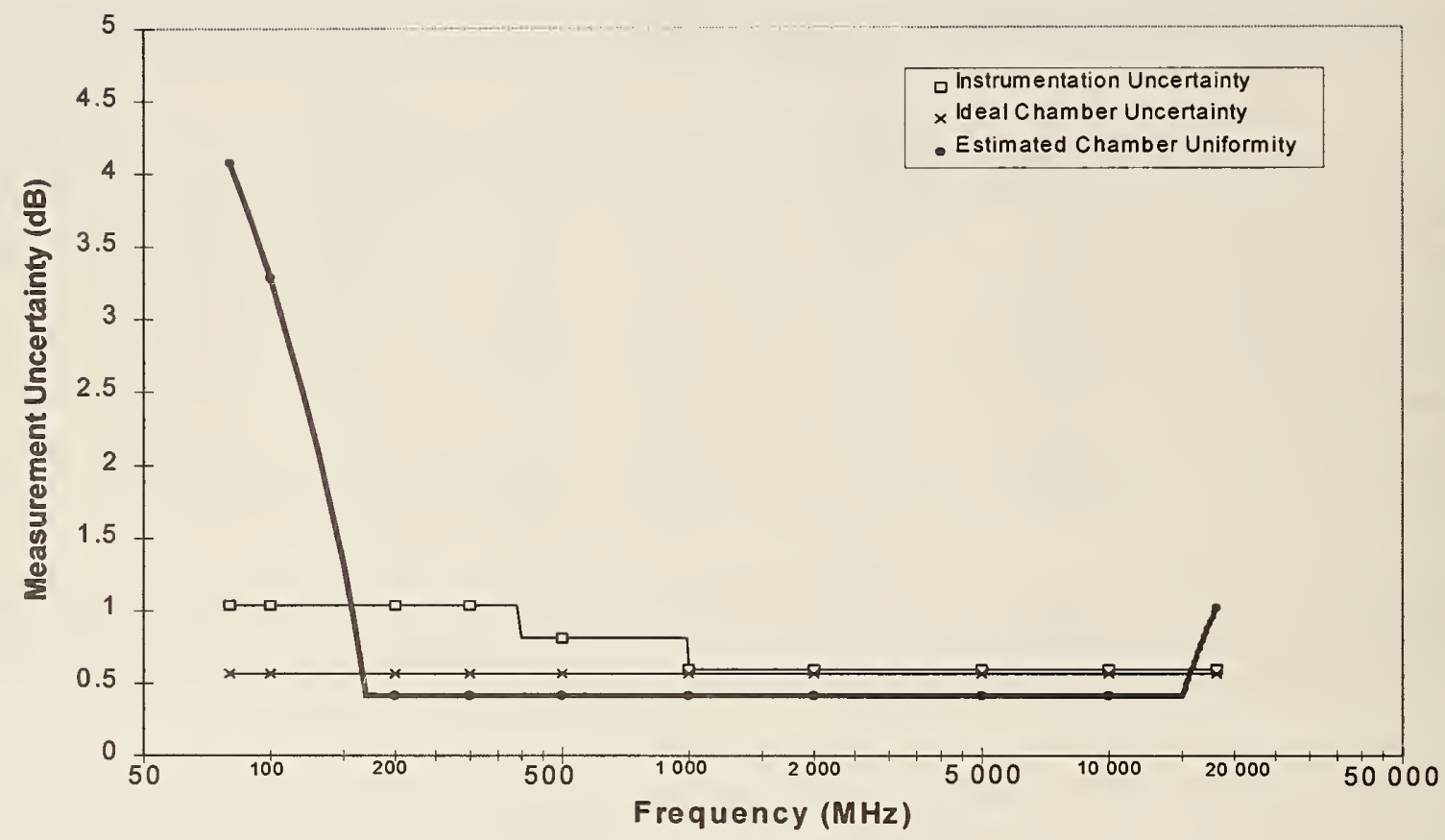

(a)

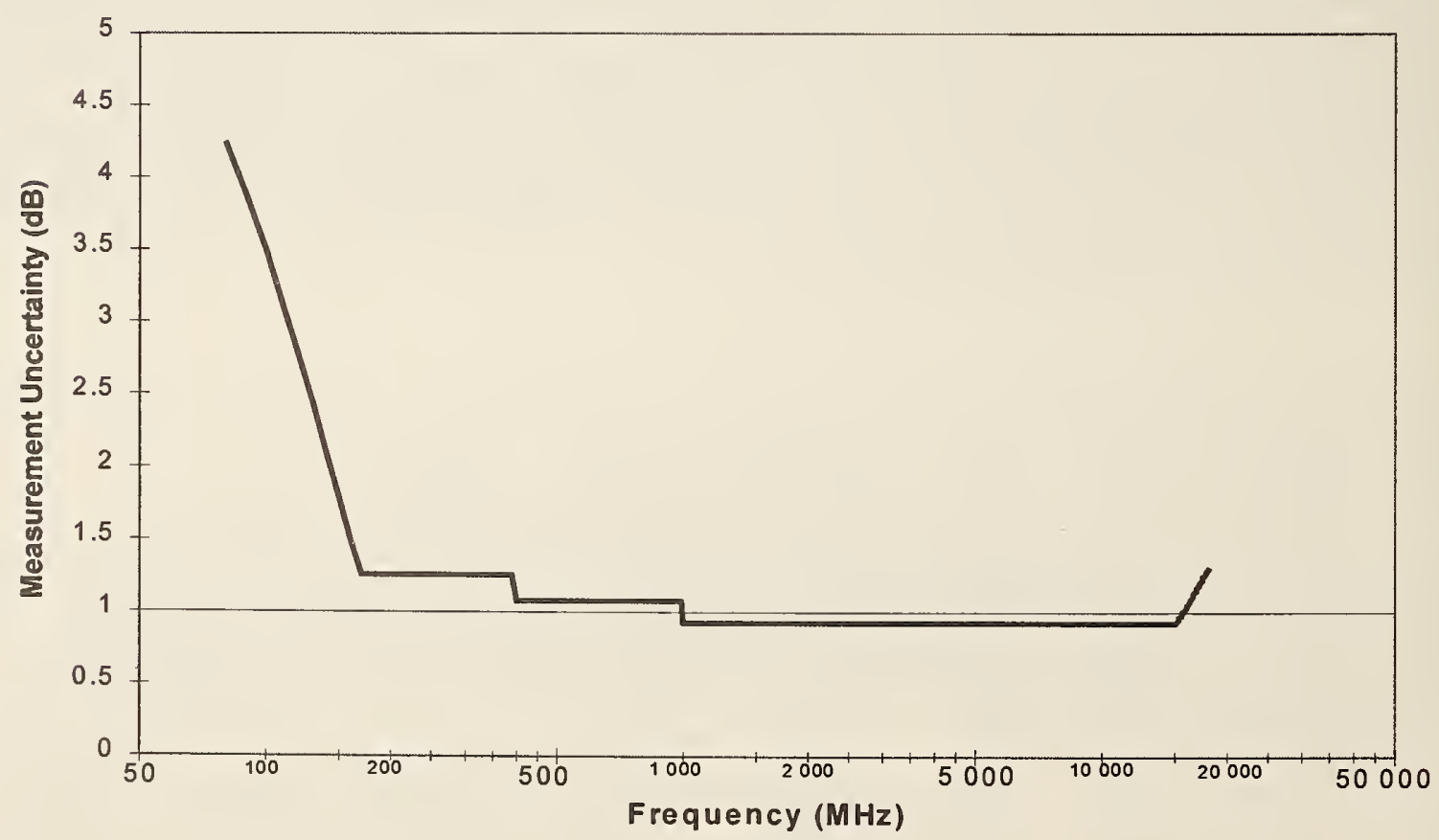

(b)

Figure 143. Uncertainty ( $95 \%$ confidence level) in measurements of average received power at multiple locations in chamber A using vector network analyzer. (a) Three main components of the measurement uncertainty. (b) Combined uncertainty. 


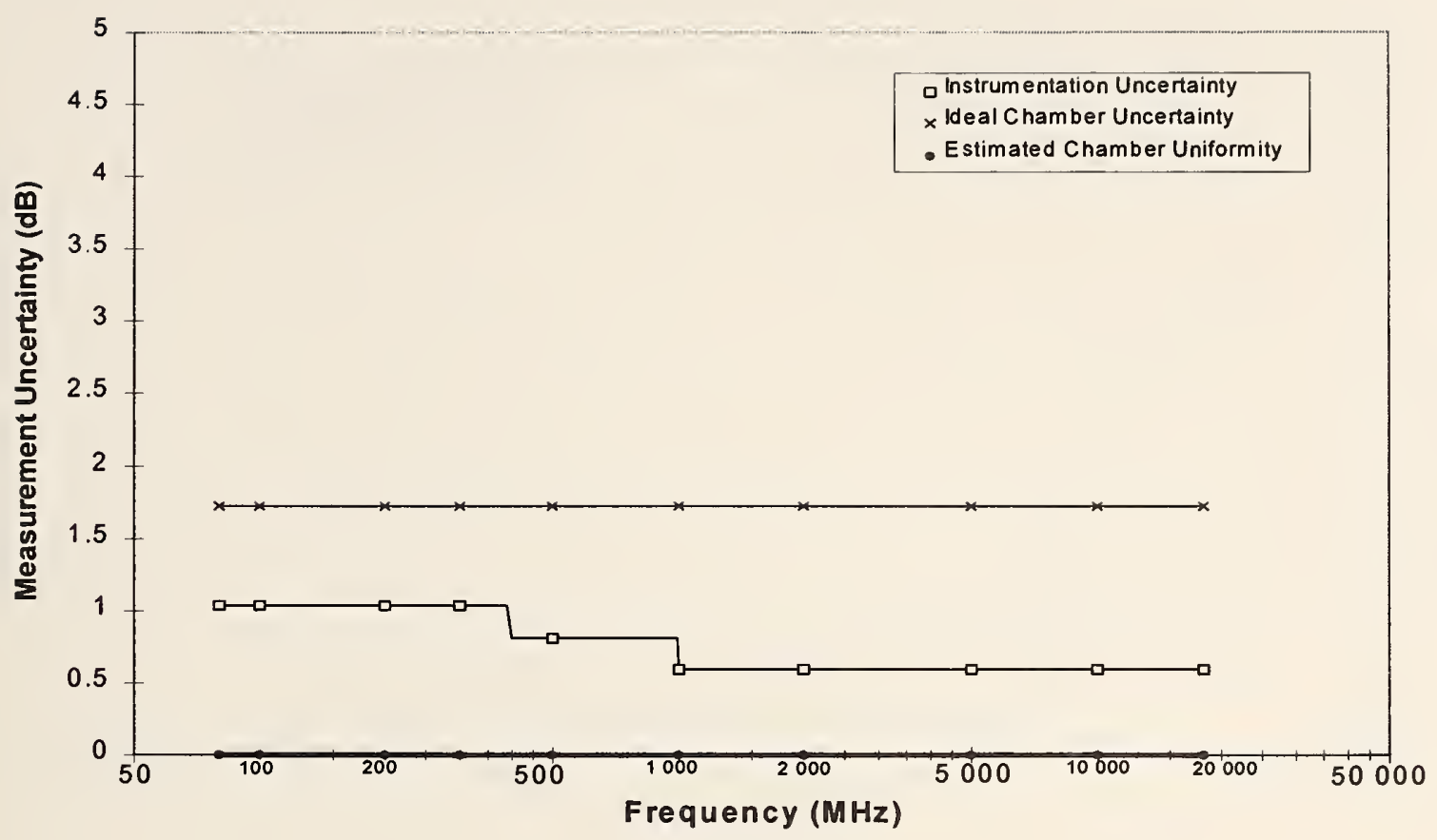

(a)

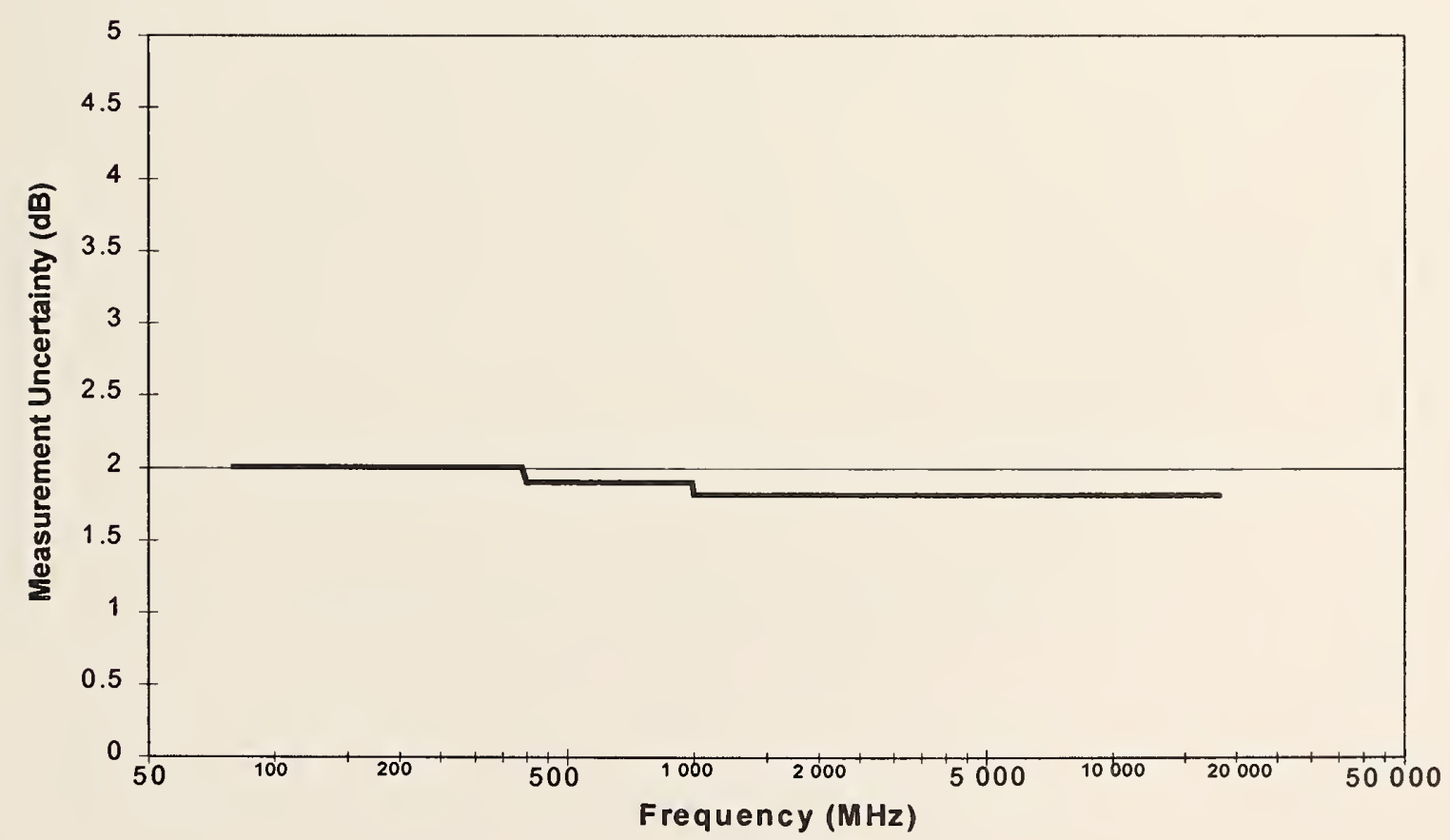

(b)

Figure 144. Uncertainty ( $95 \%$ confidence level) in measurements of maximum received power at multiple locations in chamber A using vector network analyzer. (a) Three main components of the measurement uncertainty. (b) Combined uncertainty. 


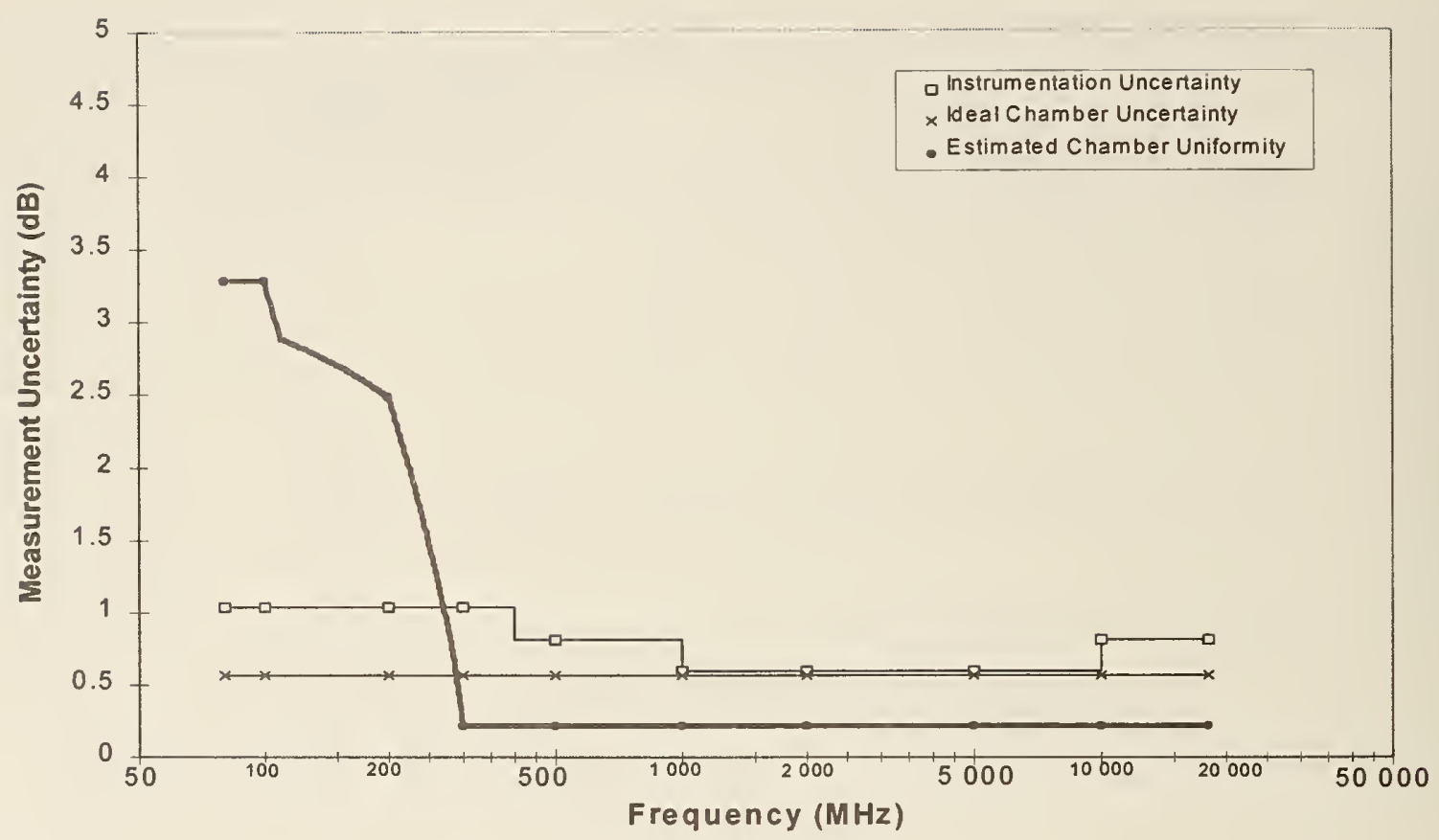

(a)

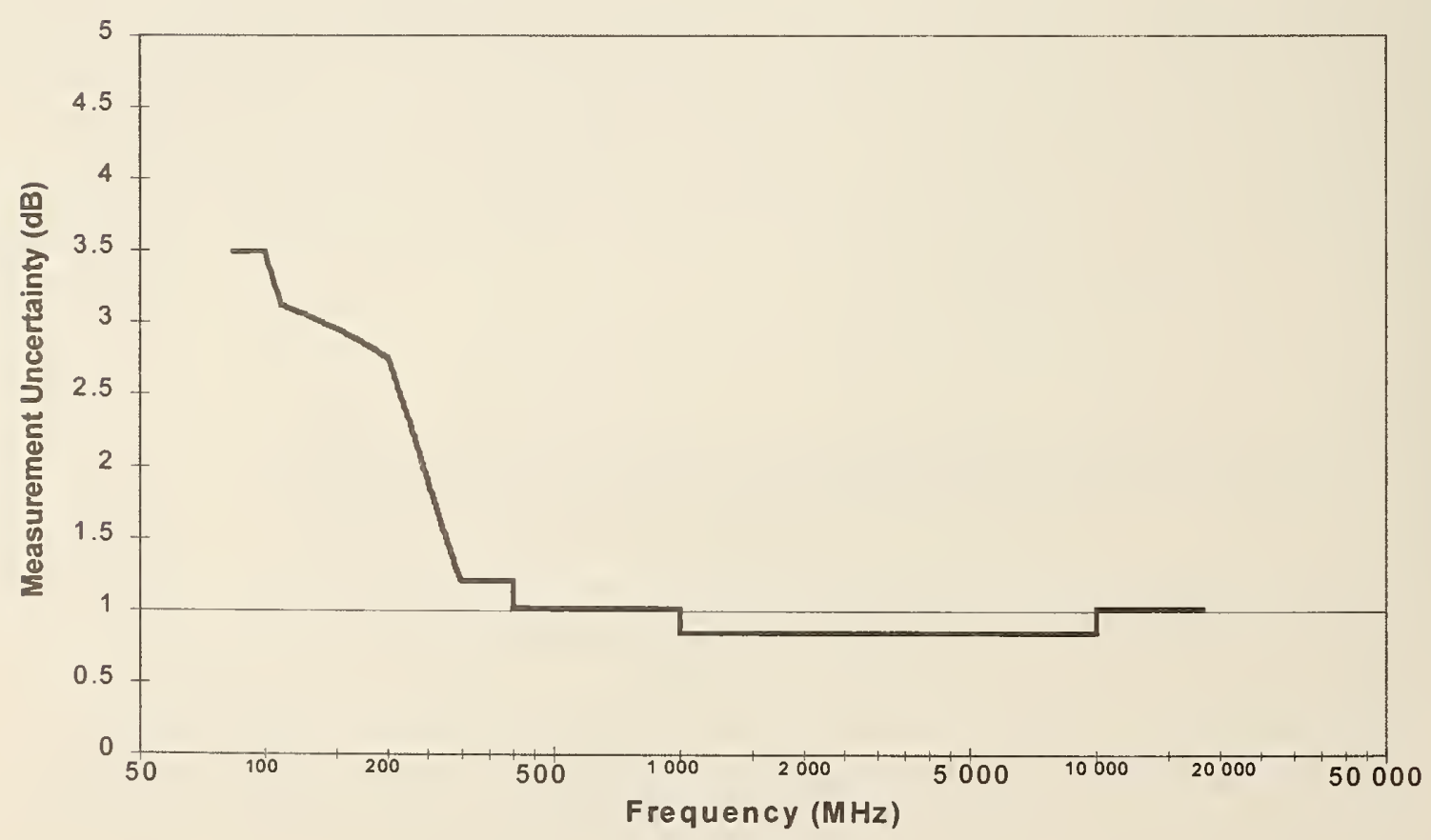

(b)

Figure 145. Uncertainty ( $95 \%$ confidence level) in measurements of average received power using "moving standard deviation" technique in chamber A using vector network analyzer. (a) Three main components of the measurement uncertainty.

(b) Combined uncertainty. 


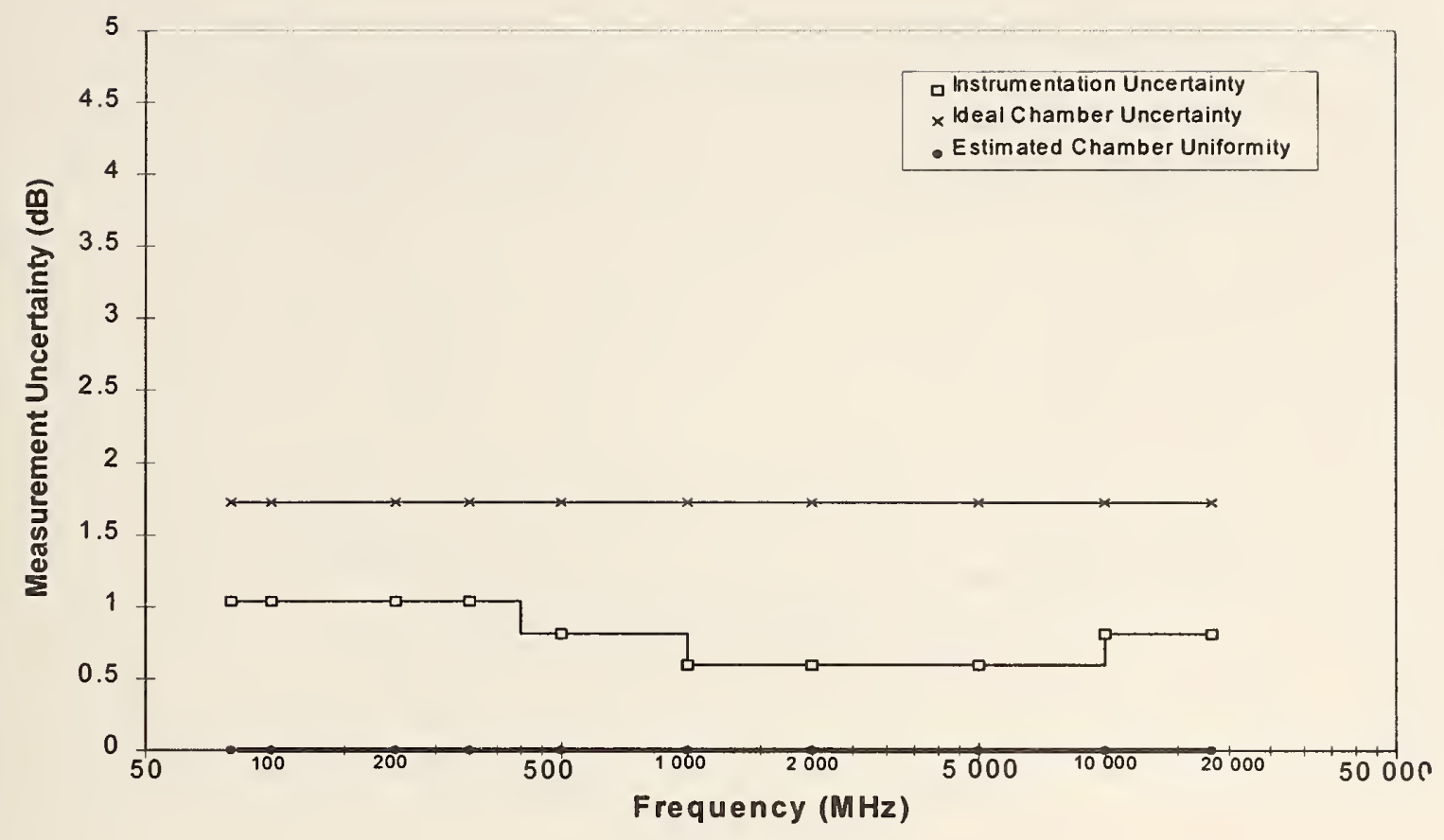

(a)

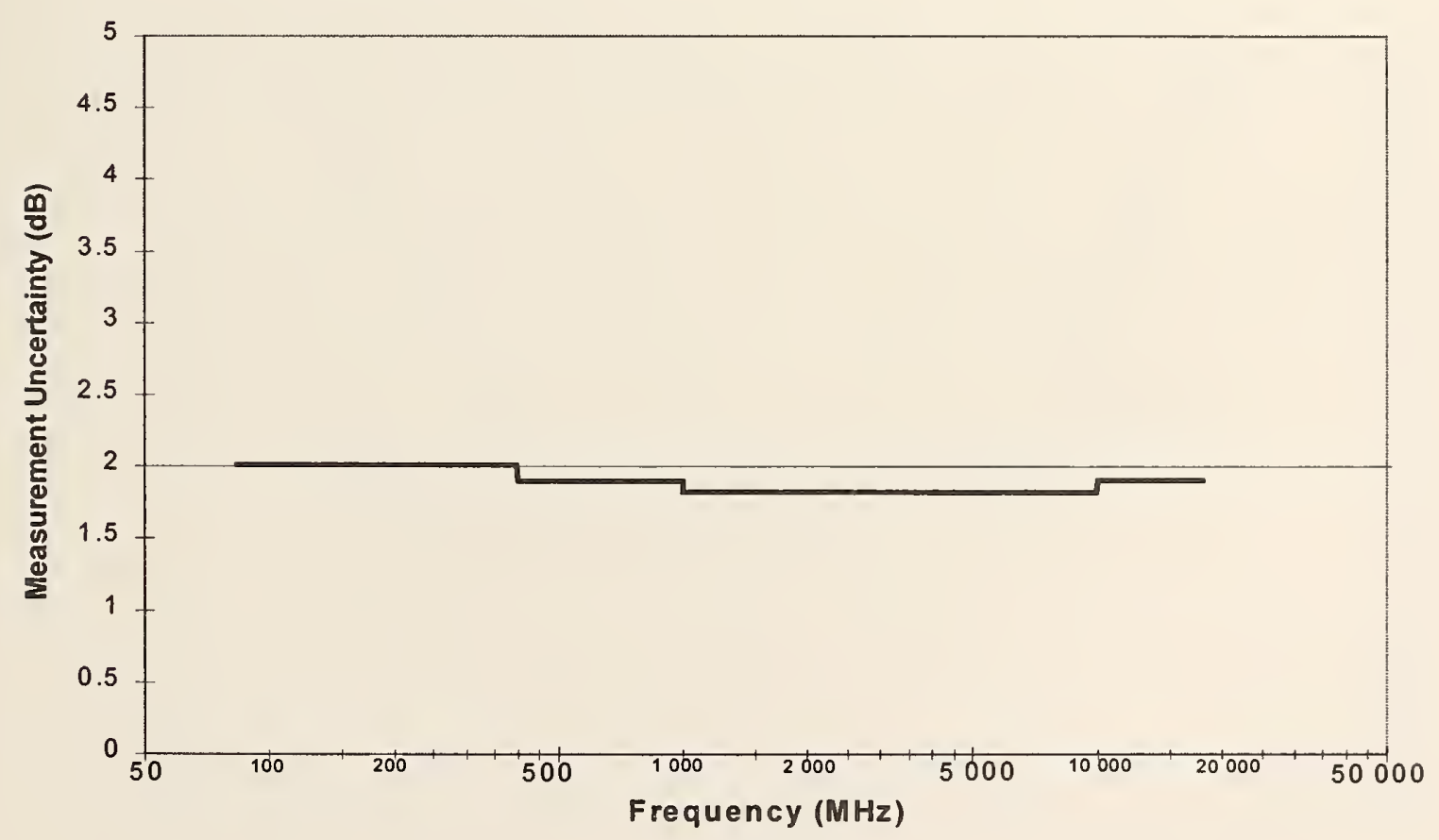

(b)

Figure 146. Uncertainty ( $95 \%$ confidence level) in measurements of maximum received power using "moving standard deviation" technique in chamber A using vector network analyzer. (a) Three main components of the measurement uncertainty.

(b) Combined uncertainty. 


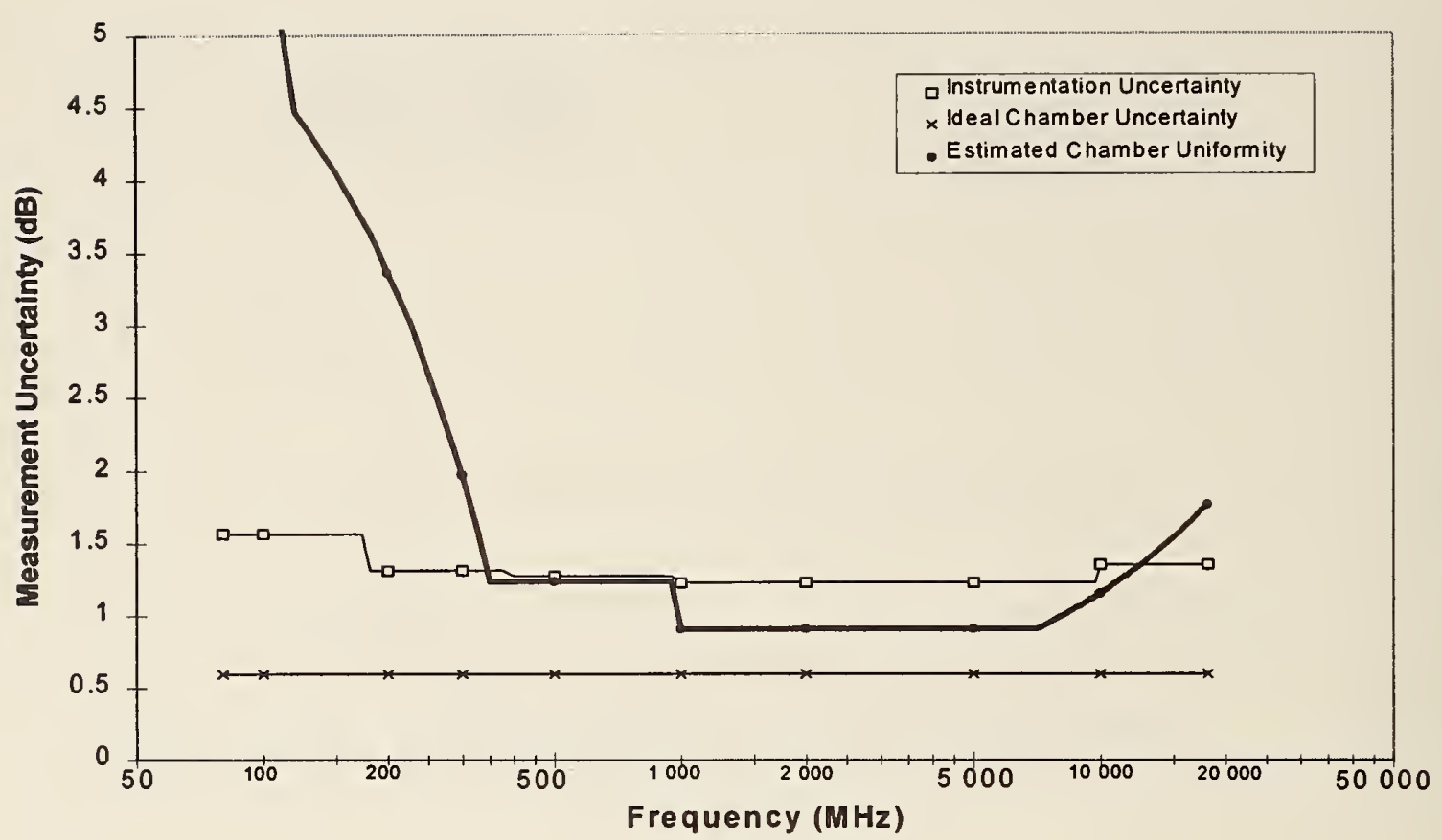

(a)

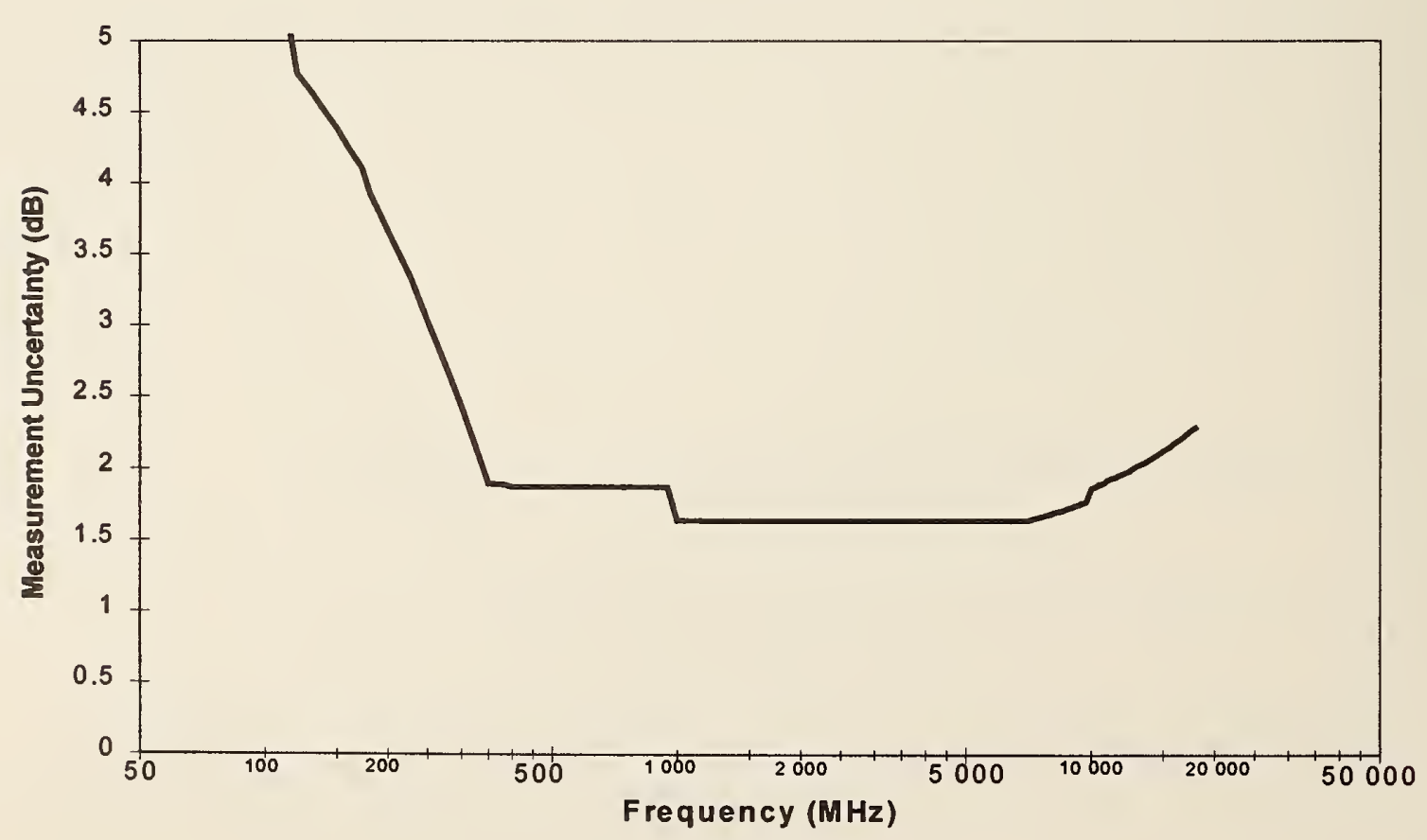

(b)

Figure 147. Uncertainty ( $95 \%$ confidence level) in measurements of average electric field (rectangular component) at multiple locations in chamber A. (a) Three main components of the measurement uncertainty. (b) Combined uncertainty. 


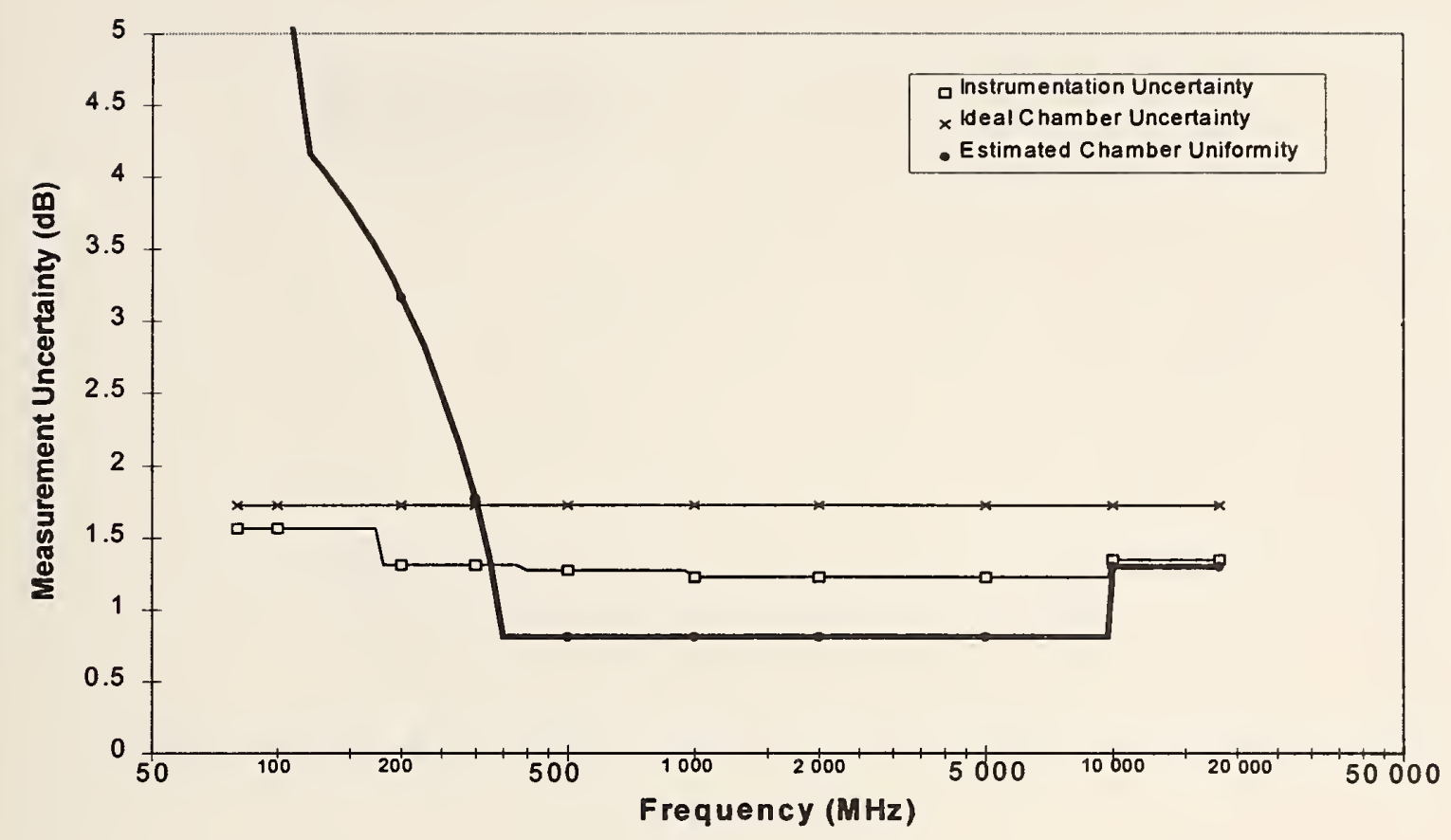

(a)

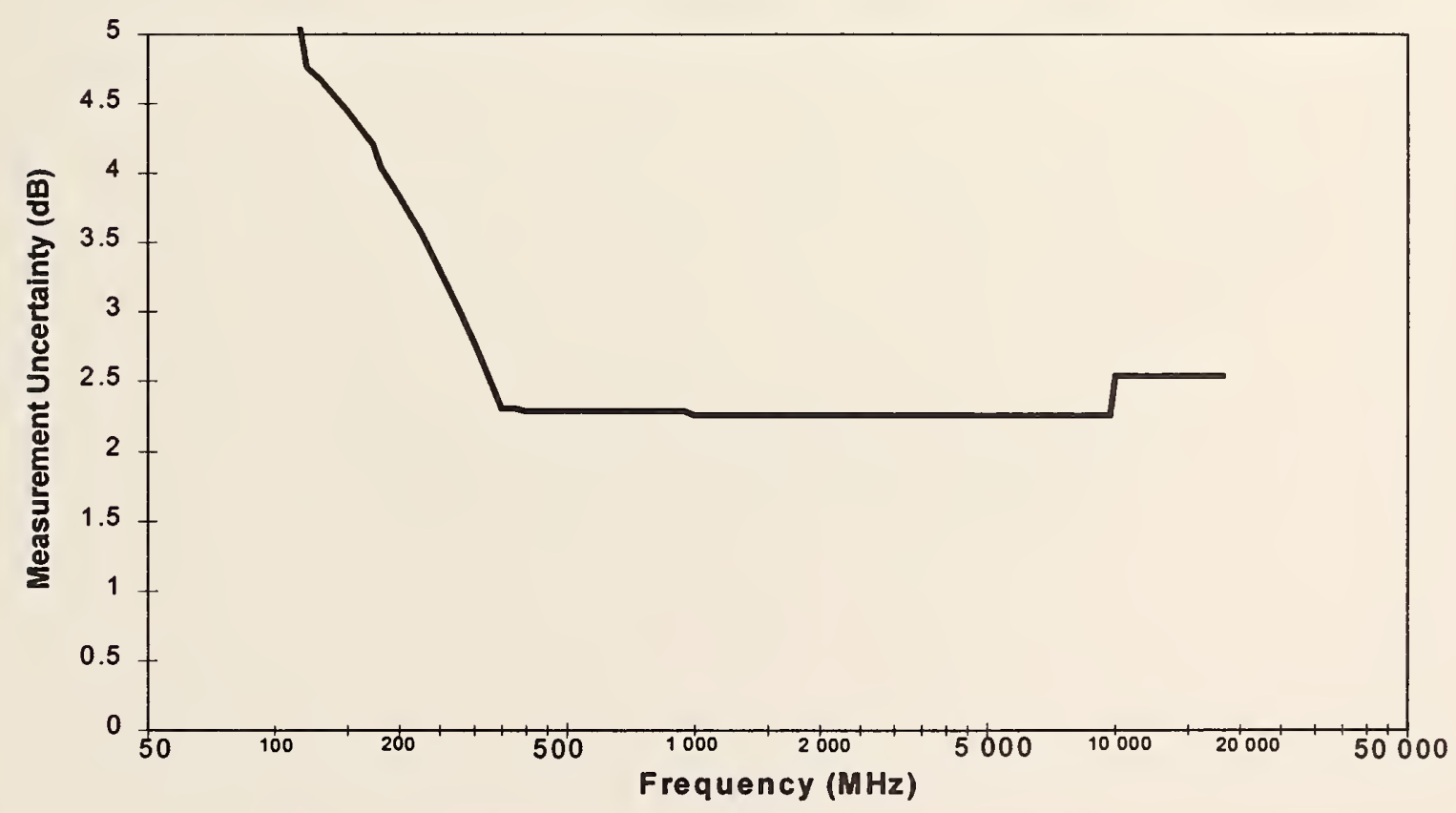

(b)

Figure 148. Uncertainty ( $95 \%$ confidence level) in measurements of maximum electric field (rectangular component) at multiple locations in chamber A. (a) Three main components of the measurement uncertainty. (b) Combined uncertainty. 


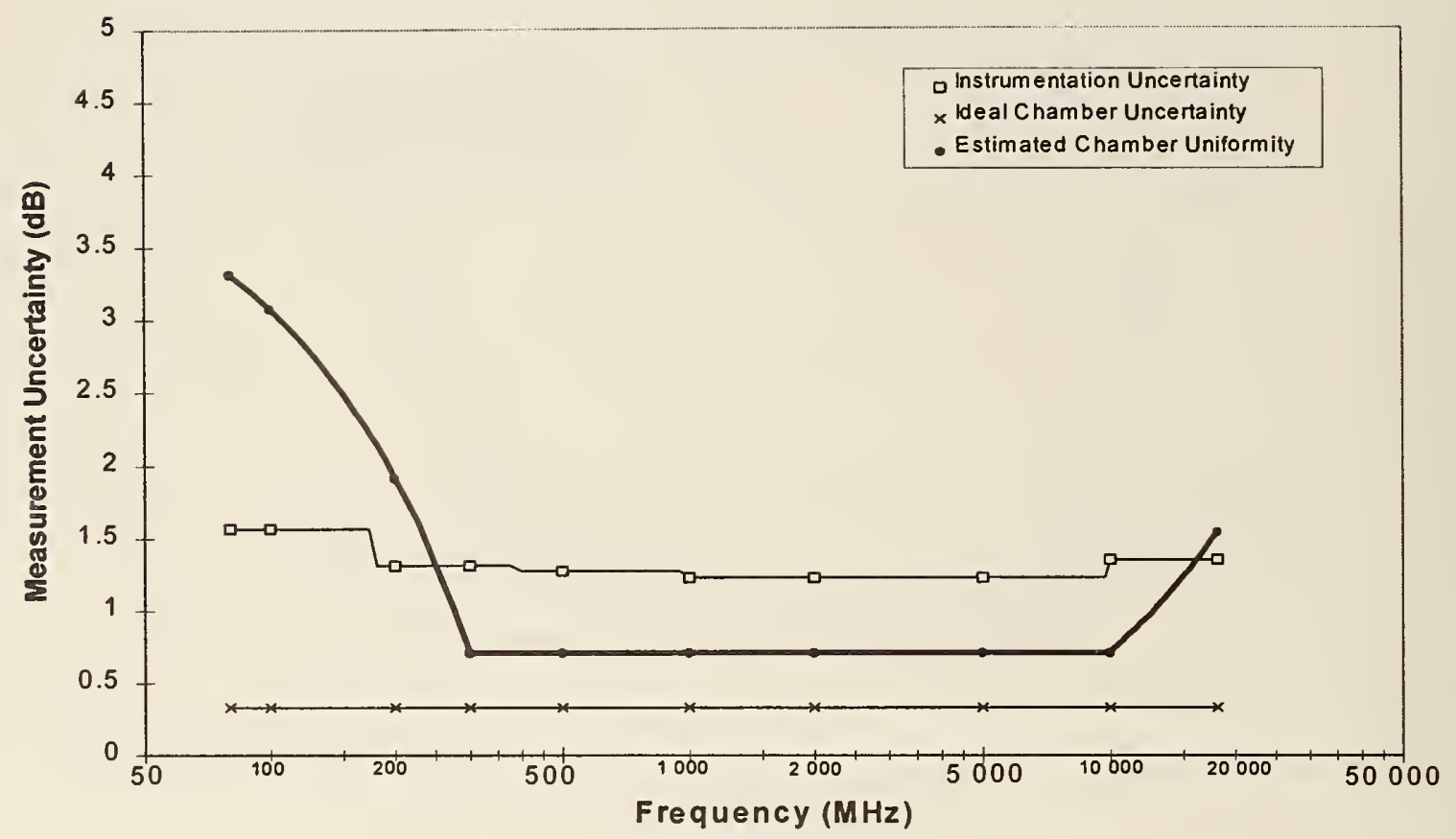

(a)

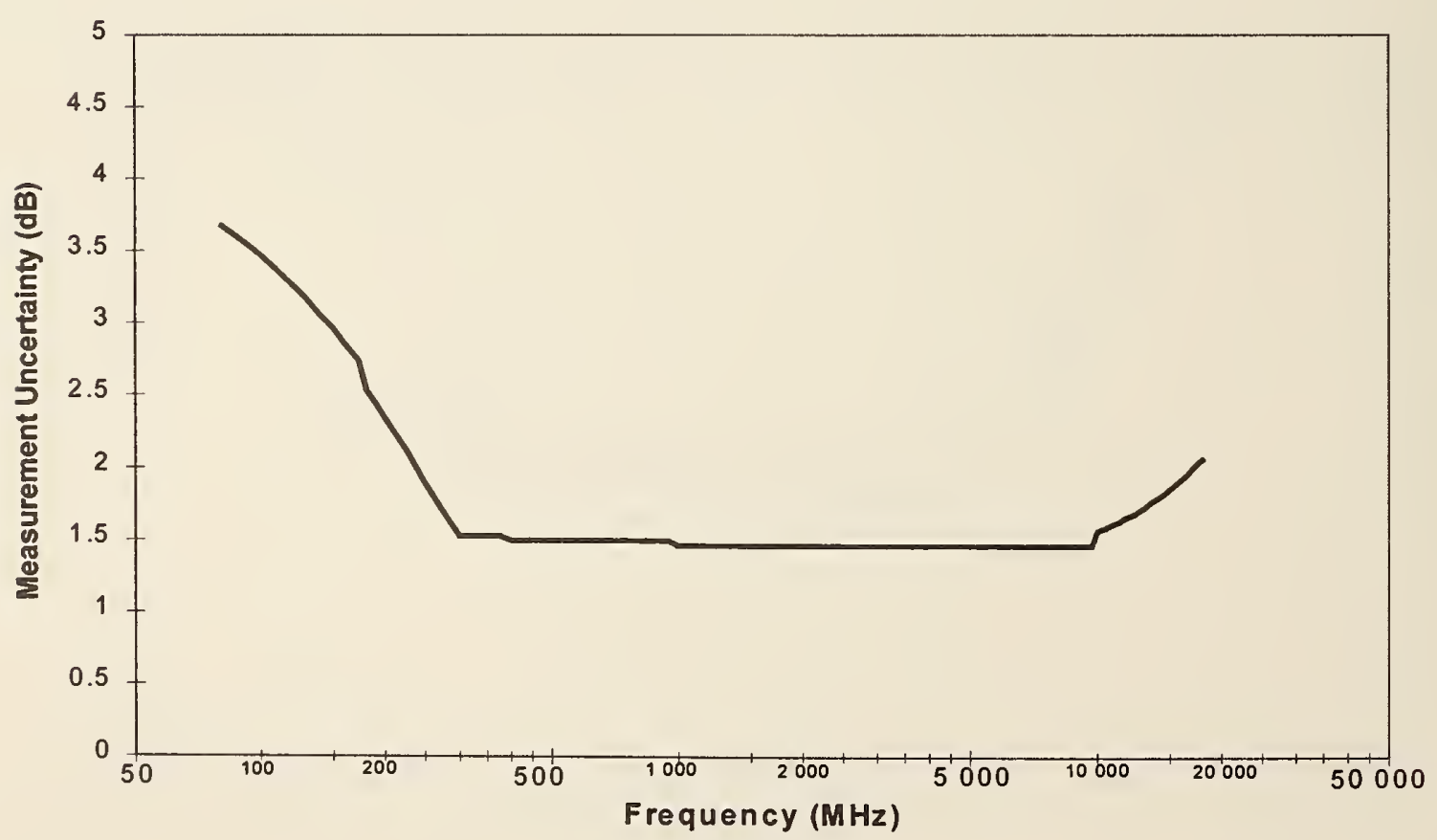

(b)

Figure 149. Uncertainty ( $95 \%$ confidence level) in measurements of average total electric field at multiple locations in chamber A. (a) Three main components of the measurement uncertainty. (b) Combined uncertainty. 


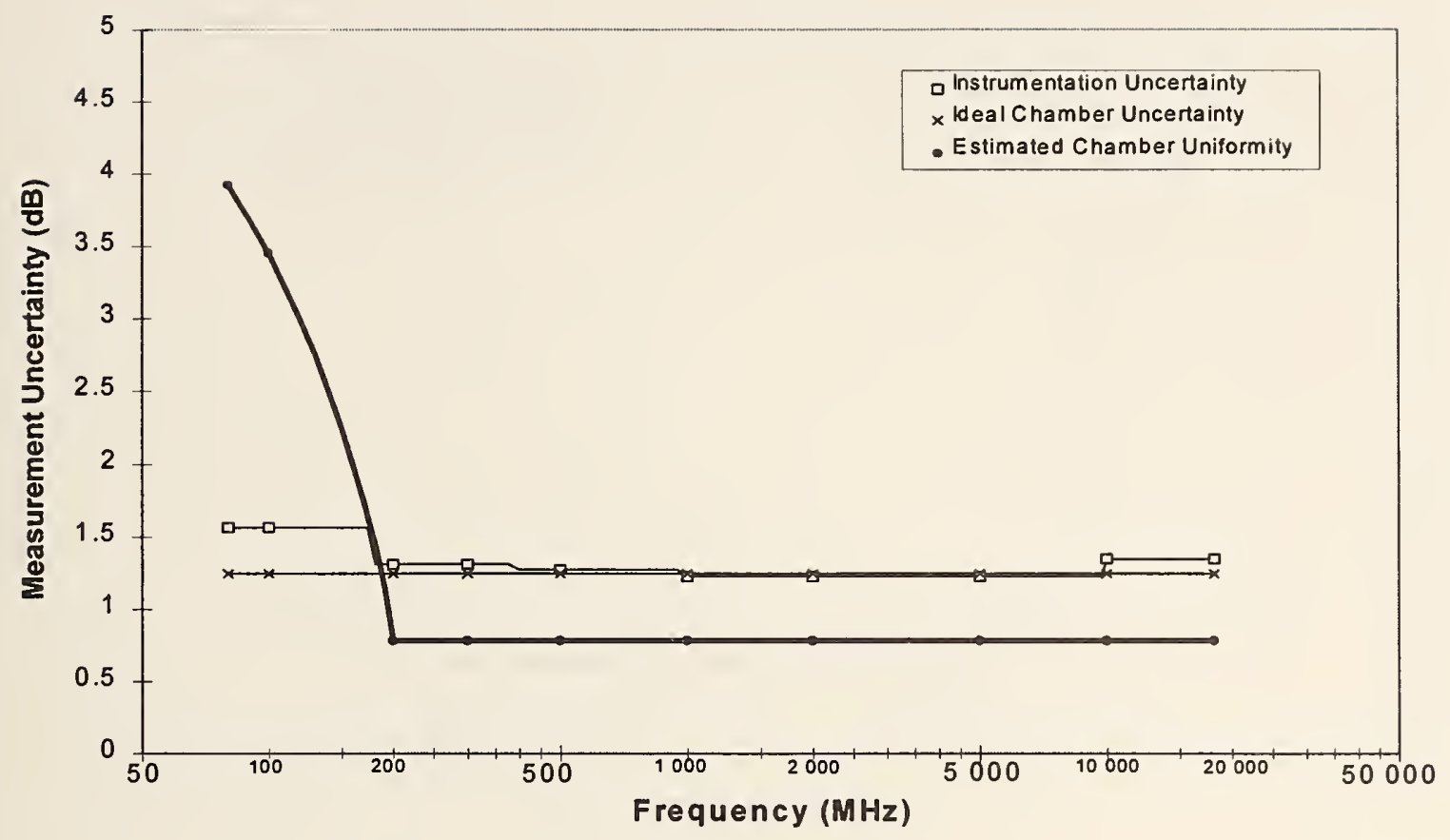

(a)

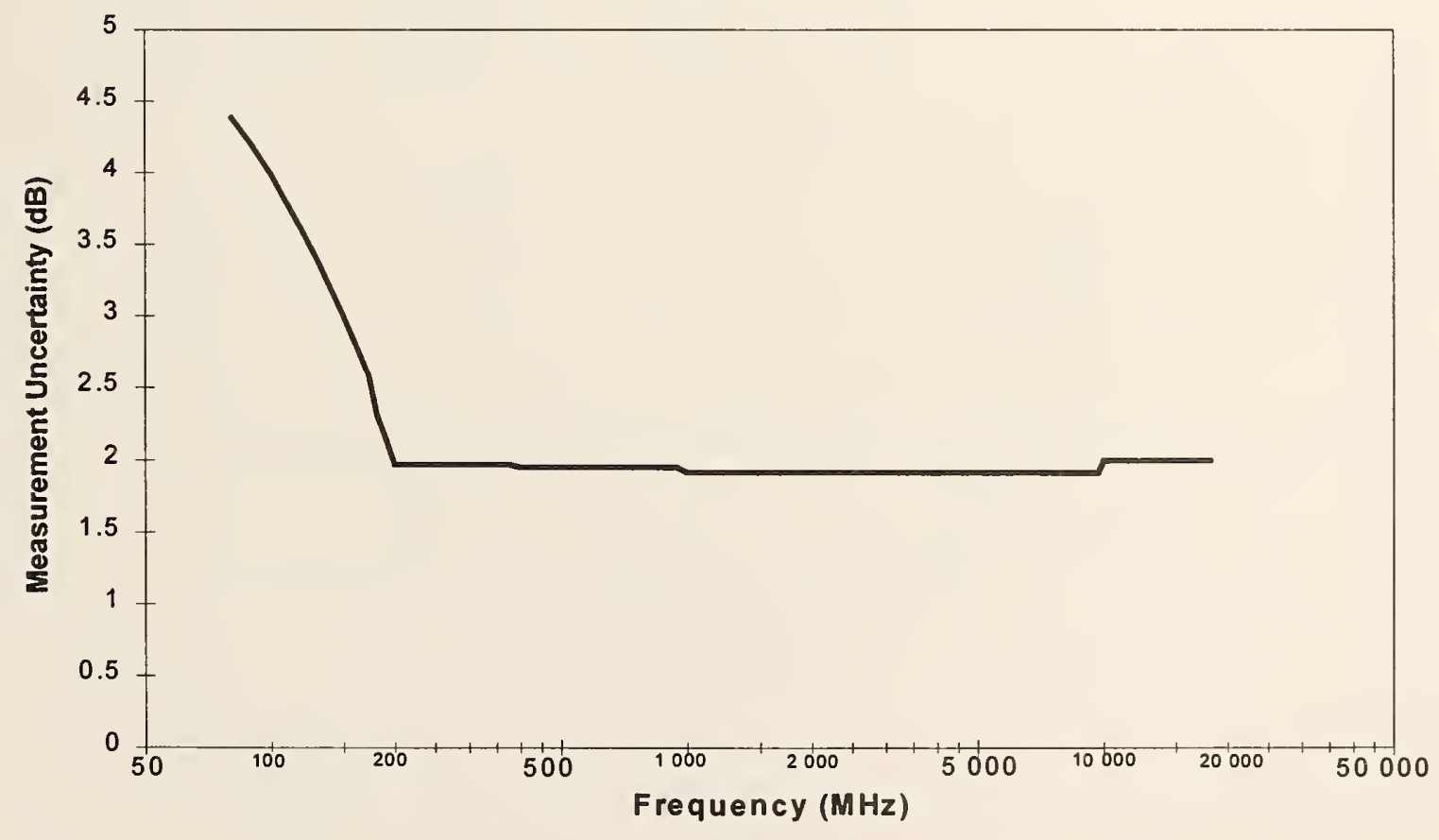

(b)

Figure 150. Uncertainty ( $95 \%$ confidence level) in measurements of maximum total electric field at multiple locations in chamber A. (a) Three main components of the measurement uncertainty. (b) Combined uncertainty. 


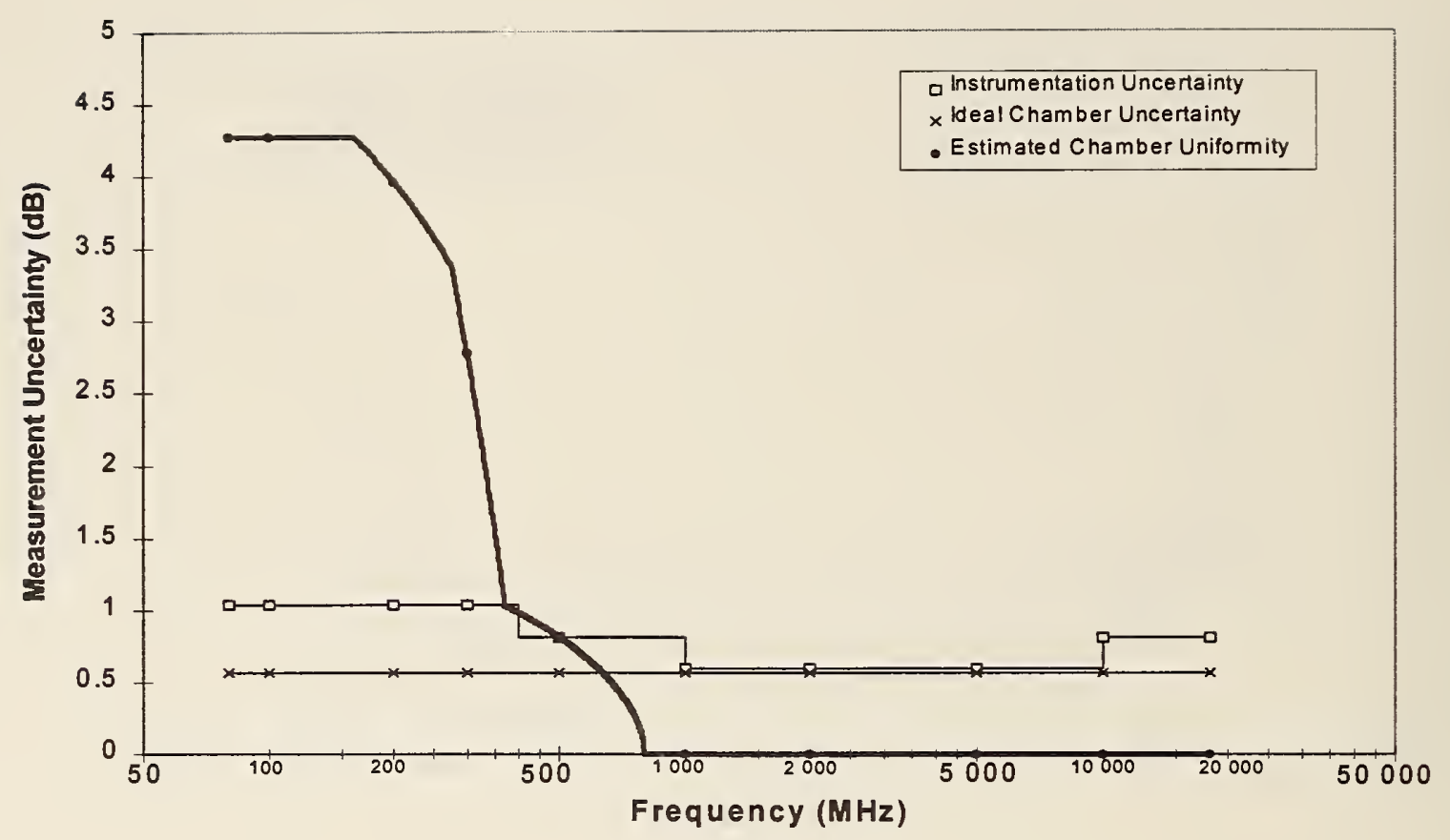

(a)

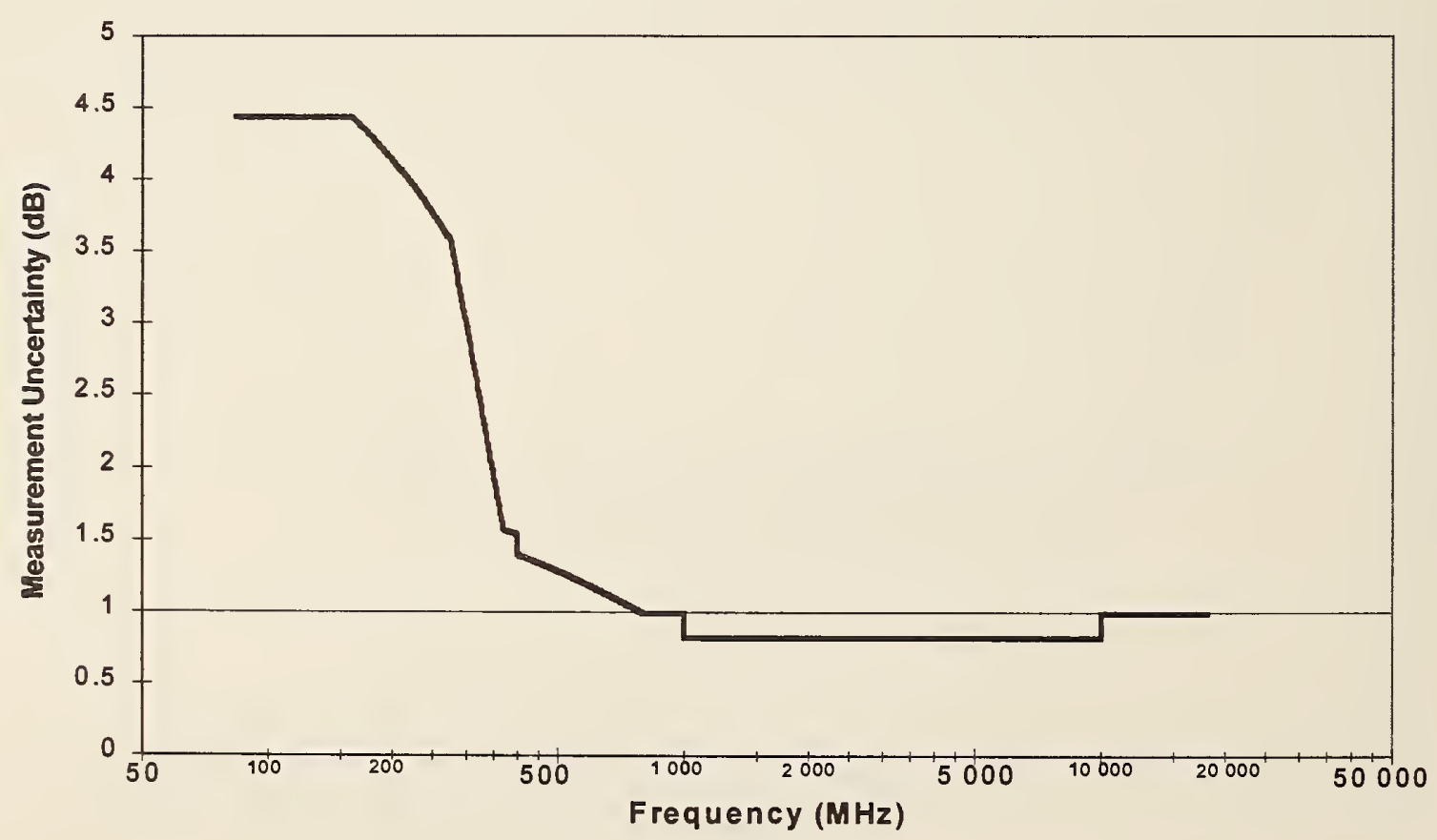

(b)

Figure 151. Uncertainty ( $95 \%$ confidence level) in measurements of average received power using "moving standard deviation" technique in chamber B using vector network analyzer. (a) Three main components of the measurement uncertainty.

(b) Combined uncertainty. 


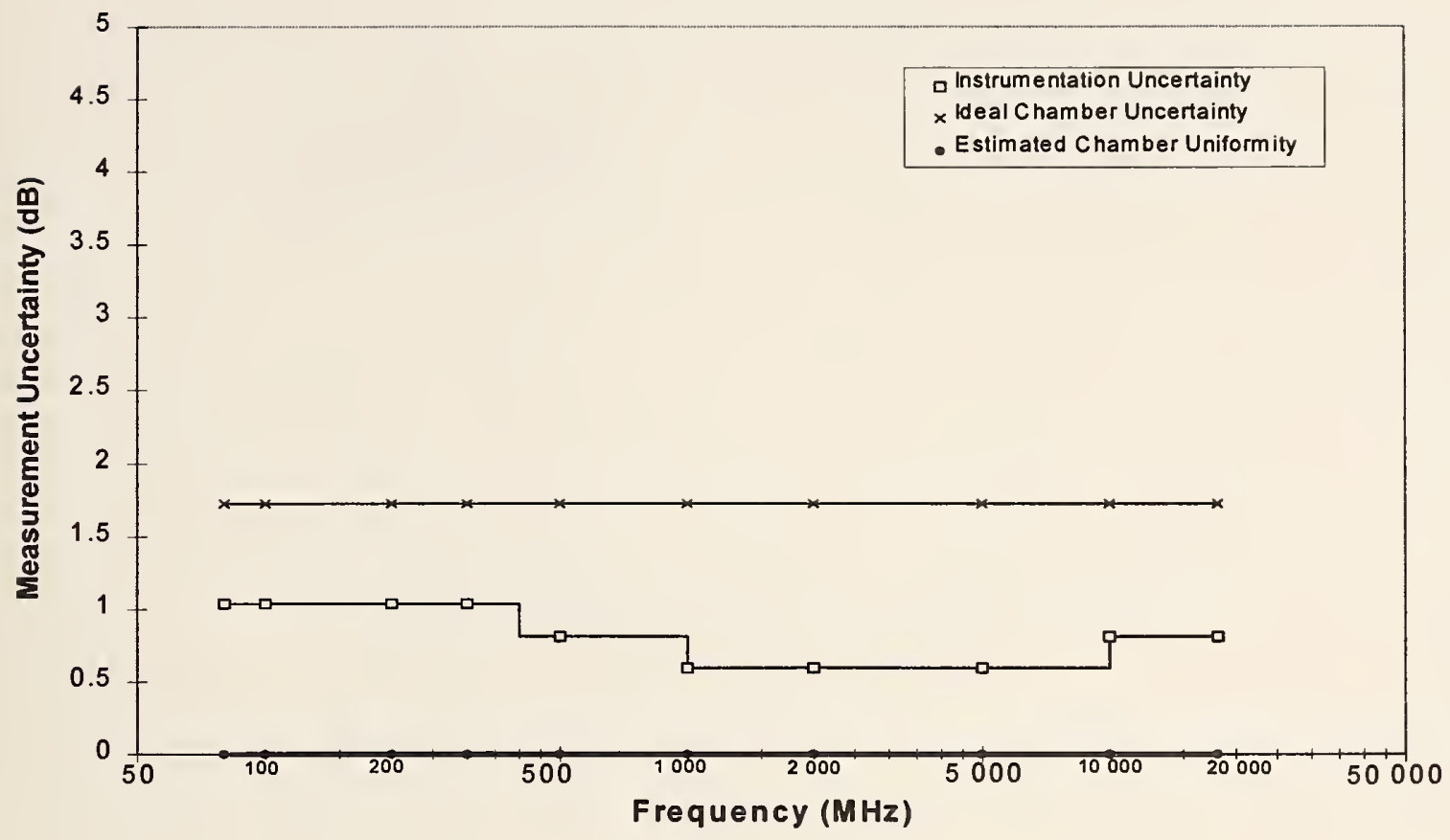

(a)

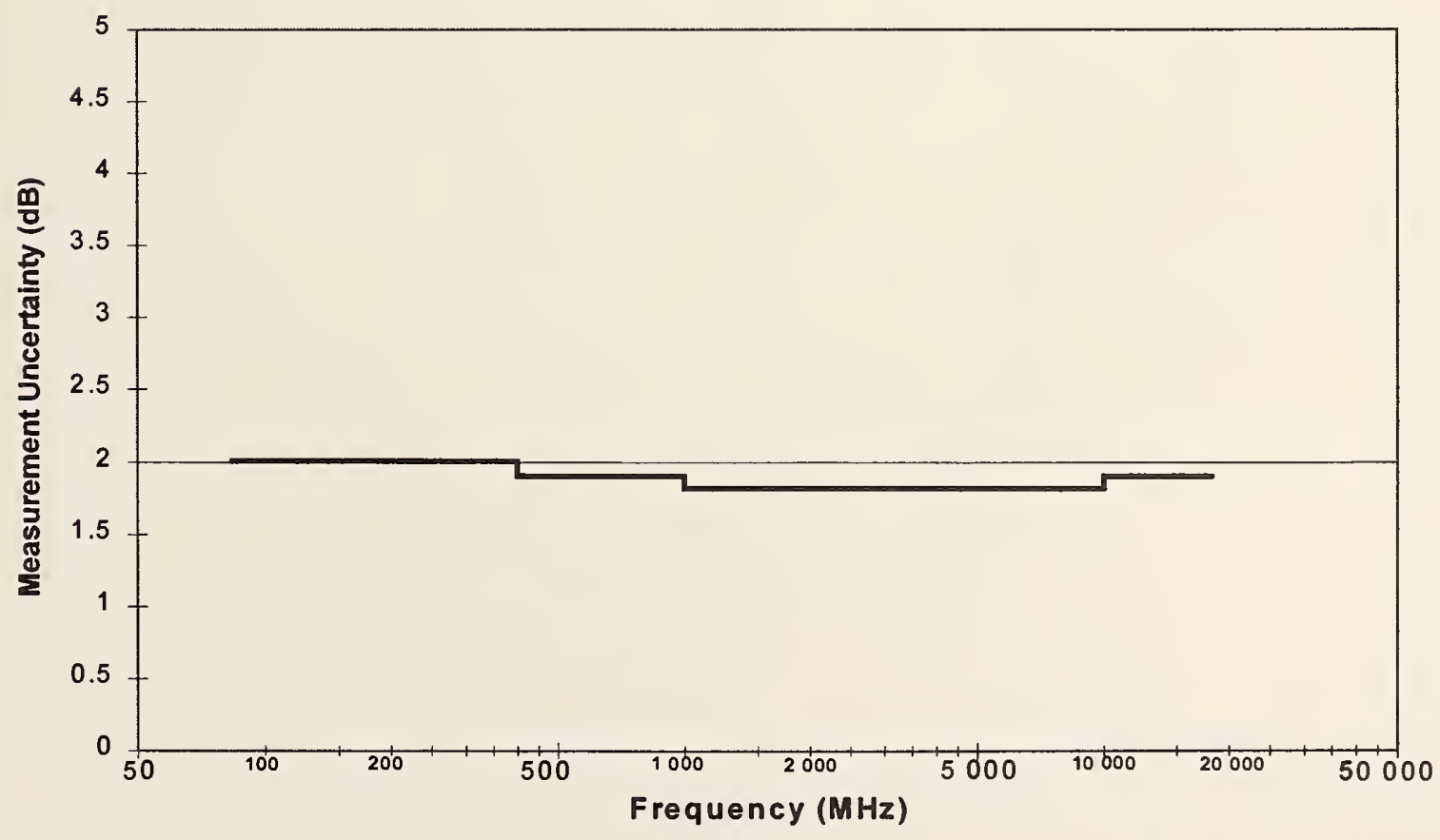

(b)

Figure 152. Uncertainty ( $95 \%$ confidence level) in measurements of maximum received power using "moving standard deviation" technique in chamber B using vector network analyzer. (a) Three main components of the measurement uncertainty.

(b) Combined uncertainty. 


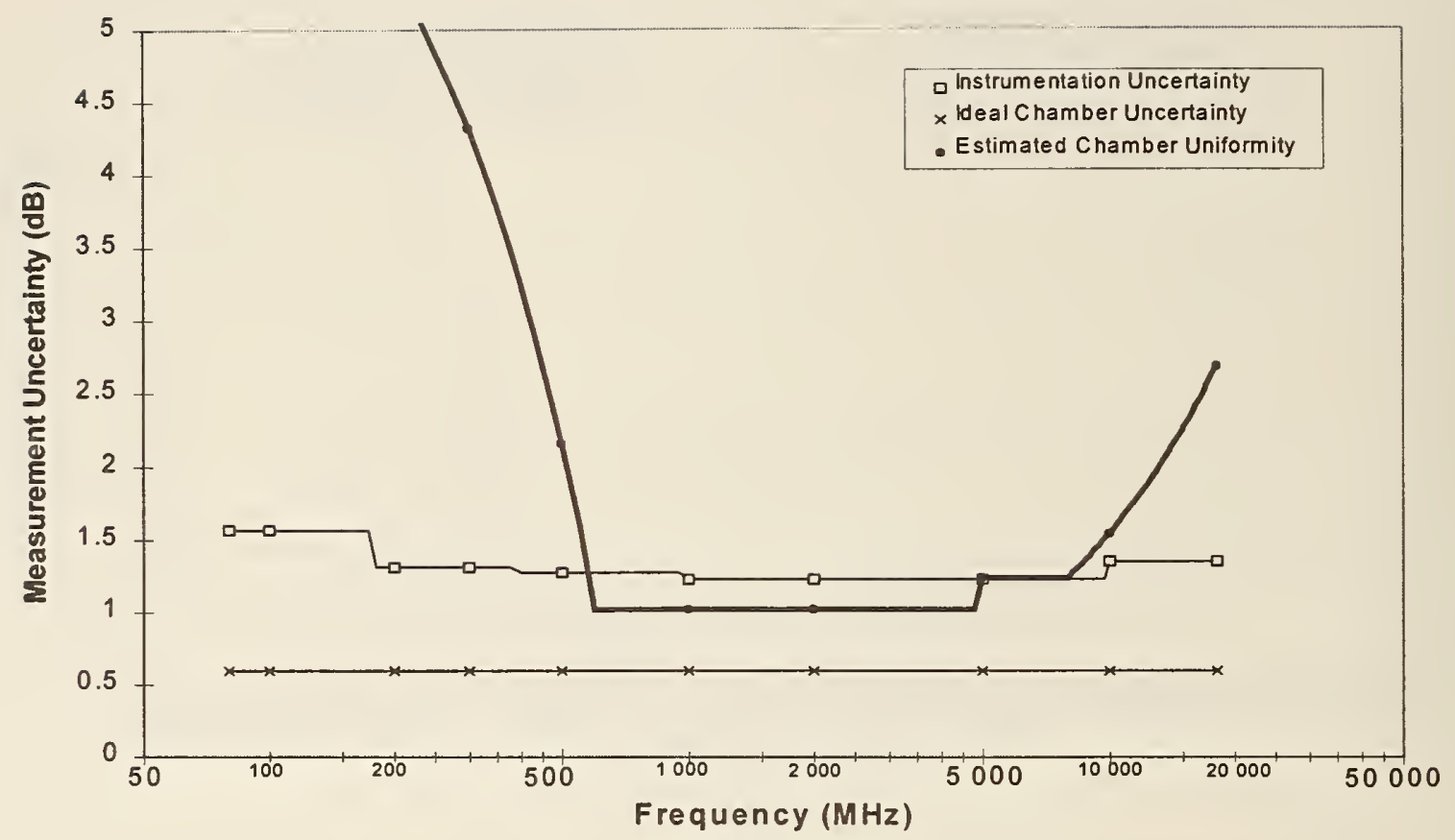

(a)

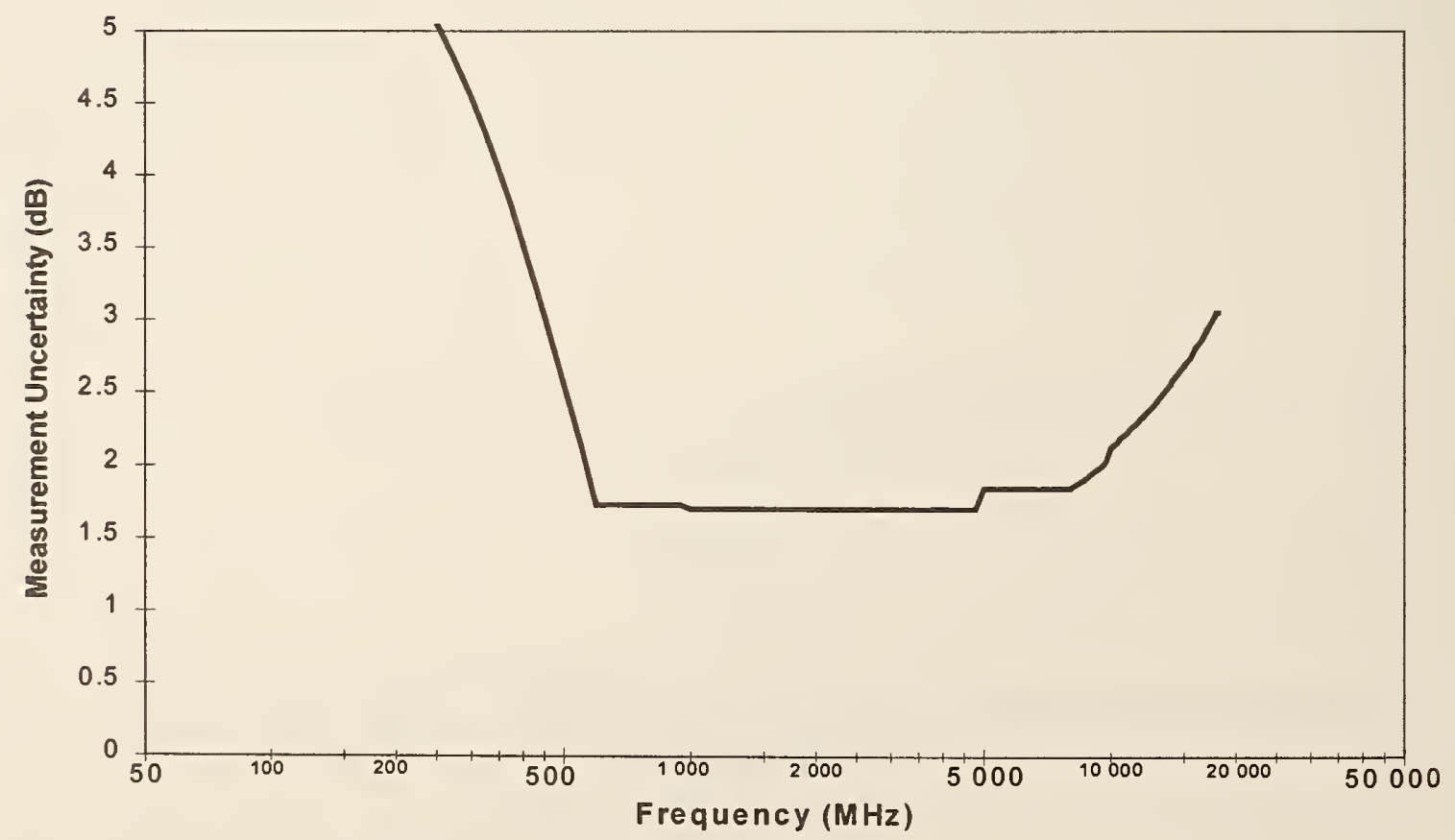

(b)

Figure 153. Uncertainty ( $95 \%$ confidence level) in measurements of average electric field (rectangular component) at multiple locations in chamber B. (a) Three main components of the measurement uncertainty. (b) Combined uncertainty. 


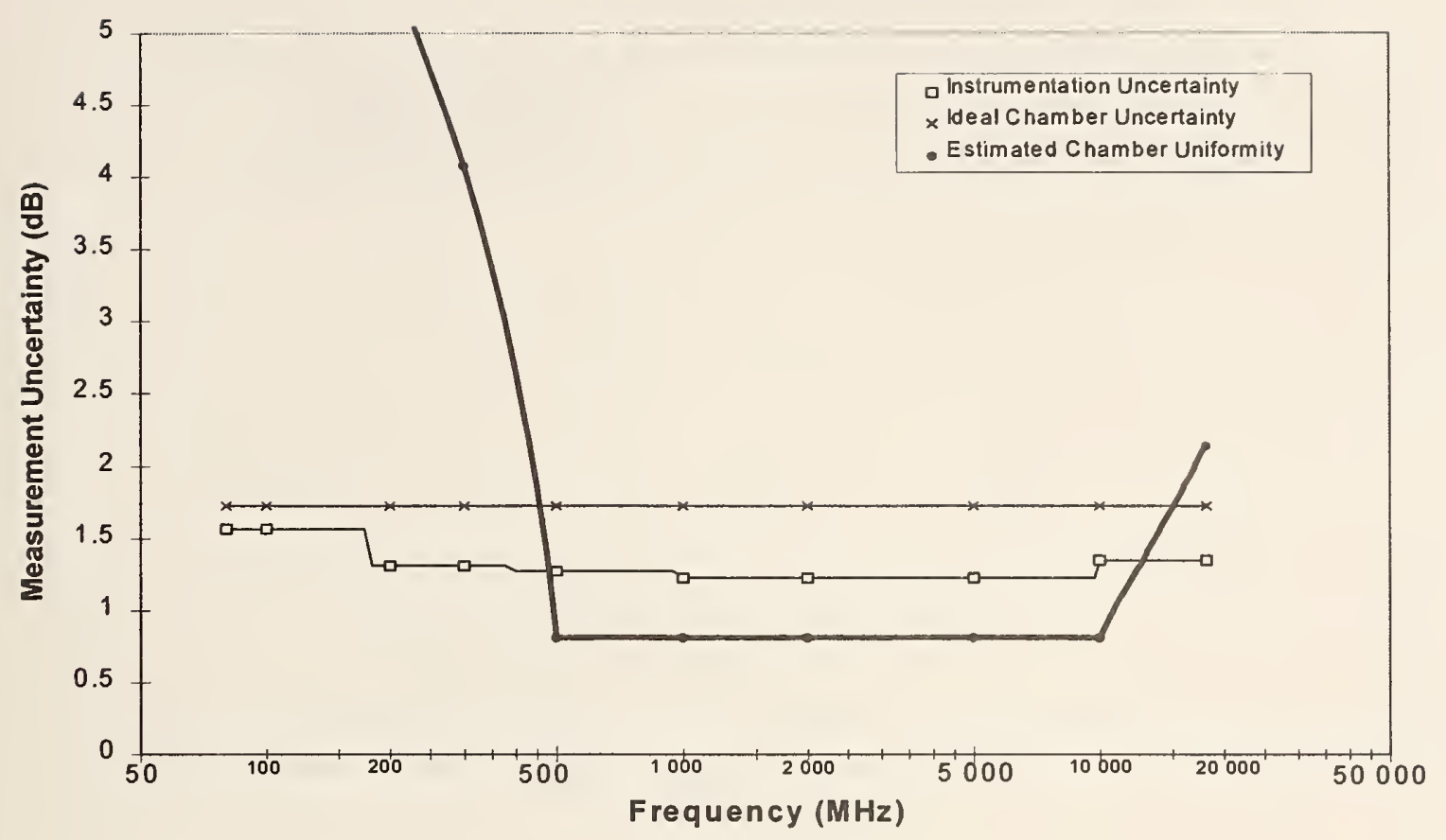

(a)

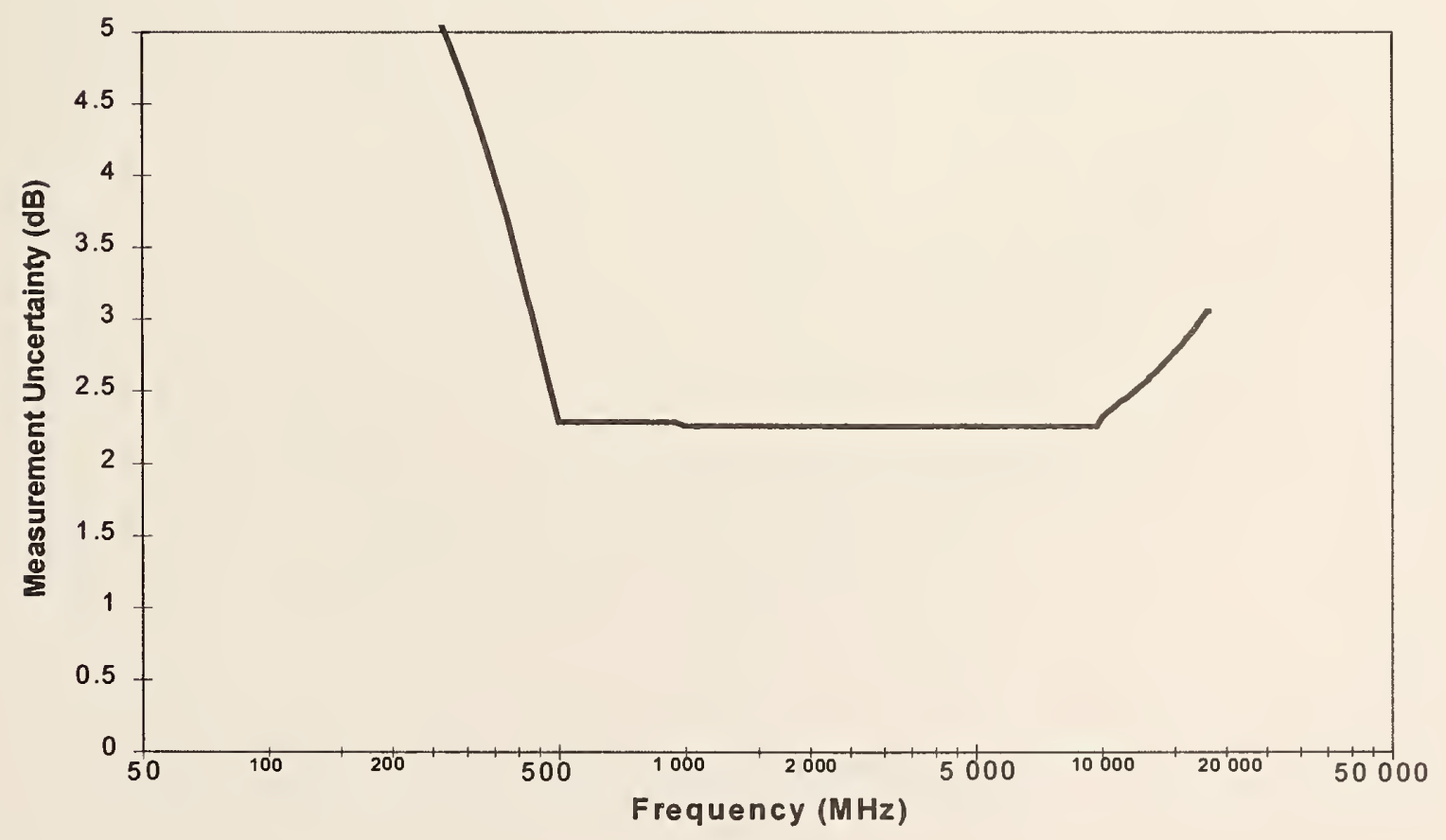

(b)

Figure 154. Uncertainty ( $95 \%$ confidence level) in measurements of maximum electric field (rectangular component) at multiple locations in chamber B. (a) Three main components of the measurement uncertainty. (b) Combined uncertainty. 


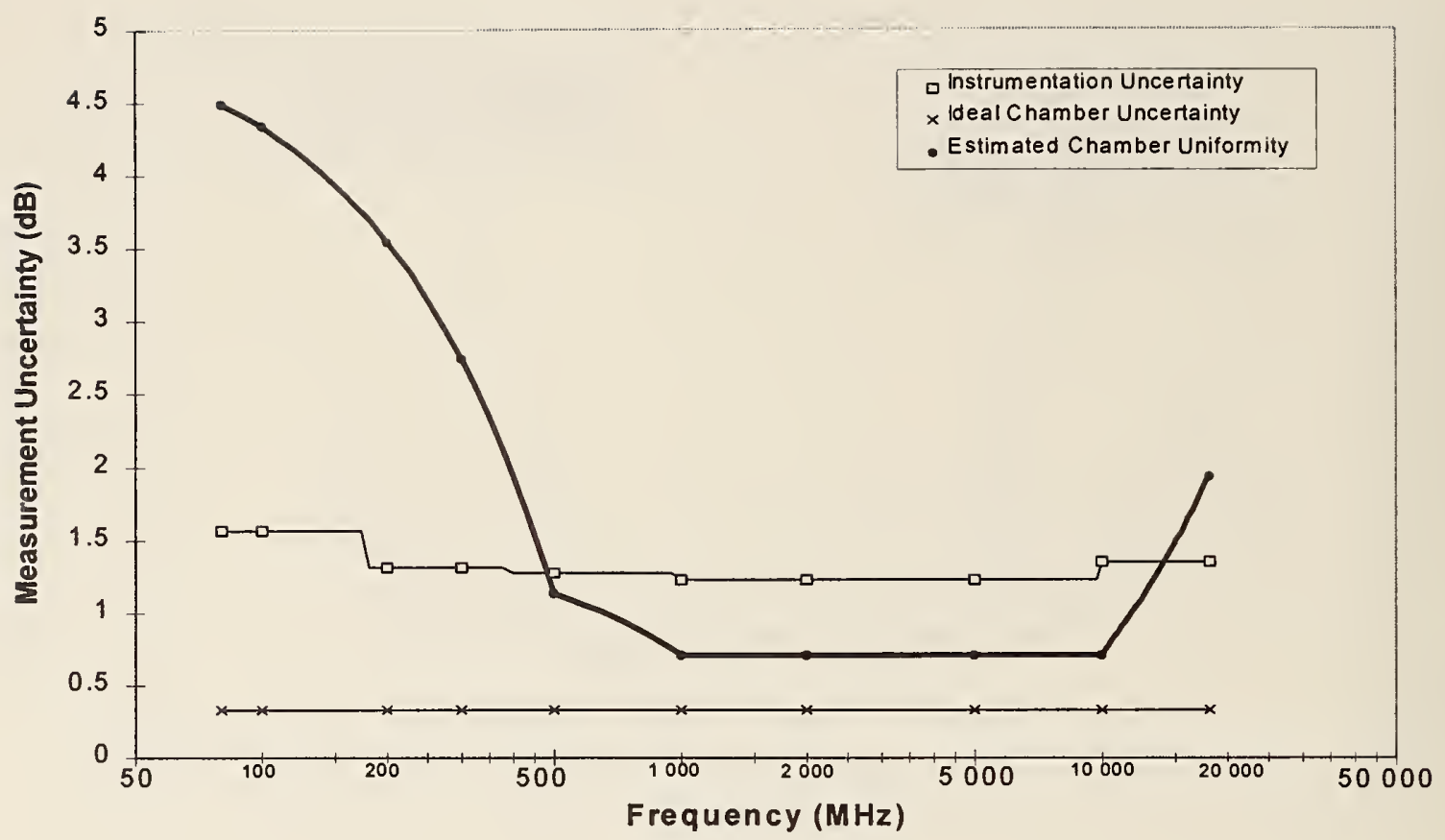

(a)

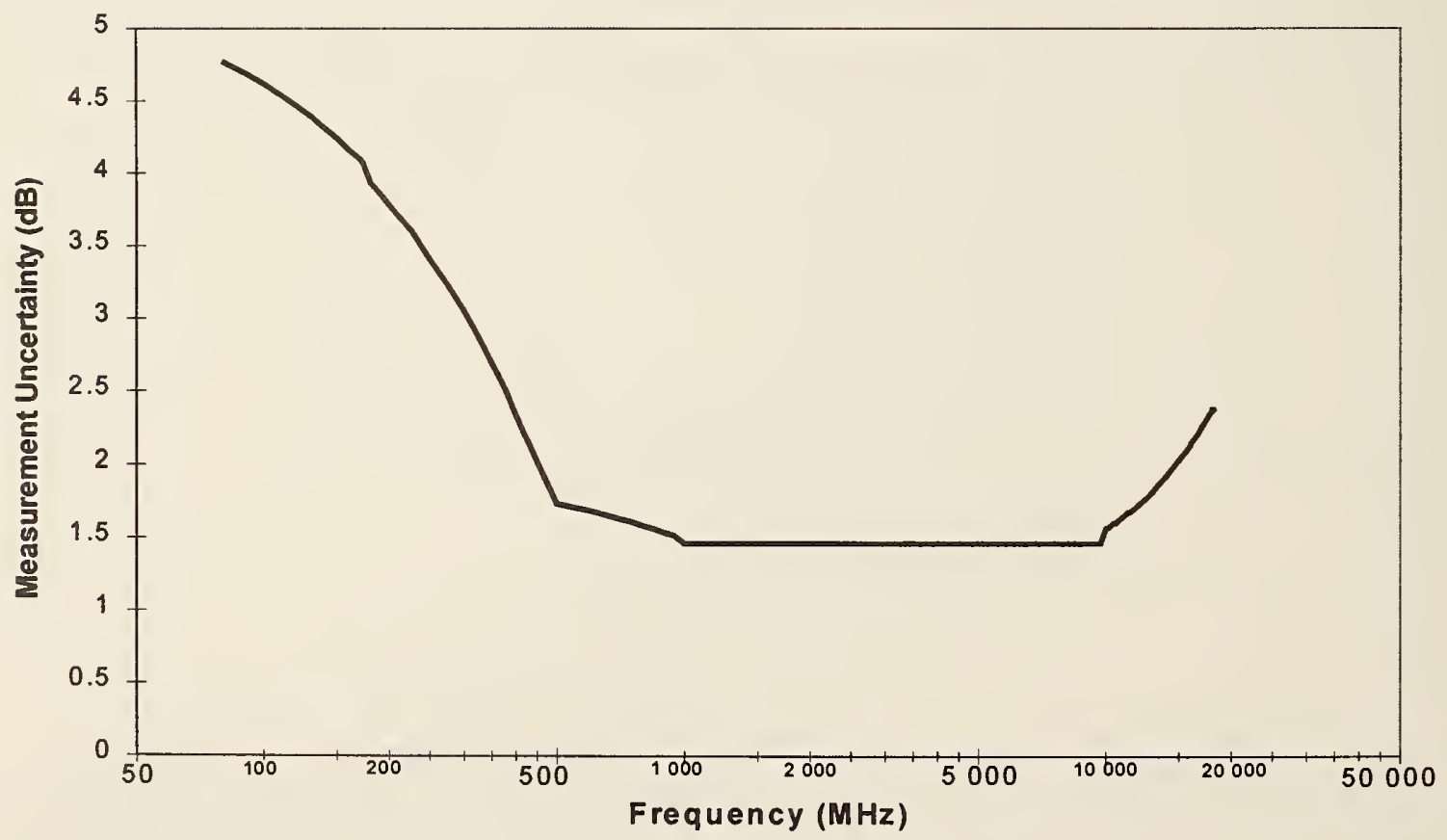

(b)

Figure 155. Uncertainty ( $95 \%$ confidence level) in measurements of average total electric field at multiple locations in chamber B. (a) Three main components of the measurement uncertainty. (b) Combined uncertainty. 


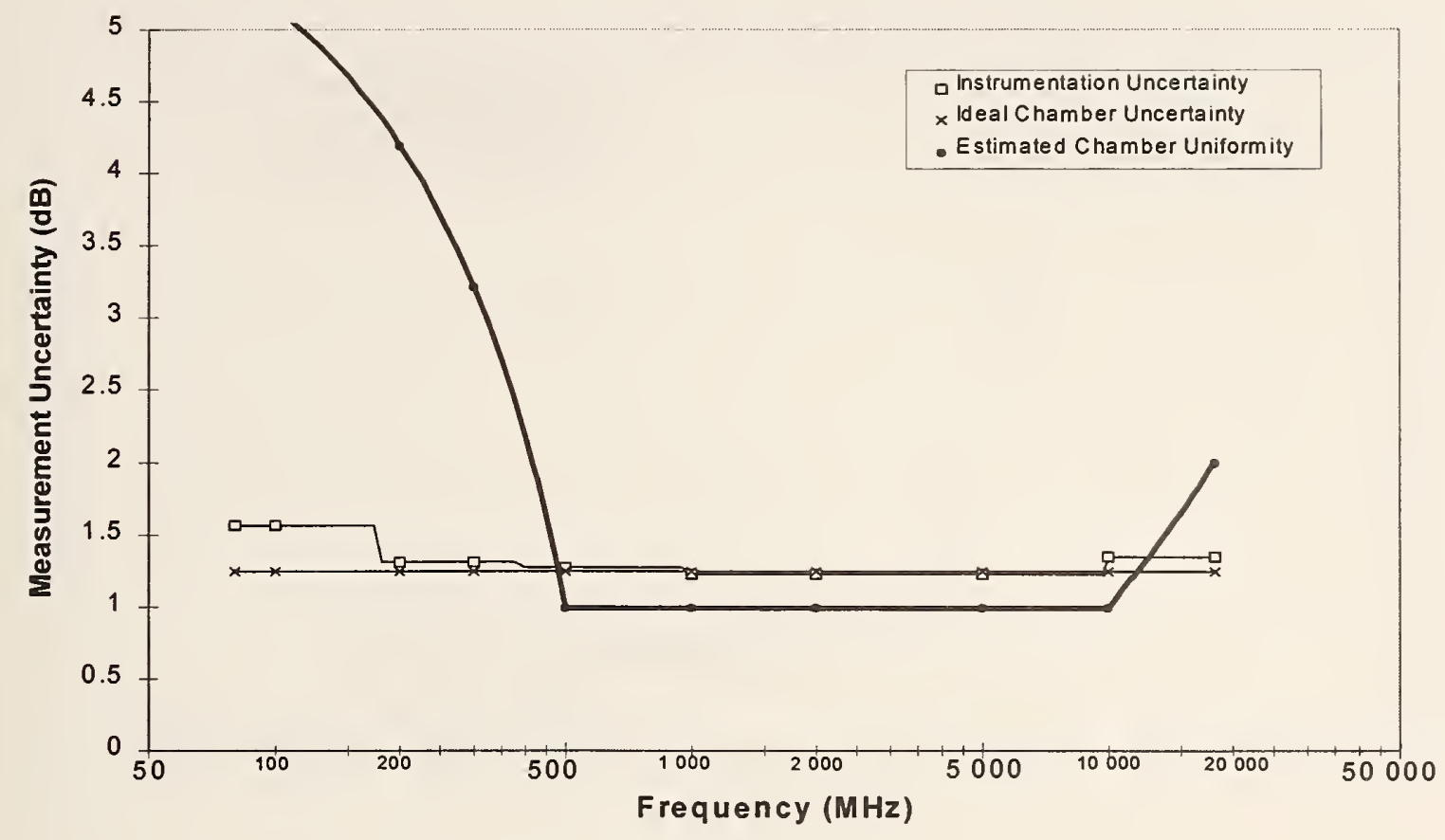

(a)

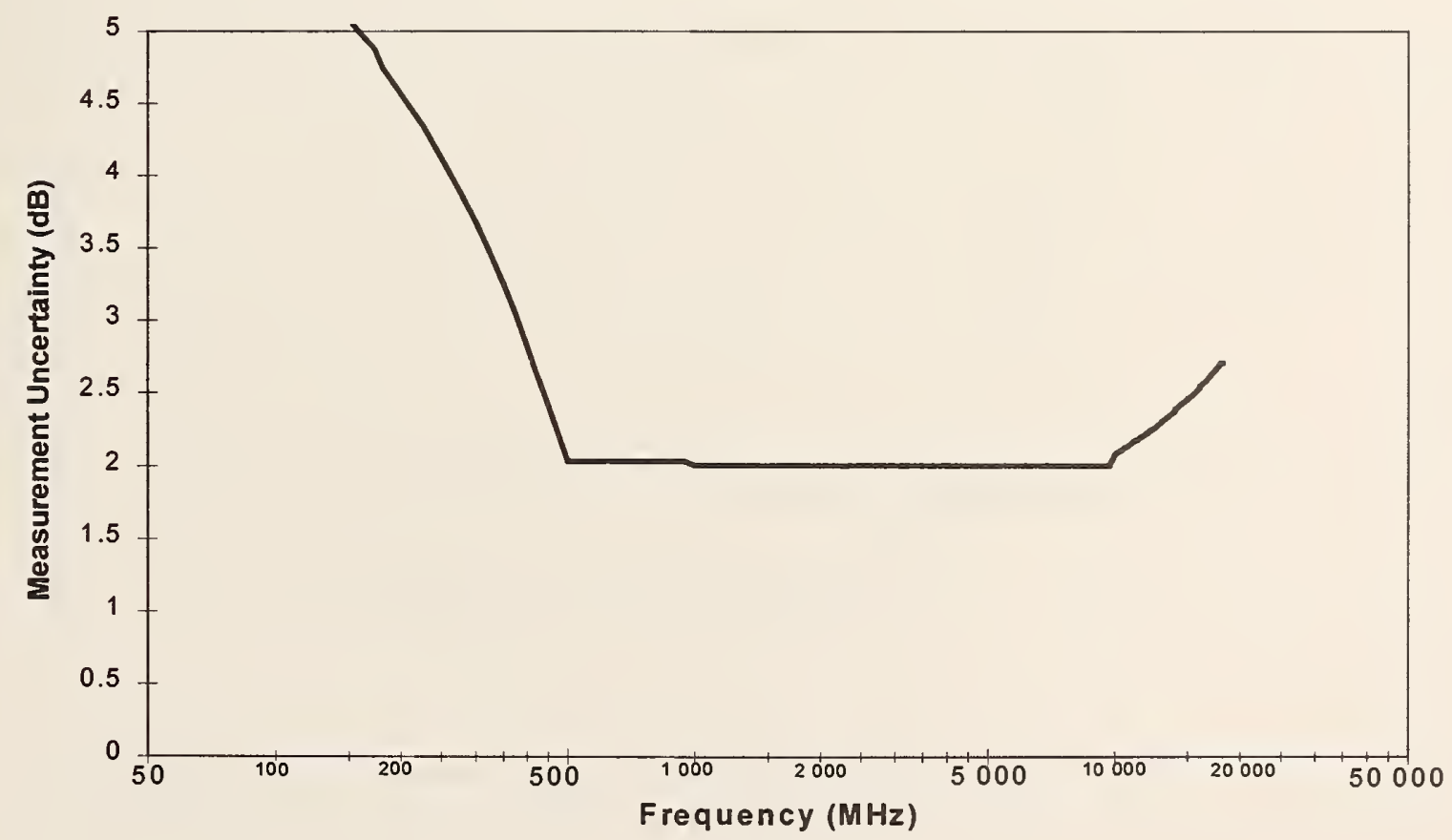

(b)

Figure 156. Uncertainty ( $95 \%$ confidence level) in measurements of maximum total electric field at multiple locations in chamber B. (a) Three main components of the measurement uncertainty. (b) Combined uncertainty. 


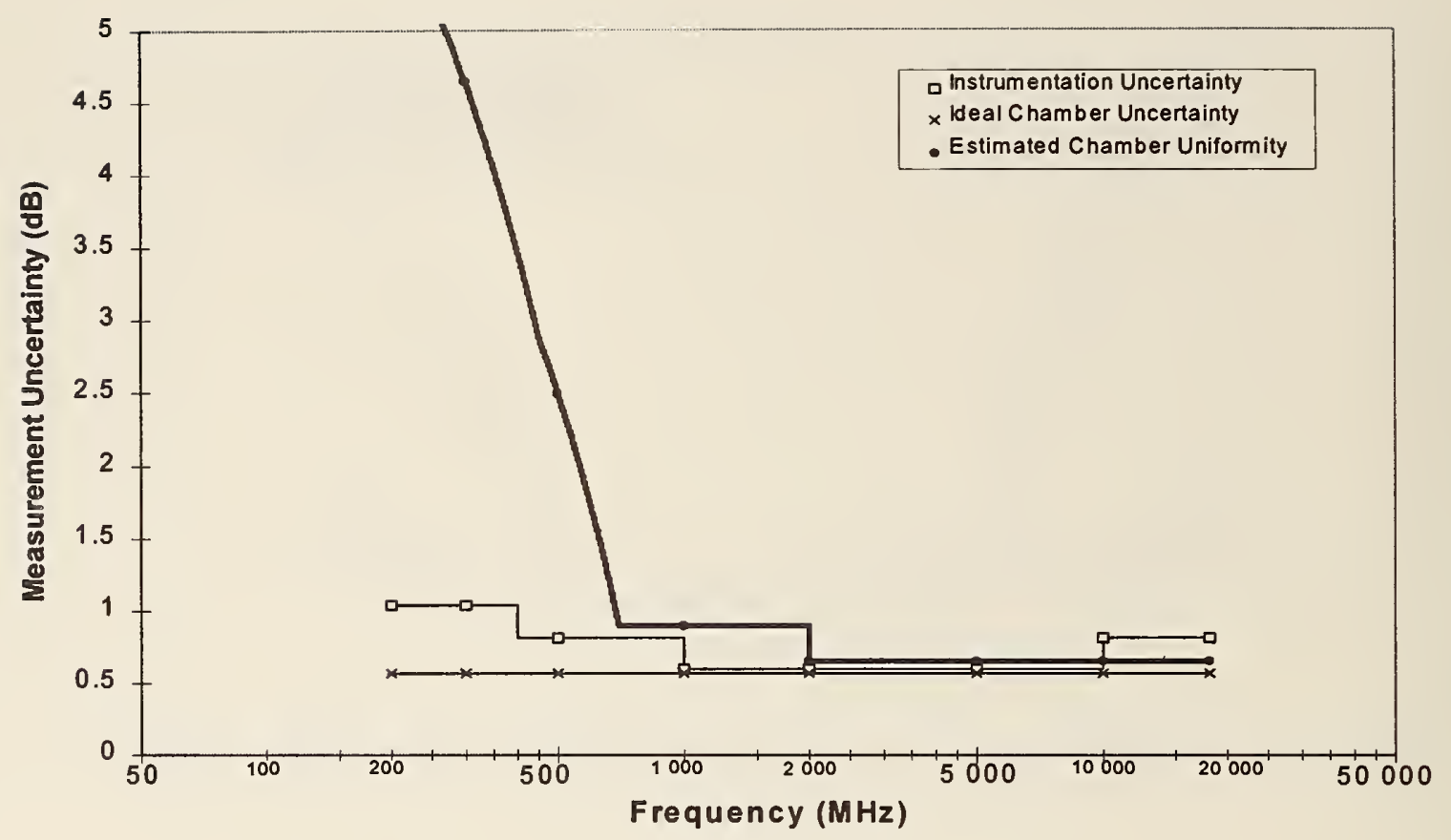

(a)

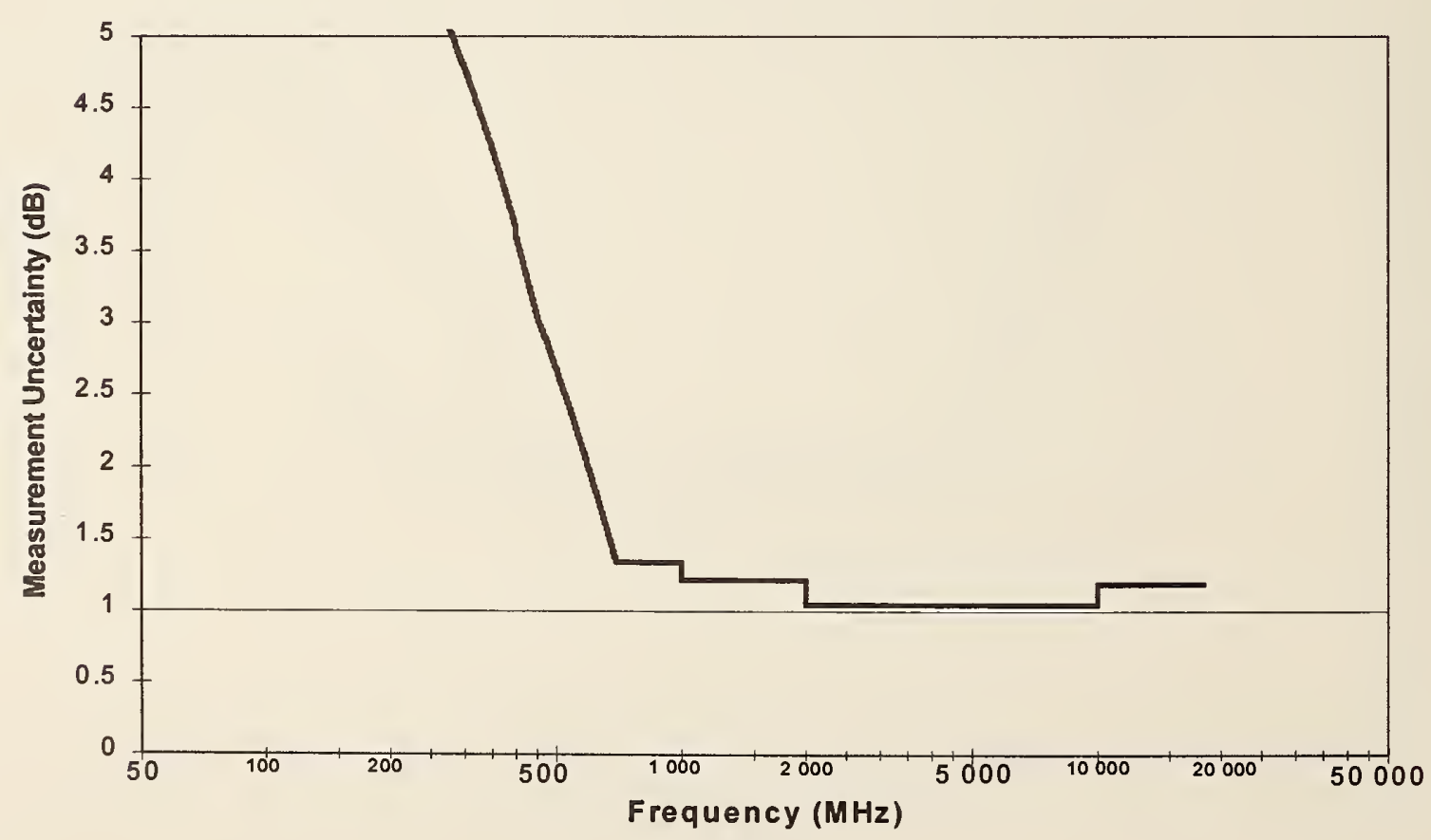

(b)

Figure 157. Uncertainty ( $95 \%$ confidence level) in measurements of average received power using "moving standard deviation" technique in chamber $\mathrm{C}$ using vector network analyzer. (a) Three main components of the measurement uncertainty.

(b) Combined uncertainty. 


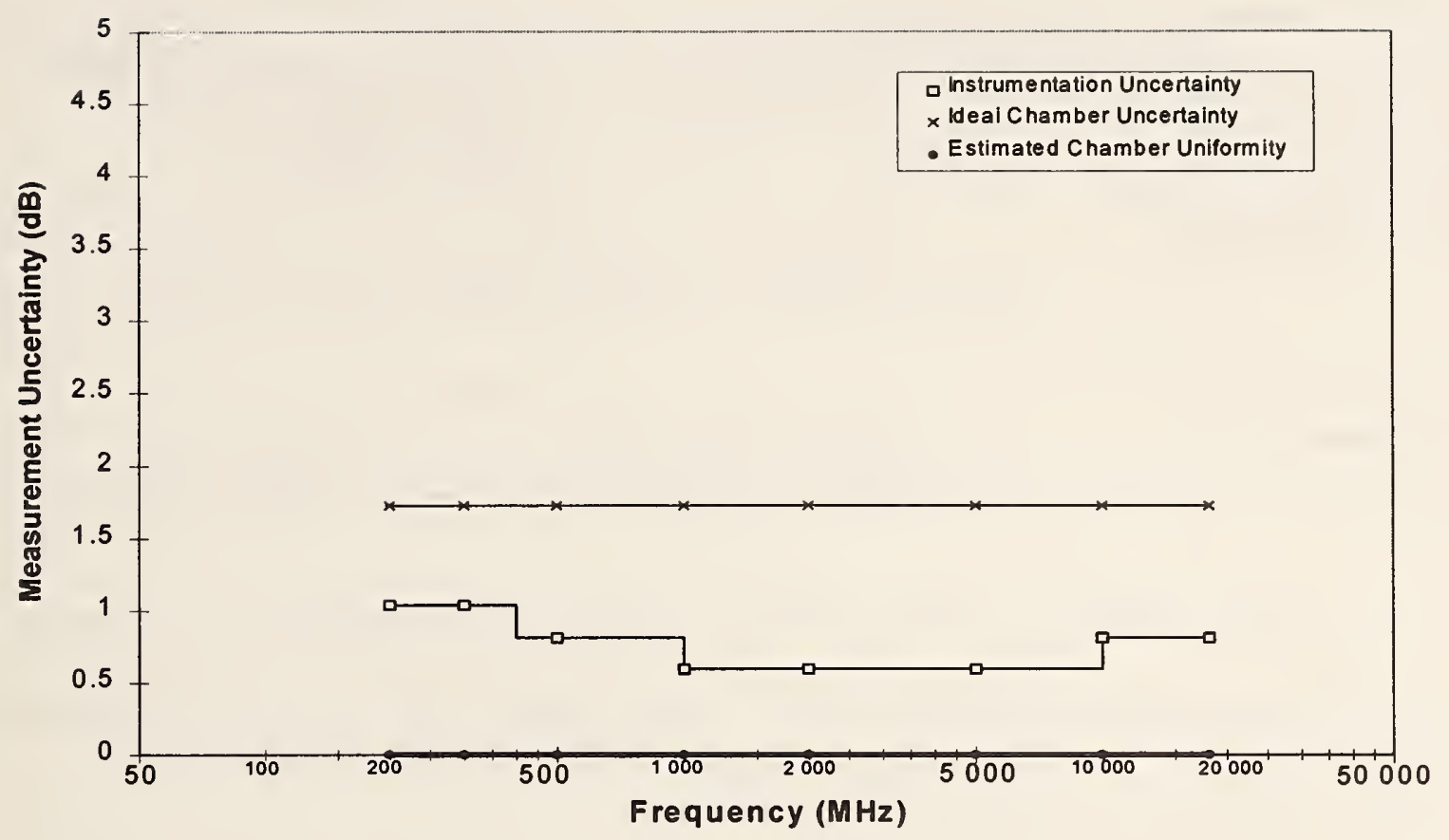

(a)

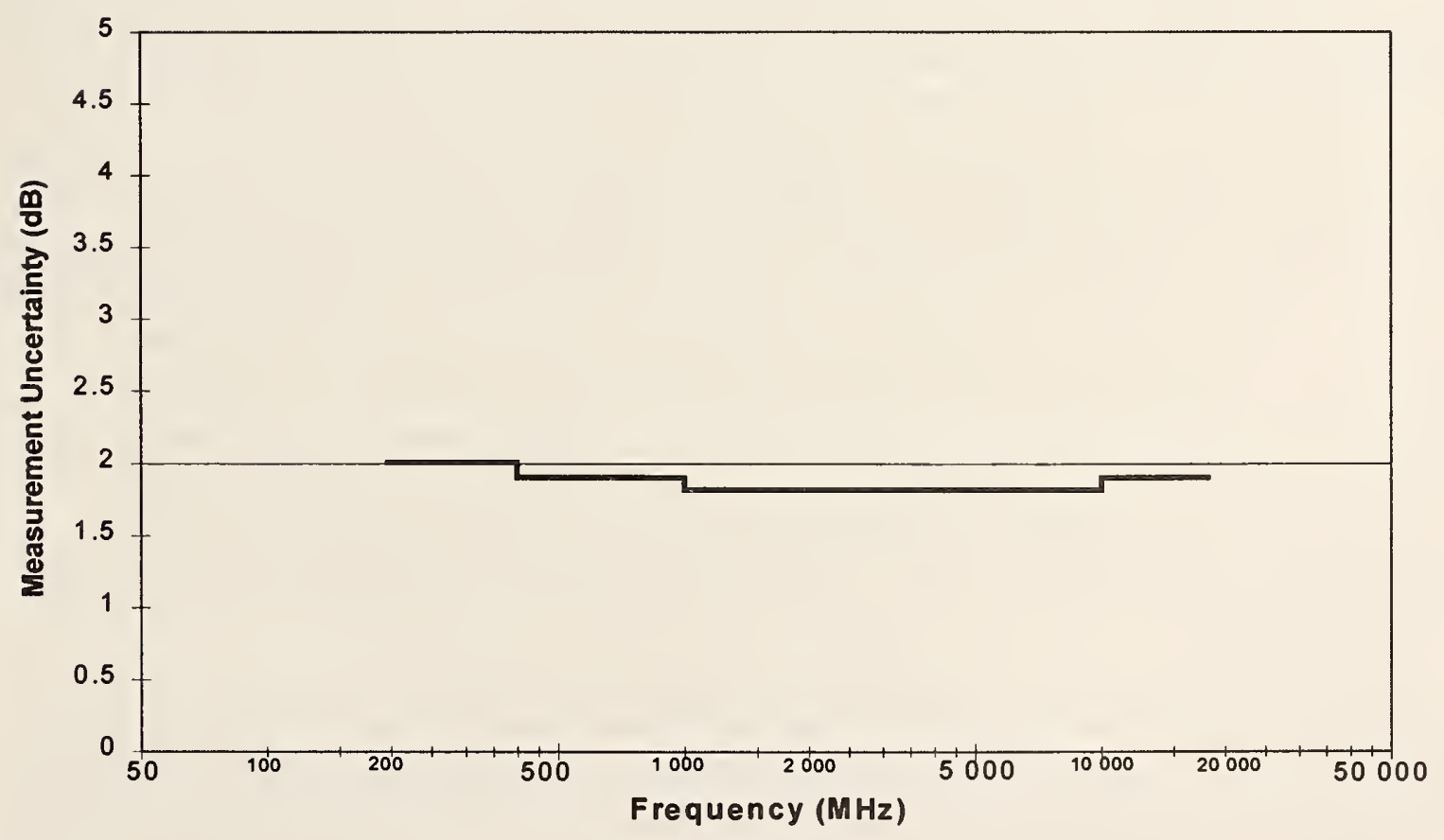

(b)

Figure 158. Uncertainty ( $95 \%$ confidence level) in measurements of maximum received power using "moving standard deviation" technique in chamber $\mathrm{C}$ using vector network analyzer. (a) Three main components of the measurement uncertainty.

(b) Combined uncertainty. 
Table 19. Definition of uncertainty terms.

\begin{tabular}{|c|c|c|}
\hline Uncertainty component & Standard uncertainty & Expanded uncertainty $(95 \%)$ \\
\hline Ideal chamber uncertainty & $\sigma_{\text {ideal }}$ & $u_{\text {ideal }}=2 \sigma_{\text {ideal }}$ \\
\hline Chamber uniformity & $\sigma_{\text {unif }}$ & $u_{\text {unif }}=2 \sigma_{\text {unif }}$ \\
\hline Observed uncertainty & $\sigma_{o b s}=\sqrt{\left(\sigma_{\text {ideal }}\right)^{2}+\left(\sigma_{\text {unif }}\right)}$ & $u_{o b s}=2 \sigma_{o b s}=\sqrt{\left(u_{i d e a l}\right)^{2}+\left(u_{u n i f}\right)^{2}}$ \\
\hline $\begin{array}{l}\text { Instrumentation uncertainty } \\
\text { Combined uncertainty }\end{array}$ & $\begin{array}{l}\sigma_{i n s t} \\
\sigma_{c}=\sqrt{\left(\sigma_{o b s}\right)^{2}+\left(\sigma_{i n s t}\right)^{2}}\end{array}$ & $\begin{array}{l}u_{i n s t}=2 \sigma_{i n s t} \\
u_{c}=2 \sigma_{c}=\sqrt{\left(u_{o b s}\right)^{2}+\left(u_{i n s t}\right)^{2}}\end{array}$ \\
\hline
\end{tabular}

A description of the three components of uncertainty and the combined uncertainty is given in Table 19.

In all subsequent plots of uncertainty, we will present the expanded uncertainty instead of the standard uncertainty because an expanded uncertainty with a confidence level of $95 \%$ is typically used in the final presentation of uncertainty.

The uncertainty in repeated measurements of average received power using a network analyzer in Chamber A is presented in Figure 143, with the three components of uncertainty given in Figure 143a, and the combined uncertainty given in Figure 143b. This figure shows that it is possible to perform measurements inside a mode-stirred chamber above $1 \mathrm{GHz}$ with uncertainties less than $1 \mathrm{~dB}$ (this is approximately the same uncertainty that can be achieved in a good anechoic chamber). Additionally, it should be possible to perform measurements with uncertainties of less than $1 \mathrm{~dB}$ down to $200 \mathrm{MHz}$ and below if we can remove the uncertainty associated with the measurement antennas. In fact, over the majority of the measured spectrum, the largest contributor to the combined uncertainty is instrumentation uncertainty, not chamber uncertainty. In Figure 144, however, we show that the combined uncertainty in repeated measurements of the maximum received power using a network analyzer in Chamber $\mathrm{A}$ is dominated by the ideal chamber uncertainty, with the instrumentation uncertainty contributing very little to the combined uncertainty, and the chamber uniformity contributing nothing (the observed uncertainty was indistinguishable from the ideal uncertainty). The uncertainty in measurements of the maximum received power is approximately $2 \mathrm{~dB}$ or less over the entire measured spectrum.

Similar plots for the uncertainties in other measurements are also provided. The uncertainty in measurements of average received power using the moving standard deviation technique are given in Figure 145, and the uncertainty in measurements of maximum received power using the moving standard deviation technique are given in Figure 146. These estimates are similar to those for repeated measurements, although there are some minor differences at low frequencies. The uncertainty in measurements of maximum received power are surprisingly low at low frequencies, and substantially lower than the uncertainties in measurements of the average received power. This is probably due to received power compression discussed above. Due to this low measurements uncertainty, we claim that the uncertainty in measurements of maximum received power are not indicative of the uncertainties in measurements of any other parameter in 
the chamber. To verify that this is the case, we examine the uncertainty in measurements of the electric field.

The uncertainties associated with measurements of the electric field are given in Figure 147 for measurements of the average magnitude of a rectangular component of the electric field, in Figure 148 for measurements of the maximum magnitude of a rectangular component of the electric field, Figure 149 for measurements of the average magnitude of the total electric field, and in Figure 150 for measurements of the maximum magnitude of the total electric field. In all cases the uncertainty at low frequencies is greater than that for the maximum received power, indicating that a chamber must be evaluated with probes if the qualities of the chamber, including uncertainties, are to be adequately characterized. At high frequencies, too, we see that the uncertainty in measurements of the electric field are greater than would be expected for a perfect chamber, although the uncertainties in measurements of received power are very similar to those expected in an ideal chamber. This indicates either that the receiving antennas are performing spatial averaging and the probes are not, or that the additional uncertainty is attributable to the measurement system. Even if the probe system behaves perfectly, the uncertainty due to the other instrumentation still contributes significantly to the overall uncertainty in our measurements.

The uncertainties in the measurements performed in Chamber B were processed similarly to those in Chamber A, and with similar results. Once again, the only real difference is in the low frequency response of the chambers. The uncertainty in measurements of the average received power are given in Figure 151, and the uncertainty in measurements of the maximum received power are given in Figure 152. Figure 153 and Figure 154 show the uncertainties in measurements of the average and maximum of a rectangular component of the electric field, respectively, and Figure 155 and Figure 156 show the uncertainties in measurements of the average and maximum total electric field, respectively.

Finally, the uncertainty in measurements of received power as measured in Chamber C are given in Figure 157 for measurements of the average received power, and Figure 158 for measurements of maximum received power.

\subsection{Lowest Usable Frequency}

The lowest usable frequency of a chamber is typically used to indicate a frequency above which measurements can be well characterized and the chamber behaves in a predictable manner, and below which reliable measurements cannot be made. Unfortunately, this research has shown that this is a slight oversimplification. In reality, there appears to be a "break frequency" where measurements above this frequency can be approximately characterized by a constant, and measurements below this frequency will have a higher associated standard deviation, and this standard deviation will increase as the spacing between the test frequency and the break frequency increases. This does not mean that tests cannot be performed below this break frequency, only that the uncertainties will be greater for measurements below the break frequency than above the break frequency. Because of this, the lowest usable frequency of a chamber depends on the uncertainty that is allowed in a test, and also on the number of paddle positions used in the test. If uncertainties of $\pm 6 \mathrm{~dB}$ ( $95 \%$ confidence) in the maximum rectangular component of the electric field are acceptable, then Chamber A is usable down to 80 
$\mathrm{MHz}$ and probably lower, and Chamber $\mathrm{B}$ is usable down to $200 \mathrm{MHz}$. If uncertainties of \pm 0.5 $\mathrm{dB}(95 \%$ confidence) are required, however, then no known chamber will be usable, regardless of the number of paddle positions.

Because of the ambiguity in the definition of the lowest usable frequency, we propose the lowest overmoded frequency (LOF) instead. The LOF is simply the break frequency described above. More specifically, we define the LOF as the lowest frequency above which all chamber parameters have an approximately constant uniformity. Thus, even though the maximum received power as measured in Chamber $\mathrm{A}$ and $\mathrm{B}$ has a standard deviation that is approximately constant over the entire measured frequency range, we would estimate the LOF of Chamber A as $350 \mathrm{MHz}$ (taken from a plot of the standard deviation of measurements of a rectangular component of the electric field, Figure 137), Figure 140, and the LOF of Chamber B, based on Figure 140, is $600 \mathrm{MHz}$. Once again we stress that these chambers can easily be used below these frequencies, but the uncertainty curves should be examined before a test is performed at lower frequencies to verify that the uncertainty at a lower frequency is acceptably small.

Since we did not perform measurements in Chamber $C$ using the probe system, the best we can do is estimate the LOF based on the data in Figure 142. From these data, we can state that the LOF is approximately $700 \mathrm{MHz}$.

\section{Conclusions}

This research has generated a large number of results. The most fundamental conclusion is that the chambers evaluated here are very good and very well behaved. These are the first chambers that were good enough to demonstrate that accurate measurements could be performed in a reverberation chamber and that measurement uncertainties could be reduced to the point that small errors in the underlying theory of mode-stirred chambers could be identified and corrected. We will summarize and categorize the fundamental results according to the various sections of this report: electromagnetic theory, statistical theory, measurement systems, processing techniques, and uncertainty analysis.

\subsection{Electromagnetic Theory}

We rederived many of the fundamental electromagnetic descriptions of mode-stirred chambers using a new approach, and demonstrated that our results are consistent with previous derivations. We point out, however, that the use of the term power density, as it has been used in other derivations, can be problematic and should be avoided, or the potential problems should be documented whenever this term is used.

We also showed that, although it is possible to predict the characteristics of the average squared magnitude of the electric field (either total or a rectangular component), we cannot predict the characteristics of the maximum squared magnitude of the electric field without knowledge of the probability distribution describing the fields in the chamber. In fact, we show 
that we cannot predict the characteristics of the average magnitude (not squared magnitude) of the electric field, even if we know the average squared magnitude, unless we have an adequate statistical description of the fields inside the chamber.

In addition, we showed that many of the characteristics of an empty (except for test antennas) metal cavity can be described by a simple two-parameter model and that it may be possible to predict the values of both of these parameters based on the materials characteristics of the cavity walls and the number of antennas placed in the cavity.

\subsection{Statistical Theory}

Using simple assumptions, we develop detailed statistical models of the fields inside a mode-stirred chamber and point out where these models might and might not be appropriate. Using these models, we derived the characteristics of the average and maximum magnitude of the electric field (both total and a rectangular component), as well as the average and maximum power received by an antenna placed in such a facility. These derivations show that calculations of electric field based on measurements of received power given in other documents (including NBS Tech. Note 1092 [3]) are incorrect and may overestimate the maximum electric field in the chamber by several decibels.

Finally, we show how to predict the maximum electric field in a chamber as a function of the number of paddle positions used in the measurement and the average electric field (or average received power). These calculations should be valid as long as the statistical models are accurate.

\subsection{Measurement System}

In addition to the traditional probe system, which has been used to characterize a variety of chambers, we describe how we used a vector network analyzer to evaluate a mode-stirred chamber. This tool gives us new and unusual ways of looking at the data, and enabled us to significantly reduce the uncertainty in measurements of chamber gain and received power. The use of a network analyzer also allowed us to develop new processing techniques that allowed us to characterize some of the parameters of the antennas used in the measurements. These processing techniques are described below.

\subsection{Processing Techniques}

Based on measurements using a network analyzer, we showed that there was a step in the estimates of the chamber gain at $1 \mathrm{GHz}$ and that this step could not be explained by the mismatch characteristics of the measurement antennas. We then argued that the step is most likely due to differences in the efficiencies of the antennas used below and above $1 \mathrm{GHz}$. We developed a method for estimating correcting for the efficiencies of these antennas. These corrections not only improved the agreement between estimates of the chamber gain below and above $1 \mathrm{GHz}$, 
but also resulted in a much smoother estimate of the chamber gain over the entire measurement spectrum. This appears to justify these corrections.

The data measured with the network analyzer indicate that the maximum power is compressed at low frequencies. This is caused by decreasing power absorption by the walls at low frequencies, resulting in increase power absorption by the antennas. In the extreme case, almost all power transmitted into the chamber is received by the receiving antenna, resulting in a maximum received power that is comparable to the transmitted power. This implies that the distributions describing the behavior of the received power may not be accurate at low frequencies and that the received power may not allow us to adequately predict the electric field.

The reflection characteristics of the receiving antenna appear to cause significant biases in the data and should be corrected for, if possible. Unfortunately, this correction can be performed only if the measurements are performed with a network analyzer (difficult when high power is required), a pretest is performed with a network analyzer (time consuming), or the freespace characteristics of the receiving antenna are used (may not describe the characteristics of the antenna at low frequencies when it is placed in a cavity). Given that none of the options is appealing, more research needs to be done.

We also show that the average reflection characteristics of the measurement antennas are similar to those of the same antennas placed in free space. Because of this, if may be possible to perform measurements by maintaining a constant incident power and correcting for the average reflections characteristics, instead of maintaining a constant net input power, thus simplifying the measurement process.

\subsection{Uncertainty Analysis}

We present a thorough analysis of the uncertainties associated with mode-stirred chamber measurements in general, and with the NASA chambers in particular. We show that uncertainty in the measurement instrumentation can be a significant contributor to the overall uncertainty in a measurement, and that with the proper choice of instrumentation, the uncertainties can be greatly reduced. In some measurements, the uncertainty is as low as can be expected, given the number of paddle positions used in the measurement.

We also suggest a definition for the term "chamber uniformity" that is less dependent on the number of paddle positions used in the measurement and more of a fundamental characteristic of the chamber.

We examined the concept of the lowest usable frequency of a chamber and commented that the concept of a lowest usable frequency is application specific, and not necessarily a fundamental characteristic of a chamber. Because of this, we suggest the lowest overmoded frequency as a less ambiguous parameter.

\section{Suggestions for Further Research}

Although the research described in this report answers a number of questions, there are still a large number of problems that require additional research. We list some of these topics below. 
1. Mode-stirring. Although chamber processes are most easily described by performing measurements at a large number of fixed tuner locations (mode tuning), there are potential advantages to using a continuously rotating tuner (mode stirring). Such tuners are generally easier to implement, and tests performed with such tuners can take less time to complete. These advantages have their price, however. The fields in the chamber are no longer static, and the time-variant nature of the field can influence the response of the monitoring equipment as well as any test devices inside the chamber. More research is needed to address the interaction between device response time, bandwidth, and tuner rotation speed.

2. Frequency stirring. This method is closely related to mode stirring, but instead of using a tuner to change the boundary conditions, the drive frequency is changed by some small amount. If this is done at discrete frequencies, then this analysis should be similar to that presented here, as long as the bandwidths of the test equipment and devices are large relative to the change in frequency, and that the frequency change is sufficient to give a sufficiently different measurement. Additional research is required to determine the required change in frequency and the effects of small bandwidths on test results. If frequencies are changed continuously, device bandwidths may be even more significant, and this also requires further investigation.

3. Antenna characterization. The antenna characterizations presented in this report are still preliminary, and additional research is required to verify these results. Assuming that these results can be verified, methods need to be developed to characterize the antennas at lower frequencies.

4. Field monitoring methods. Alternative methods need to be developed for monitoring fields in the chamber during a test. The reflection characteristics of a reference antenna have not been sufficiently characterized, and the compression effects indicate that other alternatives should be investigated. These include the use of a monitoring probe or possibly a monopole antenna mounted on the wall of the chamber.

5. Pulse testing. Many specifications require some type of pulsed if or other transient testing. Although some transient tests have been performed in mode-stirred chambers, this type of test is still not well understood, and the uncertainties in such tests are difficult to estimate.

6. Test artifacts. A set of test artifacts that are simple, repeatable, and easy to manufacture needs to be developed so that different test methods can be compared. Typical parameters that need to be controlled are upset level (the field required to cause a device to fail), response time, and bandwidth. Other parameters that should be considered are directivity characteristics of the device, response to transient signals, and response to either the total electric field or a rectangular component of the electric field.

7. Low frequency statistical analysis. The statistical model is not appropriate for measurements of received power at low frequencies, and measurements of the electric field show larger variations than can be explained by the simple statistical models given here. 
We express our appreciation to the management and staff of the Electromagnetics Research Branch, Information and Electromagnetic Technology Division at NASA Langley Research Center (Thomas Campbell, Branch Head) for the technical and financial support given to this project. We especially thank Reuben Williams, Truong Nguyen, and Kenneth Dudley for technical assistance and after-hours access to the laboratory which helped expedite the measurements.

We also appreciate the technical support of present and former members of the Fields and Interference Metrology Group, Electromagnetic Fields Division, NIST. We thank Motohisa Kanda, Allen Newell, and Dennis Friday for their continuing support of Mode-Stirred Chamber research.

The people who have contributed in some way to this research and to our understanding of mode-stirred chambers are too numerous to list in detail, but several deserve special mention. We thank Kenneth Cavcey, who spent long hours helping to calibrate the rf field probes. We would also like to thank Dave Hill, who had the patience and knowledge to explain both simple and intricate details of electromagnetic field theory. The present and former members of the Statistical Engineering Division of NIST, specifically Dominic Vecchia, Kevin Coakley, and Brad Biggerstaff, have been extremely helpful in explaining statistical analysis techniques and suggesting tests and test methods. They had no idea what they were getting themselves into when they allowed us into their offices to answer "just one quick question."

\section{References}

[1] Hatfield, M.O.; Freyer, G.J.; Johnson, D.M.; Farthing, C.L.. Demonstration test of the electromagnetic reverberation characteristics of a transport size aircraft, Naval Surface Warfare Center, Dahlgren Division, Dahlgren, VA; Report No. NSWCDD/TR-93/339.

[2] RTCA/DO-160D Environmental conditions and test procedures for airborne equipment. Radio Technical Commission for Aeronautics. 1140 Connecticut Avenue, N.W., Suite 1020, Washington, D.C. 20036; July 29, 1997.

[3] Crawford, M.L.; Koepke, G.H. Design, evaluation, and use of a reverberation chamber for performing electromagnetic susceptibility/vulnerability measurements. Nat. Bur. Stand. (U.S.), Tech. Note 1092; 1986 April. 139 p.

[4] Lehman, T.H. Statistics of MSCs. Presented at the anechoic chamber and reverberation chamber operators group meeting, Naval Surface Warfare Center, Dahlgren, VA; November 1992.

[5] Hill, D.A. Electronic mode stirring for reverberation chambers. IEEE Trans. Electromag. Compat., EMC-36:294-299; 1994.

[6] Crawford, M.L.; Loughry, T.A.; Hatfield, M.O.; Freyer, G.J. Band-limited, white gaussian noise excitation for reverberation chambers and applications to radiated susceptibility testing. Natl. Inst. Stand. Technol., Tech. Note 1375; 1996 January. 116 p. 
[7] Lehman, T.H. A statistical theory of electromagnetic fields in complex cavities. EMP Interaction Note 494, May 1993.

[8] Corona, P.; Ferrara, G.; Migliaccio, M. Reverberation chambers as sources of stochastic electromagnetic fields. IEEE Trans. Electromag. Compat., EMC-38:348-356; 1996.

[9] Page, J. Not shaken, not stirred. Approval:26-27; Nov./Dec. 1997.

[10] Hill, D.A.; Ma, M.T.; Ondrejka, A.R.; Riddle, B.F.; Crawford, M.T.; Johnk, R.T. Aperture excitation of electrically large, lossy cavities. IEEE Trans. Electromag. Compat. 36: 169-178; 1994.

[11] Dunn, J.M. Local, high frequency analysis of the fields in a mode-stirred chamber. IEEE Trans. Electromag. Compat., EMC-32:53-58; 1990.

[12] Bozorth, R. M. Ferromagnetism. Toronto: D. Van Nostrand Company, Inc. 1951.

[13] Otoshi, T.Y.; Franco, M.M. The electrical conductivities of steel and other candidate materials for shrouds in a beam-waveguide antenna system. IEEE Trans. Instrum. Meas. 45: 77-83; 1996.

[14] Papoulis, A. Probability, Random Variables, and Stochastic Processes, Second Edition. New York: McGraw-Hill Book Company; 1984.

[15] Kerns, D.M.; Beatty, R.W. Basic Theory of Waveguide Junctions and Introductory Microwave Network Analysis. Oxford: Pergamon Press Inc.; 1967.

[16] Zehna, Peter W. Probability distributions and statistics. Boston: Allyn and Bacon, Inc. 1970.

[17] Johnson, N.L.; Kotz, S. Continuous Univariate Distributions-1. New York: John Wiley and Sons; 1970.

[18] Page, J. Stirred mode reverberation chambers for EMC emission measurements and radio type approvals or organised chaos. IEE Intl. Conf. on Electromagnetic Compatibility: 313-320; 1994.

[19] Taylor, J.R. An Introduction to Error Analysis. Sausalito, CA: University Science Books; 1982.

[20] Fryer, G.J.; Hatfield, M.O.; Johnson, D.M.; Slocum, M.B. Comparison of measured and theoretical statistical parameters of complex cavities. IEEE Intl. Symp. on EMC, Santa Clara, CA; August 1996.

[21] Gradshteyn, I.S.; Rhyzhik, I.M.. Tables of Integrals, Series, and Products. New York: Academic Press; 1965.

[22] Kanda, M. Analytical and numerical techniques for anlyzing an electrically short dipole with a nonlinear load. IEEE Trans. Antennas Propag. AP-28(1): 71-78; 1980 January.

[23] Bensema, W.D.; Koepke, G.H.; Medley, H.W. Handbook for operation and maintenance of an NBS multisensor automated em field measurement system. Nat. Bur. Stand. (U.S.), Internal Report 86-3056; 1986 October. 70 p.

[24] Hewlett Packard HP 8510B network analyzer operating and programming manual. Manual No. 08510-90070. 1987.

[25] IEEE standard definitions of terms for antennas. IEEE Std. 145-1983.

[26] Pozar, D.M.; Kaufman, B. Comparison of three methods for the measurement of printed antenna efficiency. IEEE Trans. Antennas Propag. AP-36(1): 71-78; 1988 January. 
[27] Ladbury, J.M.; Johnk, R.T.; Ondrejka, A.R. Rapid evaluation of mode-stirred chambers using impulsive waveforms. Natl. Inst. Stand. Technol., Tech. Note 1381; 1996 June. $40 \mathrm{p}$.

[28] Wu, D.I.; Chang, D.C. The effect of an electrically large stirrer in a mode-stirred chamber. IEEE Trans. Electromag. Compat. 31: 164-169; 1989.

[29] Taylor, B.N.; Kuyatt, C.E. Guidelines for evaluating and expressing the uncertainty of NIST measurement results. Natl. Inst. Stand. Technol., Tech. Note 1297; 1994 September. $20 \mathrm{p}$. 




\section{NIST Technical Publications}

\section{Periodical}

Journal of Research of the National Institute of Standards and Technology-Reports NIST research and development in those disciplines of the physical and engineering sciences in which the Institute is active. These include physics, chemistry, engineering, mathematics, and computer sciences. Papers cover a broad range of subjects, with major emphasis on measurement methodology and the basic technology underlying standardization. Also included from time to time are survey articles on topics closely related to the Institute's technical and scientific programs. Issued six times a year.

\section{Nonperiodicals}

Monographs-Major contributions to the technical literature on various subjects related to the Institute's scientific and technical activities.

Handbooks-Recommended codes of engineering and industrial practice (including safety codes) developed in cooperation with interested industries, professional organizations, and regulatory bodies.

Special Publications-Include proceedings of conferences sponsored by NIST, NIST annual reports, and other special publications appropriate to this grouping such as wall charts, pocket cards, and bibliographies.

Applied Mathematics Series-Mathematical tables, manuals, and studies of special interest to physicists, engineers, chemists, biologists, mathematicians, computer programmers, and others engaged in scientific and technical work.

National Standard Reference Data Series-Provides quantitative data on the physical and chemical properties of materials, compiled from the world's literature and critically evaluated. Developed under a worldwide program coordinated by NIST under the authority of the National Standard Data Act (Public Law 90-396). NOTE: The Journal of Physical and Chemical Reference Data (JPCRD) is published bimonthly for NIST by the American Chemical Society (ACS) and the American Institute of Physics (AIP). Subscriptions, reprints, and supplements are available from ACS, 1155 Sixteenth St., NW, Washington, DC 20056.

Building Science Series-Disseminates technical information developed at the Institute on building materials, components, systems, and whole structures. The series presents research results, test methods, and performance criteria related to the structural and environmental functions and the durability and safety characteristics of building elements and systems.

Technical Notes-Studies or reports which are complete in themselves but restrictive in their treatment of a subject. Analogous to monographs but not so comprehensive in scope or definitive in treatment of the subject area. Often serve as a vehicle for final reports of work performed at NIST under the sponsorship of other government agencies.

Voluntary Product Standards-Developed under procedures published by the Department of Commerce in Part 10, Title 15, of the Code of Federal Regulations. The standards establish nationally recognized requirements for products, and provide all concerned interests with a basis for common understanding of the characteristics of the products. NIST administers this program in support of the efforts of privatesector standardizing organizations.

Consumer Information Series-Practical information, based on NIST research and experience, covering areas of interest to the consumer. Easily understandable language and illustrations provide useful background knowledge for shopping in today's technological marketplace.

Order the above NIST publications from: Superintendent of Documents, Government Printing Office, Washington, DC 20402.

Order the following NIST publications-FIPS and NISTIRs-from the National Technical Information Service, Springfield, VA 22161.

Federal Information Processing Standards Publications (FIPS PUB)-Publications in this series collectively constitute the Federal Information Processing Standards Register. The Register serves as the official source of information in the Federal Government regarding standards issued by NIST pursuant to the Federal Property and Administrative Services Act of 1949 as amended, Public Law 89-306 (79 Stat. 1127), and as implemented by Executive Order 11717 (38 FR 12315, dated May 11, 1973) and Part 6 of Title 15 CFR (Code of Federal Regulations).

NIST Interagency Reports (NISTIR)-A special series of interim or final reports on work performed by NIST for outside sponsors (both government and non-government). In general, initial distribution is handled by the sponsor; public distribution is by the National Technical Information Service, Springfield, VA 22161 , in paper copy or microfiche form. 
U.S. Department of Commerce

National Institute of Standards and Technology

325 Broadway

Boulder, Colorado 80303-3328

\section{Official Business}

Penalty for Private Use, $\$ \mathbf{3 0 0}$ 